\title{
Isolierung und Strukturaufklärung neuer Naturstoffe aus Bakterien und endophytischen Pilzen durch chemisches Screening
}

\author{
Dissertation \\ zur Erlangung des Doktorgrades \\ der Mathematisch-Naturwissenschaftlichen Fakultäten \\ der Georg-August-Universität zu Göttingen
}

vorgelegt von

Jens Bitzer

aus Ulm

Göttingen 2005 
D7

Referent: Prof. Dr. A. Zeeck

Korreferent: Prof. Dr. H. Laatsch

Tag der mündlichen Prüfung: 29.06.2005 
Die vorliegende Arbeit wurde im Institut für Organische und Biomolekulare Chemie der Universität Göttingen in der Zeit von April 2001 bis Mai 2005 unter Anleitung von Herrn Prof. Dr. A. Zeeck durchgeführt.

Herrn Prof. Dr. A. Zeeck danke ich für die interessante Aufgabenstellung und das entgegengebrachte Vertrauen. 



\section{Inhaltsverzeichnis}

\section{A. Theoretischer Teil}

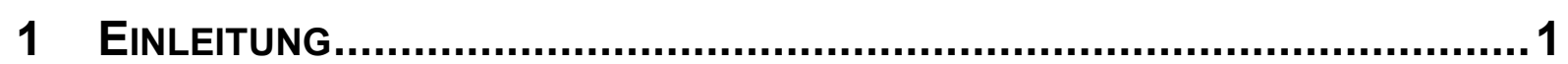

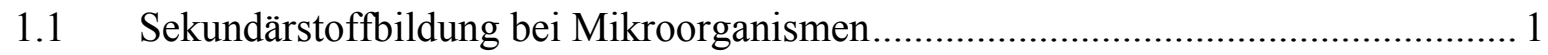

1.2 Endophytische Pilze als Quelle neuer Sekundärstoffe ............................................ 5

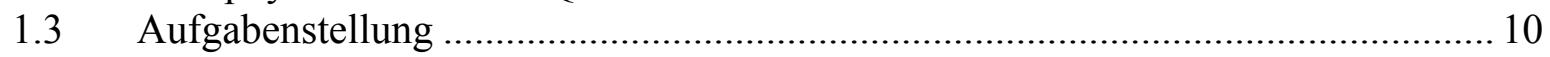

2 NeUe ACtinomycine Aus ACtINomyCES SP. CS210 ….....................11

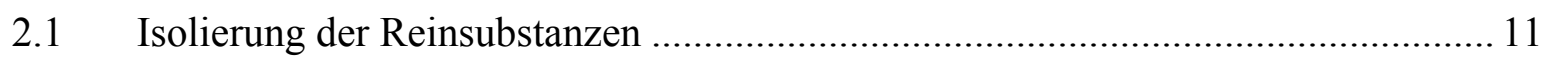

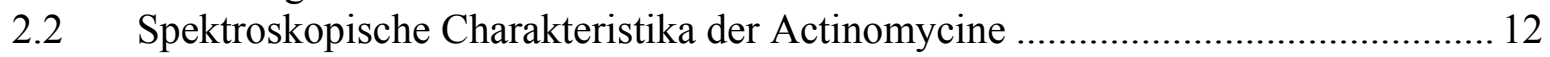

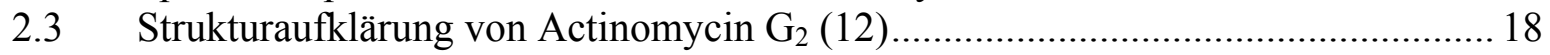

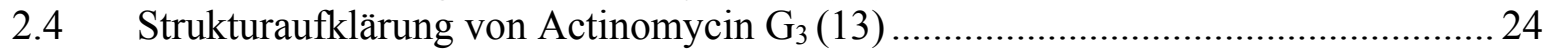

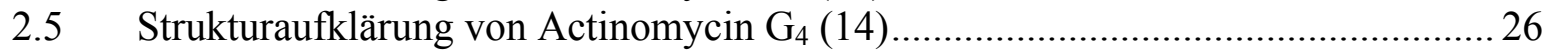

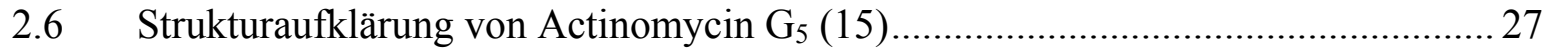

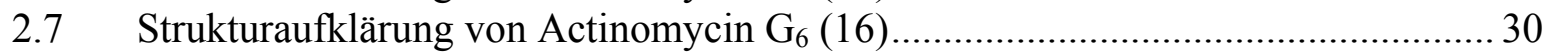

2.8 Weitere Sekundärmetaboliten aus Actinomyces sp. CS210 ................................. 34

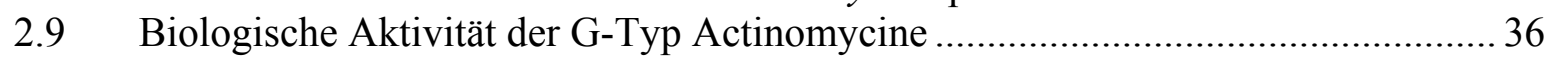

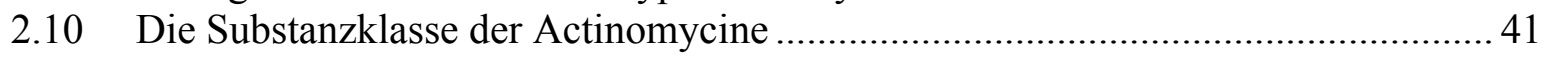

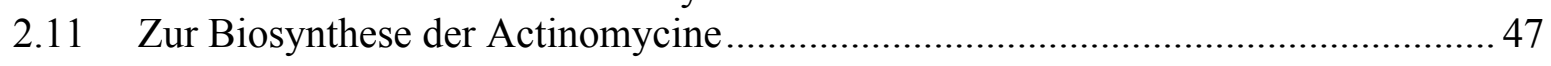

3 Neue Aminophenoxazone aus Halomonas Sp. Rk377a ..................50

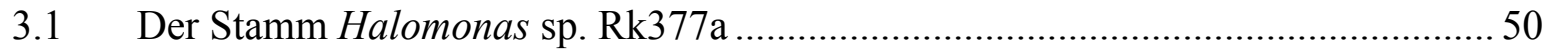

3.2 Neue Aminophenoxazon-Derivate durch veränderte Kultivierungsbedingungen ... 51

3.3 Biologische Aktivitäten der Aminophenoxazone ..................................................59

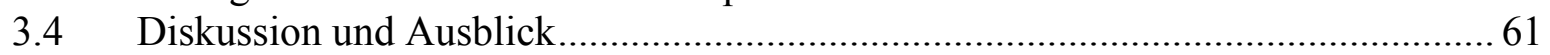

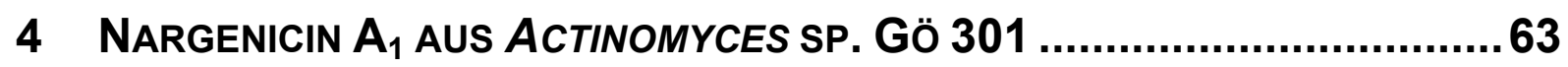

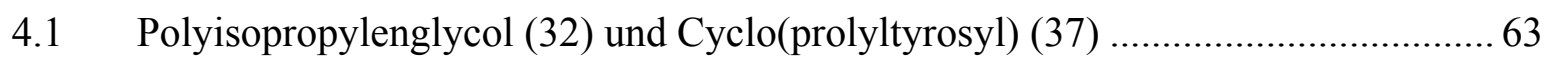

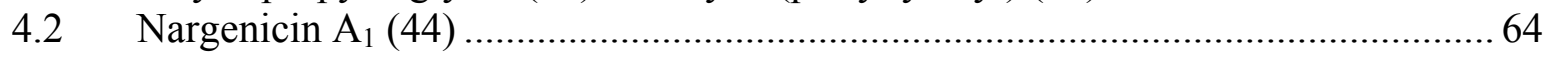

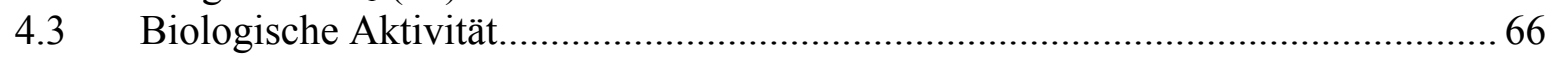

5 6-Desoxytalopyranoside AUs ActinomycES SP. GÖ M1 ................ 67

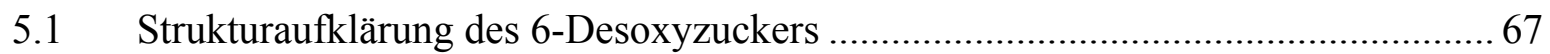

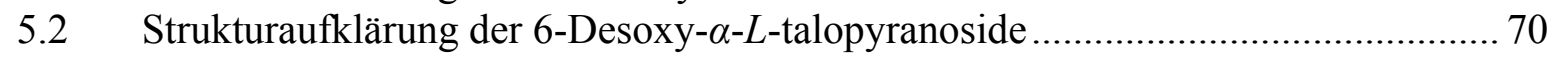

5.3 Vorläufer-dirigierte Biosynthese mit Actinomyces sp. Gö M1 ............................... 75

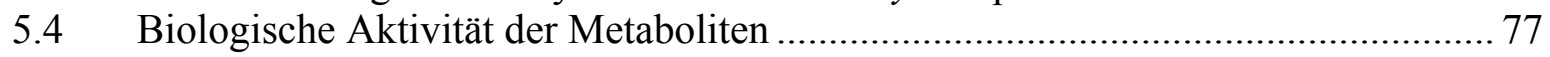

5.5 6-Desoxytalose - ein ungewöhnlicher Zuckerbaustein...................................... 78

5.6 Vergleich mit den bekannten 1-O-Acyl- $\alpha$ - $L$-rhamnopyranosiden ......................... 79

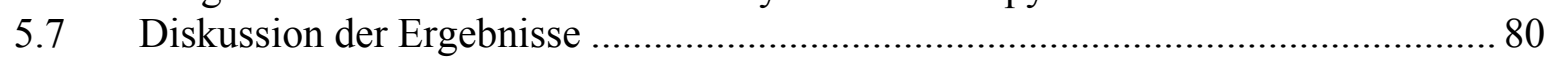

6 BMBF-Verbundprojekt „ENDOPhytische Pilze aus Algen UND PfLANZEN Verschiedener MEERESBIOTOPE“" 


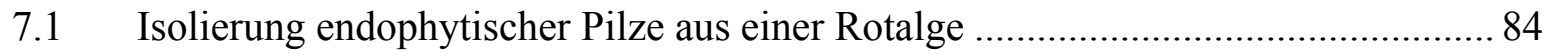

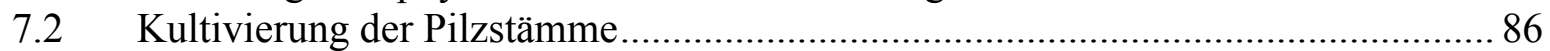

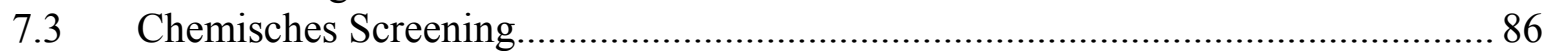

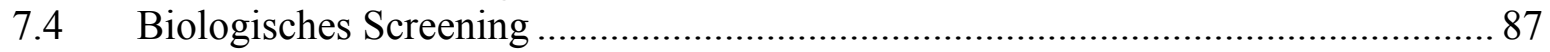

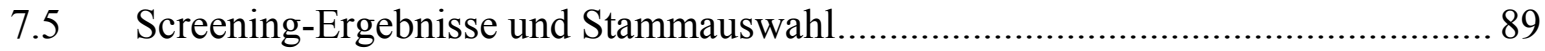

8 HäUfIG VORKOMMENDE MetABOLITEN ..........................................92

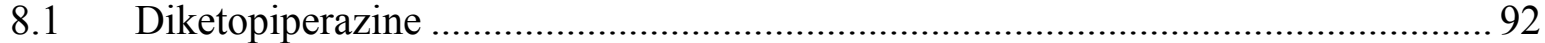

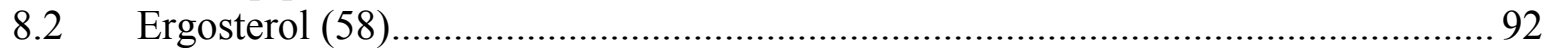

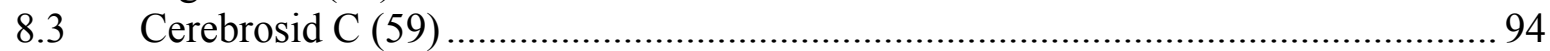

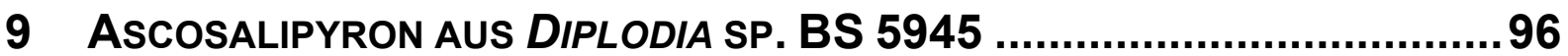

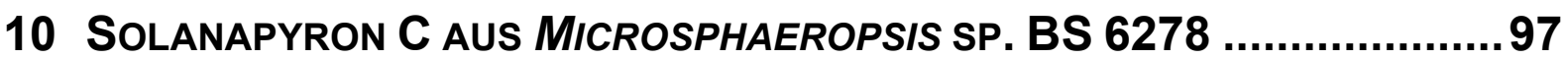

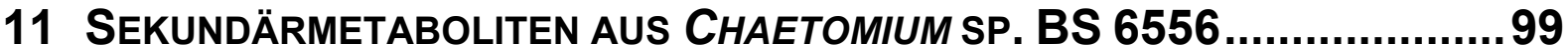

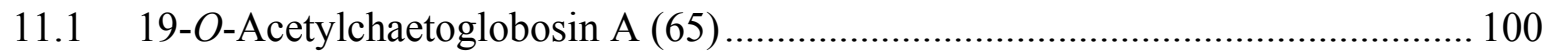

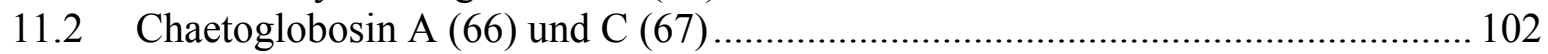

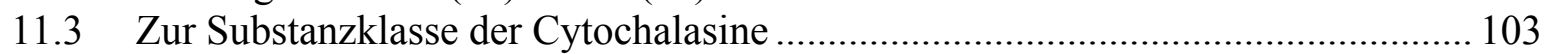

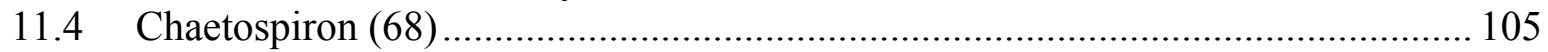

11.5 Biologische Aktivität der Sekundärmetaboliten ................................................ 113

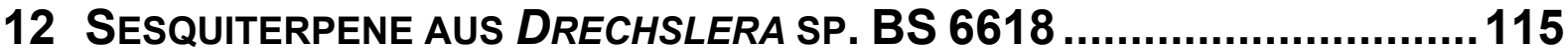

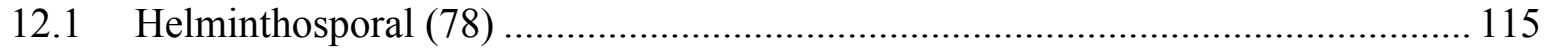

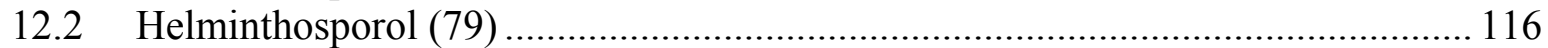

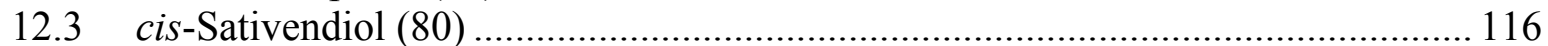

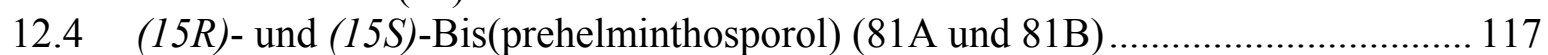

12.5 Zur biologischen Aktivität der Sesquiterpene................................................... 120

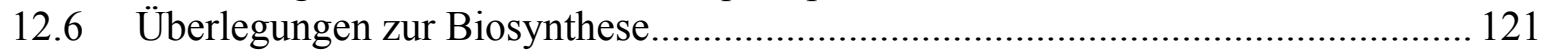

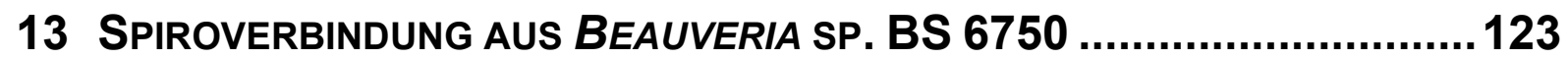

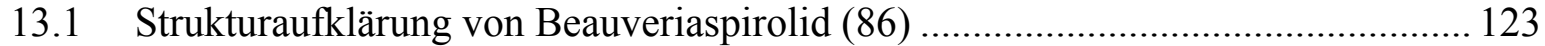

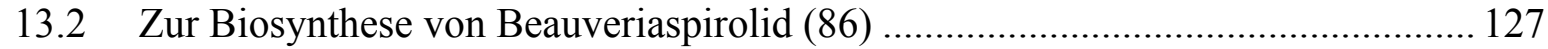

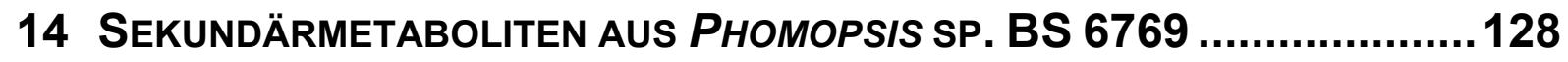

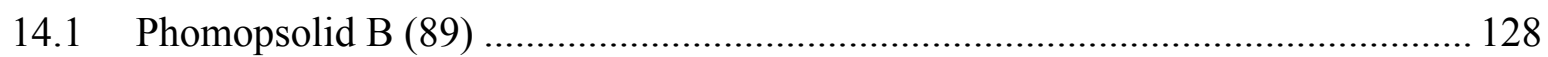

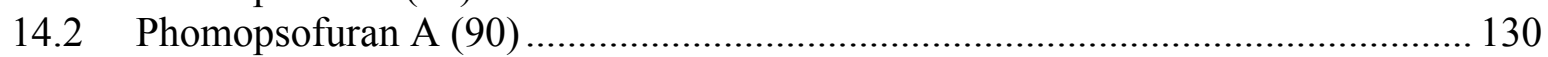

14.3 Zur Biosynthese von Phomopsolid B (89) und Phomopsofuran A (90) ................ 131

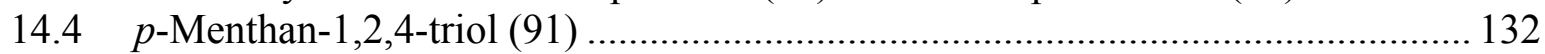

15 Biologische Aktivität der Pilzmetaboliten ...............................134

15.1 Biologische Aktivität der bekannten Substanzen ............................................... 134

15.2 Biologische Aktivität der neuen Sekundärmetaboliten ........................................ 135

\section{Diskussion deR im Screening mit endophytischen Pilzen eRZielten}

ERGEBNISSE ...................................................................... 137

17 ZUSAMMENFASSUNG DER ERGEBNISSE .......................................140

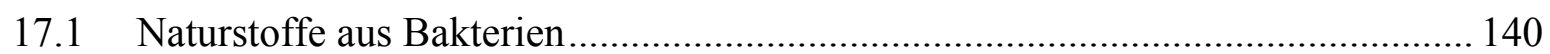

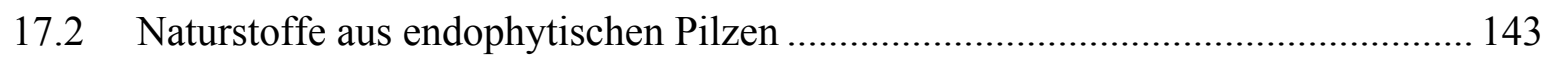




\section{B. Experimenteller Teil}

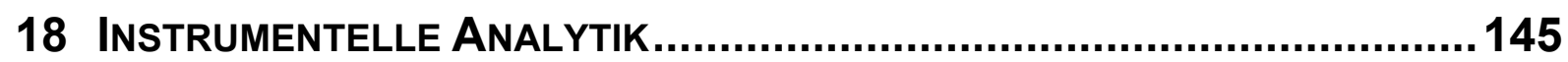

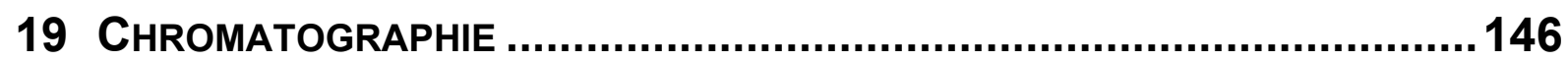

20 Mikrobiologische Methoden UNd GeRÄTE..................................150

21 Chemisches Screening von endophytischen Pilzen ....................153

22 Kultivierung, Aufarbeitung und Isolierung der Metaboliten... 154

22.1 Neue Actinomycine aus Actinomyces sp. CS210 ……........................................ 154

22.2 Neue Aminophenoxazone aus Halomonas sp. Rk377a ........................................ 155

22.3 6-Desoxy- $\alpha$-L-talopyranoside aus Actinomyces sp. Gö M1 .................................. 156

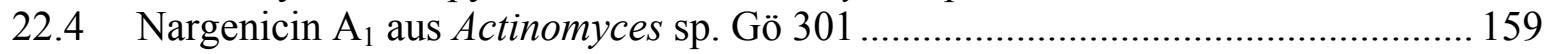

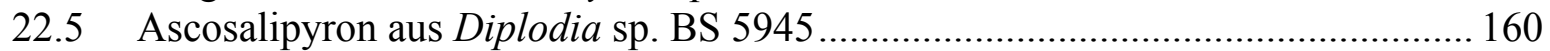

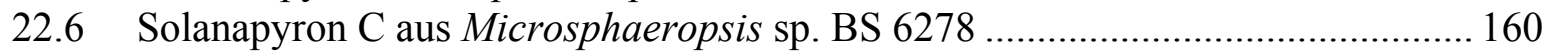

22.7 Sekundärmetaboliten aus Chaetomium sp. BS 6556........................................... 161

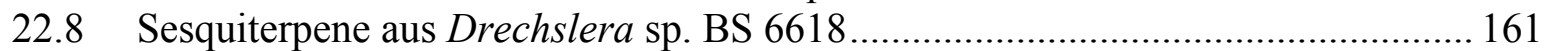

22.9 Spiroverbindung aus Beauveria sp. BS 6750 .................................................. 163

22.10 Sekundärmetaboliten aus Phomopsis sp. BS 6769............................................. 163

23 Charakterisierung der Metaboliten.......................................165

23.1 Substanzen aus Actinomyces sp. Stamm CS210 ……………………………..... 165

23.2 Substanzen aus Halomonas sp. Stamm Rk377a.................................................. 177

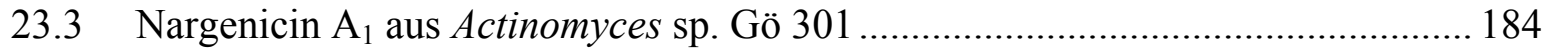

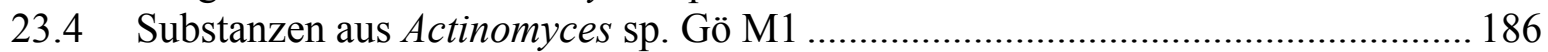

23.5 Häufig aus Pilzstämmen isolierte Metaboliten........................................................ 193

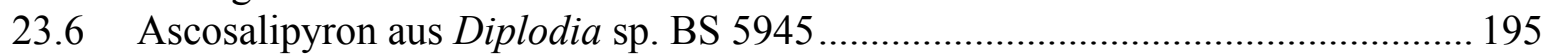

23.7 Solanapyron C aus Microsphaeropsis sp. BS 6278 ………………………….... 196

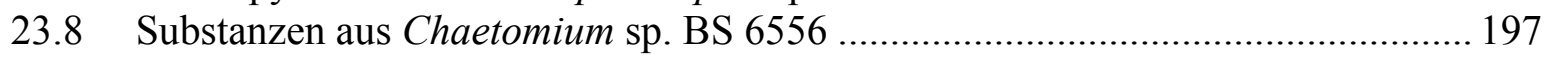

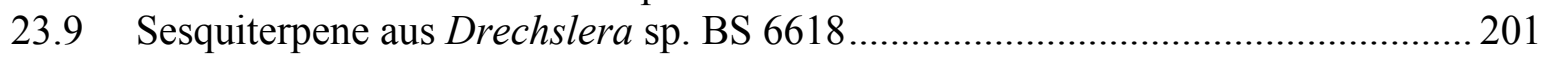

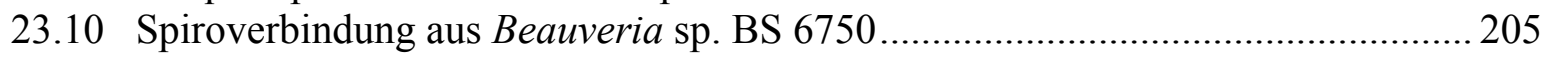

23.11 Sekundärmetaboliten aus Phomopsis sp. BS 6769............................................. 206

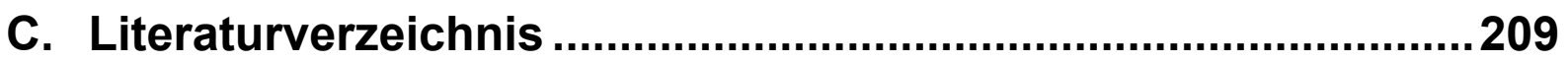





\section{A. Theoretischer Teil}

\section{$1 \quad$ Einleitung}

\subsection{Sekundärstoffbildung bei Mikroorganismen}

Bakterien und Pilze produzieren eine faszinierende Vielfalt an strukturell interessanten und biologisch aktiven Sekundärmetaboliten. Hierunter fallen Antibiotika, Immunsuppressiva, antitumorale und antivirale Wirkstoffe, Enzym-Inhibitoren, Rezeptor-Antagonisten und -Agonisten, Siderophore, Toxine, Pflanzenwachstums-Regulatoren, Insektizide und Herbizide sowie Signalstoffe. Den Sekundärstoffen gemeinsam ist, dass sie für das Wachstum und Überleben des jeweiligen Organismus nicht essentiell sind. Sie besitzen eine beschränkte Molekülgröße und sind meist das Produkt ungewöhnlich komplexer Biosynthesewege. Im Unterschied zu den Primärmetaboliten wie z. B. Aminosäuren oder Zuckern werden sie nur von bestimmten Spezies einer biologischen Gattung, und innerhalb dieser Spezies nur von bestimmten Subspezies oder Stämmen produziert ${ }^{[1,2]}$.

Über die Funktion der Sekundärmetaboliten für den Produzenten existieren verschiedene Theorien. Da es sich um hoch komplexe, genetisch wie energetisch aufwendige Stoffwechselprozesse handelt, muss die Biosynthese dieser Substanzen für den jeweiligen Organismus einen evolutionären Vorteil erbringen. Die Entwicklung dieser Fähigkeiten unter einem hohen Selektions- und Konkurrenzdruck wäre andernfalls nicht denkbar. Dies impliziert zugleich eine stets vorhandene biologische Wirkung bzw. Funktion dieser Substanzen ${ }^{[3]}$.

\section{Funktions- und evolutionsbasierte Erklärungsmodelle}

Die Verteidigungs-Hypothese geht davon aus, dass viele Sekundärstoffe zur chemischen Verteidigung gegen konkurrierende Organismen dienen. Diese Funktion liegt bei antibiotisch wirkenden Substanzen nahe und wird durch die Beobachtung unterstützt, dass Sekundärstoffe fast ausschließlich von Organismen produziert werden, die kein eigenes Immunsystem besitzen ${ }^{[4]}$. Als besonders produktiv haben sich neben Pflanzen vor allem solche Bakterienoder Pilzgattungen herausgestellt, die beispielsweise im Erdboden einem besonders hohen Selektionsdruck unterliegen ${ }^{[3]}$. So wurde in dem natürlichen Substrat verschiedener Basidiomyceten eine Antibiotika-Konzentration nachgewiesen, die konkurrierende Pilze zu hemmen vermochte $^{[5]}$. Deutlich weniger Sekundärstoffe sind hingegen von Organismen bekannt, die ökologische Nischen einnehmen wie z. B. Extremophile. Ein weiterer Hinweis auf eine Verteidigungs-Funktion ist das Auftreten von Resistenz-Genen auch in Mikroorganismen, die 
das entsprechende Antibiotikum überhaupt nicht bilden. Die hieraus folgende Immunität dieser Organismen gegen ansonsten letal wirkende Antibiotika gewährt ihnen einen Vorteil gegenüber Konkurrenten ${ }^{[6]}$. In dieses Bild passt die Beobachtung, dass die AntibiotikaProduktion einiger Mikroorganismen durch die Anwesenheit anderer Keime gesteigert wird. So erhöht der Ascomycet Oudemansiella mucida die Biosynthese des fungizid wirkenden Strobilurin A (1) um ein Vielfaches bei Zugabe von Sporen oder Myzel von Penicillium-

Stämmen zur Kultur ${ }^{[7]}$. Die Funktion einer<smiles>COC=C(C(=O)OC)/C(C)=C\C=C\c1ccccc1</smiles>
chemischen Verteidigung ist auch plausibel für die in der vorliegenden Arbeit beschriebenen Antibiotika wie die Actinomycine (Kapitel 2), Nargenicin $A_{1}$ (Kapitel 4) oder die Chaetoglobosine (Kapitel 11).

Gegen eine generelle Gültigkeit des VerteidigungsKonzepts spricht, dass viele Antibiotika in natürlicher Umgebung nicht in einer Konzentration vorliegen, die eine Wachstumshemmung anderer Keime bewirken könnte. Interessanterweise zeigen sie in subinhibitorischer Konzentration dennoch eine Wirkung auf Bakterien, indem sie die Transkription bestimmter Gene regulieren. So ist Erythromycin A (2, ein Inhibitor der ribosomalen Proteinsynthese) ebenso wie Rifampicin (3, ein RNA-Polymerase Inhibitor) in der Lage, in niedriger

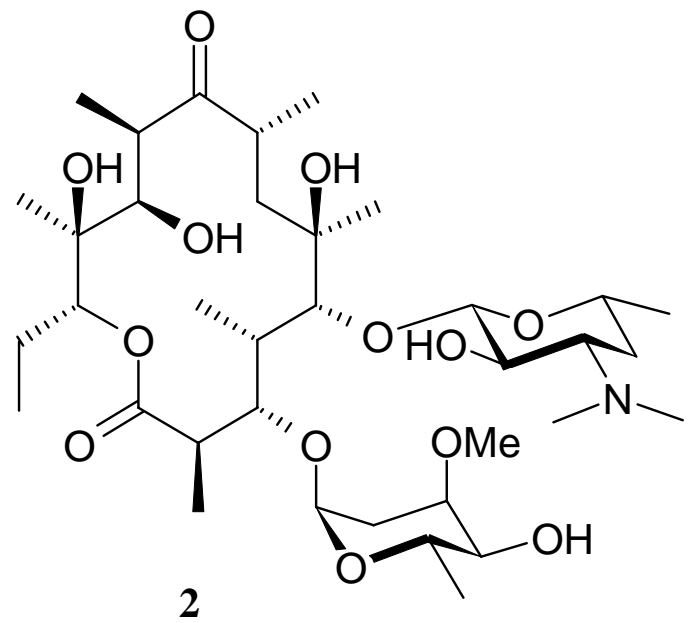<smiles></smiles>

3

Konzentration die Promotoren von Genen mit sehr unterschiedlicher Funktion zu aktivieren oder deaktivieren. Dies wurde anhand einer Klon-Bibliothek nachgewiesen, deren Stämme verschiedene Promotor-Gene vor einem lux-Operon enthielten. Die Regulierung der Transkription bei Zugabe der Antibiotika konnte so anhand der Lichtbildung beobachtet werden. Da resistente Stämme keine Reaktion auf das entsprechende Antibiotikum zeigten, 
dient vermutlich derselbe Wirkort sowohl der antibiotischen als auch der regulatorischen Aktivität ${ }^{[8]}$. Man könnte in diesem Zusammenhang von einer Kommunikations-Hypothese bezüglich der Funktion von Sekundärmetaboliten sprechen. Bevor dieser Effekt bei Antibiotika nachgewiesen wurde, waren bereits eine Reihe anderer Metaboliten als Autoinducer in Quorum-Sensing Systemen bekannt. Hierunter versteht man Signalstoffe, die biologische Funktionen steuern, wenn mikrobielle Populationen eine bestimmte Zelldichte erreichen $^{[9,10]}$. Unter anderem wird über Quorum-Sensing bei manchen Bakterien die Fähigkeit zur Aufnahme von Fremd-DNA gesteuert ${ }^{[11]}$. Die Produktion von Pflanzenwachstums-Wachstumsregulatoren wie Gibbellerinsäure (4, Seite 120) durch Pilze stützt die Kommunikations-Hypothese ebenso wie Wechselbeziehungen zwischen symbiotischen Mikroorganismen und ihrem Wirt. Auf die Beziehung zwischen endophytischen Pilzen und ihren Wirtspflanzen wird im nächsten Unterkapitel näher eingegangen.

Einen anderen Erklärungsansatz verwendet die so genannte Screening-Hypothese. Sie geht davon aus, dass eine starke und selektive biologische Aktivität für ein durch zufällige Mutation neu entstehendes Biosyntheseprodukt eine sehr außergewöhnliche und demnach unwahrscheinliche Eigenschaft ist. Um dennoch im Laufe der Evolution solche Leistungen entwickeln zu können, müssen die Organismen auch die Produktion von Metaboliten ohne einen direkten Nutzen akzeptieren. Im Gegenzug erhöht die Produktion möglichst diverser Naturstoffe die Wahrscheinlichkeit, durch Weiterentwicklung schließlich doch ein bioaktives Produkt zu erhalten. Damit würde die Anpassungs- und Überlebensfähigkeit dieses Organismus zwar kurzfristig durch den erhöhten Energieaufwand beeinträchtigt, auf lange Sicht jedoch gestärkt werden. Dieses Modell erklärt elegant die im Vergleich zum Primärstoffwechsel geringere Substrat- und Produktspezifität der Biosyntheseenzyme, da diese Eigenschaft eine möglichst breite Produktpalette begünstigt ${ }^{[12]}$. Auch das Entstehen neuer Strukturen wird erleichtert, was im Labor im Rahmen der kombinatorischen und Vorläufer-dirigierten Biosynthese ausgenutzt wird ${ }^{[13,14]}$. Die geringe Spezifität bestimmter Glykosyltransferasen wird auch im Kapitel 5 thematisiert (6-Desoxytalopyranoside aus Actinomyces sp. Gö M1, ab Seite 67) und zur Vorläufer-dirigierten Biosynthese genutzt. Die Screening-Hypothese sagt weiterhin eine Bevorzugung verzweigter Biosynthesewege gegenüber linearen sowie die mögliche Synthese mehrerer Produkte durch dasselbe Enzym voraus, da beides dem primären Ziel der Erzeugung chemischer Diversität dient. Dies kann beispielsweise bei der Biosynthese von Terpenoiden beobachtet werden ${ }^{[15]}$. So ermöglicht u. a. die Substrattoleranz der beteiligten Enzyme die Bildung der bis zu 400 flüchtigen Substanzen, die für den Duft von Rosenblüten verantwortlich sind ${ }^{[12]}$. 


\section{Erkenntnisse aus der Genomanalyse von Streptomyceten}

Bis heute wurden über 150 mikrobielle Genome sequenziert, darunter mit Streptomyces coelicolor $^{[16]}$ und Streptomyces avermitilis ${ }^{[17]}$ zwei bekannte Sekundärstoff-Produzenten. Eine Analyse dieser Daten kann alle drei erwähnten Hypothesen anteilig unterstützen. Auffällig ist eine unerwartet hohe Anzahl regulatorischer Gene, die sowohl der Anpassung an wechselnde Umweltbedingungen als auch der Kommunikation dienen könnten. Im Genom von S. coelicolor wurden 23 für Sekundärstoffe codierende Gencluster gefunden, obwohl zuvor nur sechs der Substanzen bekannt waren ${ }^{[18]}$. Bei S. avermitilis sind es sogar 30 Gencluster ${ }^{[19]}$. Es gibt also im Einklang mit der Screening-Hypothese eine große Zahl unterschiedlicher Biosynthese-Gene, die potentiell zu bioaktiven Naturstoffen führen können. Einige dieser Gene sind erhalten geblieben, obwohl sie keine funktionsfähigen Genprodukte hervorbringen oder nur unter spezifischen, bisher nicht bekannten Bedingungen transkribiert werden. Die Gencluster sind nicht gleichmäßig über das Genom verteilt, sondern treten gehäuft am Anfang und Ende des linearen Chromosoms auf (Abbildung 1), was eine erhöhte Variabilität implizieren könnte. Alle essentiellen Gene liegen hingegen im mittleren, hell gezeichneten Bereich. Der hohe Anteil der Gene für Sekundärstoffbildung am gesamten Genom von bis zu $6 \%$ kann nur durch einen signifikanten evolutionären Vorteil erklärt werden, den die Organismen hierdurch erhalten ${ }^{[16,19]}$.

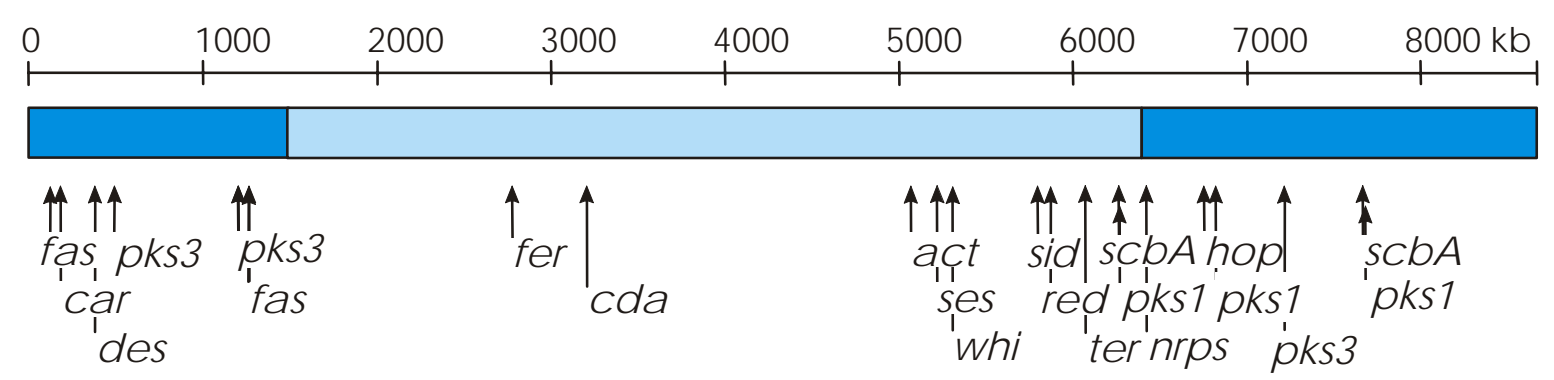

Abbildung 1: Verteilung der Sekundärstoff-Gene im Genom von Streptomyces coelicolor.

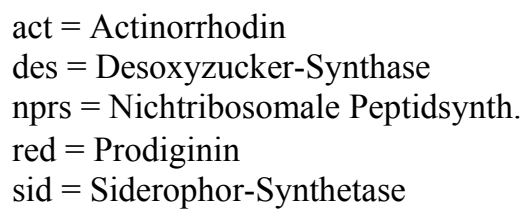




\subsection{Endophytische Pilze als Quelle neuer Sekundärstoffe}

Was sind endophytische Pilze?

Unter Endophyten versteht man Mikroorganismen, die im Inneren von Pflanzen leben, ohne dass diese hierdurch einen sofortigen, erkennbaren Schaden erleiden ${ }^{[20]}$. Diese Definition legt die Beziehung zwischen Pflanze und Mikroorganismus nicht fest, sie kann von symbiotisch über saprophytisch bis hin zu pathogen reichen. In den interzellularen Räumen aller Pflanzen finden sich sowohl Bakterien als auch Pilze ${ }^{[21]}$.

Über die Anzahl der existierenden endophytischen Pilzgattungen gibt es keine genauen Angaben, Schätzungen gehen von bis zu einer Million aus. In einer Pflanzenart werden an unterschiedlichen Standorten häufig dieselben Pilzarten gefunden, was ein Anzeichen für eine spezifische Interaktion zwischen Wirtspflanze und Endophyt sein könnte. Eine generelle Korrelation auf Gattungs-Ebene ist bisher jedoch nur selten möglich. Einige Gattungen wie Fusarium, Pestalotiopsis oder Phomopsis werden besonders häufig in Pflanzen gefunden ${ }^{[21]}$.

\section{Produktion von Wirkstoffen als Anpassung an ein Habitat}

Viele Endophyten produzieren bioaktive Naturstoffe, die ihnen zur Interaktion mit der Wirtspflanze oder zur Verteidigung ihrer ökologischen Nische dienen können. Möglicherweise verschaffen sie auch dem Wirt Vorteile, indem sie ihn vor pathogenen Erregern, Insekten oder anderen Schädlingen schützen ${ }^{[7,21]}$.

Im Hinblick auf diese Funktionen schlagen Strobel, DAisy, CASTILlo und HARPer vier Kriterien für eine rationale Pflanzenauswahl vor, deren Endophyten besonders produktiv sein sollten $^{[21,22]}$.

\section{1) Pflanzen aus ungewöhnlichen Habitaten}

Bei der Entwicklung besonderer Anpassungs- und Überlebensstrategien könnten auch endophytisch lebende Mikroorganismen einen Beitrag geleistet haben. Dies lässt auf einen besonderen Sekundärstoffwechsel und damit die Möglichkeit schließen, neuartige bioaktive Substanzen zu finden. In diese Kategorie fallen auch die in der vorliegenden Arbeit untersuchten Endophyten aus marinen Habitaten.

\section{2) Endemische Pflanzen}

Mit derselben Argumentation wie unter Punkt 1 werden bei diesen oft erdgeschichtlich alten Pflanzen interessante Sekundärstoffbildner erwartet. 


\section{3) Pflanzen mit bekannter biologischer Wirkung}

Von vielen Pflanzen ist eine biologische Wirkung bekannt, die für medizinische oder andere Zwecke ausgenutzt wird. STROBEL ET AL. sprechen hier von Pflanzen mit ethnobotanischer Geschichte. Für ihre biologische Aktivität ist manchmal nicht die Pflanze selbst verantwortlich, sondern von Epi- oder Endophyten produzierte Sekundärstoffe. Die Isolierung und Kultivierung der mikrobiellen WirkstoffProduzenten sollte das Auffinden neuer bioaktiver Substanzen ermöglichen und die Substanzbeschaffung von zuvor nur aus Pflanzen bekannten Metaboliten vereinfachen.

\section{4) Pflanzen aus Habitaten mit besonders hoher Biodiversität}

Eine hohe biologische Diversität impliziert eine ebenfalls hohe chemische Diversität, da nur diese das Überleben einer Spezies unter einem besonders hohen Konkurrenzdruck sichert. Diese These steht im Einklang mit den in der Verteidigungs- und Screening-Hypothese geäußerten Annahmen über die evolutionäre Funktion der Sekundärmetaboliten. Tatsächlich konnte eine Studie mehr biologisch aktive Naturstoffe in Endophyten nachweisen, die aus Pflanzen des tropischen Regenwalds isoliert wurden, im Vergleich zu solchen, die aus Pflanzen der gemäßigten Klimazonen stammten.

\section{Fungizide Wirkstoffe aus endophytischen Pilzen}

Die toxische Pflanze Tripterygeum wilfordii wird in der chinesischen Medizin verwendet, ihre Auswahl zur Untersuchung von Endophyten entspricht dem obigen Kriterium 3. Aus ihr wurde der endophytische Pilzstamm Cryptosporiopsis cf. quercina isoliert ${ }^{[23]}$, der mit dem Lipopeptid Cryptocandin und dem Tetramsäure-Derivat Cryptocin (5) zwei neue, hochpotente Fungizide produziert $^{[24]}$. Neben der ökologischen Funktion (Schutz der Wirtspflanze vor phytopathogenen Pilzen) wird hier ein häufig bei Sekundärstoff-Produzenten anzutreffendes

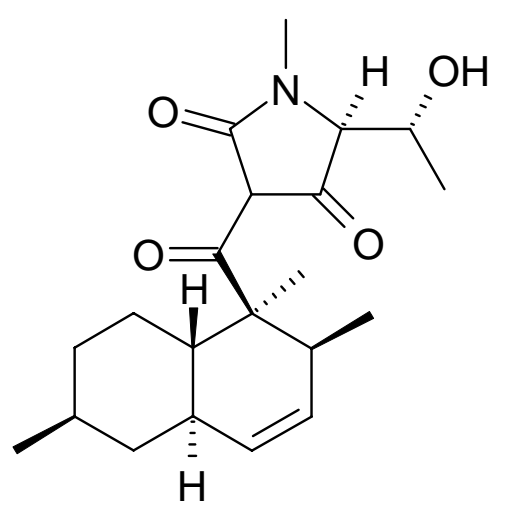

5 Prinzip sichtbar: das synergistische Zusammenwirken mehrere Substanzen. Dieses ist auch bei dem bereits erwähnten Actinomyceten Streptomyces coelicolor zu erkennen, der mehrere verschiedene Antibiotika produziert. HoPwOOD und CHALLIS sehen in einem solchen Synergismus eine generelle Triebkraft für die Evolution von Sekundärmetaboliten ${ }^{[18]}$. 


\section{Cytotoxische Substanzen aus endophytischen Pilzen}

Ein weiteres Beispiel für einen Naturstoff aus einem Endophyten, der in einer biologisch wirksamen Pflanze vorkommt, ist das bekannte Antitumor-Mittel Paclitaxel (6, Taxol $\left.{ }^{\circledR}\right)$. Es wurde erstmals 1971 aus der Rinde der pazifischen Eibe (Taxus brevifolia) isoliert, wird jedoch von allen bekannten Eiben-Arten gebildet. 1993 fand man heraus, dass auch der aus T. brevifolia isolierte endophytische Pilz Taxomyces andreanae 6 produziert. Seither wurden zahlreiche weitere endophytische und nicht-endophytische Pilzstämme gefunden, die Taxane bilden $^{[25]}$. Der Nutzen für die Wirtspflanzen liegt in der fungiziden Wirkung von Paclitaxel (6), die besonders stark gegenüber phytopathogenen Pilzen wie Phytophthora sp. ausgeprägt ist $^{[21]}$. Interessant ist, dass<smiles>CC(=O)O[C@H]1C(=O)[C@]2(C)[C@@H](O)C[C@@H]3OC[C@]3(OC(C)=O)[C@H]2[C@@H](OC(=O)c2ccccc2)[C@]2(O)C[C@@H](OC(=O)[C@H](O)[C@H](NC(=O)c3ccccc3)c3ccccc3)C(C)=C1C2(C)C</smiles>
dieser Metabolit sowohl von der Pflanze selbst als auch dem Endophyten biosynthetisiert wird. Aufgrund der weiten Verbreitung Paclitaxelproduzierender Stämme im Pilzreich nimmt man an, dass dort der evolutionäre Ursprung der Biosynthese-Gene liegt, die letztlich auch auf die Pflanzen der Gattung Taxus übertragen wurden ${ }^{[26]}$.

Die in Südamerika beheimateten Büsche Baccharis coridifolia und Baccharis artemisiodis sind dort häufig Ursache für Vergiftungen von Weidetieren. Ihre toxische Wirkung geht auf den Gehalt an Roridinen sowie Verrucarin A (7) und B zurück, bei denen es sich um zur Familie der Trichothecene gehörende Mykotoxine handelt. Sie hemmen bereits in

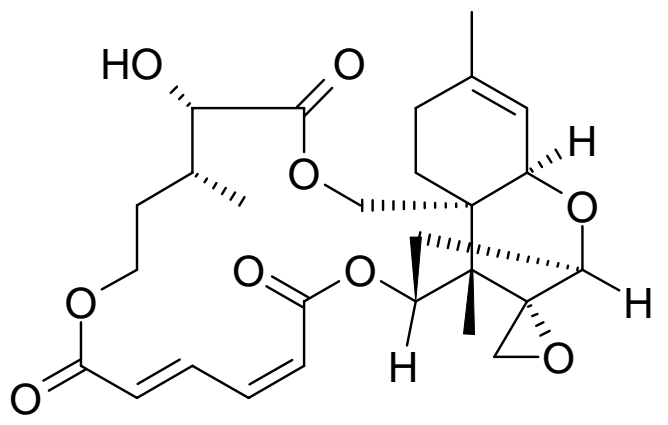
7 nanomolaren Konzentrationen die Proteinbiosynthese von Säugetierzellen. B. coridifolia und B.artemisiodis produzieren die Trichothecene nicht selbst, ihr Gehalt in den Pflanzen ist vielmehr auf Pilze zurückzuführen. Endophyten der Spezies Ceratopicnidium baccharidicola bilden die Rodirine und Verrucarine und konnten in beiden 
Pflanzenarten nachgewiesen werden. Zusätzlich erfolgt auch eine Aufnahme der Toxine aus dem Boden, wo sie von in der Umgebung der Pflanzen lebenden Pilzen gebildet werden. Die Mykotoxine bieten der Pflanze einen umfassenden Schutz sowohl gegen pathogene Pilze und Bakterien als auch gegen Fraßfeinde ${ }^{[27]}$.

\section{Insektizide und nematozide Wirkstoffe aus endophytischen Pilzen}

Eine Schutzfunktion für ihre Wirtspflanze erfüllen auch endophytische Pilze, deren Metaboliten gegen Insekten und Parasiten schützen. So produziert ein aus der tropischen Pflanze Bontia daphnoides isolierter Pilz der Gattung Nodulisporium ein insektizid wirksames Indol-Diterpen. Die Nodulisporic Acid A (8) genannte Substanz blockiert selektiv Glutamatgesteuerte Chlorid-Ionenkanäle von Invertebraten und ist besonders stark wirksam gegen Fliegenlarven $^{[28]}$. Die Wirtspflanze entstammte in diesem Fall einem Habitat mit besonders großer Biodiversität. Entsprechend der zuvor erläuterten Erwartung erhöht der mikrobielle Endophyt durch seine Sekundärstoffproduktion die Durchsetzungsfähigkeit und Überlebenschancen der Pflanze in einer kompetitiven Umgebung.

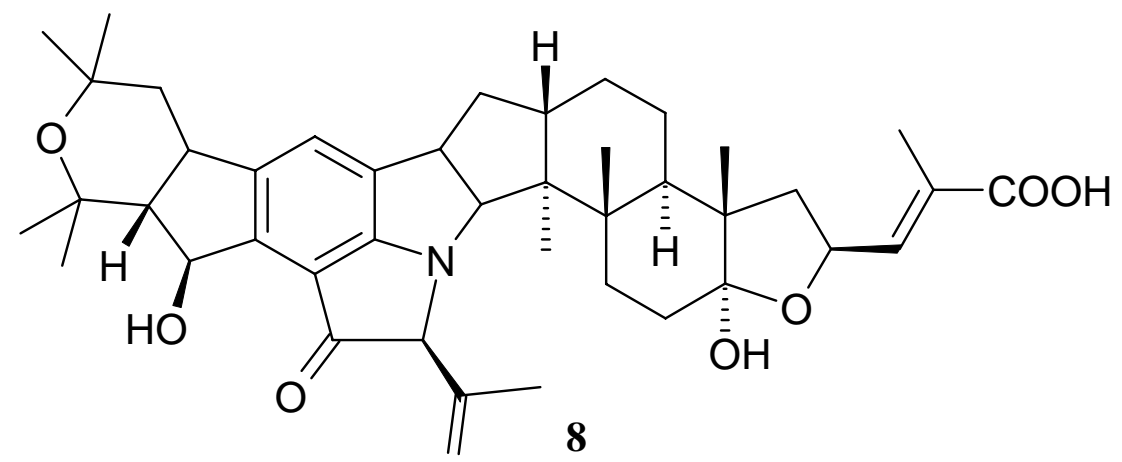

Es gibt auch Beispiele für Endophyten mit nematozider Aktivität. Bei einem biologischen Screening von 500 Pilzstämmen aus Pflanzen unterschiedlicher Habitate wurden 17 nematozid aktive Isolate gefunden. Die Mehrzahl der aktiven Stämme gehörte zur Gattung Phomopsis, allen gemeinsam war die Produktion von 3-Hydroxypropionsäure. Diese wirkt nematozid gegen den Pflanzenparasiten Meloidogyne incognita ( $\left.\mathrm{LD}_{50}=15 \mu \mathrm{g} / \mathrm{L}\right)$, ihre Aktivität gegen den saprophytisch lebenden Fadenwurm Caenorhabditis elegans ist fünffach geringer. Die besonders starke Wirkung gegen den Pflanzen-Schädling sowie die unterschiedliche Herkunft der Produzenten lassen hier ein weit verbreitetes biologisches Prinzip vermuten, mit dem endophytische Pilze der Wirtspflanze einen biologischen Vorteil verschaffen $^{[29]}$. 


\section{Entzündungshemmende Substanzen aus endophytischen Pilzen}

Eine weitere Pflanze, deren biologische Wirksamkeit teilweise auf einen endophytischen Pilz zurückgeführt werden kann, ist der südamerikanische Korallenstrauch (Erythina crista-galli). Die Pflanze ist stark neurotoxisch und wirkt in hoher Konzentration ähnlich wie Curare, bei niedriger Dosierung stehen jedoch medizinisch nutzbare entzündungshemmende Eigenschaften im Vordergrund. Aus einem hieraus isolierten endophytischen Pilzstamm der Gattung Phomopsis wurde das neue Antibiotikum Phomol (9) erhalten, das neben<smiles>CCCCCC(OC(=O)CC(O)C(O)C=CC(C)O)C(O)OC(=O)C(C)=CC(C)CC</smiles>

9 antibakteriellen, fungiziden und schwach cytotoxischen Eigenschaften eine starke entzündungshemmende Wirkung besitzt ${ }^{[30]}$. Es erscheint plausibel, dass 9 für die pharmakologische Wirkung des Pflanzenextrakts mit verantwortlich ist, was die zuvor geäußerte These 3 zur Auswahl viel versprechender Wirtspflanzen unterstützt. 


\subsection{Aufgabenstellung}

Ziel dieser Arbeit war das Auffinden und Isolieren neuer mikrobieller Sekundärmetaboliten mit interessanter chemischer Struktur und biologischer Aktivität. An die Strukturaufklärung und spektroskopische Charakterisierung der Naturstoffe sollte sich deren breite biologische Testung anschließen.

Als Quelle für neue Naturstoffe dienten einerseits im chemischen Screening aufgefallene Actinomyceten, andererseits im Rahmen eines BMBF-geförderten Projekts endophytische Pilze aus unterschiedlichen Habitaten. Neben aus Algen isolierten Pilzstämmen sollten auch Endophyten aus terrestrischen Pflanzen sowie Pflanzen der Brackwasser- und Küstenregion untersucht werden.

Um das Biosynthesepotential der Pilzstämme zu erschließen, war eine umfangreiche Variation der Kultivierungsbedingungen geplant. Die Extrakte sollten sowohl einem chemischen als auch einem biologischen Screening unterzogen werden, um eine Auswahl produktiver Stämme und geeigneter Kultivierungsbedingungen treffen zu können. Das Testung hinsichtlich einer für den Pflanzenschutz interessanten biologischen Aktivität wurde von der BASF A. G. als Kooperationspartner durchgeführt, die Isolierung endophytischer Pilze vom Arbeitskreis AUST/SCHULZ an der TU Braunschweig (siehe Projektbeschreibung ab Seite 82).

Bei dem im Verlauf der vorliegenden Arbeit aufgefallenen Produzenten der neuartigen 6-Desoxytalopyranoside, Actinomyces sp. Gö M1, sollte durch Vorläufer-dirigierte Biosynthese die Ausbeute der Glykoside erhöht und das Metabolitenspektrum erweitert werden.

Im Rahmen einer Kooperation mit dem Arbeitskreis LANG (TU Braunschweig) wurde der Extrakt des aus der Nordsee isolierten Bakteriums Halomonas sp. Rk377a im Hinblick auf Sekundärmetaboliten untersucht, deren Produktion bei Zugabe von Anthranilsäure zum Kulturmedium erfolgte. Hierbei waren eine starke Zunahme der Melaninbildung sowie eine orange Färbung des Extrakts aufgefallen. 


\section{Neue Actinomycine aus Actinomyces sp. CS210}

Der Stamm Actinomyces sp. CS210 fiel im chemischen Screening durch eine grüne Färbung der Kulturbrühe auf, auch die Dünnschichtchromatogramme von Kulturfiltrat- und Myzelextrakt zeigten neben mehreren gelben Zonen einen grünen $\operatorname{Spot}^{\mathrm{a}}$. Die chromatographische Auftrennung der Extrakte ergab, dass die grüne Farbe aus der Mischung einer blauen Substanz (10) mit mehreren orangen Substanzen (12 - 17) resultierte.

Anhand ihres charakteristischen UV-Spektrums und der hohen Molmasse wurden 12 - 16 als neue Actinomycine identifiziert, deren Strukturaufklärung durch hochauflösende Massenspektrometrie und NMR-Spektroskopie gelang. Die Struktur des blauen Farbstoffs 10 konnte aufgrund der sehr geringen Substanzmenge nicht bestimmt werden. Vermutlich handelt es sich ebenfalls um eine nicht in der Literatur beschriebene Verbindung, da es zu ihrer Molmasse von $651 \mathrm{~g} / \mathrm{mol}$ keinen passenden Eintrag in den Datenbanken gab. Zusätzlich wurden die bereits bekannten Metaboliten 1-Phenazinol (17) und N-(2-Hydroxyphenyl)acetamid (18) isoliert.

\subsection{Isolierung der Reinsubstanzen}

Für die Isolierung der Actinomycine erwies sich die Kombination aus Chromatographie an Kieselgel, Sephadex LH-20 und Nucleodur RP-18 in der angegebenen Reihenfolge als optimal. Nach einer groben Abtrennung sehr polarer (Zucker etc.) und unpolarer (Fettsäuren etc.) Bestandteile des Extrakts durch Chromatographie an Kieselgel erfolgte die Anreicherung der höhermolekularen Actinomycine mittels Gelchromatographie. Die Trennung in die Einzelkomponenten gelang schließlich durch semipräparative RP-HPLC (siehe S. 154, Experimenteller Teil).

Wichtig bei der Chromatographie ist die Verwendung säurefreier Lösungsmittel, da Actinomycine unter sauren Bedingungen rasch umgewandelt werden. Besonders leicht findet ein Austausch der Aminogruppe des Chromophors gegen eine Hydroxygruppe statt ${ }^{[31,32]}$, bei längerer Reaktionszeit oder höherer Säurekonzentration kommt es zur Hydrolyse von Amidbindungen sowie Umwandlungen des Chromophors ${ }^{[33]}$.

\footnotetext{
${ }^{\text {a }}$ Frau Dr. V. Gesheva (Institute of Microbiology, Bulgarian Academy of Science, Sofia) danke ich für die Überlassung des Stammes und Frau M. Klingebiel (Institut für Organische und Biomolekulare Chemie, Universität Göttingen) für die Durchführung des chemischen Screenings.
} 


\subsection{Spektroskopische Charakteristika der Actinomycine}

Alle Actinomycine haben einen Actinoyl-Chromophor und unterscheiden sich in der Zusammensetzung der Pentapeptidlactone. Charakteristische Absorptionsmaxima ermöglichen die Zuordnung zur Substanzklasse anhand der UV-Spektren. Einen raschen Überblick über die Aminosäure-Zusammensetzung und das Vorliegen der nativen Konformation (A-Konformation ${ }^{\mathrm{b},[34]}$ ) erhält man aus den ${ }^{1} \mathrm{H}$ - und ${ }^{13} \mathrm{C}-\mathrm{NMR}-$ Spektren.

Abbildung 2 zeigt als Beispiel die Struktur von Actinomycin $G_{2}$ (12), dessen Strukturaufklärung in Kapitel 2.3 ab Seite 18 beschrieben wird.

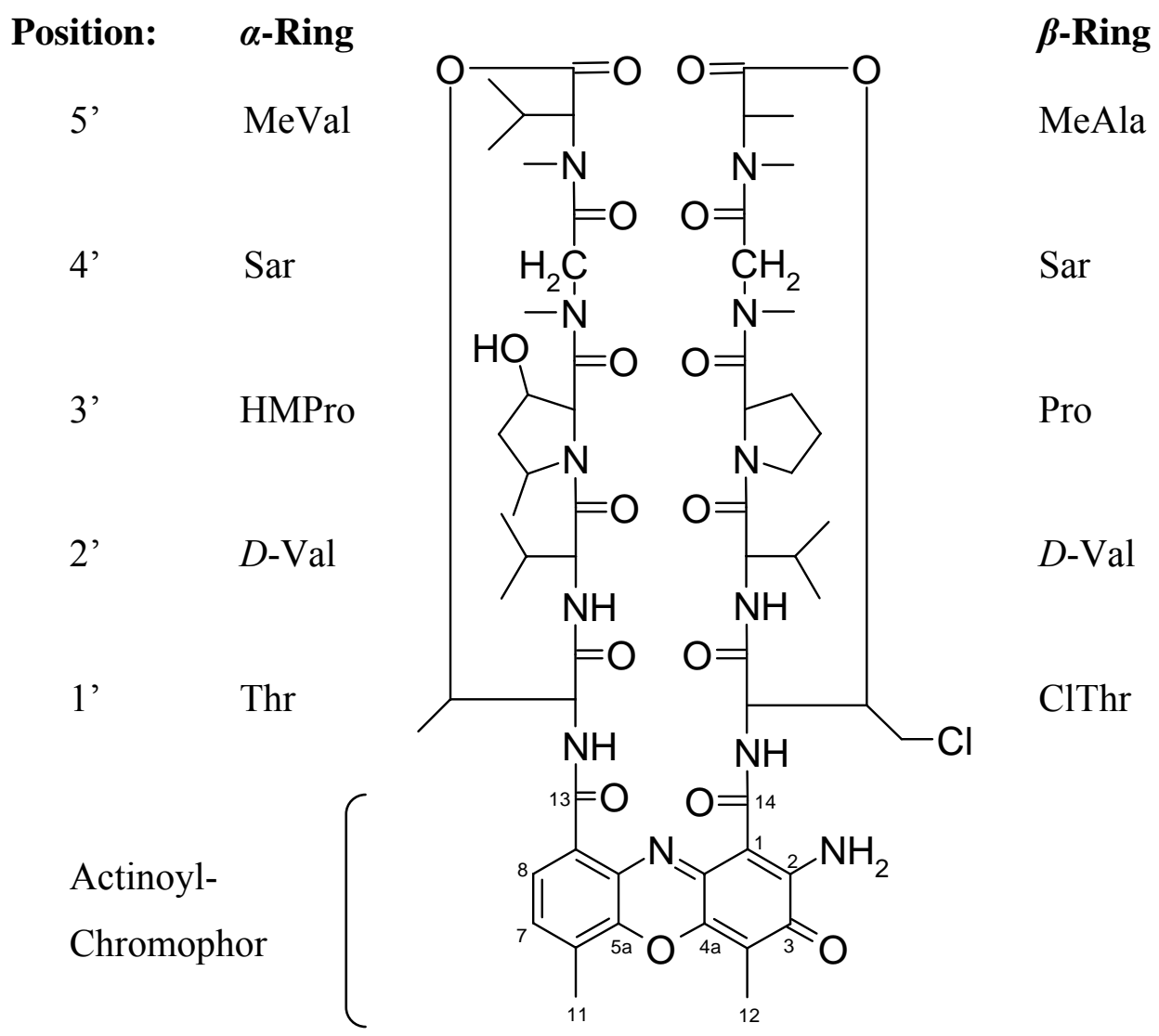

Abbildung 2: Struktur von Actinomycin $\mathrm{G}_{2}$ (12) mit Bezifferung der Aminosäuren.

Nicht-proteinogene Aminosäuren: $\mathrm{MeVal}=N$-Methylvalin, MeAla $=N$-Methylalanin,

HMPro = trans-3-Hydroxy-cis-5-methylprolin, $D$-Val = D-Valin, ClThr $=4$-Chlorthreonin

\footnotetext{
${ }^{\mathrm{b}}$ Die Bezeichnung „A-Konformation“ leitet sich von der Konformation des isolierten Pentapeptidlacton-Rings in Aceton $\mathrm{ab}$ und beinhaltet von MeVal ausgehend eine cis-cis-trans-trans-Konfiguration der Amidbindungen. In Chloroform („C-Konformation“) kommt es zur Ausbildung eines all-trans verknüpften Rings. Beim vollständigen Actinomycin-Molekül liegt unabhängig vom Lösungsmittel eine A-Konformation der Ringe vor, da sie durch interannulare Wasserstoffbrückenbindungen stabilisiert wird.
} 


\subsubsection{UV-Spektrum}

Das charakteristische UV-Spektrum eignet sich gut zur Identifizierung der Actinomycine und ermöglicht in HPLC-DAD-Chromatogrammen die einfache Zuordnung von Peaks zu dieser Substanzklasse. Das UV-Spektrum von Actinomycinen mit intaktem Chromophor zeigt starke Absorptionsmaxima bei 240, 426 und $440 \mathrm{~nm}$.

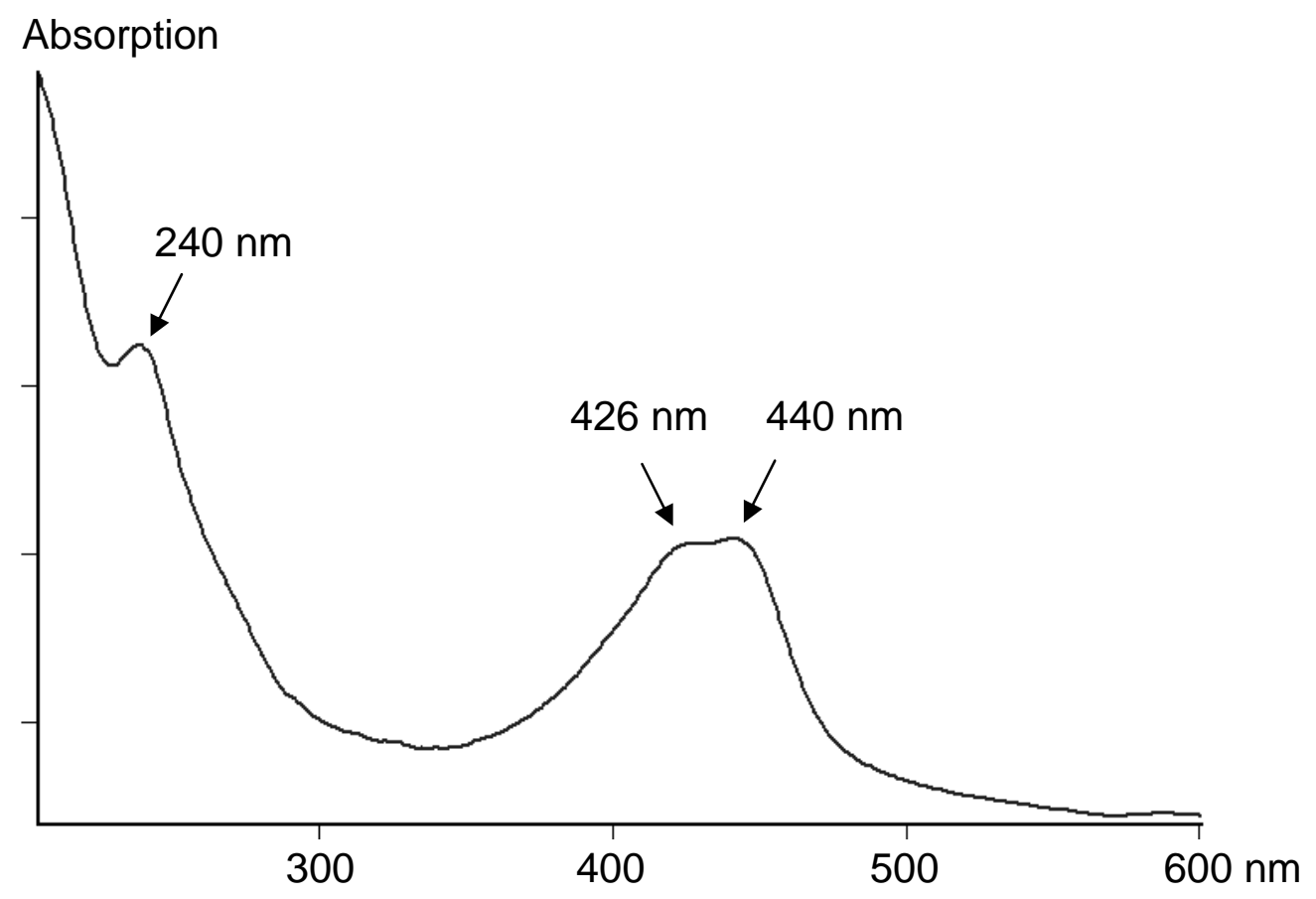

Abbildung 3: UV-Spektrum von Actinomycin $\mathrm{G}_{2}(\mathbf{1 2})$.

\subsection{2 ${ }^{1} \mathrm{H}-\mathrm{NMR}-$ Spektrum}

Die ${ }^{1}$ H-NMR-Spektren der Actinomycine weisen eine Reihe typischer Signale auf, die unmittelbar Rückschlüsse auf vorkommende Aminosäuren und die vorliegende Konformation erlauben:

- Chromophor

Die Methylgruppen zeigen eine Resonanz bei $\delta_{\mathrm{H}} \approx 2.1$ und 2.5, die Signale der beiden aromatischen Protonen an C-7 und C-8 sind bei $\delta_{\mathrm{H}} \approx 7.2-7.5 \mathrm{zu}$ Dubletts aufgespalten $\left({ }^{3} J_{\mathrm{HH}}=8 \mathrm{~Hz}\right)$. Diese Signale sind einfach zuzuordnen, erlauben jedoch keine detaillierte Aussage über Veränderungen am Chromophor. Mehr Informationen über den Chromophor lassen sich dem ${ }^{13} \mathrm{C}-\mathrm{NMR}-$ Spektrum entnehmen. 
- Amidprotonen und N-Methylgruppen

Vier der Amidbindungen besitzen Wasserstoffatome, weitere vier tragen Methylgruppen. Beide sind für die Strukturaufklärung von besonderem Wert, da sie im HMBC-Spektrum neben den $\alpha$-Wasserstoffatomen Auskunft über die Verknüpfung der Aminosäuren geben können. Die Amidprotonen liegen im Bereich zwischen $\delta_{H}=6.8$ und 8.3 und sind durch Kopplung mit $\mathrm{H}-\alpha$ zum Dublett aufgespalten. Bei Verwendung von $\mathrm{CDCl}_{3}$ als Lösungsmittel sind alle vier Amidprotonen zu sehen, in Methanol tauschen die NH-Gruppen der beiden Threoninreste (Position 1') rasch aus. Die amidischen Protonen von $D$-Valin (Position 2') sind hingegen für das Lösungsmittel weniger gut zugänglich. Sie bilden antiparallele Wasserstoffbrückenbindungen zur Carbonylgruppe des Valins am jeweils anderen Ring aus ${ }^{[35]}$ und bleiben daher auch in protischen Lösungsmitteln sichtbar.

- 2-H von Prolin

Das Signal des Wasserstoffatoms an C-2 der Proline (Position 3') ist stark ins Hochfeld verschoben $\left(\delta_{\mathrm{H}} \approx 5.8-6.2\right)$. Ursache hierfür ist die räumliche Nähe zur Carbonylgruppe von Threonin an Position 1' und deren magnetische Anisotropie. Bei nicht modifiziertem Prolin spaltet das Signal zum Dublett auf $\left({ }^{3} J_{\mathrm{HH}}=9 \mathrm{~Hz}\right)$. Ursache hierfür ist ein Diederwinkel nahe $90^{\circ}$ zum (pro-S)-Proton der benachbarten Methylengruppe. Der Betrag der Kopplungskonstanten ist hierdurch so gering, dass sie nicht sichtbar ist. Bei trans3-Hydroxy-cis-5-methylprolin (HMPro) fehlt die Kopplung zum (pro-R)-Proton, wodurch ein Singulett für 2-H zu sehen ist.

- 2-H und 3-H von Threonin

Das Proton in $\alpha$-Position erscheint bei $\delta_{\mathrm{H}} \approx 4.4-4.6$ als Dublett $\left({ }^{3} J_{\mathrm{HH}}=3 \mathrm{~Hz}\right)$ in Methanol, in $\mathrm{CDCl}_{3}$ kommt die $7 \mathrm{~Hz}$ große Kopplung zum Amidproton hinzu. Bei hydroxyliertem oder chloriertem Threonin erfolgt eine Tieffeldverschiebung dieses Signals zu $\delta_{\mathrm{H}} \approx 5.2-5.4$. In diesem Bereich liegt auch das Proton an C-3 der Seitenkette, dessen komplexere Signalaufspaltung es von 2-H unterscheidet. Die hohe chemische Verschiebung erklärt sich hier durch die Anisotropie der Ester-Carbonylgruppe, die in räumlicher Nachbarschaft liegt.

- 2- $\mathrm{H}_{2}$ von Sarkosin

Die diastereotopen Protonen der Methylengruppen haben eine auffällig hohe geminale Kopplungskonstante von ${ }^{2} J_{\mathrm{HH}}=18 \mathrm{~Hz}$, sofern der Pentapeptidlactonring in der Standard- 
konformation vorliegt (A-Konformation ${ }^{\mathrm{c}}$ ). Hier liegt eine cis-Amidbindung zwischen Sarkosin und Prolin vor. Kommt es bei einer veränderten Konformation zu einer transAmidbindung, so sinkt der Betrag der Kopplungskonstante auf ${ }^{2} J_{\mathrm{HH}}=14.5 \mathrm{~Hz}$.

Diese und weitere auffällige Signale wie die Methylgruppen von Valin und Alanin sind in Abbildung 3 am Beispiel des ${ }^{1} \mathrm{H}-\mathrm{NMR}-$ Spektrums von Actinomycin $\mathrm{G}_{2}$ (12) gezeigt.

\subsection{3 ${ }^{13}$ C-NMR-Spektrum}

Durch Vergleich der chemischen Verschiebungen im ${ }^{13} \mathrm{C}-\mathrm{NMR}-\mathrm{Spektrum}$ mit bekannten Daten lassen sich Veränderungen des Chromophors nachweisen. Das Auftreten von Prolinresten mit Oxofunktion kann durch die Resonanz der Carbonylgruppe bei $\delta_{\mathrm{C}} \approx 210$ erkannt werden. Weitere typische Signale im ${ }^{13} \mathrm{C}-\mathrm{NMR}-$ Spektrum von Actinomycin $\mathrm{G}_{2}(\mathbf{1 2})$ siehe Abbildung 5.

${ }^{\mathrm{c}}$ Zur Bezeichnung A-Konformation siehe Fußnote auf Seite 12. 


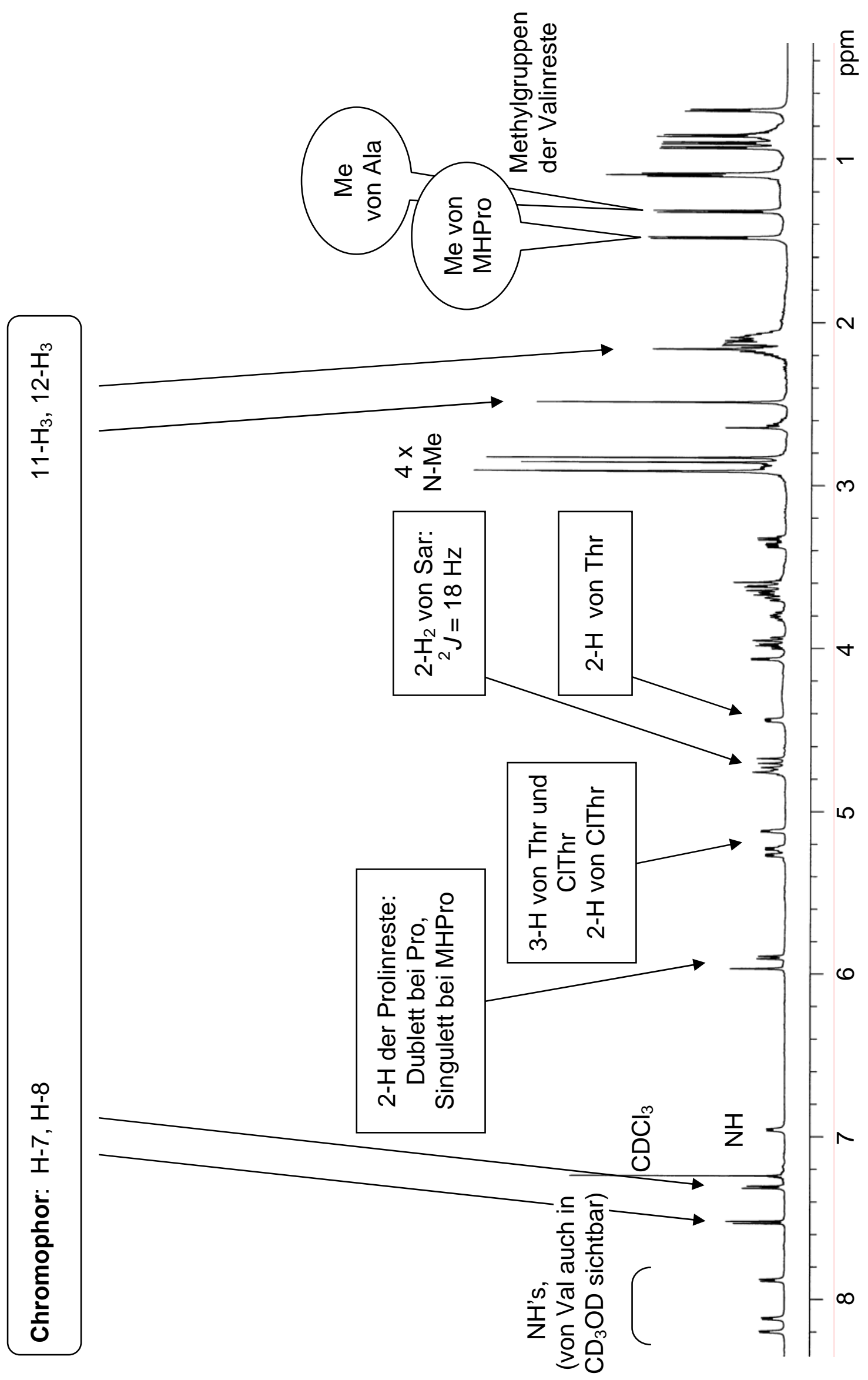

Abbildung 4: Charakteristische Signale im ${ }^{1} \mathrm{H}-\mathrm{NMR}-$ Spektrum von Actinomycin $\mathrm{G}_{2}(\mathbf{1 2})$ in $\mathrm{CDCl}_{3}(600 \mathrm{MHz})$. 


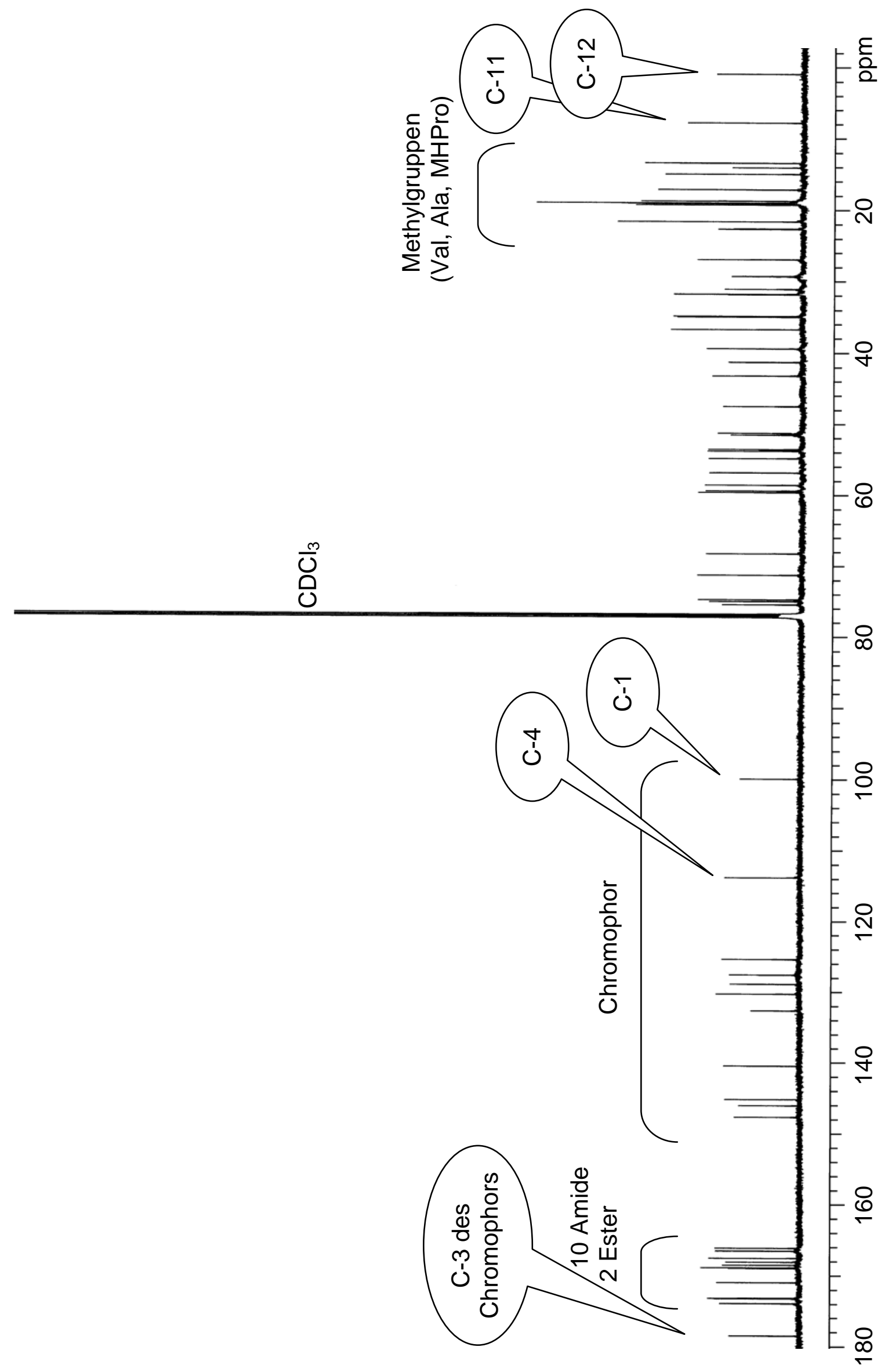

Abbildung 5: Charakteristische Signale im ${ }^{13} \mathrm{C}-\mathrm{NMR}-$ Spektrum von Actinomycin $\mathrm{G}_{2}(\mathbf{1 2})$ in $\mathrm{CDCl}_{3}(150.8 \mathrm{MHz})$. 


\subsection{Strukturaufklärung von Actinomycin $G_{2}(12)$}

\section{Summenformel}

Durch hochauflösende ESI-Massenspektrometrie lässt sich bei den Actinomycinen die Summenformel eindeutig ermitteln, wenn man bei der Berechnung die mögliche Anzahl der Stickstoffatome auf 5 bis 15 beschränkt. Für Actinomycin $G_{2}$ (12) wurde die nominale Molmasse 1290 und die Summenformel $\mathrm{C}_{61} \mathrm{H}_{83} \mathrm{~N}_{12} \mathrm{O}_{17} \mathrm{Cl}$ erhalten, das Vorhandensein eines Chloratoms ergibt sich auch aus dem Isotopenmuster (siehe Abbildung 6).
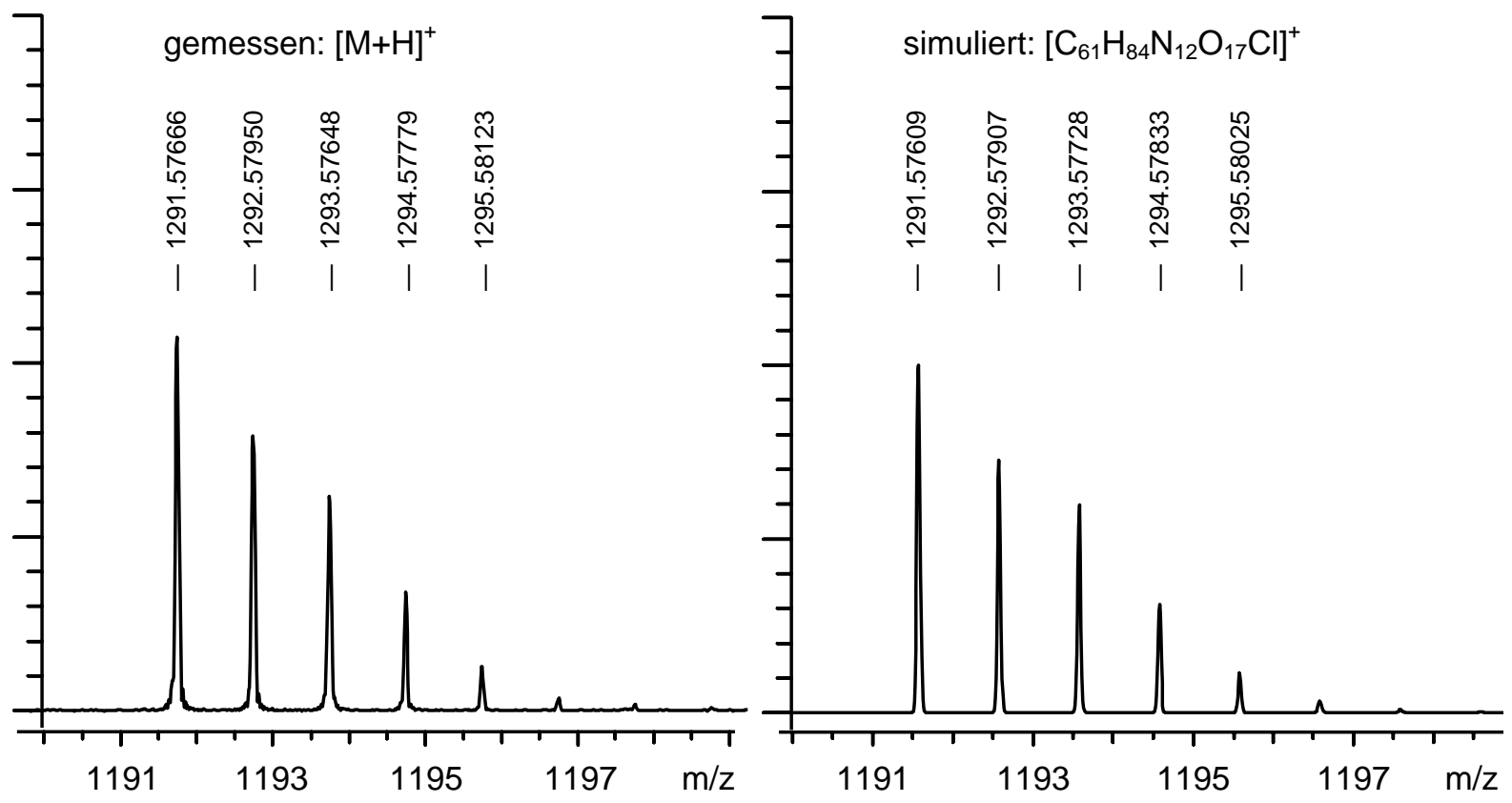

Abbildung 6: Gemessenes (links) und berechnetes (rechts) Isotopenmuster von 12 im ESIMassenspektrum.

\section{Chromophor}

Das ${ }^{13}$ C-NMR-Spektrum von 12 zeigt Signale für 12 Carbonylgruppen, das zusätzliche Signal bei $\delta_{\mathrm{C}}=178.5$ stammt von der Ketogruppe an C-3 des Chromophors. Ein Vergleich der elf aromatischen Signale des Chromophors zwischen $\delta_{C}=100-150$ mit Literaturwerten $^{[36]}$ beweist, dass der Actinoyl-Chromophor intakt ist und als 2-Amino-4,6-dimethyl-3-oxo-3Hphenoxazin-1,9-dicarbonsäure-Derivat vorliegt. Die Zuordnung der Kohlenstoffatome gelingt anhand des HMBC-Spektrums, das auch die Unterscheidung von C-4a und C-10a erlaubt, die 
eine sehr ähnliche chemische Verschiebung haben (Abbildung 7). Offen bleibt zunächst die chemische Verschiebung der Carbonylgruppe C-14, diese wird durch eine HMBC-Kopplung mit 2-H der gebundenen Aminosäure festgelegt.

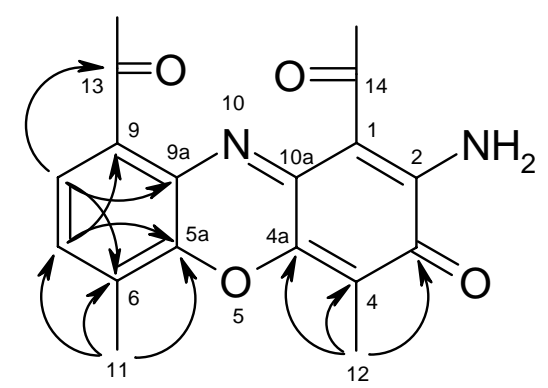

Abbildung 7: HMBC-Kopplungen im Chromophor von Actinomycin $\mathrm{G}_{2}$ (12).

\section{Aminosäuren}

Nach Korrelation der Signale des ${ }^{1} \mathrm{H}-\mathrm{NMR}$-Spektrums mit den ${ }^{13} \mathrm{C}$-chemischen Verschiebungen durch ein HSQC-Spektrum gelingt die Zuordnung der Signale der zehn einzelnen Aminosäuren größtenteils durch die Auswertung des COSY-Spektrums, da innerhalb der Aminosäuren außer der Carbonylgruppe keine quartären Kohlenstoffatome vorkommen. Unvollständig bleibt die Zuordnung der Prolinringe, da die Kreuzsignale des COSY-Spektrums hier teils sehr schwach ausfallen. Mittels eines HMBC-Spektrums lassen sich auch diese Resonanzen eindeutig den jeweiligen Positionen im Prolinring zuweisen (Abbildung 9). Die Zuordnung der Protonen am Stickstoff ist ebenfalls anhand des COSYSpektrums möglich, da sie vicinale Kopplungen von $6-7 \mathrm{~Hz}$ mit 2-H zeigen. Die $N$-Methylgruppen koppeln nicht mit anderen Protonen, im HMBC jedoch mit dem C-2 der jeweiligen Aminosäure.

Die Bestimmung des Amid-Kohlenstoffatoms kann bei einigen Aminosäuren anhand der im HMBC sichtbaren ${ }^{3} J_{\mathrm{CH}}-$ Kopplung von $3-\mathrm{H}$ auf C-1 geschehen. Im Falle des Fehlens der Seitenkette (Sarkosin) oder des Fehlens der erwähnten Korrelation im HMBC (Valin, 4-Chlorthreonin, 3-Hydroxy-5-methylprolin) muss auf die ${ }^{2} J_{\mathrm{CH}}-\mathrm{Kopplung}$ von 2-H auf C-1 zurückgegriffen werden. Die Protonen in dieser Position zeigen jedoch zusätzlich eine interresiduale ${ }^{3} J_{\mathrm{CH}}$-Kopplung zur Carboxylgruppe der nächsten Aminosäure. Die Unterscheidung zwischen der ${ }^{2} J_{\mathrm{CH}}$ - (intraresidual) und der ${ }^{3} J_{\mathrm{CH}}$-Kopplung (interresidual) gelingt im Fall von Sarkosin durch den Vergleich mit der HMBC-Korrelation der $N$-Methylgruppe, da diese nur zur Carboxylgruppe der nächsten Aminosäure geht. Bei den anderen 
Aminosäuren hilft die Kombination mit zuvor ermittelten Informationen (wenn eines der beiden Kohlenstoffatome bereits einer anderen Aminosäure zugeordnet wurde) sowie die HMBC-Korrelationen der Amidprotonen: ${ }^{2} J_{\mathrm{CH}}$ (interresidual) ist deutlich größer als ${ }^{3} J_{\mathrm{CH}}$ (intraresidual).

Neben den von Actinomycin D (19) bekannten Aminosäuren $N$-Methylvalin (MeVal), Sarkosin (Sar, 2x vorkommend), Prolin (Pro), Valin (Val, 2x vorkommend) und Threonin (Thr) ergibt die Analyse der NMR-Spektren von Actinomycin $G_{2}$ (12) drei veränderte Untereinheiten:

N-Methylalanin (MeAla): Eine im Vergleich zu Valin zu tieferem Feld verschobene Methylgruppe bei $\delta_{\mathrm{H}}=1.32$ koppelt mit einer zum Quartett aufgespaltenen Methingruppe $\left(\delta_{\mathrm{H}}=3.33\right)$. Sie gehören zu einem Alaninrest, dessen $N$-Methylgruppe das HMBC-Spektrum anzeigt.

trans-3-Hydroxy-cis-5-methyl-prolin (HMPro): Eine weitere Methylgruppe mit Resonanz bei relativ tiefem Feld gehört zu einem modifizierten Prolinring. Dieser ist an Position drei zusätzlich hydroxyliert, was durch die chemischen Verschiebungen deutlich wird $\left(\delta_{\mathrm{H}}=4.06\right.$, $\left.\delta_{\mathrm{C}}=75\right)$. Da das H-Atom in $\alpha$-Position als Singulett erscheint, muss der Diederwinkel $\mathrm{H}-\mathrm{C}(2)-\mathrm{C}(3)-\mathrm{H}$ nahe $90^{\circ}$ liegen. Die Größe der vicinalen Kopplungskonstante, beschrieben durch die Karpluskurve, ist hier minimal ${ }^{[37]}$. Die Konfiguration der Hydroxygruppe an C-3 ist folglich trans zur Amidgruppe an C-2 (Abbildung 8). Die cis-Konfiguration der Methylgruppe ergibt sich aus dem NOESY-Spektrum, das eine räumliche Nähe von 3-H und der Methylgruppe an C-5 anzeigt. Dies ist nur möglich, wenn sie auf derselben Seite des Rings stehen. Der zwischen 5-H und 2-H auftretende NOE bestätigt die Konfiguration.

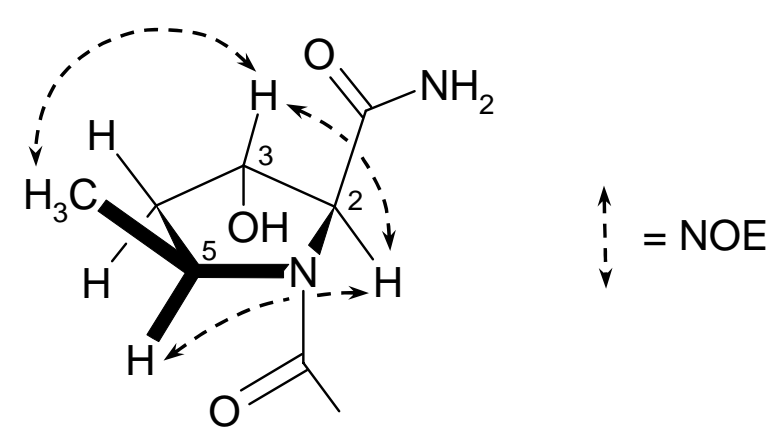

Abbildung 8: Konfiguration des 3-Hydroxy-5-methylprolins (HMPro). 
4-Chlorthreonin (ClThr): Das ${ }^{1} \mathrm{H}-\mathrm{NMR}-S p e k t r u m$ von 12 enthält keine Methylgruppe eines zweiten Threonins, stattdessen tritt eine zusätzliche Methylengruppe bei $\delta_{H}=3.94 / 3.99$ auf. Die chemische Verschiebung des dazugehörigen Kohlenstoffatoms ist mit $\delta_{\mathrm{C}}=43$ niedriger als für die Bindung an ein Sauerstoffatom zu erwarten wäre. Sie zeigt die Position des Chloratoms an, dessen Vorhandensein sich aus der Summenformel ergibt.

Alle drei ungewöhnlichen Aminosäuren sind von den Actinomycinen des Z-Typs ${ }^{[36]}$ sowie von Actinomycin $G_{1}{ }^{d,[38]}$ (11) bekannt. Ein Vergleich der NMR-Daten zeigt weitgehende Übereinstimmung mit den Literaturwerten.
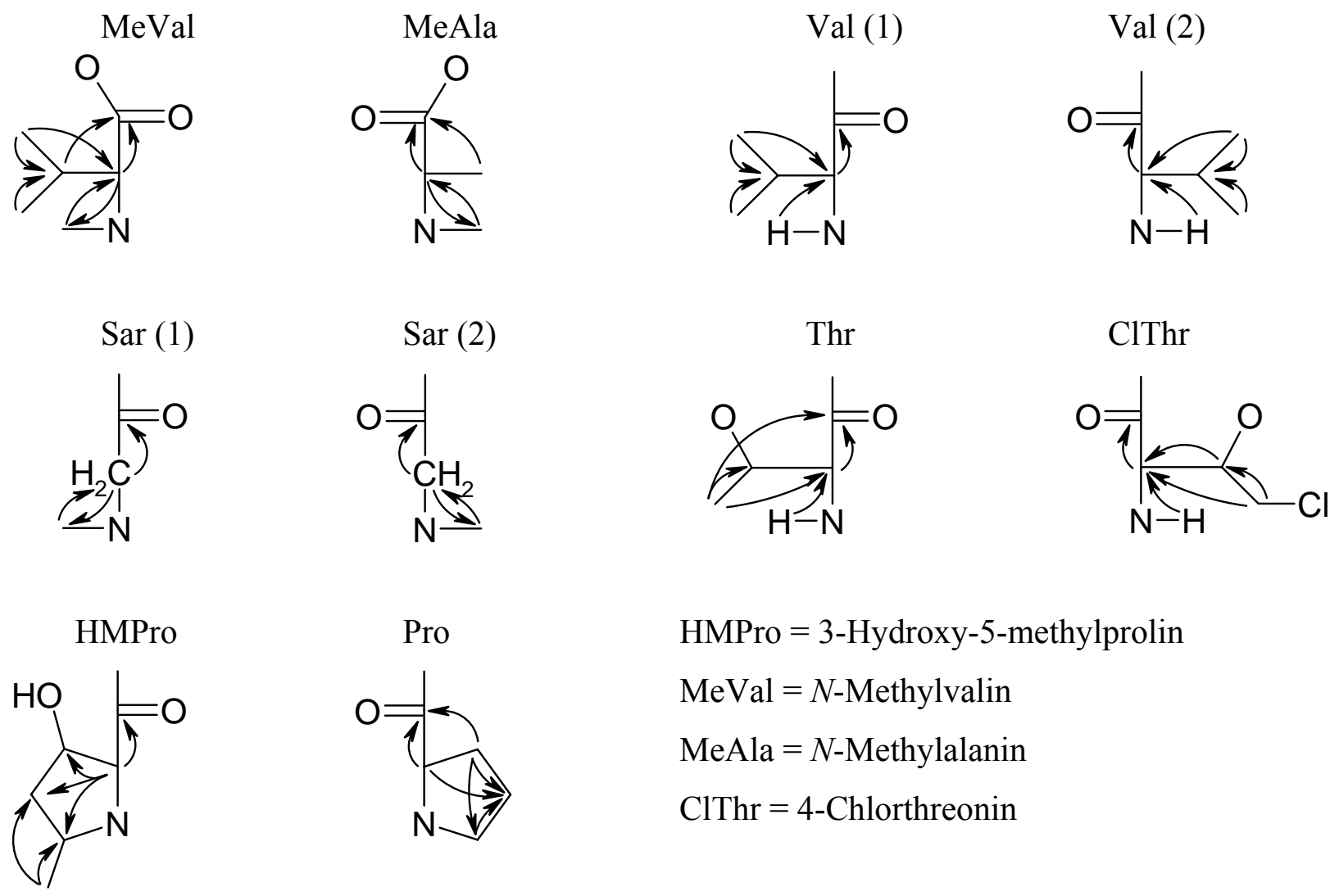

$$
\begin{aligned}
& \text { HMPro }=3 \text {-Hydroxy-5-methylprolin } \\
& \text { MeVal }=N \text {-Methylvalin } \\
& \text { MeAla }=N \text {-Methylalanin } \\
& \text { ClThr }=4 \text {-Chlorthreonin }
\end{aligned}
$$

Abbildung 9: Wichtige HMBC-Korrelationen innerhalb der Aminosäuren von 12.

\footnotetext{
${ }^{\mathrm{d}}$ Zur Bezeichnung von Actinomycin $\mathrm{G}_{1}$ (11) siehe Fußnote auf Seite 23.
} 


\section{Verknüpfung der Aminosäuren}

Die Verknüpfung der Aminosäuren folgt aus dem HMBC-Spektrum (Abbildung 10). Hierbei werden die ${ }^{3} J_{\mathrm{CH}}-$ Kopplungen der Protonen in $\alpha$-Position $\mathrm{zu}$ den Carboxylgruppen der nächsten Aminosäure bzw. des Chromophors verwendet. Unterstützt wird dies durch ${ }^{3} J_{\mathrm{CH}^{-}}$ Kopplungen der $N$-Methylgruppen, ${ }^{3} J_{\mathrm{CH}}$-Kopplungen ausgehend von $\mathrm{C}-5$ an den Prolinringen und die ${ }^{2} J_{\mathrm{CH}}-$ Kopplung der Amidprotonen. Der Ringschluss durch eine Esterbindung zur fünften Aminosäure ausgehend von C-3 der Threoninreste wird ebenfalls durch eine entsprechende HMBC-Korrelation bewiesen. Das NOESY-Spektrum bestätigt zusätzlich die ermittelten Konnektivitäten.

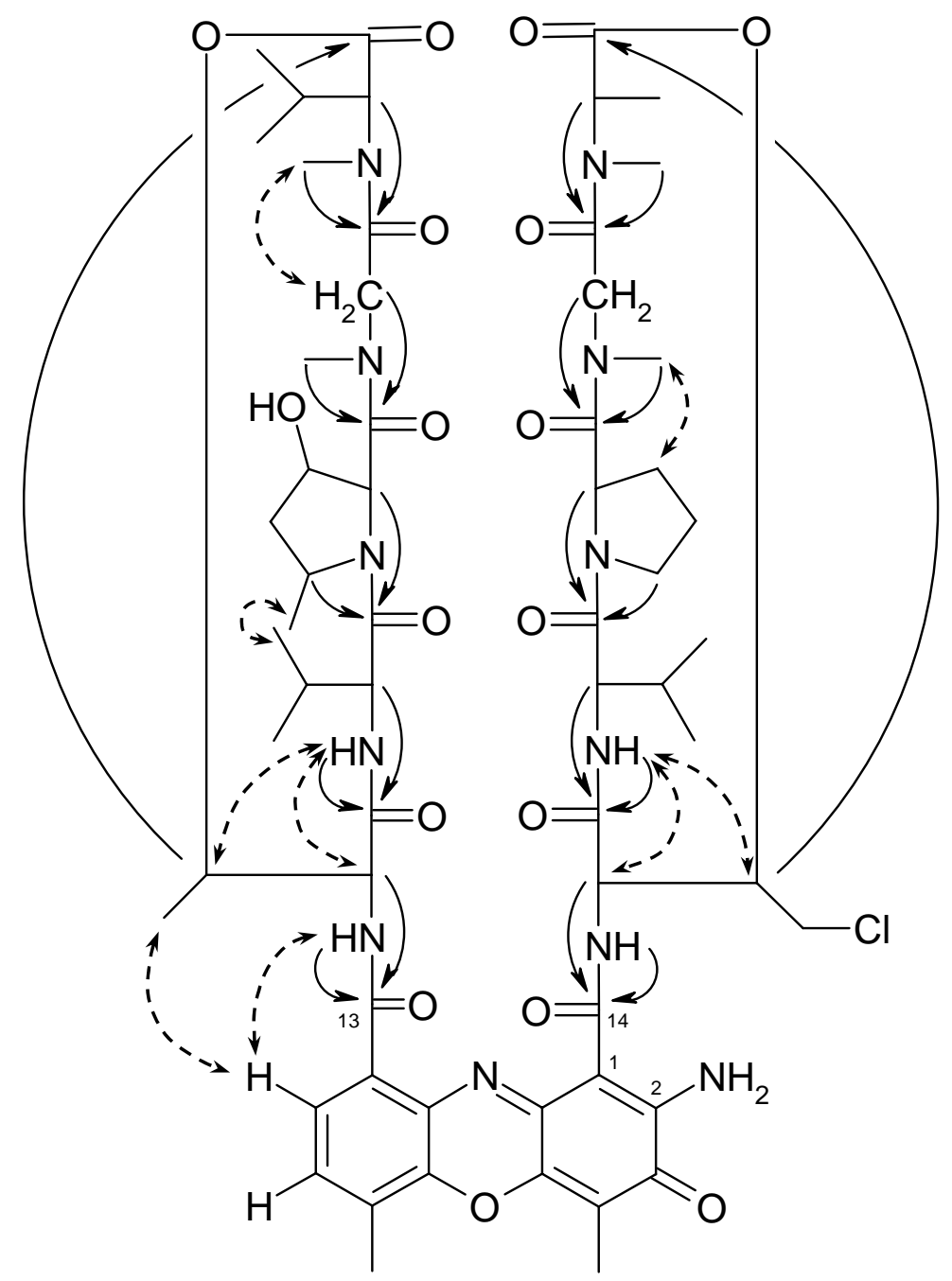

Abbildung 10: Struktur von Actinomycin $G_{2}$ (12) und wichtige interresiduale Kopplungen (HMBC: durchgehende Linie, NOESY: gestrichelte Linie). 


\section{Absolute Stereochemie}

Die absolute Stereochemie der einzelnen Aminosäuren von 12 wurde nicht bestimmt. Es ist jedoch anzunehmen, dass sie mit der bei anderen Actinomycinen ermittelten Konfiguration übereinstimmt. Dies ergibt sich aus der beibehaltenen A-Konformation (vgl. Fußnote S. 12), die u. a. durch die im ${ }^{1}$ H-NMR-Spektrum ins Tieffeld verschobenen Protonen in $\alpha$-Position der Prolinringe sowie die hohe geminale Kopplungskonstante der Sarkosine bewiesen wird. Eine Änderung der Stereochemie einzelner Aminosäuren würde zum Verlust dieser Konformation führen ${ }^{[33,34]}$. Ein weiterer Hinweis ist die biologische Aktivität von 12, die einen intakten Chromophor und die A-Konformation der Pentapeptidringe zur Voraussetzung hat (vgl. Seite 38$)^{[33,39]}$.

\section{Struktur von Actinomycin $G_{2}(12)$}

Die Struktur und Aminosäuresequenz von Actinomycin $G_{2}$ (12) zeigt Abbildung 11. Die Namensgebung erfolgte aufgrund der Ähnlichkeit $\mathrm{zu}$ dem bekannten Actinomycin $\mathrm{G}_{1}$ (11) $^{\mathrm{e},[38,40]}$. Im Unterschied zu 11 befindet sich bei 12 3-Hydroxy-5-methylprolin im $\alpha$ - anstatt im $\beta$-Ring, und der $\beta$-Threoninrest ist an C-4 chloriert anstatt hydroxyliert.
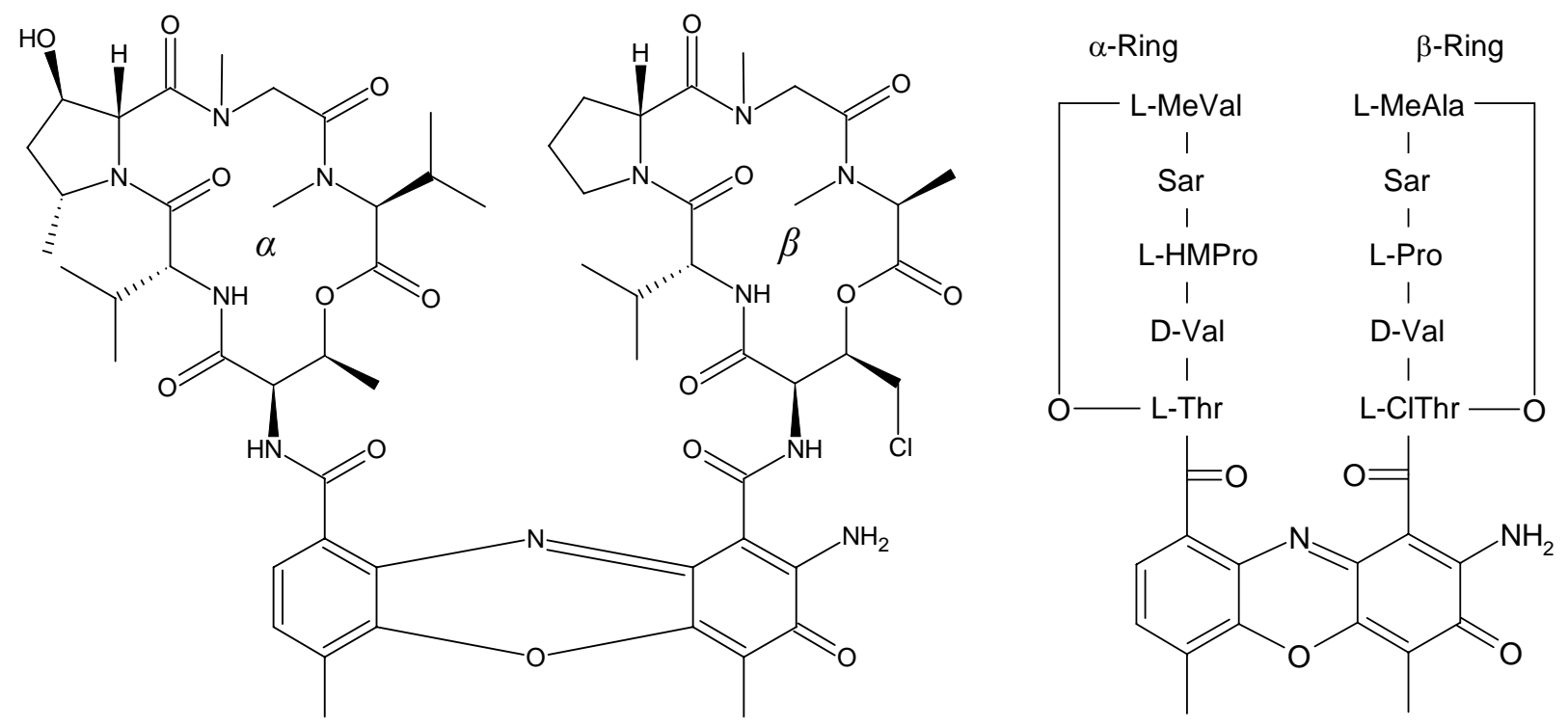

Abbildung 11: Struktur und Aminosäuresequenz von Actinomycin $G_{2}$ (12).

\footnotetext{
e 11 wurde unter der Bezeichnung Actinomycin HKI-0155 veröffentlicht. Der Name Actinomycin $\mathrm{G}_{1}$ ist ein Vorschlag von H. Lackner und A. B. Mauger in Erinnerung an den Naturstoffchemiker U. Gräfe (HKI Jena, $†$ 2003), in dessen Arbeitskreis 11 gefunden wurde. Dieser Name wird auch in einem demnächst erscheinenden Review über Actinomycine verwendet werden ${ }^{[10]}$.
} 

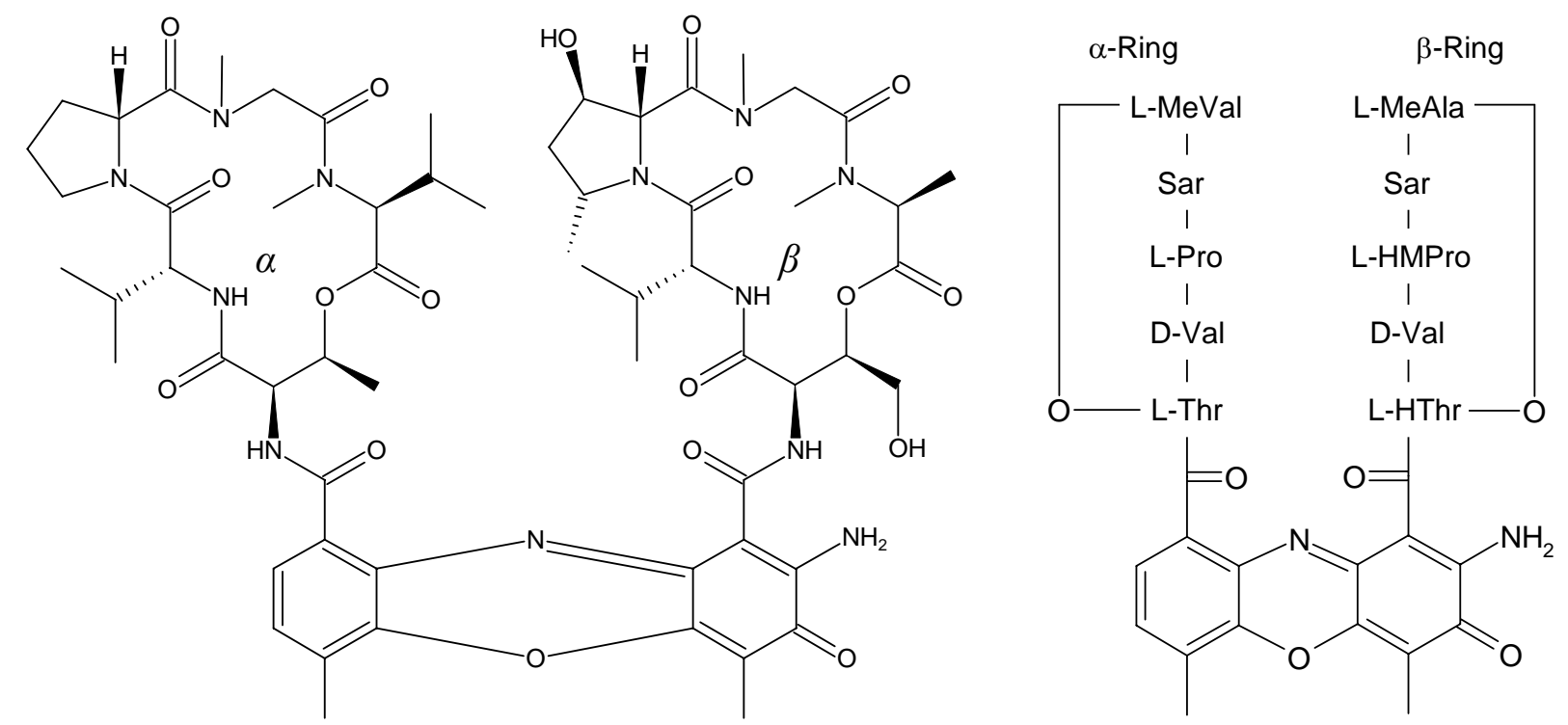

Abbildung 12: Struktur und Aminosäuresequenz von Actinomycin $\mathrm{G}_{1}(\mathbf{1 1})^{[38]}$.

\subsection{Strukturaufklärung von Actinomycin $G_{3}(13)$}

\section{Summenformel}

Actinomycin $\mathrm{G}_{3}(\mathbf{1 3})$ besitzt eine nominale Masse von 1272, die Hochauflösung des ESIMassenspektrums liefert die Summenformel $\mathrm{C}_{61} \mathrm{H}_{84} \mathrm{~N}_{12} \mathrm{O}_{18}$. Im Vergleich zu Actinomycin $\mathrm{G}_{2}$ (12) entspricht dies dem Austausch des Chloratoms gegen eine OH-Gruppe, die Actinomycine $G_{3}(\mathbf{1 3})$ und $G_{1}(\mathbf{1 1})$ sind Isomere.

\section{NMR-Spektren und Struktur}

Die ${ }^{1} \mathrm{H}$ - und ${ }^{13} \mathrm{C}-\mathrm{NMR}-$ Spektren von 13 sind denen von 12 sehr ähnlich. Aus der Analyse der 2D-NMR-Daten (HSQC, COSY und HMBC) ergeben sich dieselben Aminosäuren wie für 12, einziger Unterschied ist das Vorkommen von 4-Hydroxythreonin (HThr) anstelle von 4-Chlorthreonin (ClThr). Diese Änderung wird an der höheren chemischen Verschiebung des Kohlenstoffatoms C-4 der Aminosäure deutlich (Tabelle 1). Die Sequenz der Aminosäuren im $\alpha$ - und $\beta$-Ring ist gleich der von 12, Actinomycin $\mathrm{G}_{3}$ (13) besitzt daher die in Abbildung 13 gezeigte Struktur. Die Aminosäure-Zusammensetzung von 13 ist identisch mit Actinomycin $\mathrm{G}_{1}(\mathbf{1 1}$, Abbildung 12), der modifizierte Prolinrest befindet sich jedoch im anderen Ring. 
Tabelle 1: NMR-Daten von 4-Chlorthreonin und 4-Hydroxythreonin $\left(\mathrm{CDCl}_{3}, 600 \mathrm{MHz}\right)$

\begin{tabular}{r|rrl|rll} 
& \multicolumn{2}{|l|}{ ClThr (12) } & & HThr (13) & \\
Atom & $\delta_{\mathrm{C}}$ & $\delta_{\mathrm{H}}$ & $J[\mathrm{~Hz}]$ & \multicolumn{1}{|c}{$\delta_{\mathrm{C}}$} & $\delta_{\mathrm{H}}$ & $J[\mathrm{~Hz}]$ \\
\hline 1 & 168.07 & & & 168.40 & & \\
2 & 53.54 & 5.27 & $\mathrm{dd}, 7.6,2.7$ & 51.96 & 5.20 & $\mathrm{dd}, 7.0,2.0$ \\
3 & 74.98 & 5.12 & $\mathrm{ddd}, 5.0,2.5,2.5$ & 75.39 & 5.05 & $\mathrm{ddd}, 8.5,5.0,2.5$ \\
4 & 43.21 & 3.94 & $\mathrm{dd}, 11.4,2.5$ & 59.40 & 3.23 & $\mathrm{dd}, 12.0,9.0$ \\
& & 3.99 & $\mathrm{dd}, 11.4,6.0$ & & 3.76 & $\mathrm{dd}, 12.0,4.5$ \\
$\mathrm{NH}$ & & 7.88 & $\mathrm{~d}, 7.3$ & & 8.28 & $\mathrm{~d}, 6.5$ \\
\hline
\end{tabular}

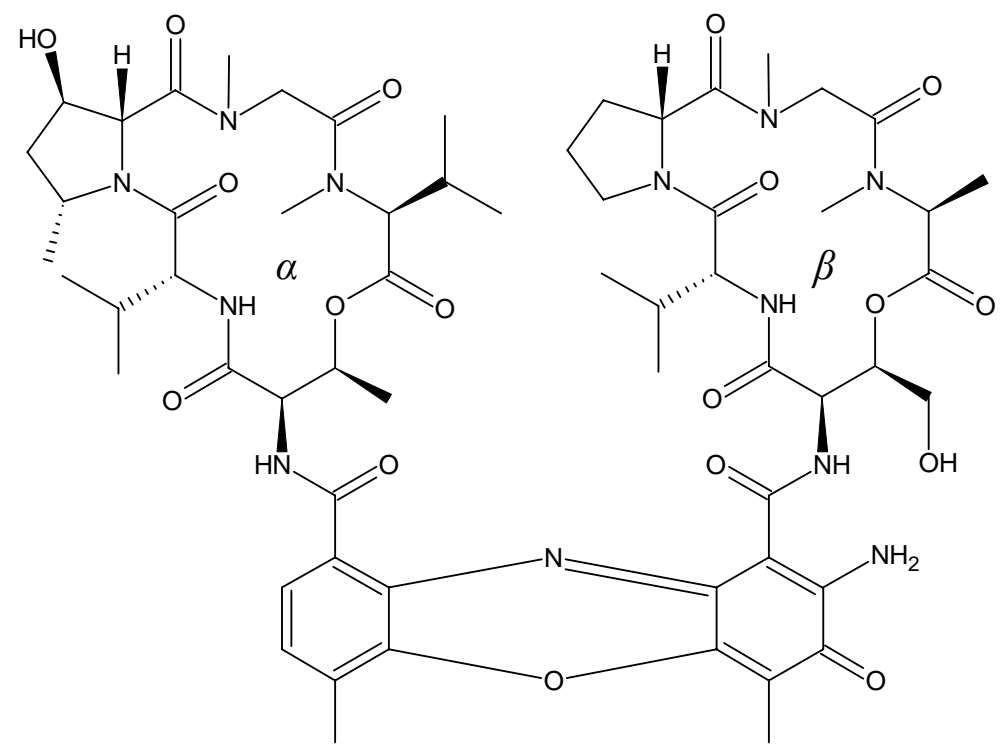

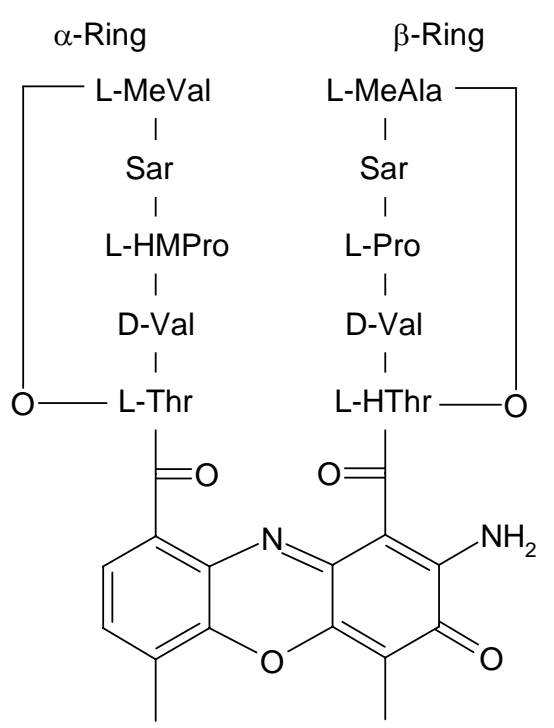

Abbildung 13: Struktur und Aminosäuresequenz von Actinomycin $G_{3}$ (13). 


\subsection{Strukturaufklärung von Actinomycin $\mathrm{G}_{4}(14)$}

\section{Summenformel}

Die nominale Masse von 1256 wurde durch ein ESI-Massenspektrum bestimmt, die Hochauflösung ergab die Summenformel $\mathrm{C}_{61} \mathrm{H}_{84} \mathrm{~N}_{12} \mathrm{O}_{17}$. Actinomycin $\mathrm{G}_{4}$ (14) besitzt demnach ein Sauerstoffatom weniger als 13, im Vergleich zu 12 ist ein Chloratom durch ein Wasserstoffatom ersetzt.

\section{NMR-Spektren und Struktur}

Die Summenformel lässt das Vorliegen eines unsubstituierten Threonins im $\beta$-Ring erwarten bei ansonsten im Vergleich zu 12 und 13 unveränderter Grundstruktur. Diese Annahme wird durch das ${ }^{1} \mathrm{H}-\mathrm{NMR}-$ Spektrum bestätigt: es fehlt das auffällige Signal bei $\delta_{\mathrm{H}} \approx 5.3$ für 3-H des in Position 4 substituierten Threonins. Stattdessen gibt es eine zusätzliche Methylgruppe $\left(\delta_{\mathrm{H}}=\right.$ 1.30, 4- $\mathrm{H}_{3}$ ), die eine Änderung des Kopplungsmusters von H-3 zu einem Quartett von Dubletts (qd) bewirkt. Da die weiteren Signale nahezu unverändert sind, ergibt sich folgende Struktur für Actinomycin $\mathrm{G}_{4}(\mathbf{1 4})$ :
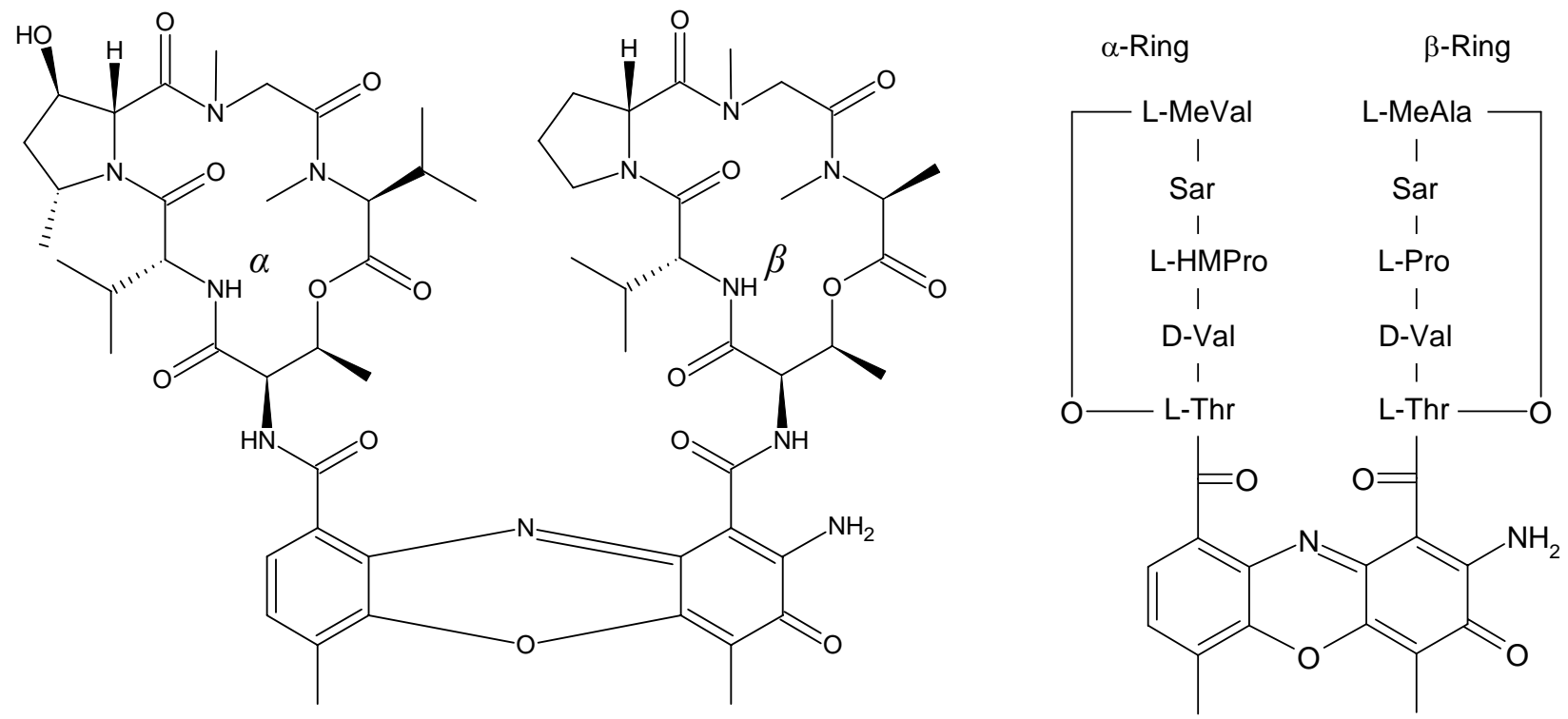

Abbildung 14: Struktur und Aminosäuresequenz von Actinomycin $\mathrm{G}_{4}$ (14). 


\subsection{Strukturaufklärung von Actinomycin $\mathrm{G}_{5}(15)$}

\section{UV-Spektrum}

Das UV-Spektrum von Actinomycin $\mathrm{G}_{5}$ (15) zeigt eine zusätzliche Bande bei $260 \mathrm{~nm}$, die bei den Actinomycinen $\mathrm{G}_{2}$ bis $\mathrm{G}_{4}(\mathbf{1 2}$ - 14) nicht auftritt. Zusammen mit der geringeren Extinktion $(\log \varepsilon=4.2$ statt 4.5$)$ weist dies auf einen veränderten Chromophor hin.

\section{Summenformel}

Die durch hochauflösende ESI-Massenspektrometrie bestimmte Summenformel von 15 lautet $\mathrm{C}_{61} \mathrm{H}_{81} \mathrm{~N}_{11} \mathrm{O}_{18}$ (nominale Masse 1255). Es fallen zwei Besonderheiten auf:

(1) Die Substanz hat ein Stickstoffatom weniger als die anderen Actinomycine. Diese besitzen zehn Stickstoffatome in Amidbindungen und zwei im Chromophor, 15 hat insgesamt nur elf. Am leichtesten zu ersetzen ist die Aminogruppe des Chromophors, hier findet schon unter schwach sauren Bedingungen ein Austausch gegen eine Hydroxygruppe statt (vgl. S. 11) ${ }^{[31,32]}$.

(2) Die Anzahl der Doppelbindungsäquivalente berechnet sich für $15 \mathrm{zu}$ 27, die der Actinomycine $\mathrm{G}_{2}$ bis $\mathrm{G}_{4}\left(\mathbf{1 2}\right.$ - 14) $\mathrm{zu}$ 26. Da die Zahl der $\mathrm{sp}^{2}$-hybridisierten Kohlenstoffatome im ${ }^{13} \mathrm{C}-\mathrm{NMR}-$ Spektrum nicht gestiegen ist und demnach keine weiteren Doppelbindungen vorliegen, muss ein zusätzlicher Ringschluss im Molekül vorhanden sein.

\section{NMR-Spektren}

Das ${ }^{1}$ H-NMR-Spektrum zeigt die Signale der Protonen an C-2 von Prolin und Hydroxymethylprolin $\left(\delta_{\mathrm{H}}=6.19,6.34\right)$, auch die charakteristisch aufgespaltenen Methylengruppen von zwei Sarkosinresten fallen auf $\left({ }^{2} J_{\mathrm{HH}}=17.5 \mathrm{~Hz}\right)$. Die Interpretation der 2D-NMRSpektren (HSQC, COSY, HMBC) erbringt dieselbe Aminosäure-Zusammensetzung wie für Actinomycin $\mathrm{G}_{3}$ (13). Auffällig sind die von 13 abweichenden chemischen Verschiebungen des Hydroxythreonins (insbesondere eine Tieffeldverschiebung der Methylengruppe in Position 4, siehe Tabelle 2) und die veränderten Resonanzen des AminophenoxazonChromophors im ${ }^{13} \mathrm{C}-\mathrm{NMR}-$ Spektrum (Tabelle 3). 
Tabelle 2: Vergleich der chemischen Verschiebungen der Hydroxythreonine von Actinomycin $\mathrm{G}_{3}(\mathbf{1 3})$ und Actinomycin $\mathrm{G}_{5}(\mathbf{1 5})\left(\mathrm{CD}_{3} \mathrm{OD}, 600\right.$ bzw. $\left.150.8 \mathrm{MHz}\right)$

\begin{tabular}{r|rl|rl} 
& \multicolumn{2}{|c|}{ HThr (13) } & \multicolumn{2}{|c}{ HThr (15) } \\
Atom & \multicolumn{1}{|c|}{$\delta_{\mathrm{C}}$} & $\delta_{\mathrm{H}}$ & \multicolumn{1}{c}{$\delta_{\mathrm{C}}$} & $\delta_{\mathrm{H}}$ \\
\hline $1^{\prime}$ & 168.40 & - & 165.80 & - \\
$2^{\prime}$ & 51.96 & 5.27 & 54.04 & 4.07 \\
$3^{\prime}$ & 75.39 & 5.12 & 64.71 & 5.53 \\
$4^{\prime}$ & 59.40 & $3.94,3.99$ & 68.48 & $4.64,4.86$ \\
\hline
\end{tabular}

Tabelle 3: Vergleich der ${ }^{13} \mathrm{C}-\mathrm{NMR}-\mathrm{Daten}$ des rechten Rings im Actinoyl-Chromophor von 13 und $15\left(\mathrm{CD}_{3} \mathrm{OD}, 150.8 \mathrm{MHz}\right)$

\begin{tabular}{r|rr} 
Atom & $\delta_{\mathrm{C}}(\mathbf{1 3})$ & $\delta_{\mathrm{C}}(\mathbf{1 5})$ \\
\hline 1 & 102.32 & 94.74 \\
2 & 148.54 & 167.98 \\
3 & 180.30 & 182.64 \\
\hline
\end{tabular}

\begin{tabular}{r|rr} 
Atom & $\delta_{\mathrm{C}}(\mathbf{1 3})$ & $\delta_{\mathrm{C}}(\mathbf{1 5})$ \\
\hline 4 & 114.14 & 117.42 \\
$4 \mathrm{a}$ & 146.52 & 149.35 \\
$10 \mathrm{a}$ & 146.91 & 150.17 \\
\hline
\end{tabular}

Struktur von Actinomycin $G_{5}(\mathbf{1 5})$

Die genannten Abweichungen der spektralen Daten erklären sich durch einen Ringschluss zwischen der 4'-OH-Gruppe des Hydroxythreonins und C-2 des Chromophors, der durch eine HMBC-Korrelation zwischen den Strukturteilen angezeigt wird (siehe Abbildung 15). Diese Etherbindung bewirkt die starke Tieffeldverschiebung der ${ }^{13} \mathrm{C}-$ Resonanz von C-2 und der ${ }^{1}$ H-Resonanz der diastereotopen Methylengruppe des HThr. Die Struktur und Aminosäuresequenz von Actinomycin $\mathrm{G}_{5}$ (15) zeigt Abbildung 16.

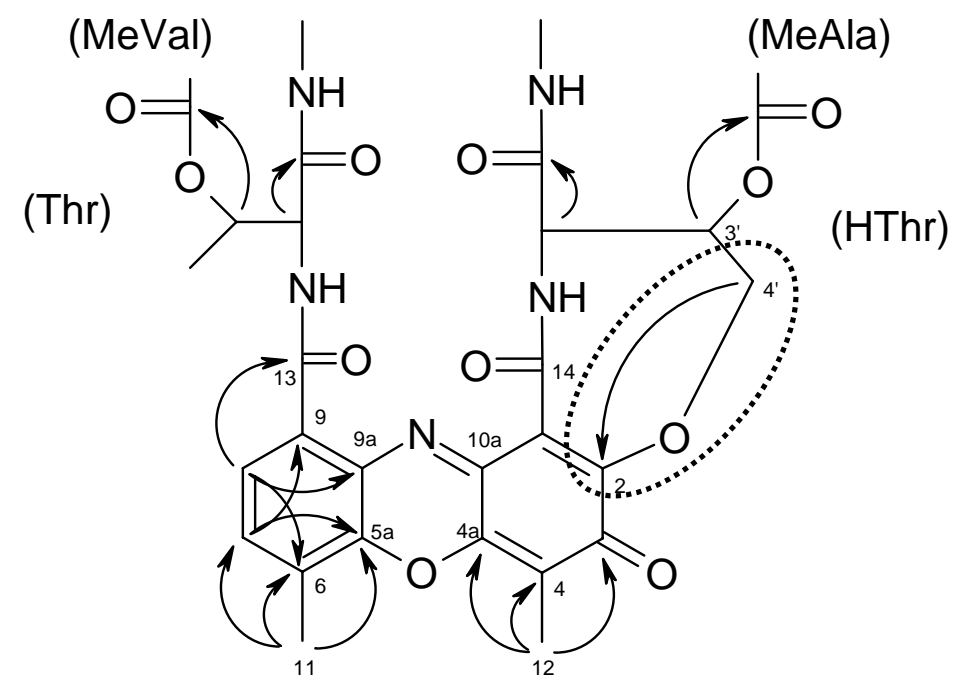

Abbildung 15: Wichtige HMBC-Korrelationen in 15. 

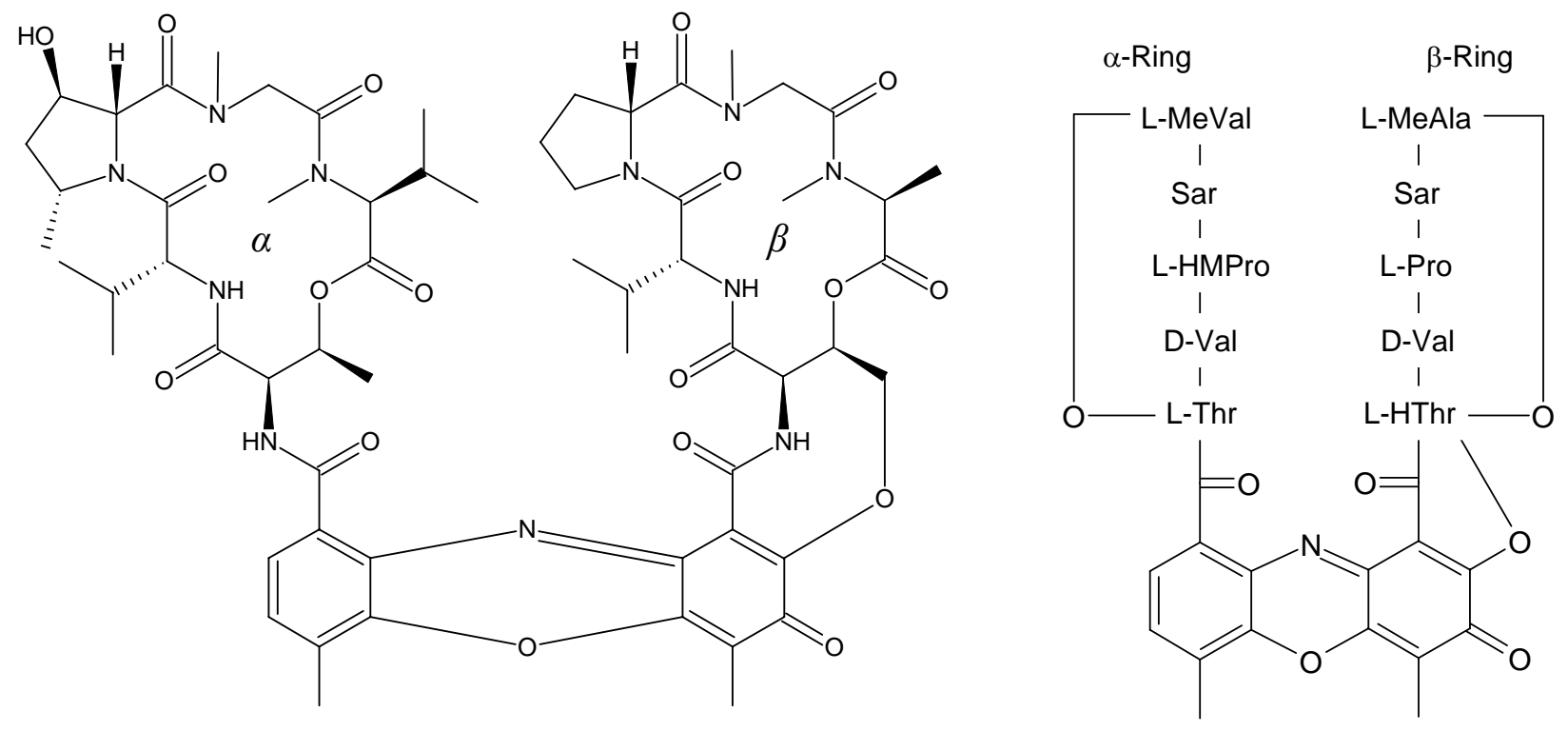

Abbildung 16: Struktur und Aminosäuresequenz von Actinomycin $G_{5}$ (14).

Die zusätzliche Verknüpfung des $\beta$-Pentapeptidlacton-Rings mit dem Actinoyl-Chromophor ist in der Literatur noch nicht berichtet worden, obwohl die Actinomycine des Z-Typs teilweise ebenfalls Hydroxythreonin an Position 1' des $\beta$-Rings besitzen ${ }^{[36]}$ und somit grundsätzlich die Möglichkeit zu einem solchen Ringschluss gegeben wäre. Auch synthetisch ist ein solches Produkt nicht bekannt. Actinomycin $\mathrm{G}_{5}$ (15) stellt daher eine neuartige Strukturvariante mit einem zusätzlichen Achtring zwischen Chromophor und Peptidlacton auf der $\beta$-Seite dar.

Der Produzent von Actinomycin $G_{1}$ (11) bildet ein Actinomycin mit gleicher Molmasse und Summenformel wie 15, dessen Struktur bisher nicht vollständig aufgeklärt wurde ${ }^{\mathrm{f}}$. Es ist zu vermuten, dass hier ein analoger Ringschluss erfolgte. Hydroxymethylprolin gehört jedoch wie bei 11 zum $\beta$-Ring, so dass die Verbindungen nicht identisch sein können.

\section{Überlegungen zur Biosynthese}

Biosynthetisch entsteht 15 wahrscheinlich durch einen nucleophilen Angriff von 4'-OH auf C-2 unter Abspaltung von Ammoniak aus Actinomycin $\mathrm{G}_{3}$ (13). Ob dieser Schritt enzymatisch katalysiert wird oder spontan verläuft, ist ungeklärt. Für eine Enzymbeteiligung spricht die Tatsache, dass ein derartiges Produkt bei den Z-Actinomycinen nicht gefunden wurde, obwohl dort ebenfalls 4-Hydroxythreonin als Aminosäure vorkommt. 15 kann bereits

\footnotetext{
${ }^{\mathrm{f}}$ H. Lackner, persönliche Mitteilung unveröffentlichter Ergebnisse
} 
im Rohextrakt mittels analytischer HPLC nachgewiesen werden, das Vorliegen eines bei der Isolierung entstandenen Artefakts kann daher ausgeschlossen werden (möglich ist jedoch die Entstehung im Zellmilieu oder bei der Aufarbeitung der Kulturbrühe).

\subsection{Strukturaufklärung von Actinomycin $\mathrm{G}_{6}(\mathbf{1 6})$}

\section{Summenformel und Polarität}

Die Verbindung 16 hat dieselbe Molmasse und Summenformel wie die Actinomycine $\mathrm{G}_{1}(\mathbf{1 1})$ und $\mathrm{G}_{3}(13)\left(\mathrm{M}=1272, \mathrm{C}_{61} \mathrm{H}_{84} \mathrm{~N}_{12} \mathrm{O}_{18}\right)$, ist jedoch deutlich polarer. Dadurch ist die Substanz in Chloroform nur schlecht löslich, in Methanol, DMSO oder Wasser jedoch gut.

\section{Strukturaufklärung}

Das UV- und das ${ }^{13} \mathrm{C}-\mathrm{NMR}-$ Spektrum beweisen einen intakten, unveränderten ActinoylChromophor. Die Auswertung der COSY-, HSQC- und HMBC-Spektren zeigt, dass 16 dieselbe Aminosäure-Zusammensetzung besitzt wie Actinomycin $\mathrm{G}_{3}$ (13). Die Aminosäuren des $\beta$-Rings weisen jedoch auffällige Signal-Veränderungen in den eindimensionalen NMRSpektren auf:

(1) Die geminale Kopplung der Methylengruppe des Sarkosins beträgt nicht wie gewohnt $18 \mathrm{~Hz}$, sondern nur noch ${ }^{2} J_{\mathrm{HH}}=14.5 \mathrm{~Hz}$, offensichtlich liegt hier eine trans- anstelle der üblichen cis-Amidbindung vor.

(2) Das $\alpha$-Wasserstoffatom des Prolins ist von $\delta_{\mathrm{H}}=6.1 \mathrm{zu} 4.9$ deutlich Hochfeld-, das des Methylalanins hingegen von $\delta_{\mathrm{H}}=3.5 \mathrm{zu} 5.4$ stark Tieffeld-verschoben. Diese und weitere abweichende chemische Verschiebungen beweisen die starke konformative Veränderung im Vergleich zur normalen A-Konformation des Pentapeptidlactons.

Aus der Analyse der HMBC-Korrelationen der Protonen des Hydroxythreonins ergibt sich die Ursache für die Veränderungen: die Aminosäure ist nicht über eine Amidbindung an den Chromophor gebunden, sondern über eine Esterbindung ausgehend von der Hydroxygruppe an C-4'. Die Aminogruppe von HThr formt nun den Ringschluss zum Methylalanin, das Sauerstoffatom an Position 3 ist zur freien Hydroxygruppe geworden. Bewiesen wird dies durch die HMBC-Korrelation der Methylengruppe (C-4') von HThr zur Carboxylgruppe (C-14) des Chromophors und die Korrelation von 2'-H über den Stickstoff zur Carboxylgruppe von Methylalanin (Abbildung 17). Die Esterbindung bewirkt eine Tieffeld- 
verschiebung der Protonen an C-4', die Methingruppe in Position 3 ist durch das Fehlen einer solchen Bindung ins Hochfeld verschoben (Tabelle 4).

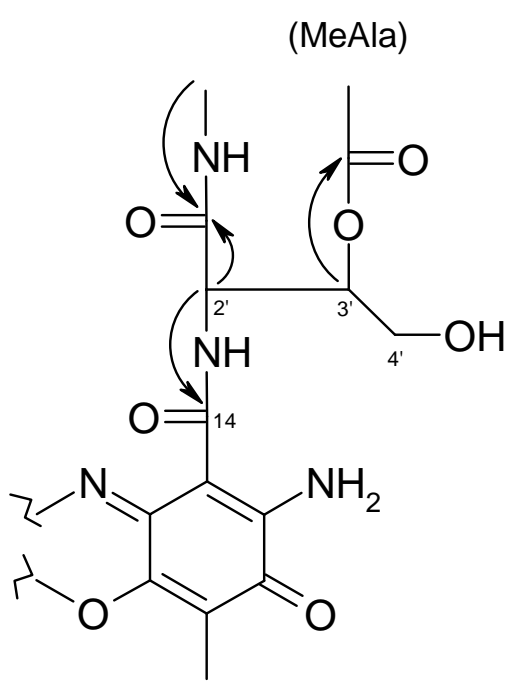

$13($,normal)

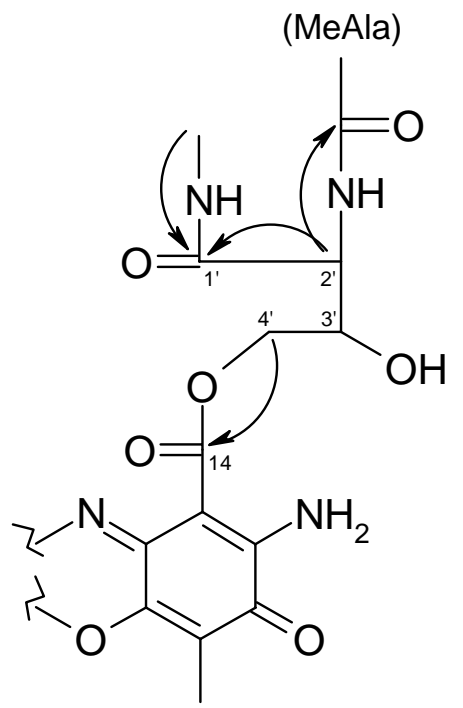

16 (,umgelagert”)

Abbildung 17: Wichtige HMBC-Kopplungen von 13 und 16 im Vergleich.

Tabelle 4: Vergleich der NMR-Daten der 4-Hydroxythreonine von 13 und 16

\begin{tabular}{r|rl|rl} 
& \multicolumn{2}{|c|}{$\mathbf{1 3}$} & \multicolumn{2}{c}{$\mathbf{1 6}$} \\
Atom & \multicolumn{1}{|c|}{$\delta_{\mathrm{C}}$} & $\delta_{\mathrm{H}}$ & \multicolumn{1}{c}{$\delta_{\mathrm{C}}$} & $\delta_{\mathrm{H}}$ \\
\hline $1^{\prime}$ & 169.92 & & 171.63 & \\
$2^{\prime}$ & 53.39 & 5.13 & 54.93 & 4.75 \\
$3^{\prime}$ & 78.49 & 5.07 & 70.10 & 4.40 \\
$4^{\prime}$ & 60.50 & $3.51,3.69$ & 67.13 & $4.24,4.47$ \\
\hline
\end{tabular}

Die vollständige Struktur von Actinomycin $\mathrm{G}_{6}(\mathbf{1 6})$ zeigt Abbildung 18. 16 ist neben 12 das Hauptprodukt des Stamms CS210. Im Unterschied zu allen anderen bekannten Actinomycinen befindet sich auf der $\beta$-Seite die Esterbindung nicht im Ring, sondern stellt die Verbindung zum Chromophor dar. Der Ring wird nicht als Lacton geschlossen, sondern durch die Aminogruppe als Lactam. 

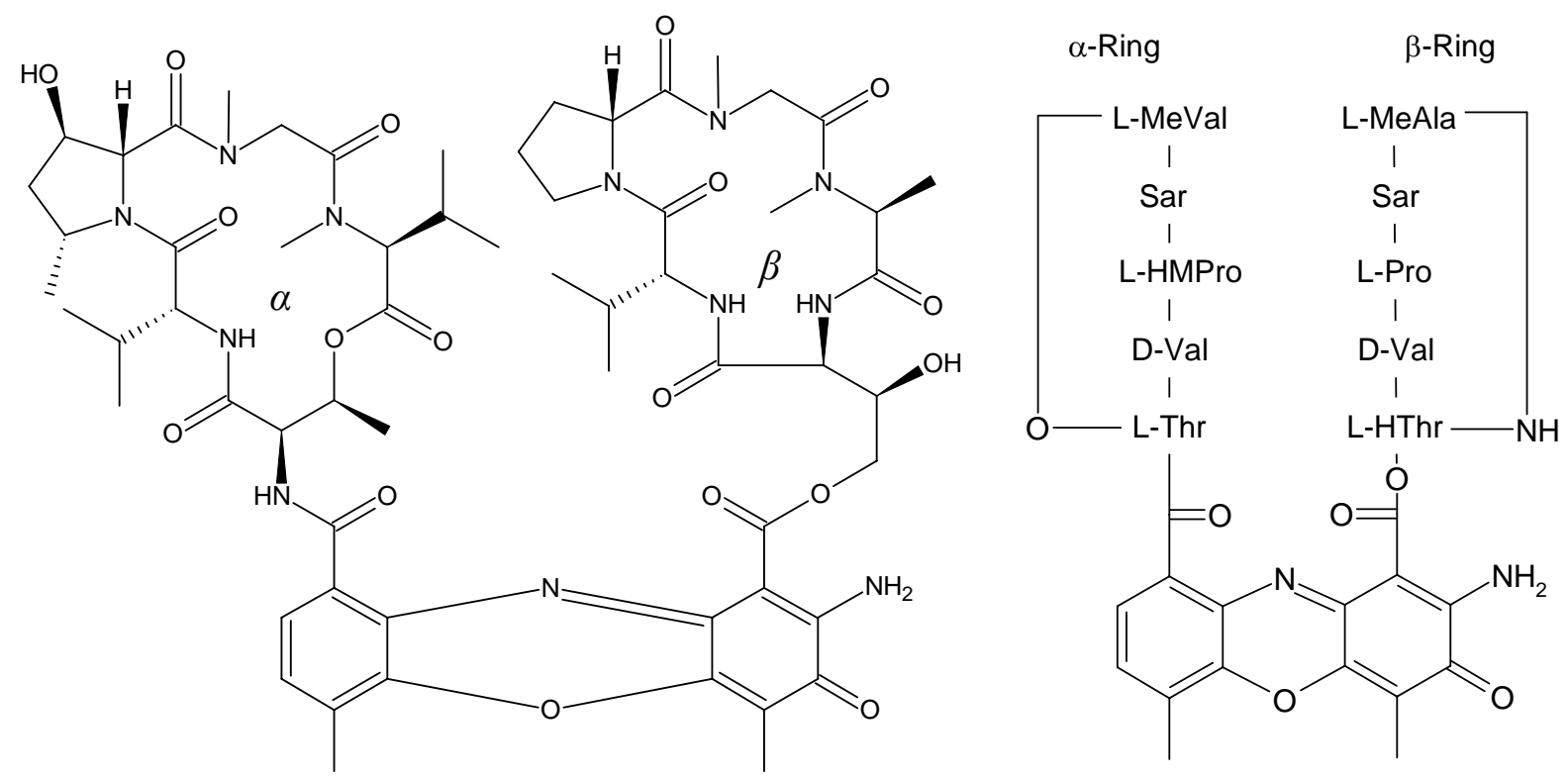

Abbildung 18: Struktur und Aminosäuresequenz von Actinomycin $\mathrm{G}_{6}(\mathbf{1 6})$.

\section{Überlegungen zur Biosynthese}

Biosynthetisch geht Actinomycin $G_{6}(\mathbf{1 6})$ vermutlich aus Actinomycin $G_{3}$ (13) durch eine zweifache Acylverschiebung hervor. Durch einen nucleophilen Angriff an C-14 verdrängt die terminale Hydroxygruppe des Hydroxythreonins die Aminogruppe aus der Amidbindung, für diese Reaktion lässt sich eine sechsgliedrige zyklische Zwischenstufe formulieren. Die freie Aminogruppe an C-2' kann anschließend als Nucleophil die Esterbindung zum Methylalanin angreifen und die 3'-OH-Gruppe als Bindungspartner ersetzen (Abbildung 19).

Es ist unklar, ob die Umwandlung von 13 zu 16 durch Acyltransferasen katalysiert wird oder nicht-enzymatisch ein thermodynamisch begünstigtes Produkt entsteht. Ebenso wie bei Actinomycin $G_{5}$ (15) spricht für eine Enzymbeteiligung, dass bei den Actinomycinen vom Z-Typ keine zu 16 analoge Verbindung gefunden wurde, obwohl bei $Z_{1}$ ebenfalls 4-Hydroxythreonin im $\beta$-Ring vorkommt und demnach eine Acylverschiebung möglich wäre. Beim Produzenten CS210 der G-Actinomycine fällt auf, dass Actinomycin $\mathrm{G}_{3}$ (13) eine Minderkomponente ist. Sie ist offensichtlich Vorläufer für die Actinomycine $\mathrm{G}_{5}(\mathbf{1 5})$ und $\mathrm{G}_{6}$ (16), möglicherweise auch für das Chlorthreonin enthaltende Actinomycin $\mathrm{G}_{2}$ (12). 


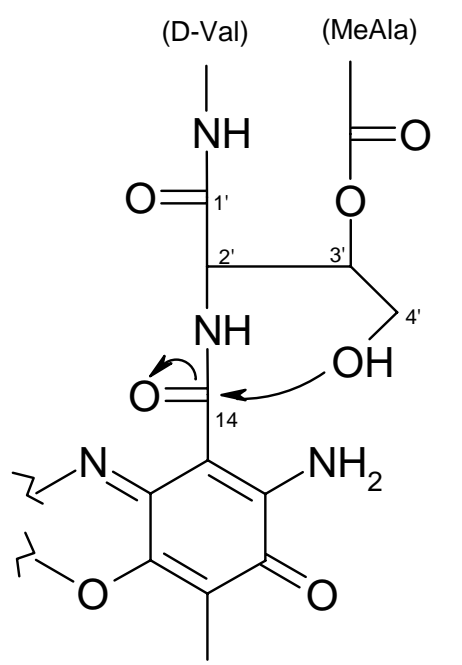

13

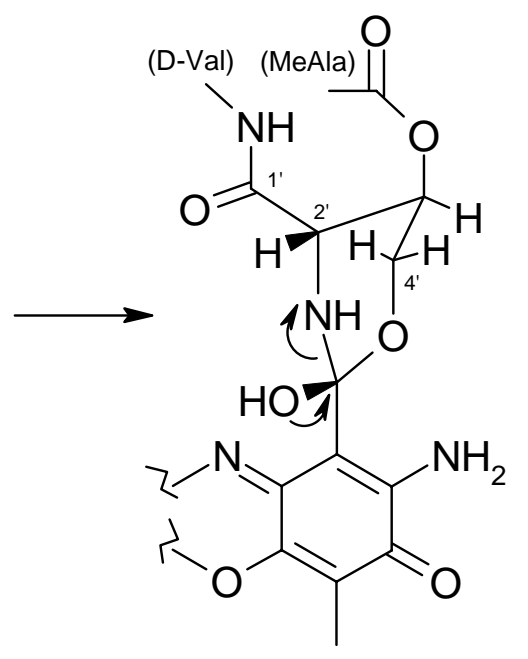

16

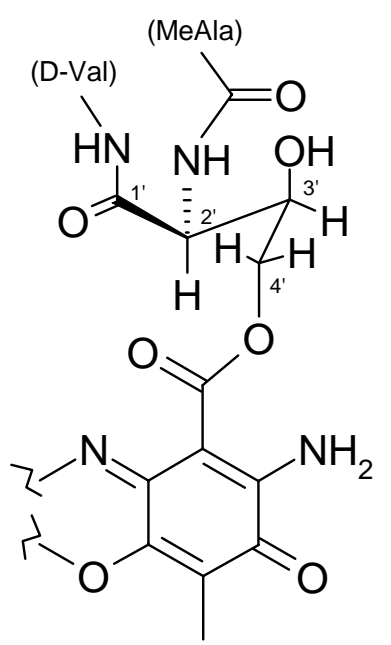

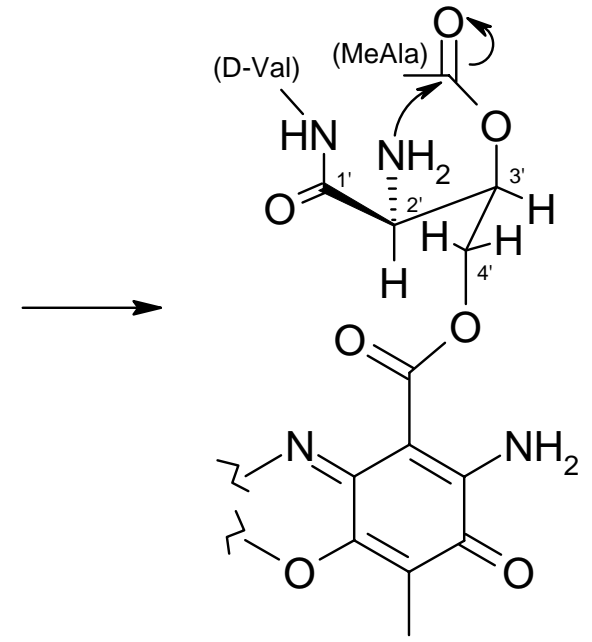

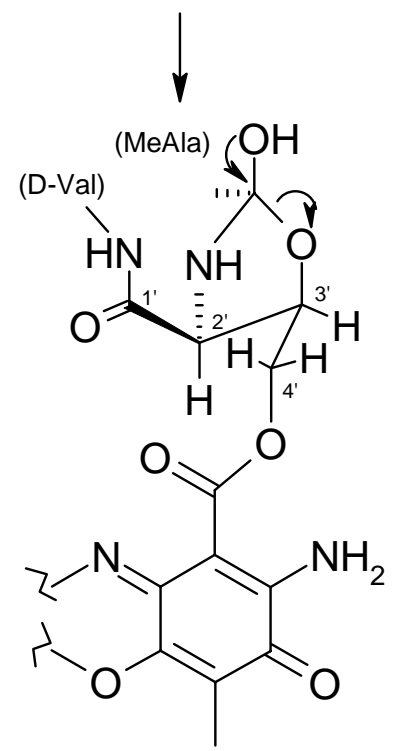

Abbildung 19: Hypothetischer Biosyntheseweg von Actinomycin $G_{3}(\mathbf{1 3}) z^{\prime} G_{6}(\mathbf{1 6})$. 


\subsection{Weitere Sekundärmetaboliten aus Actinomyces sp. CS210}

\subsubsection{1-Phenazinol (17)}

Die lipophile Substanz mit intensiver gelber Eigenfarbe lässt sich durch Gelchromatographie an Sephadex LH-20 leicht von den höhermolekularen Actinomycinen abtrennen. Das im Vergleich zu den Actinomycinen komplexere, hypsochrom verschobene UV-Spektrum weist auf einen veränderten Chromophor hin und die sehr stark verbreiterte Bande bei $420 \mathrm{~nm}$ deutet Wasserstoffbrückenbindungen mit dem Lösungsmittel an. Die 1D-NMR-Spektren zeigen Signale für sieben aromatische Protonen und 12 aromatische Kohlenstoffatome. 17 ergibt im EI-Massenspektrum ein Ion höchster Masse und Intensität bei m/z=196, woraus sich gemeinsam mit der Anzahl der Kohlenstoffatome die Summenformel $\mathrm{C}_{12} \mathrm{H}_{8} \mathrm{~N}_{2} \mathrm{O}$ ableiten lässt. Eine Suche mit diesen Informationen in der Datenbank AntiBase ${ }^{[41]}$ ergab 1- und 2-Phenazinol als mögliche Strukturen. Ein Vergleich mit publizierten ${ }^{13} \mathrm{C}-\mathrm{NMR}-\mathrm{Daten}{ }^{[42]}$ legte die Verbindung 17 als 1-Phenazinol fest, das als Sekundärmetabolit des PhenazinProduzenten Pseudomonas aeruginosa bekannt ist.

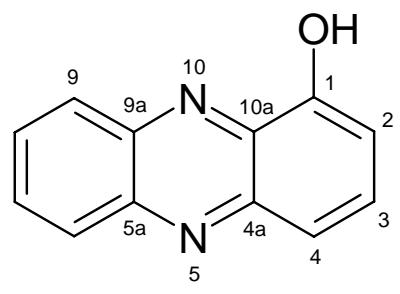

17

\subsubsection{N-(2-Hydroxyphenyl)acetamid (18)}

Der farblose Feststoff 18 wurde als Nebenprodukt bei der Trennung der Actinomycine $\mathrm{G}_{3}$ (13) und $\mathrm{G}_{5}$ (15) durch semipräparative HPLC erhalten. Die ungerade Molmasse von $151 \mathrm{~g} / \mathrm{mol}$ weist auf das Vorhandensein eines Stickstoffatoms hin. Das ${ }^{1}$ H-NMR-Spektrum zeigt vier Protonen im Tieffeldbereich, die anhand ihres typischen Kopplungsmusters einem ortho-disubstituierten Aromaten zugeordnet werden können (zwei Dubletts und zwei Tripletts mit einer Kopplungskonstanten von je $8 \mathrm{~Hz}$ ). Bei $\delta_{\mathrm{H}}=2.16$ ist die Resonanz einer Acetylgruppe zu sehen, weitere Signale treten nicht auf. Eine Datenbanksuche ${ }^{[41]}$ führte $\mathrm{zu}$ $N$-(2-Hydroxyphenyl)acetamid, das bereits mehrfach als Metabolit von Streptomyceten beschrieben wurde ${ }^{[43]}$. Die Übereinstimmung des ${ }^{1} \mathrm{H}-\mathrm{NMR}-$ Spektrums mit publizierten Daten $^{[43]}$ bestätigte die Zuordnung. 
<smiles>CC(=O)Nc1ccccc1O</smiles>

17

Ein plausibler Biosyntheseweg für 18 geht von 3-Hydroxyanthranilsäure aus, einem aus Tryptophan hervorgehenden Intermediat bei der Biosynthese des Actinoyl-Chromophors der Actinomycine. Nach Decarboxylierung zu ortho-Aminophenol erfolgt die Acetylierung zu $N$-(2-Hydroxyphenyl)acetamid (18), siehe Abbildung 20.

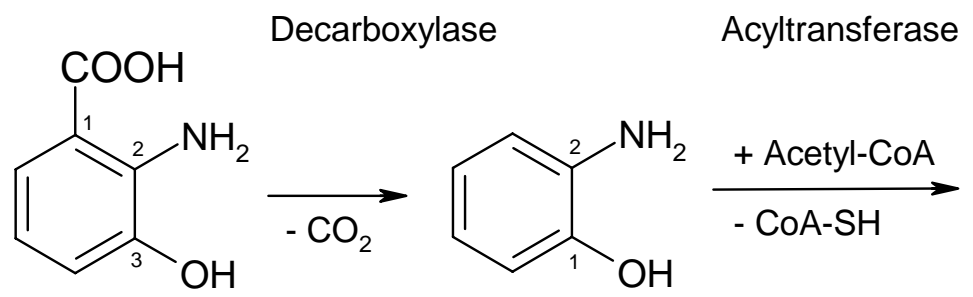<smiles>CC(=O)Nc1ccccc1O</smiles>

Abbildung 20: Hypothetische Biosynthese von 18 ausgehend von 3-Hydroxyanthranilsäure. 


\subsection{Biologische Aktivität der G-Typ Actinomycine}

\subsubsection{Wirkmechanismus der Actinomycine}

Actinomycine interkalieren reversibel mit doppelsträngiger DNA, der Chromophor schiebt sich dabei selektiv zwischen zwei Guanosin/Cytosin - Basenpaare (Abbildung 21). Eine pseudo $\mathrm{C}_{2}$-symmetrische Konformation der Actinomycine ist Voraussetzung für ihre Affinität zu DNA ${ }^{[33,34]}$. Die Selektivität von Actinomycin D (19) bezüglich der Nukleinbasen (5'-GpC-3') beruht auf der Ausbildung von vier Wasserstoffbrückenbindungen zwischen den Amidgruppen der Threonine und den N2/N3-Aminogruppen von Guanin ${ }^{[39,44]}$. Die Peptidringe binden auf beiden Seiten in der kleinen Furche und bewirken eine Abhängigkeit der Bindungsstärke von der flankierenden Sequenz ${ }^{[45]}$. Neben der Interkalation wird auch die Bindung an Einzelstrang-DNA berichtet ${ }^{[46]}$, für die ein sequenzabhängiger Bindungsmechanismus unter Ausbildung von Haarnadel-Schleifen diskutiert wird ${ }^{[47]}$.

In Folge der Interkalation werden Replikation und Transkription gestört, da die Enzyme DNA als Templat verwenden. Die Transkription wird wesentlich stärker und daher schon bei geringeren Konzentrationen inhibiert als die Replikation. Diese Präferenz entsteht durch die unterschiedliche Konformation der DNA im aktiven Zentrum von DNA- und RNAPolymerase, die im Falle der RNA-Polymerase eine stärkere Bindung von Actinomycin bewirkt $^{[48]}$.
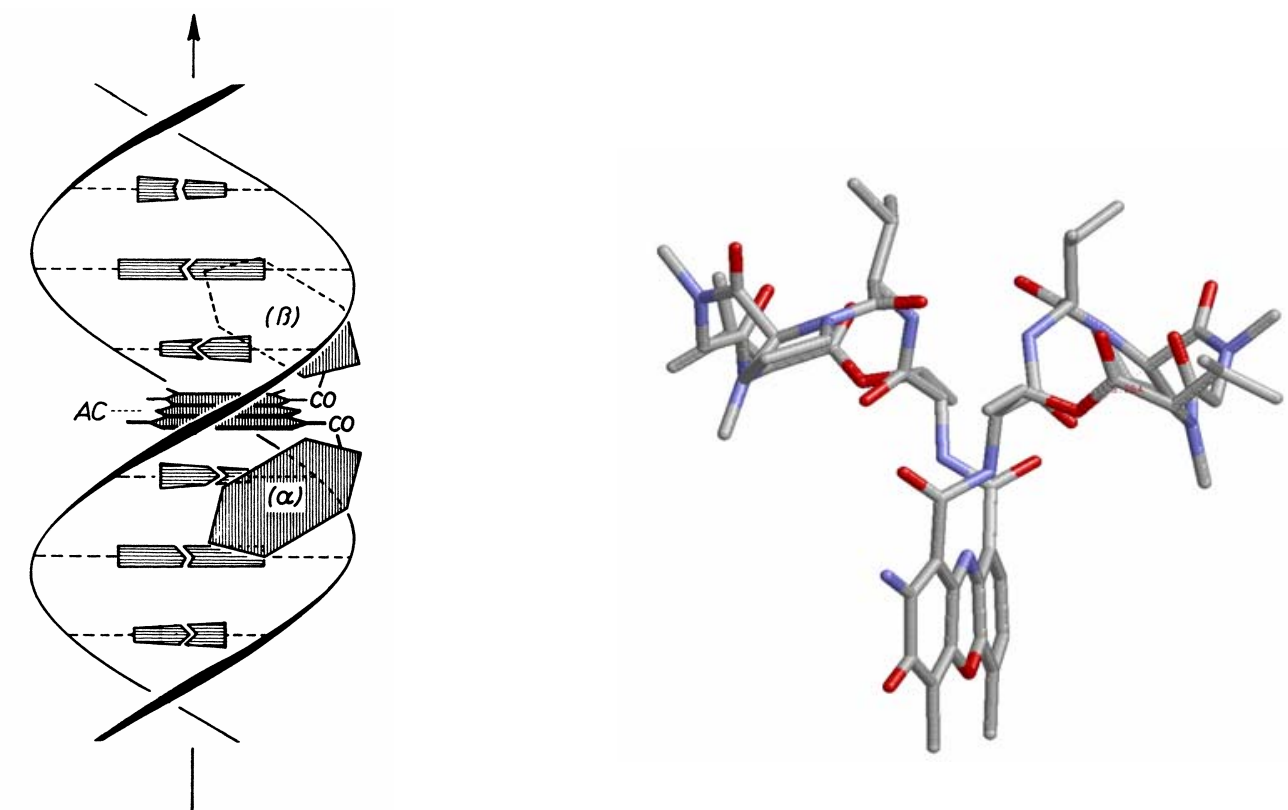

Abbildung 21: Interkalation mit DNA (links, übernommen aus ${ }^{[34]}$ ). Die Kristallstruktur von Actinomycin D (19, rechts) zeigt die pseudo $\mathrm{C}_{2}$-symmetrische Konformation ${ }^{[35,49]}$. 
Aufgrund des nicht-selektiven Wirkmechanismus ist die antitumorale und antibakterielle Aktivität mit einer hohen Toxizität verknüpft. Trotzdem kommt Actinomycin D als Chemotherapeutikum bei einigen Krebsarten zur Anwendung, so z. B. in Kombination mit Vincristin zur Behandlung bestimmter Nierentumore bei Kindern (Wilms Tumor) ${ }^{[50]}$. Bei in vivo Tests einiger Actinomycin-Analoga konnte eine durch pharmakokinetische Effekte verursachte Selektivität für Leukämie-Zellen festgestellt werden ${ }^{[51]}$.

\subsubsection{Testung der neuen Actinomycine}

Die in dieser Arbeit isolierten Actinomycine wurden mittels Plattendiffusionstests auf antibakterielle und antifungische Aktivität getestet. Die cytotoxische Wirkung gegenüber Tumorzelllinien wurde an der Medizinischen Hochschule Hannover geprüft. ${ }^{\mathrm{g}}$

Antimikrobielle Wirkung: Eine Hemmung trat bei Gram-positiven und Gram-negativen Bakterien auf (Tabelle 5), eine antifungische Wirkung wurde nicht festgestellt. Die stärkste antibakterielle Wirkung besitzt Actinomycin $\mathrm{G}_{2}$ (12), das 4-Chlorthreonin im $\beta$-Ring enthält. Steht an dieser Stelle 4-Hydroxythreonin wie bei 13, ist die Aktivität deutlich geringer. Eine weitere Verringerung der Wirkung tritt bei Actinomycin $G_{5}$ (15) auf, während das umgelagerte Actinomycin $G_{6}$ (16) inaktiv ist. Keines der G-Actinomycine erreicht die Wirkhöhe der Vergleichssubstanz Actinomycin D (19).

Cytotoxische Wirkung: Unter den natürlich vorkommenden Actinomycinen ist $\mathrm{X}_{2}$ (20) dasjenige mit der höchsten cytotoxischen Aktivität ${ }^{[52]}$. Es wurde daher neben dem in der klinischen Anwendung befindlichen Actinomycin D (19) als Vergleichssubstanz gewählt. Die stärkste Wirkung unter den G-Actinomycinen zeigte wie bei den antibakteriellen Tests die chlorierte Verbindung 12. Die GI50- und TGI-Werte für 12 bei den Zelllinien HM02 und Hep2G (Magen- bzw. Leberkarzinom) liegen zwischen denen von Actinomycin D (19) und $\mathrm{X}_{2}$ (20) und beweisen eine sehr hohe Cytotoxizität (Tabelle 6). Die nicht-chlorierte Substanz 14 hemmte das Wachstum der Zellen deutlich schlechter, jedoch immer noch besser als die am Threoninrest hydroxylierte Komponente 13. Nur noch eine schwache Restwirkung zeigten die beiden strukturell andersartigen Actinomycine $G_{5}$ (15) und $G_{6}(\mathbf{1 6})$, in der gleichen Größenordnung liegt auch die Wirkung von 1-Phenazinol (17).

\footnotetext{
${ }^{\mathrm{g}}$ Herrn Prof. Dr. W. Beil (Institut für Pharmakologie, Medizinische Hochschule Hannover) danke ich für die Durchführung der Cytotoxizitäts-Tests.
} 
Tabelle 5: Antibakterielle Wirkung der Actinomycine. Angegeben ist der HemmhofDurchmesser beim Plattendiffusionstest in [mm].

\begin{tabular}{|c|c|c|c|}
\hline & Escherichia coli & $\begin{array}{c}\text { Staphylococcus } \\
\text { aureus }\end{array}$ & Bacillus subtilis \\
\hline Actinomycin D (19) & 20 & 30 & 55 \\
\hline Actinomycin $\mathrm{G}_{2}(\mathbf{1 2})$ & 15 & 27 & 45 \\
\hline Actinomycin $\mathrm{G}_{3}(\mathbf{1 3})$ & 0 & 21 & 33 \\
\hline Actinomycin $\mathrm{G}_{5}(\mathbf{1 5})$ & 0 & 10 & 20 \\
\hline Actinomycin $\mathrm{G}_{6}(\mathbf{1 6})$ & 0 & 0 & 0 \\
\hline
\end{tabular}

$50 \mu \mathrm{g}$ Substanz auf Filterpapier-Plättchen, Ø $6 \mathrm{~mm}$ 
Tabelle 6: Ergebnisse der Cytotoxizitäts-Tests

\begin{tabular}{|c|c|c|}
\hline & $\mathrm{GI} 50[\mu \mathrm{g} / \mathrm{mL}]$ & TGI $[\mu \mathrm{g} / \mathrm{mL}$ \\
\hline Tumorzelllinie & \multicolumn{2}{|c|}{ HM02 (Magenkarzinom) } \\
\hline Actinomycin D (19) & 0.002 & 0.008 \\
\hline Actinomycin $\mathrm{X}_{2}(\mathbf{2 0})$ & 0.0012 & 0.005 \\
\hline Actinomycin $\mathrm{G}_{2}(\mathbf{1 2})$ & 0.0017 & 0.0055 \\
\hline Actinomycin $\mathrm{G}_{3}(\mathbf{1 3})$ & 0.16 & 0.7 \\
\hline Actinomycin $\mathrm{G}_{4}(\mathbf{1 4})$ & 0.082 & 0.65 \\
\hline Actinomycin $\mathrm{G}_{5}(\mathbf{1 5})$ & 2.7 & 5.5 \\
\hline Actinomycin $\mathrm{G}_{6}(\mathbf{1 6})$ & 1.3 & 2.6 \\
\hline 1-Phenazinol (17) & 5.6 & 9.5 \\
\hline Tumorzelllinie & \multicolumn{2}{|c|}{ HepG2 (Leberkarzinom) } \\
\hline Actinomycin D (19) & 0.0015 & 0.065 \\
\hline Actinomycin $X_{2}(\mathbf{2 0})$ & 0.0021 & 0.018 \\
\hline Actinomycin $\mathrm{G}_{2}(\mathbf{1 2})$ & 0.0038 & 0.023 \\
\hline Actinomycin $\mathrm{G}_{3}(\mathbf{1 3})$ & 1.4 & $>10$ \\
\hline Actinomycin $\mathrm{G}_{4}(\mathbf{1 4})$ & 0.8 & 1.8 \\
\hline Actinomycin $\mathrm{G}_{5}(\mathbf{1 5})$ & 3.4 & 9.8 \\
\hline Actinomycin $\mathrm{G}_{6}(\mathbf{1 6})$ & 2.1 & $>10$ \\
\hline 1-Phenazinol (17) & 4.2 & $>10$ \\
\hline Tumorzelllinie & \multicolumn{2}{|c|}{ MCF7 (Mammakarzinom) } \\
\hline Actinomycin D (19) & 0.0024 & 0.011 \\
\hline Actinomycin $X_{2}(20)$ & 0.0024 & 0.01 \\
\hline Actinomycin $\mathrm{G}_{2}(\mathbf{1 2})$ & 0.005 & 0.016 \\
\hline Actinomycin $\mathrm{G}_{3}(\mathbf{1 3})$ & 0.58 & 2.0 \\
\hline Actinomycin $\mathrm{G}_{4}(\mathbf{1 4})$ & 0.50 & 1.9 \\
\hline Actinomycin $\mathrm{G}_{5}(\mathbf{1 5})$ & 1.7 & 3.1 \\
\hline Actinomycin $\mathrm{G}_{6}(\mathbf{1 6})$ & 1.8 & 5.8 \\
\hline 1-Phenazinol (17) & 3.4 & $>10$ \\
\hline
\end{tabular}




\subsubsection{Diskussion der biologischen Aktivität}

Vergleich der Wirkung mit Actinomycin $G_{1}$ und $Z_{1}$ bis $Z_{5}$

Von dem zu 13 sehr ähnlichen Actinomycin $G_{1}$ (11) wird in der Literatur eine schwache Wirkung gegen Gram-positive Bakterien berichtet, es konnte allerdings keine Interkalation mit doppelsträngiger DNA festgestellt werden ${ }^{[38]}$. Unklar bleibt, wie diese beiden scheinbar widersprüchlichen Informationen zusammen passen. Möglicherweise ist die antibiotische Wirkung durch eine im Vergleich zu Actinomycin D (19) schwächere, jedoch nicht ausgebliebene Interkalation zu erklären.

Die Ergebnisse der vorliegenden Arbeit korrelieren besser mit den für die Z-Actinomycine veröffentlichten Daten ${ }^{[36]}$. Die cytotoxische und antibakterielle Wirkung der Chlorthreoninhaltigen Verbindungen $Z_{3}$ und $Z_{5}$ ist etwas stärker als die der Vergleichssubstanz Actinomycin D (19), die Aktivität der Hydroxythreonin-haltigen Verbindung $Z_{1}$ ist deutlich schwächer.

\section{Struktur-Wirkungsbeziehungen}

Die nur schwache antibiotische Wirkung der Actinomycine $G_{5}(\mathbf{1 5})$ und $G_{6}(\mathbf{1 6})$ ist vermutlich auf deren „gestörte“ Raumstruktur zurückzuführen. Sowohl die Veränderung des Chromophors bei $\mathbf{1 5}$ als auch die vollkommen veränderte Konformation des $\beta$-Rings von 16 behindern die Einlagerung der Substanz in die DNA und damit ihre biologische Wirkung. Ein Austausch der Aminogruppe des Chromophors bewirkt ebenfalls eine signifikant geringere Affinität zu doppelsträngiger $\mathrm{DNA}^{[33]}$.

Als Ursache für die besonders starke Wirkung der Chlorthreonin enthaltenden Actinomycine wie 12 und Actinomycin $Z_{3}$ wird eine partiell auftretende kovalente Bindung an die DNA durch nucleophile Substitution vorgeschlagen ${ }^{[53]}$, jedoch können optimierte nicht-kovalente Wechselwirkungen nicht ausgeschlossen werden. Die nur schwache Aktivität bei Actinomycinen mit hydroxyliertem Threoninrest am $\alpha$-Ring ist vermutlich auf die Ausbildung einer intramolekularen Wasserstoffbrückenbindung von der zusätzlichen Hydroxyfunktion zur Aminogruppe des Chromophors zurückzuführen. Letztere ist wie oben erwähnt für die Affinität zur DNA essentiell, das Molekül wird daher schwächer gebunden. 


\subsection{Die Substanzklasse der Actinomycine}

\subsubsection{Entdeckung der Actinomycine und Nomenklatur}

Actinomycin A wurde 1940 von WAKSMAN aus Streptomyces antibioticus isoliert und war das erste aus Actinomyceten erhaltene Antibiotikum ${ }^{[54]}$.

Die weiteren Untersuchungen zeigten, dass es sich um ein Gemisch aus mehreren Einzelkomponenten handelte. Diese als Komplexe bezeichneten Gemische wurden in unterschiedlicher Zusammensetzung auch in anderen Streptomyceten gefunden und mit lateinischen Buchstaben gekennzeichnet, die Benennung der Einzelkomponenten eines Komplexes erfolgte durch eine Zahl als Index ${ }^{[33]}$. Da die gefundenen Gemische oft dieselben Substanzen enthielten, jedoch in unterschiedlichen Verhältnissen, existiert für die meisten Actinomycine eine Reihe synonymer Bezeichnungen (siehe Tabelle 7). Versuche zur Einführung einer systematischen Nomenklatur durch Nummerierung der Actinomycine oder Beschreibung veränderter Aminosäuren im Vergleich zu Actinomycin D haben sich nicht durchgesetzt ${ }^{[33]}$.

Tabelle 7: Wichtige Actinomycine und ihre Synonyme (die linke Spalte enthält die gebräuchlichste Bezeichnung) ${ }^{[55]}$.

\begin{tabular}{l|l} 
Actinomycin & Synonyme \\
\hline Actinomycin D & $\begin{array}{l}\text { Actinomycin IV / } \mathrm{A}_{4} / \mathrm{B}_{4} / \mathrm{C}_{1} / \mathrm{D}_{4} / \mathrm{F}_{0} / \mathrm{I}_{1} / \mathrm{S}_{2} / \mathrm{X}_{1} / \mathrm{Au}_{3} \\
\text { Auranthin } \mathrm{C} / \mathrm{A} 3, \text { Cosmogen, Dactinomycin, Oncostatin } \mathrm{K}\end{array}$ \\
Actinomycin $\mathrm{C}_{2}$ & Actinomycin VI / $\mathrm{I}_{2} / \mathrm{Au}_{2}$, Auranthin B $/ \mathrm{A} 2$ \\
Actinomycin $\mathrm{C}_{3}$ & Actinomycin VII $/ \mathrm{I}_{3} / \mathrm{Au}_{1}$, Auranthin A \\
Actinomycin $\mathrm{X}_{2}$ & Actinomycin V / $\mathrm{A}_{5} / \mathrm{B}_{5} / \mathrm{D}_{5} / \mathrm{S}_{3}$ \\
\hline
\end{tabular}

\subsubsection{Struktureller Aufbau}

Actinomycine sind Chromopeptide. Sie besitzen als gemeinsamen Actinoyl-Chromophor ein 2-Aminophenoxazin-3-on-System, das zweifach methyliert und über zwei Amidbindungen mit Pentapeptidlacton-Ringen verknüpft ist.

Als Grundstruktur kann Actinomycin D (19) angesehen werden (Abbildung 22), von dem sich durch Abwandlung oder Modifikation einzelner Aminosäuren die anderen Strukturen ableiten 
lassen. Neben den proteinogenen Aminosäuren $L$-Threonin, $L$-Valin und $L$-Prolin sind in 19 auch die nicht-proteinogenen Aminosäuren Sarkosin ${ }^{\mathrm{h}}$ und $D$-Valin enthalten. Bei zwei gleichen Peptidlactonringen wie bei 19 spricht man von iso-Actinomycinen, bei unterschiedlichen Ringen von aniso-Actinomycinen.

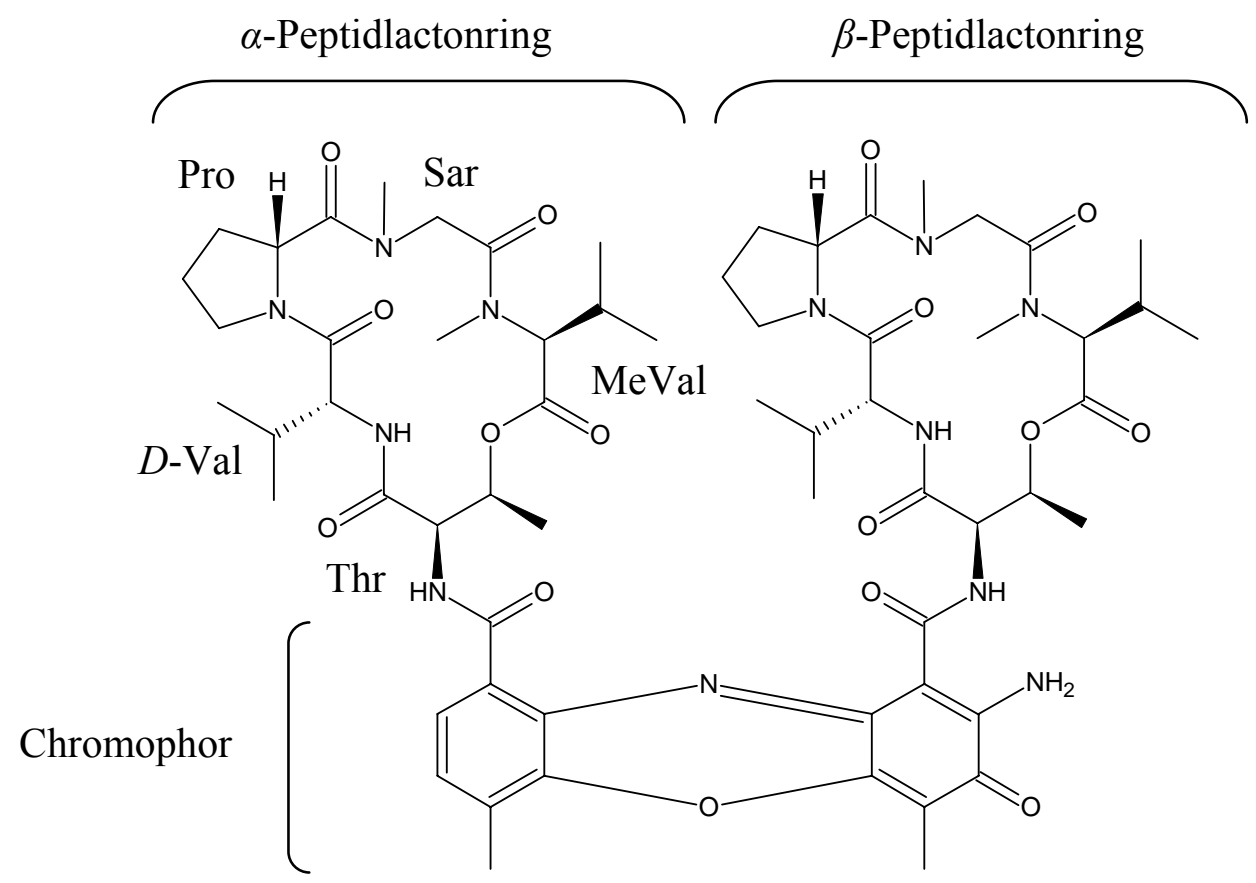

Abbildung 22: Struktur von Actinomycin D (19).

Der erste Strukturbeweis eines Actinomycins gelang BROCKMANN und LACKNER 1960 durch die Totalsynthese von Actinomycin $\mathrm{C}_{3}{ }^{[56]}$. Heute sind insgesamt 19 Actinomycine natürlicher Herkunft bekannt (siehe Tabelle 8), hinzu kommen die fünf in dieser Arbeit beschriebenen neuen Strukturen vom G-Typ.

\subsubsection{Vorläuferdirigierte Biosynthese}

Durch vorläuferdirigierte Biosynthese konnten zahlreiche neue Actinomycine gewonnen werden, bis heute sind über 25 Strukturen veröffentlicht worden. Als besonders variabel hat sich die Position 3' in den Peptidringen herausgestellt, neben modifizierten Prolinringen werden hier auch Sechsringe wie Pipecolinsäure oder Vierringe wie Azetidincarbonsäure

\footnotetext{
${ }^{\text {h }}$ Als Sarkosin bezeichnet man $N$-Methylglycin. Der Name geht zurück auf Justus von Liebig, der die Aminosäure erstmals aus dem Kreatin der Fleischbrühe gewann (griech.: sarx = Fleisch).
} 
eingebaut. Eine weitere Modifikationsmöglichkeit bietet die Position 2' der Pentapeptide. Fütterungsexperimente ergaben, dass $D$-Valin durch $D$-Leucin und $D$-allo-Isoleucin austauschbar ist. Tabelle 9 listet alle bisher veröffentlichten Actinomycine auf, die aus vorläuferdirigierter Biosynthese erhalten wurden. 
Tabelle 8: Systematische Ordnung der natürlich vorkommenden Actinomycine.

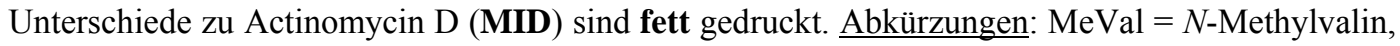
$a \mathrm{Ile}=$ allo-Isoleucin, MeAla $=N$-Methylalanin, HMPro $=$ trans-3-Hydroxy-cis-5-methylprolin, OPro = 3-Oxoprolin, MOPro = cis-5-Methyl-3-oxoprolin, Hyp = trans-4-Hydroxyprolin, $a$ Hyp = cis-4-Hydroxyprolin, HThr = 4-Hydroxythreonin, ClThr = 4-Chlorthreonin.

\section{(1) Actinomycin D}

$\begin{array}{lll} & \boldsymbol{\alpha} \text {-Ring } & \boldsymbol{\beta} \text {-Ring } \\ \text { Actinomycin D } & \text { Thr- } D \text {-Val-Pro-Sar-MeVal } & \text { Thr- } D \text {-Val-Pro-Sar-MeVal }\end{array}$

\section{(2) Actinomycine der C-Reihe (Variationen in Position 2' durch D-aIle) ${ }^{[57-59]}$}

Actinomycin $\mathrm{C}_{2}$ Actinomycin $\mathrm{C}_{2 \mathrm{a}}$ Actinomycin $\mathrm{C}_{3}$
Thr- $D$-Val-Pro-Sar-MeVal Thr-D-aIle-Pro-Sar-MeVal Thr-D-aIle-Pro-Sar-MeVal
Thr-D-aIle-Pro-Sar-MeVal Thr- $D$-Val-Pro-Sar-MeVal Thr-D-aIle-Pro-Sar-MeVal

(3) Actinomycine der F-Reihe (Variation in Position 3' durch Sarkosin) ${ }^{[60-62]}$

$\begin{array}{lll}\text { Actinomycin } \mathrm{F}_{8} & \text { Thr- } D \text {-Val-Sar-Sar-MeVal } & \text { Thr- } D \text {-Val-Sar-Sar-MeVal } \\ \text { Actinomycin } \mathrm{F}_{9} & \text { Thr- } D \text {-Val-Sar-Sar-MeVal } & \text { Thr- } D \text {-Val-Pro-Sar-MeVal } \\ & \text { Thr- } D \text {-Val-Pro-Sar-MeVal } & \text { Thr- } D \text {-Val-Sar-Sar-MeVal }\end{array}$

Anmerkung: Actinomycin $\mathrm{F}_{9}$ besteht aus zwei Isomeren, die auch als Actinomycin III und IIIA bezeichnet werden (Sarkosin in $\beta$ - bzw. $\alpha$-Kette) ${ }^{[63]}$. $\mathrm{F}_{0}$ bis $\mathrm{F}_{7}$ wurden mittels vorläuferdirigierter Biosynthese erhalten, hier tritt zusätzlich eine Variation in Position 2' durch alle auf.

(4) Actinomycine der X-Reihe (Var. in Pos. 3' durch oxidiertes Prolin oder Sarkosin) ${ }^{[52,64-66]}$

$\begin{array}{lll}\text { Actinomycin } \mathrm{X}_{0 \alpha} & \text { Thr- } D \text {-Val-Sar-Sar-MeVal } & \text { Thr- } D \text {-Val-Hyp-Sar-MeVal } \\ \text { Actinomycin } \mathrm{X}_{0 \beta} & \text { Thr- } D \text {-Val-Pro-Sar-MeVal } & \text { Thr- } D \text {-Val-Hyp-Sar-MeVal } \\ \text { Actinomycin } \mathrm{X}_{0 \delta} & \text { Thr- } D \text {-Val-Pro-Sar-MeVal } & \text { Thr- } D \text {-Val-aHyp-Sar-MeVal } \\ \text { Actinomycin } \mathrm{X}_{1 \mathrm{a}} & \text { Thr- } D \text {-Val-Sar-Sar-MeVal } & \text { Thr- } D \text {-Val-OPro-Sar-MeVal } \\ \text { Actinomycin } \mathrm{X}_{2} & \text { Thr- } D \text {-Val-Pro-Sar-MeVal } & \text { Thr- } D \text {-Val-OPro-Sar-MeVal }\end{array}$

(5) Actinomycine der G- ${ }^{[38]}$ und Z-Reihe $^{[36]}$ (Var. in Pos. 1' durch hydroxyliertes/chloriertes Threonin, in Pos. 3' durch methyliertes und oxidiertes Prolin und in Pos. 5' durch MeAla)

$\begin{array}{lll}\text { Actinomycin } \mathrm{G}_{1} & \text { Thr- } D \text {-Val-Pro-Sar-MeVal } & \text { HThr- } D \text {-Val-HMPro-Sar-MeAla } \\ \text { Actinomycin } \mathrm{Z}_{1} & \text { Thr- } D \text {-Val-HMPro-Sar-MeVal } & \text { Thr- } D \text {-Val-MOPro-Sar-MeAla } \\ \text { Actinomycin } \mathrm{Z}_{2} & \text { Thr- } D \text {-Val-HMPro-Sar-MeVal } & \text { HThr- } D \text {-Val-MOPro-Sar-MeAla } \\ \text { Actinomycin } \mathrm{Z}_{3} & \text { Thr- } D \text {-Val-HMPro-Sar-MeVal } & \text { CIThr- } D \text {-Val-MOPro-Sar-MeAla } \\ \text { Actinomycin } \mathrm{Z}_{4} & \text { Thr- } D \text {-Val-MOPro-Sar-MeVal } & \text { Thr- } D \text {-Val-MOPro-Sar-MeAla } \\ \text { Actinomycin } \mathrm{Z}_{5} & \text { Thr- } D \text {-Val-MOPro-Sar-MeVal } & \text { CIThr- } D \text {-Val-MOPro-Sar-MeAla }\end{array}$

(6) $\mathrm{N}$-desmethylierte Actinomycine (Variation in Position 4' durch Glycin) ${ }^{[55,67]}$

Actinomycin $\mathrm{D}_{0} \quad$ Thr- $D$-Val-Pro-Sar-MeVal

$N, N$-Didemethyl-

actinomycin D
Thr-D-Val-Pro-Gly-MeVal
Thr-D-Val-Pro-Gly-MeVal

Thr-D-Val-Pro-Gly-MeVal 
Tabelle 9: Actinomycine, die aus vorläuferdirigierter Biosynthese erhalten wurden.

\section{Fütterung von $\boldsymbol{L}$-Isoleucin: ${ }^{[68,69]}$}<smiles>CC[C@H](C)C(N)C(=O)O</smiles>

Actinomycin $\mathrm{E}_{1}$

$\alpha$-Kette

Actinomycin $\mathrm{E}_{2}$

Thr-D-aIle-Pro-Sar-MeVal

$\beta$-Kette

$($ Melle $=N$-Methyl- $L$-isoleucin, $a$ Ile $=$ allo- $L$-Isoleucin $)$

Thr-D-aIle-Pro-Sar-MeIle

Thr-D-aIle-Pro-Sar-MeIle

\section{Fütterung von $L$-Leucin und $L$-Isoleucin:}<smiles>CC(C)CC(N)C(=O)O</smiles><smiles>CC[C@H](C)C(N)C(=O)O</smiles>

Actinomycin $\mathrm{A}_{\mathrm{u} 5}{ }^{[70]}$

Actinomycin $\mathrm{A}_{\mathrm{u} 6 \mathrm{a}}$ Actinomycin $\mathrm{A}_{\mathrm{u} 6 \mathrm{~b}}{ }^{[71]}$

Actinomycin $\mathrm{A}_{\mathrm{u} 7 \mathrm{a}}$ Actinomycin $\mathrm{A}_{\mathrm{u} 7 \mathrm{~b}}{ }^{[72]}$.
Thr-D-Leu-Pro-Sar-MeVal

Thr-D-aIle-Pro-Sar-MeVal

Thr-D-Leu-Pro-Sar-MeVal

Thr-D-Leu-Pro-Sar-MeVal

Thr- $D$-Val-Pro-Sar-MeVal
Thr-D-Leu-Pro-Sar-MeVal

Thr-D-Leu-Pro-Sar-MeVal

Thr-D-aIle-Pro-Sar-MeVal

Thr-D-Val-Pro-Sar-MeVal

Thr-D-Leu-Pro-Sar-MeVal

Anmerkung: Actinomycin $\mathrm{Au}_{7 \mathrm{~b}}$ wurde unter der Bezeichnung Actinomycin $\mathrm{C}_{3 \mathrm{a}}$ auch ohne Zufütterung von Aminosäuren gefunden, jedoch nicht veröffentlicht ${ }^{[73]}$.

\section{Fütterung von Sarkosin: ${ }^{[61]}$}<smiles>CNCC(=O)O</smiles>

Actinomycin $\mathrm{F}_{1}$ Actinomycin $\mathrm{F}_{2}$ Actinomycin $\mathrm{F}_{3}$ Actinomycin $\mathrm{F}_{4}$ Anmerkung: Die Struk
Thr- $D$-Val-Sar-Sar-MeVal

Thr- $D$-Val-Pro-Sar-MeVal

Thr-D-aIle-Sar-Sar-MeVal

Thr-D-aIle-Pro-Sar-MeVal
Thr-D-aIle-Sar-Sar-MeVal

Thr-D-aIle-Sar-Sar-MeVal

Thr-D-aIle-Sar-Sar-MeVal

Thr-D-aIle-Sar-Sar-MeVal

\section{Fütterung von (4R)- und (4S)-4-Methyl- $L$-prolin: ${ }^{[74]}$}

cis-4-Methyl- $L$-prolin:

Actinomycin $\mathrm{K}_{1 \mathrm{c}}$ Actinomycin $\mathrm{K}_{2 \mathrm{c}}$ trans-4-Methyl- $L$-prolin: Actinomycin $\mathrm{K}_{1 \mathrm{t}}$ Actinomycin $\mathrm{K}_{2 \mathrm{t}}$
Thr- $D$-Val-Pro-Sar-MeVal Thr- $D$-Val-MPro-Sar-MeVal

Thr- $D$-Val-Pro-Sar-MeVal Thr- $D$-Val-MPro-Sar-MeVal<smiles>C[C@@H]1CN[C@H](C(=O)O)C1</smiles>

Thr- $D$-Val-MPro-Sar-MeVal Thr- $D$-Val-MPro-Sar-MeVal

Thr- $D$-Val-MPro-Sar-MeVal Thr- $D$-Val-MPro-Sar-MeVal 
... Fortsetzung Tabelle 9: Actinomycine aus vorläuferdirigierter Biosynthese

\section{Fütterung von (4R)- und (4S)-4-Chlor- $L$-prolin: ${ }^{[33]}$}

cis-4-Chlor-L-prolin:

unbenannt $(*)$

unbenannt

trans-4-Chlor-L-prolin:

unbenannt $\left(^{*}\right)$
Thr- $D$-Val-Pro-Sar-MeVal

Thr- $D$-Val-CIPro-Sar-MeVal

Thr-D-Val-Pro-Sar-MeVal<smiles>O=C(O)C1C[C@@H](Cl)CN1</smiles>

Thr- $D$-Val-CIPro-Sar-MeVal Thr- $D$-Val-CIPro-Sar-MeVal

Thr- $D$-Val-CIPro-Sar-MeVal

Fütterung von Pipecolinsäure: ${ }^{[75]}$<smiles>O=C(O)C1CCCCN1</smiles>

Pipecolinsäure (Pip)<smiles>O=C(O)C1CC(O)CCN1</smiles>

4-Hydroxy- (HPip)<smiles>O=C1CCNC(C(=O)O)C1</smiles>

4-Oxo- (OPip)
Actinomycin Pip $1_{\alpha}(*)$ Actinomycin Pip $1_{\beta}\left(^{*}\right)$ Actinomycin Pip $1_{\gamma}(*)$ Actinomycin Pip $1_{\S}(*)$ Actinomycin Pip $1_{\xi}(*)$ Actinomycin Pip 2 (*) $^{*}$
Thr- $D$-Val-Pip-Sar-MeVal Thr- $D$-Val-Pip-Sar-MeVal Thr- $D$-Val-Pip-Sar-MeVal Thr- $D$-Val-Pro-Sar-MeVal Thr- $D$-Val-Pro-Sar-MeVal Thr- $D$-Val-Pip-Sar-MeVal
Thr-D-Val-OPip-Sar-MeVal Thr-D-Val-Pro-Sar-MeVal Thr- $D$-Val-HPip-Sar-MeVal Thr-D-Val-OPip-Sar-MeVal Thr-D-Val-HPip-Sar-MeVal Thr- $D$-Val-Pip-Sar-MeVal

\section{Fütterung von Thiazolidin-4-carbonsäure: ${ }^{[76]}$}

Der Einbau von Thiazolidin-4-carbonsäure in zwei neue Actinomycine konnte durch ${ }^{35}$ S-Isotopenmarkierung nachgewiesen werden. Die Struktur der Verbindungen wurde nicht geklärt, vermutlich handelt es sich analog zu den Azetomycinen um einen einfachen und doppelten<smiles>O=C(O)C1CSCN1</smiles>
Einbau von Thiazolidin-4-carbonsäure anstelle von Prolin.

Fütterung von Azetidin-2-carbonsäure: ${ }^{[77,78]}$<smiles>O=C(O)C1CCN1</smiles>

Azetomycin I (*) Azetomycin II
Thr- $D$-Val-Pro-Sar-MeVal Thr- $D$-Val-AzC-Sar-MeVal
Thr- $D$-Val-AzC-Sar-MeVal Thr- $D$-Val-AzC-Sar-MeVal

(*) bei den gekennzeichneten Substanzen wurde nicht untersucht, ob der Einbau im $\alpha$ - oder im $\beta$-Ring erfolgte. Wahrscheinlich ist das Vorliegen von Gemischen. 


\subsection{Zur Biosynthese der Actinomycine}

\subsubsection{Biosynthese von Actinomycin D (19)}

Die Biosynthese von Actinomycin D (19) lässt sich in drei Schritte unterteilen:

1. Die Bildung von 4-Methyl-3-hydroxyanthranilsäure (4-MHA) aus L-Tryptophan ... erfolgt in mehreren enzymatisch katalysierten Schritten ${ }^{[79]}$ (Abbildung 23).<smiles>CC(=O)CC(N)Cc1c[nH]c2ccccc12</smiles>

L-Tryptophan<smiles>NC(CC(=O)c1ccccc1NC=O)C(=O)O</smiles>

<smiles>Nc1ccccc1C(=O)CC(N)C(=O)O</smiles>

Kynurenin $\mid \begin{aligned} & +\mathrm{O}_{2}, \mathrm{NADPH} / \mathrm{H}^{+} \\ & -\mathrm{H}_{2} \mathrm{O}, \mathrm{NADP}^{+}\end{aligned}$<smiles>Cc1ccc(C(=O)O)c(N)c1O</smiles>

4-Methyl-3-hydroxyanthranilsäure (4-MHA)<smiles>Nc1c(O)cccc1C(=O)O</smiles><smiles>Nc1c(O)cccc1C(=O)CC(N)C(=O)O</smiles>

3-Hydroxyanthranilsäure

Abbildung 23: Biosynthese von 4-Methyl-3-hydroxyanthranilsäure.

2. Die nichtribosomale Synthese des Pentapeptidlactons mit 4-MHA als Starteinheit

... benötigt insgesamt vier Multienzymkomplexe. Zunächst wird 4-MHA durch die Actinomycin-Synthetase I (ACMSI) als Adenylat aktiviert. Dieses wird auf das Actinomycin-Acyl-Carrier-Protein $(A c m A C P)$ übertragen, 4-MHA ist nun als Thioester gebunden.

Der Aufbau des Pentapeptids geschieht durch fünf NRPS-Module, die auf die beiden multifunktionellen Enzyme Actinomycin-Synthetase II und III (ACMS II und III) verteilt sind. ACMS II besitzt zwei der Module und katalysiert die Aktivierung und Kondensation von Threonin und Valin an die als Thioester vorliegende Starteinheit. Das zweite Modul enthält zusätzlich eine Epimerisierungs-Funktion, die das Substrat $L$-Valin nach dem Einbau zu D-Valin epimerisiert. Die drei Module von ACMS III bewirken die 
Verlängerung der Peptidkette durch Prolin, Glycin und Valin, die beiden letzteren werden jeweils durch eine Methylierungs-Domäne zu Sarkosin bzw. $N$-Methylvalin umgesetzt. Das letzte Modul enthält zusätzlich eine Thioesterase-Funktion, die wahrscheinlich neben der Abspaltung vom Protein auch die Ausbildung der Lactonbindung katalysiert ${ }^{[80]}$.

\section{Oxidative Kupplung von zwei 4-MHA-Pentapeptidlactonen zu Actinomycin}

Der letzte Schritt der Actinomycin-Biosynthese ist bisher nicht geklärt. Lange Zeit wurde vermutet, dass hierfür das Enzym Phenoxazin-Synthase (PHS) verantwortlich sei. Tatsächlich wurde ein solches Enzym bei Streptomyces antibioticus gefunden und es katalysiert auch die oxidative Kondensation von Aminophenolen zu Aminophenoxazonen. Bei anderen Actinomycin-Produzenten wie S. chrysomallus fehlt dieses Enzym jedoch, und Inaktivierung der PHS-Gene in S. antibioticus bewirkt keine Verringerung der Actinomycin-Produktion. Es muss daher ein anderer Kupplungsmechanismus vorliegen, der sowohl enzymatischer als auch nicht-enzymatischer Natur sein könnte ${ }^{[80,81]}$.

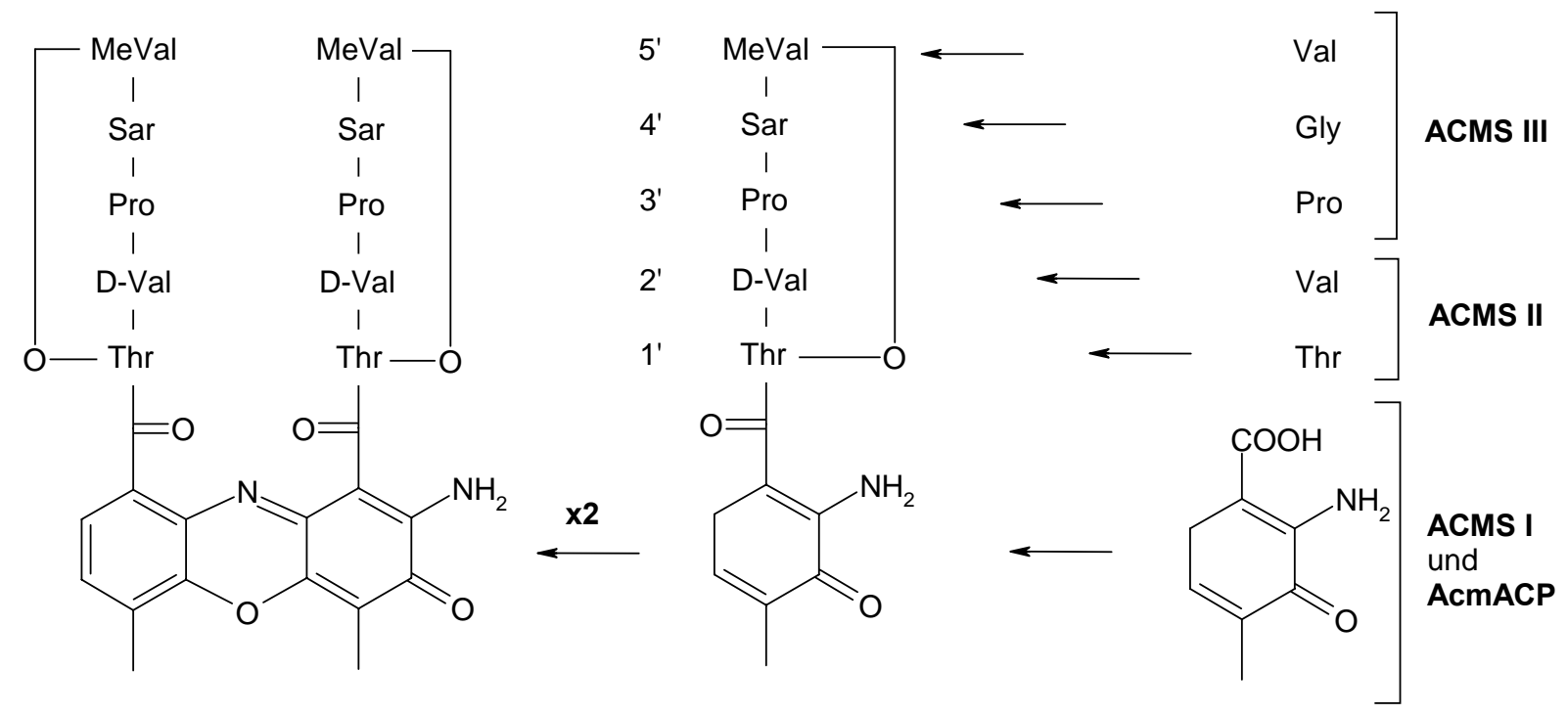

18

Abbildung 24: Biosynthetischer Aufbau der Pentapeptid-Einheit und anschließende Kupplung zum Actinomycin D (19). 


\subsubsection{Diskussion der Biosynthese der G-, X- und Z-Actinomycine}

Während bei den C- und F-Actinomycinen wahrscheinlich eine geringe Substratspezifität der zweiten und dritten Adenylierungsdomäne den Einbau anderer Aminosäuren ermöglicht, gibt es bisher keine Untersuchungen zur Biosynthese der übrigen aniso-Actinomycine. Diese zeigen eine klare Präferenz bezüglich der Seitenkette, in der sich variierte Aminosäuren befinden. Bei den X-Actinomycinen findet man einen oxidierten Prolinring ausschließlich im $\beta$-Ring, bei den G-Actinomycinen hingegen im $\alpha$-Ring. Den G- und Z-Actinomycinen ist gemeinsam, dass sowohl $N$-Methylalanin als auch 4-Chlor- oder 4-Hydroxythreonin nur im $\beta$-Ring vorkommen.

Diese Präferenz für einen bestimmten Ring macht einen direkten Einbau modifizierter Aminosäuren durch die NRPS-Enzyme unwahrscheinlich. In diesem Fall wäre zu erwarten, dass nach der Kondensation zweier 4-MHA-Pentapeptidlactone alle denkbaren Regioisomere gefunden würden, also z. B. auch $N$-Methylalanin im $\alpha$-Ring der G-Actinomycine. Da die Oxidation zum Aminophenoxazon bereits durch Luftsauerstoff erfolgen kann, ist eine vollständige Kontrolle der Dimerisierung durch die Phenoxazin-Synthase $(P H S)$ oder ein ähnliches Enzym nur schwierig erreichbar.

An der Modifikation der Aminosäuren sind daher wahrscheinlich post-NRPS-Enzyme beteiligt, deren Substrate nicht die einzelnen Aminosäuren oder das Pentapeptidlacton sind, sondern das zunächst entstandene iso-Actinomycin. Fraglich ist diese Hypothese im Hinblick auf die Biogenese des Methylalanins in Position 5', da bisher weder ein Abbau des an dieser Stelle üblichen Valins zu Alanin noch ein Selektivität erlaubender Kondensationsmechanismus von zwei Monomereinheiten zum Actinomycin beschrieben wurde. Ein besseres Verständnis des dritten Biosynthese-Schritts (Kupplung der ortho-Aminophenole) ist eine essentielle Voraussetzung für die Aufklärung der Biogenese der aniso-Actinomycine. 


\section{$3 \quad$ Neue Aminophenoxazone aus Halomonas sp. Rk377a}

\subsection{Der Stamm Halomonas sp. Rk377a}

Der Stamm Rk377a gehört zur Gattung Halomonas und damit zu den $\gamma$-Proteobakterien. Er wurde war in einer Wasserprobe enthalten, die der Nordsee bei Norddeich-Mole entnommen wurde $^{\mathrm{i}}$. L. LIANG konnte im Rahmen ihrer Dissertation ${ }^{[82]}$ aus diesem Stamm 13 Substanzen isolieren, unter ihnen viele Indol-Derivate (Abbildung 25).<smiles>O=C1Nc2ccccc2C1=O</smiles>

21<smiles>O=C(O)Cc1c[nH]c2ccccc12</smiles>

23<smiles>O=C(O)c1c[nH]c2ccccc12</smiles>

25<smiles>O=C(O)C(=O)CC1(O)CNc2ccccc21</smiles>

$22(*)$<smiles>O=C(CO)c1c[nH]c2ccccc12</smiles>

24<smiles>[R]c1ccc(-c2c(C(=O)O)[nH]c(C(=O)O)c2-c2ccc(O)cc2)cc1</smiles>

29: $\mathrm{R}=\mathrm{H}, 30 \mathrm{R}:=\mathrm{OH}$<smiles>O=C1CSc2cc(O)ccc2N1</smiles>

31<smiles>O=C(O)Cc1c(S)c(=O)ncn1C(=O)c1c[nH]c2ccccc12</smiles>

27 (*)<smiles>O=Cc1c[nH]c2ccccc12</smiles>

26

Abbildung 25: Bekannte Metaboliten aus Halomonas sp. Rk377a.<smiles>O=C(O)CC1N=C(S)C(CC(=O)O)N1C(=O)c1c[nH]c2ccccc12</smiles>

28 (*)

21 = Isatin, $\quad 22$ = 3-(3-Hydroxy-2,3-dihydroindol-3-yl)-2-oxopropionsäure,$\quad 23$ = Indol-3-essigsäure, $\mathbf{2 4}$ = 3-Hydroxyacetylindol, $\quad 25$ = Indol-3-carbonsäure,$\quad \mathbf{2 6}=\quad$ Indol-3-carboxaldehyd, $27=$ Glusun I, $\mathbf{2 8}$ = Glusun II, 29 = 3-(4'-Hydroxyphenyl)-4-phenylpyrrol-2,5-dicarbonsäure, 30 = 3,4-Di(4'-hydroxyphenyl)pyrrol-2,5-dicarbonsäure, $31=7$-Hydroxybenzo[1,4]thiazin-3-on. Nicht gezeigt sind die ebenfalls beschriebene Phenylessigsäure und Bis(isooctylphthalat).

(*) Die Strukturen 22, 27 und 28 sind nicht gesichert und als Vorschläge anzusehen.

\footnotetext{
${ }^{\text {i }}$ Der Stamm wurde von Frau Dr. H. Steven (Arbeitskreis Prof. Simon, ICBM, Universität Oldenburg) im Rahmen des vom Land Niedersachsen und der VW-Stiftung geförderten Niedersächsischen Forschungsschwerpunkts Meeresbiotechnologie isoliert und charakterisiert.
} 


\subsection{Neue Aminophenoxazon-Derivate durch veränderte Kultivierungsbedingungen}

Die Auswirkungen von veränderten Kultivierungsbedingungen auf Wachstum und Metabolitenspektrum des Stamms Rk377a wurden in der Diplomarbeit von T. GROßE ${ }^{[83]}$ untersucht. Der Zusatz von Biosynthesevorläufern zum Kulturmedium führte zu einer Ausbeutesteigerung der entsprechenden Substanzen. So bewirkte die Zugabe von Tyrosin eine vermehrte Produktion von 29 und 30, und die Zugabe von Indol steigerte die Biosynthese von Indol-Derivaten.

Die Anwesenheit von Anthranilsäure bei der Fermentation führte zu einer Schwarzfärbung der Zellen und des Überstands. Neben unlöslichen dunklen Pigmenten, bei denen es sich möglicherweise um Melanine handelt, wurde ein orange-roter Farbstoff produziert, der im Ethylacetatextrakt enthalten war und eine UV-Absorption bei $430 \mathrm{~nm}$ zeigte. Die interessante Veränderung des Metabolitenspektrums bei Zugabe von Anthranilsäure wurde in dieser Arbeit durch Isolierung und Strukturaufklärung der im Extrakt enthaltenen Substanzen untersucht und wird im Folgenden beschrieben.

\subsubsection{Isolierung der Reinsubstanzen}

Der vollständig in Methanol lösliche Extrakt wurde durch Gelchromatographie in zwölf Fraktionen getrennt. Diese wurden, soweit erforderlich, durch weitere chromatographische Schritte getrennt bzw. gereinigt (siehe Abbildung 74 auf Seite 156). Es wurden insgesamt zwölf Substanzen in reiner Form erhalten. Ca. 80\% des Extrakts bestanden aus Polyisopropylenglycol (32), das bei der Fermentation als Antischaummittel verwendet wurde. Weiterhin enthielt der Extrakt fünf Diketopiperazine (33 - 37) und den Weichmacher Di(isooctylphthalat) (38). Die orange Färbung konnte auf fünf Aminophenoxazone (39 - 43) zurückgeführt werden, von denen drei bisher nicht beschriebene Strukturen darstellen.

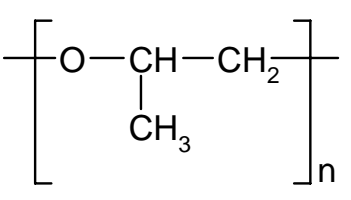

32

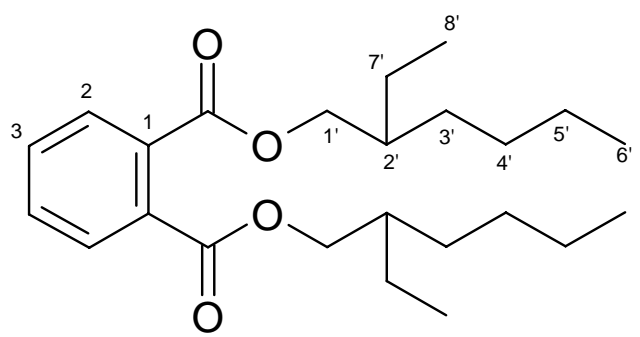

38 


\subsubsection{Cyclo(prolylvalyl) (33)}

Der farblose Feststoff färbt auf dem Dünnschichtchromatogramm mit Anisaldehyd oder Orcin-Sprühreagenz in der Wärme braun an und hat eine Molmasse von $196 \mathrm{~g} / \mathrm{mol}$. Das ${ }^{1}$ H-NMR-Spektrum zeigt das Vorhandensein einer Isopropylgruppe, deren diastereotope Methylgruppen jeweils zu einem Dublett aufgespalten sind $\left(\delta_{\mathrm{H}}=0.93\right.$ und 1.10). Aromatische oder olefinische Protonen sind nicht vorhanden. Die Suche mit diesen Informationen in AntiBase ${ }^{[41]}$ erbrachte Cyclo(prolylvalyl) (33) als Strukturvorschlag, der durch den Vergleich mit Literaturdaten ${ }^{[84]}$ bestätigt wurde.

Das Fragmentierungsmuster des EI-Massenspektrums von 33 ist charakteristisch für ein Prolin enthaltendes Diketopiperazin. Die Spektren enthalten ein Fragment bei $\mathrm{m} / \mathrm{z}=154$, das durch Abspaltung des Rests der zweiten Aminosäure entsteht (Abbildung 26).

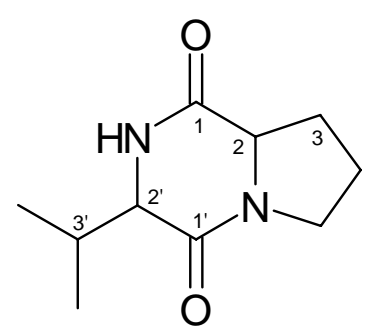

33

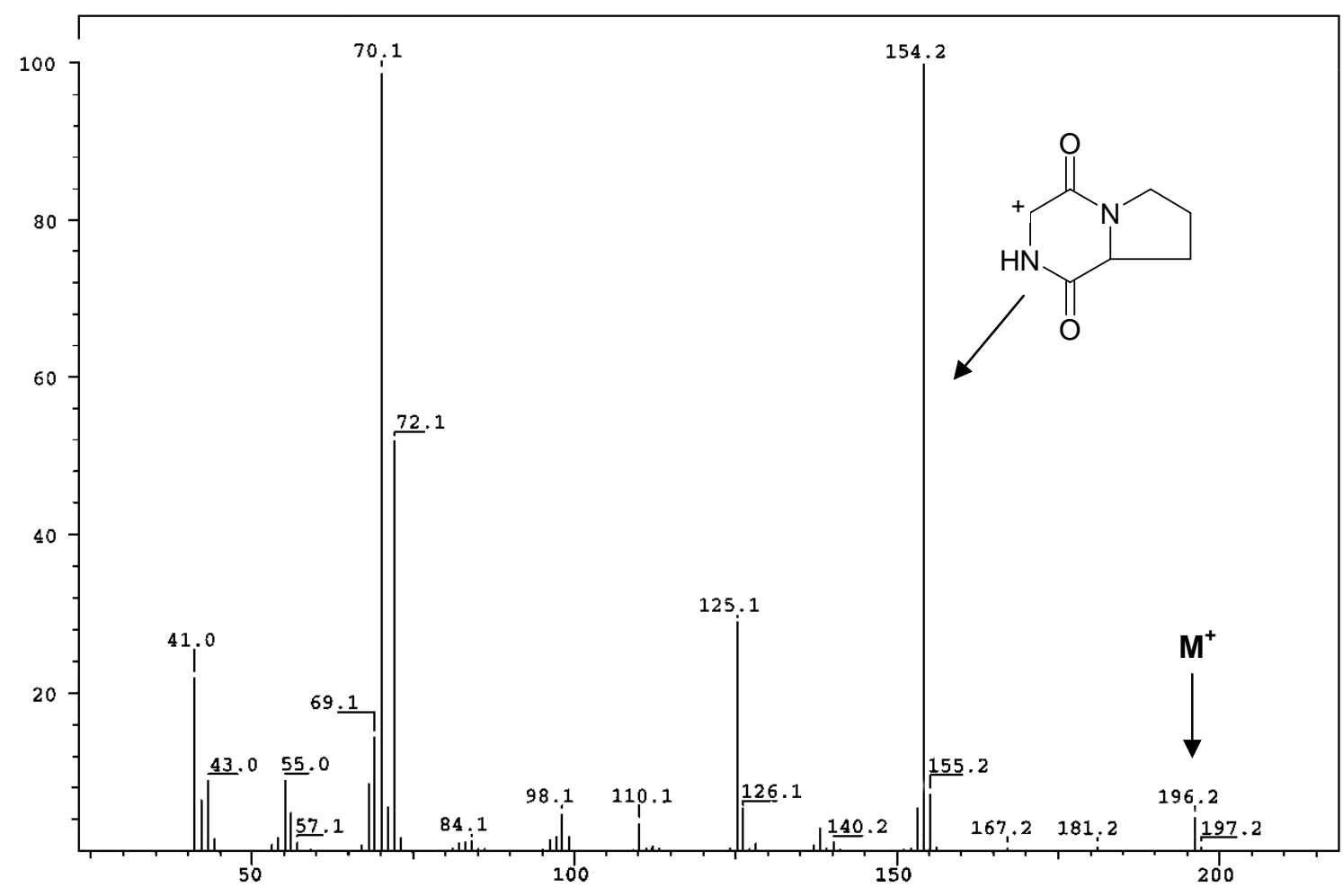

Abbildung 26: EI-Massenspektrum von Cyclo(prolylvalyl) (33). 
Diketopiperazine wie 33 entstehen durch Kondensation von zwei Aminosäuren und sind sowohl aus Bakterien als auch aus Pilzen bekannt. Sie sind biosynthetisch ebenso wie synthetisch $^{[85]}$ leicht zugänglich und werden häufig beim Screening von mikrobiellen Extrakten gefunden. Auffällig ist eine Präferenz für die Aminosäure Prolin als Baustein ${ }^{[86]}$.

Vertretern dieser Substanzklasse wird in der Literatur eine ganze Palette biologischer Aktivitäten zugeschrieben, unter anderem antibakteriell ${ }^{[84]}$, antifungisch ${ }^{[87]}$, phytotoxisch $^{[88]}$, als Signalstoff in Quorum-Sensing Systemen ${ }^{[89]}$ und als Inhibitoren verschiedener Enzyme (Bsp. in ${ }^{[90,91]}$ ). Diese Vielzahl an biologischen Wirkungen korreliert sowohl mit dem häufigen Auffinden dieser Substanzklasse als auch mit ihrem einfachen Aufbau aus zwei Aminosäuren, der eine hohe Affinität zu Biomolekülen erwarten lässt.

Natürlich vorkommende Diketopiperazine bestehen in der Regel aus zwei L-Aminosäuren, dies wird auch für die aus Halomonas sp. Rk377a isolierten Verbindungen 33 - 37 angenommen. Es sind jedoch auch $D L$-, $L D$ - und $D D$-Kombinationen bekannt. Diese können aus dem $L L$-Isomer durch nicht-enzymatische Epimerisierung entstehen ${ }^{[86]}$, die durch Nachbargruppeneffekte begünstigt wird ${ }^{[92]}$. Leider erfolgte in der Literatur häufig eine fehlerhafte Bestimmung der Stereozentren ${ }^{[86]}$, so dass keine genaue Aussage bezüglich der Häufigkeit von $D$-Aminosäuren in Diketopiperazinen gemacht werden kann.

\subsubsection{Cyclo(leucylprolyl) (34)}

Das EI-Massenspektrum des farblosen Feststoffs 34 zeigt ein Ion höchster Masse bei $\mathrm{m} / \mathrm{z}=$ 210 und das typische Fragment eines Prolin enthaltenden Diketopiperazins bei $\mathrm{m} / \mathrm{z}=154$. Im ${ }^{1}$ H-NMR-Spektrum finden sich Signale für zwei Methylgruppen, zwei Methin- und eine Methylengruppe neben Heteroatomen sowie drei weitere Methylengruppen. Die im Vergleich zu 33 um 14 g/mol höhere Molmasse kann somit auf eine zusätzliche Methylengruppe zurückgeführt werden. Die hieraus folgende Annahme, dass Leucin anstelle von Valin vorliegt, konnte durch die Aufnahme eines ${ }^{13} \mathrm{C}$-NMR-Spektrums und den Vergleich mit Literaturdaten ${ }^{[87]}$ bestätigt werden. Bei der Substanz 34 handelt es sich um das Diketopiperazin<smiles>CC(C)CC1NC(=O)C2CCCN2C1=O</smiles>
Cyclo(leucylprolyl). 


\subsubsection{Cyclo(isoleucylprolyl) (35)}

Substanz 35 fiel als farbloser Feststoff an, dessen Molmasse zu 210 g/mol bestimmt wurde. Das von 33 und 34 schon bekannte Fragment bei m/z=154 im EI-Massenspektrum und das ähnliche chromatographische Verhalten ließen vermuten, dass es sich hier ebenfalls um ein Diketopiperazin und somit ein Isomer von $\mathbf{3 4}$ handelt. Die Übereinstimmung der chemischen Verschiebungen des ${ }^{1} \mathrm{H}-\mathrm{NMR}-$ Spektrums mit Literaturdaten $^{[87]}$ ermöglichte die Identifizierung als Cyclo(isoleucylprolyl) (35).<smiles>CCC(C)C1NC(=O)C2CCCN2C1=O</smiles>

35

\subsubsection{Cyclo(phenylalanylprolyl) (36)}

Die Molmasse wurde durch EI-Massenspektrometrie zu $244 \mathrm{~g} / \mathrm{mol}$ bestimmt, das Fragmentierungsmuster wies auf ein Prolin enthaltendes Diketopiperazin hin. Die zwei Heteroatom-benachbarten Methingruppen bei $\delta_{\mathrm{H}}=4.06$ und 4.44 erinnern an die ${ }^{1}$ H-NMR-Spektren der Diketopiperazine 33 und 34, ebenso finden sich die drei<smiles>O=C1NC(Cc2ccccc2)C(=O)N2CCCC12</smiles>

36 Methylengruppen des Prolins wieder. Zusätzlich enthält das Spektrum von 36 die Resonanzen fünf aromatischer Protonen, die zu einer Phenylgruppe gehören $\left(\delta_{H} \approx 7.25\right)$, und einer ins Tieffeld verschobenen Methylengruppe $\left(\delta_{H}=3.14 / 3.17\right)$. Die zweite Aminosäure konnte dem entsprechend als Phenylalanin und das gesamte Diketopiperazin als Cyclo(phenylalanylprolyl) (36) identifiziert werden, dessen chemische Verschiebungen im ${ }^{1} \mathrm{H}-\mathrm{NMR}-$ Spektrum mit publizierten Werten ${ }^{[87]}$ übereinstimmen. 


\subsubsection{Cyclo(prolyltyrosyl) (37)}

Das ${ }^{1}$ H-NMR-Spektrum von 37 stimmt im aliphatischen Bereich sehr gut mit dem Spektrum von Cyclo(phenylalanylprolyl) (36) überein. Anstelle einer Phenylgruppe finden sich im aromatischen Bereich die Resonanzen eines para-disubstituierten Aromaten, erkennbar an zwei Dubletts, die für je zwei Protonen integrieren. Deren chemische Verschiebung $\left(\delta_{\mathrm{H}}=\right.$ 6.69 und 7.03) legt Sauerstoff als einen Substituenten nahe, übereinstimmend hiermit wird im EI-Massenspektrum eine im Vergleich zu 36 um 16 Einheiten höhere Molmasse von $260 \mathrm{~g} / \mathrm{mol}$ gefunden. Diese Daten charakterisieren 37 als Cyclo(prolyltyrosyl), es ist das fünfte aus dem Extrakt des Stamms Halomonas sp. Rk377a isolierte Diketopiperazin.<smiles>O=C1NC(Cc2ccc(O)cc2)C(=O)N2CCCC12</smiles>

37

\subsubsection{2-Aminophenoxazin-3-on (39)}

Der rote kristalline Feststoff hat eine Molmasse von $212 \mathrm{~g} / \mathrm{mol}$, das EI-Massenspektrum lieferte durch Abgleich mit der Wiley EI-MS-Datenbank 2-Aminophenoxazin-3-on (39) als beste Übereinstimmung. Diese Zuordnung wurde unterstützt durch die<smiles>Nc1cc2nc3ccccc3oc-2cc1=O</smiles>

39 Absorptionsmaxima bei 237, 421 und $431 \mathrm{~nm} \mathrm{im}$ UV-Spektrum von 39 (vgl. UV-Spektren der Actinomycine auf Seite 13). Eine zweifelsfreie Bestätigung konnte durch die NMR-Spektren gewonnen werden, die mit publizierten Daten ${ }^{[93-}$ ${ }^{95]}$ übereinstimmen.

Als nicht trivial gestaltete sich die Zuordnung der NMR-Daten zu den jeweiligen Atomen, da diese bei jeder der hier zitierten Literaturstellen anders erfolgt. Aufgrund ihrer chemischen Verschiebung im NMR-Spektrum sind die Carbonylgruppe $\left(\delta_{\mathrm{C}}=180\right)$ sowie das hierzu in $\beta$-Position befindliche Kohlenstoffatom C-1 $\left(\delta_{\mathrm{C}}=98\right)$ zweifelsfrei erkennbar. Die Zuordnung der weiteren Kohlenstoffatome erfolgte anhand eines HMBC-Spektrums (siehe Abbildung $27)$, wobei die Unterscheidung zwischen den quartären Kohlenstoffatomen C-5a $\left(\delta_{\mathrm{C}}=142\right)$ und C-9a $\left(\delta_{\mathrm{C}}=134\right)$ durch Zuordnung des höheren $\delta$-Wertes zum Sauerstoff-substituierten Atom erfolgte. Unsicher bleibt die Differenzierung zwischen C-4a und C-10a $\left(\delta_{\mathrm{C}}=147\right.$ und 148), Inkrementberechnungen ${ }^{[96]}$ legen eine höhere chemische Verschiebung von C-10a nahe. 


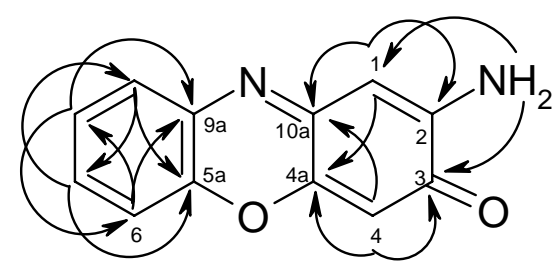

Abbildung 27: HMBC-Korrelationen in 39.

Die sehr intensiv farbige Substanz 39 wurde in einer Ausbeute von $6 \mathrm{mg} / \mathrm{L}$ erhalten und ist hauptsächlich verantwortlich für die rot-orange Färbung des Rohextraktes. Daneben wurden geringere Mengen ebenfalls farbiger Derivate von 39 gefunden (40-43). Ein 2-Aminophenoxazon-Gerüst liegt auch dem Chromophor der Actinomycine zu Grunde (siehe Kapitel 2).

Ausgangspunkt der Biosynthese ist vermutlich die dem Nährmedium zugegebene Anthranilsäure. Durch Einführung einer Hydroxygruppe in Position 3 (diese Reaktion kann durch das Enzym Anthranilat-3-monooxidase katalysiert werden ${ }^{[97]}$ ) und Decarboxylierung entsteht ortho-Aminophenol, das durch oxidative Kupplung zu 2-Aminophenoxazon umgesetzt wird. Für den letzten Schritt kommt eine Phenoxazin-Synthase als Enzym in Frage, auch die nicht-enzymatische Oxidation durch reaktive Sauerstoffspezies ${ }^{[98]}$ oder Elektronenazeptoren wie Flavin ${ }^{[99]}$ ist möglich (vgl. Kapitel 2.11). 


\subsubsection{2-Amino-6-hydroxyphenoxazin-3-on (40)}

Die Verbindung 40 ist ebenso wie 39 ein roter Feststoff, dessen UV-Spektrum ein Aminophenoxazon-Grundgerüst erkennen lässt. Ein zusätzliches Absorptionsmaximum bei $278 \mathrm{~nm}$ weist zugleich auf eine Veränderung im Chromophor hin. Anhand der im Vergleich zu 39 um $16 \mathrm{~g} / \mathrm{mol}$ höheren Molmasse und der durch ESI-<smiles>Nc1cc2nc3cccc(O)c3oc-2cc1=O</smiles>

40 Hochauflösung erhaltenen Summenformel $\mathrm{C}_{12} \mathrm{H}_{8} \mathrm{~N}_{2} \mathrm{O}_{3}$ konnte auf eine Hydroxygruppe am Chromophor geschlossen werden. Deren Position ergab sich aus dem Protonenresonanzspektrum, in dem drei aromatische Protonen in ortho-Stellung zueinander zu erkennen sind. Die Unterscheidung zwischen C-6 und C-9 als Träger der Hydroxygruppe erfolgte anhand der chemischen Verschiebungen: wäre die Hydroxygruppe in Position 9, so würde C-9a aufgrund der +M-Effekte von O-5 und O-9 eine Resonanz bei hohem Feld zeigen ( $<130$ ppm), C-5a und C-9 hingegen wegen der -I-Effekte bei tiefem Feld $(>140 \mathrm{ppm})$. Bei einer Hydroxygruppe an C-6 ständen beide sauerstoff-substituierten C-Atome ortho zueinander, infolge dessen sich $+\mathrm{M}$ - und -I-Effekte teilweise ausgleichen würden. Auf C-5a wirkt zusätzlich der + M-Effekt von N-10. Die beobachteten Resonanzen bei $\delta_{\mathrm{C}}=131(\mathrm{C}-9 \mathrm{a})$ und $135(\mathrm{C}-5 \mathrm{a})$ entsprechen der letzteren Annahme und legen die Struktur als 2-Amino-6-hydroxyphenoxazin-3-on (40) fest.

Dieses mit einer Ausbeute von ca. $1 \mathrm{mg} / \mathrm{L}$ erhaltene Aminophenoxazon-Derivat wurde in der Literatur bisher nicht beschrieben. Ähnliche Verbindungen sind z. B. Chandrananimycin A ${ }^{[94]}$ (Hydroxygruppe an C-9, Aminogruppe acetyliert), 2-Amino-7-hydroxy-phenoxazin-3-on ${ }^{[100]}$ und 2-Amino-7-methoxy-phenoxazin-3-on ${ }^{[101]}$. An C-6 monosubstituierte Aminophenoxazone sind nicht bekannt, die Substitution an C-6 geht stattdessen meist mit einer zweiten funktionellen Gruppe an C-9 einher. Dieses Substitutionsmuster findet man auch beim Actinoyl-Chromophor der Actinomycine, der an C-6 und C-4 je eine Methylgruppe trägt, an C-9 und C-1 je eine Carboxylgruppe. 


\subsubsection{Chandrananimycin C (41)}

Der rote Feststoff $\mathbf{4 1}$ wurde in Ausbeuten von $1.5 \mathrm{mg} / \mathrm{L}$ erhalten und seine Molmasse durch EI-Massenspektrometrie zu $296 \mathrm{~g} / \mathrm{mol}$ bestimmt. Das UV-Spektrum ließ analog zu 40 einen modifizierten AminophenoxazonChromophor erwarten. Eine Datenbanksuche mit diesen Informationen in AntiBase ${ }^{[41]}$ führte $\mathrm{zu}$ Chandrananimycin C (41), dessen publizierte ${ }^{1}$ H-NMR-Daten ${ }^{[94]}$ mit<smiles>COC1CC(C)NC2=C1C1=Nc3ccccc3OC2=CC1=O</smiles>

41 den gemessenen Werten übereinstimmen.

Als Produzent von $\mathbf{4 1}$ wird in der Literatur ebenfalls ein Bakterium aus einem marinen Habitat genannt (Actinomadura sp.). Für Chandrananimycin C (41) wird eine Wirkung gegen Gram-positive Bakterien, Algen und Tumorzelllinien beschrieben ${ }^{[94]}$.

\subsubsection{2-Amino-8-benzoyl-6-hydroxyphenoxazin-3-on (42)}

Eine weitere rote Nebenkomponente wurde mit einer Ausbeute von ca. $1 \mathrm{mg} / \mathrm{L}$ erhalten. Ihre Molmasse beträgt $332 \mathrm{~g} / \mathrm{mol}$, durch eine ESI-MS-Hochauflösung wurde die Summenformel $\mathrm{C}_{19} \mathrm{H}_{12} \mathrm{~N}_{2} \mathrm{O}_{4}$ ermittelt. Neben dem UV-Spektrum bestätigen auch die NMR-Daten das Vorliegen eines Aminophenoxazin-Grundgerüsts. Das ${ }^{13} \mathrm{C}-\mathrm{NMR}-$ Spektrum zeigt im Vergleich zu 40 eine zusätzliche Ketogruppe $\left(\delta_{C}=194\right)$ und sechs weitere aromatische Kohlenstoffatome, die von einem Phenylrest stammen. Die beiden Protonen des in der Strukturformel mit „A“ gekennzeichneten Rings erscheinen als Dubletts, aufgrund ihrer Kopplungskonstante $\left({ }^{4} J_{\mathrm{HH}}=2.0 \mathrm{~Hz}\right)$ müssen sie sich in meta-Position zueinander befinden. Beide zeigen ebenso wie die ortho-ständigen Protonen der Phenylgruppe im HMBCSpektrum eine Kopplung zur Ketogruppe bei $\delta_{C}=194$. In Übereinstimmung mit der Struktur von 40 konnte 42 daher als 2-Amino-8-benzoyl-6-hydroxyphenoxazin-3-on bestimmt werden.

42 ist weder als Naturstoff noch als Syntheseprodukt bekannt. Generell wurden in der Literatur bisher keine 6,8-disubstituierten Aminophenoxazone beschrieben.<smiles>Nc1cc2nc3cc(C(=O)c4ccccc4)cc(O)c3oc-2cc1=O</smiles> 


\subsubsection{2-Amino-6-hydroxy-8-(4-hydroxybenzoyl)-phenoxazin-3-on (43)}

Das vierte Pigment mit intensiv roter Eigenfarbe wurde in einer Ausbeute von $0.5 \mathrm{mg} / \mathrm{L}$ isoliert. Das ${ }^{1} \mathrm{H}-\mathrm{NMR}-$ Spektrum zeigt die von 42 bekannten vier Protonen des 6,8-disubstituierten Aminophenoxazon-Grundgerüsts: $1-\mathrm{H}$ und 4-H erscheinen als Singuletts bei<smiles>Nc1cc2nc3cc(C(=O)c4ccc(O)cc4)cc(O)c3oc-2cc1=O</smiles>

43 relativ hohem Feld $\left(\delta_{\mathrm{H}}=6.34\right.$ und 6.37), 7-H und 9-H sind durch eine meta-Kopplung zu Dubletts aufgespalten $\left(\delta_{\mathrm{H}}=7.11\right.$ und 7.17). Anstelle eines Phenylrests wie bei 42 ist ein paradisubstituierter Aromat enthalten, erkennbar an zwei für je zwei Protonen integrierenden Signalen $\left(\delta_{\mathrm{H}}=6.81\right.$ und 7.65), die eine ortho-Kopplung von $9 \mathrm{~Hz}$ zeigen. Die chemischen Verschiebungen lassen auf einen über Sauerstoff gebundenen Substituenten schließen, der anhand der Molmasse als Hydroxygruppe festgelegt werden kann. Hochauflösende ESIMassenspektrometrie bestätigt die Summenformel der Verbindung 43, deren systematischer Name 2-Amino-6-hydroxy-8-(4'-hydroxybenzoyl)-phenoxazin-3-on lautet. 43 ist neben 42 ein weiterer Vertreter der bisher nicht bekannten 6,8-disubstituierten Aminophenoxazone.

\subsection{Biologische Aktivitäten der Aminophenoxazone}

Die erstmalig beschriebenen Aminophenoxazon-Derivate 40, 42 und 43, Chandranamimycin C (41) und das allen Derivaten zugrunde liegende 2-Aminophenoxazin-3on (39) wurden auf antibakterielle, antifungische und cytotoxische Wirkung getestet.

Antimikrobielle Wirkung: Die stärkste Hemmwirkung gegen Gram-positive Bakterien zeigte das hydroxylierte Aminophenoxazon 40, etwas schwächer wirkte die strukturell einfachste Substanz 39 und eine weitere Abschwächung war bei 41 zu beobachten. Die Wirkung aller drei Substanzen war deutlich schwächer als die von Actinomycin D (19), das ebenfalls ein Aminophenoxazon-System besitzt und daher als Vergleich gewählt wurde. Die in der Literatur ${ }^{[94]}$ berichtete antibakterielle Wirkung von 41 konnte nicht reproduziert werden, auch 43 zeigte keine Aktivität gegen die Testkeime. 39 konnte als einzige Testsubstanz das Wachstum des Hefepilzes hemmen. 
Cytotoxizität: Die cytotoxische Wirkung ${ }^{\mathrm{j}}$ der getesteten Aminophenoxazone 39, $\mathbf{4 0}$ und 42 ist im Vergleich zu Actinomycin D (19) gering (Tabelle 11). Die ermittelten GI50-Werte liegen bei der HM02-Zelllinie (Magenkarzinom) etwa um den Faktor 500 (39) bis 1000 (40) höher als bei 19, die TGI-Werte sind jeweils ca. 300fach höher. Bei den Zelllinien HepG2 und MCF7 (Leber- bzw. Mammakarzinom) sind die Unterschiede zu 19 ähnlich groß, jedoch kann man eine deutlich stärkere Aktivität von 2-Aminophenoxazin-3-on (39) im Vergleich zu den in Position 6 hydroxylierten Derivaten 40 und 42 erkennen. Der Benzoylrest in Position 8 bei 42 hat hingegen kaum Einfluss auf die Wirkung der Substanz. Die stärkste Wirkung zeigte 39 bei der MCF7-Zelllinie: GI50- und TGI-Werte sind um eine Größenordnung kleiner als bei den anderen Aminophenoxazonen, trotzdem noch ca. 40mal höher als die entsprechenden Werte von 19.

Tabelle 10: Antimikrobielle Wirkung der Aminophenoxazone. Angegeben ist der HemmhofDurchmesser beim Plattendiffusionstest in [mm], Actinomycin D diente als Vergleich.

\begin{tabular}{l|cccc} 
& $\begin{array}{c}\text { Escherichia } \\
\text { coli }\end{array}$ & $\begin{array}{c}\text { Bacillus } \\
\text { subtilis }\end{array}$ & $\begin{array}{c}\text { Staphylococcus } \\
\text { aureus }\end{array}$ & $\begin{array}{c}\text { Candida } \\
\text { albicans }\end{array}$ \\
\hline Actinomycin D (19) & 16 & 35 & 25 & 0 \\
39 & 0 & 20 & 15 & 25 \\
$\mathbf{4 0}$ & 0 & 25 & 16 & 0 \\
$\mathbf{4 1}$ & 0 & 0 & 0 & 0 \\
$\mathbf{4 2}$ & 0 & 16 & 10 & 0 \\
$\mathbf{4 3}$ & 0 & 0 & 0 & 0 \\
\hline
\end{tabular}

$50 \mu \mathrm{g}$ Substanz auf Filterpapier-Plättchen, Ø $6 \mathrm{~mm}$

\footnotetext{
${ }^{\mathrm{j}}$ Für die Durchführung der Cytotoxizitäts-Tests bedanke ich mich bei Herrn Prof. Dr. W. Beil (Institut für Pharmakologie, Medizinische Hochschule Hannover).
} 
Tabelle 11: Cytotoxische Wirkung der Aminophenoxazone im Vergleich zu Actinomycin D.

\begin{tabular}{l|cc} 
& GI50 $[\mu \mathrm{g} / \mathrm{mL}]$ & TGI $[\mu \mathrm{g} / \mathrm{mL}]$ \\
\hline Tumorzelllinie & \multicolumn{2}{|c}{ HM02 (Magenkarzinom) } \\
\hline Actinomycin D (19) & 0.002 & 0.008 \\
39 & 0.95 & 2.2 \\
$\mathbf{4 0}$ & 1.6 & 2.6 \\
$\mathbf{4 2}$ & 1.35 & 2.6 \\
\hline Tumorzelllinie & HepG2 (Leberkarzinom) \\
\hline Actinomycin D (19) & 0.0015 & 0.065 \\
39 & 1.4 & 5.6 \\
$\mathbf{4 0}$ & 4.3 & $>10$ \\
$\mathbf{4 2}$ & 3.2 & 10.0 \\
\hline Tumorzellinie & MCF7 (Mammakarzinom) \\
\hline Actinomycin D (19) & 0.0024 & 0.011 \\
39 & 0.13 & 0.42 \\
$\mathbf{4 0}$ & 1.6 & 2.7 \\
$\mathbf{4 2}$ & 2.0 & 3.2 \\
\hline
\end{tabular}

GI50 = Konzentration, die eine Abnahme des Zellwachstums um die Hälfte bewirkt.

TGI = Konzentration, die eine vollständige Hemmung des Zellwachstums bewirkt.

\subsection{Diskussion und Ausblick}

Neue Metaboliten durch veränderte Kultivierungsbedingungen

Die Zugabe von Anthranilsäure zum Kulturmedium von Halomonas sp. Rk377a führte zur Produktion neuer chemischer Verbindungen. Bemerkenswert ist, dass sich das Metabolitenspektrum so vollständig änderte, dass keine der zuvor produzierten Substanzen mehr nachgewiesen werden konnte. Neben der Produktion verschiedener Diketopiperazine wurde stattdessen der zugefütterte Baustein vom Stamm zum Aufbau des bekannten 2-Aminophenoxazin-3-ons (39) genutzt, welches regioselektiv an Position 6 hydroxyliert wurde (40). Eine weitere Substitution erfolgte an Position 8 durch einen Benzoylrest (42), der zusätzlich in para-Stellung hydroxyliert werden konnte (43). Weder die Monosubstitution an C-6 noch die Disubstitution an C-6 und C-8 sind bisher in der Literatur beschrieben worden. 40, 42 und 43 stellen somit neuartige Strukturvarianten eines bekannten Grundkörpers und die

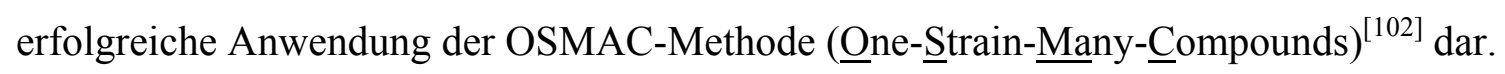




\section{Induktion der Melanin-Bildung}

Die wahrscheinlich auf Melanin-Bildung zurückzuführende Schwarzfärbung der Zellen könnte sowohl durch die Anthranilsäure als auch durch die hieraus entstandenen Aminophenoxazone induziert worden sein. Zur Klärung dieser Frage steht der Versuch aus, ob die Zufütterung von 2-Aminophenoxazon (39) ebenfalls zur vermehrten MelaninProduktion führt.

\section{Zur biologischen Wirkung der Aminophenoxazon-Derivate}

Die etwas stärkere antimikrobielle Wirkung des 6-Amino-6-hydroxyphenoxazin-3-ons (40) im Vergleich zu 39 ist überraschend, wenn man von einem Wirkmechanismus ähnlich dem der Actinomycine ausgeht. Bei letzteren erschweren Modifikationen am Chromophor die Interkalation mit dem DNA-Doppelstrang (siehe Kapitel 2.9). Möglicherweise wirkt sich hier die bessere Löslichkeit von $\mathbf{4 0}$ gegenüber 39 positiv aus, oder die Hydroxylierung hat eine Verbesserung der Pharmakodynamik wie z. B. einen erleichterten Transport ins Zellinnere zur Folge. Eine weitere Substitution in Position 8 bewirkt eine deutliche Abschwächung der Aktivität. Da zudem sowohl die achiralen Strukturen selbst als auch ihr hypothetischer Wirkmechanismus keine Selektivität in der Wirkung erwarten lassen, ist von einer hohen Toxizität der Verbindungen auszugehen.

Ebenso wie die antibakterielle Aktivität ist auch die cytotoxische Wirkung der Aminophenoxazone 39 - 42 schwächer als die von Actinomycin D (19), allerdings ist hier der Unterschied zur Referenzsubstanz noch wesentlich größer. Dies lässt zwar ihren Einsatz als Antitumor-Mittel unattraktiv erscheinen, jedoch könnte die geringere Cytotoxizität die therapeutische Breite für eine potentielle Anwendung gegen pathogene Keime erhöhen. 


\section{$4 \quad$ Nargenicin $A_{1}$ aus Actinomyces sp. Gö 301}

Der Stamm Actinomyces sp. Gö 301 wurde aus einer afrikanischen Bodenprobe isoliert ${ }^{\mathrm{k}}$. Er fiel im chemischen Screening durch eine mittelpolare, mit Anisaldehyd in der Wärme schwarz-braun anfärbende Zone auf dem Dünnschichtchromatogramm auf. Nach Kultivierung des Stamms im 2L-Maßstab wurde die entsprechende Substanz chromatographisch aufgereinigt und durch massenspektrometrische und NMR-spektroskopische Untersuchung als Nargenicin $A_{1}$ (44) identifiziert. Eine zweite, etwas

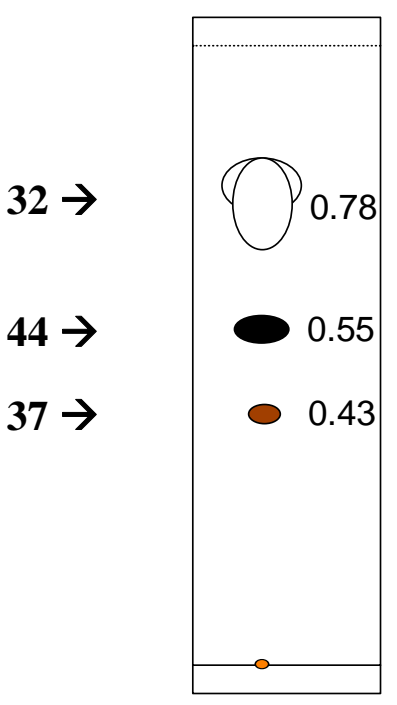

Abbildung 28: Skizze des

Dünnschichtchromatogramms des Extrakts, angegeben sind $\mathrm{R}_{\mathrm{f}}$-Werte $\left(\mathrm{CHCl}_{3} / \mathrm{MeOH} 9: 1\right)$. polarere Substanz erwies sich als das Diketopiperazin Cyclo(prolyltyrosyl) (37), das auch aus dem Stamm Halomonas sp. Rk377a erhalten wurde (siehe S. 55). Eine dritte Substanz hinterließ auf dem Dünnschichtchromatogramm bei Anfärbung mit Orcin in der Wärme einen ungefärbten, weißen Bereich. Hierbei handelt es sich um Polyisopropylenglycol (32), ein Polymer, das auch als Antischaum-Mittel bei Fermentationen Anwendung findet. Bei Actinomyces sp. Gö 301 wurde es nicht der Kultur zugesetzt, es handelt sich daher um eine vom Actinomyceten produzierte Komponente.

\subsection{Polyisopropylenglycol (32) und Cyclo(prolyltyrosyl) (37)}

Die Strukturaufklärung des Diketopiperazins Cyclo(prolyltyrosyl) (37) erfolgte anhand des EI-Massenspektrums und des ${ }^{1} \mathrm{H}-\mathrm{NMR}$-Spektrums wie auf Seite 55 beschrieben und wurde durch einen Vergleich mit Literaturdaten ${ }^{[84]}$ verifiziert.

Die nicht-anfärbende, lipophile Komponente des Extrakts zeigte im EI-Massenspektrum beginnend bei $\mathrm{m} / \mathrm{z}=59$ eine Reihe von Signalen, die jeweils eine Massendifferenz von $\mathrm{m} / \mathrm{z}=$ 58 haben. Dies entspricht einem Baustein mit der Summenformel $\mathrm{C}_{3} \mathrm{H}_{6} \mathrm{O}$, dessen Verwendung

\footnotetext{
${ }^{\mathrm{k}}$ Nähere Angaben zum Ort fehlen. Frau M. Klingebiel danke ich für die Isolierung des Stamms.
} 
zum Aufbau eines Polymers durch ein ESI-Massenspektrum bestätigt wird. Es zeigt ein Gemisch von Polymeren unterschiedlicher Länge, deren mittlere Molmasse etwa $1045 \mathrm{~g} / \mathrm{mol}$ beträgt. Das ${ }^{1}$ H-NMR-Spektrum legt durch die Resonanzen einer Methyl-, einer Methylenund einer Methingruppe das Polymer als Polyisopropylenglycol (32) fest.<smiles>O=C1N[C@@H](Cc2ccc(O)cc2)C(=O)N2CCC[C@H]12</smiles>

37<smiles>CCCCC(C)C</smiles>

32

\subsection{Nargenicin $A_{1}(44)$}

Der farblose Feststoff zeigt im EI-Massenspektrum ein Ion höchster Masse und Intensität bei $\mathrm{m} / \mathrm{z}=515$. Das ${ }^{13} \mathrm{C}-\mathrm{NMR}-$ Spektrum beinhaltet die Resonanzen von $28 \mathrm{C}$-Atomen, darunter zwei Ester- oder Amidgruppen und acht Signale für olefinische oder aromatische C-Atome. Im ${ }^{1}$ H-NMR-Spektrum (Abbildung 29) erkennt man eine Methoxygruppe $\left(\delta_{\mathrm{H}}=3.29\right)$, drei zum Dublett aufgespaltene Methylgruppen $\left(\delta_{\mathrm{H}}=0.95,1.24,1.30\right)$ und eine, die als Singulett erscheint $\left(\delta_{\mathrm{H}}=1.77\right)$. Eine Suche mit der Molmasse, der Anzahl der C-Atome und den genannten Strukturelementen in der Datenbank AntiBase ${ }^{[41]}$ führte zu zwei Makroliden: Nargenicin $A_{1}$ und Isonargenicin $A_{1}$. Ein Vergleich der NMR-Daten mit publizierten Werten ${ }^{[103,104]}$ bestätigte das Vorliegen von Nargenicin $A_{1}$ (44).

44 wurde erstmals 1977 von der Fa. Pfizer als Metabolit von Nocardia argentinensis beschrieben $^{[105]}$ und ist aufgrund seiner antibiotischen Wirkung Gegenstand mehrerer Patente. Nargenicin $A_{1}(44)$ hat ein enges Wirkspektrum und hemmt bestimmte Gram-positive Bakterien, insbesondere Staphylococcen. Es ist auch gegen multiresistente Stämme wirksam und in der Wirkstärke etwa mit Erythromycin

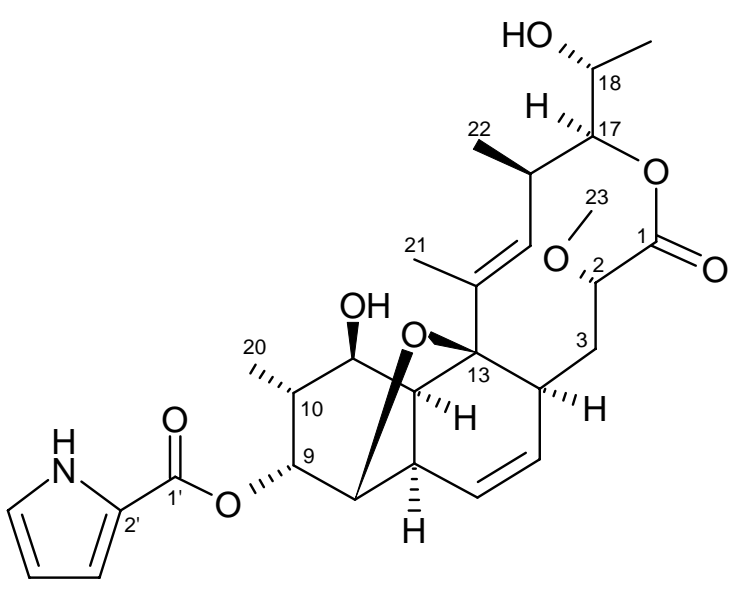

44 vergleichbar $^{[106]}$. Wirkort und -mechanismus sind bisher nicht publiziert worden. 


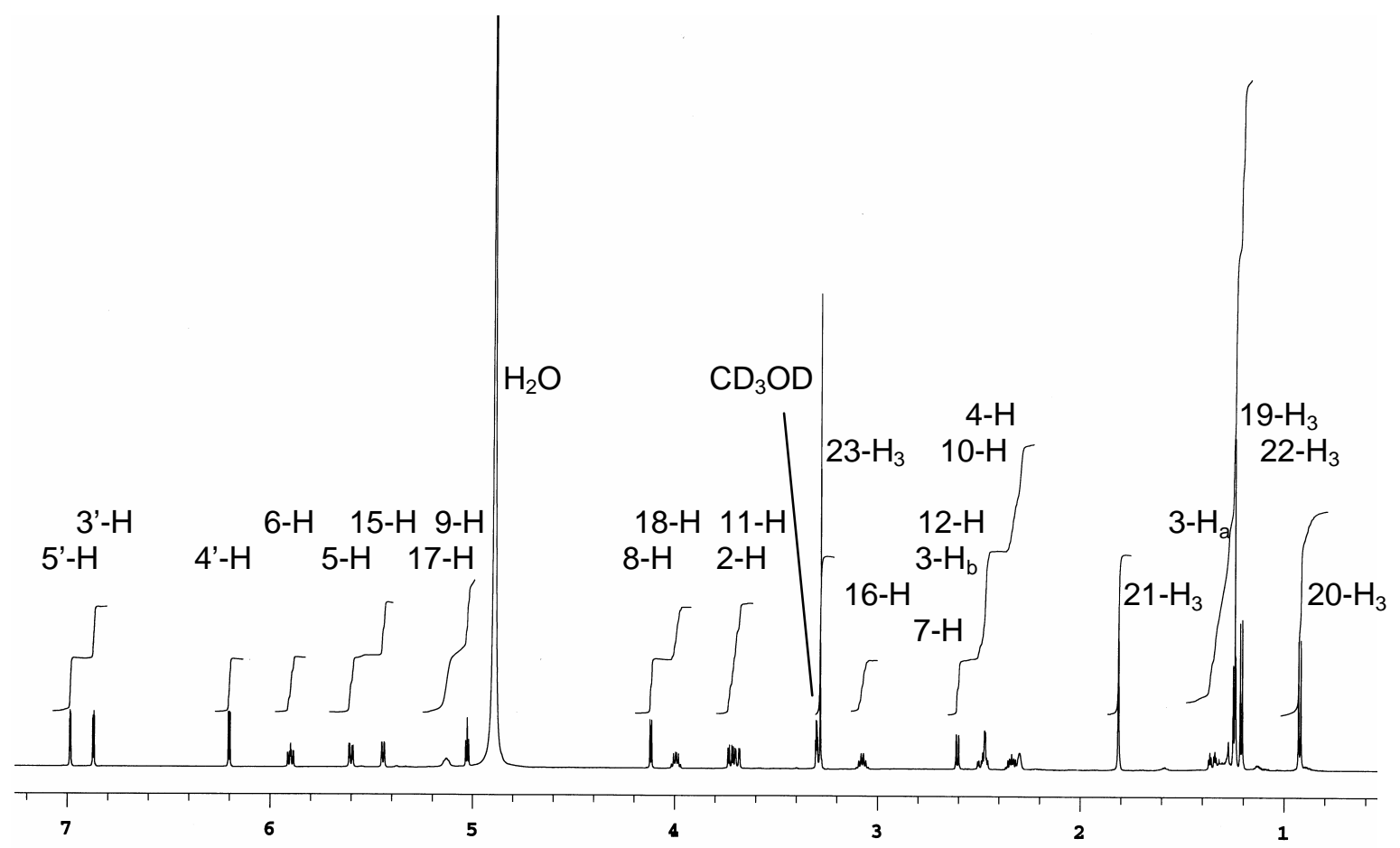

Abbildung 29: ${ }^{1} \mathrm{H}-\mathrm{NMR}-\mathrm{Spektrum}$ von Nargenicin $\mathrm{A}_{1}(\mathbf{4 4})$ in $\mathrm{CD}_{3} \mathrm{OD}(300 \mathrm{MHz})$.

Die 1980 veröffentlichte Struktur ${ }^{[103]}$ von Nargenicin $A_{1}$ (44) ist der Ester aus Pyrrol-2carbonsäure und dem ebenfalls antibiotisch wirksamen Nodusmicin. Biosynthetisch entsteht das Makrolid als Produkt einer Polyketidsynthase vom Typ I aus fünf Acetat- und vier Propionat-Bausteinen ${ }^{[104]}$. Die Zyklisierung des Nonaketids unter Ausbildung des cisverknüpften Octalin-Systems erfolgt vermutlich durch eine enzymatisch katalysierte DielsAlder-Reaktion (Abbildung 30). Im Zuge der späten Biosynthese finden Oxidationen an C-2, C-8, C-13 und C-18, die Methylierung von 2-OH zur Methoxygruppe und die Veresterung von 9-OH mit Pyrrolcarbonsäure statt ${ }^{[107]}$.

Intramolekulare Diels-Alder-Reaktion werden auch bei der Biosynthese einiger in dieser Arbeit aus Pilzstämmen isolierter Polyketide angenommen ${ }^{[108]}$ : Solanapyron C (61, Seite 97) und die Chaetoglobosine 65, 66 und 67 (ab Seite 100). 


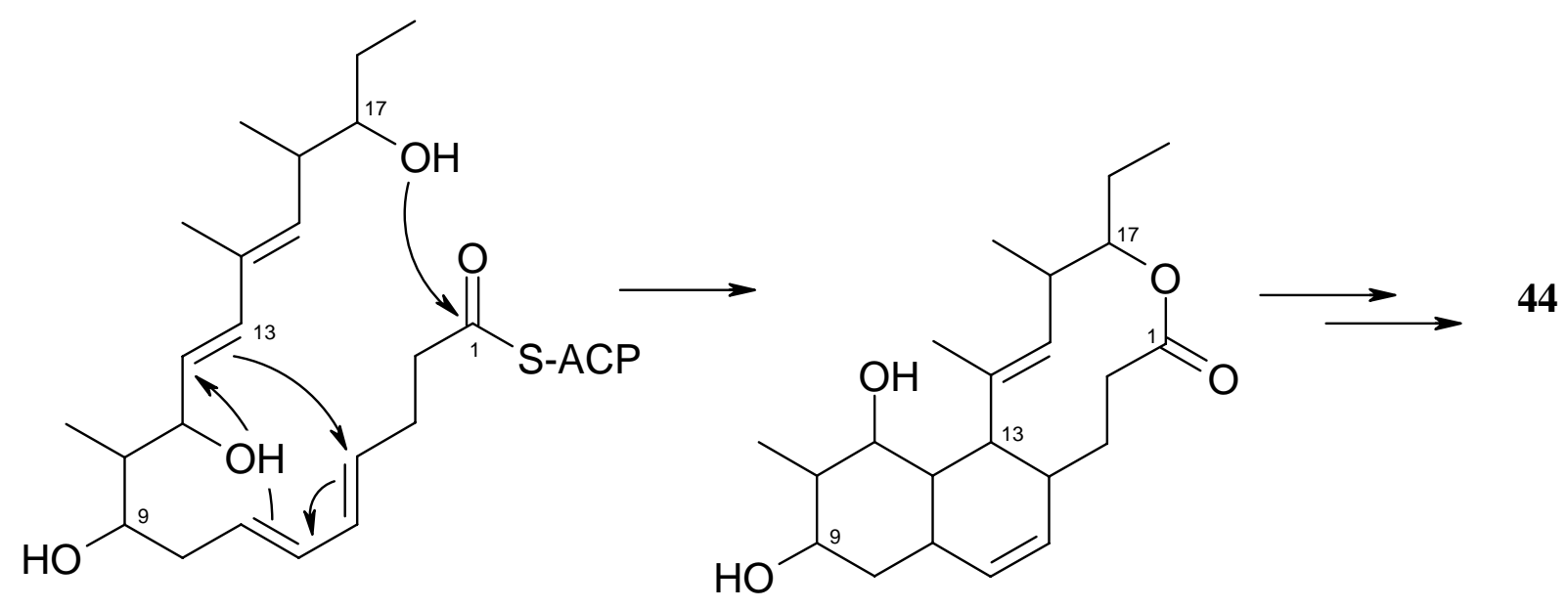

Abbildung 30: Diels-Alder-Reaktion bei der Biosynthese von Nargenicin $A_{1}$ (44).

\subsection{Biologische Aktivität}

Die antibiotische Wirkung von $\mathbf{4 4}$ wurde in Plattendiffusionstests überprüft, die Ergebnisse sind in Tabelle 12 zusammengefasst. Nargenicin $\mathrm{A}_{1}$ (44) zeigte keine Wirksamkeit gegen das Gram-negative Bakterium E. coli und den Hefepilz C. albicans. Bei den Gram-positiven Bakterien wurde selektiv S. aureus mit einer in etwa der Vergleichssubstanz Penicillin G entsprechenden Stärke gehemmt. Das Wachstum von B. subtilis wurde hingegen nicht beeinflusst. Das Ergebnis entspricht den Literaturangaben und bestätigt die hohe Selektivität der Wirkung von 44.

Eine cytotoxische Wirkung von $\mathbf{4 4}$ gegen Tumorzelllinien wurde nicht beobachtet ${ }^{1}$.

Tabelle 12: Antibiotische Wirkung von Nargenicin $A_{1}$ (44). Angegeben ist der HemmhofDurchmesser beim Plattendiffusionstest in [mm], Penicillin G diente als Vergleich.

\begin{tabular}{l|cccc} 
& $\begin{array}{c}\text { Escherichia } \\
\text { coli }\end{array}$ & $\begin{array}{c}\text { Bacillus } \\
\text { subtilis }\end{array}$ & $\begin{array}{c}\text { Staphylococcus } \\
\text { aureus }\end{array}$ & $\begin{array}{c}\text { Candida } \\
\text { albicans }\end{array}$ \\
\hline Penicillin G & 16 & 35 & 25 & 0 \\
$\mathbf{4 4}$ & 0 & 0 & 23 & 0 \\
\hline
\end{tabular}

$50 \mu \mathrm{g}$ Substanz auf Filterpapier-Plättchen, Ø $6 \mathrm{~mm}$

\footnotetext{
${ }^{1}$ Herrn Prof. Dr. W. Beil (Institut für Pharmakologie, Medizinische Hochschule Hannover) danke ich für die Durchführung der Cytotoxizitäts-Tests.
} 


\section{6-Desoxytalopyranoside aus Actinomyces sp. Gö M1}

Der Stamm Actinomyces sp. Gö M1 wurde aus einer Sandprobe isoliert, die auf vulkanischem Gebiet nahe Mexico-City gesammelt wurde ${ }^{\mathrm{m}}$. Im chemischen Screening fielen mehrere mit Orcin in der Wärme braun-schwarz anfärbende Zonen auf. Die Isolierung und Strukturaufklärung der Reinsubstanzen führte zu neuen Sekundärstoffen, die 6-Desoxytalose als gemeinsamen Baustein enthalten. Dieser seltene Desoxyzucker ist $\alpha$-glykosidisch mit der Carboxylgruppe kleiner, meist aromatischer Aglykone (O-Acyl-6-desoxytalopyranoside) oder mit phenolischen OH-Gruppen (O-Aryl-6-desoxytalopyranoside) verknüpft. Die drei Hauptmetaboliten sind 1-O-Phenylacetyl-6-desoxy- $\alpha$ - $L$-talopyranosid $\quad(46, \quad 19 \mathrm{mg} / \mathrm{L}), \quad 1-O-$ Isovaleryl-6-desoxy- $\alpha$ - $L$-talopyranosid (45, $6 \mathrm{mg} / \mathrm{L})$ und 1-O-(4-Hydroxyphenyl)-6-desoxy- $\alpha$ $L$-talopyranosid (48, $6 \mathrm{mg} / \mathrm{L})$. Des Weiteren wurden vier Verbindungen (47, 49-51) in Ausbeuten von 0.3 bis $1 \mathrm{mg} / \mathrm{L}$ erhalten. Alle Strukturen sind zusammenfassend in Abbildung 34 gezeigt.

\subsection{Strukturaufklärung des 6-Desoxyzuckers}

Die Strukturaufklärung der Zuckerkomponente erfolgte für alle sieben Verbindungen aus Actinomyces sp. Gö M1 analog und wird im Folgenden am Beispiel von 1-O-(4-Hydroxyphenyl)-6-desoxy- $\alpha$ - $L$-talopyranosid (48) diskutiert.

Das ${ }^{1} \mathrm{H}-\mathrm{NMR}-$ Spektrum der Verbindung (Abbildung 31$)$ zeigt eine Methylgruppe $\left(\delta_{\mathrm{H}}=1.21\right)$ und vier Methingruppen $\left(\delta_{\mathrm{H}}=3.65,3.86,3.90,4.01\right)$, die aufgrund ihrer Tieffeldverschiebung an Sauerstoff gebunden sein müssen. Eine weitere Methingruppe $\left(\delta_{\mathrm{H}}=5.33\right)$ konnte aufgrund ihrer ${ }^{13}$ C-Resonanz bei $\delta_{\mathrm{C}}=102$ als anomeres Kohlenstoffatom eines Zuckers erkannt werden. Mittels eines COSY-Spektrums gelang die Verknüpfung der funktionellen Gruppen zu einer Hexose, die an Position 6 eine Methylgruppe besitzt (Abbildung 32, links).

Eine Korrelation im NOESY-Spektrum zwischen 3'-H und 5'-H spricht für eine axiale Anordnung dieser Protonen (Abbildung 32, rechts). Da keine diaxialen Kopplungen beobachtet werden können (alle Kopplungskonstanten sind kleiner als $6.5 \mathrm{~Hz}$ ), müssen die zu 3'-H und 5'-H benachbarten Protonen 2'-H und 4'-H eine äquatoriale Position einnehmen. Eine zwischen 2'-H und 4'-H auftretende W-Kopplung $\left({ }^{4} J_{\mathrm{HH}}=1.5 \mathrm{~Hz}\right)$ bestätigt diese

\footnotetext{
${ }^{\mathrm{m}}$ Frau M. Klingebiel danke ich für die Isolierung des Stamms und den Teilnehmer/innen des Praktikums Biomolekulare Chemie im WS 2002/2003 für die Durchführung des chemischen Screenings.
} 
Annahme. Die Konfiguration am anomeren C-Atom wurde anhand der ${ }^{1} J_{\mathrm{CH}}-$ Kopplungskonstanten bestimmt ${ }^{\mathrm{n},[109]}$. Der gemessene Wert von $170.9 \mathrm{~Hz}$ beweist das Vorliegen des $\alpha$-Anomers, was durch die kleine geminale Kopplungskonstante von ${ }^{3} J_{\mathrm{HH}}=2 \mathrm{~Hz}$ unterstützt wird. Der Desoxyzucker kann somit eindeutig als 6-Desoxy- $\boldsymbol{\alpha}$-talose in der Pyranosid-Form beschrieben werden.

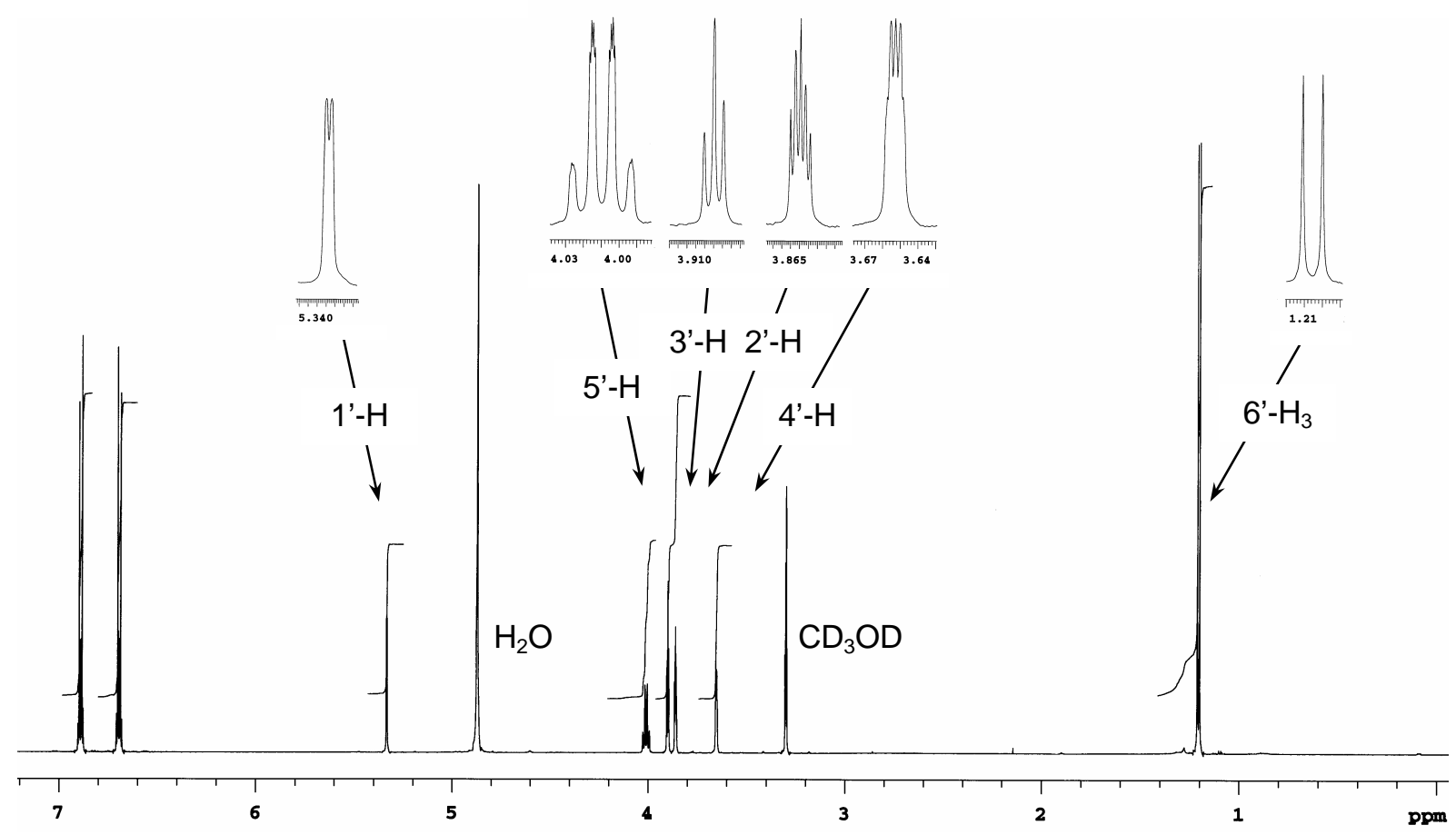

Abbildung 31: ${ }^{1} \mathrm{H}-\mathrm{NMR}-\mathrm{Spektrum}$ von 1-O-(4-Hydroxyphenyl)-6-desoxy- $\alpha$ - $L$-talopyranosid (48) mit Spreizungen der Signale des Desoxyzuckers (600 MHz, $\mathrm{CD}_{3} \mathrm{OD}$ ).
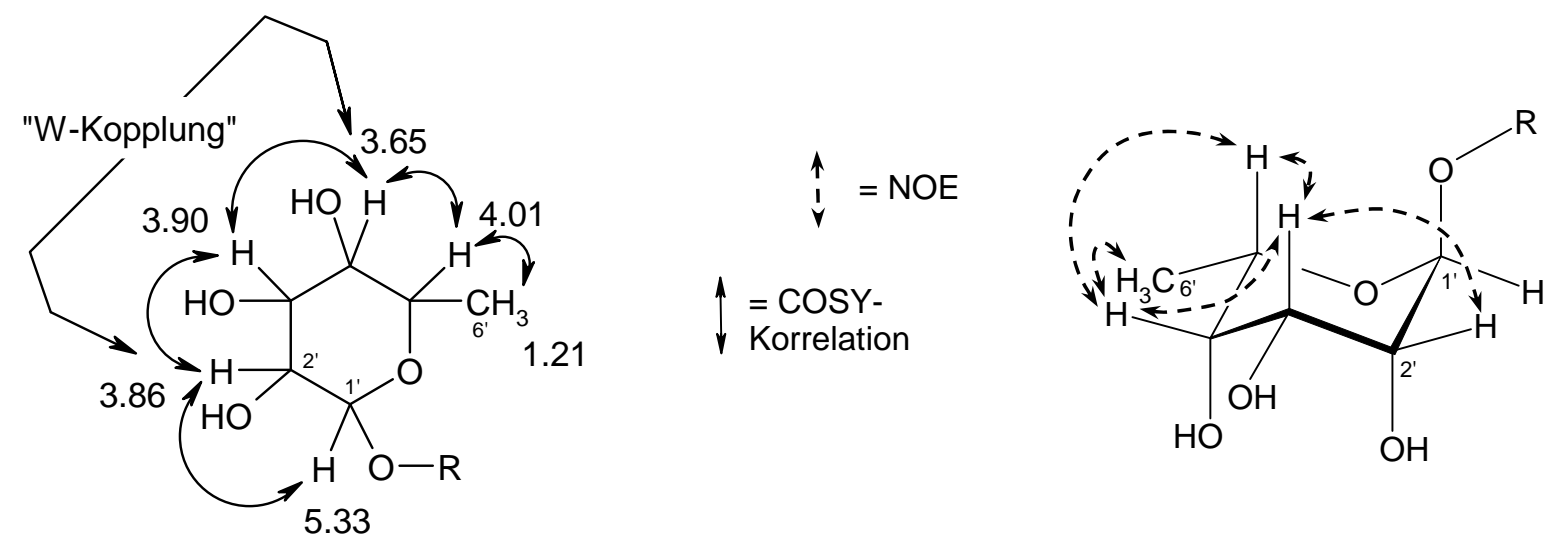

Abbildung 32: COSY- (links) und NOESY-Korrelationen (rechts) des Desoxyzuckers von 48. Die Zahlen geben die chemische Verschiebung der Protonen in ppm an.

${ }^{\mathrm{n}} \alpha$-Anomer: ${ }^{1} J_{\mathrm{CH}}=169-175 \mathrm{~Hz}, \beta$-Anomer: ${ }^{1} J_{\mathrm{CH}}=158-162 \mathrm{~Hz}$ 


\section{Absolute Stereochemie}

Die empirisch gefundene KLYNE-Regel ${ }^{[110]}$ besagt, dass in bakteriellen Naturstoffen Zucker der $D$-Reihe in der $\beta$-Form und Zucker der $L$-Reihe in der $\alpha$-Form vorliegen. Entsprechend dieser Regel kann davon ausgegangen werden, dass hier das $L$-Enantiomer der 6-Desoxytalose vorliegt.

Diese Annahme wird durch die Drehwerte der isolierten 6-Desoxytalopyranoside unterstützt. Für alle Verbindungen (45 - 51) wurden negative Drehwerte ermittelt, was übereinstimmt mit den negativen Drehwerten der synthetisch dargestellten Verbindungen Benzyl-6-desoxy- $\alpha-L$ talopyranosid (52) ${ }^{[111]}$ und Methyl-6-desoxy- $\alpha-L$-talopyranosid (53) ${ }^{[112,113]}$.

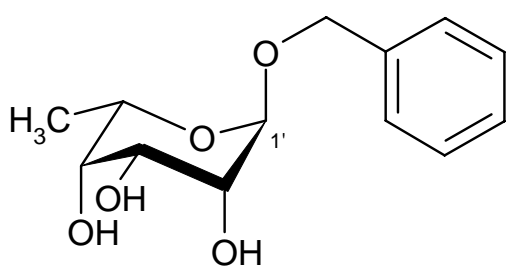

52

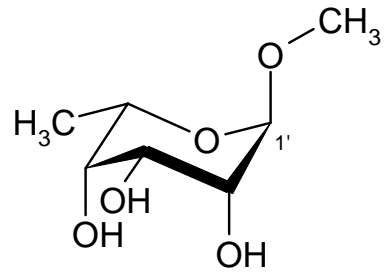

53

Zur Absicherung der absoluten Stereochemie ist eine Methanolyse der Verbindungen geplant, um die Drehwerte der entstehenden Methylglykoside mit den Literaturdaten zu vergleichen.

\section{GC-MS-Analytik}

Bei 6-Desoxyzuckern von Sekundärstoffen handelt es sich in der Regel um Rhamnose oder Fucose ${ }^{[114]}$. Zur zusätzlichen Bestätigung der Zuordnung als 6-Desoxytalose und um Rhamnose und Fucose auszuschließen, wurde der Zucker der Hauptprodukte 45, 46 und 48 durch Methanolyse abgespalten. Die Methylglykoside wurden nach Silylierung mittels GC-MS analysiert ${ }^{[115]}$ und mit entsprechend derivatisierter Rhamnose und Fucose als Referenzen verglichen. Ein direkter Vergleich mit 6-Desoxytalose konnte nicht durchgeführt werden, da diese weder kommerziell erhältlich noch synthetisch einfach zugänglich ist. Die GC-MS-Experimente zeigten, dass alle drei Naturstoffe denselben Baustein enthalten, dessen EI-Massenspektren mit Fucose und Rhamnose übereinstimmen, nicht aber die Retentionszeiten. Als Ergebnis können Rhamnose und Fucose wie erwartet als Desoxyzucker von 45, 46 und 48 ausgeschlossen werden. 


\subsection{Strukturaufklärung der 6-Desoxy- $\alpha$-L-talopyranoside}

Die Strukturen der sieben verschiedenen 6-Desoxy- $\alpha-L$-talopyranoside zeigt Abbildung 34 auf Seite 74, ihre Aufklärung wird in den folgenden Unterkapiteln beschrieben.

\subsubsection{1-O-Isovaleryl-6-desoxy- $\alpha-L-t a l o p y r a n o s i d ~(45)$}

Das ${ }^{1}$ H-NMR-Spektrum der farblosen, öligen Substanz zeigt das Vorhandensein von 6-Desoxy- $\alpha$ - $L$-talose (siehe vorhergehendes Kapitel), deren anomeres Proton ins Tieffeld verschoben ist $\left(\delta_{\mathrm{H}}=6.03\right)$. Weiterhin sind die Signale von zwei Methylgruppen $\left(\delta_{\mathrm{H}}=0.96\right.$ und 0.97), einer Methin- $\left(\delta_{\mathrm{H}}=2.07\right)$ und einer Methylengruppe $\left(\delta_{\mathrm{H}}=2.22\right)$ vorhanden, die mittels eines COSY-Spektrums zu einer Isobutylgruppe verknüpft werden können. Im ${ }^{13} \mathrm{C}$-NMR-Spektrum fällt neben den Resonanzen des Zuckers und der Isopropylgruppe das Signal einer Ester-Carbonylgruppe auf. Das Vorliegen eines $\alpha$-glykosidisch gebundenen Isovaleriansäure-Esters erklärt die hohe chemische Verschiebung des Protons am anomeren C-Atom und steht im Einklang mit der Molmasse von $248 \mathrm{~g} / \mathrm{mol}$. Durch ein hochaufgelöstes ESI-Massenspektrum ließ sich die Summenformel $\mathrm{C}_{11} \mathrm{H}_{20} \mathrm{O}_{6}$ ermitteln, die die Substanz als 1-O-Isovaleryl-6-desoxy- $\alpha$ - $L$-talopyranosid (45) bestätigt.

\subsubsection{1-O-Phenylacetyl-6-desoxy- $\alpha$-L-talopyranosid (46)}

Der farblose Feststoff wurde mit einer Ausbeute $19 \mathrm{mg} / \mathrm{L}$ als Hauptmetabolit des Stammes Actinomyces sp. Gö M1 erhalten. 46 besitzt eine Molmasse von 282 g/mol und die durch ESIMS-Hochauflösung bestimmte Summenformel $\mathrm{C}_{14} \mathrm{H}_{18} \mathrm{O}_{6}$. Abzüglich der 6-Desoxytalose, deren Vorhandensein die ${ }^{1} \mathrm{H}$ - und ${ }^{13} \mathrm{C}-\mathrm{NMR}-$ Spektren beweisen, bleibt für den Rest die Formel $\mathrm{C}_{8} \mathrm{H}_{7} \mathrm{O}$. Auf die im Protonenresonanzspektrum erkennbare Phenylgruppe entfallen hiervon sechs Kohlenstoff- und fünf Wasserstoffatome, ein weiteres Kohlenstoff- und zwei Wasserstoffatome gehören zu einer Methylengruppe $\left(\delta_{\mathrm{C}}=42, \delta_{\mathrm{H}}=3.68\right)$. Das übrig bleibende Kohlenstoff- und das Sauerstoffatom können einer Ester-Carbonylgruppe $\left(\delta_{C}=171\right)$ zugeordnet werden, deren Bindung an das anomere C-Atom des Zuckers durch die Tieffeldverschiebung des dortigen Protons $\left(\delta_{\mathrm{H}}=6.00\right)$ angezeigt wird. Die Position der Methylengruppe zwischen Aromat und Carbonylgruppe erklärt ihre hohe chemische Verschiebung, als Struktur ergibt sich 1-O-Phenylacetyl-6-desoxy- $\alpha-L$-talopyranosid (46). 


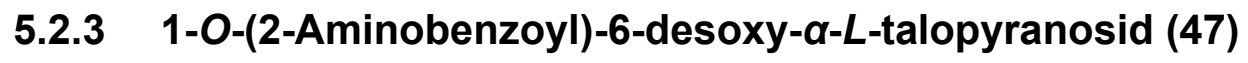

Die ungerade Molmasse dieser Minderkomponente (283 g/mol) ließ ein Stickstoffatom im Molekül erwarten, aus dem hochaufgelösten Massenspektrum wurde die Summenformel $\mathrm{C}_{13} \mathrm{H}_{17} \mathrm{NO}_{6}$ ermittelt. Zusätzlich zu den Signalen von 6-Desoxytalose enthält das ${ }^{1} \mathrm{H}-\mathrm{NMR}$ Spektrum die Resonanzen vier aromatischer Protonen, deren Kopplungsmuster (zwei Dubletts, zwei Tripletts) einen ortho-disubstituierten Aromaten anzeigen. Die Substituenten lassen sich aus der Summenformel ableiten: eine Amino- und eine Ester-Carbonylgruppe. Es handelt sich demnach bei der Substanz um 1-O-(2-Aminobenzoyl)-6-desoxy- $\alpha-L$ talopyranosid (47). Die Zuordnung der Protonen im Aromaten erfolgte anhand ihrer chemischen Verschiebung. Durch ihren -M-Effekt bewirkt die Carbonylgruppe in orthoPosition eine starke Entschirmung, folglich befindet sich dort das Proton mit der höchsten chemischen Verschiebung $\left(\delta_{\mathrm{H}}=7.74\right)$. Die Positionen der anderen drei Protonen folgen aus der Auswertung der Kopplungskonstanten.

\subsubsection{1-O-(4-Hydroxyphenyl)-6-desoxy- $\alpha-L-t a l o p y r a n o s i d ~(48)$}

Das in $\mathrm{CD}_{3} \mathrm{OD}$ gemessene ${ }^{1} \mathrm{H}-\mathrm{NMR}-$ Spektrum (siehe Abbildung 31, Seite 68) zeigt neben dem Vorhandensein des Desoxyzuckers zwei aromatische Signale, die für je zwei Protonen integrieren und zu einem para-disubstituierten Aromaten gehören. Die Hochfeldverschiebung dieser Protonen $\left(\delta_{\mathrm{H}}=6.70\right.$ und 6.94) lässt auf Sauerstoff als zweifachen Substituenten schließen, was von der durch hochauflösende Massenspektrometrie erhaltenen Summenformel $\mathrm{C}_{12} \mathrm{H}_{16} \mathrm{O}_{6}$ unterstützt wird. Die im Vergleich zu 45 - 47 um ca. 0.7 ppm geringere chemische Verschiebung des Protons am anomeren Zentrum $\left(\delta_{\mathrm{H}}=5.33\right)$ steht im Einklang mit der Tatsache, dass eine normale glykosidische Bindung anstelle eines Esterglykosids an C-1' vorliegt. Der systematische Name dieser Verbindung lautet 1-O-(4-Hydroxyphenyl)-6desoxy- $\alpha$ - $L$-talopyranosid (48).

\subsubsection{1-O-(4-Hydroxy-2-methoxyphenyl)-6-desoxy-a-L-talopyranosid (49)}

Das Aglykon dieses farblosen Feststoffs zeigt im ${ }^{1} \mathrm{H}-\mathrm{NMR}-$ Spektrum die Signale von einer Methoxygruppe und drei aromatischen Protonen. Die Molmasse ist mit $286 \mathrm{~g} / \mathrm{mol}$ um $30 \mathrm{~g} / \mathrm{mol}$ höher als die von 48, die Differenz entspricht der Methoxygruppe. Das Substitutionsmuster des Aromaten ließ sich durch die Kombination von NOESY - und COSYKorrelationen ermitteln (Abbildung 33). 1'-H befindet sich in räumlicher Nähe zum Proton 
bei $\delta_{\mathrm{H}}=6.87$, das hierdurch als 6-H festgelegt wird. Das zu letzterem in ortho-Position befindliche 5-H $\left(\delta_{\mathrm{H}}=6.28\right)$ zeigt eine meta-Kopplung zu dem Proton bei $\delta_{\mathrm{H}}=6.46$, welches sich demnach in Position 3 befindet. Der Nuclear-Overhauser-Effekt zwischen 3-H und der Methoxygruppe legt schließlich die Struktur als 1-O-(4-Hydroxy-2-methoxyphenyl)-6desoxy- $\alpha$ - $L$-talopyranosid (49) fest.

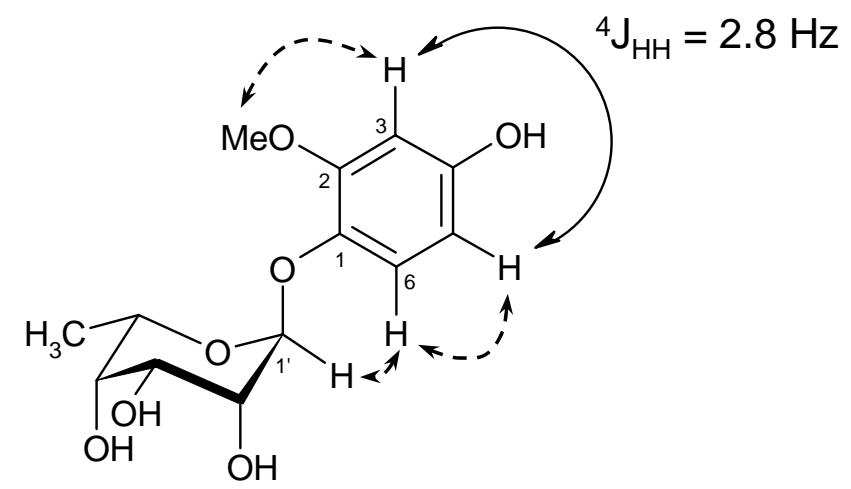

J-Kopplung (COSY)

NOE (1D-NOESY)

Abbildung 33: Wichtige COSY- und NOESY-Korrelationen in 49.

\subsubsection{1-O-(2-Pyrrolylcarbonyl)-6-desoxy- $\alpha$-L-talopyranosid (50)}

Der glykosidisch gebundene Rest dieses 6-Desoxy- $\alpha$ - $L$-talopyranosids zeigt im ${ }^{13} \mathrm{C}$-NMRSpektrum die Signale fünf $\mathrm{sp}^{2}$-hybridisierter Kohlenstoffatome, darunter eine EsterCarbonylgruppe. Drei der aromatischen C-Atome tragen ein Proton und weisen eine deutliche Hochfeldverschiebung auf. Anhand der charakteristischen kleinen ${ }^{3} J_{\mathrm{HH}}-$ Kopplungskonstanten der zugehörigen Signale (3.0 bzw. $3.5 \mathrm{~Hz})$ sowie der durch hochauflösende ESIMassenspektrometrie ermittelten Summenformel $\mathrm{C}_{11} \mathrm{H}_{15} \mathrm{NO}_{6}$ kann auf Pyrrolcarbonsäure geschlossen werden, die $\alpha$-glykosidisch mit dem Zucker verestert ist. Ein Vergleich der chemischen Verschiebungen und Kopplungskonstanten mit Literaturwerten ${ }^{[109]}$ zeigt, dass sich die Carbonylgruppe in Position 2 des Pyrrolrings befindet. Die Substanz wird daher als 1-O-(2-Pyrrolylcarbonyl)-6-desoxy- $\alpha$ - $L$-talopyranosid (50) identifiziert.

\subsubsection{1-O-(4-Hydroxybenzoyl)-6-desoxy- $\alpha$-L-talopyranosid (51)}

Das ${ }^{1} \mathrm{H}-\mathrm{NMR}-\mathrm{Spektrum}$ von 51 enthält neben den Resonanzen des 6-Desoxyzuckers zwei Signale im aromatischen Bereich, die für je zwei Protonen integrieren. Die ortho-Kopplung 
von ${ }^{3} J_{\mathrm{HH}}=9 \mathrm{~Hz}$ zwischen ihnen kennzeichnet einen para-disubstituierten Aromaten. Die beiden Substituenten (eine Hydroxygruppe, eine Carbonylgruppe) können aus der Summenformel $\mathrm{C}_{13} \mathrm{H}_{16} \mathrm{O}_{7}$ abgeleitet werden und stehen im Einklang mit der beobachteten Tieffeldverschiebung des einen aromatischen ${ }^{1} \mathrm{H}$-Signals (benachbart zur Carbonylgruppe, $\delta_{\mathrm{H}}=7.89$ ) und der Hochfeldverschiebung des anderen (benachbart zur Hydroxygruppe, $\left.\delta_{\mathrm{H}}=6.83\right)$. Die hohe chemische Verschiebung des anomeren Protons des Zuckers $\left(\delta_{\mathrm{H}}=6.24\right)$ spricht für eine $\alpha$-glykosidische Esterbindung zwischen Aromat und 6-Desoxytalose. Die Komponente 51 kann zusammenfassend als 1-O-(4-Hydroxybenzoyl)-6-desoxy- $\alpha-L$ talopyranosid charakterisiert werden. 
<smiles>CC(C)CC(=O)O[C@H]1O[C@H](C)[C@@H](O)[C@H](O)[C@H]1O</smiles>

45<smiles>CC1O[C@H](OC(=O)c2ccccc2N)[C@@H](O)[C@H](O)C1O</smiles>

47<smiles>COc1cc(O)ccc1O[14C]1O[C@H](C)[C@@H](O)[C@H](O)[C@H]1O</smiles>

49<smiles>CC1O[C@H](OC(=O)c2ccc(O)cc2)[C@@H](O)[C@H](O)[C@H]1O</smiles><smiles>CC1O[C@H](OC(=O)Cc2ccccc2)[C@@H](O)[C@H](O)[C@H]1O</smiles>

46<smiles>CC1O[C@H](Oc2ccc(O)cc2)[C@@H](O)[C@H](O)[C@H]1O</smiles>

48<smiles>CC1O[C@H](OC(=O)c2ccc[nH]2)[C@@H](O)[C@H](O)[C@H]1O</smiles>

50

51

Abbildung 34: Aus Actinomyces sp. Gö M1 isolierte 6-Desoxy- $\alpha$ - $L$-talopyranoside. 


\subsection{Vorläufer-dirigierte Biosynthese mit Actinomyces sp. Gö M1}

Das Produktspektrum von Actinomyces sp. Gö M1 zeigt, dass die für den Aufbau der 6-Desoxytalopyranoside verantwortliche Glykosyltransferase nur eine geringe Substratspezifität hinsichtlich der Carbonsäure bzw. des Phenols besitzt ${ }^{\circ}$. Dies ließ erwarten, dass der Stamm auch andere aromatische Carbonsäuren als Substrate akzeptieren und mit dem Desoxyzucker zu den entsprechenden $\alpha$-Pyranosiden umsetzen würde.

Für die Experimente zur Vorläufer-dirigierten Biosynthese wurde der Stamm im 1L-Rührblattfermenter kultiviert. Nach der 12. Stunde wurden $7.3 \mathrm{mmol}$ der jeweiligen Carbonsäure als Lösung über $12 \mathrm{~h}$ kontinuierlich zugepumpt. Die zugegebene Stoffmenge wurde zuvor nicht optimiert, sie hatte sich jedoch in analogen Experimenten mit dem Rhamnosid-Produzenten Streptomyces griseoviridis Tü 3634 (Kapitel 5.6) als geeignet erwiesen $^{[116,117]}$ und wurde übernommen. Durch Zugabe von Pyrrolcarbonsäure oder Phenylessigsäure konnte die Ausbeute an den jeweiligen 1-O-Acyl-glykosiden erheblich gesteigert werden. Die Fütterung von 4-Hydroxybenzoesäure führte zur erwarteten Verbindung 51, zusätzlich wurden das 1-O-Aryl-pyranosid $\mathbf{4 8}$ sowie die neue diglykosylierte Verbindung $\mathbf{5 4}$ gebildet. Einen Überblick über die Fütterungsexperimente gibt Abbildung 35.
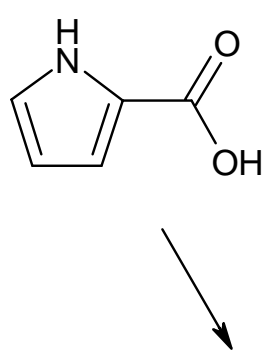

$50(33 \mathrm{mg} / \mathrm{L})$

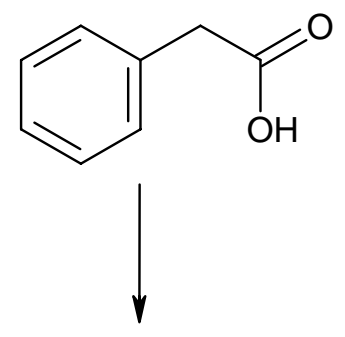

46 (60 mg/L)

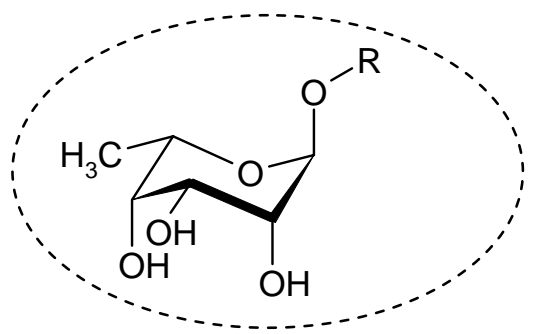<smiles>O=C(O)c1ccc(O)cc1</smiles>

51 (75 mg/L), $48(50 \mathrm{mg} / \mathrm{L})$, 54 (11 mg/L)

Abbildung 35: Durch Vorläufer-dirigierte Biosynthese erhaltene 1-O-Acyl- und 1-O-Aryl6-desoxy- $\alpha$ - $L$-talopyranoside.

\footnotetext{
${ }^{\circ}$ Es kann nicht ausgeschlossen werden, dass der Stamm mehrere Glykosyltransferasen mit Spezifität für 6-Desoxytalose besitzt. Unter evolutionären Gesichtspunkten erscheint ein einzelnes Enzym ökonomischer und damit wahrscheinlicher, Auskunft hierüber könnten Untersuchungen mit entsprechenden Gensonden geben.
} 


\subsubsection{Fütterung von Pyrrolcarbonsäure oder Phenylessigsäure}

Die Zugabe von Pyrrolcarbonsäure oder Phenylessigsäure zur Kultur von Actinomyces sp. Gö M1 führte zur vermehrten Produktion der 1-O-Acyl-6-desoxy- $\alpha$ - $L$-talopyranoside 50 bzw. 46. Die Ausbeute an 50 wurde nahezu um den Faktor 30 auf $33 \mathrm{mg} / \mathrm{L}$ gesteigert, die Ausbeute an 46 erhöhte sich um den Faktor drei auf ca. $60 \mathrm{mg} / \mathrm{L}$. Die im normalen Produktspektrum ebenso wie bei der Vorläufer-dirigierten Biosynthese zu beobachtende vermehrte Produktion von 1-O-Phenylacetyl-6-desoxy- $\alpha$ - $L$-talopyranosid (46) im Vergleich zum Glykosid der Pyrrolcarbonsäure (50) könnte auf die Größe des aromatischen Rings zurückzuführen sein. Der größere Abstand der Carboxylgruppe vom Aromaten scheint hingegen nur eine untergeordnete Rolle zu spielen, wie der ebenfalls hohe Einbau von 4-Hydroxybenzoesäure zeigt (siehe nächstes Unterkapitel).

\subsubsection{Fütterung von 4-Hydroxybenzoesäure}

4-Hydroxybenzoesäure wurde bei Zufütterung ebenfalls als Substrat akzeptiert und führte zur Bildung des 1-O-Acyl-glykosids 51, des 1-O-Aryl-glykosids 48 und der zweifach glykosylierten Verbindung 54 (Abbildung 36).<smiles>O=C(O)c1ccc(O)cc1</smiles><smiles>CC(O)[C@H](O)[C@H](O)[C@H](OC(=O)c1ccc(O)cc1)OC(C)(C)C</smiles><smiles>CC1C(O)[C@H](O)[C@@H](O)[C@@H](Oc2ccc(O)cc2)C1O</smiles><smiles>CI</smiles>

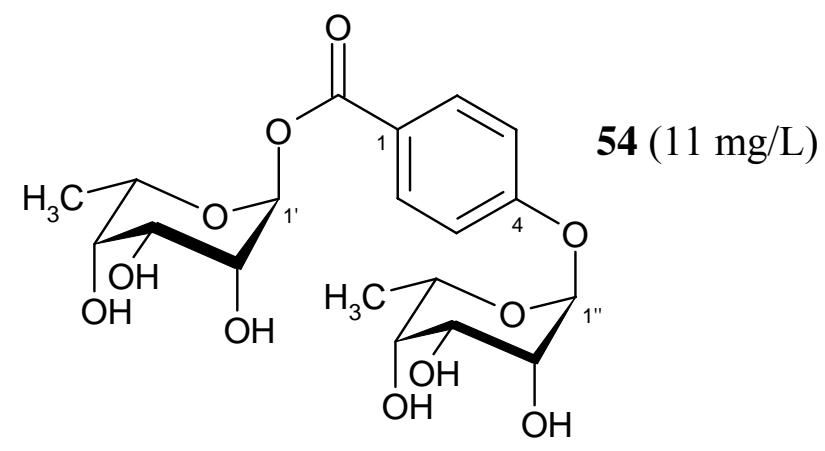

Abbildung 36: Durch Fütterung von 4-Hydroxybenzoesäure erhaltene 6-Desoxy- $\alpha$ - $L$-talopyranoside. 
Während die Biosynthese von $\mathbf{5 1}$ der Erwartung entspricht, unterscheidet sich 1-O-(4-Hydroxyphenyl)-6-desoxy- $\alpha-L$-talopyranosid (48) durch die durch eine Hydroxygruppe ersetzte Carboxylgruppe von dem erwarteten 4-Hydroxybenzoesäure-Derivat. Scheinbar findet vor oder nach der Glykosylierung der phenolischen OH-Gruppe eine oxidative Decarboxylierung am Aromaten statt $^{\mathrm{p}}$. Eine solche Reaktion ist für 4-Hydroxybenzoesäuren bei Pilzen bekannt, z. B. bei der Biosynthese von Shikonin ${ }^{[118]}$.

51 wurde in einer Ausbeute von $75 \mathrm{mg} / \mathrm{L}$ erhalten, ohne Zugabe des Vorläufers waren es nur $0.5 \mathrm{mg} / \mathrm{L}$. Die Vorläufer-dirigierte Biosynthese führte demnach zu einer Ausbeutesteigerung um das 150fache. Die Bildung von 48 wurde um den Faktor 4 auf $50 \mathrm{mg} / \mathrm{L}$ erhöht. Das Mengenverhältnis 1-O-Acyl- zu 1-O-Aryl-glykosid beträgt etwa $5: 4$, die Glykosylierung von Carboxylgruppen scheint leicht bevorzugt gegenüber phenolischen $\mathrm{OH}-\mathrm{Gruppen}$ zu sein. Eine genaue Aussage über die Spezifität der Glykosyltransferase kann jedoch nicht getroffen werden, da nicht bekannt ist, wie rasch die Umwandlung der 4-Hydroxybenzoesäure zum Hydrochinon erfolgt. Für das Produktverhältnis könnten daher auch unterschiedliche SubstratKonzentrationen ursächlich sein.

Als neue Verbindung wurde der farblose Feststoff $\mathbf{5 4}$ isoliert, dessen ${ }^{1}$ H-NMR-Spektrum neben den Protonen des para-disubstituierten Aromaten das Vorliegen von zwei Desoxyzuckern zeigt. Eines der anomeren Protonen zeigt ein Signal bei $\delta_{\mathrm{H}}=6.24$ (kennzeichnend für eine glykosidische Esterbindung), das andere erscheint bei höherem Feld $\left(\delta_{\mathrm{H}}=5.65\right)$, was für eine normale Glykosidbindung spricht. Das ${ }^{13} \mathrm{C}-\mathrm{NMR}-$ Spektrum belegt mit der Resonanz bei $\delta_{\mathrm{C}}=166$, dass keine Decarboxylierung am Aromaten stattgefunden hat. Die Molmasse von $430 \mathrm{~g} / \mathrm{mol}$ sowie die durch hochauflösende Massenspektrometrie verifizierte Summenformel $\mathrm{C}_{19} \mathrm{H}_{26} \mathrm{O}_{11}$ sichern die Struktur von 4-O-(6-Desoxy- $\alpha-L$-talopyranosyl)-benzoesäure(6-desoxy- $\alpha$ - $L$-talopyranosyl)ester (54) ab.

\subsection{Biologische Aktivität der Metaboliten}

Die 6-Desoxy- $\alpha$ - $L$-talopyranoside 45 - 51 und 54 wurden auf antimikrobielle Aktivität gegen Escherichia coli, Bacillus subtilis, Staphylococcus aureus und Candida albicans getestet. Es konnte keine hemmende Wirkung der Substanzen festgestellt werden.

\footnotetext{
${ }^{\mathrm{p}}$ Der tatsächliche Einbau des Vorläufermoleküls in $\mathbf{4 8}$ ist zwar aufgrund der Ausbeutesteigerung zu vermuten, ein Beweis wäre jedoch nur durch die Fütterung einer markierten Verbindung zu erlangen.
} 
Die Hauptmetaboliten 46 und 48 wurden gemäß der NCI-Richtlinien auf ihre cytotoxische Wirkung gegen die Krebszelllinien HM02 (Magenkarzinom), HepG2 (Leberkarzinom) und MCF7 (Mammakarzinom) untersucht, wiesen hier jedoch ebenfalls keine Aktivität auf ${ }^{\mathrm{q}}$.

Die mögliche biologische Funktion der Substanzen wird in Kapitel 5.7 (Seite 80) diskutiert.

\subsection{6-Desoxytalose - ein ungewöhnlicher Zuckerbaustein}

6-Desoxyzucker sind bei Sekundärmetaboliten häufig vorkommende Bausteine, meist handelt es sich um Rhamnose (=6-Desoxymannose) oder Fucose (=6-Desoxygalaktose) ${ }^{[114]}$. Aus Mikroorganismen ist lediglich ein Sekundärstoff in der Literatur beschrieben, der 6-Desoxytalose enthält: Phenazoviridin (55), ein aus einem Streptomyceten isoliertes Phenazin-Derivat (Abbildung 37). Ebenso wie bei 45 - 51 und 54 liegt der Desoxyzucker hier als $\alpha$-L-Pyranose vor ${ }^{[119]}$.

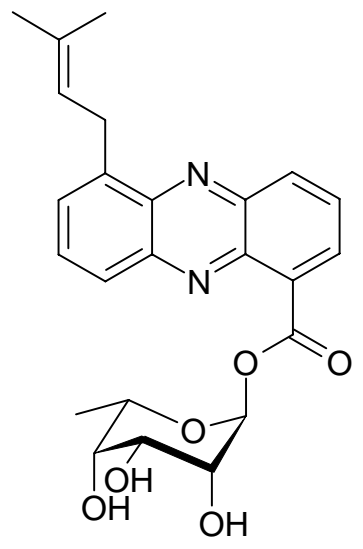

55

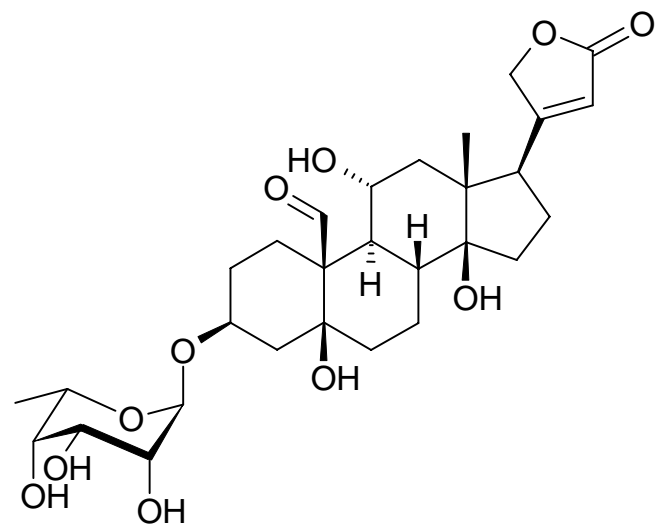

56

Abbildung 37: Phenazoviridin A (55) und Sarmentosid A (56).

Ebenfalls in der $\alpha$ - $L$-Form liegt 6-Desoxytalose in einigen Strophantidinen vor, einer Familie hochwirksamer Herzglykoside. Diese pflanzlichen Sekundärstoffe sind aus den Samen afrikanischer Heilpflanzen der Gattung Strophanthus bekannt und werden ähnlich wie die Digitalis-Glykoside therapeutisch angewandt. Abbildung 37 zeigt als Beispiel das aus S. sarmentosus isolierte Sarmentosid A (56) ${ }^{[120]}$. Weiterhin ist $\alpha$-L-Desoxytalose Bestandteil

\footnotetext{
${ }^{\mathrm{q}}$ Für die Durchführung der Cytotoxizitäts-Tests bedanke ich mich bei Herrn Prof. Dr. W. Beil (Institut für Pharmakologie, Medizinische Hochschule Hannover).
} 
von Glykolipiden verschiedener Bakterienstämme der Gattungen Mycobacterium, Pseudomonas und Rhodopseudomonas ${ }^{[121,122]}$.

Das $D$-Enantiomer der 6-Desoxytalose wurde in einigen bakteriellen Lipopolysacchariden (LPS), die in der Zellwand Gram-negativer Bakterien vorkommen ${ }^{[123]}$, sowie bei Glykolipiden aus Mycobacterium-Arten nachgewiesen ${ }^{[124]}$. In beiden Fällen wurde nicht berichtet, ob der Desoxyzucker in der $\alpha$ - oder $\beta$-Form vorliegt.

\subsection{Vergleich mit den bekannten 1-O-Acyl- $\alpha-L-r h a m n o-$ pyranosiden}

Von verschiedenen Streptomyceten ist bekannt, dass sie 1-O-Acyl- $\alpha$ - $L$-Rhamnopyranoside bilden $^{[117,125,126]}$. Abgesehen von dem veränderten Desoxyzucker gleichen sie den hier vorgestellten 6-Desoxy- $\alpha$ - $L$-talopyranosiden. So ist der Hauptmetabolit des Stamms Streptomyces griseoviridis Tü 3634 ebenfalls eine glykosylierte Phenylessigsäure (57, Abbildung 38). Auch die Rhamnose-Analoga der anderen 1-O-Acyl-6-desoxy- $\alpha$ - $L$-talopyranoside werden von diesem Stamm gebildet ${ }^{[125]}$ (50) oder sind durch Vorläufer-dirigierte Biosynthese zugänglich ${ }^{[127]}(\mathbf{4 7}, \mathbf{5 1})$.
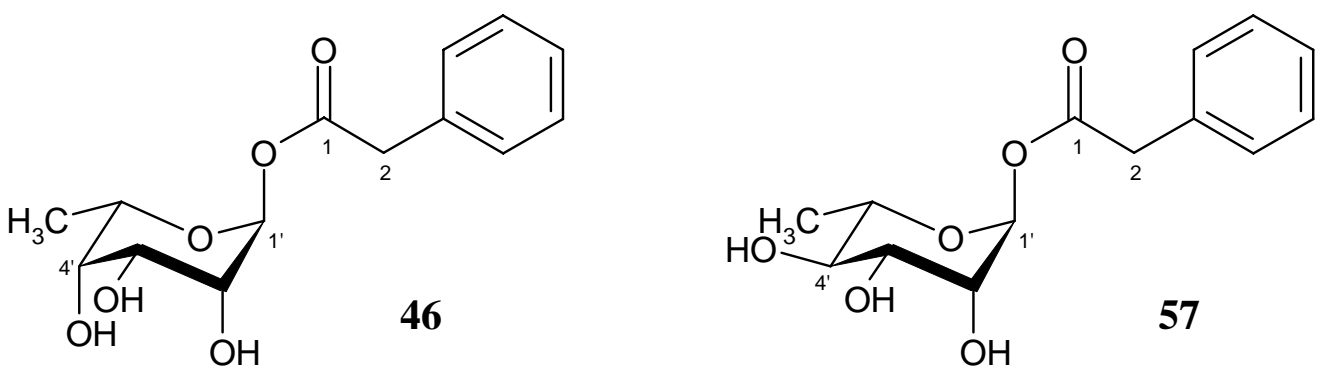

Abbildung 38: 1-O-Phenylacetyl-6-desoxy- $\alpha-L$-talopyranosid (46) und 1-O-Phenylacetyl- $\alpha$ $L$-rhamnopyranosid (57).

Die bakteriellen Produzenten der Rhamnopyranoside zeigten in Versuchen zur Vorläuferdirigierten Biosynthese eine breite Akzeptanz verschiedener Carbonsäuren, wodurch die Anzahl bekannter 1-O-Acyl- $\alpha$ - $L$-Rhamnopyranoside auf über 20 erhöht werden konnte ${ }^{[116,117]}$. Als essentiell stellte sich die Bindung der Carboxylgruppe an einen Aromaten oder das Vorkommen einer Doppelbindung in $\beta$-Position heraus ${ }^{[16]}$. Isovaleriansäure (wie in 
Verbindung 45 vorliegend) würde demnach von der Glykosyltransferase der Rhamnopyranosid-Bildner nicht als Substrat akzeptiert werden.

Die geringe Substratspezifität der Glykosyltransferasen bezüglich der Carbonsäure geht einher mit einer hohen Spezifität für die Zuckerkomponente, Zufütterung von z. B. L-Fucose zu S. griesoviridis Tü 3634 führte zu keiner Umsetzung ${ }^{[127]}$.

Als einziger natürlich vorkommender 1-O-Arylether der Rhamnose ist das entsprechende Salicylsäure-Derivat bekannt ${ }^{[127]}, p$-Hydroxybenzylalkohol konnte durch Zufütterung zu Kulturen von S. griseoviridis Tü 3634 rhamnosyliert werden ${ }^{[16]}$. 1-O-Aryl- $\alpha$ - L-rhamnoside mit den in 48 und 49 vorkommenden Aromaten wurden bisher nicht beschrieben. Ebenso ist eine mehrfach rhamnosylierte Verbindung analog zu 54 nicht bekannt.

\subsection{Diskussion der Ergebnisse}

\section{Zur Struktur und biologischen Funktion der Substanzen}

6-Desoxytalose ist ein ungewöhnlicher Zuckerbaustein, der im Sekundärstoffwechsel von Mikroorganismen zuvor nur von einer einzigen Substanz bekannt war ${ }^{[119]}$. Die in dieser Arbeit beschriebenen neuen 1-O-Acyl-6-desoxy- $\alpha$-talopyranoside 45 - 51 besitzen ähnliche glykosidisch gebundene Bausteine wie die bekannten 1-O-Acyl- $\alpha-L$-Rhamnopyranoside ${ }^{[125]}$. Unabhängig vom Zuckerbaustein sind die Moleküle weder antimikrobiell noch cytotoxisch wirksam, ihre biologische Funktion ist bisher nicht bekannt. Für einige Rhamnopyranoside wurde eine moderate inhibierende Wirkung gegen das Enzym 3a-HydroxysteroidDehydrogenase ( $3 \alpha-\mathrm{HSD}$ ) festgestellt, das an der Biosynthese von Arachidonsäure beteiligt ist $^{[126]}$.

Im Sekundärmetabolismus von Pflanzen sind Acyltransferasen bekannt, die acylierte Zucker anstelle von CoA-Thioestern als Substrate akzeptieren ${ }^{[128]}$. Die Acyl-Donoren sind hier 1-O- $\beta$-Glucose-Ester, die Enzyme selbst weisen überraschenderweise eine hohe Ähnlichkeit zu Carboxypeptidasen und damit Enzymen des Primärstoffwechsels auf. Das Wissen über die Verwendung von 1-O-Acyl- $\beta$-glucopyranosiden in Pflanzen kann sicherlich nicht direkt auf 1-O-Acyl- $\alpha$-Desoxyzuckerpyranoside in Mikroorganismen übertragen werden. Dennoch bietet die Idee, die acylierten Zucker als energiereiche Intermediate anzusehen, eine plausible Erklärung für ihr wiederholtes Vorkommen in Bakterien. Die große Anzahl an akzeptierten Substraten und die hieraus abzuleitende geringe Spezifität der entsprechenden Glykosyltransferase lassen sogar die Verwendung als Energiespeicher möglich erscheinen, da eine 
Vielzahl an acylierten Verbindungen für den Stamm wichtiger zu sein scheint als die genaue Kontrolle des Produkts.

Interessant wäre das Auffinden der bakteriellen Enzyme, die die acylierten Pyranoside als Substrat akzeptieren und deren biochemische und molekularbiologische Charakterisierung. Möglicherweise gibt es hier, ebenso wie bei den Pflanzen, eine enge Verwandtschaft zwischen Primär- und Sekundärmetabolismus zu entdecken.

\section{Vorläufer-dirigierte Biosynthese}

Die Zufütterung aromatischer Carbonsäuren zur Kultur von Actinomyces sp. Gö M1 bewirkte eine erhebliche Steigerung der Ausbeute an den entsprechenden Glykosiden sowie die Bildung eines neuen 6-Desoxytalosids (54). Durch Vorläufer-dirigierte Biosynthese dürfte eine Vielzahl an weiteren neuen 1-O-Acyl- und 1-O-Aryl-6-desoxy- $\alpha$ - $L$-talopyranosiden zugänglich sein. Es erscheint lohnenswert, das Glykosylierungs-Potential des Stammes intensiver zu erschließen.

\section{Zur Biosynthese}

Die Biosynthese der 6-Desoxy- $\alpha$ - $L$-talopyranoside lässt sich unterteilen in den Aufbau des Desoxyzuckers und die Biosynthese der glykosidisch gebundenen, meist aromatischen Reste. Die Enzyme und katalytischen Mechanismen, die zur Bildung von 6-Desoxyzuckern führen, wurden ausführlich untersucht. Als Ausgangsverbindung dient Glucose, die zunächst in Position 6 reduziert und anschließend epimerisiert wird ${ }^{[129,130]}$.

Die aromatischen Bausteine dürften unterschiedlicher Herkunft sein. Ausgehend von Glucose entsteht zunächst Shikimat, welches weiter zu Chorismat umgesetzt wird. Bei Pflanzen und Pilzen führt der Biosyntheseweg weiter über Phenylalanin als Zwischenstufe zu $p$-Hydroxybenzoesäure (PHB). Ein analoger Weg wurde bei dem marinen Bakterium Streptomyces maritimus $^{[131]}$ und dem Myxobakterium Sorangium cellulosum gefunden ${ }^{[132]}$. Von dem Bakterium E. coli ist hingegen die direkte Umsetzung von Chorismat zu PHB durch das Enzym Chorismat-Lyase bekannt ${ }^{[133]}$. Eine direkte Bildung von Benzoat aus Shikimat ohne Phenylalanin-Zwischenstufe wurde auch bei dem Rhamnosidbildner S. griseoviridis Tü 3634 nachgewiesen $^{[134]}$ und kann daher ebenso für 47 - 49 und 51 vermutet werden. Für die Bildung von Phenylessigsäure erscheint hingegen die Transaminierung von Phenylalanin zu Phenylpyruvat und anschließende Decarboxylierung wahrscheinlich ${ }^{[131]}$. Beide Hypothesen wären durch geeignete Fütterungsexperimente mit markierten Verbindungen zu beweisen, was jedoch nicht Gegenstand der vorliegenden Arbeit war. 


\section{BMBF-Verbundprojekt „Endophytische Pilze aus Algen und Pflanzen verschiedener Meeresbiotope“}

Im Rahmen des Forschungsschwerpunkts „Marine Naturstoffforschung“, der vom Bundesministerium für Bildung und Forschung (BMBF) im Juli 2000 zum zweiten Mal ausgeschrieben wurde, wird das Projekt „Endophytische Pilze aus Algen und Pflanzen verschiedener Meeresbiotope“ gefördert (Förder-Kennzeichen 03F0360A). Der Förderzeitraum beträgt 3.5 Jahre, beginnend mit dem 1. Juli 2002.

Ziel des Projekts ist die Identifizierung neuer Naturstoffe und das Auffinden neuer Leitstrukturen für den Pflanzenschutz. Hierfür sollten endophytische Pilze aus Meeresalgen sowie aus Pflanzen der Brackwasserzonen und Küstenregionen hinsichtlich ihrer Inhaltsstoffe untersucht werden. Aufgrund ihrer speziellen Lebensbedingungen (Interaktion zwischen Endophyt und Pflanze, Salzgehalt, Gezeiten) wurde erwartet, dass die dort isolierten Pilze eine viel versprechende Quelle für neue Naturstoffe sind. Über die Inhaltsstoffe von Endophyten dieser Biotope war bisher vergleichsweise wenig bekannt.

Am Verbundprojekt sind vier Projektpartner beteiligt (siehe auch Abbildung 39):

1) Technische Universität Braunschweig (Arbeitsgruppe Prof. Dr. H.-J. AUST / PD Dr. B. SCHULZ, Institut für Mikrobiologie)

Der Arbeitskreis ist zuständig für die Isolierung der Pilze und ihre taxonomische Bestimmung. Die Isolate werden einem biologischen Screening unterzogen, die Kultivierungsbedingungen optimiert und die Extrakte der BASF zur Testung zugesandt. Dort aufgefallene Pilzstämme sollten im größeren Maßstab kultiviert und den Arbeitskreisen KROHN und ZEECK zur Verfügung gestellt werden.

2) Universität-GH Paderborn (Arbeitsgruppe Prof. Dr. K. KROHN, Organische Chemie) In diesem Arbeitskreis erfolgt die Isolierung und Strukturaufklärung von Reinsubstanzen aus einem Teil der Pilze, deren Extrakte biologische Aktivität zeigten oder im chemischen Screening auffielen. Zudem soll durch chemische Synthese und Variation besonders interessanter Strukturen das Spektrum der zur Verfügung stehenden Substanzen vergrößert werden. Reinsubstanzen werden den Pflanzenschutztests der BASF zugeführt. 
3) Universität Göttingen (Arbeitsgruppe Prof. Dr. A. ZEECK, Institut für Organische und Biomolekulare Chemie)

Vom Kooperationspartner in Braunschweig erhaltene Pilzstämme sowie selbst isolierte Endophyten werden einer Variation und Optimierung der Kultivierungsbedingungen unterzogen (OSMAC-Methode ${ }^{[102]}$ ). In den Pflanzenschutz-Tests der BASF und im chemischen Screening aufgefallene Stämme werden im größeren Maßstab kultiviert, die Sekundärmetaboliten isoliert und in ihrer Struktur aufgeklärt. Reinsubstanzen werden durch Plattendiffusionstests auf antimikrobielle Wirkung untersucht und ebenso wie die Extrakte zur Testung an die BASF gegeben.

4) BASF AG, Ludwigshafen

Die erhaltenen Rohextrakte und Reinsubstanzen werden in Mikrotestsystemen auf fungizide, insektizide und herbizide Wirkung geprüft. Biologisch aktive Extrakte werden HPLC-chromatographisch aufgetrennt und die Fraktionen auf biologische Aktivität hin getestet. Diese Korrelation zwischen Retentionszeit und biologischer Aktivität dient als Orientierung für die Isolierung der Reinsubstanzen.

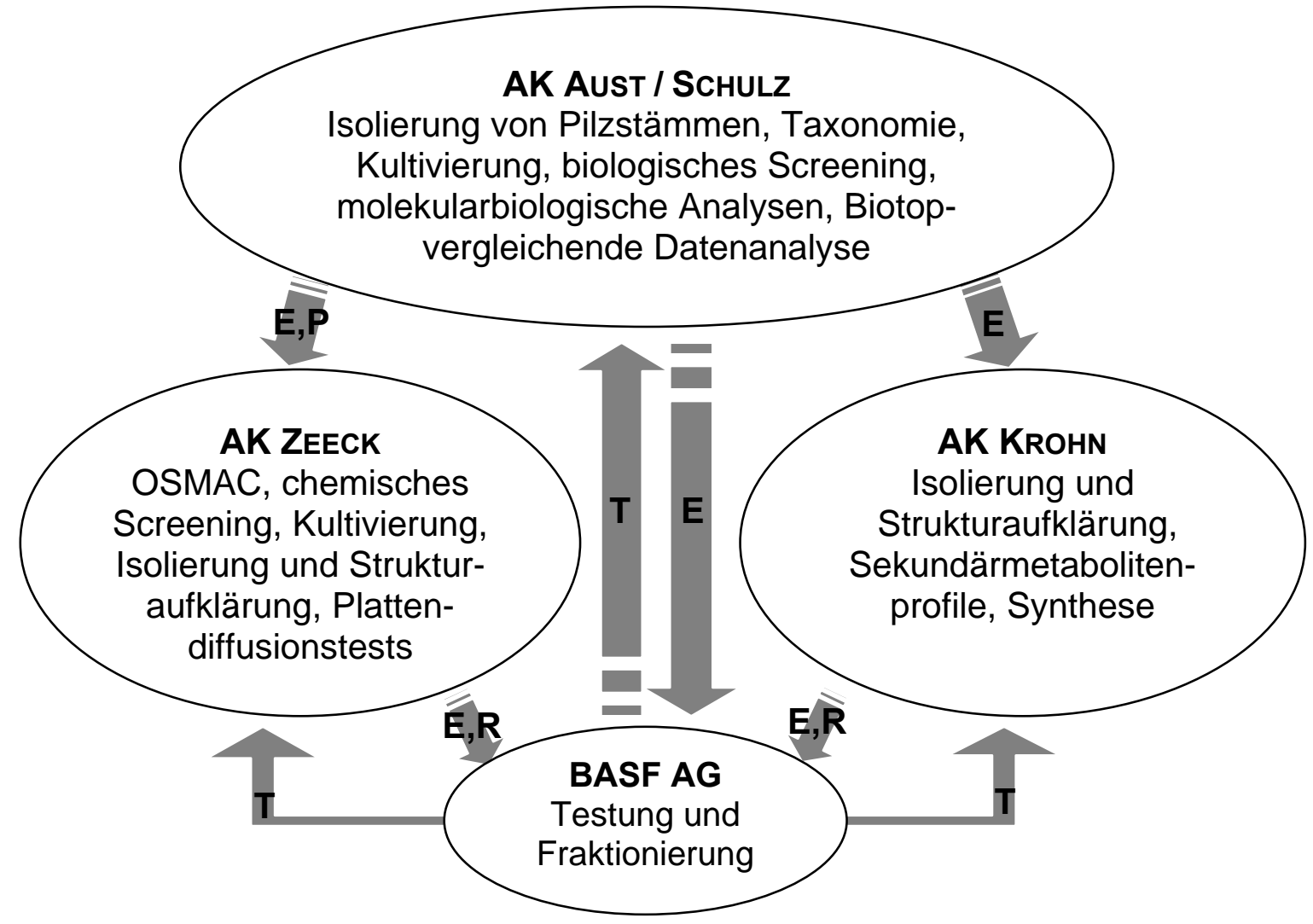

Abbildung 39: Arbeitsaufteilung im Verbundprojekt ( $\mathbf{E}=$ Extrakte, $\mathbf{R}=$ Reinsubstanzen, $\mathbf{P}=$ Pilzstämme, $\mathbf{T}=$ Testergebnisse). 


\section{$7 \quad$ Isolierung von Pilzen, Screening und Stammauswahl}

\subsection{Isolierung endophytischer Pilze aus einer Rotalge}

Im Rahmen des BMBF-Projekts wurden hauptsächlich Pilzstämme bearbeitet, die an der TU Braunschweig isoliert und charakterisiert wurden. Um die zur Verfügung stehende Anzahl an Pilzstämmen zu erhöhen, wurden zusätzlich eigene Pilzstämme aus einer Rotalge der Spezies Hypnea cervicornis ${ }^{r}$ isoliert. Diese auf Kreta gesammelte Algenart ist weltweit in tropischen und subtropischen Gebieten verbreitet. Sie wächst in flachem Wasser und wird auch zur Gewinnung von Agar und Carrageen genutzt ${ }^{[135]}$.

Zur Isolierung endophytischer Pilze nach einer von SCHULZ ET. AL. entwickelten Methode ${ }^{[136]}$ wurde die Oberfläche der Alge mit Ethanol sterilisiert. Anschließend zerschnitt man die Alge in ca. 2-5 mm große Stücke, legte diese auf Agarplatten aus und inkubierte bei $25^{\circ} \mathrm{C}$ für ein bis drei Tage, bis Kolonien sichtbar wurden (Abbildung 40). Die Kolonien wurden durch mehrfaches Überimpfen und Verdünnungsausstriche vereinzelt.

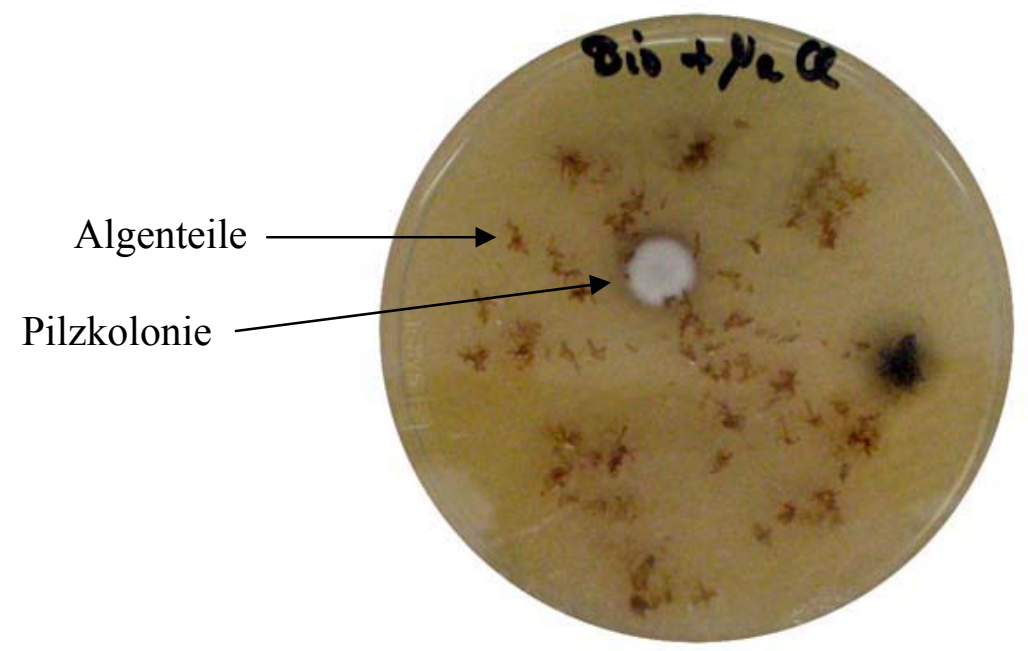

Abbildung 40: Agarplatte mit aus Algenteilen herauswachsenden Pilzkolonien.

Insgesamt wurden aus der Alge 36 Pilzstämme isoliert (Tabelle 13). Ein Unterschied zwischen Nährmedien mit und ohne Zugabe von Natriumchlorid wurde nicht festgestellt, alle

\footnotetext{
${ }^{\mathrm{r}}$ Für die Bestimmung von Gattung und Art der Alge bedanke ich mich bei Frau PD Dr. B. Schulz (Institut für Mikrobiologie, Technische Universität Braunschweig).
} 
Stämme sind ohne Zugabe von Salz kultivierbar. Die auffällig große Anzahl an Stämmen der Gattung Aspergillus weist darauf hin, dass die angewandte Isolierungsmethode schnell wachsende Stämme bevorzugt. Stachybotrys, Chaetomium, Paecilomyces, Arthrinium, Phoma und Trichoderma stellen ebenfalls weit verbreitete Taxa dar. Daneben finden sich mehrere Stämme der vor allem aus marinen Habitaten bekannten Gattung Dendryphiella ${ }^{[137]}$ und je ein Stamm der weniger häufig anzutreffenden Gattungen Thelebolus und Nodulisporium.

Tabelle 13: Taxonomische Einordnung der endophytischen Pilzstämme ${ }^{\mathrm{s}}$

\begin{tabular}{llll} 
Stamm- & Taxonomische & Stamm- & Taxonomische \\
Bezeichnung & Bestimmung & Bezeichnung & Bestimmung \\
\hline Gö 201 & Dendryphiella sp. & Gö 219 & Chaetomium sp. \\
Gö 202 & Stachybotrys sp. & Gö 220 & Stachybotrys sp. \\
Gö 203 & Stachybotrys sp. & Gö 221 & Aspergillus flavus \\
Gö 204 & Aspergillus niger & Gö 222 & Cladosporium sp. \\
Gö 205 & Aspergillus niger & Gö 223 & Nodulisporium sp. \\
Gö 206 & Chaetomium sp. & Gö 224 & Dendryphiella sp. \\
Gö 207 & n. b. & Gö 225 & Phoma sp. \\
Gö 208 & Thelebolus sp. & Gö 226 & Aspergillus niger \\
Gö 209 & Coprinus sp. & Gö 227 & Aspergillus niger \\
Gö 210 & n. b. & Gö 228 & Aspergillus niger \\
Gö 211 & Aspergillus flavus & Gö 229 & Trichoderma sp. \\
Gö 212 & Chaetomium sp. & Gö 230 & Aspergillus niger \\
Gö 213 & n. b., a) & Gö 231 & n. b. \\
Gö 214 & Aspergillus niger & Gö 232 & Eurotium/Aspergillus sp. \\
Gö 215 & Aspergillus niger & Gö 233 & Arthrinium sp. \\
Gö 216 & Aspergillus niger & Gö 234 & Aspergillus niger \\
Gö 217 & Paecilomyces farinosus & Gö 235 & Aspergillus niger \\
Gö 218 & Dendryphiella sp. & Gö 236 & Aspergillus niger \\
\hline
\end{tabular}
n. b. = nicht bestimmt, a) = nicht sporulierend

\footnotetext{
${ }^{\text {s }}$ Herrn Dr. S. Draeger (Institut für Mikrobiologie, Technische Universität Braunschweig) danke ich für die taxonomische Bestimmung der Pilzstämme und die Auskünfte bezüglich seltener Gattungen.
} 


\subsection{Kultivierung der Pilzstämme}

Die Kultivierung der Endophyten erfolgte durch vier verschiedene Verfahren:

- An der TU Braunschweig wurden alle dort isolierten Stämme auf Festagar mit Biomalz-Nährmedium und einem Feststoffmedium auf Dinkel-Gerste Basis (DiGe) kultiviert. Die Extrakte wurden einem biologischen Screening sowie den Testsystemen der BASF zugeführt (siehe Kapitel 7.4).

- Ausgewählte aus Braunschweig erhaltene Stämme wurden unter variierten Bedingungen kultiviert. Die Inkubation der Schüttelkulturen erfolgte in sechs verschiedenen Nährmedien (1158, G20, E2, 1549, SGG, Biomalz). Einige der Stämme wurden zusätzlich unter Zugabe von $33 \mathrm{~g} / \mathrm{L}$ Natriumchlorid oder $58 \mathrm{~g} / \mathrm{L}$ Natriumbromid zur Nährlösung kultiviert. Ausgehend von den Ergebnissen der Schüttelkultur wurde der jeweilige Stamm mit zwei bis sechs verschiedenen Nährmedien in P-Kolben inkubiert. Die Extrakte wurden einem chemischen Screening unterzogen sowie den BASF-Pflanzenschutztests zugeführt.

- Die Endophyten aus der Rotalge Hypnea cervicornis wurden in den erwähnten sechs Nährlösungen in Schüttelkolben kultiviert und die Extrakte durch chemisches und biologisches Screening untersucht. Bei der Kultivierung blieben Isolate der Gattung Aspergillus weitgehend unberücksichtigt, da diese sehr umfangreich untersucht ist und somit die Gefahr der Reisolierung bekannter Substanzen als besonders hoch angesehen wurde.

\subsection{Chemisches Screening}

Das chemische Screening erfolgte durch eine dünnschichtchromatographische Auftrennung der Kulturfiltrat- und Myzelextakte an Kieselgel, als Laufmittel diente Chloroform/Methanol (9:1). Der Nachweis der Metaboliten geschah unter UV-Licht (254 und $366 \mathrm{~nm}$ ) sowie durch Anfärbereagenzien (Anisaldehyd $/ \mathrm{H}_{2} \mathrm{SO}_{4}$, Orcin-Sprühreagenz, Ehrlich's Reagenz). Ausgewählte Extrakte, die auf dem Dünnschichtchromatogramm ein interessantes Metabolitenspektrum zeigten, wurden zusätzlich mittels HPLC-MS untersucht. Nachfolgend wird eine Zusammenfassung der erhaltenen Ergebnisse gegeben, die Resultate einzelner Stämme werden in Kapitel 7.5 dargestellt.

Extrakte von Festagar- und Feststoffmedium-Kulturen („Tüpfelproben“): Von 235 untersuchten Extrakten wurden im chemischen Screening 29 als interessant und 37 als 
eingeschränkt interessant beurteilt. Bei 169 Proben konnte keine Sekundärstoffproduktion in größerem Umfang festgestellt werden.

Schüttelkulturen und P-Kolben der aus Braunschweig erhaltenen Endophyten: Von 14 in Schüttelkolben kultivierten Pilzstämmen zeigten vier ein interessantes Metabolitenmuster. In P-Kolben war die Erfolgsrate höher: sechs der elf bearbeiteten Stämme wiesen eine verfolgenswerte Sekundärstoffbildung auf.

Schüttelkulturen der Pilzstämme Gö 201 - Gö 233: Neun der bisher 18 kultivierten Pilzstämme wurden als interessant beurteilt.

\subsection{Biologisches Screening}

Bei der BASF erfolgte die Untersuchung hinsichtlich einer für den Pflanzenschutz relevanten biologischen Aktivität. Die Extrakte und Reinsubstanzen wurden in Mikrotestsystemen auf eine hemmende Wirkung gegen phytopathogene Pilze (Tabelle 14) und schädigende Insekten (Tabelle 15) geprüft. Bei größeren Probenmengen erfolgte zusätzlich die Testung der fungiziden Wirkung im Gewächshaus und der herbiziden Eigenschaften gegen bestimmte Pflanzenarten (Tabelle 16).

Tabelle 14: In den Testsystemen verwendete phytopathogene Pilze

\begin{tabular}{lll} 
Wissenschaftlicher Name & wächst auf & verursachte Krankheit \\
\hline Phytophthora infestans & Tomate & Kraut- und Knollenfäule \\
Alternaria solani & Tomate & Dürrfleckenkrankheit \\
Botrytis cinerea & Paprika/ Tomate & Grauschimmel \\
Pyricularia oryzae & Reis & Reisbrand \\
Erysiphe segetum & Weizen & Getreidemehltau \\
Puccinia recondita & Weizen & Braunrost \\
Septoria tritici & Weizen & Blattdürre des Weizens \\
Septoria nodorum & Weizen & Blatt- und Spelzenbräune \\
Pyrenophora teres & Gerste & Netzfleckenkrankheit der Gerste \\
\hline
\end{tabular}

Standard-Set für Mikrotests: $P$. infestans, $B$. cinerea, $P$. oryzae, $S$. tritici

Standard-Set für Gewächshaus-Tests: P. infestans, A. solani, B. cinerea, E. segetum, $P$. recondita, $S$. nodorum, $P$. teres 
Tabelle 15: Insekten des BASF Insektizid-Eingangstests

\begin{tabular}{ll} 
Wissenschaftlicher Name & Deutsche Bezeichnung \\
\hline Anthonomus grandis & Baumwollkapselrüßler \\
Certitis capitata & Mittelmeerfruchtfliege \\
Heliothis virescens & Eulenfalter \\
Megoura viciae & Bohnenblattlaus \\
\hline
\end{tabular}

Tabelle 16: Im Herbizid-Eingangstest verwendete Pflanzen

\begin{tabular}{ll} 
Wissenschaftlicher Name & Deutsche Bezeichnung \\
\hline Abuliton theophrasti & Samtpappel (Malvengewächs) \\
Alopecurus myosuroides & Acker-Fuchsschwanz \\
Avena fatua & Flughafer \\
Echinochloa crus-galli & Hühnerhirse \\
Setaria faberi & Fabers Borstenhirse \\
Seraria italica & Kolbenhirse \\
\hline
\end{tabular}

Schüttel- und Ruhekulturen der aus Braunschweig erhaltenen Pilzstämme: 14 Pilzstämme wurden unter verschiedenen Bedingungen in Schüttelkolben (ca. 300 Extrakte) kultiviert, zusätzlich wurden von 11 dieser Pilzstämme Ruhekulturen in P-Kolben angelegt (ca. 100 Extrakte). Proben von sieben der Stämme wiesen signifikante Aktivitäten auf (siehe Tabelle 17 auf Seite 90). Hierbei zeigte sich, dass bei interessanten Stämmen meist mehrere (jedoch nicht alle) Extrakte, die aus Kulturen mit verschiedenen Nährmedien gewonnen wurden, eine biologische Wirkung zeigten. Ein deutlicher Unterschied hingegen wurde stets zwischen den Extrakten aus Schüttel- und Ruhekultur beobachtet.

Schüttelkulturen der selbst isolierten Endophyten: Von bisher 18 kultivierten Pilzstämmen (insgesamt 216 Extrakte) zeigten Extrakte von drei Stämmen eine gute fungizide Wirkung und von einem Stamm eine starke insektizide Wirkung. Weitere drei Stämme ergaben schwächer fungizide bzw. insektizide Extrakte. Eine detaillierte Übersicht wird in Tabelle 18 auf Seite 91 gegeben. 


\subsection{Screening-Ergebnisse und Stammauswahl}

Die Ergebnisse des chemischen und biologischen Screenings sind in Tabelle 17 (aus Braunschweig erhaltene Endophyten) und Tabelle 18 (selbst isolierte Endophyten) zusammengefasst.

In der ersten Hälfte des Projektzeitraums wurden mangels der Verfügbarkeit anderer Pilzstämme die Stämme BS 5943, BS 5945, BS 5954, BS 5958 und BS 6278 bearbeitet, obwohl diese weder im chemischen noch im biologischen Screening besonders auffällig waren. Einzig der Agarplatten-Extrakt des Stamms BS 5954 zeigte eine insektizide Wirkung, die jedoch in Schüttel- oder P-Kolben-Kultur nicht reproduziert werden konnte. Trotz intensiver Bearbeitung aller fünf Pilzstämme konnten nur aus den Stämmen BS 5945 und BS 6278 interessante Sekundärmetaboliten isoliert werden, deren Strukturaufklärung in Kapitel 9 (Ascosalipyron (60)) bzw. Kapitel 10 (Solanapyron C (61)) besprochen wird. Aus den anderen drei Pilzen wurden lediglich Metaboliten wie Ergosterol-5,8-epoxid (Stamm BS 5943) und 4-(2-Hydroxyethyl)-phenol (Stamm BS 5958) erhalten, so dass auf eine Beschreibung dieser Experimente verzichtet wird.

In der nachfolgenden Zeit hingegen stand durch die erhöhte Anzahl positiver ScreeningResultate eine größere Anzahl an interessanten Pilzen zur Verfügung. Die Auswahl der bearbeiteten Pilzstämme erfolgte aufgrund interessanter Metabolitenprofile im chemischen Screening (Drechslera sp. BS 6618, Beauveria sp. BS 6750, Phomopsis sp. BS 6769), die teilweise auch mit einer signifikanten biologischen Wirkung einher gingen (Chaetomium sp. BS 6556).

Da viele Screening-Ergebnisse erst gegen Ende dieser Arbeit zur Verfügung standen, steht die Bearbeitung einiger hoch interessanter Extrakte noch aus bzw. ist nicht abgeschlossen. $\mathrm{Zu}$ nennen sind hier insbesondere die chemisch interessanten und biologisch aktiven P-KolbenKulturen der Stämme Phoma sp. BS 6829 und Pleospora sp. BS 6631 sowie der AgarplattenExtrakt von Phoma sp. BS 6771. Der nicht bestimmte Stamm BS 6612 produzierte sowohl in Schüttel- als auch in Ruhekulturen biologisch und chemisch interessante Substanzen. Auch die Pilze BS 6666 und BS 6689 lassen interessante Sekundärstoffe erhoffen, ebenso Coprinus sp. Gö 209, Chaetomium sp. Gö 212, Aspergillus flavus Gö 221 und Nodulisporium sp. Gö 223. 
Tabelle 17: Screening-Ergebnisse der aus Braunschweig erhaltenen Pilzstämme

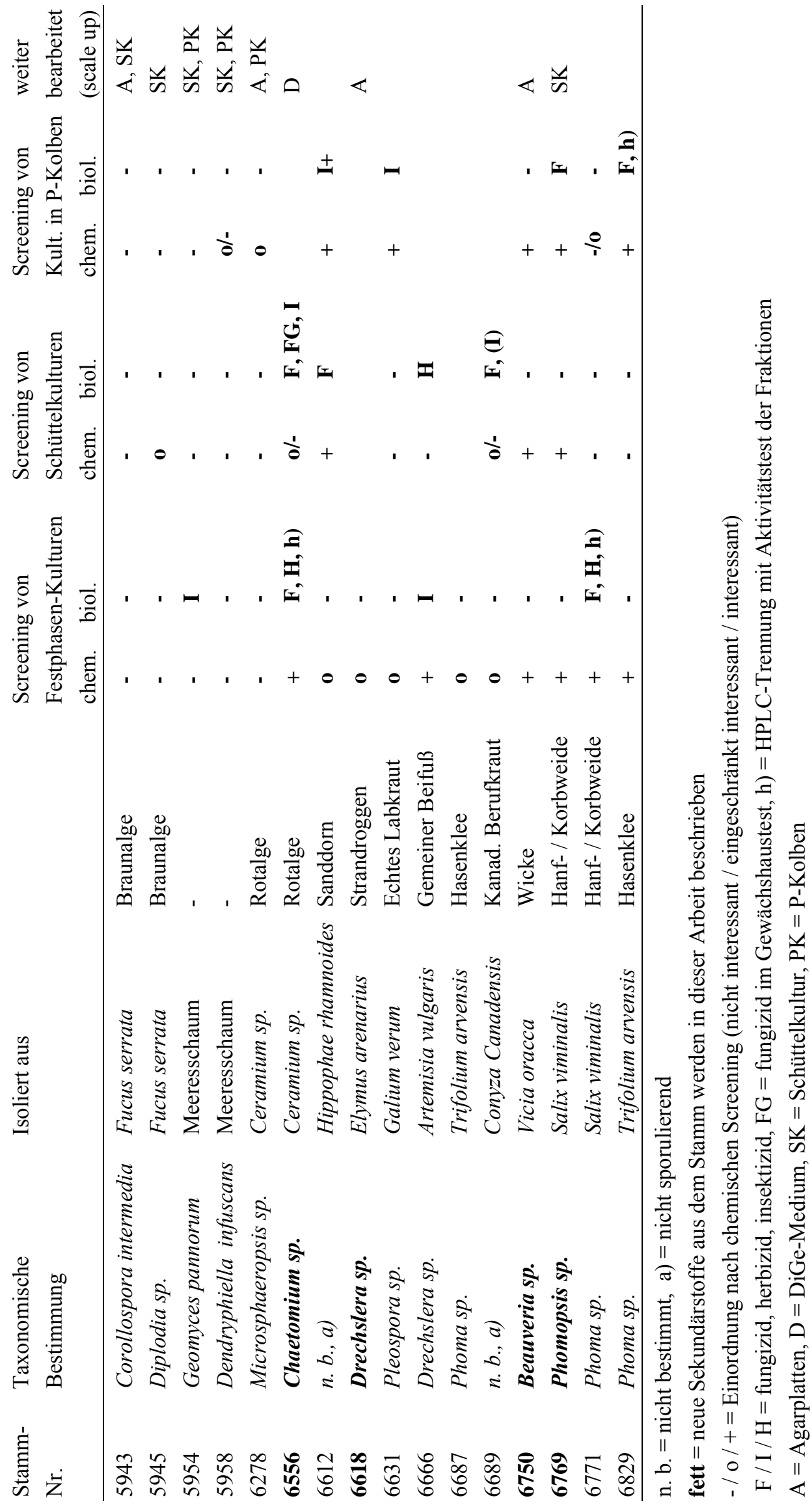


Tabelle 18: Screening-Ergebnisse der selbst isolierten Endophyten

Stamm- Taxonomische Bestimmung Ergebnisse der Schüttelkultur-Extrakte Bezeichnung

chem. Screening biol. Screening

\begin{tabular}{llcc}
\hline Gö 201 & Dendryphiella sp. & - & (F) \\
Gö 202 & Stachybotrys sp. & - & - \\
Gö 203 & Stachybotrys sp. & o & - \\
Gö 207 & n. b. & - & F \\
Gö 208 & Thelebolus sp. & - & - \\
Gö 209 & Coprinus sp. & + & - \\
Gö 212 & Chaetomium sp. & + & F, h) \\
Gö 213 & n. b., a) & $\mathbf{0}$ & I \\
Gö 217 & Paecilomyces farinosus & + & - \\
Gö 218 & Dendryphiella sp. & - & - \\
Gö 220 & Stachybotrys sp. & + & F \\
Gö 221 & Aspergillus flavus & + & - \\
Gö 223 & Nodulisporium sp. & + & - \\
Gö 224 & Dendryphiella sp. & - & - \\
Gö 225 & Phoma sp. & - & (I) \\
Gö 227 & Aspergillus niger & - & (I) \\
Gö 229 & Trichoderma sp. & $\mathbf{0}$ & - \\
Gö 233 & Arthrinium sp. & - & \\
\hline
\end{tabular}

n. b. = nicht bestimmt, a) $=$ nicht sporulierend

- / o / + = Einordnung nach chemischen Screening (nicht interessant / eingeschränkt interessant / interessant)

$\mathrm{F}=$ fungizid, $\mathrm{I}=$ insektizid, $\mathrm{h}$ ) $=$ HPLC-Trennung mit Aktivitätstest der Fraktionen 


\section{Häufig vorkommende Metaboliten}

\subsection{Diketopiperazine}

Diketopiperazine werden häufig von Pilz- und Bakterienstämmen produziert. Alle in dieser Arbeit aus Pilzkulturen isolierten Diketopiperazine wurden bereits in Kapitel 3.2 (Seite 51) als Metaboliten des Bakteriums Halomonas sp. Rk377a beschrieben (Substanzen 33 bis 37).

\subsection{Ergosterol (58)}

Ergosterol (58) ist die dominierende Sterol-Komponente der Zellmembran von Pilzen und wichtig für die Struktur und Fluidität der Membran ${ }^{[138]}$. 58 ist damit ein Produkt des Primärmetabolismus und kann in jedem aus Pilzen erhaltenen Extrakt nachgewiesen werden. Insbesondere die Myzelextrakte enthalten meist größere Mengen (z. B. $25 \mathrm{mg} / \mathrm{L}$ bei Microsphaeropsis sp. BS 6278, Kapitel 10). Eine zweite Funktion besitzt Ergosterol (58) als hormoneller Wachstumspromotor. Es konnte nachgewiesen werden, dass bei Abwesenheit von 58 kein Wachstum von Pilzen möglich ist, obwohl die Funktion als Membranlipid von strukturell ähnlichen Sterolen ersetzt werden könnte ${ }^{[139]}$.

Aufgrund des nahezu ubiquitären Vorkommens bei Pilzen kann der Nachweis von Ergosterol (58) zur qualitativen und quantitativen Bestimmung pilzlicher Biomasse in Umweltproben ${ }^{[140]}$ oder bei Lebensmitteln ${ }^{[141]}$ dienen. Hierzu wird die Probe mit einem lipophilen Lösungsmittel extrahiert und 58 HPLC-analytisch oder

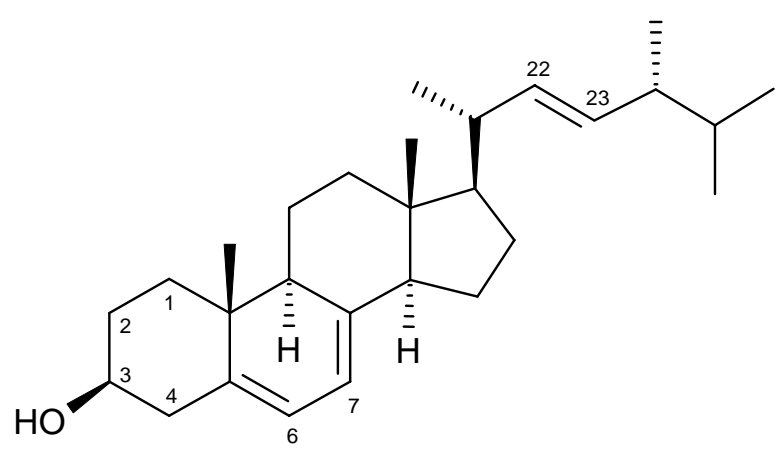

58

durch Dünnschichtchromatographie nachgewiesen $^{[142]}$.

58 färbt auf dem Dünnschichtchromatogramm mit Anisaldehyd in der Wärme violett an und löscht UV-Licht bei $254 \mathrm{~nm}$. Das ${ }^{1}$ H-NMR-Spektrum zeigt ein auffälliges Kopplungsmuster der olefinischen Protonen, weitere kennzeichnende Signale sind von $3-\mathrm{H}\left(\delta_{\mathrm{H}}=3.61\right)$ sowie den insgesamt sechs Methylgruppen (Abbildung 41). 


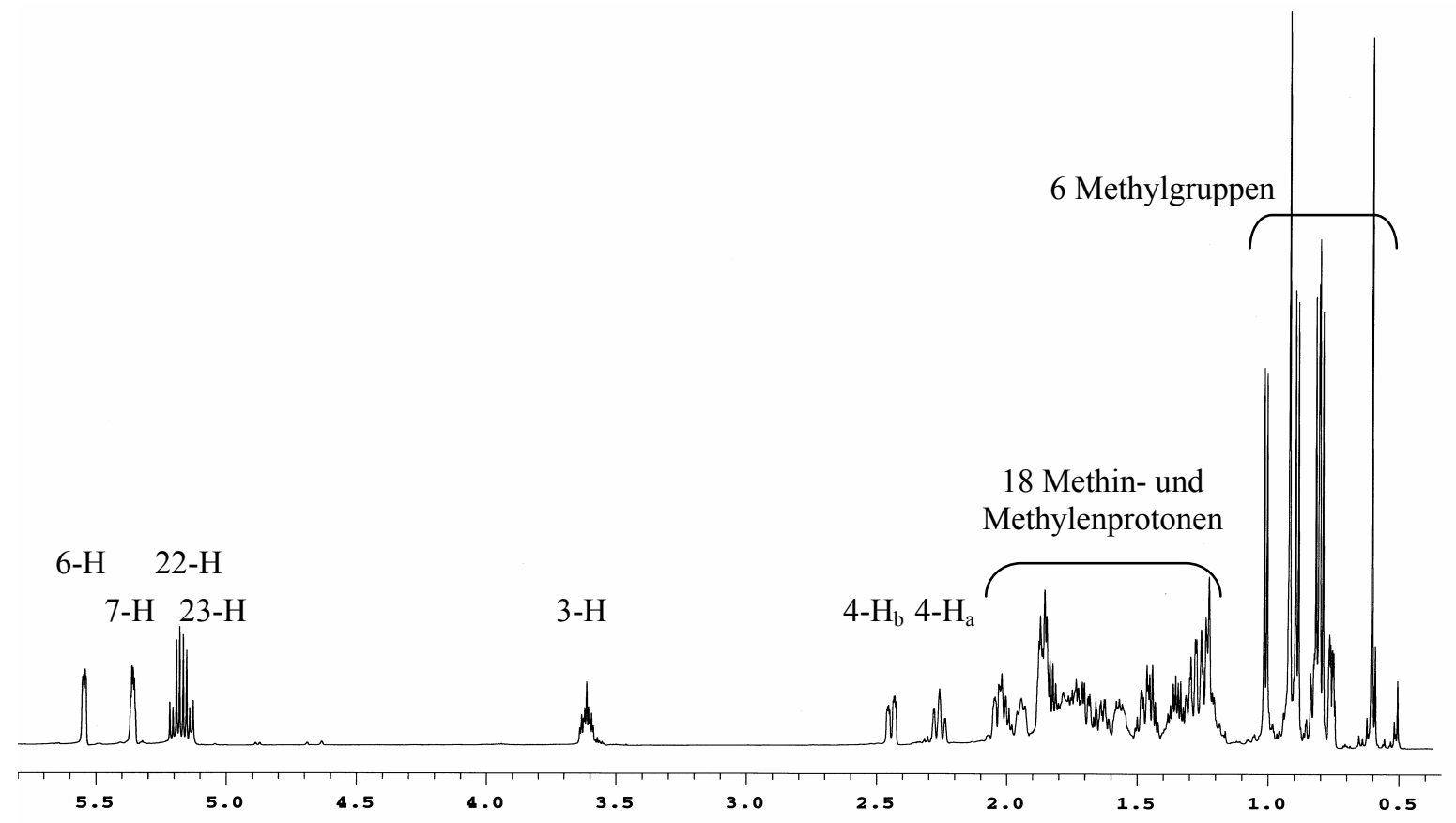

Abbildung 41: ${ }^{1} \mathrm{H}-\mathrm{NMR}-$ Spektrum $\left(600 \mathrm{MHz}, \mathrm{CDCl}_{3}\right)$ von Ergosterol (58).

Säugetiere verwenden Cholesterol anstelle von Ergosterol (58) als dominierendes Sterol in ihrer Zellwand. Dieser grundlegende Unterschied im Zellaufbau eröffnet Möglichkeiten für einen selektiven Angriff durch Antimykotika. Polyenantibiotika wie Amphotericin B oder Nystatin bewirken durch eine irreversible Komplexbildung mit 58 eine Permeabilisierung der Membran, andere Substanzklassen wie die Allylamine oder die Azol-Antimykotika inhibieren die Biosynthese von Ergosterol ${ }^{[143]}$.

Ergosterol (58) wird auch als Provitamin $\mathrm{D}_{2}$ bezeichnet, da es sich unter dem Einfluss von UV-Licht zu Vitamin $\mathrm{D}_{2}$ umwandelt ${ }^{[144]}$. Außerdem besitzt 58 antioxidative Eigenschaften, indem es mit reaktiven Sauerstoffspezies zu Ergosterol-5,8-peroxid umgesetzt wird ${ }^{[145]}$. 


\subsection{Cerebrosid C (59)}

Als Cerebroside bezeichnet man Glykolipide, die aus Sphingosin, einer Fettsäure und einem Monosaccharid ( $D$-Galactose oder $D$-Glucose) bestehen (Abbildung 42). Sie gehören zur Substanzklasse der Sphingolipide, zu denen u. a. auch Ceramide, Ganglioside und Sphingomyeline zählen. Sphingolipide kommen ubiquitär in allen eukaryotischen Organismen vor. Sie sind meist in der Plasmamembran angereichert und besitzen vielfältige biologische Funktionen $^{[144]}$. Bei Cerebrosid C (59) handelt es sich daher ebenso wie bei Ergosterol (59) um ein primäres Stoffwechselprodukt von Pilzen, das hauptsächlich im Myzelextrakt gefunden wird.

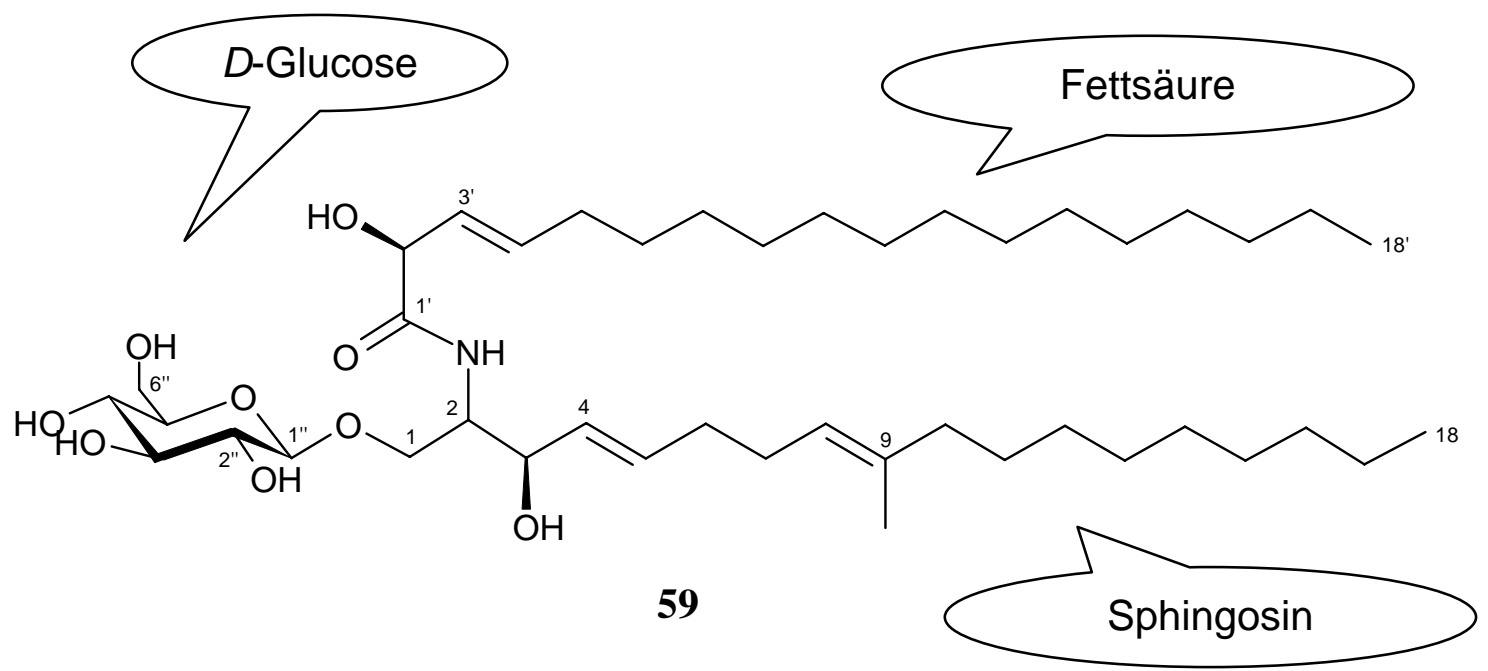

Abbildung 42: Struktureller Aufbau von Cerebrosid C (59).

Auf dem Dünnschichtchromatogramm fällt 59 als eine polare Substanz auf, die mit Anisaldehyd in der Wärme intensiv violett anfärbt. Die Strukturaufklärung gelingt rasch anhand des Massenspektrums und eines ${ }^{1} \mathrm{H}-\mathrm{NMR}$-Spektrums (Abbildung 43). Letzteres zeigt die Resonanzen fünf olefinischer Protonen $\left(\delta_{\mathrm{H}}=5.1\right.$ - 5.9) und von insgesamt elf Protonen an Sauerstoff-substituierten C-Atomen $\left(\delta_{\mathrm{H}}=3.2-4.5\right)$, die der Glucose sowie 1- $\mathrm{H}_{2}, 3-\mathrm{H}$ und 2'-H zuzuordnen sind. Auch das zum Stickstoff benachbarte Proton 2-H erscheint bei höherem Feld $\left(\delta_{\mathrm{H}}=3.97\right)$. Auffällig ist zudem das intensive Signal der zur Fettsäure und dem lipophilen Schwanz des Sphingosins gehörenden Methylengruppen, das für insgesamt 36 Protonen integriert. 


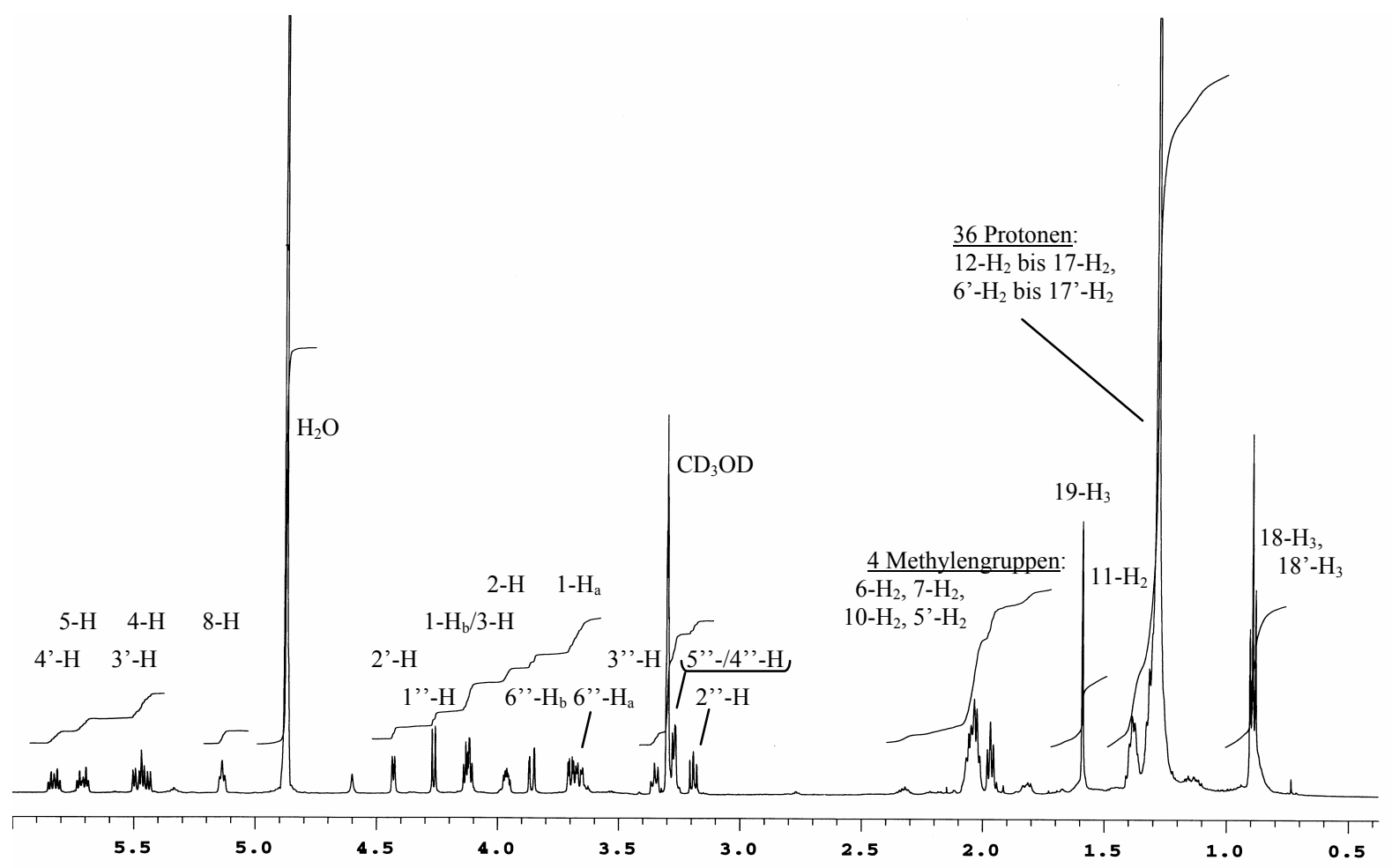

Abbildung 43: ${ }^{1} \mathrm{H}-\mathrm{NMR}-\mathrm{Spektrum}\left(600 \mathrm{MHz}, \mathrm{CD}_{3} \mathrm{OD}\right)$ von Cerebrosid $\mathrm{C}$ (59).

Es ist bekannt, dass Cerebrosid B und C (59), die sich in der Länge der Fettsäurekette und ihrem Sättigungsgrad an C-3' unterscheiden, bei dem Pilz Schizophyllum commune die Zelldifferenzierung induzieren und damit die Bildung von Fruchtkörpern auslösen ${ }^{[146]}$.

Eine ganz andere Wirkung wurde bei Pflanzen festgestellt: bei der Reispflanze bewirken die Cerebroside A und C (59) die Auslösung des hypersensitiven Zelltods, einem mit der Apoptose vergleichbaren programmierten Absterben einzelner Zellen. Mit diesem Prozess ist die Akkumulierung von Phytoalexinen verbunden, d. h. von Substanzen, die die Pflanze zur Abwehr gegen den Befall mit Erregern bildet. Interessant ist, dass die Cerebroside A und C (59) in größerem Maßstab von dem phytopathogenen Pilz Magnaporthe grisea produziert werden. Dieser ist zugleich Auslöser einer als Reisbrand bekannten Pflanzenkrankheit und unter dem Synonym Pyricularia oryzae Bestandteil der bei der BASF durchgeführten Mikrotests. Die Erkennung der Cerebroside stellt für die Pflanze daher einen wichtigen Schutzmechanismus gegen den Produzenten dieser Substanzen dar ${ }^{[147]}$. 


\section{$9 \quad$ Ascosalipyron aus Diplodia sp. BS 5945}

Der Stamm Diplodia sp. BS 5945 wurde aus einer Braunalge (Fucus serrata) isoliert. Er wurde unter Verwendung sechs verschiedener Nährmedien jeweils mit und ohne Zusatz von $33 \mathrm{~g} / \mathrm{L} \mathrm{NaCl}$ kultiviert. Keiner der Extrakte fiel in den biologischen Tests auf, im chemischen Screening jedoch zeigte der Extrakt der im Nährmedium G20 gewachsenen Schüttelkultur mehrere interessante Zonen.

Die Kultivierung des Stamms im 4L-Maßstab ermöglichte die Isolierung der Hauptkomponente mit einer Ausbeute von $7 \mathrm{mg} / \mathrm{L}$. Hierbei handelt es sich um einen farblosen Feststoff, dessen Molmasse durch ein EI-Massenspektrum zu $238 \mathrm{~g} / \mathrm{mol}$ bestimmt wurde. Das ${ }^{1}$ H-NMR-Spektrum zeigt vier Methylgruppen, eine Methylengruppe sowie drei Methinprotonen, von denen eines im olefinischen/aromatischen Bereich liegt $\left(\delta_{\mathrm{H}}=6.21\right)$. Anhand des COSY-Spektrums lassen sich die beiden anderen Methingruppen zwei Fragmenten zuordnen (Abbildung 44). Die Protonen beider Teilstrukturen zeigen im HMBC-Spektrum CH-Fernkopplungen zu einer Ketogruppe $\left(\delta_{\mathrm{C}}=211\right)$, die sie $\mathrm{zu}$ einem 1,3-Dimethyl-2pentanoyl-Rest verbindet. Eine Datenbanksuche ${ }^{[41]}$ mit diesem Strukturelement und der Molmasse führte zu Ascosalipyron (60), die Identität der spektroskopischen Daten mit publizierten Werten ${ }^{[148]}$ bestätigte die Strukturaufklärung.

Ascosalipyron (60) wird in der Literatur ebenfalls als Metabolit eines endophytischen Pilzes (Ascochyta salicorniae) beschrieben, der aus einer Alge isoliert wurde ${ }^{[148]}$. Die Autoren des Artikels konnten für $\mathbf{6 0}$ keine antimikrobielle, cytotoxische, antiplasmodische oder algizide Wirkung feststellen, auch die HIV-1-Enzyme Reverse Transkriptase sowie Tyrosinkinase (p56 $\left.{ }^{\mathrm{lck}}\right)$ wurden nicht gehemmt.

In Übereinstimmung hiermit zeigte Ascosalipyron (60) im Plattendiffusionstest keine antibiotische Aktivität. In den Pflanzenschutz-Tests wurde eine schwache Hemmwirkung gegen $S$. tritici und P. oryzae festgestellt (siehe Kapitel 15, Seite 134).

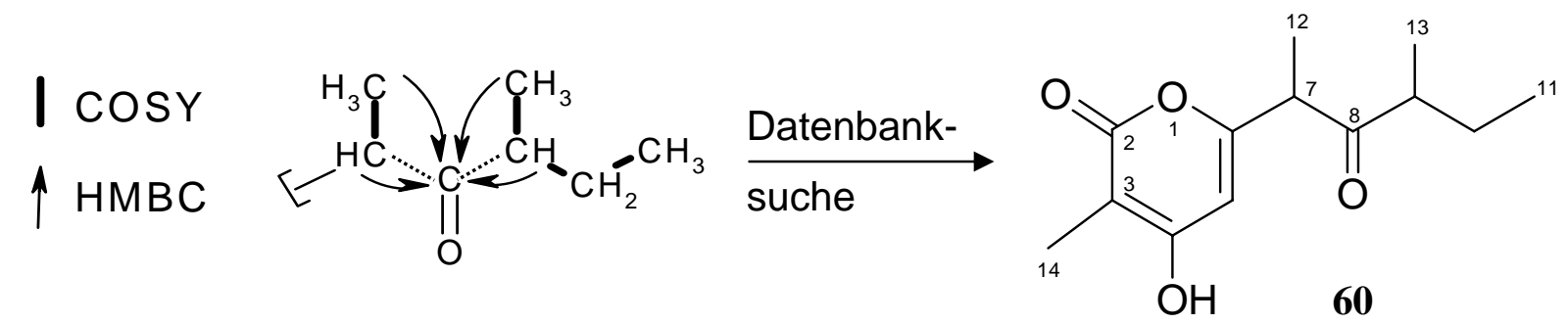

Abbildung 44: Strukturaufklärung von Ascosalipyron (60). 


\section{Solanapyron C aus Microsphaeropsis sp. BS 6278}

Der Stamm Microsphaeropsis sp. BS 6278 wurde aus der Rotalge Ceramium sp. isoliert. Die Kultivierung in P-Kolben mit dem Nährmedium SGG unter Zusatz von 58 g/L NaBr führte zu einem Extrakt, in dessen Dünnschichtchromatogramm eine mit Anisaldehyd beim Erhitzen blau anfärbenden Zone auffiel. Die Isolierung und Strukturaufklärung der hierfür ursächlichen Substanz ergab, dass der Stamm das Phytotoxin Solanapyron C (61) produziert. Die Extrakte aus Festagar- und Schüttelkulturen des Stamms BS 6278 waren hingegen weder im chemischen noch im biologischen Screening auffällig.

Die Isolierung der Substanz erfolgte aus einem 2L-Ansatz. Das ESI-Massenspektrum ergab eine Molmasse von $331 \mathrm{~g} / \mathrm{mol}$, woraus auf das Vorkommen mindestens eines Stickstoffatoms im Molekül geschlossen werden konnte. Das ${ }^{1}$ H-NMR-Spektrum zeigt eine zum Dublett aufgespaltene Methylgruppe $\left(\delta_{\mathrm{H}}=0.97\right)$, eine cis-konfigurierte Doppelbindung $\left(\delta_{\mathrm{H}}=5.46,5.68 ;{ }^{3} J_{\mathrm{HH}}=10.0 \mathrm{~Hz}\right)$ und ein Aldehydproton bei $\delta_{\mathrm{H}}=9.89$. Weiterhin sind zwei Heteroatom-substituierte Methylengruppen zu erkennen $\left(\delta_{\mathrm{H}}=3.56,3.73\right)$, die aufgrund ihrer Aufspaltung zu Tripletts $\left({ }^{3} J_{\mathrm{HH}}=5.5 \mathrm{~Hz}\right)$ benachbart sein müssen. Eine Datenbanksuche ${ }^{[55]}$ mit diesen Informationen führte zu Solanapyron C (61), dessen veröffentlichte spektroskopische Daten ${ }^{[149]}$ mit den

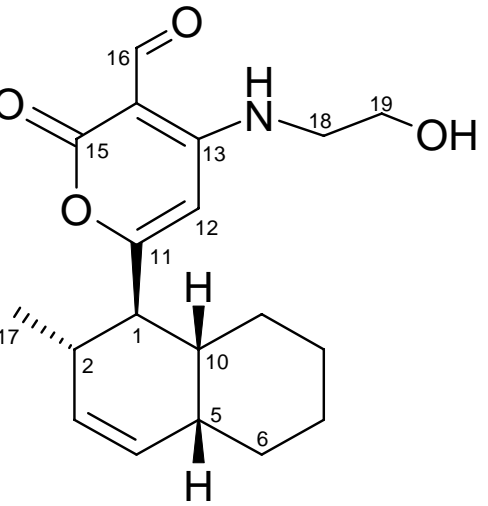

61 gemessenen Werten übereinstimmen.

Solanapyron C (61) ist ein von dem pathogenen Pilz Alternaria solani bekanntes Phytotoxin. A. solani ist Verursacher der Dürrfleckenkrankheit, die vor allem Kartoffel- und Tomatenpflanzen betrifft ${ }^{[150]}$. Neben den Hauptkomponenten Solanapyron A (62), B und C (61), die alle ein cis-Decalin-System enthalten, produziert der Pilz auch Nebenkomponenten mit einem trans-Decalin-Körper. Hierzu zählen Solanapyron D (63) und E. Die Erklärung für das Auftreten dieser unterschiedlichen Konfiguration liegt in der Biosynthese der Substanzen (Abbildung 45). Aus acht Acetat-Bausteinen sowie zwei von $L$-Methionin stammenden $\mathrm{C}_{1}$ Einheiten wird in mehreren Schritten Prosolanapyron III (64) aufgebaut, das bereits den Pyronring enthält und hieran eine aliphatische Kette trägt. Für die Ausbildung des DecalinSystems wird nun eine enzymatisch katalysierte Diels-Alder-Reaktion angenommen, deren exo-Produkt das cis-Decalin und deren endo-Produkt das trans-Decalin ist ${ }^{[151]}$. Aus dem 
zunächst vorliegenden Solanapyron A (62) entsteht durch eine nucleophile Substitution mit 2-Aminoethanol Solanapyron C (61) ${ }^{[150]}$.

In einem zellfreien Extrakt von A. solani konnte erstmals die Diels-Alderase-Aktivität eines Enzyms nachgewiesen werden ${ }^{[151]}$. Auch bei den in dieser Arbeit beschriebenen Chaetoglobosinen (ab S. 100) und Nargenicin $A_{1}$ (44, S. 63) sowie einigen anderen mikrobiellen Sekundärstoffen wird die Beteiligung einer Diels-Alderase an der Biosynthese postuliert $^{[108,152]}$.

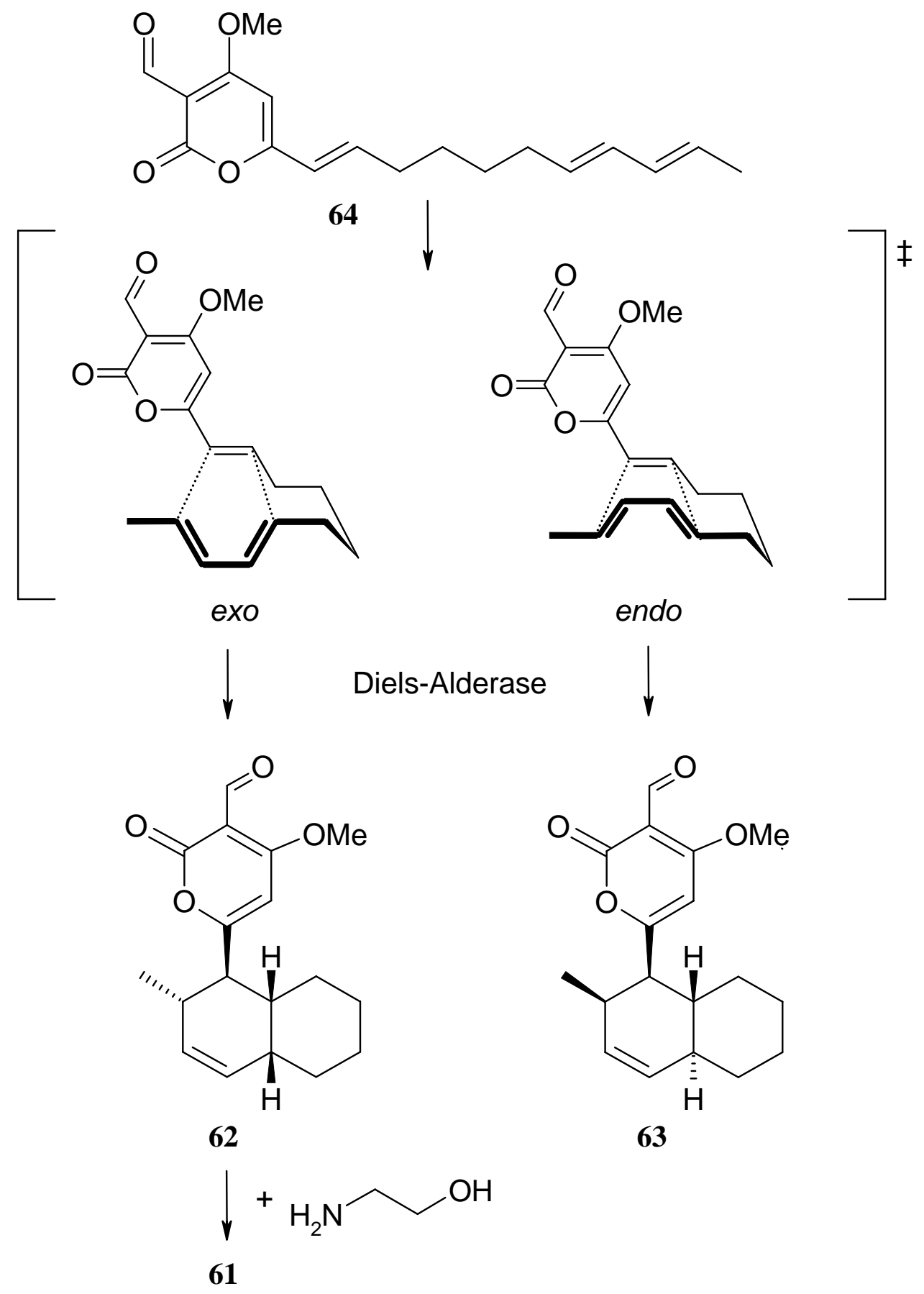

Abbildung 45: Biosynthese von Solanapyron A (62), C (61) und D (63). 


\section{Sekundärmetaboliten aus Chaetomium sp. BS 6556}

Der Stamm Chaetomium sp. BS 6556 fiel sowohl im chemischen als auch im biologischen Screening auf. Der Extrakt einer auf dem Feststoffmedium DiGe gewachsenen Kultur zeigte im Dünnschichtchromatogramm mehrere bei $254 \mathrm{~nm}$ UV-löschende Zonen, die mit Anisaldehyd beim Erhitzen braun anfärbten. In den Mikrotests der BASF wurde eine gute Wirksamkeit des Extrakts gegen die phytopathogenen Pilze B. cinerea, P. oryzae und S. tritici festgestellt, bei einer Konzentration von 8 ppm erfolgte jeweils eine vollständige Wachstumshemmung. Eine schwache herbizide Wirksamkeit gegen $A$. theophrasti konnte ebenfalls nachgewiesen werden.

Die bei der BASF erfolgte Auftrennung des Extrakts mittels HPLC und anschließende biologische Testung der Fraktionen ergab, dass mindestens drei Substanzen im Extrakt für diese Wirkung verantwortlich sein müssen. Die mit A, B und $\mathbf{C}$ markierten Bereiche fungizider Aktivität sind im HPLC-UV-Chromatogramm als Peaks erkennbar, denen sich durch HPLC-MS insgesamt vier Molmassen zuordnen ließen (Abbildung 46).

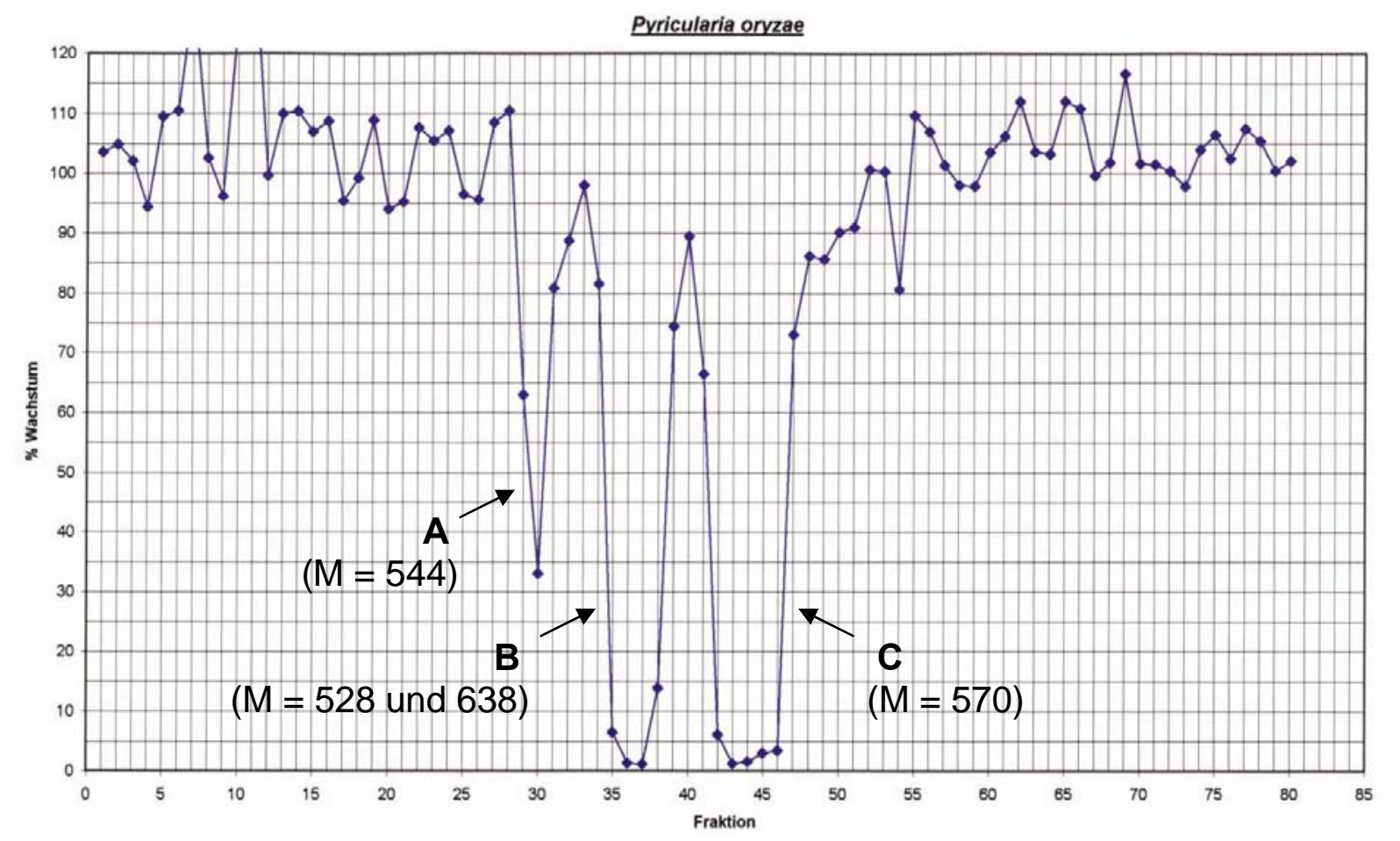

Abbildung 46: Mikrotest der Rohextrakt-Fraktionen gegen P. oryzae und HPLC-MS-Daten. 
Die HPLC-Trennung von 20 mg Rohextrakt unter gleichen Bedingungen führte zur Isolierung von $4.5 \mathrm{mg}$ der Komponente C, die anhand ihrer spektroskopischen Daten als 19-O-Acetylchaetoglobosin A (65) identifiziert wurde (die Strukturaufklärung wird im folgenden Unterkapitel beschrieben). Diese zur Familie der Cytochalasine gehörende Verbindung ist das Hauptprodukt des Stamms Chaetomium sp. BS 6556 (ca. $25 \mathrm{mg} / \mathrm{L}$ ) und vermutlich für die starke fungizide Wirkung im Bereich $\mathbf{C}$ des Extrakts verantwortlich.

Die weiteren Komponenten wurden aus dem Extrakt eines größeren Kultivierungssatzes (ca. 12 L) isoliert und aufgeklärt. Die Trennung und Strukturaufklärung der Metaboliten im Bereich B erbrachte zwei weitere Cytochalasine, Chaetoglobosin A (66) und Chaetoglobosin C (67), sowie eine nicht in der Literatur beschriebene Verbindung mit einem neuartigen Spiro-Kohlenstoffgerüst, die Chaetospiron (68) genannt wurde.

Der Bereich A konnte bei der Refermentation bisher nicht reproduziert werden. Aufgrund der im Vergleich zu 66 und 67 um 16 Masseneinheiten höheren Molmasse $(M=544)$ ist ein hydroxyliertes Chaetoglobosin zu erwarten, auch das dem HPLC-Experiment entnommene UV-Spektrum von A zeigt weitgehende Übereinstimmung mit denen der anderen Chaetoglobosine. Ein solches Cytochalasin ist in der Literatur bisher nicht beschrieben und würde daher einen neuen Naturstoff darstellen.

Die Kultivierung des Stamms Chaetomium sp. BS 6556 in Schüttelkolben führte ebenfalls zu fungizid wirksamen Extrakten, jedoch zeigte das chemische Screening, dass die Metaboliten hier in weit geringerer Konzentration gebildet wurden. Möglicherweise liegt eine Erklärung für die nicht befriedigende Ausbeute an Chaetoglobosinen in einer zu kurzen Fermentationsdauer von 6 Tagen. Durch eine Kultivierung in Schüttelkultur über 11 Tage wurden in der Literatur Ausbeuten von bis zu $700 \mathrm{mg} / \mathrm{L}_{\text {erzielt }}{ }^{[153]}$.

\subsection{9-O-Acetylchaetoglobosin A (65)}

Der gelbliche Feststoff 65 ist das Hauptprodukt von Chaetomium sp. BS 6556 und wurde in Ausbeuten von ca. $25 \mathrm{mg} / \mathrm{L}$ erhalten. Die Molmasse von $570 \mathrm{~g} / \mathrm{mol}$ wurde durch ein ESIMassenspektrum bestimmt, dessen Hochauflösung die Summenformel $\mathrm{C}_{34} \mathrm{H}_{38} \mathrm{~N}_{2} \mathrm{O}_{6}$ lieferte. Eine Suche mit der Summenformel in Naturstoff-Datenbanken ${ }^{[41,55]}$ erbrachte nur zwei Einträge: die 19-O-Acetate von Chaetoglobosin A und B. Durch Vergleich der NMR-Daten mit Literaturwerten ${ }^{[153]}$ wurde die Substanz als 19-O-Acetylchaetoglobosin A identifiziert. 
Das ${ }^{1}$ H-NMR-Spektrum (Abbildung 47) zeigt trotz der großen Anzahl an Protonen nur eine geringe Überlappung der Signale. Auffällige Resonanzen ergeben im aromatischen Bereich die Protonen des zum Indoyl-Rest gehörenden ortho-disubstituierten Aromaten (zwei Dubletts und zwei Tripletts) sowie die Protonen der trans-konfigurierten Doppelbindung in Position 21, die deutlich ins Tieffeld verschoben sind $\left(\delta_{\mathrm{H}}=6.30\right.$ bzw. 7.50) und als Dubletts $\left({ }^{3} J_{\mathrm{HH}}=16.5 \mathrm{~Hz}\right)$ erscheinen. Ein Signal im olefinischen Bereich zeigt auch das Proton 19-H $\left(\delta_{\mathrm{H}}=5.85\right)$. Seine Position zwischen der Carbonylgruppe C-20 und der Doppelbindung C-17/C-18 bewirkt eine starke Entschirmung, die durch die Acetylgruppe noch verstärkt wird.

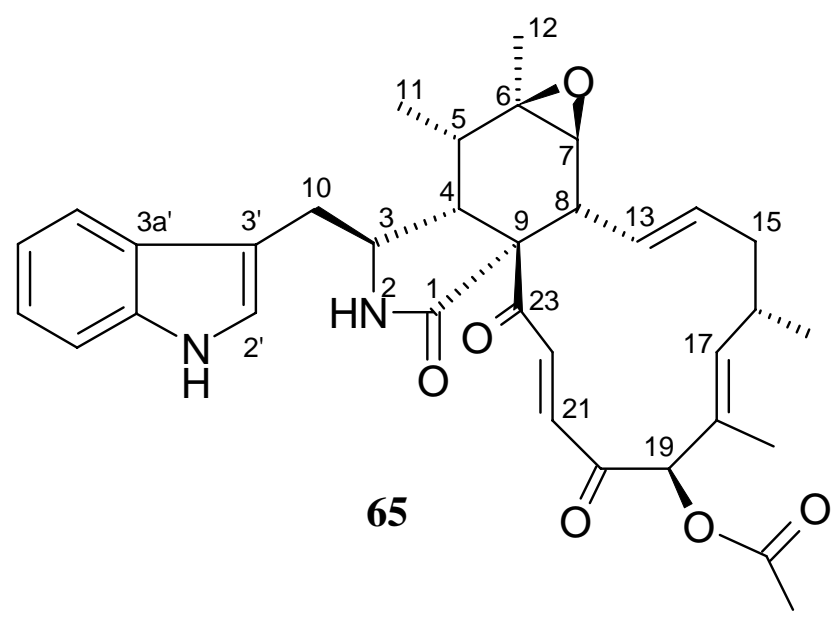

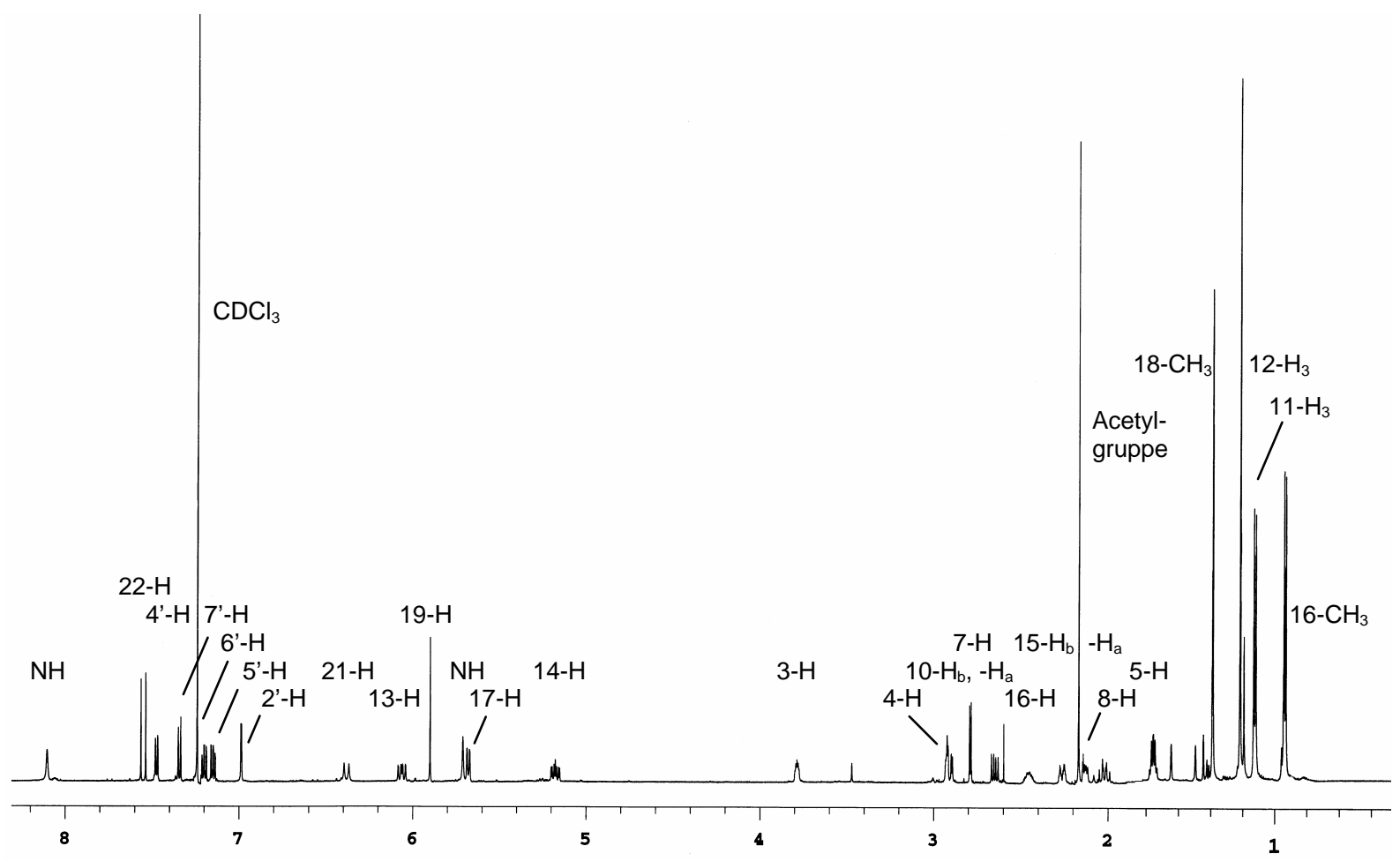

Abbildung 47: ${ }^{1} \mathrm{H}-\mathrm{NMR}-\mathrm{Spektrum}\left(600 \mathrm{MHz}, \mathrm{CDCl}_{3}\right)$ von 65. 


\subsection{Chaetoglobosin A (66) und C (67)}

Die beiden Metaboliten 66 und 67 des Stamms BS 6556 sind ebenso wie 65 gelbliche Feststoffe und wurden als Nebenprodukte in einer Ausbeute von 5 bzw. $2 \mathrm{mg} / \mathrm{L}$ isoliert. Ihre UV-Spektren stimmen mit dem von 65 überein, weshalb davon ausgegangen werden konnte, dass es sich ebenfalls um Chaetoglobosine handelt.

Das ${ }^{1} \mathrm{H}-\mathrm{NMR}-\mathrm{Spektrum}$ von 66 ist nahezu identisch mit dem von 19-O-Acetylchaetoglobosin A (65), einziger Unterschied ist das Fehlen des Signals der Acetylgruppe. Den gleichen Befund zeigt das ${ }^{13} \mathrm{C}-\mathrm{NMR}$-Spektrum. In Übereinstimmung mit der Molmasse von $528 \mathrm{~g} / \mathrm{mol}$ konnte die Substanz somit als Chaetoglobosin A (66) identifiziert werden ${ }^{[154]}$.

67 besitzt dieselbe Molmasse wie 66, jedoch fehlen im ${ }^{1} \mathrm{H}-\mathrm{NMR}-\mathrm{Spektrum}$ die Signale der Doppelbindung in Position 21 ebenso wie die Methingruppe in Position 19. Stattdessen zeigt das ${ }^{13} \mathrm{C}$-NMR-Spektrum zwei zusätzliche aliphatische Kohlenstoffatome sowie eine vierte Carbonylgruppe. Geht man von einer Oxidation der Hydroxygruppe an C-19 aus, so ergibt sich die Struktur von Chaetoglobosin C (67), dessen spektroskopische Daten mit Literaturwerten $^{[154]}$ übereinstimmten.
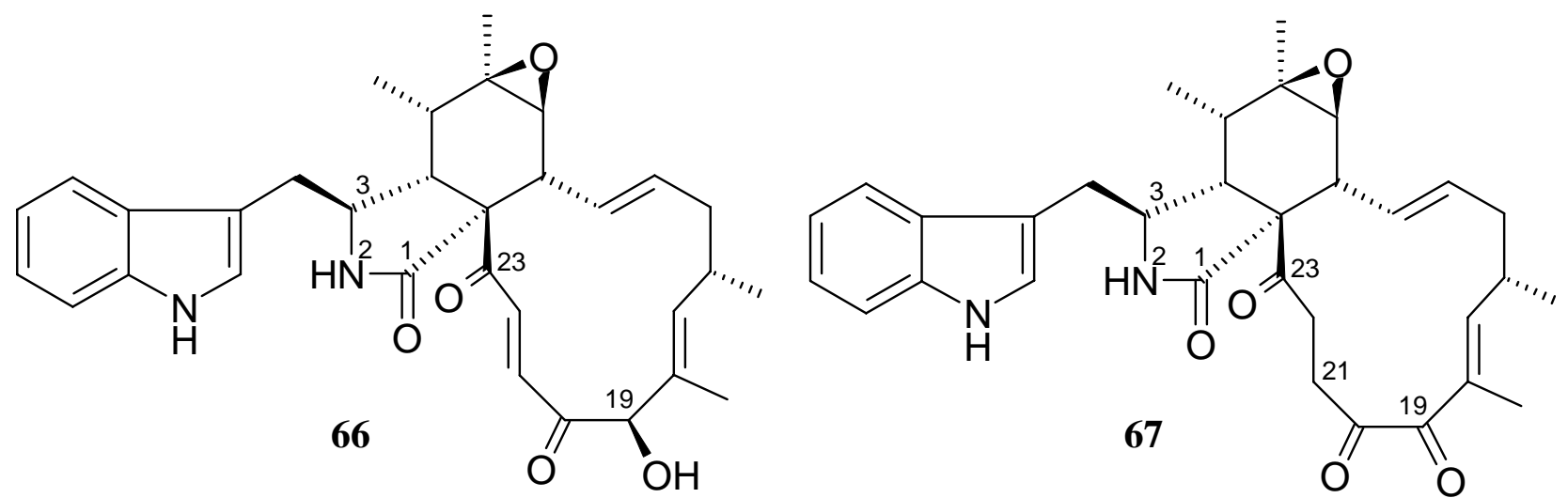

Chaetoglobosin A (66) und seine Struktur wurden erstmals 1973 beschrieben ${ }^{[155]}$. Die Isolierung erfolgte aus dem Pilz Chaetomium globosum, der als Namensgeber für die Substanz diente. In derselben Publikation wird auch 19-O-Acetylchaetoglobosin A (65) erwähnt, jedoch nur als Produkt chemischer Derivatisierung. Drei Jahre später wurde die Struktur von Chaetoglobosin C (67) aufgeklärt ${ }^{[156]}$ und durch Röntgenstrukturanalyse bestätigt $^{[157]}$. 1981 schließlich fand man 65 als Metabolit eines Pilzes derselben Gattung und Art, wo die Substanz ebenso wie bei Chaetomium sp. BS 6556 das Hauptprodukt darstellte ${ }^{[153]}$. 


\subsection{Zur Substanzklasse der Cytochalasine}

Chaetoglobosine gehören zur Substanzklasse der Cytochalasine ${ }^{\mathrm{t}}$, denen der in Abbildung 48 dargestellte Aufbau zugrunde liegt. Man kennt heute ca. 120 verschiedene Cytochalasine, die alle von Pilzen produziert werden ${ }^{[55]}$. Strukturell unterscheiden sie sich durch die verwendete Aminosäure, die Länge der Polyketidkette sowie deren Konstitution wie z. B. dem Grad der Oxidation und Methylierung.<smiles>[R]CC1NC(=O)C2(CCCCCCCCCC2)C1CC(C)C</smiles>

I<smiles>[R]CC1NC(=O)C2(CCCCCCCCCC[CH]O2)C(C)CC(C)C1C</smiles>

IV<smiles>[R]CC1NC(=O)C2CCCCCCCCCCCC(CC(C)C2C)C1(C)O</smiles>

II<smiles></smiles>

$\mathrm{V}$<smiles>[R]CC1NC(=O)C2CCCCCCCCCCCC(C(C)CC2C)C1C</smiles>

III

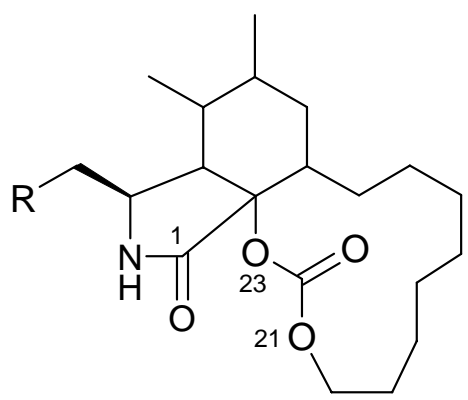

VI

Abbildung 48: Verschiedene Strukturtypen der Cytochalasine ( $\mathrm{R}=$ Aminosäure-Rest). Es sind auch Cytochalasine mit einem jeweils 2 C-Atome kleineren Makrozyklus bekannt.

\footnotetext{
${ }^{\mathrm{t}}$ Die Bezeichnung leitet sich ab von den griechischen Worten kytos (= Zelle) und chalasis (= Erschlaffung), in Anspielung auf ihre biologische Wirkung.
} 


\section{Cytochalasine mit der Aminosäure Tryptophan}

Den 30 in der Literatur beschriebenen Chaetoglobosinen ${ }^{[55,158]}$ ist die Aminosäure Tryptophan sowie ein 13 Kohlenstoffatome großer Makrozyklus gemeinsam. Sie entsprechen dem Strukturtyp III in Abbildung 48. Außer aus Chaetomium sp. wurden sie auch aus Diplodia sp., Phomopsis sp., Penicillium sp. und anderen Gattungen erhalten. Aus Penicillium sp. wurden zudem einige diesem Strukturtyp entsprechende Substanzen isoliert, die jedoch Penochalasin E, F, G und H genannt wurden. Ebenfalls Tryptophan enthalten die Penochalasine A - D, die einen Furan-Ring im Makrozyklus aufweisen, sowie Cytochalasin G, X, Y und Z, deren makrozyklischer Ring jedoch zwei C-Atome kürzer ist.

\section{Cytochalasine mit anderen Aminosäuren}

Die am häufigsten in Cytochalasinen vorkommende Aminosäure ist Phenylalanin. Hierzu zählen die meisten mit „Cytochalasin“ benannten Verbindungen, außerdem die Zygosporine, Ascochalasine sowie einige einzelne Substanznamen. Weitere auftretende Aminosäuren sind Tyrosin (Cytochalasin $\mathrm{Z}_{1}$ und $\mathrm{Z}_{5}$, Phenochalasin $\mathrm{A}$ und $\mathrm{B}$, Pyrichalasine) sowie Leucin (Aspochalasine) $)^{[55]}$.

\section{Biosynthese der Cytochalasine}

Biosynthetisch entstehen Cytochalasine aus einer acht oder neun Acetat-Einheiten langen Polyketidkette und einer Aminosäure. Ihre Aminogruppe bewirkt durch eine nucleophile Substitution an C-1 des Polyketids die Ausbildung einer Peptidbindung, die zwischen C-2 des Polyketids und der Carboxylgruppe der Aminosäure erfolgende Aldol-Kondensation führt zum Lactamring. Für die darauf folgende Formierung des Sechsrings wird eine Diels-AlderReaktion angenommen, bei der das Ende der Polyketidkette als Dien fungiert ${ }^{[159,160]}$. 


\subsection{Chaetospiron (68)}

Der farblose Feststoff 68 wurde in einer Ausbeute von ca. $6 \mathrm{mg} / \mathrm{L}$ isoliert. Das ESIMassenspektrum ergab eine Molmasse von $638 \mathrm{~g} / \mathrm{mol}$, durch hochauflösende Massenspektrometrie wurde die Summenformel $\mathrm{C}_{34} \mathrm{H}_{38} \mathrm{O}_{12}$ ermittelt. Die NMR-Spektren zeigen das Vorhandensein von drei Methyl-, vier Methylen- und zwei Methingruppen sowie von acht quartären Kohlenstoffatomen. Da die Spektren damit lediglich halb so viele Atome zeigen wie laut Summenformel vorhanden sind, muss die Verbindung als symmetrisches Dimer vorliegen. Die Summenformel einer Molekülhälfte ist $\mathrm{C}_{17} \mathrm{H}_{19} \mathrm{O}_{6}$, woraus sich acht Doppelbindungsäquivalente ergeben. Das ${ }^{13} \mathrm{C}$-NMR-Spektrum lässt vier olefinische C-Atome, eine Ester-Carbonylgruppe und zwei Ketogruppen erkennen (gemeinsam nur fünf DBÄquivalente), das Monomer muss daher ein Trizyklus sein. Die gerade Anzahl an Sauerstoffatomen schließt die Verknüpfung der Monomer-Einheiten über ein O-Atom aus, folglich ist eine C-C-Verbrückung zu erwarten.

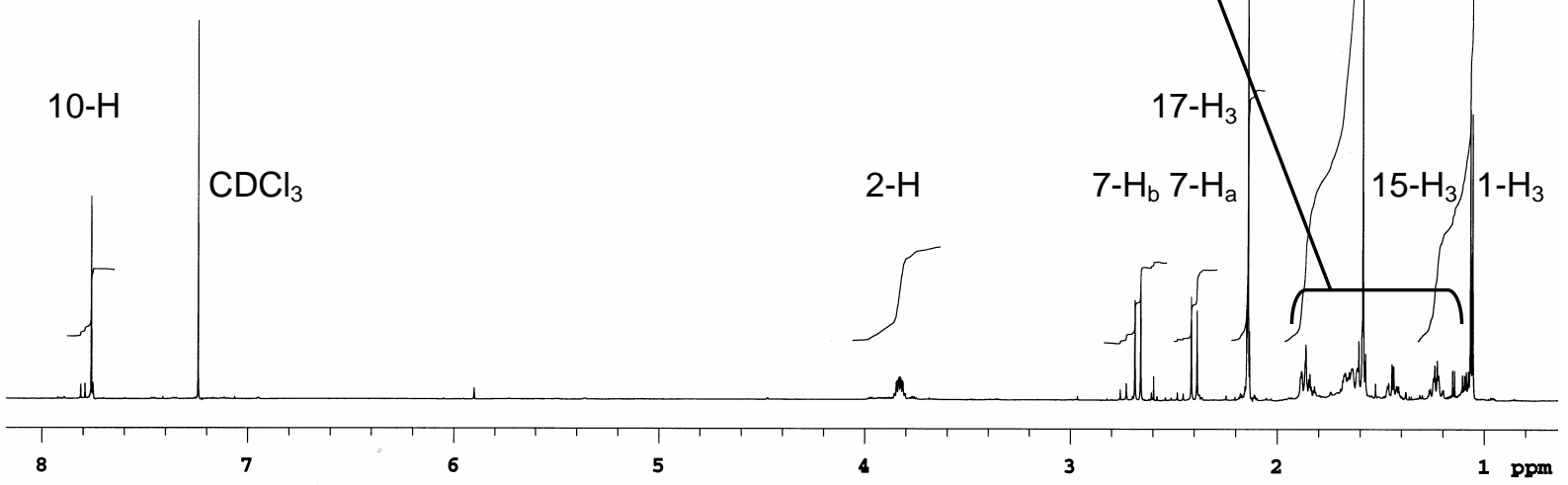

Abbildung 49: ${ }^{1} \mathrm{H}-\mathrm{NMR}-\mathrm{Spektrum}\left(600 \mathrm{MHz}, \mathrm{CDCl}_{3}\right)$ von Chaetospiron (68).

Ausgehend von der zum Dublett aufgespaltenen Methylgruppe bei $\delta_{\mathrm{H}}=1.06$ lässt sich mit Hilfe eines COSY-Spektrums eine Kette mit einer Sauerstoff-substituierten Methingruppe $\left(\delta_{\mathrm{H}}=3.86\right)$ und drei Methylengruppen erkennen. Anhand der HMBC-Korrelationen kann 
diese Kette um ein quartäres Kohlenstoffatom erweitert werden, dessen chemische Verschiebung $\left(\delta_{\mathrm{C}}=102.8\right)$ eine Acetal-Struktur erwarten lässt. Eine ${ }^{4} J_{\mathrm{CH}}-\mathrm{Korrelation}$ ausgehend von der Methylgruppe zeigt den Ringschluss zum Tetrahydropyran-Ring an. Weitere Signale im HMBC-Spektrum beweisen die Bindung einer Tieffeld-verschobenen Methylengruppe $\left(\delta_{\mathrm{H}}=2.40 / 2.67\right)$ an das acetalische C-Atom, woraus sich zusammenfassend das in Abbildung 50 gezeigte Fragment I ergibt. Die chemische Verschiebung einer zweiten Methylgruppe $\left(\delta_{\mathrm{H}}=2.14\right)$ ist typisch für einen Acetylrest, diese Zuordnung wird durch eine ${ }^{2} J_{\mathrm{CH}}-$ Korrelation zu einer Ester-Carbonylgruppe $\left(\delta_{\mathrm{C}}=169.9\right)$ bestätigt. Eine ${ }^{4} J_{\mathrm{CH}}-\mathrm{Korrelation}$ verbindet die Acetylgruppe mit einem quartären, aliphatischen $C$-Atom $\left(\delta_{C}=84.7\right)$, an das eine weitere Methylgruppe $\left(\delta_{\mathrm{H}}=1.59\right)$ gebunden ist (Fragment II). Das ${ }^{13} \mathrm{C}-\mathrm{NMR}-\mathrm{Spektrum}$ beinhaltet zwei Ketogruppen, deren chemische Verschiebung sie als $\alpha, \beta$-ungesättigt ausweist $\left(\delta_{\mathrm{C}}=191.8,192.2\right)$. Sie bilden gemeinsam mit den vier olefinischen C-Atomen die Fragmente III und IV, womit alle in den Spektren vorhandenen Atome einer Teilstruktur zugeordnet werden konnten. Die $\beta$-Position der Methin- zur Carbonylgruppe in Fragment III ergibt sich aus der Tieffeld-Verschiebung ihrer NMR-Signale $\left(\delta_{\mathrm{H}}=7.76, \delta_{\mathrm{C}}=157.6\right)$.

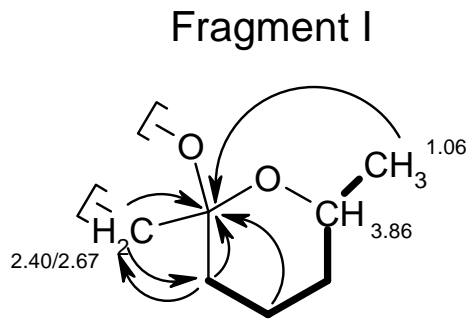

Fragment II

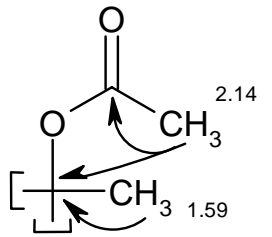

Fragmente III und IV
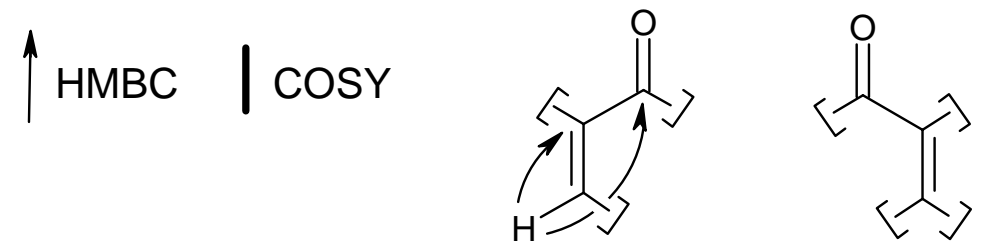

Abbildung 50: Aus den NMR-Spektren abgeleitete Fragmente I-IV von 68.

Die Verknüpfung der Fragmente anhand von HMBC-Korrelationen ist in Abbildung 51 dargestellt. Sie steht im Einklang mit den beobachteten chemischen Verschiebungen der olefinischen Kohlenstoffatome. Die Konjugation der Doppelbindungen wird durch die Lage der $\mathrm{C}=\mathrm{C}$-Streckschwingung im IR-Spektrum unterstützt $(\widetilde{v}=1608)$. 


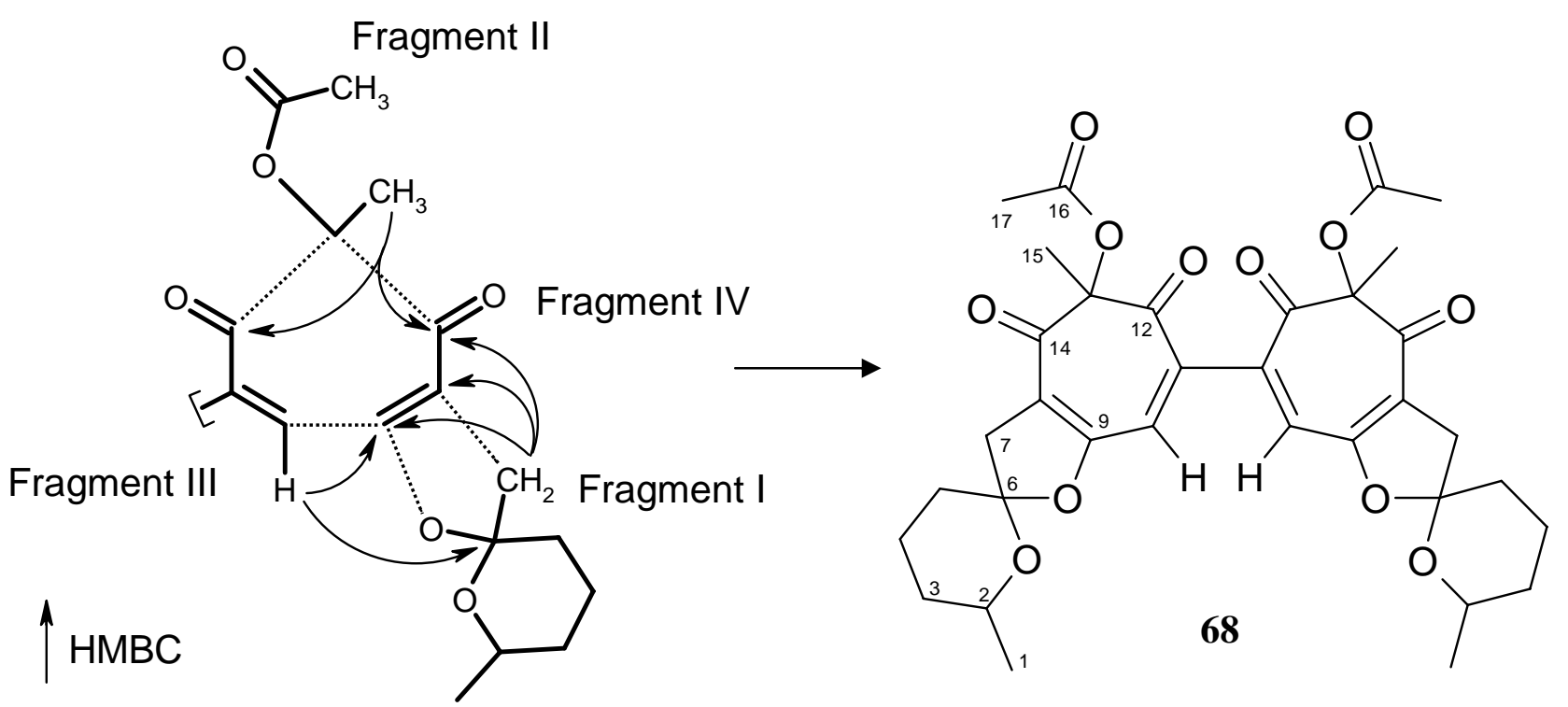

Abbildung 51: Verknüpfung der Fragmente I-IV anhand von HMBC-Korrelationen zur Struktur von 68.

Aus der erhaltenen Struktur ergibt sich, dass die C-C-Verknüpfung der beiden monomeren Einheiten zur Gesamtstruktur nur über das $\alpha$-C-Atom von Fragment III erfolgen kann (Abbildung 51). Bei dem Spiroketal 68 handelt es sich um einen bisher nicht beschriebenen Naturstoff, für den der Name Chaetospiron vorgeschlagen wird ${ }^{\mathrm{u}}$.

\section{Relative Stereochemie von Chaetospiron (68)}

68 besitzt drei Stereozentren an C-2, C-6 sowie C-13, außerdem dürfte die Rotation um die Achse C-12/C-12' wegen der benachbarten Ketogruppe stark eingeschränkt sein. Die relative Konfiguration von C-2 und C-6 lässt sich anhand von NOESY-Korrelationen bestimmen. Dies ist für C-13 aufgrund der räumlichen Entfernung nicht möglich, so dass die Konfiguration dort ungeklärt bleibt. Es ist plausibel, für den Tetrahydropyran-Ring eine Sesselform anzunehmen. Die Methylgruppe befindet sich in äquatorialer Position, wie die diaxiale Kopplung $\left({ }^{3} J_{\mathrm{HH}}=12.5 \mathrm{~Hz}\right)$ zwischen 2-H und 3-H beweist. Eines der Protonen der Methylengruppe in Position 5 zeigt NOESY-Korrelationen zu beiden Protonen in Position 7,

\footnotetext{
u in Anlehnung an die Pilzgattung (Chaetomium sp.), aus der die Verbindung isoliert wurde, sowie an ihre strukturellen Besonderheiten (Spiroverbindung mit Ketogruppen).
} 
das andere lediglich zu einem dieser Protonen. Dies ist nur bei der in Abbildung 52 gezeigten Konfiguration des Spirozentrums möglich, bei der sich das axiale Proton an C-5 in räumlicher Nähe zu beiden Methylenprotonen an C-7 befindet.
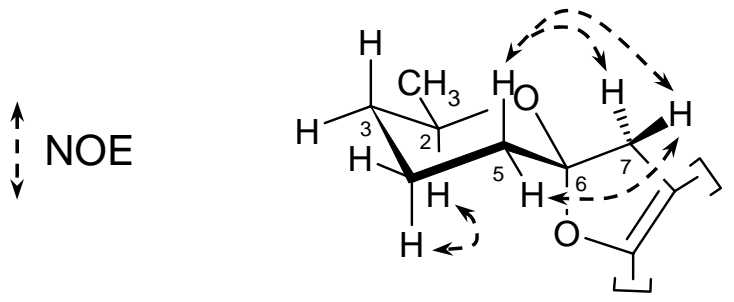

Abbildung 52: Festlegung der relativen Stereochemie an C-2 und C-6.

Daraus lässt sich folgende Struktur ableiten:

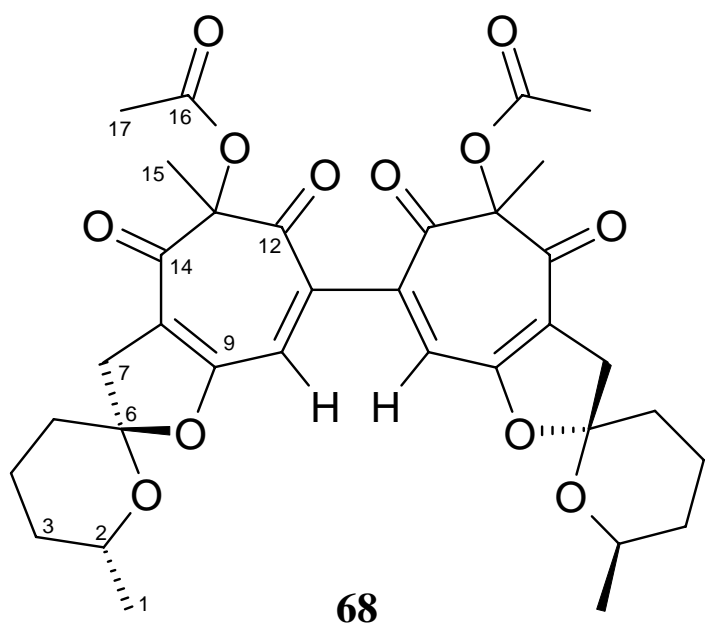

(relative Konfiguration)

\section{Strukturvergleich}

Chaetospiron (68) besitzt ein neuartiges Kohlenstoffgerüst. Weder das SpiroketalGrundgerüst (69, Abbildung 53) noch das entsprechende Octahydro-spiro[azulen-2,1'cyclohexan]-System (70) waren zuvor bei Naturstoffen oder Syntheseprodukten bekannt. Beide Strukturen existieren lediglich als Substruktur wesentlich komplexerer Systeme, so findet man 70 in einigen Fullerenen ${ }^{[161]}$. 


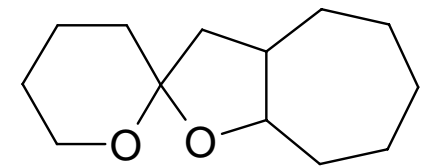

69

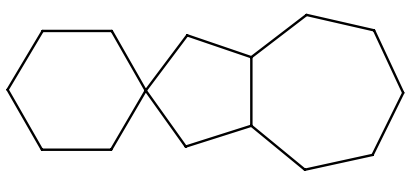

70

Abbildung 53: Grundstruktur von Chaetospiron (68).

Die neue Verbindung 68 weist eine strukturelle Ähnlichkeit zu Malettinin A (71, Abbildung 54) auf. Bei $\mathbf{7 1}$ handelt es sich um ein Tropolon-Derivat, das aus einem taxonomisch nicht näher beschriebenen, nicht sporulierenden Pilz isoliert wurde, der das Fadengeflecht eines weiteren Pilzes der Gattung Hypoxolon (Kohlenbeere) besiedelt ${ }^{[162]} .71$ und seine Derivate Malettinin B und C, die an der Ketogruppe des Fünfrings reduziert sind, besitzen eine starke antifungische Wirkung, u. a. gegen Candida albicans ${ }^{[163]}$. Im Unterschied zu Chaetospiron (68) weisen die Malettinine ein intaktes Tropolon-System ${ }^{v}$ auf (68 hat eine zweite Ketogruppe im Siebenring), der Tropolon-Ring befindet sich am Sechs- anstatt am Fünfring des bizyklischen Spiroketals und es wird keine dimere Struktur gebildet.

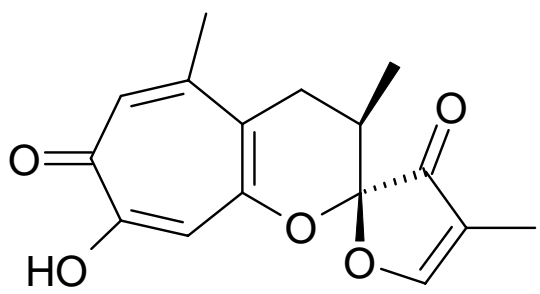

71

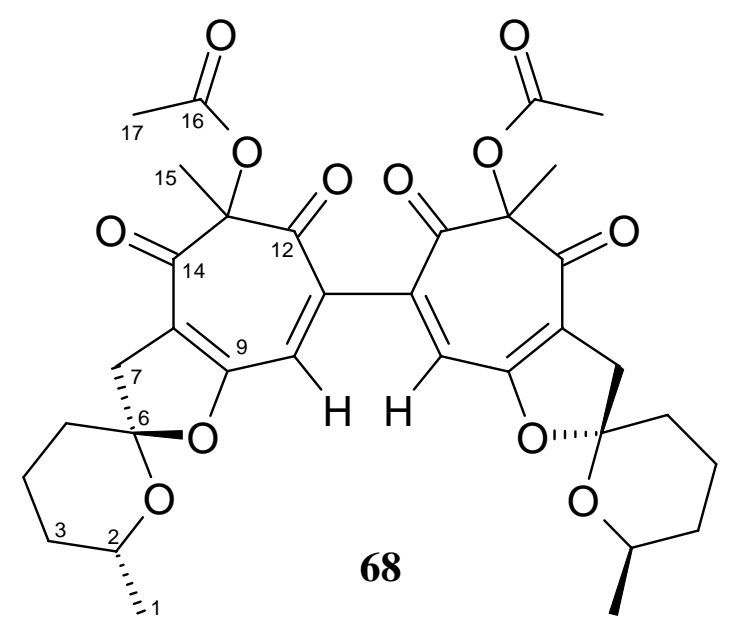

Abbildung 54: Malettinin A (71) und Chaetospiron (68) im Vergleich.

\footnotetext{
${ }^{\mathrm{v}}$ Als $(\alpha$-)Tropolon bezeichnet man 2-Hydroxy-2,4,6-cycloheptatrienon, ein bei Naturstoffen häufig vorkommender Grundkörper.
} 


\section{Überlegungen zur Biosynthese}

Für die Biosynthese von Chaetospiron (68) ist der Polyketidweg plausibel. Einen hypothetischen Verlauf zeigt Abbildung 55. Aus sieben Acetat-Bausteinen wird ein Heptaketid aufgebaut, das anschließend in Position 9 oxidiert sowie in Position 13 oxidiert und methyliert wird. Der Ringschluss zum Cycloheptan kann durch eine nucleophile Substitution am Thioester geschehen, ausgehend von einem Enolat-stabilisierten Carbanion (Schritt 2). Anschließend werden durch eine Sequenz von zwei aufeinander folgenden nucleophilen Additionen an die Carbonylgruppen C-6 und C-9 zwei weitere Ringschlüsse unter Ausbildung des Spiroketals vollzogen (Schritte 3 und 4). Eine Dehydratisierung bewirkt im nächsten Schritt die Ausbildung der Doppelbindung in Position 8. Durch Acetylierung an 13-OH und Dimerisierung unter C-C-Verknüpfung in Position 11 entsteht 68.
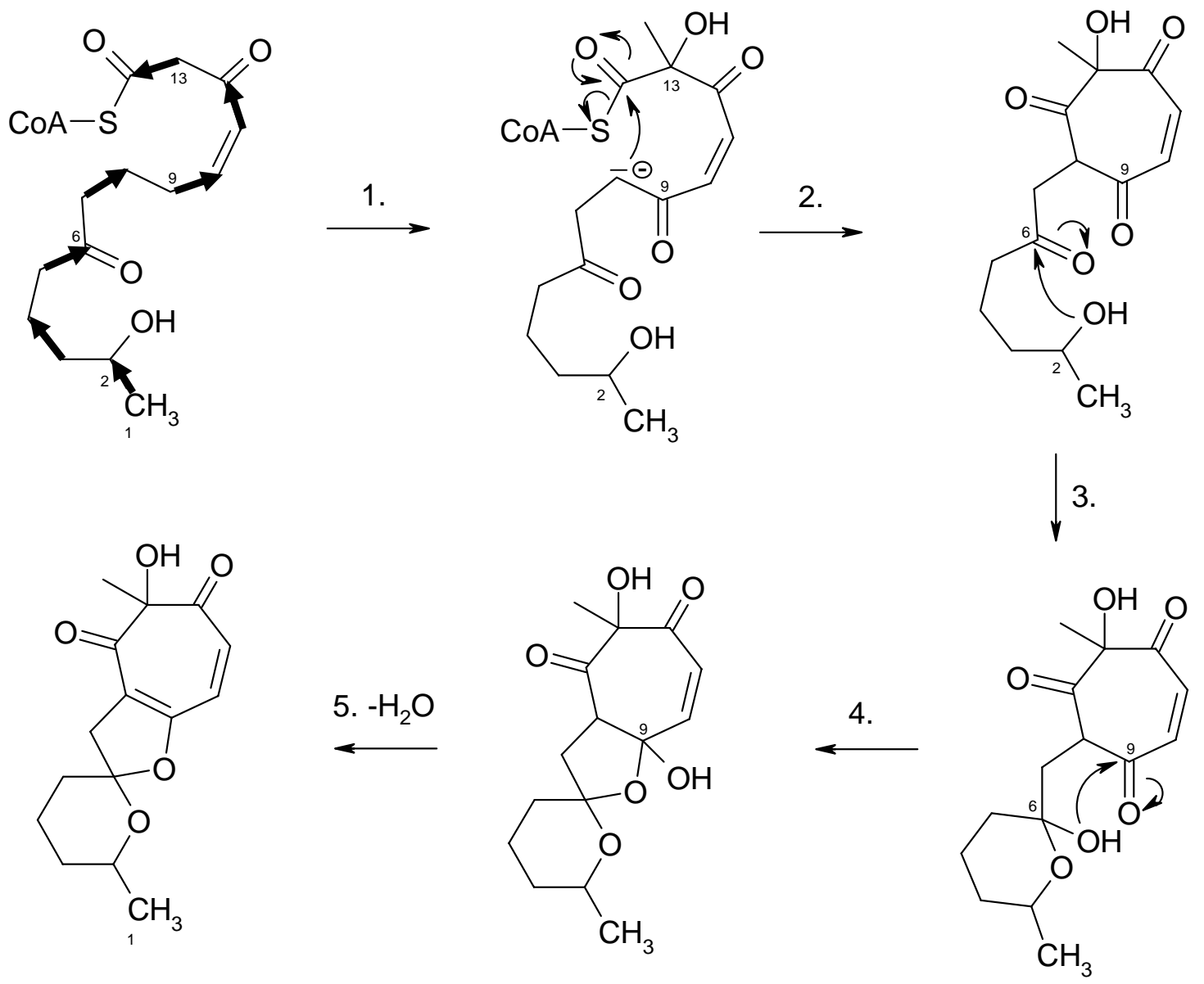

6. Acetylierung, Dimerisierung

Abbildung 55: Hypothetische Biosynthese von Chaetospiron (68). 
Diese Hypothese stellt lediglich einen von mehreren möglichen Wegen dar. Denkbar wäre auch ein Kettenaufbau in umgekehrter Richtung im Tropolon-Ring oder die Biosynthese des Tropolon-Rings durch oxidative Ringerweiterung eines zunächst entstehenden Sechsrings.

Ein solcher Mechanismus wurde bei anderen Tropolon-Derivaten nachgewiesen, die von Pilzen gebildet werden. Abbildung 56 zeigt den Biosyntheseverlauf von Stipitatsäure (72) und Stipitatonsäure (73) aus Penicillium stipitatum ${ }^{[164]}$. Aus einem Tetraketid entsteht zunächst 3-Methylorsellinsäure (74). Die Methylgruppe in Position 3 ist eine von $S$-Adenosyl- $L$ Methionin stammende $\mathrm{C}_{1}$-Einheit, die im nächsten Schritt ebenso wie C-3 durch eine Monooxygenase ${ }^{[165]}$ oxidiert wird. Durch eine Pinakol-Umlagerung wird der Tropolonring gebildet. Eine weitere Oxidation führt zu Stipitatonsäure (73), aus der letztlich Stipitatsäure (72) hervorgeht ${ }^{[166]}$.<smiles>[Z4]CSC(=O)CC(=O)[C@H](C)C(=O)CC(C)=O</smiles>

74

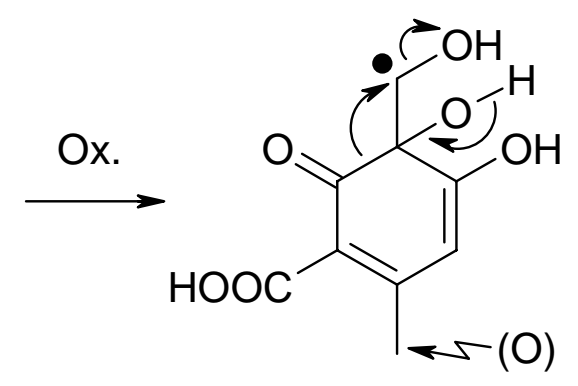

Oxidation, Pinakol$\checkmark$ Umlagerung<smiles>O=C1OC(=O)c2c(O)c[14c](=O)c(O)cc21</smiles>

72

Abbildung 56: Biosynthese von Stipitatsäure (72) und Stipitatonsäure (73). 


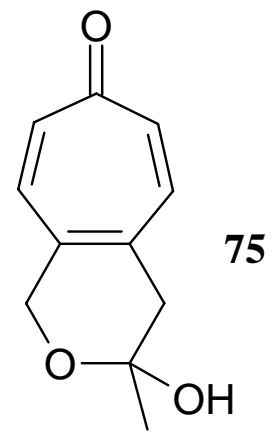

Ein analoger Biosyntheseweg führt auch zu Sepedonin (75) aus Sepedonium chrysospermum ${ }^{[167]}$ sowie Puberulic Acid und Puberulonic Acid aus Penicillium aurantio-virens. Letztere unterscheiden sich von Stipitatsäure (72) und Stipitatonsäure (73) nur durch eine zusätzliche Hydroxygruppe an C- $7^{[166]}$.

Ein entsprechender Biosyntheseweg für Chaetospiron (68) ist in Abbildung 57 gezeigt und geht von einem methylierten Heptaketid aus.

Die $\mathrm{C}_{1}$-Einheit findet sich anschließend im Tropolon-Ring wieder. Der Nachweis des tatsächlichen Verlaufs der Biosynthese müßte durch geeignete Fütterungsexperimente erfolgen, die jedoch nicht mehr Gegenstand der vorliegenden Arbeit sein konnten.
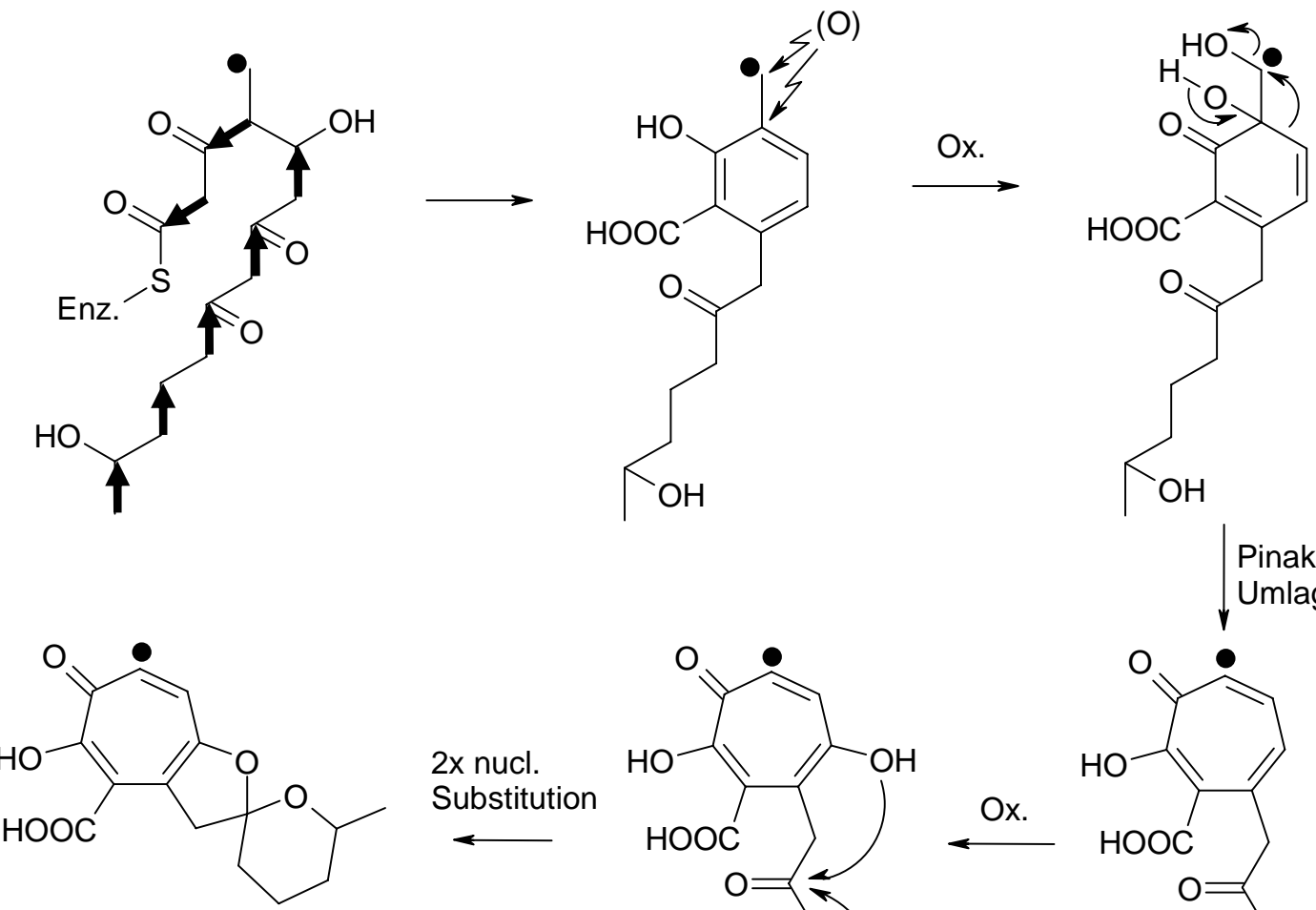

2x nucl. Substitution

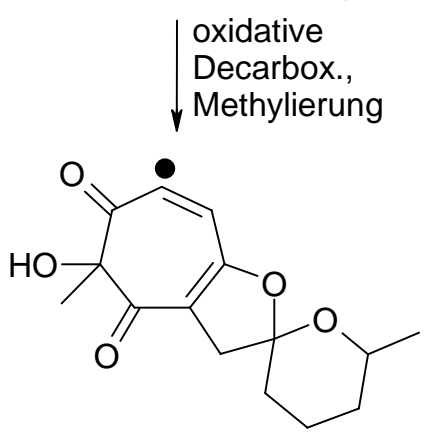

Acetylierung Dimerisierung
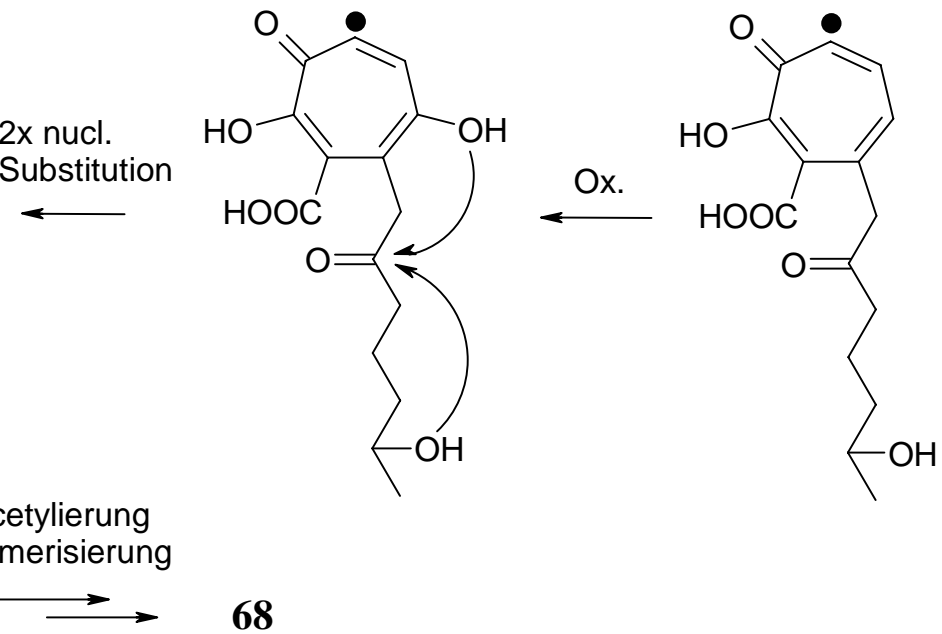

Abbildung 57: Alternativer Biosyntheseweg für Chaetospiron (68). 


\subsection{Biologische Aktivität der Sekundärmetaboliten}

\section{Zur biologischen Aktivität von Cytochalasinen}

Cytochalasine sind biologisch hoch aktiv, am auffälligsten ist ihre in vitro und in vivo nachgewiesene cytotoxische Wirkung gegen Säugetier-Zellen. Durch Hemmung der Cytoplasmateilung (Cytokinese), nicht jedoch der Kernteilung (Mitose), können sie zu vielkernigen, stark vergrößerten Zellen führen. Bei höheren Konzentrationen kommt es zum Austritt des Kerns aus der Zelle. Sie besitzen mindestens zwei Wirkorte in tierischen Zellen und hemmen in niedriger Konzentration die Aufnahme von Zuckern ${ }^{[168,169]}$. Weitere toxische Wirkungen der Cytochalasine treten gegen Bakterien, Pilze, Algen und Protozoen auf. Bei einigen Cytochalasinen wurden auch phytotoxische Eigenschaften nachgewiesen ${ }^{[170]}$.

\section{Biologische Aktivität der Chaetoglobosine 65 - 67}

Im Plattendiffusionstest hemmten die Chaetoglobosine 65 - 67 das Wachstum der bakteriellen Testkeime, wobei die Wirkung von Chaetoglobosin A (66) und seinem 19-O-Acetat (65) deutlich stärker war als die von Chaetoglobosin C (67). Eine Aktivität gegen Candida albicans wurde nicht beobachtet (siehe Tabelle 20 auf Seite 135). In der Literatur wird für alle drei Chaetoglobosine eine cytotoxische Wirkung angegeben ${ }^{[171]}$, von 66 ist zudem eine Aktivität gegen Gram-positive Bakterien und verschiedene, darunter phytopathogene Pilze bekannt $^{[158,172]}$.

Obwohl die Ergebnisse der Pflanzenschutz-Tests noch ausstehen, kann davon ausgegangen werden, dass die fungizide und herbizide Wirkung des Rohextrakts auf die Chaetoglobosine zurückzuführen ist. Das Hauptprodukt des Stamms, 19-O-Acetylchaetoglobosin A (65), bewirkt die Hemmung im Bereich $\mathbf{C}$ des Rohextrakts, 66 und 67 die im Bereich $\mathbf{B}$ (siehe Abbildung 46). Diese Annahme beruht auf der Übereinstimmung der HPLC-Retentionszeiten der Reinsubstanzen mit den aktiven Bereichen des Extrakts sowie der oben beschriebenen biologischen Aktivität von $\mathbf{6 6}$ und der Substanzklasse der Cytochalasine.

\section{Zur biologischen Aktivität von Tropolonen}

Von $\alpha$-Tropolon sind vielfältige biologische Eigenschaften bekannt. Es ist Inhibitor von Metalloproteasen, wirkt fungizid gegen phytopathogene Pilze und insektizid ${ }^{[173]}$. Eine insektizide Wirkung wird ebenfalls von verschiedenen Tropolon-Derivaten wie Cordytropolon (76) berichtet, welches das aktive Prinzip des pathogenen Pilzstamms Cordyceps sp. BCC 1681 ist. Es ist vermutlich biosynthetisch mit Stipitatonsäure (73) 
verwandt und besitzt zudem cytotoxische Eigenschaften ${ }^{[174]}$. Auch das aus Pflanzen der Gattung Thuja („Lebensbaum“) bekannte $\alpha$-Thujaplicin (77) ist giftig für Insekten. Zur Funktion von niedermolekularen Spiroacetalen bei Insekten siehe Kapitel 13.1, Seite 126.<smiles>O=c1cc(O)c2c(cc1O)COC2</smiles>

76<smiles>CC(C)c1ccccc(=O)c1O</smiles>

77

\section{Biologische Aktivität von Chaetospiron (68)}

Im Plattendiffusionstest hemmte 68 das Wachstum von Bacillus subtilis, zeigte jedoch keine antifungische Aktivität gegen Candida albicans. Ob 68 ebenso wie die Chaetoglobosine 65 67 eine Wirkung gegen phytopathogene Pilze besitzt und damit für die entsprechende Wirkung im Bereich B des Rohextrakts (Abbildung 46) mitverantwortlich ist, wird gegenwärtig geprüft (siehe Kapitel 15, Seite 134). Aufgrund der beschriebenen Wirkung anderer Tropolon-Derivate dürften auch die ausstehenden Ergebnisse des Insektizid-Tests interessant sein. 


\section{Sesquiterpene aus Drechslera sp. BS 6618}

Der Stamm Drechslera sp. BS 6618 wurde als Endophyt aus Strandroggen (Elymus arenarius) isoliert, einer typischen, halotoleranten Pflanze der Dünen- und VordünenLandschaft. Der Agarplatten-Extrakt des Stamms BS 6618 zeigte in den biologischen Tests keinen Befund. Im chemischen Screening fielen im Dünnschichtchromatogramm violette, lipophile Farbstoffe auf sowie die intensive Braunfärbung einiger Zonen unterschiedlicher Polarität beim Erhitzen mit Anisaldehyd.

Während die Struktur der Farbstoffe aufgrund der sehr geringen Substanzmengen nicht aufgeklärt werden konnte, stellten sich die mit Anisaldehyd braunfärbenden Substanzen als Sesquiterpene heraus. Vier verschiedene Terpene wurden isoliert, darunter die bereits bekannten trizyklischen Verbindungen Helminthosporal (78, 7 mg/L), Helminthosporol (79, $6 \mathrm{mg} / \mathrm{L}$ ) und cis-Sativendiol (80, $4 \mathrm{mg} / \mathrm{L})$. Mit Bis(prehelminthosporol) (81, $9.5 \mathrm{mg} / \mathrm{L})$ wurde ein dimeres Sesquiterpen vom seco-Sativentyp isoliert, das wahrscheinlich ebenso wie $\mathbf{7 8}$ und $\mathbf{7 9}$ aus $\mathbf{8 0}$ hervorgeht. Dieser neue Naturstoff wurde in zwei diastereomeren Formen erhalten (81A und 81B), die nicht voneinander getrennt werden konnten.

\subsection{Helminthosporal (78)}

Die Substanz 78 wurde als ein farbloses Öl isoliert. Mittels des EI-Massenspektrums ließ sich die Molmasse nicht eindeutig bestimmen, weshalb ein DCI-MS-Experiment durchgeführt wurde. Der erhaltene $\left[\mathrm{M}+\mathrm{NH}_{4}\right]^{+}$-Peak bei $\mathrm{m} / \mathrm{z}=252$ belegt die Molmasse $234 \mathrm{~g} / \mathrm{mol}$. Das ${ }^{13} \mathrm{C}-\mathrm{NMR}-$ Spektrum zeigt 15 Kohlenstoffatome, darunter zwei Carbonylgruppen $\left(\delta_{\mathrm{C}}=187,204\right)$. Anhand des ${ }^{1} \mathrm{H}-\mathrm{NMR}-$ Spektrums können diese als zwei Aldehydgruppen erkannt werden $\left(\delta_{\mathrm{H}}=9.48,10.00\right)$, außerdem sind insgesamt vier Methylgruppen $\mathrm{zu}$ erkennen $\left(\delta_{\mathrm{H}}=0.75,1.04,1.13,2.05\right)$. Eine Suche mit diesen Daten und Strukturelementen in Naturstoff-Datenbanken ${ }^{[41,55]}$ ergab Helminthosporal (78) als Strukturvorschlag, der durch einen Vergleich der spektroskopischen Daten mit Literaturwerten ${ }^{[175]}$

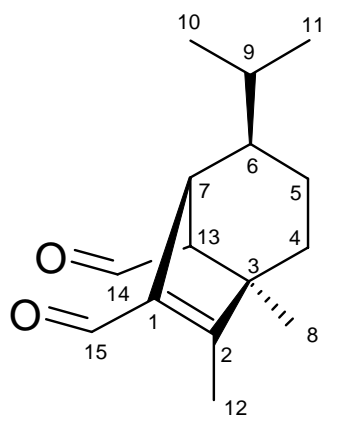

78 verifiziert wurde. 


\subsection{Helminthosporol (79)}

Das ${ }^{1}$ H-NMR-Spektrum von 79 ist dem von 78 sehr ähnlich. Es zeigt die zur quartären Doppelbindung benachbarte Aldehydgruppe $\left(\delta_{\mathrm{H}}=10.00, \mathrm{~s}\right)$, die $\mathrm{zu}$ einer Methingruppe benachbarte Aldehydgruppe fehlt jedoch. Stattdessen fällt das Signal einer neuen Methylengruppe $\left(\delta_{\mathrm{H}}=3.28,3.69\right)$ auf, deren Tieffeld-Verschiebung die Bindung an Sauerstoff vorgibt. Dieser Befund sowie die im Vergleich zu 79 um 2 Masseneinheiten höhere Molmasse von $236 \mathrm{~g} / \mathrm{mol}$ ließen das Vorliegen eines in Position 14 zum Alkohol reduzierten Derivats von 79 erwarten. Diese Vermutung bestätigte die Übereinstimmung der ${ }^{1} \mathrm{H}$ - und ${ }^{13} \mathrm{C}-\mathrm{NMR}-\mathrm{Daten}$ mit publizierten Werten für ein solches Derivat $^{[176]}$, das als Helminthosporol (79) bekannt ist.

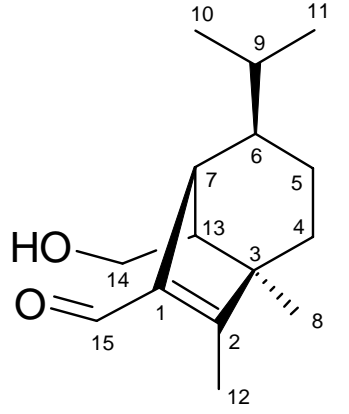

79

\section{3 cis-Sativendiol $(80)$}

Die farblose Verbindung 80 ist ebenso wie $\mathbf{7 8}$ und $\mathbf{7 9}$ von öliger Konsistenz. Ihre Molmasse von $236 \mathrm{~g} / \mathrm{mol}$ wurde durch ein DCIMassenspektrum ermittelt. Das ${ }^{13} \mathrm{C}-\mathrm{NMR}$-Spektrum beinhaltet die Signale von 15 Kohlenstoffatomen, weshalb das Vorliegen eines weiteren Sesquiterpens $\mathrm{zu}$ vermuten war. Die NMRSpektren zeigen anders als bei $\mathbf{7 8}$ und $\mathbf{7 9}$ keine Carbonylgruppen. Das HSQC-Spektrum offenbart mit der Korrelation von zwei bei relativ hohem Feld erscheinenden olefinischen Protonen

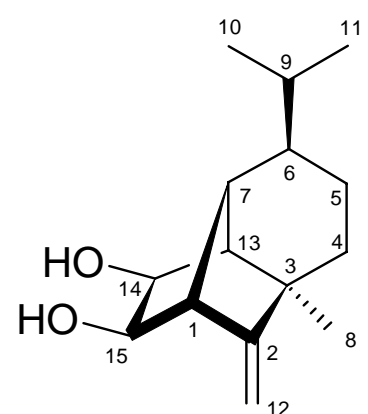

80 $\left(\delta_{\mathrm{H}}=4.58,4.89\right) \mathrm{zu}$ demselben C-Atom $\left(\delta_{\mathrm{C}}=103\right)$ das Vorliegen einer exozyklischen Doppelbindung. Die im COSY-Spektrum sichtbare Kopplung von zwei zum Dublett aufgespaltenen Methylgruppen $\left(\delta_{\mathrm{H}}=0.85,0.92\right) \mathrm{zu}$ derselben Methingruppe $\left(\delta_{\mathrm{H}}=1.34\right)$ lässt wie bei $\mathbf{7 8}$ und $\mathbf{7 9}$ eine Isopropylgruppe erkennen. Weitere auffällige Strukturelemente sind eine an ein quartäres C-Atom gebundene Methylgruppe $\left(\delta_{\mathrm{H}}=1.03\right)$ sowie zwei Sauerstoff-gebundene, benachbarte Methingruppen $\left(\delta_{\mathrm{H}}=3.63,4.02\right)$, deren vicinale Kopplungskonstante ${ }^{3} J_{\mathrm{HH}}=6.0 \mathrm{~Hz}$ beträgt. Diese Strukturelemente und Molmasse sind in der Datenbank AntiBase ${ }^{[41]}$ nur mit cis- und trans-Sativendiol in Einklang zu bringen, die erwähnte Kopplungskonstante zwischen H-14 und H-15 legt die Verbindung in

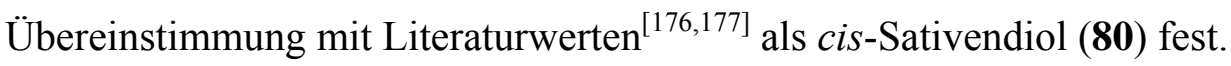




\section{4 (15R)- und (15S)-Bis(prehelminthosporol) (81A und 81B)}

Ebenso wie bei den anderen Sesquiterpenen 78 - 80 handelt es sich auch bei 81 um ein farbloses Öl. Die NMR-Spektren zeigen das Vorliegen eines Gemischs aus zwei Substanzen im Verhältnis $5: 3$, die im Folgenden mit 81A und 81B bezeichnet werden. Eine Trennung war auch durch HPLC unter den verwendeten Bedingungen nicht möglich. Die Spektren beider Substanzen weisen große Übereinstimmung mit denen von cis-Sativendiol (80) auf, wobei die Signale für 81A und 81B nur geringfügig gegeneinander verschoben sind.

Das ESI-Massenspektrum zeigt, dass 81A und 81B dieselbe Molmasse von $454 \mathrm{~g} / \mathrm{mol}$ besitzen. Durch ein hochaufgelöstes Massenspektrum konnte die Summenformel $\mathrm{C}_{30} \mathrm{H}_{46} \mathrm{O}_{3}$ für die Substanzen ermittelt werden. Durch Zugabe von Säure (TFA) zur Probe wurde stattdessen ein Fragment mit der Masse $\mathrm{m} / \mathrm{z}=219$ und der Summenformel $\mathrm{C}_{15} \mathrm{H}_{22} \mathrm{O}$ erhalten, was der Hälfe des ursprünglichen Moleküls bei vorheriger Wasserabspaltung entspricht. Da die NMR-Daten das Vorliegen eines Sesquiterpens vorschlagen, die MS-Daten jedoch eine doppelt so hohe Molmasse zeigen (minus Wasserabspaltung), müssen die Verbindungen 81A und 81B symmetrische Dimere eines terpenoiden Grundkörpers sein.

Die Zuordnung der NMR-Signale zu 81A bzw. 81B erfolgte anhand der Integrale des Protonenresonanzspektrums sowie der 2D-NMR-Spektren. Es wird zunächst die Strukturaufklärung der Hauptkomponente 81A diskutiert.

Die NMR-Spektren zeigen einige von $c i s$-Sativendiol (80) vertraute Strukturelemente: eine exozyklische Doppelbindung $\left(\delta_{\mathrm{H}}=4.77,4.87\right)$, eine Isopropylgruppe $\left(\delta_{\mathrm{H}}=0.85,0.90,1.34\right)$ und eine zu einem quartären C-Atom benachbarte Methylgruppe $\left(\delta_{\mathrm{H}}=1.13\right)$. Anhand der COSY- und HMBC-Korrelationen konnten diese Fragmente mit zwei Methylen- und vier weiteren Methingruppen zu einem zu 80 analogen Kohlenstoffgerüst verknüpft werden (Abbildung 58). Nicht von $\mathbf{8 0}$ bekannt sind eine Sauerstoff-gebundene Methylengruppe $\left(\delta_{\mathrm{H}}=3.54,3.94\right)$ und eine Acetalfunktion $\left(\delta_{\mathrm{H}}=4.64, \delta_{\mathrm{C}}=96\right)$, deren Konnektivität sich ebenfalls aus den 2D-NMR-Spektren ableiten ließ. Sie befinden sich an den Positionen im Molekül, die bei $\mathbf{8 0}$ von den Hydroxygruppen eingenommen werden. Das Fehlen einer vicinalen Kopplung zwischen den Protonen beider C-Atome beweist, dass die C-CVerknüpfung zwischen C-14 und C-15 durch eine Sauerstoff-Brücke ersetzt ist. Es liegt hier also ebenso wie bei $\mathbf{7 8}$ und $\mathbf{7 9}$ eine Verbindung vom seco-Sativentyp ${ }^{\mathrm{w}}$ vor (Abbildung 59).

${ }^{\mathrm{w}}$ lat.: $\mathrm{seco}=$ ich schneide 


\section{COSY HMBC}

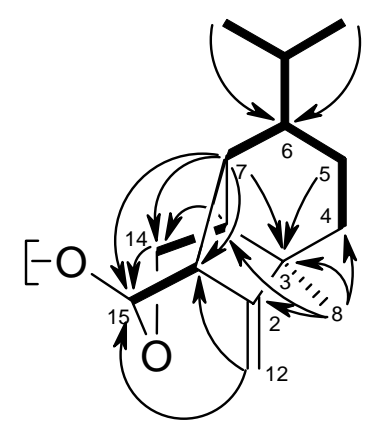

Abbildung 58: COSY-Konnektivitäten und ausgewählte HMBC-Korrelationen von 81A.

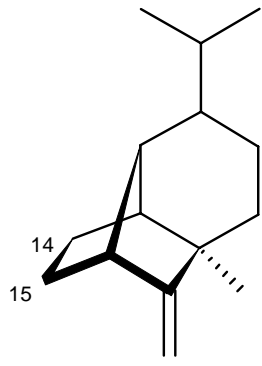

Sativen-Typ<smiles>C=C1[C@H](C)[C@H]2C(C(C)C)CC[C@@]1(C)C2C</smiles>

seco-Sativen-Typ

Abbildung 59: Sativen- und seco-Sativen-Typ bei Sesquiterpenen.

Die Konfiguration des acetalischen Kohlenstoffatoms C-15 wurde durch 1D-NOESYExperimente geklärt, bei denen auf 15-H eingestrahlt wurde. Der zwischen 15-H und 7-H auftretende Nuclear-Overhauser-Effekt ist nur bei der in Abbildung 60 (links) dargestellten Konfiguration an C-15 möglich. Nimmt man die absolute Konfiguration der anderen Stereozentren als identisch mit $\mathbf{7 8}$ und $\mathbf{7 9}$ an, so entspricht dies der (15R)-Konfiguration.

Für die Minderkomponente 81B ergibt sich auf gleichem Weg eine Struktur, deren Konnektivität mit 81A identisch ist. Die Verbindungen unterscheiden sich jedoch in der Konfiguration an C-15. Die 1D-NOESY-Spektren zeigen, dass sich das Proton 15-H von 81B in räumlicher Nähe zur exozyklischen Doppelbindung befindet, was einer (15S)-Konfiguration entspricht (Abbildung 60, rechts). 


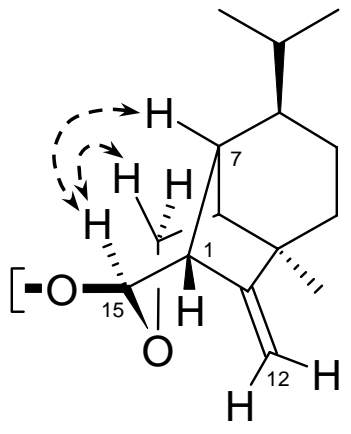

81A $(15 R)$

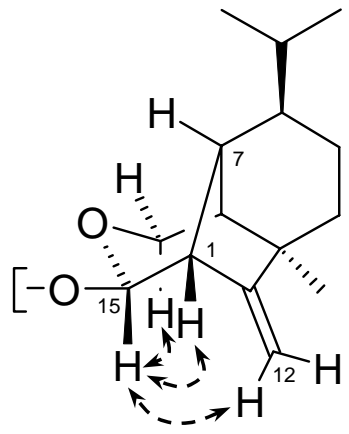

81B $(15 S)$

Abbildung 60: NOESY-Korrelationen ausgehend von 15-H in 81A (links) und 81B (rechts).

Beide Substanzen müssen aufgrund ihrer NMR-Spektren symmetrisch vorliegen, ihre Summenformel und der Zahl der Sauerstoffatome (drei) bedingen die Verknüpfung der monomeren Einheiten über das acetalische Sauerstoffatom. Diese nicht in der Literatur beschriebenen dimeren Sesquiterpene wurden (15R)-Bis(prehelminthosporol) (81A) und (15S)-Bis(prehelminthosporol) (81B) genannt.

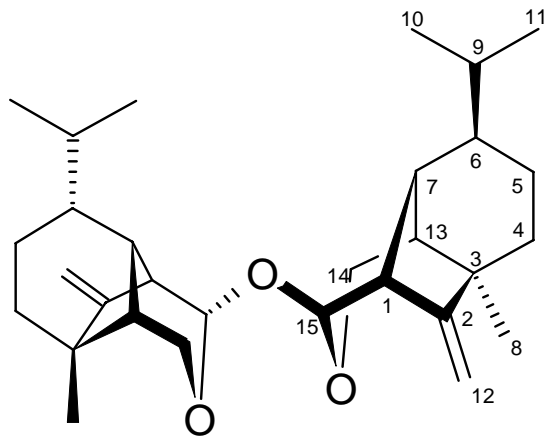

81A

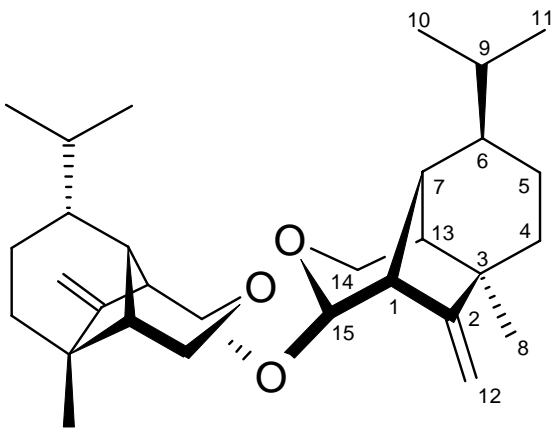

81B

Die Monomere sind unter dem Namen Prehelminthosporol (82) bekannt (siehe Abbildung 61 auf Seite 122). Sie wurden aus Pilzen isoliert, die auch 78 - 80 und ähnliche Sesquiterpene produzieren $^{[178]} .82$ wurde stets als Gemisch von epimeren Halbacetalen gefunden ${ }^{[179]}$, was einen Hinweis auf das Entstehen der beiden Isomere 81A und 81B bietet. Die für Prehelminthosporol (82) veröffentlichten NMR-Daten ${ }^{[178,180]}$ sind in guter Übereinstimmung mit den Werten von 81A und 81B. 
Es scheint wahrscheinlich, dass der Stamm BS 6618 neben den Acetalen 81A und 81B auch das gemischte Acetal aus (15R)- und (15S)-Prehelminthosporol (82) biosynthetisiert. Bei mehreren im Dünnschicht- und HPLC-Chromatogramm den beschriebenen Metaboliten ähnlichen, bisher nicht isolierten Substanzen handelt es sich vermutlich ebenfalls um Sesquiterpene. Die weitere Auftrennung des Extrakts sollte somit zu weiteren Vertretern einer Substanzklasse führen, die interessante biologische Wirkungen auf Pflanzen aufweist.

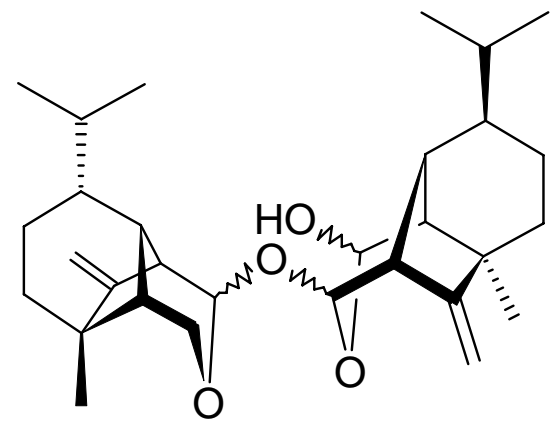

83

Strukturell sehr ähnlich ist ein von dem Pilzstamm Cochliobolus setariae IFO 6635 bekannter Aversionsfaktor (83). Hierunter versteht man Sekundärstoffe von Pilzen, die das Wachstum anderer Stämme derselben Spezies hemmen. 83 ist anders als 81 ein unsymmetrisches Dimer aus Prehelminthosporol (82) und Prehelminthosporal (85, siehe Seite 122), was durch Identifizierung der Hydrolyseprodukte nachgewiesen wurde ${ }^{[181]}$. Spektroskopische Daten von 83 sowie die Konfiguration an C-14 und C-15 sind nicht bekannt.

\subsection{Zur biologischen Aktivität der Sesquiterpene}

78, 79 und 80 sind als Produkte von Pilzen der Gattung Drechslera bekannt, die auch synonym als Helminthosporium bezeichnet wird ${ }^{[176,182]}$. Pilzstämme dieser Gattung werden zwar einerseits als phytopathogen beschrieben, andererseits besiedeln sie z. B. epiphytisch Reispflanzen, bei denen sie insbesondere durch die Produktion von cis-Sativendiol (80) eine Wachstums-Förderung der Pflanze bewirken. Die Wirkung von 80 und in einem schwächeren Ausmaß auch die von Helminthosporol (79) ist ähnlich der des bekannten Phytohormons Gibberellinsäure (4). Das ebenfalls auftretende trans-Isomer von $\mathbf{8 0}$ hingegen hat ebenso wie Helminthosporal (78) eine entgegengesetzte, phytotoxische Wirkung, wodurch dem Pilz insgesamt eine Wachstums-regulierende Funktion zukommt ${ }^{[177]}$.

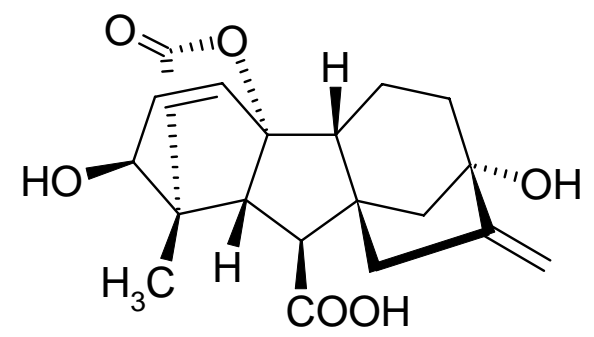


Der endophytische Pilz Drechslera dematioidea, der aus der Rotalge Liagora viscida isoliert wurde, produziert mindestens fünfzehn verschiedene Sesquiterpene (darunter auch 79 und 80 ${ }^{[176]}$, was eine ähnliche ökologische Funktion dieses Pilzes bei Algen nahe legt.

Testergebnisse bezüglich der herbiziden oder Wachstums-fördernden Wirkung der neuen Verbindungen 81A und 81B stehen noch aus. Zur Überprüfung, ob 81 analog zu 83 als Aversionsfaktor wirkt, sollte zunächst neben der Gattung (Drechslera) auch die Spezies des Produzenten bestimmt werden.

Die Verbindungen $\mathbf{7 8}$ und $\mathbf{7 9}$ zeigten im Plattendiffusionstest eine moderate Hemmwirkung gegen Bacillus subtilis, cis-Sativendiol (80) und Bis(prehelminthosporol) (81) zeigten keine antimikrobielle Aktivität (siehe Kapitel 15, Seite 134).

\section{6 Überlegungen zur Biosynthese}

Die Biosynthese der Sesquiterpene geht aus von Farnesylpyrophosphat. Durch eine Reihe von Zyklisierungen und Umlagerungen entsteht Sativen (84), das zu cis-Sativendiol (80) oxidiert wird $^{[182]}$. Dieses kann zu Prehelminthosporal (85) oder Prehelminthosporol (82) weiter oxidiert werden, wobei die Bindung zwischen C-14 und C-15 gespalten wird. Die Oxidation zur Alkohlgruppe an C-14 und zur Aldehydgruppe an C-15 bei 82 führt zur spontanen Ausbildung einer Halbacetalstruktur, die nicht stereoselektiv verläuft und so zu einem Gemisch aus zwei Epimeren führt. Dimerisierung unter Wasserabspaltung führt zu den entsprechenden Bis(prehelminthosporol)-Epimeren 81A und 81B, eine Verschiebung der Doppelbindung um eine Position ergibt Helminthosporol (79). Bei letzterem verhindert die nun zur Carbonylgruppe konjugierte Doppelbindung die Ausbildung von Isomeren wie bei 82. Eine analoge Doppelbindungs-Verlagerung führt von $85 \mathrm{zu}$ Helminthosporal (78). Die nach cis-Sativendiol (80) folgenden Biosyntheseschritte sind bisher nicht untersucht worden und stellen daher eine Hypothese dar. Auch Umwandlungen zwischen 82 und 85 sowie zwischen 78 und 79 sind denkbar. 
Farnesylpyrophosphat

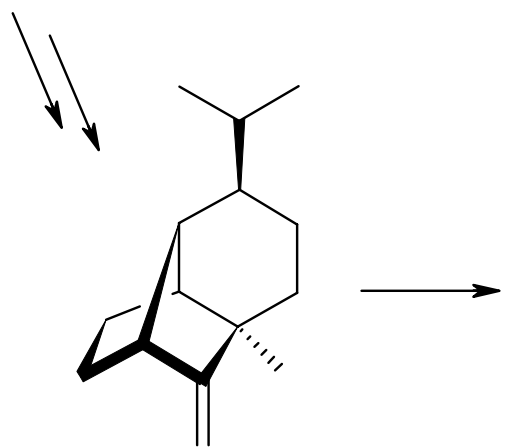

84

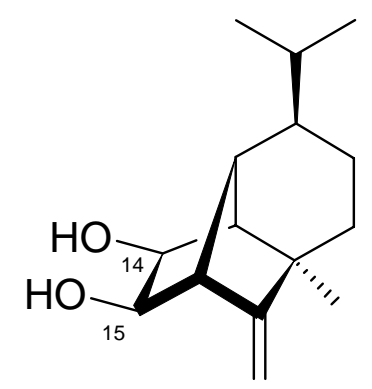

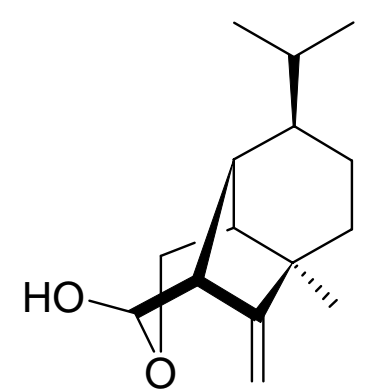

80

$\downarrow$

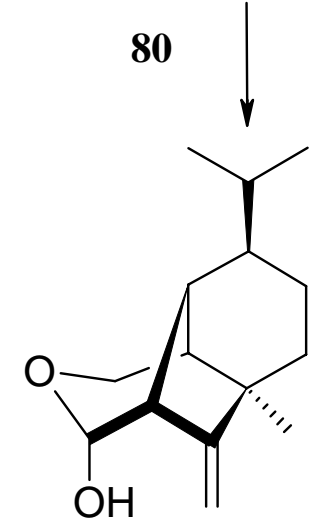

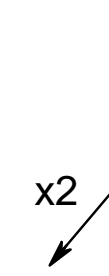

81A

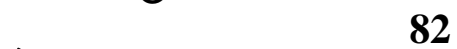

82

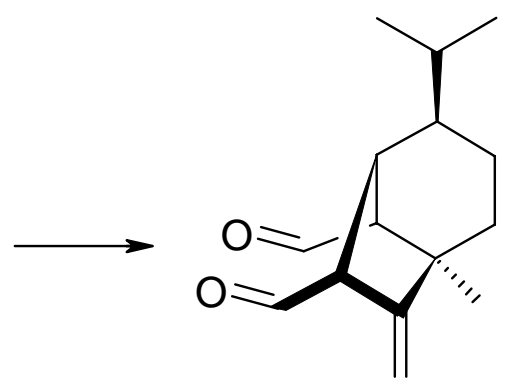<smiles>CC1=C(C=O)[C@@H]2C(C(C)C)CC[C@]1(C)C2C=O</smiles>

78

85

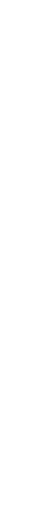




\section{Spiroverbindung aus Beauveria sp. BS 6750}

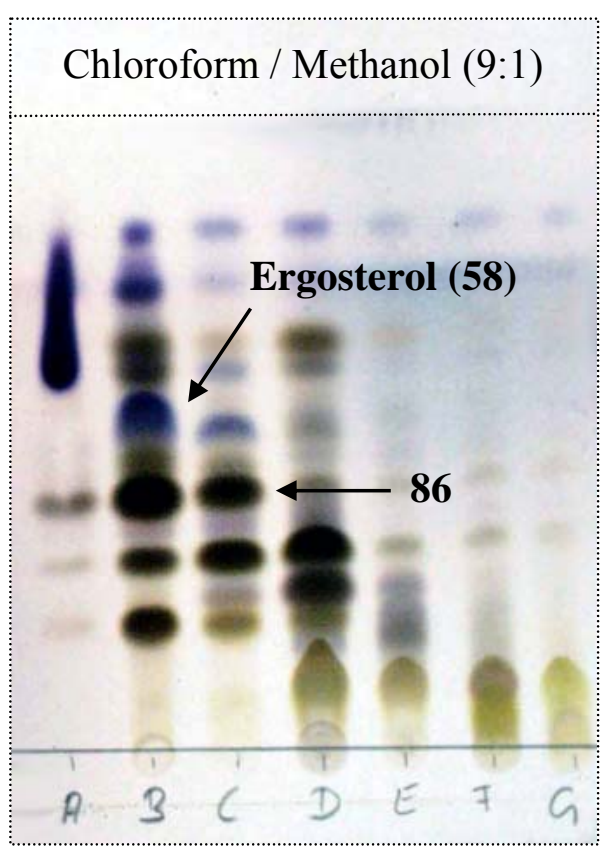

Der Stamm Beauveria sp. BS 6750 fiel im chemischen Screening durch ein interessantes Metabolitenspektrum auf. Sowohl die Festagarkultur als auch die Inkubation in Schüttelkolben und die Ruhekultur in P-Kolben führte zur Produktion mehrerer Metaboliten, die mit Anisaldehyd in der Wärme braun oder blau anfärbten. In den biologischen Tests blieben die Extrakte ohne Ergebnis. Im Folgenden wird die Strukturaufklärung einer mit Anisaldehyd braun anfärbenden Substanz aus dem Agarplatten-Extrakt beschrieben. Es handelt sich um eine neue Spiroverbindung, die Beauveriaspirolid (86) genannt wurde.

Abbildung 62: Dünnschichtchromatogramm der

Fraktionen der Rohproduktsäule (Agarplatten-

Extrakt, Anfärbung mit Anisaldehyd).

\subsection{Strukturaufklärung von Beauveriaspirolid (86)}

Das EI-Massenspektrum der farblosen, öligen Substanz 86 zeigt bei $\mathrm{m} / \mathrm{z}=196$ den Molekülpeak, dessen Fragmentierung das Vorhandensein einer Methylgruppe $(\Delta \mathrm{m} / \mathrm{z}=15)$ und einer Ester- oder Carboxylgruppe $(\Delta \mathrm{m} / \mathrm{z}=44)$ andeutet. Die NMR-Spektren beinhalten Signale von zehn Kohlenstoff- und 14 Wasserstoffatomen. Unter Berücksichtigung der Molmasse lässt sich hieraus die Summenformel $\mathrm{C}_{10} \mathrm{H}_{14} \mathrm{O}_{4}$ ableiten, welche ein hochaufgelöstes EI-Massenspektrum bestätigte. Von den sich ergebenden vier formalen Doppelbindungsäquivalenten kann nur eines einer funktionellen Gruppe zugeordnet werden (EsterCarbonylgruppe bei $\left.\delta_{\mathrm{C}}=179\right), \mathbf{8 6}$ muss daher drei Ringsysteme aufweisen. 

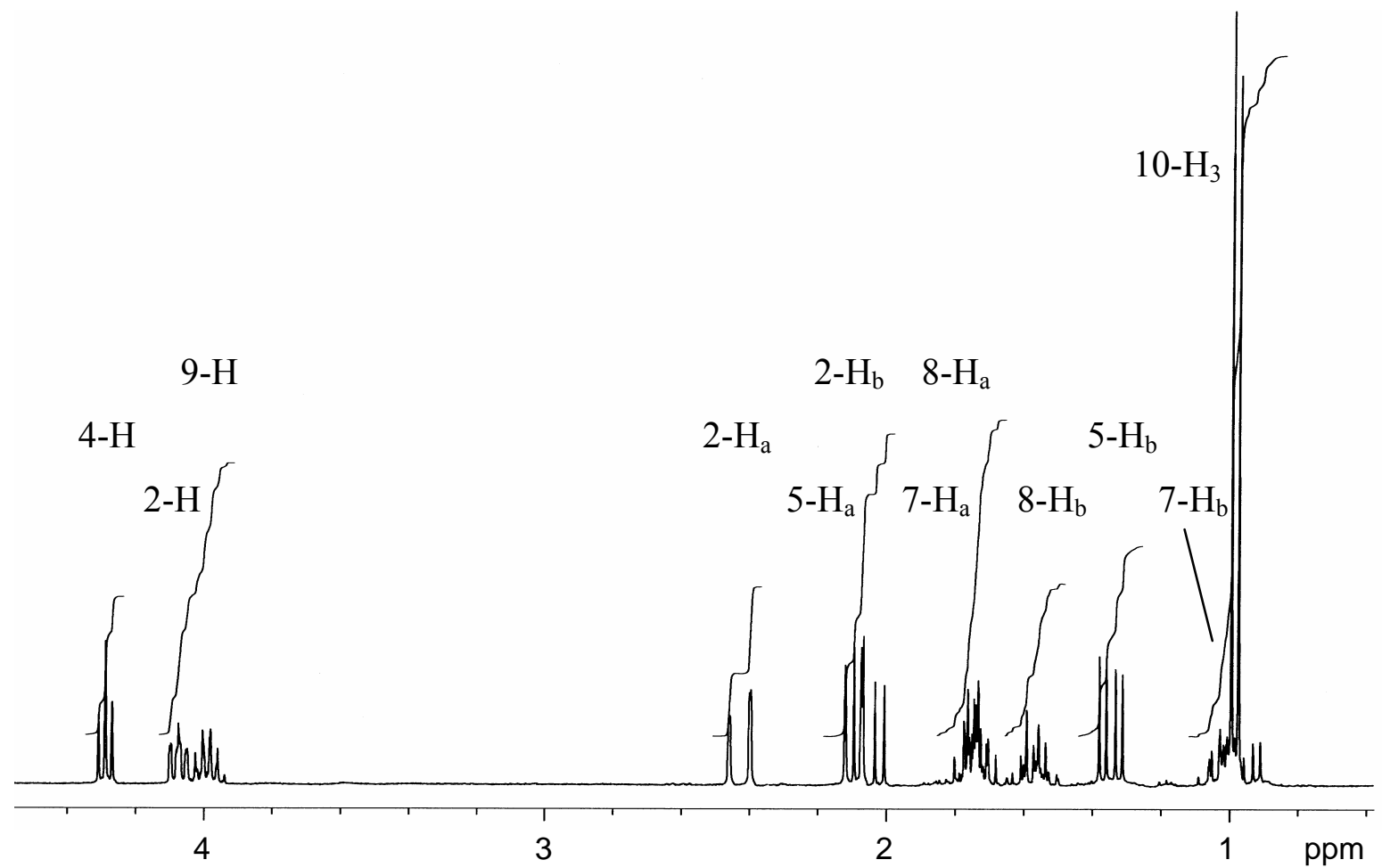

Abbildung 63: ${ }^{1} \mathrm{H}-\mathrm{NMR}-\mathrm{Spektrum}$ von $86\left(600 \mathrm{MHz}, \mathrm{C}_{6} \mathrm{D}_{6}\right)$.

Das ${ }^{1}$ H-NMR-Spektrum (Abbildung 63) zeigt eine Methylgruppe $\left(\delta_{\mathrm{H}}=0.99\right)$, die eine Sauerstoff-substituierte Methingruppe als Kopplungspartner hat $\left(\delta_{\mathrm{H}}=3.99\right)$. Mittels des COSY-Spektrums lassen sich zwei Methylengruppen ermitteln, die diese Kette fortsetzen (Abbildung 64, Fragment 1). Eine weitere Teilstruktur ergibt sich aus zwei Methylengruppen, zwischen denen sich zwei Methingruppen befinden. Letztere müssen aufgrund ihrer hohen chemischen Verschiebung $\left(\delta_{\mathrm{H}}=4.08,4.29\right)$ Sauerstoff tragen (Abbildung 64, Fragment 2).
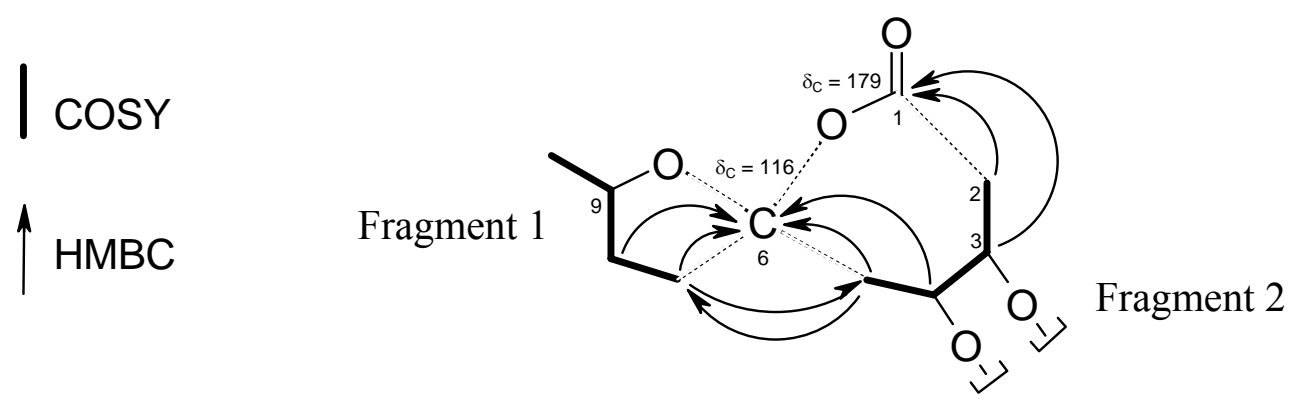

Abbildung 64: COSY-Konnektivitäten und für die Strukturaufklärung wichtige HMBCKorrelationen bei $\mathbf{8 6}$. 
Die Verknüpfung der Fragmente 1 und 2 mit den beiden quartären C-Atomen des Moleküls erfolgte anhand von HMBC-Korrelationen (ebenfalls in Abbildung 64 dargestellt) und der chemischen Verschiebungen. Das Carbonyl-Kohlenstoffatom ist direkt an Fragment 2 gebunden. Die Verbindung zwischen beiden Fragmenten bildet das zweite quartäre C-Atom, dessen starke Tieffeldverschiebung $\left(\delta_{\mathrm{C}}=116\right)$ das Vorliegen eines Acetals und zusätzlich die Bindung an die Carbonylgruppe beweist. Es ergibt sich für die rechte Hälfte des Moleküls ein siebengliedriger Lactonring. Der Ringschluss zum Tetrahydrofuran-Ring auf der linken Seite wird zwar durch keine HMBC-Korrelation angezeigt, jedoch indirekt durch das Fehlen jeglicher HMBC- und NOESY-Korrelationen zwischen den Positionen 2-5 (Fragment 2) und 8-10 (Fragment 1) bewiesen, die bei einer Verbrückung C-9-O-C-3/C-4 zu erwarten wären. Es verbleibt laut Summenformel nur ein weiteres Sauerstoffatom, so dass die Struktur durch eine Epoxidfunktion an C-3/C-4 vervollständigt wird. Man erhält ein Spiroketal-LactonSystem, das aus einem Fünf- und einem Siebenring aufgebaut ist, letzterer mit einem Epoxid (Abbildung 65).

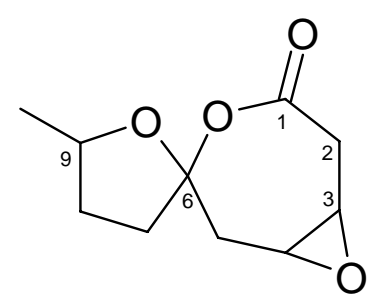

Abbildung 65: Struktur von 86 ohne Berücksichtigung der Stereochemie.

\section{Aufklärung der relativen Stereochemie}

Die Größe der vicinalen Kopplungskonstante zwischen 3-H und 4-H ( $\left.{ }^{3} J_{\mathrm{HH}}=5.5 \mathrm{~Hz}\right)$ ist kenn-

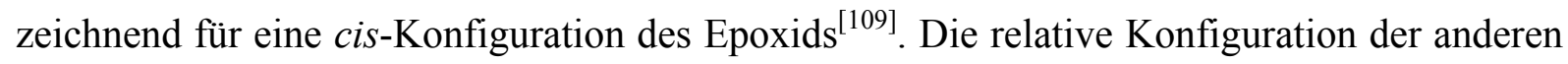
Stereozentren wurde durch ein NOESY-Spektrum aufgeklärt, wichtige Korrelationen sind in Abbildung 66 zusammengefasst. Der zwischen der Methylgruppe und 7- $\mathrm{H}_{\mathrm{a}}$ auftretende NOE weist darauf hin, dass sich beide auf derselben Seite des Fünfrings befinden. Die räumliche Nähe von $7-\mathrm{H}_{\mathrm{a}}$ zu 5- $\mathrm{H}_{\mathrm{a}}$ am Siebenring ist ebenfalls mit einer cis-Konfiguration dieser beiden Gruppen zu erklären, woraus sich auch für die Methylgruppe und C-5 eine solche Anordnung ergibt. Die beiden Methinprotonen des Epoxids hingegen zeigen ausschließlich NOESYKorrelationen zu 7- $\mathrm{H}_{\mathrm{b}}$. Hieraus lässt sich schließen, dass diese drei Protonen auf derselben Seite des Siebenrings sind, es folgt die gezeigte $\left(3 R^{*}, 4 S^{*}, 6 R^{*}, 9 S^{*}\right)$-Konfiguration von 86. 
Da die Konformation sowohl des Fünf- wie auch des Siebenrings nicht genau vorhersagbar ist, ist diese Konfiguration als Vorschlag anzusehen und sollte durch weitere Experimente belegt werden. Denkbar wäre z. B. eine oxidative Öffnung des Epoxids, welche eine Acylierung in dieser Position und anschließende Kristallisation zum Zweck der Röntgenstrukturanalyse erlauben sollte. Die Einführung eines Schweratom-haltigen Substituenten würde zudem auch die Bestimmung der absoluten Stereochemie ermöglichen.

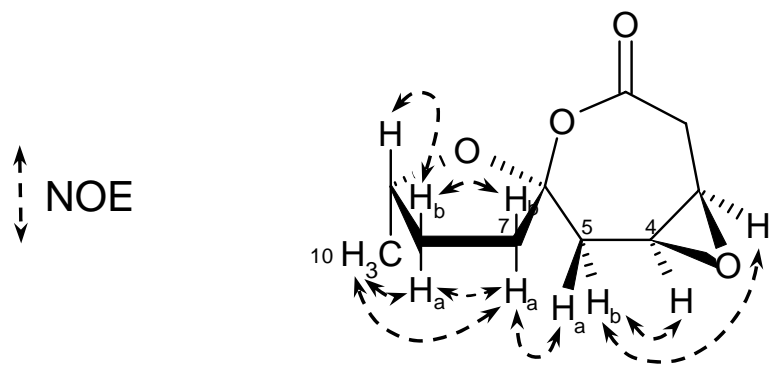

Abbildung 66: Bestimmung der relativen Stereochemie von 86.

Die Substanz wurde Beauveriaspirolid ${ }^{\mathrm{x}}(\mathbf{8 6})$ genannt. Das Grundgerüst von 86 ist strukturell sehr ungewöhnlich. Etwa 130 Naturstoffe mit einem Spiroketal-Lacton-Gerüst als Substruktur sind bekannt, keine dieser Substanzen enthält jedoch einen Fünf- und einen Siebenring. Ebenso wurde die Kombination mit einer Epoxidfunktion bisher nicht beschrieben $^{[55]}$. Meist handelt es sich um $\gamma$-Lactone, die mit

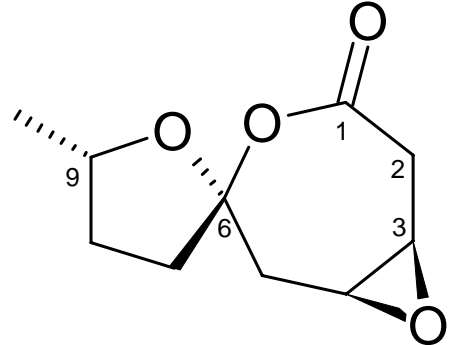

86 einem Fünf- oder Sechsring verknüpft sind wie z. B. Spiroxabovolide (87). Niedermolekulare Spiroketale ohne Lactonfunktion findet man oft bei Insekten, wo sie z. T. als Pheromone wirken. Hier gibt es auch einige wenige Vertreter mit Fünf-/Siebenring-Struktur wie z. B. 2,7-Dimethyl-1,6-dioxaspiro[4.6]undecan (88) ${ }^{[183]}$.

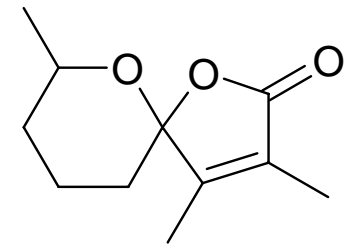

87

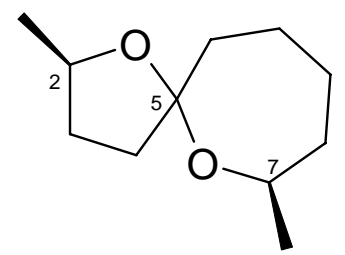

88

\footnotetext{
${ }^{\mathrm{x}}$ In Anlehnung an die Gattung des $\mathbf{8 6}$ produzierenden Endophyten (Beauveria) sowie die strukturellen Besonderheiten der Substanz (Spiroketal).
} 


\subsection{Zur Biosynthese von Beauveriaspirolid (86)}

Die Struktur von Beauveriaspirolid (86) lässt eine Biosynthese auf dem Polyketidweg vermuten. Ein hypothetischer Biosyntheseweg ist in Abbildung 67 gezeigt. Aus fünf AcetatBausteinen wird durch eine Polyketidsynthase ein Pentaketid aufgebaut (Schritt 1). In Position 6 erfolgt anschließend eine Oxidation zur Ketogruppe (Schritt 2), welche von 9-OH nucleophil angegriffen wird unter Ausbildung des Tetrahydrofuran-Rings (Schritt 3). Die entstandene Hydroxygruppe wiederum bewirkt eine nucleophile Substitution an der Carbonylgruppe, die als Thioester aktiviert vorliegt (Schritt 4). Die Oxidation der Doppelbindung in Position 3 zum Epoxid ist hier als letzter Biosyntheseschritt gezeigt, sie könnte jedoch auch zuvor erfolgen.

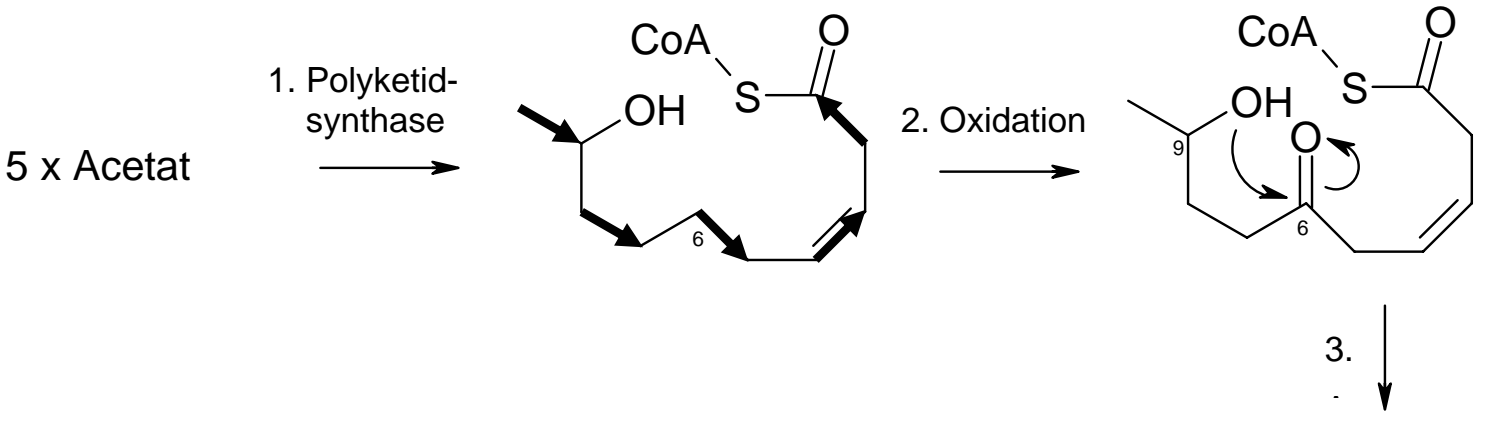<smiles>CC1CCC2(CC3OC3CC(=O)O2)O1</smiles>

86
5. Oxidation<smiles>CC1CCC2(CC=CCC(=O)O2)O1</smiles>

4.

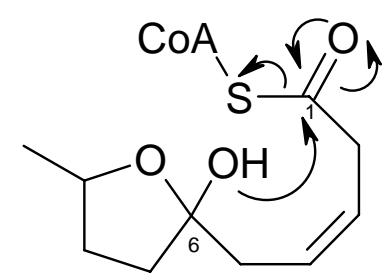

Abbildung 67: Hypothetischer Biosyntheseweg von 86. 


\section{Sekundärmetaboliten aus Phomopsis sp. BS 6769}

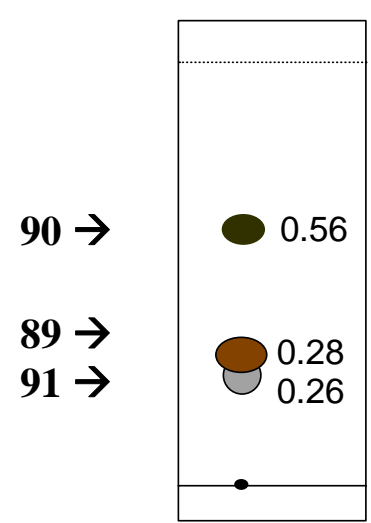

Abbildung 68: Skizze

des Dünnschichtchromatogramms des Extrakts, angegeben sind $\mathrm{R}_{\mathrm{f}}$-Werte $\left(\mathrm{CHCl}_{3} / \mathrm{MeOH}\right.$ 9:1).
Der Stamm Phomopsis sp. BS 6769 fiel im chemischen Screening durch ein interessantes Metabolitenmuster auf. Insbesondere der KulturfiltratExtrakt der Schüttelkultur mit dem Nährmedium 1158 zeigte mehrere mit Anisaldehyd beim Erhitzen anfärbende Zonen. Die Kultivierung im 4L-Maßstab ermöglichte die Isolierung von drei Reinsubstanzen. Hierbei handelt es sich um das bereits aus Phomopsis sp. bekannte Phomopsolid B (89) und eine neue, biosynthetisch verwandte Verbindung, die Phomopsofuran A (90) genannt wurde. Die dritte Substanz wurde als $p$-Menthan-1,2,4-triol (91) identifiziert, das bisher nur aus Pflanzen bekannt war und hier erstmals als Pilzmetabolit beschrieben wird.

\subsection{Phomopsolid B (89)}

Die Molmasse des farblosen Feststoffs, der in einer Ausbeute von $3.5 \mathrm{mg} / \mathrm{L}$ isoliert wurde, wurde durch ein ESI-Massenspektrum zu $296 \mathrm{~g} / \mathrm{mol}$ bestimmt. Das ${ }^{13} \mathrm{C}-\mathrm{NMR}-$ Spektrum zeigt die Signale von 15 Kohlenstoffatomen, darunter zwei Ester- oder Lactongruppen $\left(\delta_{\mathrm{C}}=164.9\right.$, 167.9) und sechs olefinische C-Atome $\left(\delta_{\mathrm{C}}=125.0\right.$ - 143.1). Ausgehend von einer im ${ }^{1} \mathrm{H}$ NMR-Spektrum zum Dublett aufspaltenden Methylgruppe $\left(\delta_{\mathrm{H}}=1.06,{ }^{3} J_{9,10}=6.2 \mathrm{~Hz}\right)$ ließ sich anhand der Korrelationen des COSY-Spektrums das in Abbildung 69 gezeigte, neun Kohlenstoffatome lange Fragment ermitteln. Die Konfiguration der Doppelbindungen ergab sich aus dem Betrag der Kopplungskonstanten der olefinischen Protonen $\left({ }^{3} J_{6,7}=15.5 \mathrm{~Hz}\right.$ für die trans- bzw. ${ }^{3} J_{2,3}=9.8 \mathrm{~Hz}$ für die cis-Kopplung). Die chemischen Verschiebungen von 4-H und 5-H $\left(\delta_{\mathrm{H}}=5.21,5.39\right)$ ließen vermuten, dass die entsprechenden C-Atome an die beiden Ester- bzw. Lactongruppen gebunden sind. Dieses wurde durch eine Suche mit der Molmasse sowie den erwähnten Fragmenten und funktionellen Gruppen in der Datenbank Dictionary of Natural Products ${ }^{[55]}$ und die hieraus erhaltene Struktur von Phomopsolid B (89) bestätigt. Die Übereinstimmung der gemessenen spektroskopischen Daten von 89 mit in der Literatur veröffentlichten Werten ${ }^{[184]}$ belegt die Strukturzuordnung. 

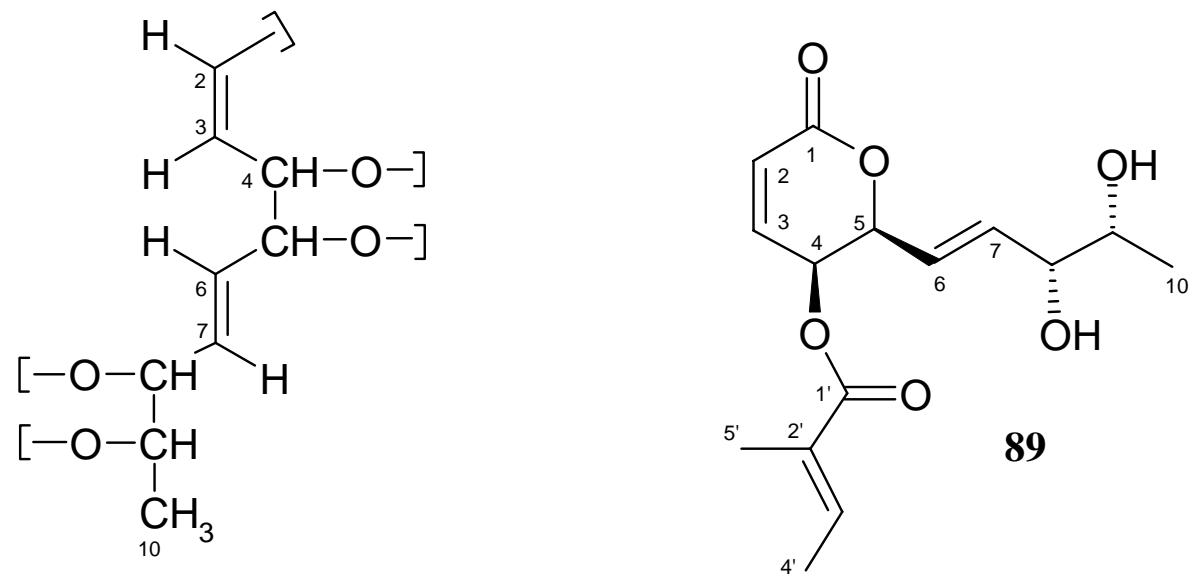

Abbildung 69: Aus dem COSY-Spektrum abgeleitetes Fragment (links) und Strukturformel von Phomopsolid B (89, rechts).

Phomopsolid B (89) wurde erstmals 1985 beschrieben, ebenfalls als Metabolit eines Pilzstamms der Gattung Phomopsis ${ }^{[184]}$. Dieser produzierte zudem eine Phomopsolid A genannte verwandte Verbindung, die an Position 8 eine Ketogruppe trägt und eine $Z$-Konfiguration der Doppelbindung zwischen C-6 und C-7 besitzt. Weitere Phomopsolid-Varianten wurden 1997 aus einem endophytischen Penicillium $s p$. Stamm erhalten ${ }^{[185]}$. Der Stamm wurde aus der Rinde einer pazifischen Eibe isoliert, auf der Suche nach einem Produzenten von Taxol. Neben Phomopsolid A und B werden hier auch die jeweiligen 6,7-Dihydro-Derivate gebildet (Phomopsolid E bzw. D). Es konnte gezeigt werden, dass die Produktion der Phomopsolide abhängig vom $\mathrm{Mg}_{3}\left(\mathrm{PO}_{4}\right)_{2}$-Zusatz zum verwendeten Glucose-Sojapepton-Nährmedium ist. Als weiterer Metabolit tritt das (6,7E)-Isomer von Phomopsolid A auf (Phomopsolid C).

Den Phomopsoliden A und B (89) wird eine abschreckende Wirkung gegen Borkenkäfer zugeschrieben $^{[184]}$. Ein endophytischer Pilz, der diese Substanzen produziert, sollte für den Wirtsbaum einen Vorteil darstellen, da er gegen die schädigenden Käfer schützt. Möglicherweise erfüllt auch Phomopsis sp. BS 6769 eine solche Funktion in dem Wirtsbaum (Weide), aus dem er isoliert wurde.

Die in Position 4 als Ester gebundene (E)-2-Methyl-2-butensäure wird auch als Tiglinsäure bezeichnet und ist bei Pflanzen weit verbreitet. Das Dictionary of Natural Products ${ }^{[55]}$ kennt über 650 pflanzliche Sekundärstoffe, die Tiglinsäure als Ester enthalten, ihr (Z)-Isomer (Angelicasäure) ist sogar Bestandteil von etwa 1500 Pflanzen-Metaboliten. Beide Carbonsäuren sind jedoch im mikrobiellen Stoffwechsel sehr selten und ungewöhnlich. Außer den Phomopsoliden sind keine weiteren Substanzen aus Pilzen beschrieben, die Tiglinsäure 
als Baustein enthalten. Als ein neuer Metabolit mit einem Tigloyl-Ester wird im nächsten Kapitel die Substanz Phomopsofuran A (90) beschrieben.

\subsection{Phomopsofuran A (90)}

Die farblose, ölige Substanz 90 mit der Molmasse $310 \mathrm{~g} / \mathrm{mol}$ wurde in einer Ausbeute von $2 \mathrm{mg} / \mathrm{L}$ erhalten. Die Summenformel $\mathrm{C}_{16} \mathrm{H}_{22} \mathrm{O}_{6}$ konnte durch ein hochaufgelöstes ESIMassenspektrum bestimmt werden. Der Vergleich des ${ }^{1}$ H-NMR-Spektrums mit dem von Phomopsolid B (89) zeigte das Vorhandensein eines (E)-2-Methyl-2-butenoyl-Substituenten, dessen zwei Methylgruppen sich überlagern $\left(\delta_{\mathrm{H}}=1.80,1.80\right)$ und dessen olefinisches Proton bei $\delta_{\mathrm{H}}=6.86$ ein Multiplett ergibt.

Das ${ }^{13}$ C-NMR-Spektrum zeigt neben den Signalen für C-1' und C-2' der Tigloylgruppe drei weitere quartäre Kohlenstoffatome: eine Ester-Carbonylgruppe $\left(\delta_{C}=175\right)$ und zwei stark ins Tieffeld verschobene aromatische C-Atome $\left(\delta_{C}=152,159\right)$. Gemeinsam mit zwei aromatischen CH-Gruppen $\left(\delta_{\mathrm{C}}=107,110\right)$ und einem Sauerstoffatom bilden sie einen Furanring, dessen Protonen $\left(\delta_{\mathrm{H}}=6.20,6.30\right)$ eine typische Kopplung von $3 \mathrm{~Hz}$ zeigen. Die weiteren Signale des ${ }^{1}$ H-NMR-Spektrums lassen sich anhand eines COSY-Spektrums drei Fragmenten zuordnen: einer Methoxygruppe $\left(\delta_{\mathrm{H}}=3.63\right)$, einer 1-Hydroxy-ethylengruppe $\left(\delta_{\mathrm{H}}=1.46,4.76\right)$ und einer Kette bestehend aus zwei Methylengruppen $\left(\delta_{H}=2.27,2.36\right)$ und einer Sauerstoffgebundenen Methingruppe $\left(\delta_{\mathrm{H}}=5.89\right)$. Deren auffällig starke Tieffeldverschiebung kann durch die Nachbarschaft zum Aromaten sowie eine Acylgruppe am Sauerstoff erklärt werden. Die Verknüpfung dieser drei Fragmente, der Tigloylgruppe und der quartären Kohlenstoffatome folgt aus dem HMBC-Spektrum und ist in Abbildung 70 illustriert.

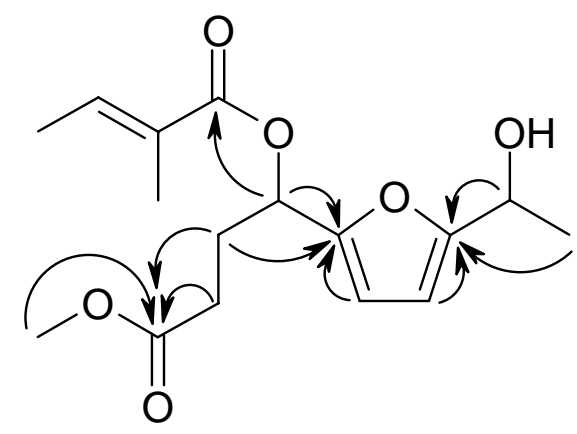

Abbildung 70: Wichtige HMBC-Korrelationen bei der Strukturaufklärung von 90. 
Hieraus ergibt sich die gezeigte Struktur der Verbindung 90, die einen neuen, in der Literatur bisher nicht beschriebenen Naturstoff darstellt und die in Anlehnung an den im Molekül vorhandenen Furanring den Namen Phomopsofuran A (90) erhält.

Die Stereochemie an C-4 und C-9 ist analog zu Phomopsolid B (89) angegeben, da beide Metaboliten einen gemeinsamen biosynthetischen Ursprung haben (siehe nächstes Unterkapitel). Diese Konfiguration ist als Vorschlag anzusehen und wäre durch z. B. durch Derivatisierung der sekundären Alkohle nach der Methode von MosHeR ${ }^{[186]}$ oder HELMCHEN ${ }^{[187]} \mathrm{zu}$<smiles>C/C=C(\C)C(=O)O[C@@H](CCC(=O)OC)c1ccc([C@H](C)O)o1</smiles>
90 beweisen.

\subsection{Zur Biosynthese von Phomopsolid B (89) und Phomopso- furan $A(90)$}

Phomopsolid B (89) und Phomopsofuran A (90) sind biosynthetisch verwandt. Dies zeigt sich zum einen in dem für Mikroorganismen ungewöhnlichen Tigloyl-Substituenten, zum anderen besitzen beide Verbindungen eine zehn Kohlenstoffatome lange unverzweigte Carbonsäure als Grundgerüst. Diese ist bei $\mathbf{8 9}$ zum Pyron zyklisiert, bei 90 liegt sie als Methylester vor. In Position 4 ist jeweils die Tigloylgruppe als Ester gebunden, auch die Oxygenierung der C-Atome C-4, C-5, C-8 und C-9 ist identisch.

Bei beiden Substanzen handelt es sich vermutlich um die Produkte eines gemischten Biosynthesewegs: die 10gliedrige Kohlenstoffkette dürfte ein durch eine Polyketidsynthase aus fünf Acetat-Einheiten aufgebautes Pentaketid sein, Tiglinsäure ist hingegen ein Hemiterpen aus dem Isoprenoid-Stoffwechsel. Das Pentaketid, das als gemeinsames Zwischenprodukt von Phomopsolid B (89) und Phomopsofuran A (90) auftreten könnte, ist in Abbildung 71 gezeigt. Es kann jedoch keine exakte Aussage hinsichtlich des Sättigungsgrads und der Position und Anzahl der OH-Gruppen gemacht werden, möglicherweise unterscheiden sich die Biosynthesewege bereits auf dieser Stufe. Die Biosynthese des Hemiterpens erfolgt vermutlich auf dem klassischen Mevalonat-Weg. Der bei Bakterien ebenfalls mögliche Nicht-Mevalonat-Weg ist bisher bei Pilzen nicht beobachtet worden ${ }^{[188]}$.

Der zur Bildung des Furanrings führende Mechanismus ist unklar. Bei Fettsäuren kennt man die so genannten F-Säuren oder furanoiden Fettsäuren, die einen methylierten Furanring 
enthalten ${ }^{[189]}$. Sie werden bei Pflanzen oder Tieren aus mehrfach ungesättigten Fettsäuren wie z. B. Linolsäure gebildet, teilweise als Reaktion auf oxidativen Stress. Die Umwandlung katalysiert eine spezielle Lipoxygenase, als Zwischenprodukt tritt das 13-Hydroperoxid der Linolsäure auf ${ }^{[190]}$. Dieser Mechanismus lässt sich jedoch nicht direkt auf die Biosynthese von Phomopsofuran (90) übertragen, da das Edukt als unabdingbare strukturelle Voraussetzung eine Methylengruppe zwischen zwei cis-konfigurierten Doppelbindungen haben muss. Dies erscheint bei dem zu 90 führenden Pentaketid unwahrscheinlich bzw. unmöglich.

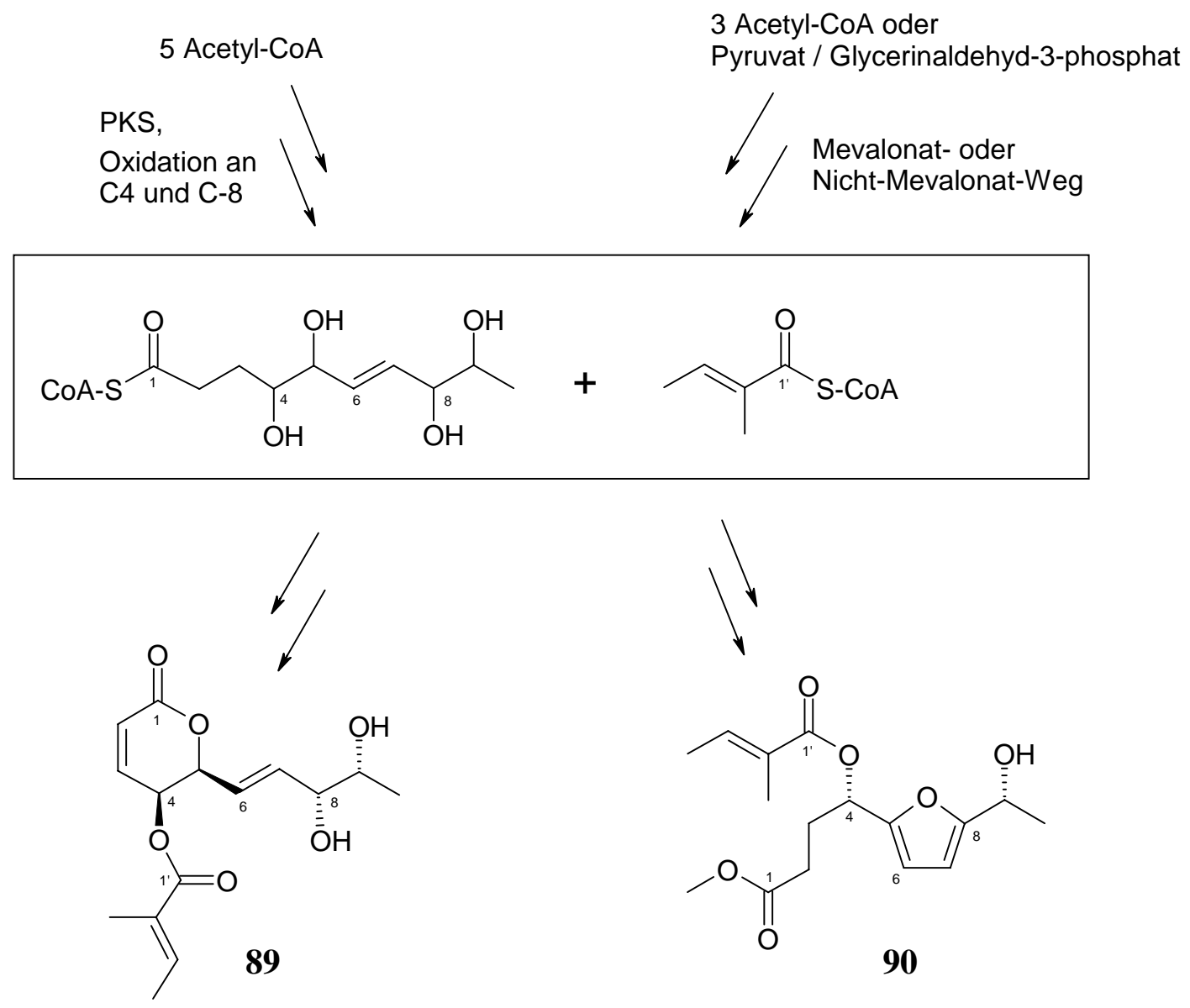

Abbildung 71: Hypothetischer Verlauf der Biosynthese von 89 und 90.

\section{4 -Menthan-1,2,4-triol (91)}

Die Substanz 91 wurde in einer Ausbeute von $2.5 \mathrm{mg} / \mathrm{L}$ als farbloses Öl erhalten. Die Molmasse von $188 \mathrm{~g} / \mathrm{mol}$ wurde durch ein ESI-Massenspektrum ermittelt. Das ${ }^{13} \mathrm{C}-\mathrm{NMR}$ Spektrum enthält die Signale von zehn Kohlenstoffatomen, die aufgrund ihrer geringen chemischen Verschiebung alle $\mathrm{sp}^{3}$-hybridisiert sein müssen. Aus der Anzahl der C-Atome und 
der Molmasse lässt sich die Summenformel $\mathrm{C}_{10} \mathrm{H}_{20} \mathrm{O}_{3}$ ableiten, die im Einklang mit der aus den Integralen des ${ }^{1} \mathrm{H}-\mathrm{NMR}-$ Spektrums ermittelten Anzahl an Protonen steht. Anhand eines zum Heptett aufgespaltenen Signals eines Methinprotons $\left(\delta_{\mathrm{H}}=1.47\right)$ kann auf das Vorhandensein einer Isopropylgruppe geschlossen werden, deren zwei Methylgruppen jeweils bei $\delta_{\mathrm{H}}=0.82$ Dubletts ergeben. Die Abspaltung der Isopropylgruppe kann auch im EI-Massenspektrum beobachtet werden $(\Delta \mathrm{m} / \mathrm{z}=45)$. Eine weitere Methylgruppe $\left(\delta_{\mathrm{H}}=1.10\right)$ erscheint als Singulett und muss daher an eines der beiden quartären Kohlenstoffatome gebunden sein $\left(\delta_{\mathrm{C}}=69.8,73.0\right)$, das andere trägt die Isopropylgruppe. Zusammen mit drei Methylengruppen und einer oxygenierten Methingruppe bilden die beiden quartären C-Atome einen Sechsring. Zwei der Methylengruppen sind benachbart, ebenso die dritte

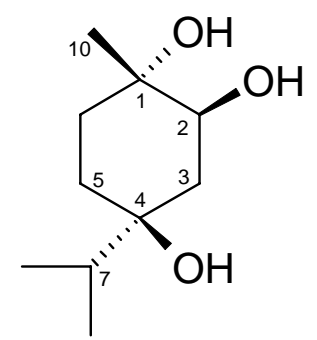

91 Methylengruppe und die Methingruppe. Als ein mögliches Isomer ergibt sich hieraus $p$-Menthan-1-2,4-triol (91), dessen Vorliegen durch die Übereinstimmung der ${ }^{1} \mathrm{H}-\mathrm{NMR}-$ Daten mit publizierten Werten ${ }^{[191]}$ bestätigt wird.

Die Isopropylgruppe wirkt als konformativer Anker des Cyclohexansystems. Das Auftreten einer W-Kopplung zwischen 2-H und 6- $\mathrm{H}_{\mathrm{eq}}$ belegt eine äquatoriale Stellung des Protons an C-2. Auf analoge Weise sowie anhand der im Vergleich zu den axialen Protonen niedrigeren chemischen Verschiebung können die äquatorialen Protonen an C-2 und C-5 bestimmt werden.

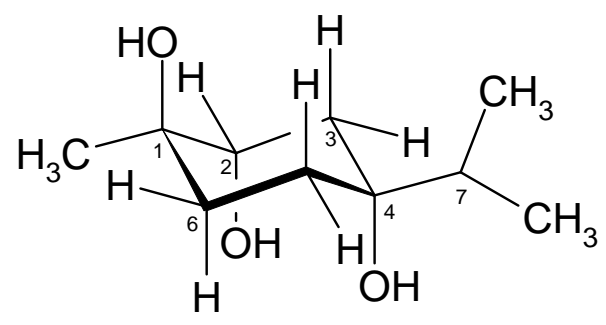

Abbildung 72: Konformation von $p$-Menthan-1-2,4-triol (91).

p-Menthan-1-2,4-triol (91) wird in der Literatur als Bestandteil bestimmter Pflanzenöle beschrieben ${ }^{[55,191]}$. Aus Mikroorganismen war dieses Monoterpen bisher nicht bekannt, jedoch wurde die regioisomere Verbindung $p$-Menthan-1,2,3-triol aus einem Pilzstamm der gleichen Gattung (Phomopsis) isoliert ${ }^{[192]}$. Das Vorkommen von 91 ist neben der in 89 und 90 enthaltenen Tiglinsäure ein weiterer Beweis für einen aktiven Isoprenoidstoffwechsel des Stamms Phomopsis sp. BS 6769. 


\section{Biologische Aktivität der Pilzmetaboliten}

Die in Plattendiffusionstests ermittelte antimikrobielle Wirkung der Metaboliten gegen Grampositive und Gram-negative Bakterien sowie den Pilz Candida albicans sind in Tabelle 19 angegeben. Tabelle 20 zeigt die Ergebnisse der Pflanzenschutz-Tests gegen phytopathogene Pilze und Insekten. Testergebnisse zur herbiziden Wirkung der Substanzen liegen noch nicht vor.

\subsection{Biologische Aktivität der bekannten Substanzen}

Die beiden Primärmetaboliten Ergosterol (58) und Cerebrosid C (59) wiesen keine Wirkung in den biologischen Tests auf, mit Ausnahme einer schwachen Aktivität von 59 gegen P. infestans (Hemmung bei $125 \mathrm{ppm}$ ).

Ascosalipyron (60) kann das Wachstum der phytopathogenen Pilze P. oryzae und S. tritici verhindern, aufgrund der geringen Wirkstärke erscheint diese Aktivität jedoch nicht verfolgenswert. Eine Wachstumshemmung von Bakterien oder C. albicans trat nicht auf.

Im Plattendiffusionstest wurde wie erwartet eine starke antibakterielle Wirkung von Chaetoglobosin A (66) und seinem 19-O-Acetat (65) festgestellt. Bei B. subtilis ist sie vergleichbar mit Penicillin G, bei E. coli und S. aureus etwas schwächer. Chaetoglobosin C (67) besitzt nur gegen B. subtilis eine moderate Hemmwirkung. Vermutlich sind sowohl die fungizide als auch die herbizide Wirkung des Extrakts aus Chaetomium sp. BS 6556 auf die Chaetoglobosine 65 - 67 zurückzuführen. Testergebnisse hierzu stehen noch aus, diskutiert wird die Wirkung der Cytochalasine auf Seite 113.

Eine hemmende Wirkung gegen B. subtilis besitzen auch die Sesquiterpene Helminthosporal (78), Helminthosporol (79) und cis-Sativendiol (80). Von ihnen ist zudem eine toxische (78) bzw. Wachstums-fördernde Wirkung $(\mathbf{7 9 , 8 0})$ gegenüber Pflanzen bekannt, diese wird in Kapitel 12.5 ab Seite 120 beschrieben.

Phomopsolid B (89) zeigte weder gegen Bakterien noch gegen phytopathogene Pilze oder Insekten eine Aktivität. Die in der Literatur angegebene starke antibakterielle Wirkung von 89 gegen $S$. aureus (vergleichbar mit z. B. Penicillin) ${ }^{[185]}$ konnte nicht reproduziert werden. Zur in der Literatur beschriebenen Aktivität gegen Borkenkäfer siehe Kapitel 14.1, Seite 128. 


\subsection{Biologische Aktivität der neuen Sekundärmetaboliten}

Chaetospiron (68) und Bis(prehelminthosporol) (81) weisen jeweils eine antibakterielle Wirkung gegen B. subtilis auf. Für Beauveriaspirolid (86) und Phomopsofuran A (90) konnte bisher keine antimikrobielle Wirkung festgestellt werden, allerdings stehen hier ebenso wie bei 68 und $\mathbf{8 1}$ die Ergebnisse der Pflanzenschutz-Tests noch aus. Bei dem dimeren Sesquiterpen 81 dürften aufgrund der bekannten Wirkungen der Sesquiterpene 78 - 80 insbesondere die Ergebnisse des Herbizid-Tests interessant sein. Bei der Spiroverbindung 86 gilt dies aufgrund der strukturellen Ähnlichkeit zu Insekten-Pheromonen für die InsektizidTests (vgl. Kapitel 13.1, Seite 123), ebenso bei 68 aufgrund seines Tropolon-Gerüsts.

Tabelle 19: Antibiotische Wirkung der aus Pilzen isolierte Sekundärmetaboliten. Angegeben ist der Hemmhof-Durchmesser beim Plattendiffusionstest in [mm], Penicillin G diente als Vergleich.

Escherichia Bacillus StaphyloCandida coli subtilis coccus aureus albicans

\begin{tabular}{lcccc}
\hline Penicillin G & 16 & 35 & 25 & 0 \\
\hline $\begin{array}{l}\text { Substanzen aus Chaetomium sp. } \\
\text { 19-OS-Acetyl- }\end{array}$ & 10 & 30 & 10 & 0 \\
chaetoglobosin A (65) & 12 & 35 & 11 & 0 \\
Chaetoglobosin A (66) & 0 & 10 & 0 & 0 \\
Chaetoglobosin C (67) & 0 & 14 & 0 & 0 \\
Chaetospiron (68) & & & & \\
\hline
\end{tabular}

Substanzen aus Drechslera sp. BS 6618

\begin{tabular}{llcll} 
Helminthosporal (78) & 0 & 10 & 0 & 0 \\
Helminthosporol (79) & 0 & 9 & 0 & 0 \\
cis-Sativendiol (80) & 0 & 8 & 0 & 0 \\
Bis(prehelminthosporol) (81) & 0 & 9 & 0 & 0 \\
\hline 50
\end{tabular}

$50 \mu \mathrm{g}$ Substanz auf Filterpapier-Plättchen, Ø $6 \mathrm{~mm}$

Keine Wirkung gegen die Testkeime zeigten Ergosterol (58), Cerebrosid C (59), Ascosalipyron (60), Beauveriaspirolid (86), Phomopsolid B (89) und Phomopsofuran A (90). 
Tabelle 20: Ergebnisse der aus Pilzen isolierten Sekundärstoffe in den Pflanzenschutz-Tests.

Mikrotest gegen phytopathogene Pilze Insektizid-Mikrotest

Häufig isolierte Metaboliten

Ergosterol (58)

nicht aktiv

Cerebrosid C (59) schwache Wirkung gegen $P$. infestans

(Hemmung bei 125 ppm)

Substanz aus Diplodia sp. BS 5945

Ascosalipyron (60)

schwache Wirkung gegen P. oryzae

und S. tritici (Hemmung bei $125 \mathrm{ppm}$ )

Substanzen aus Drechslera sp. BS 6618

Helminthosporol (79) nicht aktiv

Substanz aus Beauveria sp. BS 6750

Beauveriaspirolid (86) nicht aktiv

Substanzen aus Phomopsis sp. BS 6769

Phomopsolid B (89)

nicht aktiv

nicht aktiv

$p$-Menthan-1,2,4-triol (91)

nicht aktiv

Nicht angegebene Testergebnisse sowie die von 19-O-Acetylchaetoglobosin A (65), Chaetoglobosin A (66), Chaetoglobosin C (67), Chaetospiron (68), Helminthosporal (78), cisSativendiol (80), Bis(prehelminthosporol) (81) und Phomopsofuran A (90) stehen noch aus. 


\section{Diskussion der im Screening mit endophytischen Pilzen erzielten Ergebnisse}

\section{Endophyten als Quelle neuartiger Sekundärstoffe}

Die Ergebnisse der vorliegenden Arbeit unterstreichen, dass endophytische Pilzstämme eine reichhaltige Quelle für neue Sekundärmetaboliten sind. Vier der neun bearbeiteten Pilzstämme produzieren bisher unbekannte Verbindungen. Berücksichtigt man lediglich die im Screening aufgefallenen Pilzstämme, so erhöht sich die Quote der Stämme mit neuen Sekundärstoffen auf 100\%. Unter den Metaboliten befinden sich mit Chaetospiron (68) und Beauveriaspirolid (86) zwei Spiroverbindungen mit neuartigen Kohlenstoff-Grundgerüsten.

Ein Unterschied zwischen Endophyten aus Algen und solchen aus terrestrischen Pflanzen kann aufgrund der nicht-signifikanten Anzahl an bearbeiteten Stämmen in dieser Arbeit nicht festgestellt werden. Es kann jedoch vermutet werden, dass die als typisch für marine Habitate geltenden Gattungen Dendryphiella und Corollospora, von denen insgesamt fünf Stämme untersucht wurden, über keine signifikante Sekundärstoffbildung verfügen. Dies lässt jedoch weder Rückschlüsse auf andere Pilzgattungen zu, noch ist die Möglichkeit auszuschließen, dass die geeigneten Kultivierungsbedingungen bisher nicht gefunden wurden.

Neben dieser Biotop-vergleichenden Datenanalyse wäre auch ein Vergleich zwischen Endophyten und nicht-endophytisch lebenden Pilzen interessant. Leider ist hierzu in der Literatur keine solide Datengrundlage erhältlich, da die Ergebnisse ,unproduktiver“ Stämme und die Reisolierung bekannter Substanzen selten veröffentlicht werden. Somit ist keine abgesicherte Aussage darüber möglich, wie hoch der Anteil an Pilzstämmen einer spezifischen Gattung ist, in dem neue Sekundärmetaboliten gefunden werden können. Dies verdeutlicht, dass die Fixierung der Arbeiten auf neue Strukturen wichtige Informationen unterdrückt, die unter heutiger Fragestellung dringend gebraucht würden.

\section{Variation der Kultivierungsbedingungen}

Die Ergebnisse des Screenings zeigen, dass die Produktion von Sekundärstoffen bei Pilzen in erheblichem Maße von der Art der Kultivierung beeinflusst wird. Die größten Unterschiede zeigten die Extrakte aus Schüttel- und Ruhekulturen. Ob diese Ruhekulturen auf Festagar, auf DiGe-Feststoffmedium oder in P-Kolben in Flüssigmedium durchgeführt wurden, hatte ebenfalls deutliche Auswirkungen. Das Nährmedium schließlich bewirkte eine weitere Variation der Sekundärstoff-Biosynthese. Die Resultate des Screenings zeigen jedoch, dass 
positive Befunde eines Stamms zumeist in mehreren Nährmedien erhalten werden. Es wäre daher aus Gründen der Effizienz zu überlegen, die Anzahl der verwendeten Nährlösungen zu verringern. Es könnte eine Beschränkung auf drei oder vier Nährmedien erfolgen, wobei auf unterschiedliche Kohlenstoffquellen wie Glucose, Malz, Glycerin und Stärke geachtet werden sollte.

Die Zugabe von Natriumchlorid oder -bromid zur Nährlösung führte zur Beeinflussung des Wachstums und des Metaboliten-Musters einiger Stämme. Dieser Effekt war jedoch nicht so ausgeprägt, dass eine routinemäßige Variation der Salzkonzentration empfohlen werden kann. Lohnenswert ist sie sicherlich bei bereits als interessant eingestuften Stämmen.

Für eine Erhöhung der taxonomischen Vielfalt wäre eine Isolierung der Pilzstämme mit Nährmedien interessant, die dem jeweiligen biologischen Habitat angepasst sind. So haben beispielsweise Algen einen hohen Anteil an Zuckeralkoholen, diese werden in den üblichen Nährmedien jedoch nicht verwendet. Diese Komponenten könnten auch bei der Variation der Kultivierungsbedingungen berücksichtigt werden.

\section{Chemisches und biologisches Screening}

Eine sorgfältige Stammauswahl durch ein vorgeschaltetes chemisches oder biologisches Screening ist für das Auffinden neuer Naturstoffe unerlässlich. Dies zeigt sehr deutlich ein Vergleich der Ergebnisse aus den fünf nicht im Screening aufgefallenen Pilzstämmen mit denen der vier Stämme, die nach chemischen und biologischen Gesichtspunkten ausgewählt worden waren. Während aus Ersteren nur zwei bekannte Sekundärstoffe erhalten wurden, waren Letztere eine ergiebige Quelle auch neuartiger Strukturen.

Auffällig ist eine häufige, jedoch nicht ausnahmslose Übereinstimmung der Ergebnisse des chemischen und biologischen Screenings. Da die biologischen Tests auf Aktivität gegen phytopathogene Pilze und Insekten beschränkt waren, war die Anzahl der chemisch interessanten Extrakte höher als die der biologisch wirksamen.

Um eine Reisolierung bereits bekannter Substanzen effektiver zu vermeiden, wäre die Verwendung einer HPLC-gestützten Datenbank sinnvoll. Da eine solche kommerziell nicht erhältlich ist, müsste sie zunächst aufgebaut werden. Hierfür wäre eine Kooperation verschiedener Arbeitsgruppen erstrebenswert, um eine möglichst große Anzahl an Reinsubstanzen zur Verfügung zu haben. Die effektivste Methode wäre sicherlich eine HPLC-MS Kopplung, häufig würde jedoch auch eine HPLC-UV Kopplung mit Dioden-Array-Detektor ausreichen. Effizient wäre weiterhin die direkte Verbindung mit einer alle bekannten Pilzmetaboliten 
enthaltenden Datenbank. Die kommerziell erhältlichen Naturstoff-Datenbanken AntiBase ${ }^{[41]}$ und Dictionary of Natural Products ${ }^{[55]}$ enthalten diese zwar, jedoch ist bei einer Suche die Unterscheidung zwischen bakteriellen, pilzlichen und pflanzlichen Metaboliten nicht ohne Weiteres möglich. Ebenso fehlt die direkte Einbindung experimenteller spektroskopischer Daten. Letztere bietet das Handbook of Fungal Metabolites ${ }^{[179]}$, das jedoch nicht in elektronischer Form erhältlich ist und nur einen Teil der bekannten Sekundärmetaboliten enthält.

\section{Projektplanung und-koordination}

Die Konzeption des Projekts sah einen gleichzeitigen Arbeitsbeginn aller Projektpartner vor. Im offensichtlichen Widerspruch hierzu steht die Tatsache, dass die Arbeiten der Gruppen aufeinander aufbauen. Die am Anfang stehende Isolierung der endophytischen Pilze und ihre taxonomische Charakterisierung ermöglichen erst die hierauf folgende Kultivierung der Stämme und deren chemisches und biologisches Screening. Wie bereits erwähnt sind die Ergebnisse dieser Tests eine essentielle Voraussetzung für eine Erfolg versprechende Stammauswahl. Nach den Erfahrungen dieses Projekts ist ein Zeitraum von etwa einem Jahr einzuplanen, bis eine Kultivierung ausgewählter Stämme im größeren Maßstab erfolgen kann und die Arbeiten zur Isolierung und Strukturaufklärung sinnvoll beginnen können.

Als sehr hilfreich erwies sich die von der BASF durchgeführte Auftrennung von Rohextrakten durch HPLC mit biologischer Testung der Fraktionen, wofür jedoch weitere Zeit notwendig war. Dies führte zu der Situation, dass erst ab der zweiten Hälfte, insbesondere jedoch gegen Ende des Projektzeitraums eine große Anzahl interessanter Stämme zur Verfügung stand, teils mit zusätzlichen Informationen durch HPLC-Trennungen. Diese können jedoch im Rahmen des eigentlichen Projekts nicht mehr vollständig bearbeitet werden.

Sinnvoll wären ein zeitlich um ein Jahr versetzter Projektbeginn der einzelnen Arbeitsgruppen sowie eine insgesamt um mindestens ein Jahr längere Projektlaufzeit gewesen. Eine schnellere Verfügbarkeit der Ergebnisse aus den Pflanzenschutz-Mikrotests wäre für einen Vergleich der biologischen Aktivität isolierter Substanzen mit der des jeweiligen Extrakts erstrebenswert.

Eine zentrale Sammlung der Ergebnisse aller Projektpartner dieses Forschungsverbunds würde vergleichende Datenanalysen mit einer maximalen Zahl an Informationen ermöglichen. Wie oben erwähnt, könnte der Aufbau einer entsprechenden Datenbank einen großen Beitrag auch für zukünftige Projekte leisten. 


\section{Zusammenfassung der Ergebnisse}

\subsection{Naturstoffe aus Bakterien}

Neue Actinomycine aus Actinomyces sp. CS 210

Die Strukturen von fünf neuen Actinomycinen des G-Typs wurden durch spektroskopische Methoden aufgeklärt. Damit wurde die Zahl der bekannten natürlich vorkommenden Actinomycine von 19 auf 24 erweitert. Abweichend von Actinomycin D (19) enthalten sie die Aminosäuren 3-Hydroxy-5-methylprolin im $\alpha$-Ring und $N$-Methylalanin sowie 4-Chlor- bzw. 4-Hydroxythreonin im $\beta$-Ring. Erstmals wurde bei Actinomycin $\mathrm{G}_{5}$ (15) ein Ringschluss

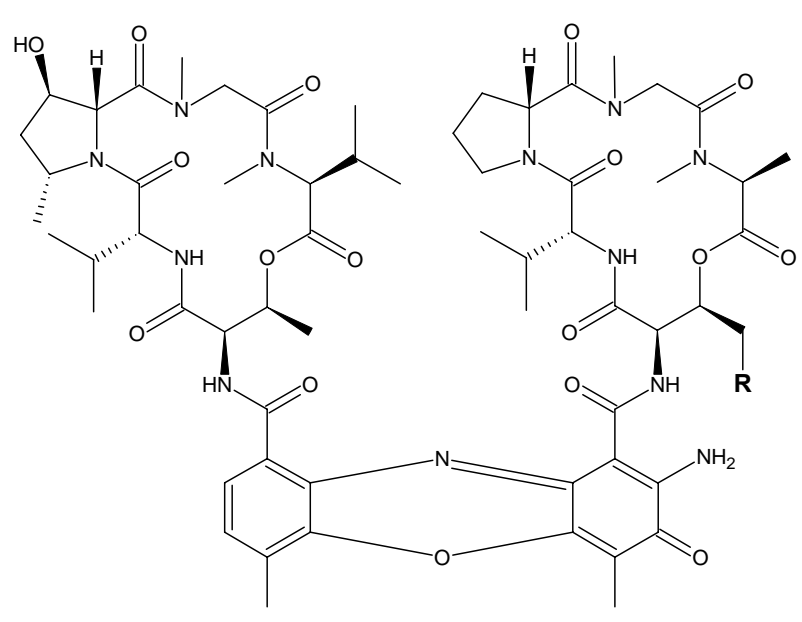
zwischen $\quad \beta$-Pentapeptidring und Chromophor festgestellt. Actinomycin $\mathrm{G}_{6}$ (16) hingegen entsteht biosynthetisch vermutlich durch zweifache Acylverschiebung aus $\mathrm{G}_{3}(\mathbf{1 3})$.

$$
\begin{aligned}
& \mathrm{R}=\mathrm{Cl}: \quad \text { Actinomycin } \mathrm{G}_{2}(\mathbf{1 2}) \\
& \mathrm{R}=\mathrm{OH}: \text { Actinomycin } \mathrm{G}_{3}(\mathbf{1 3}) \\
& \mathrm{R}=\mathrm{H}: \quad \text { Actinomycin } \mathrm{G}_{4}(\mathbf{1 4})
\end{aligned}
$$

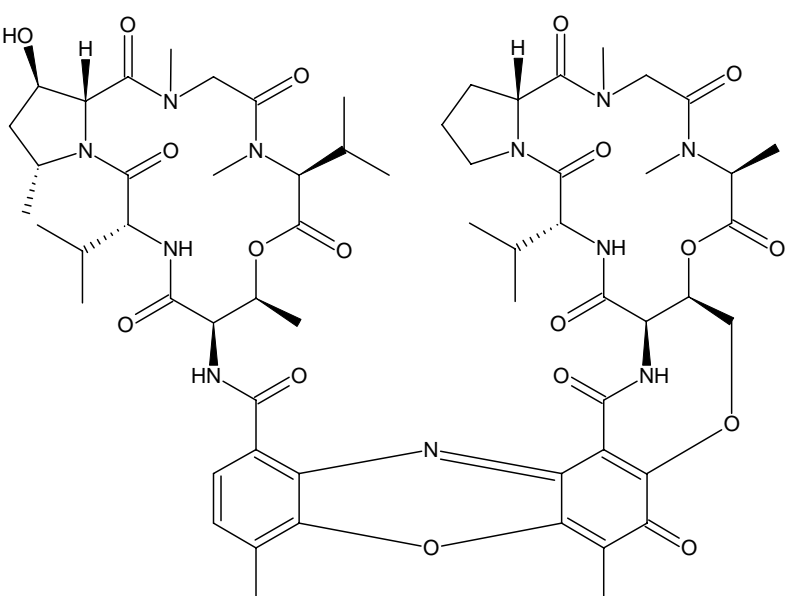

Actinomycin $\mathrm{G}_{5}(\mathbf{1 5})$

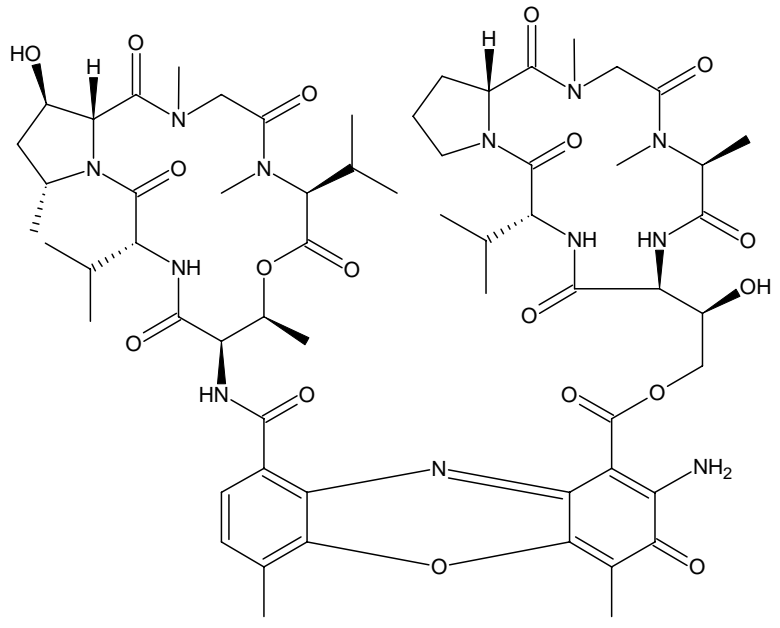

Actinomycin $\mathrm{G}_{6}(\mathbf{1 6})$

12 - 16 wurden auf ihre Cytotoxizität gegen verschiedene Zelllinien und im Plattendiffusionstest auf antimikrobielle Wirkung untersucht. Das 4-Chlorthreonin enthaltende 
Actinomycin $\mathrm{G}_{2}$ (12) wirkt ebenso stark cytotoxisch, jedoch etwas schwächer antibakteriell als Actinomycin D (19). Deutlich schwächer ist die biologische Wirksamkeit der 4-Hydroxythreonin enthaltenden Verbindungen 13, 15 und 16, insbesondere im Fall des veränderten Chromophors (15) oder des Verlusts der nativen Konformation des $\beta$-Rings (16). Struktur-Wirkungsbeziehungen wurden herausgestellt. Die Biosynthese der Actinomycine vom G-, X- und Z-Typ wurde diskutiert und für die Bildung von 15 und 16 aus 13 Reaktionsmechanismen aufgezeigt.

Es wurde ein Überblick über die Substanzklasse der Actinomycine und die durch Vorläuferdirigierte Biosynthese erhaltenen Derivate gegeben.

\section{Neue Aminophenoxazone aus Halomonas sp. Rk377a}

Die Zugabe von Anthranilsäure zum Kulturmedium von Halomonas sp. Rk377a führte zu einer vollständigen Änderung des Metabolitenspektrums des Stamms. Die orange-rote Färbung des Extraktes ist auf die Produktion von 2-Aminophenoxazon (39) und vier Derivaten hiervon zurückzuführen. Drei dieser Derivate besitzen eine in der Literatur nicht beschriebene Substitution in Position 6 bzw. 6 und 8: 2-Amino-6-hydroxy-phenoxazin-3-on (40), 2-Amino-8-benzoyl-6-hydroxy-phenoxazin-3-on (42) und 2-Amino-8-(4-hydroxybenzoyl)-6-hydroxy-phenoxazin-3-on (43). Die antimikrobiellen sowie cytotoxischen Aktivitäten der Verbindungen wurden ermittelt und diskutiert, ebenso der mögliche Zusammenhang zwischen Aminophenoxazon-Produktion und der beobachteten verstärkten Melanin-Bildung.

\begin{tabular}{l|l|l} 
& $\mathbf{R}_{1}$ & $\mathbf{R}_{2}$ \\
\hline 39 & $\mathrm{H}$ & $\mathrm{OH}$ \\
40 & $\mathrm{OH}$ &
\end{tabular}<smiles>[R]c1cc([R])c2oc3cc(=O)c(N)cc-3nc2c1</smiles>

Nargenicin $A_{1}$ aus Actinomyces sp. Gö 301

Mit dem Stamm Actinomyces sp. Gö 301 wurde ein effektiver Produzent des Antibiotikums Nargenicin $A_{1}$ (44) gefunden, der die Verbindung in Ausbeuten von $50 \mathrm{mg} / \mathrm{L}$ verfügbar 
macht. Die selektive Wirkung von 44 gegen Staphylokokken wurde im Plattendiffusionstest bestätigt.

\section{6-Desoxy- $\alpha$-L-talopyranoside aus Actinomyces sp. Gö M1}

Aus Actinomyces sp. Gö M1 wurden sieben 1-O-Aryl- und 1-O-Acyl-glykoside isoliert, deren gemeinsamer Zuckerbaustein 6-Desoxy- $\alpha$ - $L$-talose ist. Dieser ungewöhnliche Desoxyzucker war zuvor bei nur einem anderen mikrobiellen Sekundärstoff gefunden worden.<smiles>[R]OC1O[C@H](C)[C@@H](O)[C@H](O)[C@H]1O</smiles>

1-O-Isovaleryl-... (45)<smiles>CC(=O)CC(C)C</smiles>

1-O-Phenylacetyl-... (46)<smiles>CC(=O)Cc1ccccc1</smiles>

1-O-(2-Aminobenzoyl)-... (47)<smiles>CC(=O)c1ccccc1N</smiles>

$\ldots$-6-desoxy- $\alpha$ - $L$-talopyranosid
$\mathbf{R}=$ 1-O-(4-Hydroxyphenyl)-...

1-O-(4-Hydroxy-2-methoxyphenyl)-... (49)

1-O-(2-Pyrrolylcarbonyl)-...

1-O-(4-Hydroxybenzoyl)-...
$\mathbf{R}=$<smiles>Cc1ccc(O)cc1</smiles><smiles>COc1cccc(O)c1</smiles><smiles>CC(=O)c1ccc[nH]1</smiles><smiles>CC(=O)c1ccc(O)cc1</smiles>

Durch Vorläufer-dirigierte Biosynthese konnte die Ausbeute an 46 um das Dreifache und an 50 um das Dreißigfache erhöht werden. Die Zugabe von 4-Hydroxybenzoesäure zur Kultur führte zur vermehrten Bildung des 1-O-Acyl-glykosids 51 und des 1-O-Aryl-glykosids 48, außerdem wurde der neue zweifach glykosylierte 4-O-(6-Desoxy- $\alpha-L$-talopyranosyl)benzoesäure-(6-desoxy- $\alpha$ - $L$-talopyranosyl)ester (54) erhalten.

Die Biosyntheseleistung und das Produktspektrum des Stamms wurden mit den bekannten Rhamopyranosid-Bildnern verglichen und Annahmen zur Biosynthese gemacht. Die biologische Bedeutung der Verbindungen wurde im Hinblick auf ihre mögliche Funktion als energiereiche Intermediate im bakteriellen Stoffwechsel diskutiert. 


\subsection{Naturstoffe aus endophytischen Pilzen}

\section{Chemisches und biologisches Screening}

Im Rahmen eines BMBF-Forschungsverbunds wurden endophytische Pilzstämme aus verschiedenen Habitaten auf ihre Sekundärstoffproduktion hin untersucht. Aus der Rotalge Hypnea cervicornis wurden eigenständig 36 endophytische Pilzstämme isoliert. 18 dieser Stämme sowie 16 von B. ScHUlz (TU Braunschweig) erhaltene Endophyten wurden einem intensiven chemischen und biologischen Screening unter Variation der Kultivierungsbedingungen unterzogen (OSMAC-Methode), in dessen Verlauf jeweils über 600 Proben getestet wurden. Beim chemischen Screening erwiesen sich die Extrakte von 16 Pilzen als interessant, in den biologischen Tests fielen 14 Stämme auf.

\section{Endophyten als Produzenten von Sekundärmetaboliten}

Neun Pilzstämme wurden im Rahmen dieser Arbeit im größeren Maßstab kultiviert, so dass ihre Sekundärstoffe isoliert und in ihrer Struktur aufgeklärt werden konnten. Die Endophyten erwiesen sich als potente Produzenten strukturell vielfältiger, teils neuartiger Naturstoffe. Voraussetzung für das Auffinden interessanter Metaboliten war eine sorgfältige Stammauswahl nach chemischen und biologischen Gesichtspunkten.

\section{Sekundärmetaboliten aus Diplodia sp. BS 5945 und Microsphaeropsis sp. BS 6278}

Aus dem Stamm BS 5945 wurde Ascosalipyron (60) isoliert, welches in der Literatur bereits als Metabolit eines anderen Endophyten beschrieben wurde. Das aus dem Stamm BS 6278 erhaltene Solanapyron C (61) ist ein bekanntes Toxin phytopathogener Pilzstämme.

\section{Chaetoglobosine und Chaetospiron aus Chaetomium sp. BS 6556}

Die im biologischen Screening aufgefallene starke fungizide Wirkung des DiGe-Extrakts konnte auf die Produktion von Cytochalasinen zurückgeführt werden. Als Hauptprodukt bildet der Stamm 19-O-Acetylchaetoglobosin A (65), Nebenprodukte sind Chaetoglobosin A (66) und C (67). Aus dem lipophilen Anteil des Extrakts wurde Chaetospiron (68) isoliert, welches ein neuartiges Kohlenstoffgerüst besitzt. $\mathbf{6 8}$ wirkt antibakteriell, der hypothetische Verlauf der Biosynthese von 68 auf dem Polyketidweg wurde dargestellt.

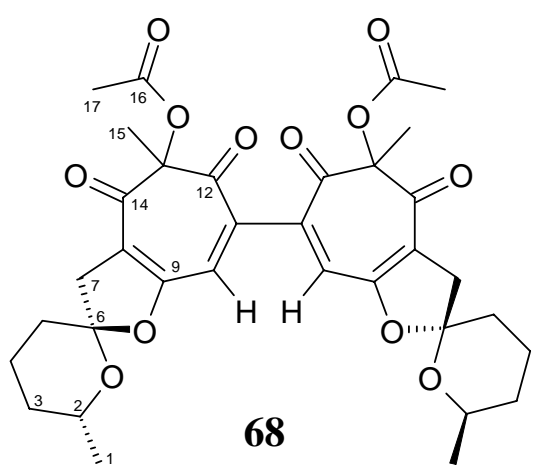


Sesquiterpene aus Drechslera sp. BS 6618

Aus dem Agarplatten-Extrakt des Stamms BS 6618 wurden die drei Sesquiterpene Helminthosporal (78), Helminthosporol (79) und cis-Sativendiol (80) isoliert. Die neue Verbindung Bis(prehelminthosporol) (81) wurde als Gemisch von zwei nicht-trennbaren Diastereomeren erhalten (81A und 81B). Es liegt das dimere Acetal von Prehelminthosporol (82) vor, einem mutmaßlichen biosynthetischen Vorläufer von 79. Die biologische Aktivität der Metaboliten wurde evaluiert und ihre biosynthetische Verwandtschaft aufgezeigt.

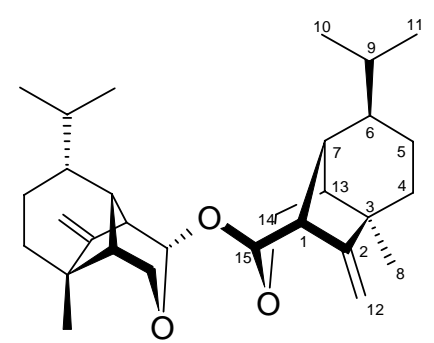

81A

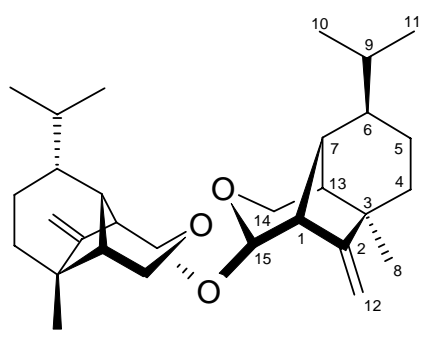

81B

Spiroverbindung aus Beauveria sp. BS 6750

Der Stamm BS 6750 fiel im chemischen Screening auf und produziert eine neuartige Spiroverbindung, die Beauveriaspirolid (86) genannt wurde. Vermutlich ist $\mathbf{8 6}$ ebenso wie $\mathbf{6 8}$ ein Polyketid.

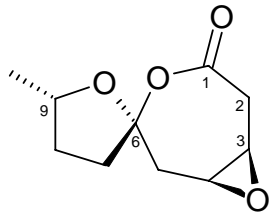

86

\section{Sekundärmetaboliten aus Phomopsis sp. BS 6769}

Neben der literaturbekannten Verbindung Phomopsolid B (89) biosynthetisiert Phomopsis sp. BS 6769 in Schüttelkultur die neue, biosynthetisch verwandte Substanz Phomopsofuran A (90). Weiterhin wurde das zuvor nur aus Pflanzen bekannte p-Menthan-1,2,4-triol (91) erhalten.<smiles>C/C=C(\C)C(=O)O[C@H](CCC(=O)OC)c1ccc([C@H](C)O)o1</smiles>

90

\section{Biologische Aktivität der neuen Sekundärmetaboliten}

Im Plattendiffusionstest wurde die antimikrobielle Wirkung aller Metaboliten bestimmt. 68 und 81 wirken antibakteriell gegen $B$. subtilis, für 86 und 90 wurde keine antimikrobielle Aktivität festgestellt. Alle Substanzen wurden den Pflanzenschutz-Tests der BASF zugeführt, deren Ergebnisse größtenteils noch ausstehen. 


\section{B. Experimenteller Teil}

\section{Instrumentelle Analytik}

Infrarotspektren: Alle IR-Spektren wurden als KBr-Presslinge mit einem FT-IRSpektrometer der Firma Perkin-Elmer Modell 1600 aufgenommen. Abkürzungen: br = breit, $\mathrm{sh}=$ Schulter.

UV-Spektren: Varian Cary 3E. Alle Spektren wurden mit Methanol als Lösungsmittel aufgenommen, soweit nicht anders vermerkt. Die Wellenlänge $\lambda$ ist in [nm] angegeben, der molare Absorptionskoeffizient $\varepsilon$ hat die Dimension $\left[1000 \mathrm{~cm}^{2} \mathrm{~mol}^{-1}\right]$ und berechnet sich wie folgt: $\varepsilon=\frac{A b s * M}{c * d}$

mit $\mathrm{Abs}=$ Absorption, $\mathrm{M}=$ Molmasse, $\mathrm{c}=$ Konzentration, $\mathrm{d}=$ Schichtdicke

Bei einer Schichtdicke von $\mathrm{d}=1 \mathrm{~cm}$ und einer Lösung mit dem Volumen $\mathrm{V}=10 \mathrm{~mL}$ ergibt sich somit: $\varepsilon\left[1000 \mathrm{~cm}^{2} \mathrm{~mol}^{-1}\right]=\frac{A b s * M[\mathrm{~g} / \mathrm{mol}]^{*} 10}{\text { Einwaage }[\mathrm{mg}]}$

Methanol/HCl bzw. Methanol/NaOH: Zu $2 \mathrm{~mL}$ methanolischer Lösung wurde jeweils ein Tropfen 1 M Salzsäure bzw. 1 M Natronlauge gegeben.

Circulardichroismus-Spektren: Jasco Modell J-500. Die molaren Elliptizitäten $\theta$ sind in $\left[10^{-1} \mathrm{grad} \mathrm{cm}^{2} \mathrm{~mol}^{-1}\right]$ angegeben.

Drehwerte: Polarimeter Perkin-Elmer Modell 241, die Drehwerte $[\alpha]_{\mathrm{D}}^{20}$ sind in $\left[10^{-1} \mathrm{deg} \mathrm{cm}^{2} \mathrm{~g}^{-1}\right]$, die Konzentrationen in $[\mathrm{mg} / \mathrm{mL}]$ angegeben.

Massenspektren: EI-MS: Finnigan MAT 95, $70 \mathrm{eV}$, Hochauflösungen wurden mit Perfluorkerosin als Vergleichssubstanz gemessen, die relativen Intensitäten beziehen sich auf den Peak höchster Intensität und sind in Klammern angegeben. DCI-MS: Finnigan MAT 95, $200 \mathrm{eV}$, Reaktandgas $\mathrm{NH}_{3}$. ESI-MS: Finnigan LC-Q. HR-ESI-MS: Bruker Apex-Q III, Feldstärke 7 Tesla. 
${ }^{1}$ H-NMR-Spektren: Varian Inova $600(600 \mathrm{MHz})$, Varian Inova 500 (500 MHz), Varian Mercury 300 (300 MHz), Varian Unity 300 (300 MHz), Bruker AMX 300 (300 MHz). Chemische Verschiebungen sind in $\delta$-Werten (ppm) relativ zum jeweiligen Lösungsmittel als internem Standard angegeben, Kopplungskonstanten $J$ in [Hz]. Alle ${ }^{1} \mathrm{H}-\mathrm{NMR}-\mathrm{Spektren}$ wurden als Spektren erster Ordnung interpretiert, soweit Signalaufspaltung und Kopplungskonstanten angegeben sind. Abkürzungen: $\mathrm{s}=$ Singulett, $\mathrm{d}=$ Dublett, $\mathrm{t}=$ Triplett, $\mathrm{q}=$ Quartett, quint $=$ Quintett, sext $=$ Sextett, hept $=$ Heptett, $\mathrm{m}=$ Multiplett, br $=$ breit.

${ }^{13}$ C-NMR-Spektren: Varian Inova 600 (150.8 MHz), Varian Inova 500 (125.7 MHz), Varian Mercury 300 (75.5 MHz), Varian Unity 300 (75.5 MHz), Bruker AMX 300 (75.5 MHz), Varian Mercury 200 (50.3 MHz). Chemische Verschiebungen in $\delta$-Werten (ppm) relativ zum jeweiligen Lösungsmittel als internem Standard. Multiplizitäten wurden aus ${ }^{1} J_{\mathrm{CH}^{-}}$ Korrelationsexperimenten oder APT-Spektren entnommen. Abkürzungen: $s=$ Singulett, $d=$

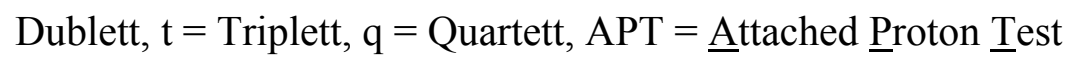

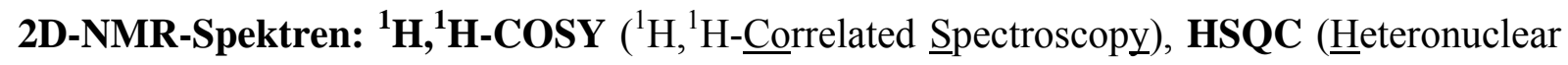

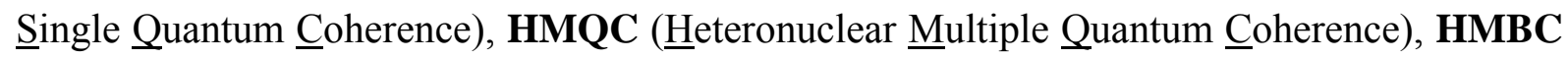
(ㅂeteronuclear Multiple $\underline{B}$ ond Connectivity), NOESY (Nuclear $\underline{\text { Overhauser }}$ Effect

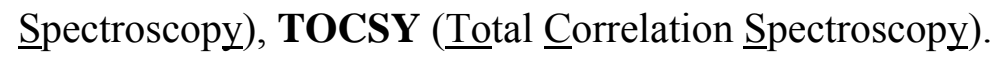

\section{Chromatographie}

Lösungsmittel: Die Lösungsmittel für die Chromatographie wurden zuvor destilliert. Für die HPLC wurden analysenreine Lösungsmittel und bidestilliertes Wasser verwendet, die zuvor im Ultraschallbad (10 min) und durch anschließendes Durchleiten von Helium (10 min) entgast wurden.

Dünnschichtchromatographie: Merck HPTLC-Fertigplatten Kieselgel 60 F254, 10 x 10 cm, Schichtdicke $0.2 \mathrm{~mm}$; Merck DC-Alufolien Kieselgel $60 \mathrm{~F}_{254}, 20 \times 20 \mathrm{~cm}$, Schichtdicke $0.2 \mathrm{~mm}$; Merck DC-Alufolien RP-18 $\mathrm{F}_{254 \mathrm{~s}}, 20$ x $20 \mathrm{~cm}$, Schichtdicke $0.2 \mathrm{~mm}$. Angegeben sind $\mathrm{R}_{\mathrm{f}}$-Werte (Laufhöhe relativ zur Laufmittelfront). 
Sprühreagenzien: Nach Merck, Anfärbereagenzien für die Dünnschichtchromatographie ${ }^{[193]}$. Die DC-Platten wurden nach dem Ansprühen auf ca. $100^{\circ} \mathrm{C}$ erwärmt.

Anisaldehyd (Nr. 21): $1.0 \mathrm{~mL}$ Anisaldehyd wird in eine Lösung aus $85 \mathrm{~mL}$ Methanol, $10 \mathrm{~mL}$ Eisessig und $5 \mathrm{~mL}$ konz. Schwefelsäure gegeben.

Ehrlichs Reagenz (Nr. 91): $1 \mathrm{~g}$ 4-Dimethylaminobenzaldehyd wird in einer Lösung aus $25 \mathrm{~mL}$ Salzsäure (36\%) und 75 mL Methanol gelöst.

Orcin-Sprühreagenz (Nr. 120-122): $1 \mathrm{~g}$ Eisen-(III)-chlorid wird in $100 \mathrm{~mL}$ Schwefelsäure gelöst und mit dem gleichen Volumen einer Orcinlösung (6\% in Ethanol) versetzt.

Adsorberharz: Serva Amberlite ${ }^{\circledR}$ XAD-2

Säulenchromatographie: Für Schwerkraftsäulen wurde Kieselgel 60, 0.2 - 0.05 mm (Macherey-Nagel, ICN) verwendet; für Flash-Chromatographie ${ }^{[194]}$ kam Kieselgel 60, 0.04 $0.063 \mathrm{~mm}$ (Macherey-Nagel) zum Einsatz. Sephadex ${ }^{\circledR}$ LH-20 (Sigma Aldrich) wurde zur Gelchromatographie benutzt.

Mitteldruckchromatographie: Pumpe: Knauer; Säule: Merck LiChroprep ${ }^{\circledR}$ RP-18, 40 $63 \mu \mathrm{m}$.

\section{Hochleistungs-Flüssigchromatographie Anlagen:}

Analytische HPLC mit DAD-Kopplung:

HPLC 1: Pumpe: Kontron 322; Mischkammer: Kontron HPLC 360; Dioden-Array-Detektor: Kontron 440; Autosampler: Kontron 360 mit analytischer Aufgabeschleife; Software: Kontron Kromasystem 2000 ${ }^{\mathrm{TM}}$ Version 1.60.

HPLC 2: Pumpe: Jasco PU-2080plus; Mischkammer: Jasco LG-2080-02; Entgaser: Jasco DG-2080-53; PDA-Detektor: Jasco MD-210plus; Autosampler: Jasco AS-2055plus mit Rheodyne $100 \mu \mathrm{L}$ Aufgabeschleife; Säulenofen: Jasco CO-2065; Fraktionensammler: Jasco SF-3120; Chromatographiesoftware: Jasco Borwin ${ }^{\mathrm{TM}}$ mit Jasco Borwin ${ }^{\mathrm{TM}}$ PDA Version 1.50; Steuersoftware: Jasco Borwin ${ }^{\mathrm{TM}}$ HSS-2000 Version 3.5.2.

\section{Analytische HPLC mit MS-Kopplung:}

HPLC 3: Pumpe und Mischkammer: Flux Instruments Rheos 4000; Entgaser: Flux Instruments ERC-3415 ; Autosampler: Jasco 851-AS mit variabler Aufgabeschleife 0-100 $\mu$ L; PDA-Detektor: Finnigan Surveyor; Massendetektor: Finnigan LC-Q; Steuersoft- 
ware HPLC: Flux Instruments Janeiro; Chromatographie- und Steuersoftware PDA und MSDetektor: Finnigan Xcalibur ${ }^{\mathrm{TM}}$ Version 1.3.

\section{Semipräparative HPLC:}

HPLC 4: Pumpe: Jasco PU-1587; Mischkammer: Jasco $1000 \mu \mathrm{L}$; UV-Detektor: Jasco UV-1575; manuelles Probenaufgabeventil: Rheodyne mit $2.0 \mathrm{~mL}$ Aufgabeschleife; Chromatographiesoftware: Jasco Borwin ${ }^{\mathrm{TM}}$ Version 1.50; Steuersoftware: Jasco HSS-2000 Version 3.5.2.

\section{Hochleistungs-Flüssigchromatographie Säulen:}

Analytisch: Säule 1 (Standardsäule für Analytik): Jasco Nucleosil 100 C18, $5 \mu \mathrm{m}$, $250 \times 3 \mathrm{~mm}$, Flussrate: $0.5 \mathrm{~mL} / \mathrm{min}$. Säule 2 (Standardsäule für HPLC-MS): Grom Supersphere 100 RP-18 endcapped, $4 \mu \mathrm{m}, 100$ x 2 mm mit Vorsäule Grom Supersphere 100 RP-18 endcapped, $4 \mu \mathrm{m}, 10$ x $2 \mathrm{~mm}$, Flussrate: $0.3 \mathrm{~mL} / \mathrm{min}$. Säule 3 (BASF-Fraktionierung): Merck Chromolith Performance RP18-e, $2 \mu \mathrm{m}, 100 \times 4.6 \mathrm{~mm}$, Flussrate: $1.4 \mathrm{~mL} / \mathrm{min}$. Säule 4: Jasco Nucleodur $100 \mathrm{C} 18,5 \mu \mathrm{m}, 250$ x 3 mm, Flussrate: $0.5 \mathrm{~mL} / \mathrm{min}$.

Semipräparativ: Säule 5: Jasco Nucleosil $100 \mathrm{C} 18,5 \mu \mathrm{m}, 250 \times 8 \mathrm{~mm}$, Flussrate: $2.6 \mathrm{~mL} / \mathrm{min}$. Säule 6: Macherey-Nagel Nucleodur 100-5 C18 endcapped, $5 \mu \mathrm{m}, 250 \times 8 \mathrm{~mm}$, Flussrate: $2.6 \mathrm{~mL} / \mathrm{min}$. Säule 7: Macherey-Nagel Nucleodur 100-5 C18 endcapped, $5 \mu \mathrm{m}$, 250 x $16 \mathrm{~mm}$, Flussrate $17 \mathrm{~mL} / \mathrm{min}$.

\section{Gradientenprogramme und Laufmittelsysteme für die HPLC:}

Programm 1 (Standardprogramm für Analytik): Laufmittelsystem: Wasser (A) / Acetonitril (B), jeweils mit $0.1 \%$ TFA; Gradient: von $10 \%$ auf $100 \%$ B in $25 \mathrm{~min}, 8 \mathrm{~min}$ bei $100 \% \mathrm{~B}$, von $100 \%$ auf $10 \% \mathrm{~B}$ in $2 \mathrm{~min}, 5 \min$ bei $10 \% \mathrm{~B}$.

Programm 2 (Standardprogramm für HPLC-MS): Laufmittelsystem: Wasser (A) / Methanol (B), jeweils mit $0.05 \%$ Ameisensäure; Gradient: von 20\% auf $100 \%$ B in 20 min, 10 min bei $100 \% \mathrm{~B}$, in $2 \mathrm{~min}$ auf $20 \% \mathrm{~B}, 5 \mathrm{~min}$ bei $20 \% \mathrm{~B}$.

Programm 3 (BASF-Programm): Laufmittelsystem: Wasser (A) / Acetonitril (B); Gradient: von $10 \%$ auf $50 \%$ B in $20 \mathrm{~min}$, von $50 \%$ auf $100 \%$ B in $7.5 \mathrm{~min}, 18.5 \mathrm{~min}$ bei $100 \% \mathrm{~B}$, von $100 \%$ auf $10 \% \mathrm{~B}$ in $0.5 \mathrm{~min}, 3.5 \mathrm{~min}$ bei $10 \% \mathrm{~B}$.

Programm 4 (Trennung der Actinomycine 13 und 15): Laufmittelsystem: Wasser (A) / Acetonitril (B); isokratische Trennung mit 54\% B, UV-Detektion bei $254 \mathrm{~nm}$.

Programm 5 (Trennung der Actinomycine 12, 13 und 14): Laufmittelsystem: Wasser (A) / Acetonitril (B); isokratische Trennung mit 51\% B; UV-Detektion bei $254 \mathrm{~nm}$. 
Programm 6 (Trennung der Diketopiperazine 33, 34, 35): Laufmittelsystem: Wasser (A) / Acetonitril (B), jeweils mit 0.1\% TFA; Gradient: 13 min bei $17.4 \%$ B, in 1.5 min auf $90 \%$ B, 5 min bei 90\% B, in 1.5 min auf 17.4\% B, 2 min bei 17.4\% B; UV-Detektion bei $215 \mathrm{~nm}$.

Programm 7 (Aufreinigung des Aminophenoxazonderivats 42): Laufmittelsystem: Wasser (A) / Acetonitril (B), jeweils mit 0.1\% TFA; Gradient: 7 min bei $22 \%$ B, in 1 min auf $44 \%$ B, $10 \mathrm{~min}$ bei $44 \% \mathrm{~B}$, in $1 \mathrm{~min}$ auf $88 \% \mathrm{~B}, 5 \mathrm{~min}$ bei $88 \% \mathrm{~B}$, in $2 \min$ auf $22 \% \mathrm{~B}, 2 \mathrm{~min}$ bei $22 \% \mathrm{~B}$; UV-Detektion bei $254 \mathrm{~nm}$.

Programm 8 (Aufreinigung des Desoxytalopyranosids 47): Laufmittelsystem: Wasser (A) / Acetonitril (B), jeweils mit 0.1\% TFA; Gradient: in 25 min von 40\% auf 75\% B, 9 min bei $75 \% \mathrm{~B}$, in $1 \mathrm{~min}$ auf 40\% B, $5 \mathrm{~min}$ bei 40\% B; UV-Detektion bei $254 \mathrm{~nm}$.

Programm 9 (Trennung der Desoxytalopyranoside 48 - 51): Laufmittelsystem: Wasser (A) / Acetonitril (B), jeweils mit 0.1\% TFA; Gradient: in 25 min von 10\% auf 20\% B, 5 min bei 20\% B, in 1 min auf 10\% B, 5 min bei 10\% B; UV-Detektion bei $254 \mathrm{~nm}$.

Programm 10 (Trennung der Sesquiterpene 80 und 81): Laufmittelsystem: Wasser (A) / Acetonitril (B); Gradient: in $25 \mathrm{~min}$ von $72 \%$ auf $80 \% \mathrm{~B}, 5 \mathrm{~min}$ bei $80 \% \mathrm{~B}$, in $1 \mathrm{~min}$ auf $72 \%$ B, 3 min bei 72\% B; UV-Detektion bei $215 \mathrm{~nm}$.

Programm 11 (Trennung der Sesquiterpene 78 und 79): Laufmittelsystem: Wasser (A) / Acetonitril (B); isokratische Trennung mit 65\% B; UV-Detektion bei $215 \mathrm{~nm}$.

Programm 12 (Trennung der Chaetoglobosine 65 - 67 und Chaetospiron (68)): Laufmittelsystem: Wasser (A) / Acetonitril (B); Gradient: $11 \mathrm{~min}$ bei $58 \% \mathrm{~B}$, in $1 \mathrm{~min}$ auf $65 \% \mathrm{~B}$, in $14 \mathrm{~min}$ auf $70 \% \mathrm{~B}$, in $1 \mathrm{~min}$ auf $58 \% \mathrm{~B} ; 3 \mathrm{~min}$ bei $58 \% \mathrm{~B}$; UV-Detektion bei $254 \mathrm{~nm}$.

\section{Gaschromatographie (GC):}

GC-MS: Gaschromatograph: ThermoFinnigan Trace GC; Massendetektor: ThermoFinnigan Trace MS (EI-Ionisierung, $70 \mathrm{eV}$ ); Autosampler: ThermoFinnigan AS2000; Säule: $J \& W$ DB-5MS, 25 m x 0.25 mm, Filmdicke $0.25 \mu \mathrm{m}$; Trägergas: Helium; Flussrate: $1 \mathrm{~mL} / \mathrm{min}$; Software: ThermoFinnigan Xcalibur ${ }^{\mathrm{TM}}$ Version 1.2, EI-MS-Datenbank: NIST/EPA/NIH Mass Spectral Library Version 2.0a.

Programm: Der Säulenofen wurde von $40^{\circ} \mathrm{C}$ ( 2 min isotherm) bis $280^{\circ} \mathrm{C}$ (4 min isotherm) mit einer Rate von $10 \mathrm{Grad} / \mathrm{min}$ geheizt. Die Temperatur des Injektors und der direkten Kopplung zum Massenspektrometer betrug $250{ }^{\circ} \mathrm{C}$. Jeweils $0.1 \mu \mathrm{L}$ Probenlösung wurden mit einem Split von 1:50 injiziert. 


\section{Mikrobiologische Methoden und Geräte}

Nährmedienbestandteile: Die verwendeten Nährmedienbestandteile wurden von folgenden Firmen bezogen: Malzextrakt, Fleischpepton und Caseinpepton von der Fa. Merck, D-Glucose und Stärke aus Kartoffeln von der Fa. Roth, D-Mannit von der Fa. Riedel-deHaën, D-Maltose von der Fa. Fluka, Marine Broth, Bacto Nutrient Broth und Agar von der Fa. Difco, Glycerin von der Fa. AppliChem, Hefeextrakt von der Fa. Oxoid, Cornsteep Powder von der Fa. Marcor, Hafermehl (Holo ${ }^{\circledR}$ Hafergold) von der Fa. Neuform, Sojamehl fettarm und Sojamehl vollfett von der Fa. Henselwerk GmbH, Sojapepton von der Fa. Sigma, Perlgraupen von der Fa. Brüggen, Vollkorn-Dinkelflocken von der Fa. Grünes Land, Biomalt Gerstenmalzextrakt von der Fa. Villa Natura.

\section{Nährmedien für Bakterienkulturen:}

M2: Malzextrakt $10 \mathrm{~g} / \mathrm{L}$, Hefeextrakt $4 \mathrm{~g} / \mathrm{L}, D$-Glucose $4 \mathrm{~g} / \mathrm{L}, \mathrm{pH} 7.0$

Soja/Mannit: Sojamehl 20 g/L, D-Mannit 20 g/L, pH 7.0

Hafer: Hafermehl $20 \mathrm{~g} / \mathrm{L}$, ,Spur 11“ $2.5 \mathrm{~mL} / \mathrm{L}$, pH 7.0

Spurenelementelösung „Spur 11“: $\mathrm{CaCl}_{2}$ x $2 \mathrm{H}_{2} \mathrm{O} 3 \mathrm{~g} / \mathrm{L}, \mathrm{Fe}(\mathrm{III})$-citrat $1 \mathrm{~g} / \mathrm{L}, \mathrm{MnSO}_{4} 0.2 \mathrm{~g} / \mathrm{L}$, $\mathrm{ZnCl}_{2} 0.1 \mathrm{~g} / \mathrm{L}, \quad \mathrm{CuSO}_{4} \times 5 \mathrm{H}_{2} \mathrm{O} 25 \mathrm{mg} / \mathrm{L}, \mathrm{Na}_{2} \mathrm{~B}_{4} \mathrm{O}_{7} \times 10 \mathrm{H}_{2} \mathrm{O} 20 \mathrm{mg} / \mathrm{L}, \mathrm{CoCl}_{2} 4 \mathrm{mg} / \mathrm{L}$, $\mathrm{Na}_{2} \mathrm{MoO}_{4}$ × $2 \mathrm{H}_{2} \mathrm{O} 10 \mathrm{mg} / \mathrm{L}$

SGG: D-Glucose $10 \mathrm{~g} / \mathrm{L}$, Glycerin $10 \mathrm{~g} / \mathrm{L}$, Stärke $10 \mathrm{~g} / \mathrm{L}$, Cornsteep Powder $2.5 \mathrm{~g} / \mathrm{L}$, Caseinpepton $5 \mathrm{~g} / \mathrm{L}$, Hefeextrakt $2 \mathrm{~g} / \mathrm{L}, \mathrm{NaCl} 1 \mathrm{~g} / \mathrm{L}, \mathrm{CaCO}_{3} 3 \mathrm{~g} / \mathrm{L}, \mathrm{pH} 7.0$

\section{Nährmedien für Pilzkulturen:}

1158: Malzextrakt 20g/L, $D$-Glucose $10 \mathrm{~g} / \mathrm{L}$, Hefeextrakt $2 \mathrm{~g} / \mathrm{L}, \mathrm{NH}_{4} \mathrm{HSO}_{4} 0.5 \mathrm{~g} / \mathrm{L}, \mathrm{pH} 6.0$

E2: D-Glucose $20 \mathrm{~g} / \mathrm{L}$, Hafermehl $20 \mathrm{~g} / \mathrm{L}$, entfettetes Sojamehl 20g/L, keine pH-Einstellung G20: Glycerin $20 \mathrm{~g} / \mathrm{L}$, Malzextrakt $10 \mathrm{~g} / \mathrm{L}$, Hefeextrakt $4 \mathrm{~g} / \mathrm{L}$, keine pH-Einstellung 1549: $D$-Glucose $10 \mathrm{~g} / \mathrm{L}, D$-Maltose $10 \mathrm{~g} / \mathrm{L}$, Caseinpepton $5 \mathrm{~g} / \mathrm{L}$, Fleischpepton $5 \mathrm{~g} / \mathrm{L}, \mathrm{pH} 5.4$ SGG: siehe Nährmedien für Bakterienkulturen

1405: $D$-Glucose $30 \mathrm{~g} / \mathrm{L}, \mathrm{NaNO}_{3} 3 \mathrm{~g} / \mathrm{L}, \mathrm{K}_{2} \mathrm{HPO}_{4} 1 \mathrm{~g} / \mathrm{L}, \mathrm{MgSO}_{4}$ x $7 \mathrm{H}_{2} \mathrm{O} 0.5 \mathrm{~g} / \mathrm{L}, \mathrm{KCl} 0.5 \mathrm{~g} / \mathrm{L}$, $\mathrm{FeSO}_{4} 0.01 \mathrm{~g} / \mathrm{L}, \mathrm{pH} 7.3$

Marine Broth: Marine Broth $37.4 \mathrm{~g} / \mathrm{L}$, keine pH-Einstellung

Biomalz: Biomalt Gerstenmalzextrakt 20g/L, pH 5.6

DiGe (Feststoffmedium): Perlgraupen 200 g, Vollkorn-Dinkelflocken 200 g, Sojapepton 2 g, $\mathrm{MnCl}_{2}$-Lösung $1 \mathrm{~mL}\left(\mathrm{c}=20 \mathrm{mg} \mathrm{MnCl}_{2} \times 4 \mathrm{H}_{2} \mathrm{O} / \mathrm{L}\right), 250 \mathrm{~mL}$ Wasser, keine $\mathrm{pH}$-Einstellung 
Für alle Nährmedien wurde demineralisiertes Wasser verwendet und der $\mathrm{pH}-$ Wert vor dem Autoklavieren mit 0.5 N Natronlauge bzw. 0.5 N Salzsäure eingestellt. Zur Herstellung von Agarplatten wurden jeweils $20 \mathrm{~g} / \mathrm{L}$ Agar zugesetzt. Die Nährmedien wurden durch Autoklavieren (feuchte Hitze, $121^{\circ} \mathrm{C}, 1$ bar Überdruck, $30 \mathrm{~min}$ ) sterilisiert.

Schüttler: Braun Inkubationsschüttelschrank BS4, Braun Certomat $^{\circledR}$ RM.

Fermenter: Rührblattfermenter Braun Biostat M (1.5 L), Braun Biostat B (5 L), Braun Biostat E (10 L), Braun Biostat U (50 L), Airlift-Fermenter (10 L), gebaut: Uni Dortmund, Fischer \& Porter Gasflowmeter (45711M), The Analytical Development Co. Ltd. $\mathrm{CO}_{2}$ Analysator, Ingold $\mathrm{O}_{2}$-Elektrode.

Stammhaltung: Die Stammhaltung erfolgte auf Agarplatten. Für Actinomyceten wurde das Nährmedium M2 verwendet und die beimpften Platten 4 bis 7 Tage bei $28{ }^{\circ} \mathrm{C}$ inkubiert. Pilzstämme wurden auf dem Nährmedium 1158 kultiviert, die Dauer betrug hier 7 bis 21 Tage bei $25{ }^{\circ} \mathrm{C}$. Bewachsene Agarplatten wurden mit Verschlussfolie (Parafilm ${ }^{\circledR} \mathrm{M}$, Fa. Brand) verschlossen und bei $4{ }^{\circ} \mathrm{C}$ für maximal 4 Monate gelagert.

Langzeiterhaltung in flüssigem Stickstoff: Von einer gut bewachsenen Agarplatte wurden mit einseitig zugeschweißten und mit einem Bleistück beschwerten sterilen PolypropylenHalmen Agarstückchen ausgestanzt, bis der Halm gefüllt war. Zur Einlagerung von Pilzstämmen wurde die bewachsene Agarplatte vor dem Ausstechen mit einer 15\%igen wässrigen Glycerinlösung bedeckt. Der Halm wurde in einen zweiten äußeren Halm gesteckt, der an beiden Enden zu geschmolzen wurde. Mehrere Halme in einem Cryoröhrchen wurden in einem Dewargefäß in flüssigem Stickstoff eingelagert.

Mit Ausnahme des Stamms Halomonas sp. Rk377a (Stammhaltung und Kultivierung erfolgten an der TU Braunschweig, siehe S. 155) wurden alle in der vorliegenden Arbeit beschriebenen Bakterien- und Pilzstämme nach der angegebenen Methode eingelagert.

Plattendiffusionstests: Auf die mit den verschiedenen Testkeimen beimpften Agarplatten wurden Filterpapierplättchen (Durchmesser: $6 \mathrm{~mm}$, Dicke: $0.5 \mathrm{~mm}$ ) gelegt, die mit $50 \mu \mathrm{L}$ Substanzlösung (in Aceton oder Methanol, $c=1 \mathrm{mg} / \mathrm{mL}$ ) getränkt und vor dem Auflegen unter sterilen Bedingungen getrocknet worden waren. Als Testkeime dienten Escherichia coli, 
Bacillus subtilis, Staphylococcus aureus und Candida albicans. Die Agarplatten wurden $24 \mathrm{~h}$ bei $37^{\circ} \mathrm{C}$ (E. coli, B. subtilis und S. aureus) bzw. $25^{\circ} \mathrm{C}$ (C. albicans) inkubiert.

Zusammensetzung der Nährböden für Plattendiffusionstests: B. subtilis und E. coli: $5 \mathrm{~g} / \mathrm{L}$ $D$-Glucose, $0.5 \mathrm{~g} / \mathrm{L}$ Trinatriumcitrat x $2 \mathrm{H}_{2} \mathrm{O}, 3 \mathrm{~g} / \mathrm{L} \mathrm{KH}_{2} \mathrm{PO}_{4}, 7 \mathrm{~g} / \mathrm{L} \mathrm{K}_{2} \mathrm{HPO}_{4}, 0.1 \mathrm{~g} / \mathrm{L} \mathrm{MgSO} 4$ x $7 \mathrm{H}_{2} \mathrm{O}, 1 \mathrm{~g} / \mathrm{L}\left(\mathrm{NH}_{4}\right)_{2} \mathrm{SO}_{4}$ und $15 \mathrm{~g} / \mathrm{L}$ Agar, keine $\mathrm{pH}$-Einstellung. S. aureus: $8 \mathrm{~g} / \mathrm{L}$ Bacto Nutrient Broth, $5 \mathrm{~g} / \mathrm{L} \mathrm{NaCl}$ und $15 \mathrm{~g} / \mathrm{L}$ Agar, keine pH-Einstellung. C. albicans: $4 \mathrm{~g} / \mathrm{L}$ Hefeextrakt, $10 \mathrm{~g} / \mathrm{L}$ Malzextrakt, $4 \mathrm{~g} / \mathrm{L} D$-Glucose und $15 \mathrm{~g} / \mathrm{L}$ Agar, $\mathrm{pH}=5.5$.

Zum Ansetzen der Nährböden wurde demineralisiertes Wasser verwendet, Glucose wurde erst nach dem Autoklavieren hinzu gegeben.

Isolierung endophytischer Pilze aus Algen: Die Isolierung endophytischer Pilze aus einer Alge erfolgte analog zu der von SchUlz et al. für Schwämme publizierten Methode ${ }^{[136]}$. Hierzu wurden Agarplatten mit 1158, E2-, G20-, 1549- und Biomalz-Nährmedium hergestellt, jeweils mit und ohne Zusatz von $33 \mathrm{~g} / \mathrm{L} \mathrm{NaCl}$. Zu den Nährlösungen wurden jeweils $250 \mu \mathrm{g} / \mathrm{L}$ Penicillin G und $250 \mu \mathrm{g} / \mathrm{L}$ Streptomycin zugegeben, um das Wachstum von Bakterien zu unterdrücken.

Zur Oberflächensterilisation wurden ca. $5 \mathrm{~cm}$ lange Stücke der Alge 30 Sekunden in eine 70\%ige Ethanollösung gehalten und anschließend mit sterilem demineralisiertem Wasser gewaschen. Die Algenstücke wurden in etwa $2 \mathrm{~mm}$ große Teile geschnitten, die auf die Antibiotika enthaltenden Agarplatten gelegt und mit einer sterilen Pinzette leicht angedrückt wurden. Die Agarplatten wurden für 2 bis 5 Tage bei $25^{\circ} \mathrm{C}$ inkubiert, bis sich Kolonien ausbildeten. Durch mehrfaches Überimpfen auf neue Agarplatten mit einer sterilen Impfnadel wurden die Kolonien vereinzelt.

Zur Überprüfung des Erfolgs der Oberflächensterilisation wurden mehrere nicht zerschnittene Algenstücke ebenfalls auf Agarplatten gelegt und inkubiert. Bei diesen wurde kein mikrobielles Wachstum beobachtet. 


\section{Chemisches Screening von endophytischen Pilzen}

Kultivierung auf festen Phasen und Aufarbeitung ${ }^{\mathrm{y}}$

Die Stämme wurden bei Raumtemperatur für 21-28 Tage auf Biomalz-Agarplatten oder DiGe-Feststoffmedium kultiviert. Die Aufarbeitung erfolgte durch Lyophilisieren der Kultur und anschließende Extraktion mit Ethylacetat. Die Extrakte wurden im Vakuum bis zur Trockne eingeengt.

\section{Kultivierung in Schüttelkolben}

Die verschiedenen Stämme wurden standardmäßig in sechs verschiedenen Nährmedien kultiviert (1158, G20, E2, 1549, SGG, Biomalz). Die Kultivierung erfolgte in $1 \mathrm{~L}$ Erlenmeyerkolben mit drei Schikanen, die mit $150 \mathrm{~mL}$ Nährlösung befüllt und mit einem Zellstoffstopfen verschlossen wurden. Jeweils zwei Kolben pro Stamm und Nährmedium wurden angesetzt, um eine Kontrolle der Ergebnisse zu gewährleisten. Die Kolben wurden autoklaviert, mit ca. $1 \mathrm{~cm}^{2}$ einer gut bewachsenen Agarplatte angeimpft und 6 Tage bei $28{ }^{\circ} \mathrm{C}$ und $180 \mathrm{U} / \mathrm{min}$ kultiviert.

\section{Kultivierung in P-Kolben}

Die Auswahl der Nährmedien wurde anhand der Ergebnisse der Schüttelkulturen vorgenommen. Die Kultivierung erfolgte in 5 L P-Kolben, die mit 1 L Nährlösung befüllt und mit einer Schaumstoffkappe verschlossen wurden. Jeweils zwei P-Kolben pro Stamm und Nährmedium wurden angesetzt, um eine Kontrolle der Ergebnisse zu gewährleisten. Die P-Kolben wurden sterilisiert, mit $20 \mathrm{~mL}$ einer $72 \mathrm{~h}$ alten in Schüttelkolben gewachsenen Vorkultur angeimpft und 28 Tage bei $28^{\circ} \mathrm{C}$ oder Raumtemperatur kultiviert.

\section{Aufarbeitung der Schüttel- und P-Kolben-Kulturen}

Kulturfiltrat und Myzel wurden durch Zentrifugieren (3000 U/min, 10 min) getrennt. Der pHWert des Kulturfiltrats wurde durch Zugabe von $1 \mathrm{~N}$ Salzsäure auf 5.0 eingestellt, falls er zuvor höher lag. Das Kulturfiltrat wurde dreimal mit dem gleichen Volumen an Ethylacetat extrahiert. Das Myzel wurde mit Aceton versetzt (ca. $250 \mathrm{~mL}$ Aceton je $1 \mathrm{~L}$ Kulturbrühe) und 15 min im Ultraschallbad aufgeschlossen. Durch Filtration wurden die nicht löslichen

\footnotetext{
${ }^{\mathrm{y}}$ Die Festphasen-Kultivierung erfolgte im Arbeitskreis von Frau PD Dr. B. Schulz (Institut für Mikrobiologie, TU Braunschweig).
} 
Bestandteile abgetrennt. Kulturfiltrat- und Myzelextrakt wurden im Vakuum eingeengt und der wässrige Rückstand lyophilisiert.

\section{Dünnschichtchromatographie und Nachweis der Metaboliten}

Das Lyophilisat von Kulturfiltrat- und Myzelextrakt der Schüttelkulturen wurde in je $3 \mathrm{~mL}$ Methanol/Wasser (9:1) aufgenommen, das der P-Kolben in $20 \mathrm{~mL}$. $5 \mu \mathrm{L}$ der Lösungen wurden auf HPTLC-Nanoplatten $(10 \times 10 \mathrm{~cm})$ aufgetragen und die Dünnschichtchromatogramme mit Chloroform/Methanol (9:1) als Laufmittel entwickelt. Der Nachweis der Metaboliten erfolgte unter UV-Licht (254 und $366 \mathrm{~nm}$ ) sowie durch Anfärbereagenzien (siehe Seite 147). Ausgewählte Extrakte wurden zusätzlich mittels HPLC-MS untersucht (HPLC 3, Säule 2, Programm 2).

\section{Kultivierung, Aufarbeitung und Isolierung der Metaboliten}

\subsection{Neue Actinomycine aus Actinomyces sp. CS210}

\section{Kultivierung und Aufarbeitung}

Die Kultivierung erfolgte in $300 \mathrm{~mL}$ Schüttelkolben mit drei Schikanen, die mit $100 \mathrm{~mL}$ Nährmedium M2 befüllt und mit einem Zellstoffstopfen verschlossen wurden. Nach dem Autoklavieren wurden die Kulturen mit ca. $1 \mathrm{~cm}^{2}$ einer gut bewachsenen Agarplatte beimpft und $72 \mathrm{~h}$ bei einer Temperatur von $28^{\circ} \mathrm{C}$ mit $180 \mathrm{U} / \mathrm{min}$ rotierend geschüttelt. Zum Erntezeitpunkt hatten die Kulturen eine grün-braune Färbung angenommen und wiesen einen pH-Wert von ca. 7.1 auf.

Das Myzel wurde durch Zentrifugation (4500 U/min, $10 \mathrm{~min}$ ) abgetrennt und mit Aceton extrahiert (3x $30 \mathrm{~mL}$ je Kolben). Der Kulturüberstand wurde nach Einstellung des pH-Werts durch tropfenweise Zugabe von $1 \mathrm{~N}$ Salzsäure auf 5.0 mit Ethylacetat extrahiert $(3 \mathrm{x} 100 \mathrm{~mL}$ je Kolben). Das Lösungsmittel der Extrakte wurde im Vakuum entfernt.

\section{Isolierung der Metaboliten}

Myzel- und Kulturfiltratextrakt wiesen ein ähnliches Metabolitenspektrum auf, jedoch lagen die Substanzen im Kulturfiltrat nur in geringer Konzentration vor. Durch Chromatographie an Kieselgel, Sephadex LH-20 und Nucleodur RP-18 (HPLC) konnten die Actinomycine $\mathrm{G}_{2}$ bis 
$\mathrm{G}_{6}$ (12 bis 16) sowie die Metaboliten 17 und 18 als Reinsubstanzen erhalten werden (siehe Abbildung 73).

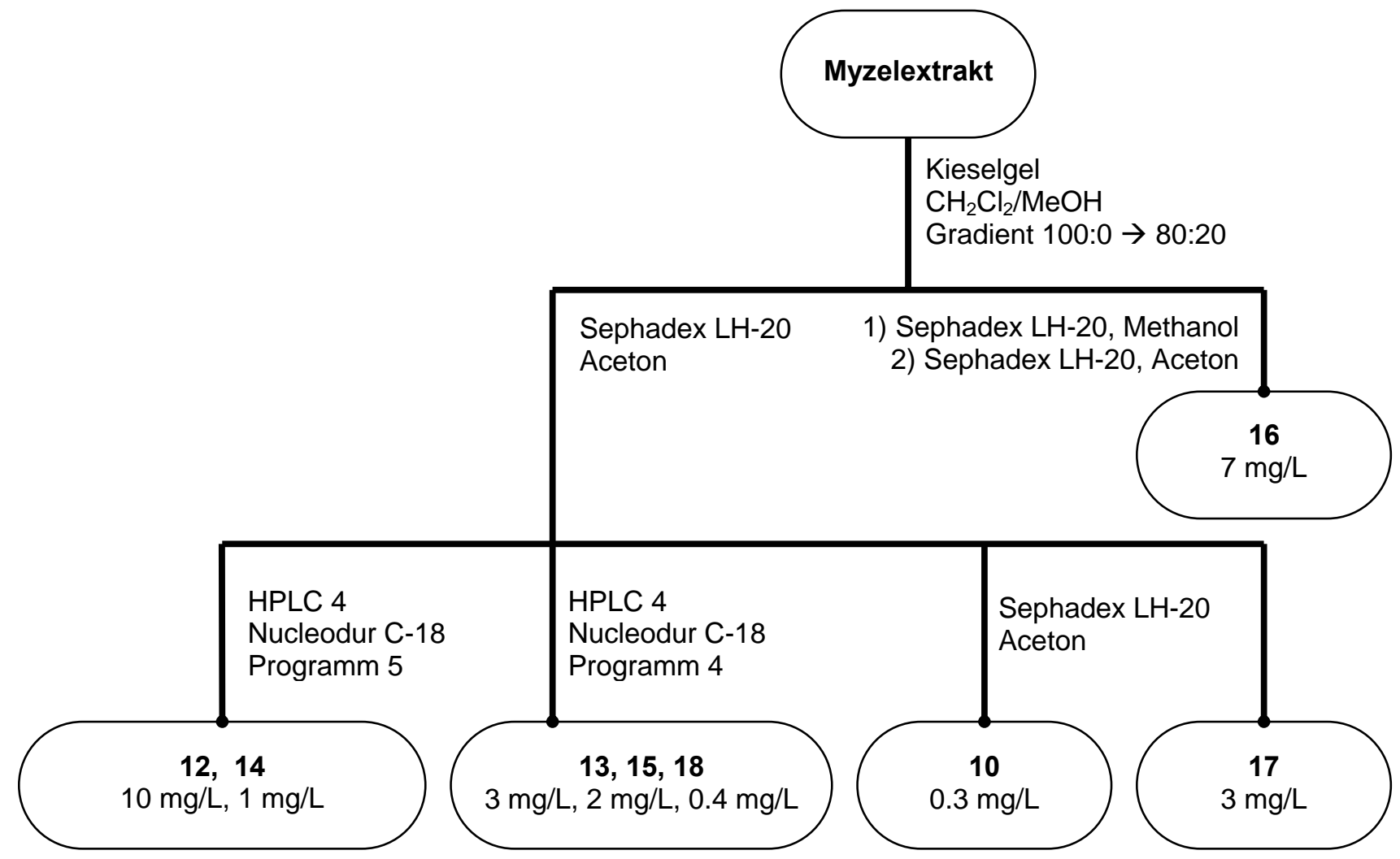

Abbildung 73: Isolierungsschema für die Actinomycine und andere Metaboliten aus dem Stamm Actinomyces sp. CS210.

\subsection{Neue Aminophenoxazone aus Halomonas sp. Rk377a}

\section{Stammhaltung. Kultivierung und Aufarbeitung ${ }^{\mathbf{z}}$}

Die Stammhaltung erfolgte auf Agarplatten mit dem Nährmedium Marine Broth, die für $72 \mathrm{~h}$ bei $27^{\circ} \mathrm{C}$ inkubiert wurden. Die Lagerung erfolgte nach Verschluss mit Parafilm ${ }^{\circledR} \mathrm{M}$ für maximal 8 Wochen bei $4{ }^{\circ} \mathrm{C}$. Für die Schüttelkultur wurden $500 \mathrm{~mL}$ Erlenmeyerkolben mit vier Schikanen verwendet, die mit $100 \mathrm{~mL}$ Nährlösung gefüllt wurden. Diese bestand aus synthetischem Meerwasser ${ }^{[195]}$ mit Zusatz von $5 \mathrm{~g} / \mathrm{L}$ Hefeextrakt, $10 \mathrm{~g} / \mathrm{L}$ Trypton und $0.5 \mathrm{~g} / \mathrm{L}$ Anthranilsäure $(\mathrm{pH}=7.4)$. Als Inokulum diente jeweils $1 \mathrm{~mL}$ einer $24 \mathrm{~h}$ alten SchüttelkolbenVorkultur. Nach einer Inkubationszeit von $48 \mathrm{~h}$ bei $27^{\circ} \mathrm{C}$ und $100 \mathrm{U} / \mathrm{min}$ wiesen die Zellen und der Überstand eine tiefe Schwarzfärbung auf, der $\mathrm{pH}-$ Wert betrug ca. 8.3. Durch

\footnotetext{
${ }^{\text {z }}$ Die Stammhaltung, Kultivierung und Aufarbeitung erfolgten am Institut für Biotechnologie der TU Braunschweig. Herrn PD Dr. S. Lang und Frau L. Wang danke ich für die Erstellung und Zusendung des Extrakts.
} 
Extraktion mit Ethylacetat bei $\mathrm{pH}=3.0$ wurden die Kulturen aufgearbeitet, das Lösungsmittel des Extrakts wurde im Vakuum entfernt.

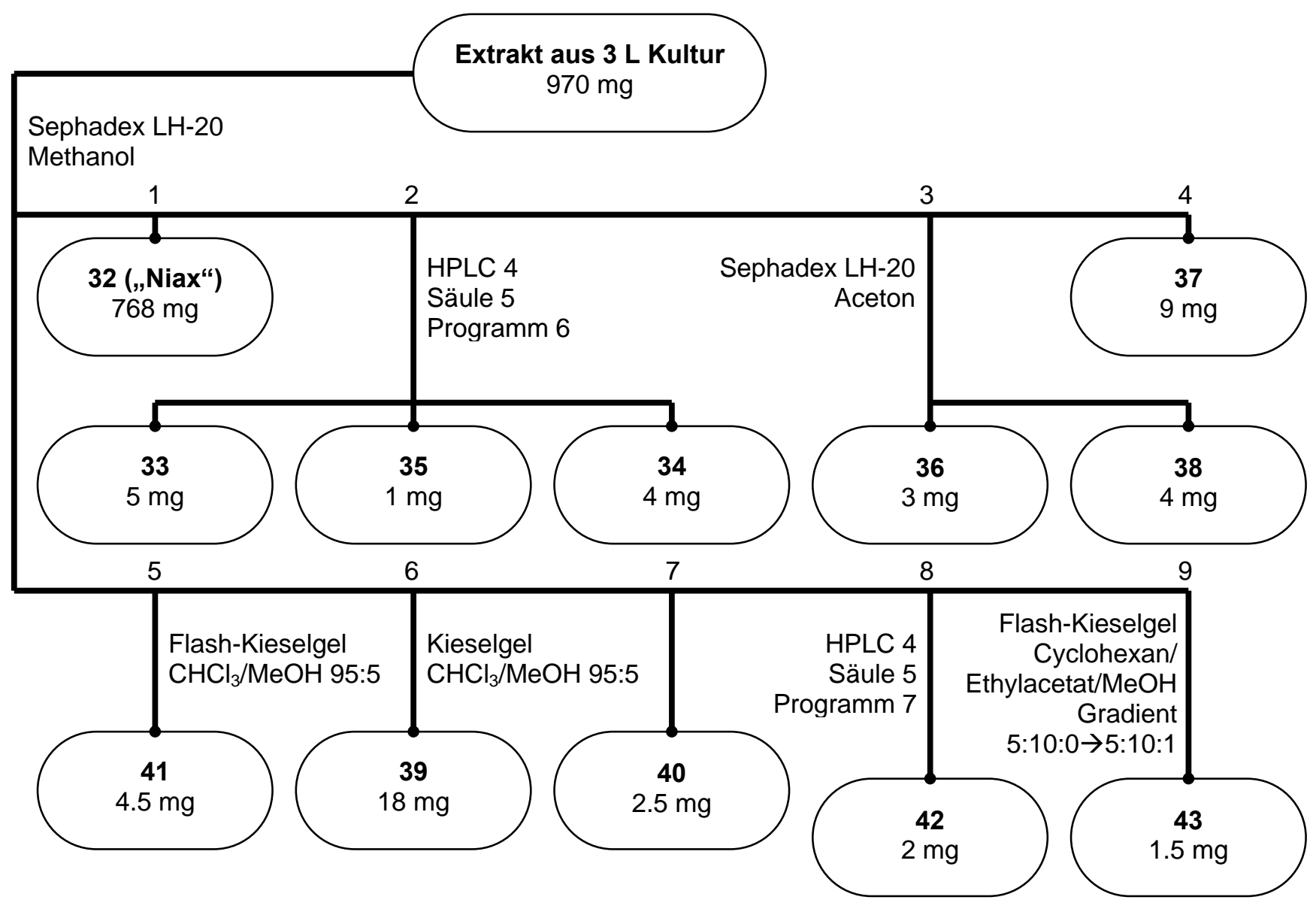

Abbildung 74: Isolierungsschema für die Aminophenoxazone und Diketopiperazine aus dem Stamm Halomonas sp. Rk377a.

\section{Isolierung der Metaboliten}

Die Auftrennung des Extraktes erfolgte durch Chromatographie an Sephadex LH-20 (MeOH).

Die hierbei erhaltenen Fraktionen wurden wie in Abbildung 74 gezeigt durch HPLC, FlashChromatographie oder Gelchromatographie weiter aufgereinigt und getrennt.

\subsection{6-Desoxy- $\alpha$-L-talopyranoside aus Actinomyces sp. Gö M1}

\section{Kultivierung und Aufarbeitung}

Die Kultivierung erfolgte in $300 \mathrm{~mL}$ Schüttelkolben mit drei Schikanen, die jeweils mit 100 mL Hafer-Nährmedium befüllt und mit einem Zellstoffstopfen verschlossen wurden. 
Nach dem Autoklavieren wurden die Kulturen mit ca. $1 \mathrm{~cm}^{2}$ einer gut bewachsenen Agarplatte angeimpft und $72 \mathrm{~h}$ bei einer Temperatur von $28^{\circ} \mathrm{C}$ mit $180 \mathrm{U} / \mathrm{min}$ rotierend geschüttelt. Zum Erntezeitpunkt hatte die Kulturbrühe eine gelbliche Färbung angenommen und wies einen $\mathrm{pH}-$ Wert von ca. 4.7 auf.

Das Myzel von 2 L Kulturansatz wurde durch Zentrifugation abgetrennt (4500 U/min, $10 \mathrm{~min}$ ) und verworfen, da der Myzelextrakt im chemischen Screening keine interessanten Metaboliten aufgewiesen hatte. Der Überstand wurde an Amberlite ${ }^{\circledR}$ XAD-2 adsorbiert $(500 \mathrm{~mL})$ und das Adsorberharz mit $500 \mathrm{~mL}$ Wasser gewaschen. Die adsorbierten Substanzen wurden mit $1000 \mathrm{~mL}$ Methanol eluiert, der Extrakt im Vakuum eingeengt und der wässrige Rückstand lyophilisiert.

\section{Isolierung der Metaboliten}

Die Isolierung der Metaboliten erfolgte wie in Abbildung 75 gezeigt.

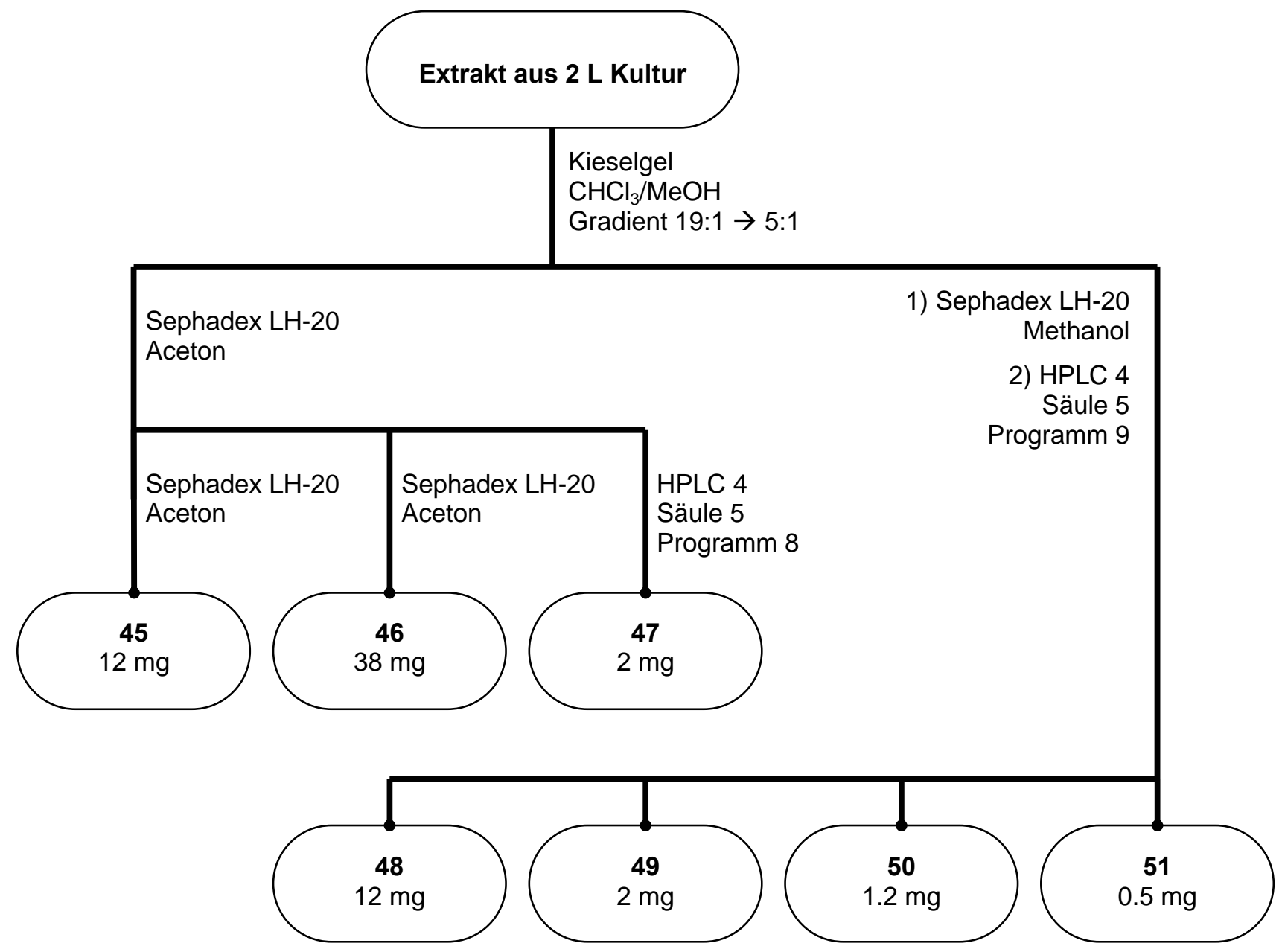

Abbildung 75: Isolierungsschema für die 6-Desoxy- $\alpha$-L-talopyranoside aus Actinomyces sp. Gö M1. 


\section{Methanolyse und Silylierung von 45, 46 und 48}

Darstellung von methanolischer Salzsäure: $7.1 \mathrm{~mL}$ (0.1 mol) Acetylchlorid (Fa. Merck) wurden bei $0{ }^{\circ} \mathrm{C}$ langsam in $25 \mathrm{~mL}$ Methanol gelöst. Die so hergestellte methanolische Salzsäure war 4 molar.

$0.3 \mathrm{mg}$ der jeweiligen Substanz wurden in ein Schmelzpunktbestimmungsröhrchen $(1=80 \mathrm{~mm}, \varnothing=2 \mathrm{~mm}$ ) gegeben, mit $4 \mathrm{M}$ methanolischer Salzsäure versetzt (Füllhöhe: $30 \mathrm{~mm}$ ) und $15 \mathrm{~min}$ im Ultraschallbad behandelt. Anschließend wurde die Probe im Vakuum bis zur Trockne eingeengt und mit 25\%iger TSIM-Lösung (Fa. Aldrich, $N$-(Trimethylsilyl)imidazol in Dimethylformamid) versetzt (Füllhöhe: $30 \mathrm{~mm}$ ). Das Schmelzpunktbestimmungsröhrchen wurde zugeschmolzen und für eine Stunde auf $65^{\circ} \mathrm{C}$ erhitzt.

\section{Vorläufer-dirigierte Biosynthese}

Zur Vorläufer-dirigierten Biosynthese wurde der Stamm im 1L-Rührblattfermenter kultiviert, der mit $800 \mathrm{~mL}$ Hafer-Nährmedium befüllt und autoklaviert wurde. Als Inokulum dienten $100 \mathrm{~mL}$ einer $72 \mathrm{~h}$ alten Schüttelkolben-Vorkultur, die wie oben beschrieben erstellt wurde. Die Rührgeschwindigkeit betrug $200 \mathrm{U} / \mathrm{min}$, die Temperatur $28{ }^{\circ} \mathrm{C}$ und die Belüftung 1.0 vvm. Die Lösung mit den jeweiligen Vorläufermolekülen (siehe Tabelle 21) wurde mittels einer Schlauchdosierpumpe ab der 12. Stunde über einen Zeitraum von 12 Stunden zugeleitet. Nach einer Fermentationsdauer von $72 \mathrm{~h}$ wurde die Kultur geerntet und wie zuvor beschrieben aufgearbeitet. Die Isolierung der Metaboliten erfolgte analog zu Abbildung 75 durch Chromatographie an Kieselgel und anschließend an Sephadex LH-20, die Ausbeuten sind Tabelle $21 \mathrm{zu}$ entnehmen.

Tabelle 21: Zur Kultur von Actinomyces sp. Gö M1 zugegebene Vorläufermoleküle. Jeweils $7.3 \mathrm{mmol}$ der Substanzen wurden in $50 \mathrm{~mL}$ sterilem demineralisierten Wasser gelöst.

\begin{tabular}{|c|c|c|c|c|c|}
\hline & Substanz & $\begin{array}{c}\text { Molmasse } \\
{[\mathrm{g} / \mathrm{mol}]}\end{array}$ & $\begin{array}{c}\text { gelöste Menge } \\
{[\mathrm{mg}]}\end{array}$ & $\begin{array}{c}\text { erhaltene } \\
\text { Metaboliten } \\
\text { (Substanz-Nr.) }\end{array}$ & $\begin{array}{c}\text { Ausbeute } \\
{[\mathrm{mg} / \mathrm{L}]}\end{array}$ \\
\hline 1. & Pyrrol-2-carbonsäure & 111.10 & 811 & 50 & 33 \\
\hline 2. & Phenylessigsäure & 138.12 & 994 & 46 & 60 \\
\hline \multirow[t]{3}{*}{3.} & 4-Hydroxybenzoesäure & 136.15 & 1008 & 54 & 11 \\
\hline & & & & 48 & 50 \\
\hline & & & & 51 & 75 \\
\hline
\end{tabular}




\subsection{Nargenicin $A_{1}$ aus Actinomyces sp. Gö 301}

\section{Kultivierung und Aufarbeitung}

Die Kultivierung erfolgte in $300 \mathrm{~mL}$ Schüttelkolben mit drei Schikanen, die jeweils mit 100 mL Nährmedium M2 befüllt und mit einem Zellstoffstopfen verschlossen wurden. Nach dem Autoklavieren wurden die Kulturen mit ca. $1 \mathrm{~cm}^{2}$ einer gut bewachsenen Agarplatte angeimpft und $96 \mathrm{~h}$ bei einer Temperatur von $28^{\circ} \mathrm{C}$ mit $180 \mathrm{U} / \mathrm{min}$ rotierend geschüttelt. Zum Erntezeitpunkt hatten die Kulturen eine bräunliche Färbung angenommen und wiesen einen pH-Wert ca. 6.5 auf.

Das Myzel von 2 L Kulturansatz wurde durch Zentrifugation abgetrennt (4500 U/min, $10 \mathrm{~min}$ ) und verworfen, da der Myzelextrakt im chemischen Screening nur eine sehr geringe Konzentration an Metaboliten aufgewiesen hatte. Der Überstand wurde an Amberlite ${ }^{\circledR}$ XAD-2 adsorbiert $(500 \mathrm{~mL})$, das Adsorberharz mit $500 \mathrm{~mL}$ Wasser gewaschen und anschließend mit $1000 \mathrm{~mL}$ Methanol eluiert. Der Extrakt wurde im Vakuum eingeengt und der wässrige Rückstand lyophilisiert.

\section{Isolierung der Metaboliten}

Das Isolierungsschema zeigt Abbildung 76.

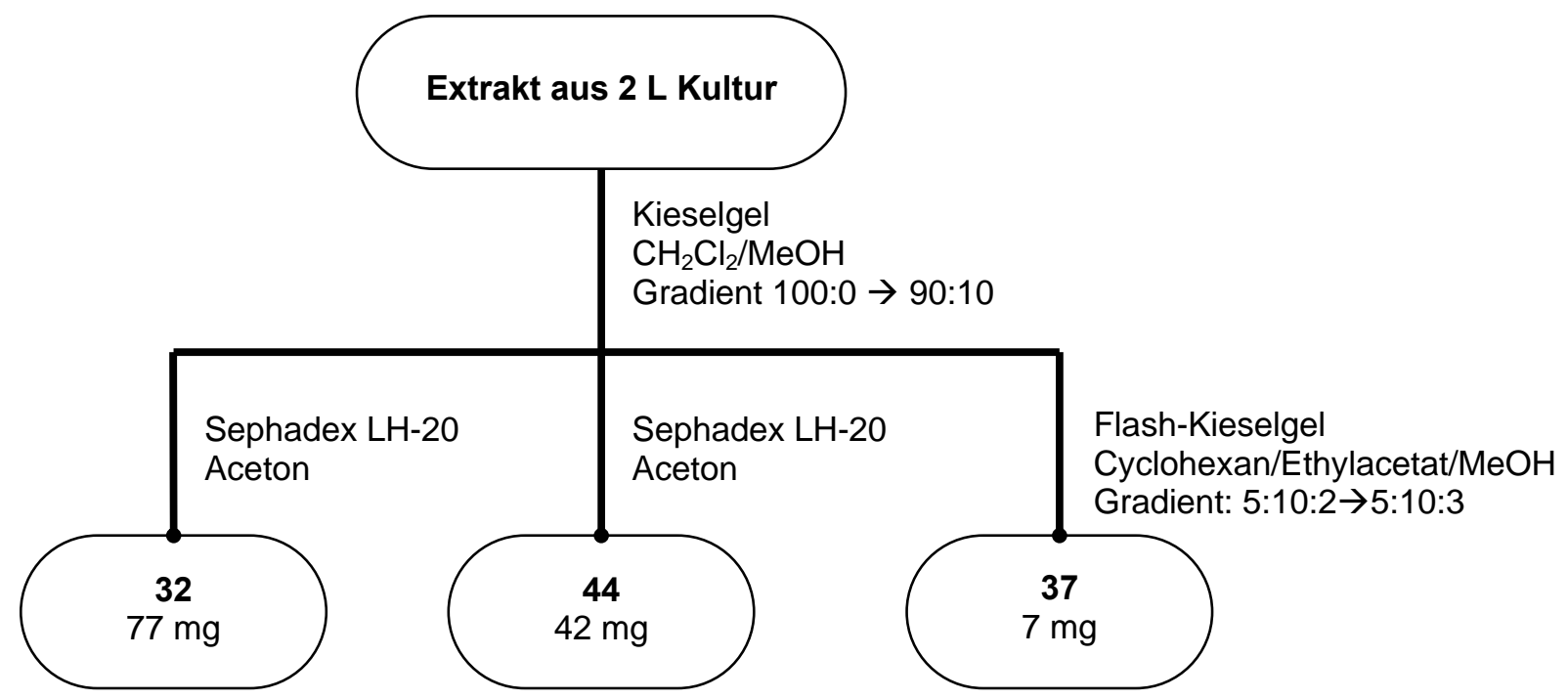

Abbildung 76: Isolierungsschema für Nargenicin $A_{1}$ (44) und andere Metaboliten aus Actinomyces sp. Gö 301. 


\subsection{Ascosalipyron aus Diplodia sp. BS 5945}

\section{Kultivierung und Aufarbeitung}

Die Kultivierung erfolgte in $1 \mathrm{~L}$ Erlenmeyerkolben mit drei Schikanen, die jeweils mit 150 mL Nährmedium G20 befüllt und mit einem Zellstoffstopfen verschlossen wurden. Nach dem Autoklavieren wurden die Kulturen mit ca. $1 \mathrm{~cm}^{2}$ einer gut bewachsenen Agarplatte angeimpft und $7 \mathrm{~d}$ bei einer Temperatur von $28^{\circ} \mathrm{C}$ mit $180 \mathrm{U} / \mathrm{min}$ rotierend geschüttelt. Zum Erntezeitpunkt hatten die Kulturen eine schwarz-grüne Färbung angenommen und wiesen einen $\mathrm{pH}-$ Wert von ca. 7.1 auf.

Das Myzel wurde durch Zentrifugation (3000 U/min, $10 \mathrm{~min}$ ) abgetrennt und verworfen. Der pH-Wert des Überstands wurde durch Zugabe von $1 \mathrm{M}$ Salzsäure auf $\mathrm{pH}=5.0$ eingestellt. Anschließend wurde der Überstand mit Ethylacetat extrahiert $(3 \times 50 \mathrm{~mL}$ je Erlenmeyerkolben) und der Extrakt im Vakuum bis zur Trockne eingeengt.

\section{Isolierung von Ascosalipyron (60)}

Der Kulturfiltrat-Extrakt von 4 L Kulturbrühe wurde durch Chromatographie an Kieselgel (Dichlormethan/Methanol, Gradient 19:1 $\rightarrow$ 4:1), Flash-Kieselgel (Dichlormethan/Methanol 9:1) und Sephadex-LH20 (Dichlormethan) aufgetrennt. Man erhielt $28 \mathrm{mg}$ der Verbindung 60 als farblosen Feststoff.

\subsection{Solanapyron C aus Microsphaeropsis sp. BS 6278}

\section{Kultivierung und Aufarbeitung}

Die Kultivierung erfolgte in vier 5 L P-Kolben, die jeweils mit 1 L Nährmedium SGG und $58 \mathrm{~g} \mathrm{NaBr}$ befüllt und mit einer Schaumstoffkappe verschlossen wurden. Die P-Kolben wurden autoklaviert und anschließend mit $20 \mathrm{~mL}$ einer $72 \mathrm{~h}$ alten Schüttelkolben-Vorkultur beimpft (Nährmedium SGG, $150 \mathrm{~mL}$ Nährlösung in einem $1 \mathrm{~L}$ Erlenmeyerkolben mit drei Schikanen). Nach einer Kultivierungsdauer von $28 \mathrm{~d}$ zeigten die Kulturen eine starke Luftmyzelbildung von gräulicher Farbe und wiesen einen pH-Wert von ca. 5.5 auf.

Das Myzel wurde durch Zentrifugation (3000 U/min, $10 \mathrm{~min}$ ) abgetrennt und zweimal mit je $1 \mathrm{~L}$ Aceton $15 \mathrm{~min}$ im Ultraschallbad aufgeschlossen. Der Überstand wurde mit Ethylacetat extrahiert ( $3 \times 2$ L). Das Lösungsmittel der Extrakte wurde im Vakuum entfernt und der Rückstand lyophilisiert. 


\section{Isolierung von Solanapyron C (61)}

Der Myzel-Extrakt von 4 L Kultur wurde durch Chromatographie an Kieselgel (Dichlormethan/Methanol, Gradient 19:1 $\rightarrow$ 4:1), Sephadex-LH20 (Aceton) und FlashKieselgel (Chloroform/Methanol 15:1) aufgetrennt. Man erhielt $5 \mathrm{mg} \mathbf{6 1}$ als farblosen Feststoff.

\subsection{Sekundärmetaboliten aus Chaetomium sp. BS 6556}

\section{Kultivierung und Aufarbeitung}

Die Kultivierung erfolgte auf DiGe-Feststoffmedium bei Raumtemperatur für $28 \mathrm{~d}$ (ca. $12 \mathrm{~L}$ ). Die Kulturen wurden anschließend lyophilisiert und dreimal mit je 1 L Ethylacetat/ Methanol (9:1) extrahiert. Der Extrakt wurde im Vakuum eingeengt.

\section{Isolierung der Sekundärmetaboliten}

Die Isolierung von $4.5 \mathrm{mg} 65$ aus $20 \mathrm{mg}$ Rohextrakt erfolgte durch semi-präparative HPLC (HPLC 2, Säule 3, Programm 3). Zur Isolierung der anderen Metaboliten sowie von 65 in größeren Mengen siehe Abbildung 77.

\subsection{Sesquiterpene aus Drechs/era sp. BS 6618}

\section{Kultivierung, Aufarbeitung und Isolierung der Metaboliten}

Die Kultivierung erfolgte auf Agarplatten mit Biomalz-Nährmedium bei Raumtemperatur für 28 d (12 L Nährmedium, ca. 250 Agarplatten). Die Agarplatten wurden lyophilisiert und anschließend dreimal mit je $1 \mathrm{~L}$ Ethylacetat/Methanol (9:1) extrahiert. Der Extrakt wurde im Vakuum eingeengt. Die Isolierung der Sekundärmetaboliten erfolgte wie in Abbildung 78 gezeigt. 


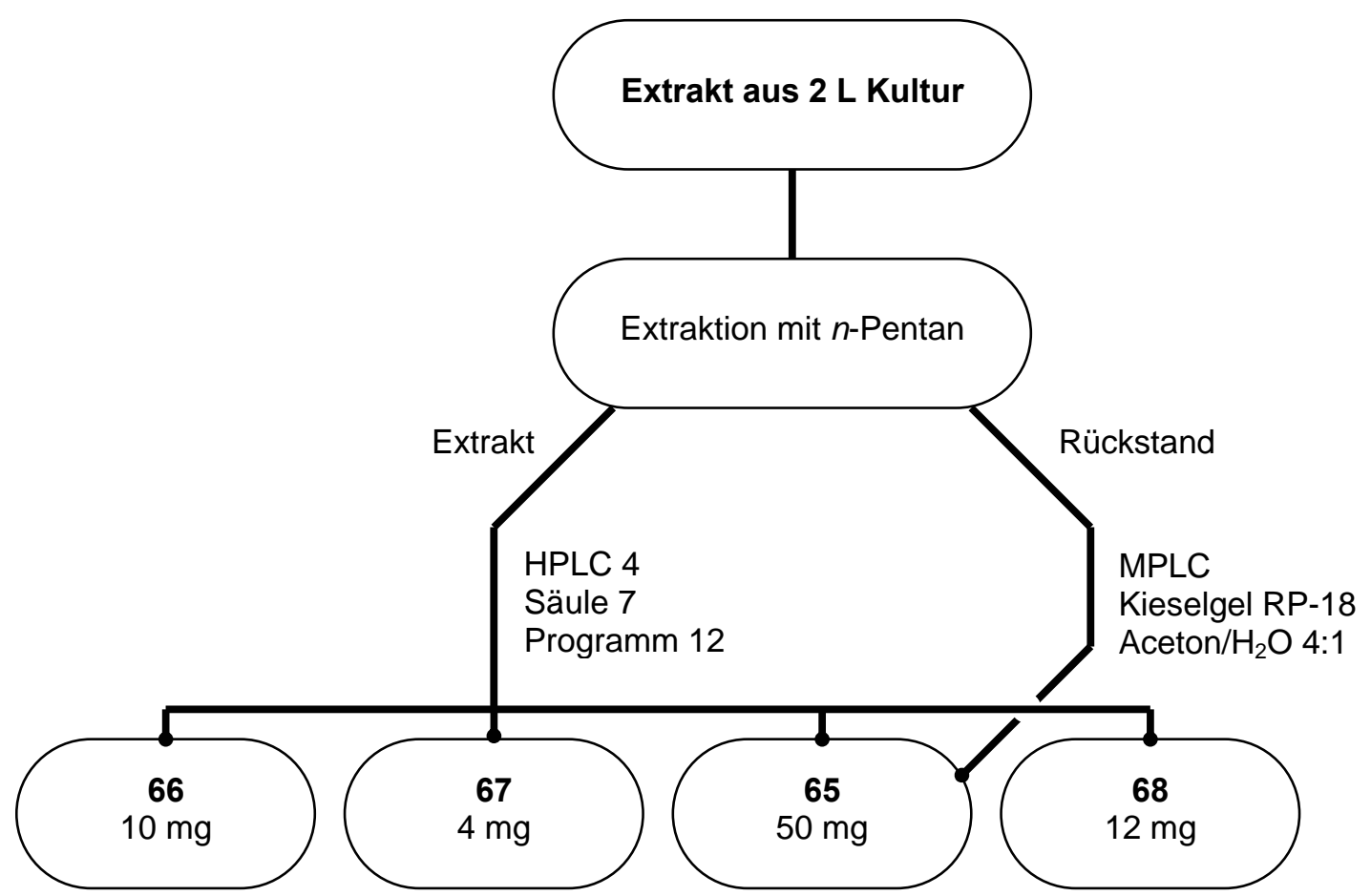

Abbildung 77: Isolierungsschema für die Sekundärmetaboliten aus Chaetomium sp. BS 6556.

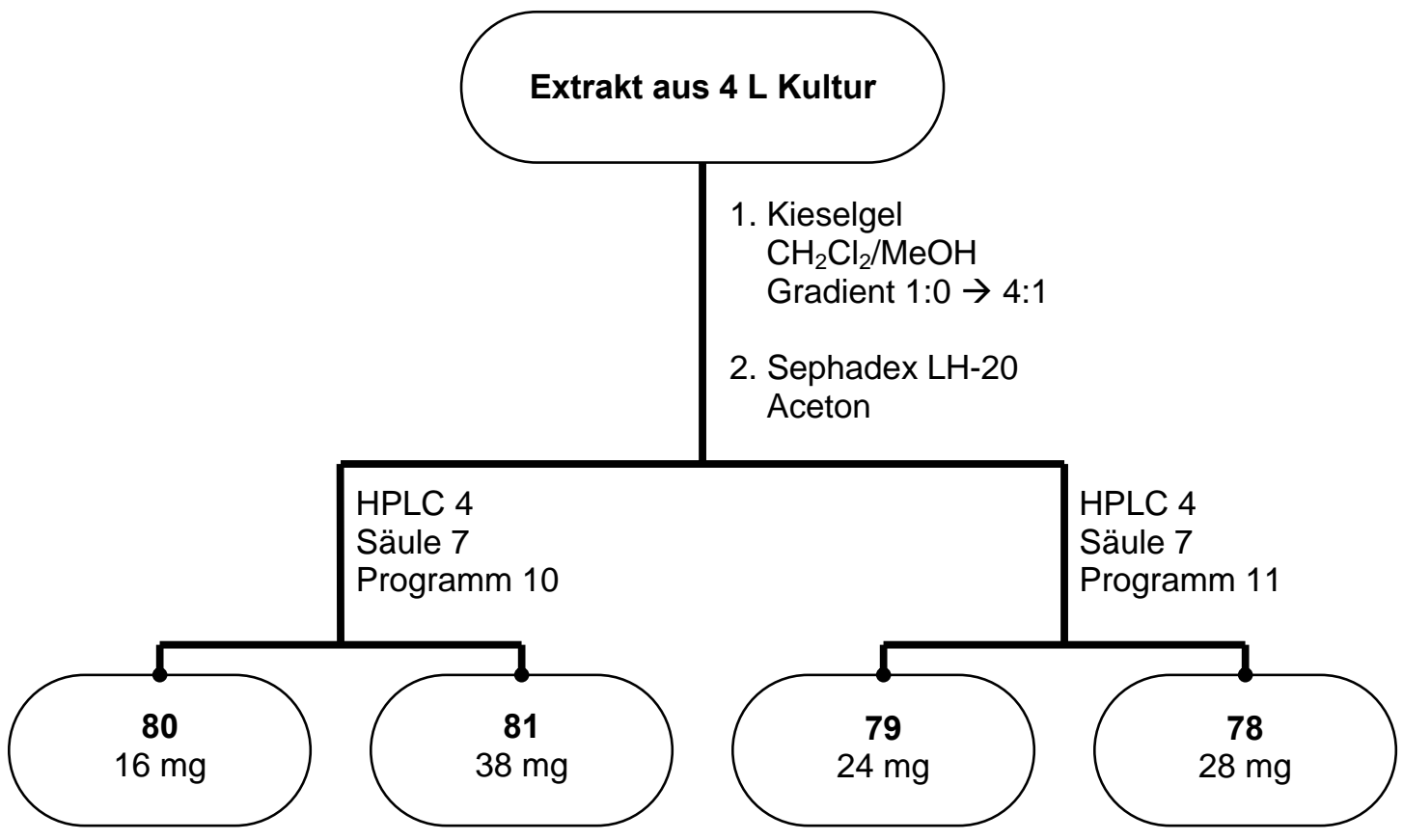

Abbildung 78: Isolierung der Sesquiterpene aus Drechslera sp. BS 6618. 


\subsection{Spiroverbindung aus Beauveria sp. BS 6750}

\section{Kultivierung und Aufarbeitung}

Die Kultivierung erfolgte auf Agarplatten mit Biomalz-Nährmedium bei Raumtemperatur für 28 d (12 L Nährmedium, ca. 250 Agarplatten). Die Agarplatten wurden lyophilisiert und anschließend dreimal mit je $1 \mathrm{~L}$ Ethylacetat/Methanol (9:1) extrahiert. Der Extrakt wurde im Vakuum eingeengt.

\section{Isolierung der Spiroverbindung}

Die Hälfte des Extrakts wurde durch zweifache Chromatographie an Kieselgel (1. $\mathrm{CH}_{2} \mathrm{Cl}_{2} / \mathrm{MeOH}$, Gradient 19:1 $\rightarrow$ 4:1; 2. Cyclohexan/Ethylacetat/MeOH, Gradient 10:5:0 $\rightarrow$ 10:5:2) aufgetrennt. Hierbei wurden $7.5 \mathrm{mg}$ der Verbindung 86 erhalten.

\subsection{Sekundärmetaboliten aus Phomopsis sp. BS 6769}

\section{Kultivierung und Aufarbeitung}

Die Kultivierung erfolgte in $1 \mathrm{~L}$ Erlenmeyerkolben mit drei Schikanen, die jeweils mit 150 mL Nährmedium 1158 befüllt und mit einem Zellstoffstopfen verschlossen wurden. Nach dem Autoklavieren wurden die Kulturen mit ca. $1 \mathrm{~cm}^{2}$ einer gut bewachsenen Agarplatte angeimpft und $5 \mathrm{~d}$ bei einer Temperatur von $28^{\circ} \mathrm{C}$ mit $180 \mathrm{U} / \mathrm{min}$ rotierend geschüttelt. Zum Erntezeitpunkt hatten die Kulturen eine gelbliche Färbung angenommen und wiesen einen pH-Wert von ca. 3.7 auf.

Das Myzel wurde durch Zentrifugation (3000 U/min, $10 \mathrm{~min}$ ) abgetrennt und der Überstand mit Ethylacetat extrahiert (3x $50 \mathrm{~mL}$ je Erlenmeyerkolben). Das Lösungsmittel der vereinigten organischen Phasen wurde im Vakuum entfernt.

\section{Isolierung der Metaboliten}

Die Isolierung der Reinsubstanzen erfolgte nach dem in Abbildung 79 gezeigten Schema. 


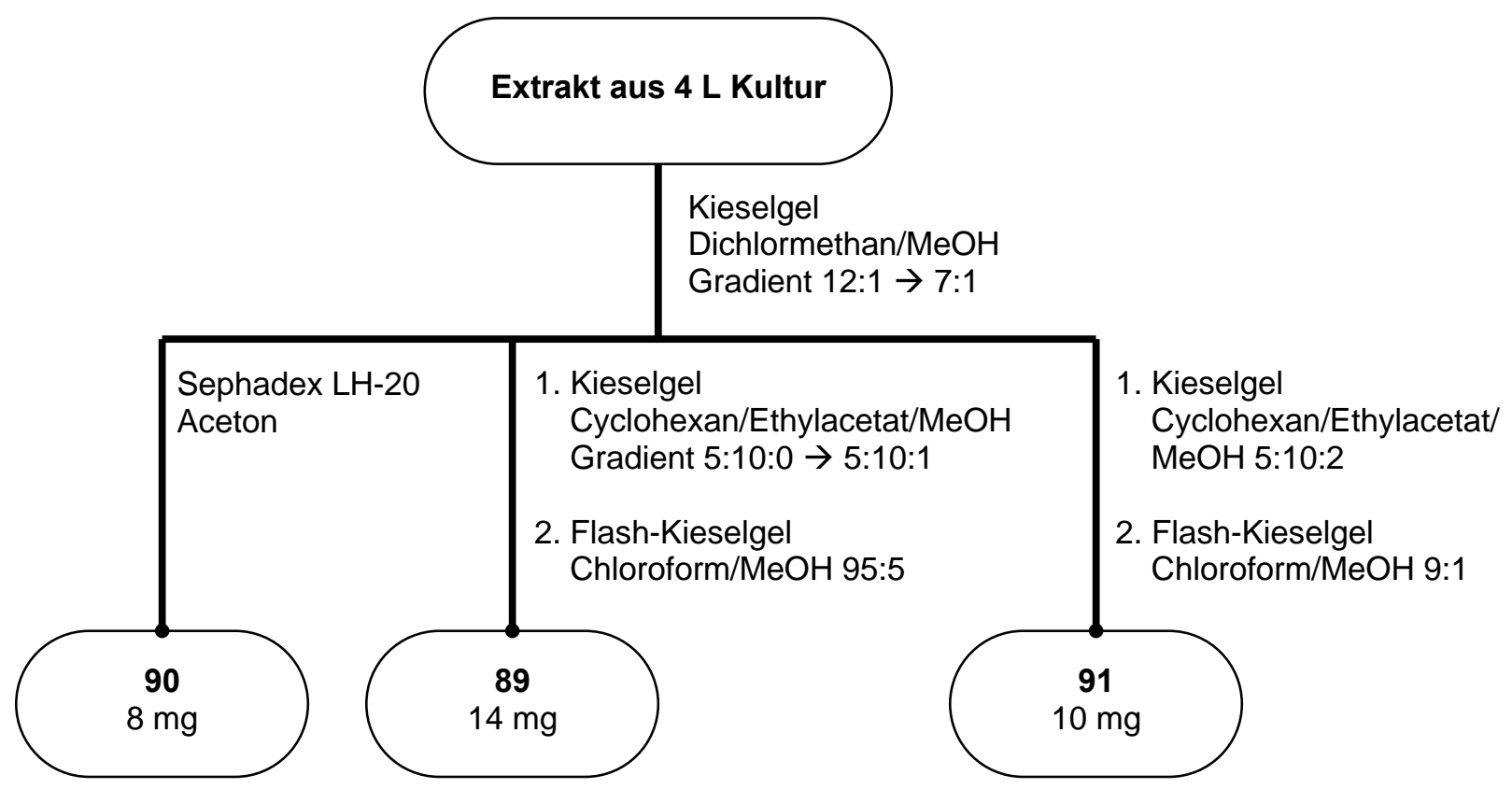

Abbildung 79: Isolierungsschema der Sekundärmetaboliten aus Phomopsis sp. BS 6769. 


\section{Charakterisierung der Metaboliten}

\subsection{Substanzen aus Actinomyces sp. Stamm CS210}

\subsubsection{Actinomycin $\mathrm{G}_{2}(12)$}

intensiv oranger Feststoff

$\mathrm{C}_{61} \mathrm{H}_{83} \mathrm{~N}_{12} \mathbf{O}_{17} \mathrm{Cl}(1291.84)$

Monoisotopische Masse: $\mathrm{M}_{\text {mono }}=1290.57$

Anfärbeverhalten: orange (Anisaldehyd)

$\mathbf{R}_{\mathbf{f}}=0.53\left(\mathrm{CHCl}_{3} / \mathrm{MeOH} 9: 1\right)$

$\mathbf{R}_{\mathbf{t}}=21.9 \min ($ HPLC 1, Säule 1, Programm 1)

ESI-MS (positive Ionen): $\mathrm{m} / \mathrm{z}=1291[\mathrm{M}+\mathrm{H}]^{+}, 1313[\mathrm{M}+\mathrm{Na}]^{+}$

ESI-MS (negative Ionen): $\mathrm{m} / \mathrm{z}=1289[\mathrm{M}-\mathrm{H}]^{-}$

HR-ESI-MS: berechnet $\mathrm{m} / \mathrm{z}=646.291660[\mathrm{M}+2 \mathrm{H}]^{2+}$

$$
\text { gefunden } \mathrm{m} / \mathrm{z}=646.291570 \text { (Abweichung } 0.14 \mathrm{ppm} \text { ) }
$$

IR $(\mathrm{KBr}): \widetilde{v}=3421,3274,3055,2963,2931,2862,2359,1749,1668,1635,1580,1507$, $1475,1360,1306,1192,1097,753 \mathrm{~cm}^{-1}$

UV $(\mathrm{MeOH}): \lambda_{\max }(\log \varepsilon)=240(4.44), 426(4.24), 440(4.25) \mathrm{nm}$

$(\mathrm{MeOH} / \mathrm{HCl}): \lambda_{\max }(\log \varepsilon)=241(4.45), 429(4.13), 441(4.14) \mathrm{nm}$

$(\mathrm{MeOH} / \mathrm{NaOH}): \lambda_{\max }(\log \varepsilon)=240(4.44), 428(4.24), 441(4.25) \mathrm{nm}$

CD $(\mathrm{MeOH}): \lambda_{\max }([\Theta])=212(-178300), 242$ (33700), $270(-73900), 378(-24600) \mathrm{nm}$

$[\alpha]_{D}^{20}=-214^{\circ}(\mathrm{c}=1$ in Methanol $)$

NMR-Daten $\left(\mathrm{CDCl}_{3}\right)$ siehe Tabelle 22, Seite 170.

NMR-Daten $\left(\mathrm{CD}_{3} \mathrm{OD}\right)$ siehe Tabelle 23, Seite 171 .

\subsubsection{Actinomycin $\mathrm{G}_{3}(13)$}

intensiv oranger Feststoff

$\mathbf{C}_{61} \mathbf{H}_{84} \mathbf{N}_{12} \mathbf{O}_{18}(1273.39)$

Monisotopische Masse: $\mathrm{M}_{\text {mono }}=1272.60$

Anfärbeverhalten: orange (Anisaldehyd)

$\mathbf{R}_{\mathbf{f}}=0.51\left(\mathrm{CHCl}_{3} / \mathrm{MeOH} 9: 1\right)$

$\mathbf{R}_{\mathbf{t}}=21.3 \min ($ HPLC 1, Säule 1, Programm 1)

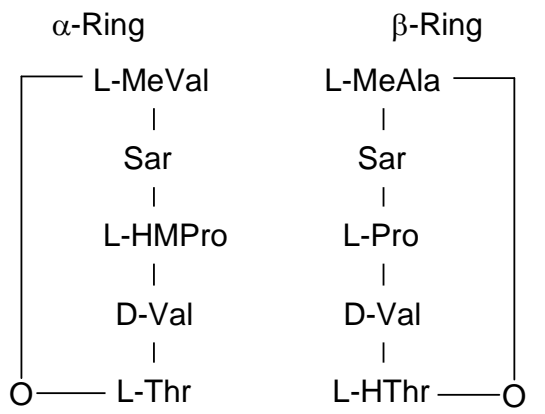<smiles>CC(=O)c1c2nc3c(C(C)=O)ccc(C)c3oc-2c(C)c(=O)c1N</smiles> 
ESI-MS (positive Ionen): $\mathrm{m} / \mathrm{z}=1273[\mathrm{M}+\mathrm{H}]^{+}, 1295[\mathrm{M}+\mathrm{Na}]^{+}$

ESI-MS (negative Ionen): $\mathrm{m} / \mathrm{z}=1271[\mathrm{M}-\mathrm{H}]^{-}$

HR-ESI-MS: berechnet $\mathrm{m} / \mathrm{z}=637.308603[\mathrm{M}+2 \mathrm{H}]^{2+}$

gefunden $\mathrm{m} / \mathrm{z}=637.308708$ (Abweichung $0.16 \mathrm{ppm}$ )

IR $(\mathrm{KBr}): \widetilde{v}=3430,2941,2354,1735,1644,1503,1467,1312,1266,1196,1115,1035$, $804,667 \mathrm{~cm}^{-1}$

UV $(\mathrm{MeOH}): \lambda_{\max }(\log \varepsilon)=236(4.39), 426(4.12), 444(4.13) \mathrm{nm}$

$(\mathrm{MeOH} / \mathrm{HCl}): \lambda_{\max }(\log \varepsilon)=236(4.41), 427(4.12), 445(4.12) \mathrm{nm}$

$(\mathrm{MeOH} / \mathrm{NaOH}): \lambda_{\max }(\log \varepsilon)=234(4.48), 425(4.14), 444(4.16) \mathrm{nm}$

CD $(\mathrm{MeOH}): \lambda_{\max }([\Theta])=210(-128500), 240(7700), 269(-26300) \mathrm{nm}$

$[\alpha]_{D}^{20}=-83^{\circ}(\mathrm{c}=1$ in Methanol $)$

NMR-Daten $\left(\mathrm{CDCl}_{3}\right)$ siehe Tabelle 24, Seite 172.

NMR-Daten $\left(\mathrm{CD}_{3} \mathrm{OD}\right)$ siehe Tabelle 25, Seite 173.

\subsubsection{Actinomycin $\mathrm{G}_{4}(14)$}

intensiv oranger Feststoff

$\mathbf{C}_{61} \mathbf{H}_{\mathbf{8 4}} \mathbf{N}_{\mathbf{1 2}} \mathbf{O}_{17}(1257.39)$

Monoisotopische Masse: $\mathrm{M}_{\text {mono }}=1256.61$

Anfärbeverhalten: orange (Anisaldehyd)

$\mathbf{R}_{\mathbf{f}}=0.31\left(\mathrm{CHCl}_{3} / \mathrm{MeOH} 9: 1\right)$

$\mathbf{R}_{\mathbf{t}}=19.5 \min ($ HPLC 1, Säule 1, Programm 1)

ESI-MS (positive Ionen): $\mathrm{m} / \mathrm{z}=1257.5[\mathrm{M}+\mathrm{H}]^{+}, 1279.6[\mathrm{M}+\mathrm{Na}]^{+}$

ESI-MS (negative Ionen): $\mathrm{m} / \mathrm{z}=1255.6[\mathrm{M}-\mathrm{H}]^{-}$

HR-ESI-MS: berechnet $\mathrm{m} / \mathrm{z}=629.311146[\mathrm{M}+2 \mathrm{H}]^{2+}$

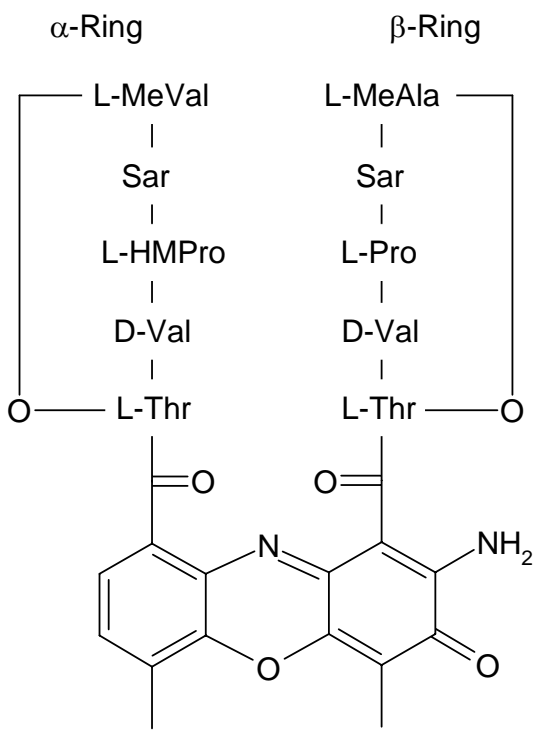

gefunden $\mathrm{m} / \mathrm{z}=629.311171$ (Abweichung $0.04 \mathrm{ppm}$ )

UV $(\mathrm{MeOH}): \lambda_{\max }(\log \varepsilon)=237(4.45), 435(4.27) \mathrm{nm}$

$(\mathrm{MeOH} / \mathrm{HCl}): \lambda_{\max }(\log \varepsilon)=233$ (4.53), $405(4.14), 458(4.24), 486(4.11) \mathrm{nm}$

$(\mathrm{MeOH} / \mathrm{NaOH}): \lambda_{\max }(\log \varepsilon)=238(4.52), 433(4.32) \mathrm{nm}$

CD $(\mathrm{MeOH}): \lambda_{\max }([\Theta])=210(-111300), 240(5000), 277(-18700) \mathrm{nm}$

NMR-Daten $\left(\mathrm{CD}_{3} \mathrm{OD}\right)$ siehe Tabelle 26, Seite 174. 
23.1.4 Actinomycin $\mathrm{G}_{5}(15)$

intensiv oranger Feststoff

$\mathbf{C}_{61} \mathbf{H}_{81} \mathbf{N}_{11} \mathbf{O}_{18}(1556.36)$

Monoisotopische Masse: $\mathrm{M}_{\text {mono }}=1255.58$

Anfärbeverhalten: orange (Anisaldehyd)

$\mathbf{R}_{\mathbf{f}}=0.36\left(\mathrm{CHCl}_{3} / \mathrm{MeOH} 9: 1\right)$

$\mathbf{R}_{\mathbf{t}}=18.7 \min ($ HPLC 1, Säule 1, Programm 1)

ESI-MS (positive Ionen): $\mathrm{m} / \mathrm{z}=1255.5[\mathrm{M}+\mathrm{H}]^{+}, 1278.6[\mathrm{M}+\mathrm{Na}]^{+}$

ESI-MS (negative Ionen): $\mathrm{m} / \mathrm{z}=1254.7[\mathrm{M}-\mathrm{H}]^{-}$

HR-ESI-MS: berechnet $\mathrm{m} / \mathrm{z}=1256.583381[\mathrm{M}+\mathrm{H}]^{+}$

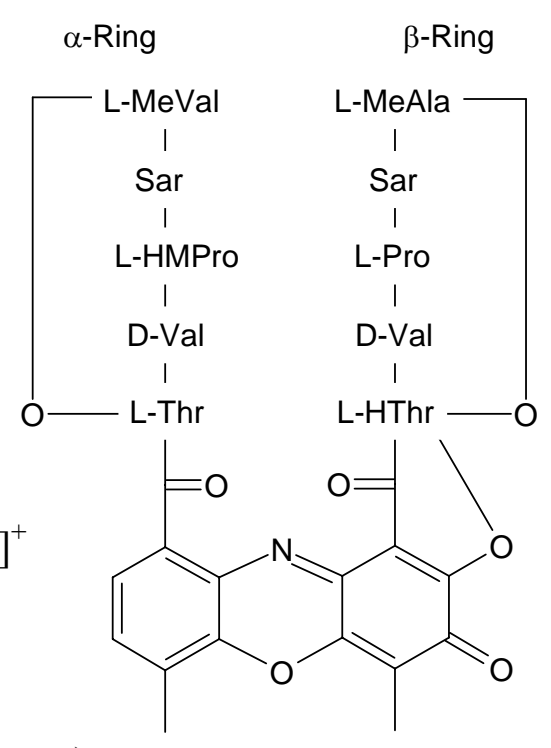
gefunden $\mathrm{m} / \mathrm{z}=1256.583064$ (Abweichung $0.25 \mathrm{ppm}$ )

IR $(\mathrm{KBr}): \widetilde{v}=3434,2931,2858,2353,1745,1654,1460,1376,1192,1135,800,668 \mathrm{~cm}^{-1}$

UV $(\mathrm{MeOH}): \lambda_{\max }(\log \varepsilon)=234$ (4.18), $260(4.04), 418(3.69), 443(3.64) \mathrm{nm}$

$(\mathrm{MeOH} / \mathrm{HCl}): \lambda_{\max }(\log \varepsilon)=233$ (4.17), 259 (4.01), 416 (3.64), $443(3.62) \mathrm{nm}$

$(\mathrm{MeOH} / \mathrm{NaOH}): \lambda_{\max }(\log \varepsilon)=261(4.35), 430(4.06) \mathrm{nm}$

CD $(\mathrm{MeOH}): \lambda_{\max }([\Theta])=212(-57900), 261(2900), 304(-9500) \mathrm{nm}$

$[\alpha]_{D}^{20}=-38^{\circ}(\mathrm{c}=1$ in Methanol $)$

NMR-Daten $\left(\mathrm{CD}_{3} \mathrm{OD}\right)$ siehe Tabelle 27, Seite 175 .

\subsubsection{Actinomycin $\mathrm{G}_{6}(16)$}

intensiv oranger Feststoff

$\mathbf{C}_{61} \mathbf{H}_{84} \mathbf{N}_{12} \mathbf{O}_{18}$ (1273.39)

Monoisotopische Masse: $\mathrm{M}_{\text {mono }}=1272.60$

Anfärbeverhalten: orange (Anisaldehyd)

$\mathbf{R}_{\mathbf{f}}=0.27\left(\mathrm{CHCl}_{3} / \mathrm{MeOH} 9: 1\right)$

$\mathbf{R}_{\mathbf{t}}=16.6 \min ($ HPLC 1, Säule 1, Programm 1)

ESI-MS (positive Ionen): $\mathrm{m} / \mathrm{z}=1273.6[\mathrm{M}+\mathrm{H}]^{+}, 1295.6[\mathrm{M}+\mathrm{Na}]^{+}$

ESI-MS (negative Ionen): $\mathrm{m} / \mathrm{z}=1271.6[\mathrm{M}-\mathrm{H}]^{-}$

HR-ESI-MS: berechnet $\mathrm{m} / \mathrm{z}=637.30860[\mathrm{M}+2 \mathrm{H}]^{2+}$

gefunden $\mathrm{m} / \mathrm{z}=637.30854$ (Abweichung $0.09 \mathrm{ppm}$ )

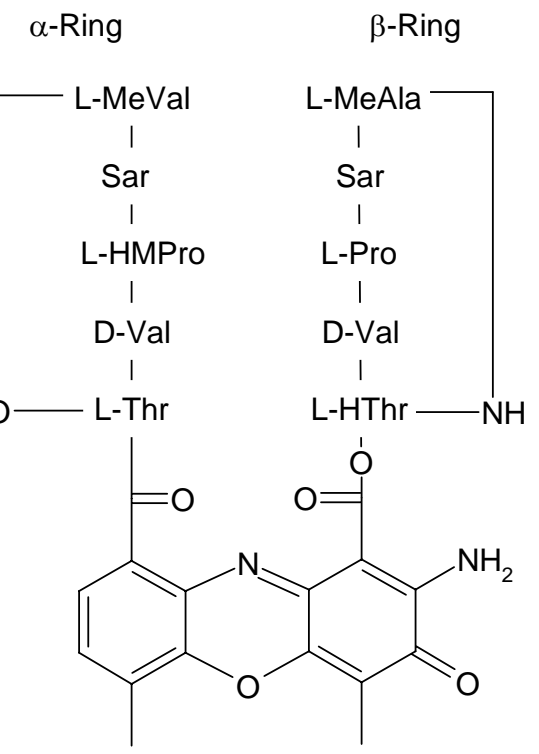


IR (KBr): $\widetilde{v}=3435,3313,2966(\mathrm{sh}), 2364,1732,1644,1582,1525,1447,1318,1475,1194$, $1101,1001 \mathrm{~cm}^{-1}$

UV $(\mathrm{MeOH}): \lambda_{\max }(\log \varepsilon)=237(4.45), 435(4.27) \mathrm{nm}$

$(\mathrm{MeOH} / \mathrm{HCl}): \lambda_{\max }(\log \varepsilon)=233(4.53), 405(4.14), 458(4.24), 486(4.11) \mathrm{nm}$

$(\mathrm{MeOH} / \mathrm{NaOH}): \lambda_{\max }(\log \varepsilon)=238(4.52), 433(4.32) \mathrm{nm}$

CD $(\mathrm{MeOH}): \lambda_{\max }([\Theta])=238(22000), 252(7600), 277(25600) \mathrm{nm}$

$[\alpha]_{D}^{20}=+16^{\circ}(\mathrm{c}=1$ in Methanol $)$

NMR-Daten $\left(\mathrm{CD}_{3} \mathrm{OD}\right)$ siehe Tabelle 28, Seite 176.

\subsubsection{1-Phenazinol (17)}

intensiv rot-brauner Feststoff

$\mathbf{C}_{12} \mathbf{H}_{8} \mathbf{N}_{2} \mathbf{O}(196.21)$

Anfärbeverhalten: rot-orange (Anisaldehyd)

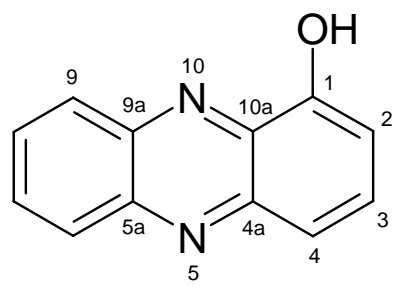

$\mathbf{R}_{\mathbf{f}}=0.75\left(\mathrm{CHCl}_{3} / \mathrm{MeOH} 9: 1\right)$

EI-MS (70 eV): m/z (\%) = $196(100)[M]^{+}, 168$ (65), 140 (8), 129 (2), 114 (4), 98 (3), 77 (10)

IR (KBr): $\widetilde{v}=3426,2924,2366,1636,1563,1520,1469,1427,1393,1359,1320,1218$, $1171,1116,1039 \mathrm{~cm}^{-1}$

UV (MeOH): $\lambda_{\max }(\log \varepsilon)=238$ (4.15), 262 (4.62), 355 (3.61), 363 (3.72), 368 (3.75), 420 , br (3.22) nm

$(\mathrm{MeOH} / \mathrm{HCl}): \lambda_{\max }(\log \varepsilon)=237$ (4.12), 264 (4.59), 368 (3.78), 383 (3.63), 423, br (3.14) $\mathrm{nm}$

$(\mathrm{MeOH} / \mathrm{NaOH}): \lambda_{\max }(\log \varepsilon)=239$ (4.35), 294 (4.56), 359 (3.34), 374 (3.35), 528, br (3.24) $\mathrm{nm}$

${ }^{1}$ H-NMR $\left(300 \mathrm{MHz}, \mathrm{CDCl}_{3}\right): 7.22(\mathrm{dd}, J=6.0,3.0 \mathrm{~Hz}, 1 \mathrm{H}, 2-\mathrm{H}), 7.75(\mathrm{~m}, 1 \mathrm{H}, 3-\mathrm{H}), 7.76(\mathrm{~m}$, 1H, 4-H), $7.81(\mathrm{~m}, 1 \mathrm{H}, 8-\mathrm{H})^{* *}, 7.84(\mathrm{~m}, 1 \mathrm{H}, 7-\mathrm{H})^{* *}, 8.20$ (m, 1H, 6-H)*, $8.24(\mathrm{~m}, 1 \mathrm{H}, 9-\mathrm{H})^{*}$. ${ }^{1}$ H-NMR (600 MHz, CD $\left.{ }_{3} \mathrm{OD}\right): 7.19$ (dd, $\left.J=7.5,1.0 \mathrm{~Hz}, 1 \mathrm{H}, 2-\mathrm{H}\right), 7.69$ (dd, $J=8.5,1.0 \mathrm{~Hz}$, 1H, 4-H), 7.78 (dd, $J=8.5,7.5 \mathrm{~Hz}, 1 \mathrm{H}, 3-\mathrm{H}), 7.89(\mathrm{~m}, 1 \mathrm{H}, 8-\mathrm{H})^{* *}, 7.92(\mathrm{~m}, 1 \mathrm{H}, 7-\mathrm{H})^{* *}, 8.18$ $(\mathrm{dd}, J=8.5,1.5 \mathrm{~Hz}, 1 \mathrm{H}, 6-\mathrm{H})^{*}, 8.32(\mathrm{~d}, J=8.0 \mathrm{~Hz}, 1 \mathrm{H}, 9-\mathrm{H})^{*}$.

${ }^{13}$ C-NMR (75.5 MHz, $\mathrm{CDCl}_{3}$ ): 108.9 (d, C-2), 119.9 (d, C-4), 129.2 (d, C-6)*, 129.6 (d, C-9)*, 130.5 (d, C-8)**, 130.8 (d, C-7)**, 131.8 (d, C-3), 134.7 (s, C-10a), 141.2 (s, C-4a), 143.8 (s, C-9a)***, 144.1 (s, C-5a)***, 151.7 (s, C-1).

$* / * * / * * *=$ Zuordnungen jeweils austauschbar 


\subsubsection{N-(2-Hydroxyphenyl)acetamid (18)}

farbloser Feststoff

$\mathrm{C}_{8} \mathrm{H}_{9} \mathrm{NO}_{2}(151.16)$

Anfärbeverhalten: braun (Anisaldehyd)<smiles>CC(=O)Nc1ccccc1O</smiles>

$\mathbf{R}_{\mathbf{f}}=0.40\left(\mathrm{CHCl}_{3} / \mathrm{MeOH} 9: 1\right)$

ESI-MS (positive Ionen): kein Spektrum erhalten

ESI-MS (negative Ionen): $\mathrm{m} / \mathrm{z}=150[\mathrm{M}-\mathrm{H}]^{-}, 301[2 \mathrm{M}-\mathrm{H}]^{-}$

UV (MeOH): $\lambda_{\max }(\log \varepsilon)=207$ (3.88), 242 (3.38), 283 (3.09) nm

$(\mathrm{MeOH} / \mathrm{HCl}): \lambda_{\max }(\log \varepsilon)=207$ (3.88), $242(3.40), 282(3.13) \mathrm{nm}$

$(\mathrm{MeOH} / \mathrm{NaOH}): \lambda_{\max }(\log \varepsilon)=220(3.69), 250(3.43), 306(3.29) \mathrm{nm}$

${ }^{1}$ H-NMR $\left(\mathrm{CDCl}_{3}, 600 \mathrm{MHz}\right): 2.16\left(\mathrm{~s}, 3 \mathrm{H}, 8-\mathrm{H}_{3}\right), 6.79(\mathrm{ddd}, J=8.0,8.0,1.5 \mathrm{~Hz}, 1 \mathrm{H}, 4-\mathrm{H})$, $6.84(\mathrm{dd}, J=8.0,1.5 \mathrm{~Hz}, 1 \mathrm{H}, 6-\mathrm{H}), 6.98(\mathrm{ddd}, J=8.0,8.0,1.5 \mathrm{~Hz}, 1 \mathrm{H}, 5-\mathrm{H}), 7.57$ (dd, $J=$ 8.0, $1.5 \mathrm{~Hz}, 1 \mathrm{H}, 3-\mathrm{H})$. 
Tabelle 22: NMR-Daten von Actinomycin $\mathbf{G}_{2} \quad{ }^{1} \mathrm{H}: 600 \mathrm{MHz},{ }^{13} \mathrm{C}: 150.8 \mathrm{MHz}, \mathrm{CDCl}_{3}$

\section{Pentapeptidlacton:}

$\begin{array}{llllllllll}\boldsymbol{\alpha} \text {-Ring } & \text { Pos. } & \delta_{\mathrm{C}} & \delta_{\mathrm{H}} & J[\mathrm{~Hz}] & \boldsymbol{\beta} \text {-Ring } & \text { Pos. } & \delta_{\mathrm{C}} & \delta_{\mathrm{H}} & J[\mathrm{~Hz}]\end{array}$

\begin{tabular}{|c|c|c|c|c|c|c|c|c|c|}
\hline \multirow[t]{6}{*}{ Thr } & 1 & 168.49 & - & - & \multirow[t]{6}{*}{ ClThr } & 1 & 168.07 & - & - \\
\hline & 2 & 54.82 & 4.44 & $\mathrm{dd}, 7.0,2.9$ & & 2 & 53.54 & 5.27 & $\mathrm{dd}, 7.6,2.7$ \\
\hline & 3 & 74.72 & 5.28 & $\mathrm{qd}, 6.3,2.9$ & & 3 & 74.98 & 5.12 & $\mathrm{dt}, 5.0,2.5$ \\
\hline & 4 & 17.15 & 1.20 & $\mathrm{~d}, 6.3$ & & 4 & 43.21 & 3.94 & $\mathrm{dd}, 11.4,2.5$ \\
\hline & \multirow[t]{2}{*}{$\mathrm{NH}$} & \multirow[t]{2}{*}{-} & \multirow[t]{2}{*}{6.96} & \multirow{2}{*}{$\mathrm{d}, 7.1$} & & & & 3.99 & $\mathrm{dd}, 11.4,6.0$ \\
\hline & & & & & & $\mathrm{NH}$ & - & 7.88 & $\mathrm{~d}, 7.3$ \\
\hline \multirow[t]{6}{*}{$D$-Val } & 1 & 173.91 & - & - & \multirow[t]{6}{*}{$D$-Val } & 1 & 173.23 & - & - \\
\hline & 2 & 59.38 & 3.37 & $\mathrm{dd}, 10.0,5.0$ & & 2 & 58.57 & 3.66 & $\mathrm{~m}$ \\
\hline & 3 & 31.77 & 2.10 & $\mathrm{~m}$ & & 3 & 31.78 & 2.08 & $\mathrm{~m}$ \\
\hline & 4 & 19.25 & 1.10 & $\mathrm{~d}, 6.7$ & & 4 & 19.28 & 0.90 & $\mathrm{~d}, 6.7$ \\
\hline & 5 & 19.04 & 0.86 & $\mathrm{~d}, 6.8$ & & 5 & 19.00 & 1.09 & $\mathrm{~d}, 6.7$ \\
\hline & $\mathrm{NH}$ & - & 8.20 & $\mathrm{~d}, 5.2$ & & $\mathrm{NH}$ & - & 8.12 & d, 6.2 \\
\hline \multirow{8}{*}{ HMPro } & 1 & 170.95 & - & - & \multirow[t]{8}{*}{ Pro } & 1 & 173.17 & - & - \\
\hline & 2 & 68.22 & 5.97 & $\mathrm{~s}$ & & 2 & 56.82 & 5.90 & $\mathrm{~d}, 9.0$ \\
\hline & 3 & 75.44 & 4.06 & $\mathrm{~d}, 5.5$ & & 3 & 31.07 & 1.84 & $\mathrm{dd}, 12.0,6.5$ \\
\hline & \multirow[t]{2}{*}{4} & \multirow[t]{2}{*}{43.21} & 2.11 & $\mathrm{~m}$ & & & & 2.91 & $\mathrm{~m}$ \\
\hline & & & 2.19 & $\mathrm{~m}$ & & 4 & 22.67 & 2.07 & $\mathrm{~m}$ \\
\hline & 5 & 53.78 & 4.76 & $\mathrm{~m}$ & & & & 2.18 & $\mathrm{~m}$ \\
\hline & 6 & 18.73 & 1.48 & $\mathrm{~d}, 6.0$ & & 5 & 47.48 & 3.68 & $\mathrm{~m}$ \\
\hline & & & & & & & & 3.80 & $\mathrm{~m}$ \\
\hline \multirow[t]{4}{*}{ Sar } & 1 & 166.12 & - & - & \multirow[t]{4}{*}{ Sar } & 1 & 166.52 & - & - \\
\hline & 2 & 51.51 & 3.64 & $\mathrm{~d}, 17.5$ & & 2 & 51.25 & 3.61 & $\mathrm{~d}, 17.5$ \\
\hline & & & 4.74 & $\mathrm{~d}, 17.5$ & & & & 4.69 & $\mathrm{~d}, 17.5$ \\
\hline & $\mathrm{NMe}$ & 34.97 & 2.86 & $\mathrm{~S}$ & & $\mathrm{NMe}$ & 34.84 & 2.83 & $\mathrm{~S}$ \\
\hline \multirow[t]{6}{*}{ MeVal } & 1 & 167.53 & - & - & \multirow[t]{6}{*}{ MeAla } & 1 & 168.85 & - & - \\
\hline & 2 & 71.27 & 2.65 & $\mathrm{~d}, 9.3$ & & 2 & 59.60 & 3.33 & $\mathrm{q}, 7.0$ \\
\hline & 3 & 26.92 & 2.63 & $\mathrm{~m}$ & & 3 & 13.42 & 1.32 & $\mathrm{~d}, 7.0$ \\
\hline & 4 & 21.63 & 0.93 & $\mathrm{~d}, 6.4$ & & $\mathrm{NMe}$ & 36.71 & 2.91 & $\mathrm{~S}$ \\
\hline & 5 & 19.00 & 0.71 & $\mathrm{~d}, 6.8$ & & & & & \\
\hline & $\mathrm{NMe}$ & 39.40 & 2.91 & $\mathrm{~s}$ & & & & & \\
\hline
\end{tabular}

\section{Chromophor:}

${ }^{1} \mathrm{H}-\mathrm{NMR}: \delta=2.13\left(\mathrm{~s}, 3 \mathrm{H}, 12-\mathrm{H}_{3}\right), 2.49\left(\mathrm{~s}, 3 \mathrm{H}, 11-\mathrm{H}_{3}\right), 7.31(\mathrm{dq}, J=7.7,0.9 \mathrm{~Hz}, 1 \mathrm{H}, 7-\mathrm{H}), 7.53(\mathrm{~d}$, $J=7.6 \mathrm{~Hz}, 1 \mathrm{H}, 8-\mathrm{H})$.

${ }^{13} \mathrm{C}-\mathrm{NMR}: \delta=7.79$ (q, C-12), 14.97 (C-11), 99.92 (s, C-1), 113.81 (s, C-4), 125.36 (d, C-8), 127.57 (s, C-6), 128.86 (s, C-9a), 130.26 (d, C-7), 132.65 (s, C-9), 140.47 (s, C-5a), 145.18 (s, C-4a), 146.04 (s, C-10a), 147.67 (s, C-2), 166.52 (s, C-13), 168.96 (s, C-14), $178.49(\mathrm{C}-3)$. 
Tabelle 23: NMR-Daten von Actinomycin $\mathbf{G}_{2} \quad{ }^{1} \mathrm{H}: 600 \mathrm{MHz},{ }^{13} \mathrm{C}: 150.8 \mathrm{MHz}, \mathrm{CD}_{3} \mathrm{OD}$

\section{Pentapeptidlacton:}

$\begin{array}{llllllllll}\boldsymbol{\alpha} \text {-Ring } & \text { Pos. } & \delta_{\mathrm{C}} & \delta_{\mathrm{H}} & J[\mathrm{~Hz}] & \boldsymbol{\beta} \text {-Ring } & \text { Pos. } & \delta_{\mathrm{C}} & \delta_{\mathrm{H}} & J[\mathrm{~Hz}]\end{array}$

\begin{tabular}{|c|c|c|c|c|c|c|c|c|c|}
\hline \multirow[t]{6}{*}{ Thr } & 1 & 169.87 & - & - & \multirow[t]{6}{*}{ ClThr } & 1 & 169.58 & - & - \\
\hline & 2 & 56.24 & 4.61 & $\mathrm{~d}, 2.5$ & & 2 & 54.05 & 5.40 & $\mathrm{~d}, 2.5$ \\
\hline & 3 & 75.59 & 5.26 & $\mathrm{qd}, 6.0,3.0$ & & 3 & 77.42 & 5.16 & ddd, $6.5,3.5$ \\
\hline & \multirow[t]{3}{*}{4} & \multirow[t]{3}{*}{17.36} & \multirow[t]{3}{*}{1.25} & \multirow[t]{3}{*}{$\mathrm{d}, 6.0$} & & & & & 2.5 \\
\hline & & & & & & 4 & 43.18 & 3.82 & $\mathrm{dd}, 11.0,6.5$ \\
\hline & & & & & & & & 3.85 & $\mathrm{dd}, 11.0,3.5$ \\
\hline \multirow[t]{6}{*}{$D$-Val } & 1 & 175.11 & - & - & \multirow[t]{6}{*}{$D$-Val } & 1 & 175.35 & - & - \\
\hline & 2 & 60.57 & 3.51 & $\mathrm{dd}, 10.0,5.0$ & & 2 & 59.91 & 3.79 & $\mathrm{dd}, 9.5,6.0$ \\
\hline & 3 & 33.13 & 2.11 & $\mathrm{~m}$ & & 3 & 33.06 & 2.11 & $\mathrm{~m}$ \\
\hline & 4 & 19.88 & 1.12 & $\mathrm{~d}, 6.5$ & & 4 & 19.92 & 0.94 & $\mathrm{~d}, 6.5$ \\
\hline & 5 & 19.67 & 0.88 & $\mathrm{~d}, 6.5$ & & 5 & 19.62 & 1.14 & $\mathrm{~d}, 6.5$ \\
\hline & $\mathrm{NH}$ & - & 8.19 & $\mathrm{~d}, 5.0$ & & $\mathrm{NH}$ & - & 8.02 & $\mathrm{~d}, 6.0$ \\
\hline \multirow[t]{8}{*}{ HMPro } & 1 & 173.22 & - & - & \multirow[t]{8}{*}{ Pro } & 1 & 175.59 & - & - \\
\hline & 2 & 69.57 & 6.23 & $\mathrm{~s}$ & & 2 & 58.85 & 6.03 & $\mathrm{~d}, 9.0$ \\
\hline & 3 & 76.62 & 4.11 & $\mathrm{~d}, 5.5$ & & 3 & 32.28 & 1.89 & $\mathrm{~m}$ \\
\hline & \multirow[t]{3}{*}{4} & \multirow[t]{3}{*}{41.82} & \multirow[t]{2}{*}{2.02} & \multirow{2}{*}{$\begin{array}{l}\text { ddd, 13.5, 10.0 } \\
5.5\end{array}$} & & & & 2.86 & $\mathrm{~m}$ \\
\hline & & & & & & 4 & 23.66 & 2.11 & $\mathrm{~m}$ \\
\hline & & & 2.29 & $\mathrm{dd}, 13.5,7.0$ & & 5 & 48.78 & 3.69 & $\mathrm{~m}$ \\
\hline & 5 & 55.16 & 5.01 & $\mathrm{~m}$ & & & & 3.90 & $\mathrm{~m}$ \\
\hline & 6 & 19.23 & 1.49 & $\mathrm{~d}, 6.0$ & & & & & \\
\hline \multirow[t]{4}{*}{ Sar } & 1 & 168.65 & - & - & \multirow[t]{4}{*}{ Sar } & 1 & 168.24 & - & - \\
\hline & 2 & 52.67 & 4.04 & $\mathrm{~d}, 18.0$ & & 2 & 52.45 & 3.95 & $\mathrm{~d}, 18.0$ \\
\hline & & & 4.72 & $\mathrm{~d}, 18.0$ & & & & 4.76 & $\mathrm{~d}, 18.0$ \\
\hline & $\mathrm{NMe}$ & 35.53 & 2.86 & $\mathrm{~s}$ & & $\mathrm{NMe}$ & 35.51 & 2.84 & $\mathrm{~s}$ \\
\hline \multirow[t]{6}{*}{ MeVal } & 1 & 169.68 & - & - & \multirow[t]{6}{*}{ MeAla } & 1 & 171.07 & - & - \\
\hline & 2 & 72.01 & 3.00 & $\mathrm{~m}$ & & 2 & 60.77 & 3.57 & $\mathrm{q}, 7.0$ \\
\hline & 3 & 28.35 & 2.58 & $\mathrm{~m}$ & & 3 & 13.59 & 1.30 & $\mathrm{~d}, 6.0$ \\
\hline & 4 & 21.78 & 0.98 & $\mathrm{~d}, 6.5$ & & $\mathrm{NMe}$ & 36.82 & 2.94 & $\mathrm{~s}$ \\
\hline & 5 & 19.38 & 0.80 & $\mathrm{~d}, 6.5$ & & & & & \\
\hline & $\mathrm{NMe}$ & 39.37 & 2.99 & $\mathrm{~s}$ & & & & & \\
\hline
\end{tabular}

\section{Chromophor:}

${ }^{1} \mathrm{H}-\mathrm{NMR}: \delta=2.19\left(\mathrm{~s}, 3 \mathrm{H}, 12-\mathrm{H}_{3}\right), 2.53\left(\mathrm{~s}, 3 \mathrm{H}, 11-\mathrm{H}_{3}\right), 7.42(\mathrm{dq}, J=7.5,1.0 \mathrm{~Hz}, 1 \mathrm{H}, 7-\mathrm{H}), 7.46$ $(\mathrm{d}, J=7.5 \mathrm{~Hz}, 1 \mathrm{H}, 8-\mathrm{H})$.

${ }^{13} \mathrm{C}-\mathrm{NMR}: \delta=7.79(\mathrm{q}, \mathrm{C}-12), 15.00(\mathrm{C}-11), 101.79$ (s, C-1), 114.48 (s, C-4), 125.91 (d, C-8), 128.98 (s, C-6), 130.78 (s, C-9a), 131.31 (d, C-7), 134.03 (s, C-9), 141.84 (s, C-5a), 146.70 (s, C-4a), 147.09 (s, C-10a), 148.73 (s, C-2), 169.09 (s, C-13), 170.04 (s, C-14), $180.11(\mathrm{C}-3)$. 
Tabelle 24: NMR-Daten von Actinomycin $\mathrm{G}_{3} \quad{ }^{1} \mathrm{H}: 600 \mathrm{MHz},{ }^{13} \mathrm{C}: 150.8 \mathrm{MHz}, \mathrm{CDCl}_{3}$

\section{Pentapeptidlacton:}

$\begin{array}{llllllllll}\boldsymbol{\alpha} \text {-Ring } & \text { Pos. } & \delta_{\mathrm{C}} & \delta_{\mathrm{H}} & J[\mathrm{~Hz}] & \boldsymbol{\beta} \text {-Ring } & \text { Pos. } & \delta_{\mathrm{C}} & \delta_{\mathrm{H}} & J[\mathrm{~Hz}]\end{array}$

\begin{tabular}{|c|c|c|c|c|c|c|c|c|c|}
\hline \multirow[t]{6}{*}{ Thr } & 1 & 168.57 & - & - & \multirow[t]{6}{*}{ HThr } & 1 & 168.40 & - & - \\
\hline & 2 & 55.01 & 4.46 & $\mathrm{dd}, 7.0,3.0$ & & 2 & 51.96 & 5.20 & $\mathrm{dd}, 7.0,2.0$ \\
\hline & 3 & 74.83 & 5.25 & $\mathrm{qd}, 6.0,3.0$ & & 3 & 75.39 & 5.05 & ddd, 8.5, 5.0, \\
\hline & 4 & 17.20 & 1.22 & $\mathrm{~d}, 6.0$ & & 4 & 59.40 & 3.23 & $\mathrm{dd}, 12.0,9.0$ \\
\hline & \multirow[t]{2}{*}{$\mathrm{NH}$} & \multirow[t]{2}{*}{-} & \multirow[t]{2}{*}{6.95} & \multirow[t]{2}{*}{$\mathrm{d}, 6.5$} & & & & 3.76 & $\mathrm{dd}, 12.0,4.5$ \\
\hline & & & & & & $\mathrm{NH}$ & - & 8.28 & $\mathrm{~d}, 6.5$ \\
\hline \multirow[t]{6}{*}{$D$-Val } & 1 & 174.03 & - & - & \multirow[t]{6}{*}{$D$-Val } & 1 & 173.46 & - & - \\
\hline & 2 & 59.50 & 3.39 & $\mathrm{dd}, 10.0,5.5$ & & 2 & 58.57 & 3.73 & $\mathrm{~m}$ \\
\hline & 3 & 31.80 & 2.14 & $\mathrm{~m}$ & & 3 & 31.88 & 2.13 & $\mathrm{~m}$ \\
\hline & 4 & 19.35 & 1.12 & $\mathrm{~d}, 6.5$ & & 4 & 19.39 & 0.94 & d, 6.5 \\
\hline & 5 & 18,98 & 0.88 & $\mathrm{~d}, 6.5$ & & 5 & 19.04 & 1.14 & d, 6.5 \\
\hline & $\mathrm{NH}$ & - & 8.30 & d, 6.0 & & $\mathrm{NH}$ & - & 7.99 & $\mathrm{~d}, 6.5$ \\
\hline \multirow[t]{8}{*}{ HMPro } & 1 & 170.84 & - & - & \multirow[t]{8}{*}{ Pro } & 1 & 173.17 & - & - \\
\hline & 2 & 68.07 & 5.92 & $\mathrm{~s}$ & & 2 & 56.80 & 5.85 & d, 9.0 \\
\hline & 3 & 75.51 & 4.07 & d, 5.0 & & 3 & 31.16 & 1.87 & $\mathrm{~d}, 12.5,7.0$ \\
\hline & \multirow[t]{2}{*}{4} & \multirow[t]{2}{*}{41.06} & 2.11 & $\mathrm{~m}$ & & \multirow{3}{*}{4} & \multirow{3}{*}{22.64} & 2.96 & $\mathrm{~m}$ \\
\hline & & & 2.15 & $\mathrm{~m}$ & & & & 2.08 & $\mathrm{~m}$ \\
\hline & 5 & 53.46 & 4.63 & $\mathrm{~m}$ & & & & 2.20 & $\mathrm{~m}$ \\
\hline & \multirow[t]{2}{*}{6} & \multirow[t]{2}{*}{18.81} & \multirow[t]{2}{*}{1.50} & \multirow[t]{2}{*}{$\mathrm{d}, 6.0$} & & \multirow[t]{2}{*}{5} & \multirow[t]{2}{*}{47.61} & 3.72 & $\mathrm{~m}$ \\
\hline & & & & & & & & 3.77 & $\mathrm{~m}$ \\
\hline \multirow[t]{4}{*}{ Sar } & 1 & 166.10 & - & - & \multirow[t]{4}{*}{ Sar } & 1 & 166.50 & - & - \\
\hline & 2 & 51.54 & 3.64 & $\mathrm{~d}, 17.5$ & & 2 & 51.31 & 3.59 & $\mathrm{~d}, 17.5$ \\
\hline & & & 4.74 & $\mathrm{~d}, 17.5$ & & & & 4.72 & d, 17.5 \\
\hline & $\mathrm{NMe}$ & 35.03 & 2.87 & $\mathrm{~s}$ & & $\mathrm{NMe}$ & 34.88 & 2.84 & $\mathrm{~s}$ \\
\hline \multirow[t]{6}{*}{ MeVal } & 1 & 167.55 & - & - & \multirow[t]{6}{*}{ MeAla } & 1 & 169.26 & - & - \\
\hline & 2 & 71.32 & 2.67 & d, 9.0 & & 2 & 59.79 & 3.21 & $\mathrm{q}, 6.5$ \\
\hline & 3 & 26.98 & 2.66 & $\mathrm{~m}$ & & 3 & 13.48 & 1.29 & $\mathrm{~d}, 6.5$ \\
\hline & 4 & 21.67 & 0.95 & $\mathrm{~d}, 6.5$ & & $\mathrm{NMe}$ & 36.63 & 2.86 & $\mathrm{~s}$ \\
\hline & 5 & 19.04 & 0.72 & $\mathrm{~d}, 6.5$ & & & & & \\
\hline & $\mathrm{NMe}$ & 39.38 & 2.93 & $\mathrm{~s}$ & & & & & \\
\hline
\end{tabular}

\section{Chromophor:}

${ }^{1} \mathrm{H}-\mathrm{NMR}: \delta=2.18\left(\mathrm{~s}, 3 \mathrm{H}, 12-\mathrm{H}_{3}\right), 2.51\left(\mathrm{~s}, 3 \mathrm{H}, 11-\mathrm{H}_{3}\right), 7.34(\mathrm{~d}, J=7.5 \mathrm{~Hz}, 1 \mathrm{H}, 7-\mathrm{H}), 7.55(\mathrm{~d}, J=$ $7.5 \mathrm{~Hz}, 1 \mathrm{H}, 8-\mathrm{H})$.

${ }^{13} \mathrm{C}-\mathrm{NMR}: \delta=7.85$ (q, C-12), 15.02 (C-11), 98.84 (s, C-1), 114.08 (s, C-4), 125.59 (d, C-8), 127.62 (s, C-6), 128.69 (s, C-9a), 130.55 (d, C-7), 132.83 (s, C-9), 140.51 (s, C-5a), 145.42 (s, C-4a), 146.12 (s, C-10a), 148.38 (s, C-2), 166.37 (s, C-13), 169.39 (s, C-14), 178.20 (C-3). 
Tabelle 25: NMR-Daten von Actinomycin $\mathbf{G}_{3} \quad{ }^{1} \mathrm{H}: 600 \mathrm{MHz},{ }^{13} \mathrm{C}: 150.8 \mathrm{MHz}, \mathrm{CD}_{3} \mathrm{OD}$

\section{Pentapeptidlacton:}

$\begin{array}{llllllllll}\boldsymbol{\alpha} \text {-Ring } & \text { Pos. } & \delta_{\mathrm{C}} & \delta_{\mathrm{H}} & J[\mathrm{~Hz}] & \boldsymbol{\beta} \text {-Ring } & \text { Pos. } & \delta_{\mathrm{C}} & \delta_{\mathrm{H}} & J[\mathrm{~Hz}]\end{array}$

\begin{tabular}{|c|c|c|c|c|c|c|c|c|c|}
\hline \multirow[t]{4}{*}{ Thr } & 1 & 169.97 & - & - & \multirow[t]{4}{*}{ HThr } & 1 & 169.92 & - & - \\
\hline & 2 & 56.16 & 4.75 & $\mathrm{~m}$ & & 2 & 53.39 & 5.13 & $\mathrm{~m}$ \\
\hline & 3 & 75.84 & 5.28 & $\mathrm{qd}, 6.0,2.5$ & & 3 & 78.49 & 5.07 & $\mathrm{~m}$ \\
\hline & 4 & 17.34 & 1.29 & $\mathrm{~d}, 6.5$ & & 4 & 60.50 & $\begin{array}{l}3.51 \\
3.69\end{array}$ & $\begin{array}{l}\mathrm{m} \\
\mathrm{dd}, 12.0,4.5\end{array}$ \\
\hline \multirow[t]{6}{*}{$D$-Val } & 1 & 175.68 & - & - & \multirow[t]{6}{*}{$D$-Val } & 1 & 175.47 & - & - \\
\hline & 2 & 60.36 & 3.55 & $\mathrm{~m}$ & & 2 & 59.95 & 3.81 & $\mathrm{~m}$ \\
\hline & 3 & 33.18 & 2.13 & $\mathrm{~m}$ & & 3 & 33.01 & 2.14 & $\mathrm{~m}$ \\
\hline & 4 & 19.93 & 1.13 & $\mathrm{~d}, 6.5$ & & 4 & 19.93 & 0.92 & $\mathrm{~d}, 6.5$ \\
\hline & 5 & 19.64 & 0.90 & $\mathrm{~d}, 6.5$ & & 5 & 19.68 & 1.16 & $\mathrm{~d}, 6.5$ \\
\hline & $\mathrm{NH}$ & - & 8.29 & d, 5.0 & & $\mathrm{NH}$ & - & 8.22 & $\mathrm{~m}$ \\
\hline \multirow[t]{7}{*}{ HMPro } & 1 & 173.32 & - & - & \multirow[t]{7}{*}{ Pro } & 1 & 175.14 & - & - \\
\hline & 2 & 68.99 & 6.20 & $\mathrm{~s}$ & & 2 & 58.73 & 6.11 & d, 9.0 \\
\hline & 3 & 76.60 & 4.18 & $\mathrm{~m}$ & & 3 & 32.20 & 1.85 & $\mathrm{~m}$ \\
\hline & \multirow[t]{2}{*}{4} & \multirow[t]{2}{*}{41.78} & 2.00 & $\mathrm{~m}$ & & & & 2.84 & $\mathrm{~m}$ \\
\hline & & & 2.28 & $\mathrm{dd}, 13.0,7.0$ & & 4 & 23.70 & 2.11 & $\mathrm{~m}$ \\
\hline & 5 & 54.86 & 4.92 & $\mathrm{~m}$ & & 5 & 48.77 & 3.68 & $\mathrm{~m}$ \\
\hline & 6 & 19.35 & 1.47 & $\mathrm{~d}, 6.0$ & & & & 3.96 & $\mathrm{~m}$ \\
\hline \multirow[t]{4}{*}{ Sar } & 1 & 168.27 & - & - & \multirow[t]{4}{*}{ Sar } & 1 & 169.15 & - & - \\
\hline & 2 & 52.71 & 4.04 & $\mathrm{~d}, 17.5$ & & 2 & 52.47 & 3.94 & $\mathrm{~d}, 17.5$ \\
\hline & & & 4.77 & $\mathrm{~d}, 17.5$ & & & & 4.71 & $\mathrm{~d}, 17.5$ \\
\hline & $\mathrm{NMe}$ & 35.60 & 2.86 & $\mathrm{~s}$ & & $\mathrm{NMe}$ & 35.47 & 2.83 & $\mathrm{~s}$ \\
\hline \multirow[t]{6}{*}{ MeVal } & 1 & 169.15 & - & - & \multirow[t]{6}{*}{ MeAla } & 1 & 170.14 & - & - \\
\hline & 2 & 71.97 & 3.01 & d, 9.0 & & 2 & 60.97 & 3.52 & $\mathrm{q}, 7.0$ \\
\hline & 3 & 28.32 & 2.58 & dhept, 9.0, 6.5 & & 3 & 13.52 & 1.29 & $\mathrm{~d}, 7.0$ \\
\hline & 4 & 21.74 & 0.98 & d, 6.5 & & $\mathrm{NMe}$ & 36.90 & 2.94 & $\mathrm{~s}$ \\
\hline & 5 & 19.37 & 0.80 & $\mathrm{~d}, 7.0$ & & & & & \\
\hline & $\mathrm{NMe}$ & 39.37 & 2.96 & $\mathrm{~s}$ & & & & & \\
\hline
\end{tabular}

\section{Chromophor:}

${ }^{1} \mathrm{H}-\mathrm{NMR}: \delta=2.01\left(\mathrm{~s}, 3 \mathrm{H}, 12-\mathrm{H}_{3}\right), 2.55\left(\mathrm{~s}, 3 \mathrm{H}, 11-\mathrm{H}_{3}\right), 7.43(\mathrm{~d}, J=7.5 \mathrm{~Hz}, 1 \mathrm{H}, 7-\mathrm{H}), 7.44(\mathrm{~d}, J=$ $7.5 \mathrm{~Hz}, 1 \mathrm{H}, 8-\mathrm{H})$.

${ }^{13} \mathrm{C}-\mathrm{NMR}: \delta=7.57$ (q, C-12), 15.00 (C-11), 102.32 (s, C-1), 114.14 (s, C-4), 125.97 (d, C-8), 128.78 (s, C-6), 130.57 (s, C-9a), 131.30 (d, C-7), 134.48 (s, C-9), 141.73 (s, C-5a), 146.52 (s, C-4a), 146.91 (s, C-10a), 148.54 (s, C-2), 168.51 (s, C-13), 171.34 (s, C-14), 180.30 (C-3). 
Tabelle 26: NMR-Daten von Actinomycin $\mathbf{G}_{4} \quad{ }^{1} \mathrm{H}: 600 \mathrm{MHz},{ }^{13} \mathrm{C}: 150.8 \mathrm{MHz}, \mathrm{CD}_{3} \mathrm{OD}$

\begin{tabular}{|c|c|c|c|c|c|c|c|c|c|}
\hline \multicolumn{10}{|c|}{ Pentapeptidlacton: } \\
\hline$\alpha$-Ring & Pos. & $\delta_{\mathrm{C}}$ & $\delta_{\mathrm{H}}$ & $J[\mathrm{~Hz}]$ & $\beta$-Ring & Pos. & $\delta_{\mathrm{C}}$ & $\delta_{\mathrm{H}}$ & $J[\mathrm{~Hz}]$ \\
\hline \multirow[t]{4}{*}{ Thr } & 1 & & - & - & \multirow[t]{4}{*}{ Thr } & 1 & & - & - \\
\hline & 2 & 55.92 & 5.08 & $\mathrm{~d}, 2.5$ & & 2 & 56.19 & 4.63 & $\mathrm{~d}, 3.0$ \\
\hline & 3 & 75.76 & 5.25 & $\mathrm{qd}, 6.5,3.0$ & & 3 & 76.43 & 5.13 & $\mathrm{qd}, 6.5,2.5$ \\
\hline & 4 & 17.37 & 1.26 & $\mathrm{~d}, 6.5$ & & 4 & 17.46 & 1.30 & d, 6.5 \\
\hline \multirow[t]{6}{*}{$D$-Val } & 1 & & - & - & \multirow[t]{6}{*}{$D$-Val } & 1 & & - & - \\
\hline & 2 & 60.45 & 3.52 & $\mathrm{~m}$ & & 2 & 59.79 & 3.79 & $\mathrm{~m}$ \\
\hline & 3 & 33.20 & 2.09 & $\mathrm{~m}$ & & 3 & 33.16 & 2.09 & $\mathrm{~m}$ \\
\hline & 4 & 19.81 & 1.12 & $\mathrm{~d}, 6.5$ & & 4 & 19.90 & 0.93 & $\mathrm{~d}, 6.5$ \\
\hline & 5 & 19.68 & 0.89 & $\mathrm{~d}, 6.5$ & & 5 & 19.57 & 1.15 & $\mathrm{~d}, 6.5$ \\
\hline & $\mathrm{NH}$ & - & 8.14 & $\mathrm{~d}, 5.0$ & & $\mathrm{NH}$ & - & 8.04 & $\mathrm{~d}, 5.5$ \\
\hline \multirow[t]{7}{*}{ HMPro } & 1 & & - & - & \multirow[t]{7}{*}{ Pro } & 1 & & - & - \\
\hline & 2 & 69.67 & 6.22 & $\mathrm{~S}$ & & 2 & 58.75 & 6.09 & $\mathrm{~d}, 9.0$ \\
\hline & 3 & 76.77 & 4.11 & $\mathrm{~d}, 5.5$ & & 3 & 32.16 & 1.87 & $\mathrm{~m}$ \\
\hline & \multirow[t]{2}{*}{4} & \multirow[t]{2}{*}{41.97} & 2.03 & $\mathrm{~m}$ & & & & 2.85 & $\mathrm{~m}$ \\
\hline & & & 2.34 & $\mathrm{~m}$ & & 4 & 23.74 & 2.12 & $\mathrm{~m}$ \\
\hline & 5 & 55.44 & 4.87 & $\mathrm{~m}$ & & 5 & 48.77 & 3.68 & $\mathrm{~m}$ \\
\hline & 6 & 19.37 & 1.52 & $\mathrm{~d}, 6.0$ & & & & 3.93 & $\mathrm{~m}$ \\
\hline \multirow[t]{4}{*}{ Sar } & 1 & & - & - & \multirow[t]{4}{*}{ Sar } & 1 & & - & - \\
\hline & 2 & 52.64 & 4.05 & $\mathrm{~d}, 17.5$ & & 2 & 52.43 & 3.95 & $\mathrm{~d}, 17.5$ \\
\hline & & & 4.71 & $\mathrm{~d}, 17.5$ & & & & 4.73 & $\mathrm{~d}, 17.5$ \\
\hline & $\mathrm{NMe}$ & 35.52 & 2.86 & $\mathrm{~s}$ & & $\mathrm{NMe}$ & 35.46 & 2.84 & $\mathrm{~s}$ \\
\hline \multirow[t]{6}{*}{ MeVal } & 1 & & - & - & \multirow[t]{6}{*}{ MeAla } & 1 & & - & - \\
\hline & 2 & 71.98 & 3.00 & $\mathrm{~d}, 10.0$ & & 2 & 61.17 & 3.47 & $\mathrm{q}, 7.0$ \\
\hline & 3 & 28.37 & 2.57 & $\mathrm{~m}$ & & 3 & 13.49 & 1.28 & $\mathrm{~d}, 7.0$ \\
\hline & 4 & 21.77 & 0.98 & $\mathrm{~d}, 6.5$ & & $\mathrm{NMe}$ & 36.90 & 2.94 & $\mathrm{~s}$ \\
\hline & 5 & 19.34 & 0.80 & $\mathrm{~d}, 6.5$ & & & & & \\
\hline & $\mathrm{NMe}$ & 39.36 & 2.99 & $\mathrm{~S}$ & & & & & \\
\hline
\end{tabular}

\section{Chromophor:}

${ }^{1} \mathrm{H}-\mathrm{NMR}: \delta=2.22\left(\mathrm{~s}, 3 \mathrm{H}, 12-\mathrm{H}_{3}\right), 2.55\left(\mathrm{~s}, 3 \mathrm{H}, 11-\mathrm{H}_{3}\right), 7.43(\mathrm{dq}, J=7.5,0.5 \mathrm{~Hz}, 1 \mathrm{H}, 7-\mathrm{H}), 7.47$ $(\mathrm{d}, J=7.5 \mathrm{~Hz}, 1 \mathrm{H}, 8-\mathrm{H})$.

${ }^{13} \mathrm{C}-\mathrm{NMR}: \delta=7.80(\mathrm{q}, \mathrm{C}-12), 15.00$ (C-11). $126.0(\mathrm{~d}, \mathrm{C}-8), 131.3(\mathrm{~d}, \mathrm{C}-7)$.

Die chemischen Verschiebungen der quartären C-Atome waren aufgrund der geringen Substanzmenge dem ${ }^{13} \mathrm{C}$-NMR-Spektrum nicht eindeutig zu entnehmen und werden daher hier nicht angegeben. 
Tabelle 27: NMR-Daten von Actinomycin $\mathbf{G}_{5} \quad{ }^{1} \mathrm{H}: 600 \mathrm{MHz},{ }^{13} \mathrm{C}: 150.8 \mathrm{MHz}, \mathrm{CD}_{3} \mathrm{OD}$

\section{Pentapeptidlacton:}

$\begin{array}{llllllllll}\boldsymbol{\alpha} \text {-Ring } & \text { Pos. } & \delta_{\mathrm{C}} & \delta_{\mathrm{H}} & J[\mathrm{~Hz}] & \boldsymbol{\beta} \text {-Ring } & \text { Pos. } & \delta_{\mathrm{C}} & \delta_{\mathrm{H}} & J[\mathrm{~Hz}]\end{array}$

\begin{tabular}{|c|c|c|c|c|c|c|c|c|c|}
\hline \multirow[t]{5}{*}{ Thr } & 1 & 171.85 & - & - & \multirow[t]{5}{*}{ HThr } & 1 & 165.80 & - & - \\
\hline & 2 & 56.46 & 4.97 & $\mathrm{~m}$ & & 2 & 54.04 & 4.07 & $\mathrm{~d}, 3.0$ \\
\hline & 3 & 74.87 & 5.36 & $\mathrm{qd}, 6.5,2.5$ & & 3 & 64.71 & 5.53 & $\mathrm{~m}$ \\
\hline & 4 & 17.30 & 1.33 & $\mathrm{~d}, 6.5$ & & 4 & 68.48 & 4.64 & $\mathrm{dd}, 11.0,1.0$ \\
\hline & & & & & & & & 4.86 & $\mathrm{~m}$ \\
\hline \multirow[t]{6}{*}{$D$-Val } & 1 & 174.99 & - & - & \multirow[t]{6}{*}{$D$-Val } & 1 & 176.97 & - & - \\
\hline & 2 & 59.58 & 3.89 & $\mathrm{~d}, 10.0$ & & 2 & 60.88 & 3.72 & $\mathrm{dd}, 10.0,5.5$ \\
\hline & 3 & 32.61 & 2.20 & $\mathrm{~m}$ & & 3 & 33.48 & 2.07 & $\mathrm{~m}$ \\
\hline & 4 & 19.76 & 0.90 & $\mathrm{~d}, 6.5$ & & 4 & 19.86 & 1.11 & $\mathrm{~d}, 6.5$ \\
\hline & 5 & 19.23 & 1.16 & $\mathrm{~d}, 6.5$ & & 5 & 19.54 & 0.90 & $\mathrm{~d}, 6.5$ \\
\hline & $\mathrm{NH}$ & - & 8.53 & s & & $\mathrm{NH}$ & - & 8.16 & $\mathrm{~d}, 6.0$ \\
\hline \multirow[t]{8}{*}{ HMPro } & 1 & 172.57 & - & - & \multirow[t]{8}{*}{ Pro } & 1 & 175.73 & - & - \\
\hline & 2 & 68.66 & 6.34 & $\mathrm{~s}$ & & 2 & 58.32 & 6.19 & $\mathrm{dd}, 9.0,3.0$ \\
\hline & 3 & 74.68 & 4.25 & $\mathrm{~d}, 3.0$ & & 3 & 32.32 & 1.83 & $\mathrm{~m}$ \\
\hline & 4 & 40.72 & 1.97 & $\mathrm{~m}$ & & & & 2.54 & $\mathrm{~m}$ \\
\hline & & & 2.12 & $\mathrm{~m}$ & & 4 & 24.23 & 2.02 & $\mathrm{~m}$ \\
\hline & 5 & 55.16 & 4.21 & $\mathrm{~m}$ & & & & 2.08 & $\mathrm{~m}$ \\
\hline & 6 & 18.73 & 1.46 & $\mathrm{~d}, 6.0$ & & 5 & 48.75 & 3.63 & $\mathrm{~m}$ \\
\hline & & & & & & & & 3.85 & $\mathrm{~m}$ \\
\hline \multirow[t]{4}{*}{ Sar } & 1 & 168.46 & - & - & \multirow[t]{4}{*}{ Sar } & 1 & 168.53 & - & - \\
\hline & 2 & 52.87 & 4.08 & $\mathrm{~d}, 18.0$ & & 2 & 52.72 & 3.97 & $\mathrm{~d}, 18.0$ \\
\hline & & & 4.84 & $\mathrm{~d}, 18.0$ & & & & 4.80 & $\mathrm{~d}, 18.0$ \\
\hline & $\mathrm{NMe}$ & 35.37 & 2.84 & $\mathrm{~s}$ & & $\mathrm{NMe}$ & 35.46 & 2.86 & $\mathrm{~s}$ \\
\hline \multirow[t]{6}{*}{ MeVal } & 1 & 169.62 & - & - & \multirow[t]{6}{*}{ MeAla } & 1 & 170.93 & - & - \\
\hline & 2 & 72.07 & 3.09 & $\mathrm{~d}, 9.0$ & & 2 & 61.01 & 3.78 & $\mathrm{q}, 7.0$ \\
\hline & 3 & 28.24 & 2.63 & $\mathrm{~m}$ & & 3 & 13.57 & 1.40 & $\mathrm{~d}, 7.0$ \\
\hline & 4 & 21.75 & 1.01 & $\mathrm{~d}, 6.5$ & & $\mathrm{NMe}$ & 37.28 & 2.84 & s \\
\hline & 5 & 18.41 & 0.82 & $\mathrm{~d}, 6.5$ & & & & & \\
\hline & NMe & 39.62 & 3.07 & s & & & & & \\
\hline
\end{tabular}

\section{Chromophor:}

${ }^{1} \mathrm{H}-\mathrm{NMR}: \delta=2.10\left(\mathrm{~s}, 3 \mathrm{H}, 12-\mathrm{H}_{3}\right), 2.48\left(\mathrm{~s}, 3 \mathrm{H}, 11-\mathrm{H}_{3}\right), 7.43(\mathrm{~d}, J=7.5 \mathrm{~Hz}, 1 \mathrm{H}, 7-\mathrm{H}), 7.44(\mathrm{~d}, J=$ $7.5 \mathrm{~Hz}, 1 \mathrm{H}, 8-\mathrm{H})$.

${ }^{13} \mathrm{C}-\mathrm{NMR}: \delta=8.02$ (q, C-12), 15.01 (C-11), 94.74 (s, C-1), 117.42 (s, C-4), 125.83 (d, C-8), 128.88 (s, C-9a), 128.99 (s, C-6), 130.05 (d, C-7), 132.11 (s, C-9), 141.47 (s, C-5a), 149.35 (s, C-4a), 150.17 (s, C-10a), 167.98 (s, C-2), 169.58 (s, C-13), 174.71 (s, C-14), 182.64 (C-3). 
Tabelle 28: NMR-Daten von Actinomycin $\mathbf{G}_{6} \quad{ }^{1} \mathrm{H}: 600 \mathrm{MHz},{ }^{13} \mathrm{C}: 150.8 \mathrm{MHz}, \mathrm{CD}_{3} \mathrm{OD}$

\begin{tabular}{|c|c|c|c|c|c|c|c|c|c|}
\hline \multicolumn{10}{|c|}{ Pentapeptidlacton: } \\
\hline$\alpha$-Ring & Pos. & $\delta_{\mathrm{C}}$ & $\delta_{\mathrm{H}}$ & $J[\mathrm{~Hz}]$ & $\beta$-Ring & Pos. & $\delta_{\mathrm{C}}$ & $\delta_{\mathrm{H}}$ & $J[\mathrm{~Hz}]$ \\
\hline \multirow[t]{5}{*}{ Thr } & 1 & 171.31 & - & - & \multirow[t]{5}{*}{ HThr } & 1 & 171.63 & - & - \\
\hline & 2 & 58.22 & 5.00 & $\mathrm{~d}, 2.2$ & & 2 & 54.93 & 4.75 & $\mathrm{~d}, 3.7$ \\
\hline & 3 & 73.69 & 5.38 & $\mathrm{qd}, 6.5,2.4$ & & 3 & 70.10 & 4.40 & $\mathrm{ddd}, 8.0,4.0,4.0$ \\
\hline & \multirow[t]{2}{*}{4} & \multirow[t]{2}{*}{17.81} & \multirow{2}{*}{1.47} & \multirow{2}{*}{$\mathrm{d}, 6.4$} & & 4 & 67.13 & 4.24 & $\mathrm{dd}, 11.1,8.5$ \\
\hline & & & & & & & & 4.47 & $\mathrm{dd}, 11.1,4.1$ \\
\hline \multirow[t]{5}{*}{$D$-Val } & 1 & 174.20 & - & - & \multirow[t]{5}{*}{$D$-Val } & 1 & 172.28 & - & - \\
\hline & 2 & 59.84 & 3.56 & $\mathrm{~d}, 10.0$ & & 2 & 59.15 & 4.43 & $\mathrm{~d}, 10.9$ \\
\hline & 3 & 32.44 & 2.03 & $\mathrm{~m}$ & & 3 & 29.60 & 2.06 & $\mathrm{~m}$ \\
\hline & 4 & 19.82 & 1.05 & $\mathrm{~d}, 6.7$ & & 4 & 19.53 & 0.90 & $\mathrm{~d}, 6.6$ \\
\hline & 5 & 19.70 & 0.84 & $\mathrm{~d}, 6.7$ & & 5 & 19.13 & 0.95 & $\mathrm{~d}, 6.7$ \\
\hline \multirow[t]{8}{*}{ HMPro } & 1 & 173.52 & - & - & \multirow[t]{8}{*}{ Pro } & 1 & 175.20 & - & - \\
\hline & 2 & 67.43 & 6.22 & $\mathrm{~s}$ & & 2 & 58.22 & 4.94 & $\mathrm{dd}, 8.5,4.5$ \\
\hline & 3 & 75.75 & 4.20 & $\mathrm{~m}$ & & 3 & 29.12 & 1.84 & $\mathrm{~m}$ \\
\hline & \multirow[t]{2}{*}{4} & \multirow[t]{2}{*}{40.88} & 1.86 & $\mathrm{~m}$ & & & & 2.28 & $\mathrm{~m}$ \\
\hline & & & 1.95 & $\mathrm{~m}$ & & 4 & 25.76 & 1.95 & $\mathrm{~m}$ \\
\hline & 5 & 54.56 & 4.31 & sext, 6.4 & & & & 2.07 & $\mathrm{~m}$ \\
\hline & 6 & 19.25 & 1.33 & $\mathrm{~d}, 6.3$ & & 5 & 48.92 & 3.64 & $\mathrm{~m}$ \\
\hline & & & & & & & & 4.02 & $\mathrm{~m}$ \\
\hline \multirow[t]{4}{*}{ Sar } & 1 & 168.78 & - & - & \multirow[t]{4}{*}{ Sar } & 1 & 172.37 & - & - \\
\hline & \multirow[t]{2}{*}{2} & \multirow[t]{2}{*}{53.09} & 4.01 & $\mathrm{~d}, 18.0$ & & 2 & 51.79 & 3.33 & $\mathrm{~d}, 14.5$ \\
\hline & & & 5.00 & $\mathrm{~d}, 18.0$ & & & & 4.58 & $\mathrm{~d}, 14.5$ \\
\hline & $\mathrm{NMe}$ & 35.79 & 2.86 & $\mathrm{~s}$ & & $\mathrm{NMe}$ & 38.71 & 3.35 & $\mathrm{~s}$ \\
\hline \multirow[t]{6}{*}{ MeVal } & 1 & 170.06 & - & - & \multirow[t]{6}{*}{ MeAla } & 1 & 173.72 & - & - \\
\hline & 2 & 71.88 & 3.20 & $\mathrm{~d}, 9.5$ & & 2 & 54.21 & 5.35 & $\mathrm{q}, 7.3$ \\
\hline & 3 & 28.19 & 2.67 & dhept, $9.5,6.5$ & & 3 & 14.74 & 1.44 & $\mathrm{~d}, 7.3$ \\
\hline & 4 & 22.17 & 1.09 & $\mathrm{~d}, 6.6$ & & $\mathrm{NMe}$ & 32.31 & 3.14 & $\mathrm{~S}$ \\
\hline & 5 & 19.47 & 0.85 & $\mathrm{~d}, 6.8$ & & & & & \\
\hline & $\mathrm{NMe}$ & 39.37 & 3.03 & $\mathrm{~S}$ & & & & & \\
\hline
\end{tabular}

\section{Chromophor:}

${ }^{1} \mathrm{H}-\mathrm{NMR}: \delta=2.06\left(\mathrm{~s}, 3 \mathrm{H}, 12-\mathrm{H}_{3}\right), 2.43\left(\mathrm{~s}, 3 \mathrm{H}, 11-\mathrm{H}_{3}\right), 7.37(\mathrm{~d}, J=7.8 \mathrm{~Hz}, 1 \mathrm{H}, 7-\mathrm{H}), 7.77(\mathrm{~d}, J=$ $7.8 \mathrm{~Hz}, 1 \mathrm{H}, 8-\mathrm{H})$.

${ }^{13} \mathrm{C}-\mathrm{NMR}: \delta=7.94$ (q, C-12), 15.19 (C-11), 98.24 (s, C-1), 114.54 (s, C-4), 127.08 (d, C-8), 129.75 (s, C-9), 130.27 (s, C-6), 131.15 (d, C-7), 131.84 (s, C-9a), 141.62 (s, C-5a), 146.98 (s, C-4a)*, 147.01 (s, C-10a)*, 149.90 (s, C-2), 168.49 (s, C-13), 167.00 (s, C-14), 179.48 (s, C-3) * = die Zuordnung der beiden Atome ist austauschbar. 


\subsection{Substanzen aus Halomonas sp. Stamm Rk377a}

\subsubsection{Polyisopropylenglycol (32)}

farbloses Öl

$\left[\mathrm{C}_{3} \mathrm{H}_{6} \mathrm{O}\right]_{\mathrm{n}}$

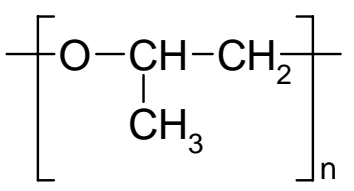

Anfärbeverhalten: weißer Fleck bei Färbung mit Anisaldehyd oder Orcin.

$\mathbf{R}_{\mathbf{f}}=$ ca. $0.78\left(\mathrm{CHCl}_{3} / \mathrm{MeOH} 9: 1\right)$

EI-MS $(70 \mathrm{eV})$ : Es sind Signale für die Summenformel $\left[\mathrm{C}_{3} \mathrm{H}_{6} \mathrm{O}\right]_{\mathrm{n}} \mathrm{H}$ von $\mathrm{n}=1$ bis $\mathrm{n}=6$ sichtbar. $\mathrm{m} / \mathrm{z}(\%)=349$ (2), 291 (4), 233 (8), 175 (17), 117 (43), 59 (100)

${ }^{1} \mathbf{H}-\mathbf{N M R}\left(300 \mathrm{MHz}, \mathrm{CDCl}_{3}\right): \delta=1.10\left(\mathrm{~m}, 3 \mathrm{H}, \mathrm{CH}_{3}\right), 3.37(\mathrm{~m}, 1 \mathrm{H}, \mathrm{CH}), 3.52\left(\mathrm{~m}, 2 \mathrm{H}, \mathrm{CH}_{2}\right)$.

${ }^{13}$ C-NMR (50.3 MHz, $\left.\mathrm{CDCl}_{3}\right)$ : es treten 3 Gruppen von Signalen auf. $\delta=17.3-17.4\left(\mathrm{q}, \mathrm{CH}_{3}\right)$, $72.8-73.3\left(\mathrm{t}, \mathrm{CH}_{2}\right), 75.0-75.4(\mathrm{~d}, \mathrm{CH})$.

\subsubsection{Cyclo(prolylvalyl) (33)}

farbloser Feststoff

$\mathbf{C}_{10} \mathbf{H}_{16} \mathbf{N}_{2} \mathbf{O}_{2}(196.25)$

Anfärbeverhalten: braun (Anisaldehyd oder Orcin)

$\mathbf{R}_{\mathbf{f}}=0.35\left(\mathrm{CHCl}_{3} / \mathrm{MeOH} 9: 1\right)$

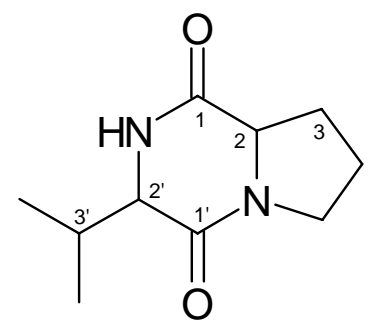

$\mathbf{R}_{\mathbf{t}}=7.7$ min (HPLC 1, Säule 1, Programm 1)

EI-MS (70 eV): m/z (\%) = $196(5)[\mathrm{M}]^{+}, 154(100)\left[\mathrm{M}-\mathrm{C}_{3} \mathrm{H}_{6}\right]^{+}, 125(30), 72(52), 70(95), 69$ (14), $55(8), 43(8), 41(21)$

${ }^{1}$ H-NMR (300 MHz, $\left.\mathrm{CD}_{3} \mathrm{OD}\right): \delta=0.93$ (d, $\left.J=7.0 \mathrm{~Hz}, 3 \mathrm{H}, 4^{\prime}-\mathrm{H}_{3}\right), 1.09$ (d, $J=7.0 \mathrm{~Hz}, 3 \mathrm{H}$, $\left.5^{\prime}-\mathrm{H}_{3}\right), 1.86-2.06\left(\mathrm{~m}, 3 \mathrm{H}, 3-\mathrm{H}_{\mathrm{a}}, 4-\mathrm{H}_{2}\right), 2.32\left(\mathrm{~m}, 1 \mathrm{H}, 3-\mathrm{H}_{\mathrm{b}}\right), 2.48$ (sept d, J = 7.0, $2.5 \mathrm{~Hz}, 1 \mathrm{H}$, 3'-H), 3.52 (m, 2H, 5- $\mathrm{H}_{2}$ ), 4.03 (m, 1H, 2'-H), 4.20 (td, $\left.J=5.0,2.0 \mathrm{~Hz}, 1 \mathrm{H}, 2-\mathrm{H}\right)$. 


\subsubsection{Cyclo(leucylprolyl) (34)}

farbloser Feststoff

$\mathrm{C}_{11} \mathbf{H}_{18} \mathbf{N}_{2} \mathbf{O}_{2}(210.28)$

Anfärbeverhalten: braun (Anisaldehyd oder Orcin)

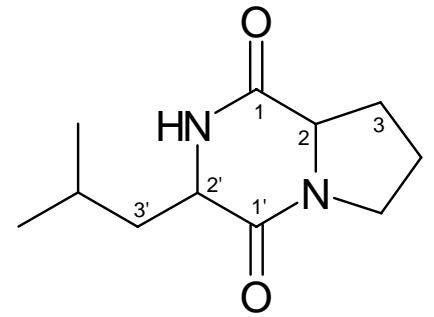

$\mathbf{R}_{\mathbf{f}}=0.45\left(\mathrm{CHCl}_{3} / \mathrm{MeOH} 9: 1\right)$

$\mathbf{R}_{\mathbf{t}}=9.8 \min ($ HPLC 1, Säule 1, Programm 1)

EI-MS $(70 \mathrm{eV}): \mathrm{m} / \mathrm{z}(\%)=210(1)[\mathrm{M}]^{+}, 195(3)\left[\mathrm{M}-\mathrm{CH}_{3}\right]^{+}, 167(5), 154(100)\left[\mathrm{M}_{-} \mathrm{C}_{4} \mathrm{H}_{8}\right]^{+}$, 139 (5), 125 (11), 96 (4), 86 (18), 70 (48), 55 (4), 43 (6), 41 (7)

${ }^{1}$ H-NMR (300 MHz, CD $\left.{ }_{3} \mathrm{OD}\right): \delta=0.94\left(\mathrm{~d}, J=6.5 \mathrm{~Hz}, 3 \mathrm{H}, 5^{\prime}-\mathrm{H}_{3}\right), 0.95(\mathrm{~d}, J=6.5 \mathrm{~Hz}, 3 \mathrm{H}$, 6'- $\left.-\mathrm{H}_{3}\right), 1.51\left(\mathrm{~m}, 1 \mathrm{H}, 3-\mathrm{H}_{\mathrm{a}}\right), 1.82-2.09$ (m, 5H, 3'- $\left.\mathrm{H}_{\mathrm{a}}, 4^{\prime}-\mathrm{H}, 3-\mathrm{H}_{\mathrm{b}}, 4-\mathrm{H}_{2}\right), 2.29\left(\mathrm{~m}, 1 \mathrm{H}, 3\right.$ '- $\left.\mathrm{H}_{\mathrm{b}}\right)$, $3.50\left(\mathrm{~m}, 2 \mathrm{H}, 5-\mathrm{H}_{2}\right), 4.12\left(\mathrm{~m}, 1 \mathrm{H}, 2^{\prime}-\mathrm{H}\right), 4.26(\mathrm{td}, J=7.5,2.0,1 \mathrm{H}, 2-\mathrm{H})$.

${ }^{1}$ H-NMR $\left(600 \mathrm{MHz}\right.$, DMSO-d $\left.{ }_{6}\right): \delta=0.86\left(\mathrm{~d}, J=6.5 \mathrm{~Hz}, 3 \mathrm{H}, 5^{\prime}-\mathrm{H}_{3}\right), 0.86$ (verdeckt, $1 \mathrm{H}$, 4- $\left.\mathrm{H}_{\mathrm{a}}\right), 0.87\left(\mathrm{~d}, J=6.5 \mathrm{~Hz}, 3 \mathrm{H}, 6^{\prime}-\mathrm{H}_{3}\right), 1.36\left(\mathrm{~m}, 1 \mathrm{H}, 3^{\prime}-\mathrm{H}_{\mathrm{a}}\right), 1.77\left(\mathrm{~m}, 1 \mathrm{H}, 3^{\prime}-\mathrm{H}_{\mathrm{b}}\right), 1.82(\mathrm{~m}, 1 \mathrm{H}$, 4- $\left.\mathrm{H}_{\mathrm{b}}\right), 1.87$ (m, 1H, 4'-H), $1.92\left(\mathrm{~m}, 1 \mathrm{H}, 3-\mathrm{H}_{\mathrm{a}}\right), 2.12$ (m, 1H, 3- $\left.\mathrm{H}_{\mathrm{b}}\right), 3.4$ (verdeckt, 2H, 5- $\mathrm{H}_{2}$ ), 3.99 (t, $J=6.0 \mathrm{~Hz}, 1 \mathrm{H}, 2$ '-H), 4.18 (t, $J=8.0 \mathrm{~Hz}, 1 \mathrm{H}, 2-\mathrm{H})$.

${ }^{13}$ C-NMR (150 MHz, DMSO-d d $_{6}$ ): $\delta=21.8$ (q, C-5'), 22.4 (t, C-4), 22.7 (q, C-6'), 24.0 (d, C-4'), 27.3 (t, C-3), 37.7 (t, C-3'), 44.8 (t, C-5), 52.6 (d, C-2'), 58.4 (d, C-2), 166.4 (s, C-1'), $170.2(\mathrm{~s}, \mathrm{C}-1)$.

\subsubsection{Cyclo(isoleucylprolyl) (35)}

farbloser Feststoff

$\mathbf{C}_{11} \mathbf{H}_{18} \mathbf{N}_{2} \mathbf{O}_{2}(210.28)$

Anfärbeverhalten: braun (Anisaldehyd oder Orcin)

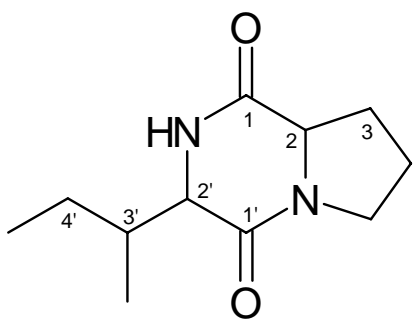

$\mathbf{R}_{\mathbf{f}}=0.36\left(\mathrm{CHCl}_{3} / \mathrm{MeOH} 9: 1\right)$

$\mathbf{R}_{\mathbf{t}}=9.4 \min ($ HPLC 1, Säule 1, Programm 1)

EI-MS $(70 \mathrm{eV}): \mathrm{m} / \mathrm{z}(\%)=154(100)\left[\mathrm{M}-\mathrm{C}_{4} \mathrm{H}_{8}\right]^{+}, 86(27), 70(46), 21$ (18)

ESI-MS (positive Ionen): $\mathrm{m} / \mathrm{z}=443[2 \mathrm{M}+\mathrm{Na}]^{+}$

${ }^{1}$ H-NMR (300 MHz, CD $\left.{ }_{3} \mathrm{OD}\right): \delta=0.92\left(\mathrm{t}, J=7.0 \mathrm{~Hz}, 5^{\prime}-\mathrm{H}_{3}\right), 1.04$ (d, $J=7.0 \mathrm{~Hz}, 6{ }^{\prime}-\mathrm{H}_{3}$ ), $1.30\left(\mathrm{~m}, 3-\mathrm{H}_{\mathrm{a}}\right), 1.86-2.20\left(\mathrm{~m}, 3^{\prime}-\mathrm{H}, 4^{\prime}-\mathrm{H}_{2}, 4-\mathrm{H}_{2}\right), 2.32\left(\mathrm{~m}, 3-\mathrm{H}_{\mathrm{b}}\right), 3.54\left(\mathrm{~m}, 5-\mathrm{H}_{2}\right), 4.06(\mathrm{~m}$, 2-H), 4.19 (m, 2'-H).

Eine Analyse der Integrale war nicht möglich, da die Substanz nicht einheitlich vorlag. Die Zuordnung der Signale erfolgte in Analogie zu 33 und 34. 


\subsubsection{Cyclo(phenylalanylprolyl) (36)}

farbloser Feststoff

$\mathrm{C}_{14} \mathrm{H}_{16} \mathbf{N}_{2} \mathbf{O}_{2}(244.30)$

Anfärbeverhalten: gelb (Anisaldehyd)

$\mathbf{R}_{\mathbf{f}}=0.50\left(\mathrm{CHCl}_{3} / \mathrm{MeOH} 9: 1\right)$

$\mathbf{R}_{\mathbf{t}}=9.0 \min ($ HPLC 1, Säule 1, Programm 1)

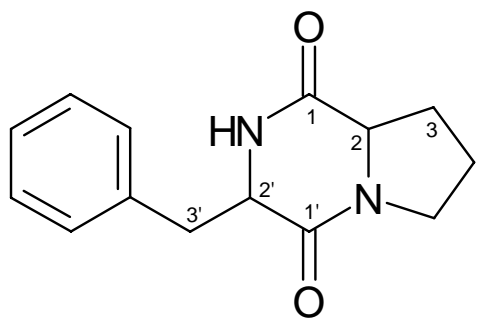

EI-MS $(70 \mathrm{eV}): \mathrm{m} / \mathrm{z}(\%)=244(36)[\mathrm{M}]^{+}, 153(24)\left[\mathrm{M}-\mathrm{Ph}-\mathrm{CH}_{2}\right]^{+}, 125$ (65), 91 (50), 70 (100), $55(10), 41(27)$

ESI-MS (positive Ionen): $\mathrm{m} / \mathrm{z}=267[\mathrm{M}+\mathrm{Na}]^{+}, 511[2 \mathrm{M}+\mathrm{Na}]^{+}$

${ }^{1}$ H-NMR $\left(600 \mathrm{MHz}, \mathrm{CD}_{3} \mathrm{OD}\right): \delta=1.20\left(\mathrm{~m}, 1 \mathrm{H}, 3-\mathrm{H}_{\mathrm{a}}\right), 1.80\left(\mathrm{~m}, 2 \mathrm{H}, 4-\mathrm{H}_{2}\right), 2.08(\mathrm{~m}, 1 \mathrm{H}$, 3- $\left.\mathrm{H}_{\mathrm{b}}\right), 3.14\left(\mathrm{dd}, J=14.0,5.0 \mathrm{~Hz}, 1 \mathrm{H}, 3^{\prime}-\mathrm{H}_{\mathrm{a}}\right), 3.17\left(\mathrm{dd}, J=14.0,5.5 \mathrm{~Hz}, 1 \mathrm{H}, 3^{\prime}-\mathrm{H}_{\mathrm{b}}\right), 3.37$ (m, $\left.1 \mathrm{H}, 5-\mathrm{H}_{\mathrm{a}}\right), 3.53\left(\mathrm{dt}, J=12.0,8.5 \mathrm{~Hz}, 1 \mathrm{H}, 5-\mathrm{H}_{\mathrm{b}}\right), 4.06(\mathrm{ddd}, J=11.0,6.0,2.0 \mathrm{~Hz}, 1 \mathrm{H}, 2-\mathrm{H})$, 4.44 (tm, $J=5.0$ Hz, 1H, 2'-H), 7.23 (m, 3H, 5'-H, 7'-H, 9'-H), 7.27 (m, 2H, 6'-H, 8'-H).

\subsubsection{Cyclo(prolyltyrosyl) (37)}

farbloser Feststoff

$\mathbf{C}_{14} \mathbf{H}_{16} \mathbf{N}_{2} \mathbf{O}_{3}(260.30)$

Anfärbeverhalten: gelb (Anisaldehyd)

$\mathbf{R}_{\mathbf{f}}=0.46\left(\mathrm{CHCl}_{3} / \mathrm{MeOH} 9: 1\right)$<smiles>O=C1NC(Cc2ccc(O)cc2)C(=O)N2CCCC12</smiles>

$\mathbf{R}_{\mathbf{t}}=7.6 \min ($ HPLC 1, Säule 1, Programm 1)

EI-MS $(70 \mathrm{eV}): \mathrm{m} / \mathrm{z}(\%)=260(18)[\mathrm{M}]^{+}, 154(100)\left[\mathrm{M}-\mathrm{PhOH}-\mathrm{CH}_{2}\right]^{+}, 107$ (43), 91 (3), 77 (4), $70(22), 55(1), 41(4)$

${ }^{1}$ H-NMR $\left(300 \mathrm{MHz}, \mathrm{CD}_{3} \mathrm{OD}\right): \delta=1.20\left(\mathrm{~m}, 1 \mathrm{H}, 3-\mathrm{H}_{\mathrm{a}}\right), 1.79\left(\mathrm{~m}, 2 \mathrm{H}, 4-\mathrm{H}_{2}\right), 2.08(\mathrm{~m}, 1 \mathrm{H}$, 3- $\left.\mathrm{H}_{\mathrm{b}}\right), 3.01\left(\mathrm{dd}, J=14.0,4.5 \mathrm{~Hz}, 1 \mathrm{H}, 3^{\prime}-\mathrm{H}_{\mathrm{a}}\right), 3.08\left(\mathrm{dd}, J=14.0,5.0 \mathrm{~Hz}, 1 \mathrm{H}, 3^{\prime}-\mathrm{H}_{\mathrm{b}}\right), 3.35$ (m, $\left.1 \mathrm{H}, 5-\mathrm{H}_{\mathrm{a}}\right), 3.54\left(\mathrm{dt}, J=12.0,8.5 \mathrm{~Hz}, 1 \mathrm{H}, 5-\mathrm{H}_{\mathrm{b}}\right), 4.04$ (ddd, $J=11.0,6.2,2.0 \mathrm{~Hz}, 1 \mathrm{H}, 2$ '- $\mathrm{H}$ ), $4.35(\mathrm{td}, J=5.0,2.0 \mathrm{~Hz}, 1 \mathrm{H}, 2-\mathrm{H}), 6.69$ (d, $\left.J=8.5 \mathrm{~Hz}, 2 \mathrm{H}, 6^{\prime}-\mathrm{H}, 8^{\prime}-\mathrm{H}\right), 7.03$ (d, $J=8.5 \mathrm{~Hz}$, 2H, 5'-H, 9'-H). 


\subsubsection{Diisooctylphthalat (38)}

$=\operatorname{Bis}(2$-ethylhexyl)phthalat

farbloses Ö1

$\mathrm{C}_{24} \mathrm{H}_{38} \mathrm{O}_{4}$ (390.57)

Anfärbeverhalten: violett (Anisaldehyd)

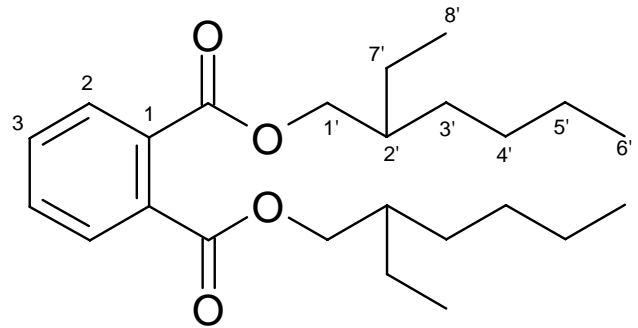

$\mathbf{R}_{\mathbf{f}}=0.45\left(\mathrm{CHCl}_{3} / \mathrm{MeOH} 9: 1\right)$

$\mathbf{R}_{\mathbf{t}}=10.6 \min ($ HPLC 1, Säule 1, Programm 1)

ESI-MS (positive Ionen): $\mathrm{m} / \mathrm{z}=413[\mathrm{M}+\mathrm{Na}]^{+}, 803[2 \mathrm{M}+\mathrm{Na}]^{+}$

${ }^{1} \mathbf{H}$-NMR (300 MHz, CD $\left.{ }_{3} \mathrm{OD}\right): \delta=0.90\left(\mathrm{t}, J=7.5 \mathrm{~Hz}, 12 \mathrm{H}, 6\right.$ ' $\left.-\mathrm{H}_{3}, 8^{\prime}-\mathrm{H}_{3}\right), 1.22-1.45(\mathrm{~m}, 16 \mathrm{H}$, 3' $-\mathrm{H}_{2}, 4^{\prime}-\mathrm{H}_{2}, 5^{\prime}-\mathrm{H}_{2}, 7^{\prime}-\mathrm{H}_{2}$ ), 1.66 (nonett, $J=6.0 \mathrm{~Hz}, 2 \mathrm{H}, 2^{\prime}-\mathrm{H}$ ), 4.19 (dd, $J=14.0,6.0,2 \mathrm{H}$, $1^{\prime}-\mathrm{H}_{\mathrm{a}}$ ), 4.21 (dd, $J=14.0,6.0,2 \mathrm{H}, 1^{\prime}-\mathrm{H}_{\mathrm{b}}$ ), 7.54 (m, 2H, 3-H, 4-H), 7.69 (m, 2H, 2-H, 5-H).

\subsubsection{2-Aminophenoxazin-3-on (39)}

intensiv roter Feststoff

$\mathrm{C}_{12} \mathrm{H}_{8} \mathrm{~N}_{2} \mathbf{O}_{2}(212.21)$

Anfärbeverhalten: braun-violett (Orcin)

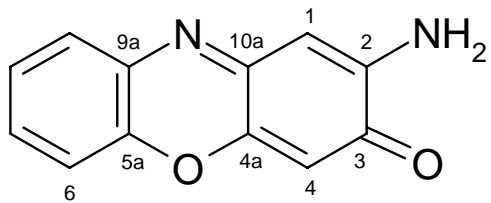

$\mathbf{R}_{\mathbf{f}}=0.54\left(\mathrm{CHCl}_{3} / \mathrm{MeOH} 9: 1\right)$

$\mathbf{R}_{\mathbf{t}}=12.9 \min ($ HPLC 1, Säule 1, Programm 1)

EI-MS $(70 \mathrm{eV}): \mathrm{m} / \mathrm{z}(\%)=212(100)[\mathrm{M}]^{+}, 185(35)$

IR (KBr): $\widetilde{v}=3407,3314,1580,1472,1420,1278,1176,1106,1054,841,755,699,575$, $542 \mathrm{~cm}^{-1}$

UV $(\mathrm{MeOH}): \lambda_{\max }(\log \varepsilon)=237(4.34), 421(4.26), 431(4.26) \mathrm{nm}$

$(\mathrm{MeOH} / \mathrm{HCl}): \lambda_{\max }(\log \varepsilon)=236(4.36), 438 \mathrm{sh}(4.07), 462(4.10), 481 \mathrm{sh}(4.06) \mathrm{nm}$ $(\mathrm{MeOH} / \mathrm{NaOH}): \lambda_{\max }(\log \varepsilon)=236(4.32), 419(4.22), 432(4.24) \mathrm{nm}$

${ }^{1} \mathrm{H}-\mathrm{NMR}\left(300 \mathrm{MHz}, \mathrm{CDCl}_{3}\right): \delta=5.10\left(\mathrm{br} \mathrm{s}, 2 \mathrm{H}, \mathrm{NH}_{2}\right), 6.40(\mathrm{~s}, 1 \mathrm{H}, 4-\mathrm{H}), 6.46(\mathrm{~s}, 1 \mathrm{H}, 1-\mathrm{H})$, 7.32-7.46 (m, 3H, 6-H, 7-H, 8-H), 7.74 (dd, $J=8.0,2.0 \mathrm{~Hz}, 1 \mathrm{H}, 9-\mathrm{H})$.

${ }^{1} \mathrm{H}-\mathrm{NMR}\left(600 \mathrm{MHz}\right.$, DMSO-d $\left.\mathrm{d}_{6}\right): \delta=6.34(\mathrm{~s}, 1 \mathrm{H}, 4-\mathrm{H}), 6.37(\mathrm{~s}, 1 \mathrm{H}, 1-\mathrm{H}), 6.76($ br s, $2 \mathrm{H}$, $\left.\mathrm{NH}_{2}\right), 7.38(\mathrm{td}, J=8.0,1.5 \mathrm{~Hz}, 1 \mathrm{H}, 8-\mathrm{H}), 7.45(\mathrm{td}, J=8.0,1.5 \mathrm{~Hz}, 1 \mathrm{H}, 7-\mathrm{H}), 7.48(\mathrm{dd}, J=8.0$, $1.5 \mathrm{~Hz}, 1 \mathrm{H}, 6-\mathrm{H}), 7.69$ (dd, $J=8.0,1.5 \mathrm{~Hz}, 1 \mathrm{H}, 9-\mathrm{H})$. 
${ }^{13}$ C-NMR (75.5 MHz, DMSO-d $\left.{ }_{6}\right): \delta=98.4$ (d, C-1), 103.3 (d, C-4), 115.8 (d, C-6), 125.2 (d, C-8), 127.9 (d, C-9), 128.7 (d, C-7), 133.7 (s, C-9a), 141.9 (s, C-5a), 147.3 (s, C-4a)*, 148.2 $(\mathrm{s}, \mathrm{C}-10 \mathrm{a})^{*}, 148.8$ (s, C-2), 180.1 (s, C-3).

* Zuordnungen austauschbar. Die angegebene Zuordnung erfolgte anhand von Inkrementberechnungen ${ }^{[6]}$.

\subsubsection{6-Hydroxy-2-aminophenoxazin-3-on (40)}

intensiv roter Feststoff

$\mathbf{C}_{12} \mathbf{H}_{8} \mathbf{N}_{2} \mathbf{O}_{3}(228.21)$

Anfärbeverhalten: braun-violett (Orcin)

$\mathbf{R}_{\mathbf{f}}=0.43\left(\mathrm{CHCl}_{3} / \mathrm{MeOH} 9: 1\right)$<smiles>Nc1cc2nc3cccc(O)c3oc-2cc1=O</smiles>

$\mathbf{R}_{\mathbf{t}}=9.9 \min$ (HPLC 1, Säule 1, Programm 1)

EI-MS $(70 \mathrm{eV}): \mathrm{m} / \mathrm{z}(\%)=228(100)[\mathrm{M}]^{+}, 201(40)$

ESI-MS (positive Ionen): $\mathrm{m} / \mathrm{z}=229[\mathrm{M}+\mathrm{H}]^{+}, 250[\mathrm{M}+\mathrm{Na}]^{+}$

HR-ESI-MS: berechnet $\mathrm{m} / \mathrm{z}=229.06077[\mathrm{M}+\mathrm{H}]^{+}$

gefunden $\mathrm{m} / \mathrm{z}=229.06079$ (Abweichung $0.09 \mathrm{ppm}$ )

IR (KBr): $\widetilde{v}=3431,2931,1643$ (sh), 1589, 1506, 1460, 1382, 1277, 1212, 1085, 1027, 834, $763,716,669 \mathrm{~cm}^{-1}$

UV $(\mathrm{MeOH}): \lambda_{\max }(\log \varepsilon)=236(3.93), 277(3.75), 430(3.72) \mathrm{nm}$

$(\mathrm{MeOH} / \mathrm{HCl}): \lambda_{\max }(\log \varepsilon)=230$ (3.91), $284(3.61), 464(3.50) \mathrm{nm}$

$(\mathrm{MeOH} / \mathrm{NaOH}): \lambda_{\max }(\log \varepsilon)=232(3.99), 298(3.72), 440(3.74) \mathrm{nm}$

${ }^{1} \mathbf{H}-\mathbf{N M R}\left(600 \mathrm{MHz}, \mathrm{CD}_{3} \mathrm{OD}\right): \delta=6.43(\mathrm{~s}, 1 \mathrm{H}, 4-\mathrm{H}), 6.44(\mathrm{~s}, 1 \mathrm{H}, 1-\mathrm{H}), 6.97(\mathrm{dd}, J=8.0$, $1.5 \mathrm{~Hz}, 1 \mathrm{H}, 7-\mathrm{H}), 7.16$ (dd, $J=8.0,1.5 \mathrm{~Hz}, 1 \mathrm{H}, 9-\mathrm{H}), 7.19$ (t, $J=8.0 \mathrm{~Hz}, 1 \mathrm{H}, 8-\mathrm{H})$.

${ }^{1}$ H-NMR (600 MHz, DMSO-d $\left.{ }_{6}\right): \delta=6.31(\mathrm{~s}, 1 \mathrm{H}, 4-\mathrm{H}), 6.37$ (s, 1H, 1-H), 6.69 (br s, 2H, $\mathrm{NH}_{2}$ ). 6.99 (d, $\left.J=8.0 \mathrm{~Hz}, 1 \mathrm{H}, 7-\mathrm{H}\right), 7.10$ (d, $\left.J=8.0 \mathrm{~Hz}, 1 \mathrm{H}, 9-\mathrm{H}\right), 7.15$ (t, $J=8.0 \mathrm{~Hz}, 1 \mathrm{H}$, $8-\mathrm{H})$.

${ }^{13}$ C-NMR (125 MHz, DMSO-d ${ }_{6}$ ): $\delta=98.4$ (d, C-1), 103.0 (d, C-4), 115.5 (d, C-7), 117.4 (d, C-9), 124.5 (d, C-8), 131.2 (s, C-9a), 134.8 (s, C-5a), 146.0 (s, C-6), 147.3 (s, C-4a)*, 147.8 $(\mathrm{s}, \mathrm{C}-10 \mathrm{a})^{*}, 148.7$ (s, C-2)*, 180.0 (s, C-3).

* Zuordnungen austauschbar. Die angegebene Zuordnung erfolgte anhand berechneter Werte ${ }^{[96]}$, eine zweifelsfreie Unterscheidung durch das HMBC-Spektrum war nicht möglich. 


\subsubsection{Chandrananimycin C (41)}

intensiv roter Feststoff

$\mathbf{C}_{17} \mathbf{H}_{16} \mathbf{N}_{2} \mathbf{O}_{3}(296.33)$

Anfärbeverhalten: orange (Orcin)

$\mathbf{R}_{\mathbf{f}}=0.78\left(\mathrm{CHCl}_{3} / \mathrm{MeOH} 9: 1\right)$

$\mathbf{R}_{\mathbf{t}}=14.9 \min ($ HPLC 1, Säule 1, Programm 1)

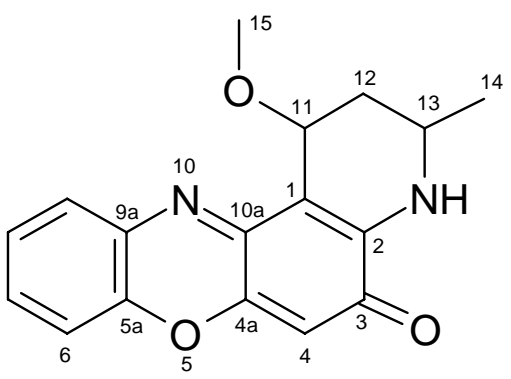

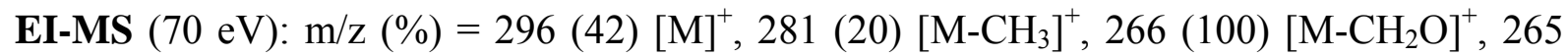
(56) $\left[\mathrm{M}-\mathrm{CH}_{3} \mathrm{O}\right]^{+}, 264$ (50) $\left[\mathrm{M}-\mathrm{CH}_{3} \mathrm{OH}\right]^{+}, 263$ (42) $\left[\mathrm{M}-\mathrm{CH}_{3}-\mathrm{H}_{2} \mathrm{O}\right]^{+}, 251$ (18), 250 (27), 249 (100), 238 (20), 221 (36), 149 (18), 132 (18), 73 (15)

${ }^{1}$ H-NMR $\left(300 \mathrm{MHz} \mathrm{CDCl}_{3}\right): \delta=1.36\left(\mathrm{~m}, 1 \mathrm{H}, 12-\mathrm{H}_{\mathrm{a}}\right), 1.37\left(\mathrm{~d}, J=6.5 \mathrm{~Hz}, 3 \mathrm{H}, 14-\mathrm{H}_{3}\right), 2.22$ (m, 1H, 12- $\left.\mathrm{H}_{\mathrm{b}}\right), 3.53$ (s, 3H, OMe), $3.73(\mathrm{~m}, 1 \mathrm{H}, 13-\mathrm{H}), 5.07$ (t, $\left.J=3.0 \mathrm{~Hz}, 1 \mathrm{H}, 11-\mathrm{H}\right), 5.88$ (br s, 1H, NH), 6.35 (s, 1H, 4-H), 7.35 (m, 3H, 6-H, 7-H, 8-H), 7.76 (dt, J= 7.0, 1.5 Hz, 1H, 9-H).

\subsubsection{2-Amino-8-benzoyl-6-hydroxy-phenoxazin-3-on (42)}

intensiv roter Feststoff

$\mathrm{C}_{19} \mathrm{H}_{12} \mathbf{N}_{2} \mathbf{O}_{4}(332.32)$

Anfärbeverhalten: braun-rot (Orcin)

$\mathbf{R}_{\mathbf{f}}=0.49\left(\mathrm{CHCl}_{3} / \mathrm{MeOH} 9: 1\right)$

$\mathbf{R}_{\mathbf{t}}=15.0 \min ($ HPLC 1, Säule 1, Programm 1)<smiles>Nc1cc2nc3cc(C(=O)c4ccccc4)cc(O)c3oc-2cc1=O</smiles>

EI-MS (70 eV): nicht erhältlich

ESI-MS (positive Ionen): $\mathrm{m} / \mathrm{z}=333[\mathrm{M}+\mathrm{H}]^{+}, 687[2 \mathrm{M}+\mathrm{Na}]^{+}, 1019[3 \mathrm{M}+\mathrm{Na}]^{+}$

ESI-MS (negative Ionen): $\mathrm{m} / \mathrm{z}=331[\mathrm{M}-\mathrm{H}]^{-}, 663$ [2M-H] $^{-}$

HR-ESI-MS: berechnet $\mathrm{m} / \mathrm{z}=333.086983[\mathrm{M}+\mathrm{H}]^{+}$ gefunden $\mathrm{m} / \mathrm{z}=333.086901$ (Abweichung $0.25 \mathrm{ppm}$ )

IR $(\mathrm{KBr}): \widetilde{v}=3435,2943,2361,1670,1638,1432,1686,1264,1202,1137,1088,1034$, $844,800,712 \mathrm{~cm}^{-1}$

UV $(\mathrm{MeOH}): \lambda_{\max }(\log \varepsilon)=257(3.81), 309(3.59), 432(3.46) \mathrm{nm}$ $(\mathrm{MeOH} / \mathrm{HCl}): \lambda_{\max }(\log \varepsilon)=254(3.73), 305(3.53), 454(3.57) \mathrm{nm}$ $(\mathrm{MeOH} / \mathrm{NaOH}): \lambda_{\max }(\log \varepsilon)=243(3.89), 438(3.67) \mathrm{nm}$ 
${ }^{1}$ H-NMR (600 MHz, DMSO-d $\left.{ }_{6}\right): \delta=6.36(\mathrm{~s}, 1 \mathrm{H}, 1-\mathrm{H}), 6.41(\mathrm{~s}, 1 \mathrm{H}, 4-\mathrm{H}), 6.88(\mathrm{br} \mathrm{s}, 2 \mathrm{H}$, $\left.\mathrm{NH}_{2}\right), 7.38$ (d, $\left.J=2.0 \mathrm{~Hz}, 1 \mathrm{H}, 7-\mathrm{H}\right), 7.42$ (d, $\left.J=2.0 \mathrm{~Hz}, 1 \mathrm{H}, 9-\mathrm{H}\right), 7.59$ (t, $J=7.5 \mathrm{~Hz}, 2 \mathrm{H}$, 4'-H, 6'-H), 7.69 (t, J = 7.5 Hz, 1H, 5'-H), 7.76 (d, J=7.5 Hz, 2H, 3'-H, 7'-H).

${ }^{13}$ C-NMR (125 MHz, DMSO-d d $_{6}$ : $\delta=98.2$ (d, C-1), 103.9 (d, C-4), 114.9 (d, C-7), 120.6 (d, C-9), 128.5 (d, C-4', C-6'), 129.3 (d, C-3', C-7'), 132.4 (d, C-5'), 132.7 (s, C-8)**, 134.0 (s, C-9a)**, 134.1 (s, C-5a), 137.1 (s, C-2'), 145.3 (s, C-6), 147.6 (s, C-4a)*, 148.4 (s, C-10a)*, 148.8 (s, C-2)* 180.2 (s, C-3), 194.4 (s, C-1').

$* / * *=$ Zuordnungen jeweils austauschbar. Die angegebene Zuordnung erfolgte anhand berechneter Werte ${ }^{[96]}$, eine zweifelsfreie Unterscheidung durch das HMBC-Spektrum war nicht möglich.

\subsubsection{2-Amino-8-(4-hydroxybenzoyl)-6-hydroxy-phenoxazin-3-on (43)}

intensiv roter Feststoff

$\mathbf{C}_{19} \mathbf{H}_{12} \mathbf{N}_{2} \mathbf{O}_{5}$ (348.32)

Anfärbeverhalten: braun-rot (Orcin)

$\mathbf{R}_{\mathbf{f}}=0.32\left(\mathrm{CHCl}_{3} / \mathrm{MeOH} 9: 1\right)$

$\mathbf{R}_{\mathbf{t}}=12.7 \min$ (HPLC 1, Säule 1, Programm 1)<smiles>Nc1cc2nc3cc(C(=O)c4ccc(O)cc4)cc(O)c3oc-2cc1=O</smiles>

EI-MS $(70 \mathrm{eV})$ : nicht erhältlich

ESI-MS (positive Ionen): $\mathrm{m} / \mathrm{z}=349[\mathrm{M}+\mathrm{H}]^{+}, 719[2 \mathrm{M}+\mathrm{Na}]^{+}$

ESI-MS (negative Ionen): $\mathrm{m} / \mathrm{z}=347.3[\mathrm{M}-\mathrm{H}]^{-}, 694.9$ [2M-H $^{-}$

HR-ESI-MS: berechnet $\mathrm{m} / \mathrm{z}=349.08190[\mathrm{M}+\mathrm{H}]^{+}$ gefunden $\mathrm{m} / \mathrm{z}=349.08176$ (Abweichung $0.40 \mathrm{ppm}$ )

IR (KBr): $\widetilde{v}=3432,2359,1618,1584,1398,1265,1176,1109,1029,662,566 \mathrm{~cm}^{-1}$

UV $(\mathrm{MeOH}): \lambda_{\max }(\log \varepsilon)=266(3.54), 300(3.52), 428(3.44), 434(3.45) \mathrm{nm}$

$(\mathrm{MeOH} / \mathrm{HCl}): \lambda_{\max }(\log \varepsilon)=249(3.62), 304(3.58), 465(3.35) \mathrm{nm}$

$(\mathrm{MeOH} / \mathrm{NaOH}): \lambda_{\max }(\log \varepsilon)=239(3.61), 346(3.56), 445(3.49) \mathrm{nm}$

${ }^{1}$ H-NMR $\left(600 \mathrm{MHz}, \mathrm{DMSO}-\mathrm{d}_{6}\right): \delta=6.34(\mathrm{~s}, 1 \mathrm{H}, 1-\mathrm{H}), 6.37(\mathrm{~s}, 1 \mathrm{H}, 4-\mathrm{H}), 6.73$ (br s, 2H, $\mathrm{NH}_{2}$ ), 6.81 (d, $J=8.5 \mathrm{~Hz}, 2 \mathrm{H}, 4^{\prime}-\mathrm{H}, 6$ '-H), 7.11 (br s, 1H, 9-H)*, 7.17 (br s, 1H, 7-H)* 7.65 (d, $\left.J=8.5 \mathrm{~Hz}, 2 \mathrm{H}, 3^{\prime}-\mathrm{H}, 7^{\prime}-\mathrm{H}\right)$.

${ }^{*}$ Zuordnung austauschbar

${ }^{1}$ H-NMR (600 MHz, CD 3 OD): $\delta=6.45$ (s, 1H, 1-H), 6.49 (s, 1H, 4-H), 6.85 (d, $J=9.0 \mathrm{~Hz}$, $\left.2 \mathrm{H}, 4^{\prime}-\mathrm{H}, 6^{\prime}-\mathrm{H}\right), 7.23$ (d, $\left.J=2.0 \mathrm{~Hz}, 1 \mathrm{H}, 9-\mathrm{H}\right)^{*}, 7.30$ (br s, 1H, 7-H)*, 7.74 (d, $J=9.0 \mathrm{~Hz}$, $\left.2 \mathrm{H}, 3^{\prime}-\mathrm{H}, 7^{\prime}-\mathrm{H}\right)$.

* Zuordnung austauschbar 


\subsection{Nargenicin $A_{1}$ aus Actinomyces sp. Gö 301}

\subsubsection{Nargenicin A1 (44)}

farbloser Feststoff

$\mathrm{C}_{28} \mathrm{H}_{37} \mathrm{NO}_{8}(\mathrm{M}=515.60)$

Anfärbeverhalten: schwarz-braun

(Anisaldehyd), braun (Orcin)

$\mathbf{R}_{\mathbf{f}}=0.55\left(\mathrm{CHCl}_{3} / \mathrm{MeOH} 9: 1\right)$

EI-MS $(70 \mathrm{eV}): \mathrm{m} / \mathrm{z}(\%)=515(100)[\mathrm{M}]^{+}, 497$

(10), 457 (12), 426 (8). 369 (10). 347 (4), 297

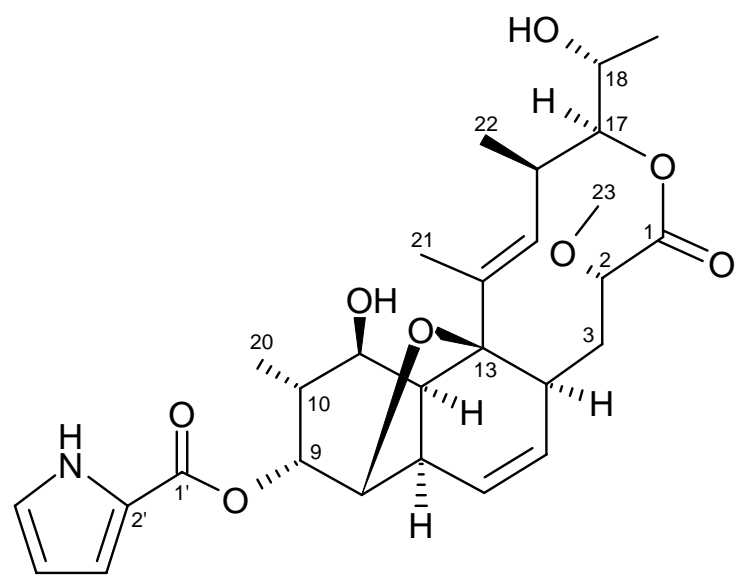

(6), 147 (14), 109 (10), 94 (50), 43 (12)

ESI-MS (positive Ionen): $\mathrm{m} / \mathrm{z}=538[\mathrm{M}+\mathrm{Na}]^{+}, 1053[2 \mathrm{M}+\mathrm{Na}]^{+}$

ESI-MS (negative Ionen): $\mathrm{m} / \mathrm{z}=514[\mathrm{M}-\mathrm{H}]^{-}$

HR-ESI-MS: berechnet $\mathrm{m} / \mathrm{z}=516.259194[\mathrm{M}+\mathrm{H}]^{+}$

$$
\text { gefunden } \mathrm{m} / \mathrm{z}=516.259245 \text { (Abweichung } 0.10 \mathrm{ppm} \text { ) }
$$

IR $(\mathrm{KBr}): \widetilde{v}=3420,2967,2927,2871,2366,2950,1701,1654,1559,1458,1410,1385$, $1307,1262,1107,1027,969,750 \mathrm{~cm}^{-1}$

UV $(\mathrm{MeOH}): \lambda_{\max }(\log \varepsilon)=227(3.68), 265(3.90) \mathrm{nm}$

$(\mathrm{MeOH} / \mathrm{HCl}): \lambda_{\max }(\log \varepsilon)=231$ (3.64), $265(3.89) \mathrm{nm}$

$(\mathrm{MeOH} / \mathrm{NaOH}): \lambda_{\max }(\log \varepsilon)=221(3.72), 265(3.91) \mathrm{nm}$

CD $(\mathrm{MeOH}): \lambda_{\max }([\Theta])=204(40800), 237(-4000) \mathrm{nm}$

$[\alpha]_{D}^{20}=+48^{\circ}(\mathrm{c}=1$ in Methanol $)$

${ }^{1}$ H-NMR (300 MHz, CD $\left.\mathrm{CD}_{3} \mathrm{OD}\right): \delta=0.93\left(\mathrm{~d}, J=7.0 \mathrm{~Hz}, 3 \mathrm{H}, 20-\mathrm{H}_{3}\right), 1.21(\mathrm{~d}, J=6.0 \mathrm{~Hz}, 3 \mathrm{H}$, 19- $\left.\mathrm{H}_{3}\right), 1.25\left(\mathrm{~d}, J=7.0 \mathrm{~Hz}, 3 \mathrm{H}, 22-\mathrm{H}_{3}\right), 1.35\left(\mathrm{~m}, 1 \mathrm{H}, 3-\mathrm{H}_{\mathrm{a}}\right), 1.82\left(\mathrm{~s}, 3 \mathrm{H}, 21-\mathrm{H}_{3}\right), 2.30(\mathrm{~m}, 1 \mathrm{H}$, 4-H), $2.34(\mathrm{~m}, 1 \mathrm{H}, 10-\mathrm{H}), 2.47(\mathrm{~m}, 1 \mathrm{H}, 12-\mathrm{H}), 2.48$ (ddd, $J=15.0,11.0,4.0 \mathrm{~Hz}, 1 \mathrm{H}, 3-\mathrm{H}_{\mathrm{b}}$ ), $2.61(\mathrm{~d}, J=7.0 \mathrm{~Hz}, 1 \mathrm{H}, 7-\mathrm{H}), 3.08(\mathrm{~m}, 1 \mathrm{H}, 16-\mathrm{H}), 3.28\left(\mathrm{~s}, 3 \mathrm{H}, 23-\mathrm{H}_{3}\right), 3.69$ (dd, $J=11.0$, $3.0 \mathrm{~Hz}, 1 \mathrm{H}, 11-\mathrm{H}), 3.72$ (dd, $J=11.0,4.5 \mathrm{~Hz}, 1 \mathrm{H}, 2-\mathrm{H}), 3.99$ (dq, $J=9.0,6.0 \mathrm{~Hz}, 1 \mathrm{H}, 18-\mathrm{H})$, $4.12(\mathrm{~d}, J=5.0 \mathrm{~Hz}, 1 \mathrm{H}, 8-\mathrm{H}), 5.03(\mathrm{t}, J=5.0 \mathrm{~Hz}, 1 \mathrm{H}, 9-\mathrm{H}), 5.14(\mathrm{dd}, J=8.5,6.0 \mathrm{~Hz}, 1 \mathrm{H}$, 17-H), 5.44 (dd, $J=7.0,1.0 \mathrm{~Hz}, 1 \mathrm{H}, 15-\mathrm{H}), 5.60$ (dd, $J=9.5,3.0 \mathrm{~Hz}, 1 \mathrm{H}, 5-\mathrm{H}), 5.90$ (ddd, $J=9.0,7.0,2.0 \mathrm{~Hz}, 1 \mathrm{H}, 6-\mathrm{H}), 6.20$ (dd, $\left.J=4.0,2.5 \mathrm{~Hz}, 1 \mathrm{H}, 4^{\prime}-\mathrm{H}\right), 6.87$ (dd, $J=4.0,1.5 \mathrm{~Hz}$, 1H, 3'-H), 6.99 (dd, $J=2.5,1.5 \mathrm{~Hz}, 1 \mathrm{H}, 5$ '-H).

${ }^{1}$ H-NMR $\left(300 \mathrm{MHz}, \mathrm{CDCl}_{3}\right): \delta=0.95\left(\mathrm{~d}, J=7.0 \mathrm{~Hz}, 3 \mathrm{H}, 20-\mathrm{H}_{3}\right), 1.24(\mathrm{~d}, J=7.0 \mathrm{~Hz}, 3 \mathrm{H}$, $\left.21-\mathrm{H}_{3}\right), 1.30\left(\mathrm{~d}, J=6.0 \mathrm{~Hz}, 3 \mathrm{H}, 19-\mathrm{H}_{3}\right), 1.38\left(\mathrm{dt}, J=15.0,3.5 \mathrm{~Hz}, 1 \mathrm{H}, 3-\mathrm{H}_{\mathrm{a}}\right), 1.77(\mathrm{~s}, 3 \mathrm{H}$, 21- $\left.\mathrm{H}_{3}\right), 2.32$ (m, 2H, 4-H, 10-H), $2.46-2.58$ (m, 3H, 3- $\left.\mathrm{H}_{\mathrm{b}}, 7-\mathrm{H}, 12-\mathrm{H}\right), 3.15$ (sext, $J=7.0 \mathrm{~Hz}$, 
$1 \mathrm{H}, 16-\mathrm{H}), 3.29\left(\mathrm{~s}, 3 \mathrm{H}, 23-\mathrm{H}_{3}\right), 3.64(\mathrm{dd}, J=11.0,2.5 \mathrm{~Hz}, 1 \mathrm{H}, 11-\mathrm{H}), 3.67(\mathrm{dd}, J=11.5$, $4.0 \mathrm{~Hz}, 1 \mathrm{H}, 2-\mathrm{H}$ ), 4.09 (quint, $J=6.5 \mathrm{~Hz}, 1 \mathrm{H}, 18-\mathrm{H}$ ), 4.20 (d, $J=5.0 \mathrm{~Hz}, 1 \mathrm{H}, 8-\mathrm{H}), 5.11$ (t, $J=4.5 \mathrm{~Hz}, 1 \mathrm{H}, 9-\mathrm{H}), 5.14$ (t, $J=7.0 \mathrm{~Hz}, 1 \mathrm{H}, 17-\mathrm{H}), 5.54$ (m, 2H, 5-H, 15-H), 5.84 (ddd, $J=9.0,7.0,1.5 \mathrm{~Hz}, 1 \mathrm{H}, 6-\mathrm{H}), 6.25$ (m, 1H, 4'-H), 6.86 (m, 1H, 3'-H), 6.97 (m, 1H, 5'-H), 9.34 (br s, $1 \mathrm{H}, \mathrm{NH})$.

${ }^{13}$ C-NMR (75.5 MHz, CD 3 OD): $\delta=13.3$ (q, C-20), 15.9 (q, C-22), 17.7 (q, C-21), 21.3 (q, C-19), 34.0 (d, C-16), 35.5 (d, C-10), 36.0 (t, C-3), 40.4 (d, C-7), 44.1 (d, C-4), 50.8 (d, C-12), 58.0 (q, C-23), 66.6 (d, C-18), 74.7 (d, C-9), 76.4 (d, C-11), 80.0 (d, C-17), 82.7 (d, C-8), 84.0 (d, C-2) , 90.6 (s, C-13), 110.8 (d, C-4'), 116.9 (d, C-3'), 123.1 (s, C-2'), 125.1 (d, C-5'), 128.9 (d, C-6), 132.3 (d, C-15), 133.9 (d, C-5), 136.2 (s, C-14), 161.9 (s, C-1'), $174.4(\mathrm{~s}, \mathrm{C}-1)$. 


\subsection{Substanzen aus Actinomyces sp. Gö M1}

\subsubsection{1-O-Isovaleryl-6-desoxy- $\alpha-L$-talopyranosid (45)}

farbloses Ö1

$\mathrm{C}_{11} \mathrm{H}_{20} \mathbf{O}_{6}$ (248.27)

Anfärbeverhalten: grau-grün (Anisaldehyd), schwarzbraun (Orcin)

$\mathbf{R}_{\mathbf{f}}=0.33\left(\mathrm{CHCl}_{3} / \mathrm{MeOH} 9: 1\right)$

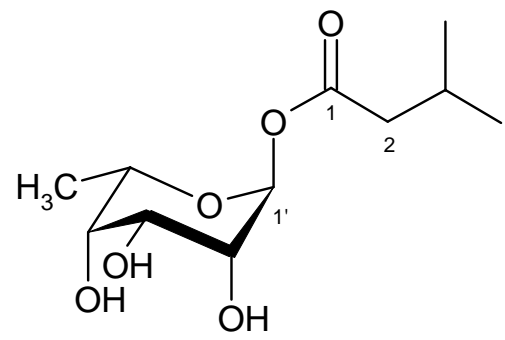

EI-MS (70 eV): nicht erhältlich

ESI-MS (positive Ionen): $\mathrm{m} / \mathrm{z}=271[\mathrm{M}+\mathrm{Na}]^{+}, 519[2 \mathrm{M}+\mathrm{Na}]^{+}$

ESI-MS (negative Ionen): $\mathrm{m} / \mathrm{z}=495[2 \mathrm{M}-\mathrm{H}]^{-}$

HR-ESI-MS: berechnet $\mathrm{m} / \mathrm{z}=271.11521[\mathrm{M}+\mathrm{Na}]^{+}$

gefunden $\mathrm{m} / \mathrm{z}=271.11515$ (Abweichung $0.22 \mathrm{ppm}$ )

IR $(\mathrm{KBr}): \widetilde{v}=3400,2966,2935,1745,1458,1387,1370,1292,1252,1169,1144,1101$, $1020,967,949,812,689 \mathrm{~cm}^{-1}$

UV $(\mathrm{MeOH}): \lambda_{\max }(\log \varepsilon)=203(3.15) \mathrm{nm}$

CD $(\mathrm{MeOH}): \lambda_{\max }([\Theta])=213(800), 247(-500) \mathrm{nm}$

$[\alpha]_{D}^{20}=-84^{\circ}(\mathrm{c}=1$ in Methanol $)$

${ }^{1}$ H-NMR $\left(600 \mathrm{MHz}, \mathrm{CD}_{3} \mathrm{OD}\right): \delta=0.96\left(\mathrm{~d}, J=6.8 \mathrm{~Hz}, 3 \mathrm{H}, 4-\mathrm{H}_{3}\right), 0.97(\mathrm{~d}, J=6.8 \mathrm{~Hz}, 3 \mathrm{H}$, 5- $\mathrm{H}_{3}$ ), $1.22\left(\mathrm{~d}, J=6.5 \mathrm{~Hz}, 3 \mathrm{H}, 6{ }^{\prime}-\mathrm{H}_{3}\right), 2.07$ (nonett, $\left.J=6.8 \mathrm{~Hz}, 1 \mathrm{H}, 3-\mathrm{H}\right), 2.22$ (dd, $J=14.0$, $\left.6.8 \mathrm{~Hz}, 1 \mathrm{H}, 2-\mathrm{H}_{\mathrm{a}}\right), 2.25$ (dd, $\left.J=14.0,7.5 \mathrm{~Hz}, 1 \mathrm{H}, 2-\mathrm{H}_{\mathrm{b}}\right), 3.65$ (m, 1H, 4'-H), 3.67 (ddd, $\left.J=3.5,2.0,1.5 \mathrm{~Hz}, 1 \mathrm{H}, 2^{\prime}-\mathrm{H}\right), 3.76\left(\mathrm{t}, J=3.5 \mathrm{~Hz}, 1 \mathrm{H}, 3^{\prime}-\mathrm{H}\right), 6.03$ (d, $\left.J=2.0 \mathrm{~Hz}, 1 \mathrm{H}, 1^{\prime}-\mathrm{H}\right)$. ${ }^{13}$ C-NMR (150.8 MHz, CD 3 OD): $\delta=16.9$ (q, C-6'), 22.6 (q, C-4), 22.7 (q, C-5), 26.9 (d, C-3), 44.1 (t, C-2), 67.1 (d, C-3’), 70.9 (d, C-5’), 71.1 (d, C-2’), 73.9 (d, C-4'), 95.8 (d, C-1'), 172.7 (s, C-1). 


\subsubsection{1-O-Phenylacetyl-6-desoxy- $\alpha-L-t a l o p y r a n o s i d(46)$}

farbloser Feststoff

$\mathrm{C}_{14} \mathrm{H}_{18} \mathbf{O}_{6}(282.29)$

Anfärbeverhalten: grau-grün (Anisaldehyd),

schwarz-blau (Orcin)

$\mathbf{R}_{\mathbf{f}}=0.43\left(\mathrm{CHCl}_{3} / \mathrm{MeOH} 9: 1\right)$

EI-MS (70 eV): nicht erhältlich

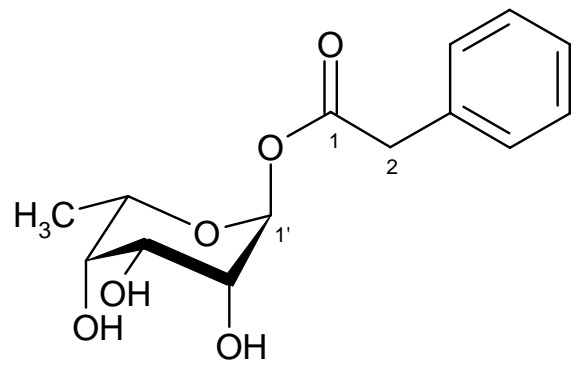

DCI-MS $(200 \mathrm{eV}): \mathrm{m} / \mathrm{z}=300\left[\mathrm{M}+\mathrm{NH}_{4}\right]^{+}, 317\left[\mathrm{M}+\mathrm{NH}_{4}+\mathrm{NH}_{3}\right]^{+}, 582\left[2 \mathrm{M}+\mathrm{NH}_{4}\right]^{+}$

ESI-MS (positive Ionen): $\mathrm{m} / \mathrm{z}=305[\mathrm{M}+\mathrm{Na}]^{+}, 587[2 \mathrm{M}+\mathrm{Na}]^{+}$

ESI-MS (negative Ionen): nicht erhältlich

HR-ESI-MS: berechnet $\mathrm{m} / \mathrm{z}=305.09956[\mathrm{M}+\mathrm{Na}]^{+}$

gefunden $\mathrm{m} / \mathrm{z}=305.09972$ (Abweichung $0.52 \mathrm{ppm}$ )

IR $(\mathrm{KBr}): \widetilde{v}=3412,2976,2933,2361,2342,1745,1456,1384,1248,1124,1102,1020$, $962,811,728,699 \mathrm{~cm}^{-1}$

UV $(\mathrm{MeOH}): \lambda_{\max }(\log \varepsilon)=206(3.83) \mathrm{nm}$

$(\mathrm{MeOH} / \mathrm{HCl}): \lambda_{\max }(\log \varepsilon)=205(3.84) \mathrm{nm}$

$(\mathrm{MeOH} / \mathrm{NaOH}): \lambda_{\max }(\log \varepsilon)=215(2.79) \mathrm{nm}$

CD $(\mathrm{MeOH}): \lambda_{\max }([\Theta])=226(4800) \mathrm{nm}$

$[\alpha]_{D}^{20}=-35^{\circ}(\mathrm{c}=1$ in Methanol $)$

${ }^{1}$ H-NMR (300 MHz, CD $\left.3 \mathrm{OD}\right): \delta=1.13$ (d, $J=6.5 \mathrm{~Hz}, 3 \mathrm{H}, 6$ 6'- $^{\prime}$ ), 3.55 (m, 1H, 4'-H), 3.63 (m, 1H, 3'-H), 3.64 (m, 1H, 2'-H), 3.68 (s, 2H, 2-H $\left.{ }^{\prime}\right), 3.69$ (q, $J=6.5$ Hz, 1H, 5'-H), 6.00 (d, $\left.J=2.0 \mathrm{~Hz}, 1 \mathrm{H}, 1^{\prime}-\mathrm{H}\right), 7.28(\mathrm{~m}, 5 \mathrm{H}, 4-\mathrm{H}$ bis $8-\mathrm{H})$.

${ }^{1}$ H-NMR (600 MHz, Pyridin-d $\left.\mathrm{d}_{6}\right): \delta=1.42\left(\mathrm{~d}, J=6.5 \mathrm{~Hz}, 3 \mathrm{H}, 6{ }^{\prime}-\mathrm{H}_{3}\right), 3.81$ (s, 2H, 2- $\left.\mathrm{H}_{2}\right), 4.03$ (ddd, $\left.J=3.5,2.0,1.5 \mathrm{~Hz}, 1 \mathrm{H}, 2^{\prime}-\mathrm{H}\right), 4.10$ (q, $\left.J=6.5 \mathrm{~Hz}, 5^{\prime}-\mathrm{H}\right), 4.20$ (dd, $J=3.0,2.5 \mathrm{~Hz}, 1 \mathrm{H}$, 3'-H), $4.26(\mathrm{~m}, 1 \mathrm{H}, 4$ '-H), 6.70 (d, $J=1.5 \mathrm{~Hz}, 1 \mathrm{H}, 1$ '-H), 7.25 (m, 1H, 6-H), 7.31 (t, $J=7.5 \mathrm{~Hz}, 2 \mathrm{H}, 5-\mathrm{H}, 7-\mathrm{H}), 7.39$ (d, $J=7.5 \mathrm{~Hz}, 2 \mathrm{H}, 4-\mathrm{H}, 8-\mathrm{H})$.

${ }^{1} \mathbf{H}-\mathbf{N M R}\left(300 \mathrm{MHz}, \mathrm{CDCl}_{3}\right): \delta=1.18\left(\mathrm{~d}, J=6.5 \mathrm{~Hz}, 3 \mathrm{H}, 6^{\prime}-\mathrm{H}_{3}\right), 3.60-3.80\left(\mathrm{~m}, 6 \mathrm{H}, 2-\mathrm{H}_{2}\right.$ und 2'-H bis 5'-H), 6.15 (br s, 1H, 1'-H), 7.26 (m, 5H, 4-H bis 8-H).

${ }^{13}$ C-NMR (75.5 MHz, CD 3 OD): $\delta=16.8$ (q, C-6'), 42.1 (t, C-2), 67.0 (d, C-3'), 70.7 (d, C-2'), 70.8 (d, C-5'), 73.8 (d, C-4'), 96.2 (d, C-1'), 128.2 (d, C-6), 129.6 (d, C-5, C-7), 130.3 (d, C-4, C-8), 135.4 (s, C-3), 171.4 (s, C-1).

${ }^{1} \boldsymbol{J}_{\mathrm{CH}}$ am anomeren C-Atom $\left(\mathrm{CD}_{3} \mathrm{OD}\right): 175.2 \mathrm{~Hz}$ 
${ }^{13}$ C-NMR (50.3 MHz, $\mathrm{CDCl}_{3}$ ): $\delta=16.8$ (q, C-6'), 42.1 (t, C-2), 67.0 (d, C-3'), 70.7 (d, C-2'), 70.8 (d, C-5'), 73.8 (d, C-4'), 96.2 (d, C-1'), 128.2 (d, C-6), 129.6 (d, C-5 und C-7), 130.3 (d, C-4 und C-8), 135.4 (s, C-3), 171.4 (s, C-1).

\subsubsection{1-O-(2-Aminobenzoyl)-6-desoxy- $\alpha-L-t a l o p y r a n o s i d ~(47)$}

farbloser Feststoff

$\mathrm{C}_{13} \mathrm{H}_{17} \mathrm{NO}_{6}(283.28)$

Anfärbeverhalten: orange-braun (Orcin)

$\mathbf{R}_{\mathbf{f}}=0.21\left(\mathrm{CHCl}_{3} / \mathrm{MeOH} 9: 1\right)$

EI-MS (70 eV): nicht erhältlich

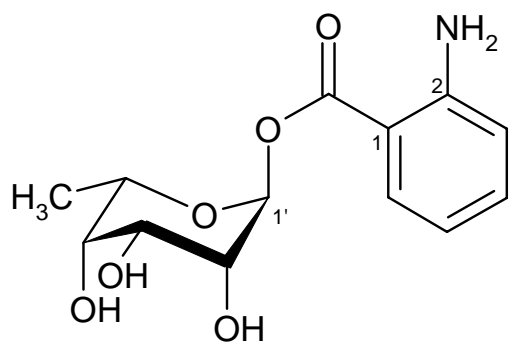

ESI-MS (positive Ionen): $\mathrm{m} / \mathrm{z}=306[\mathrm{M}+\mathrm{Na}]^{+}, 589[2 \mathrm{M}+\mathrm{Na}]^{+}$

HR-ESI-MS: berechnet $\mathrm{m} / \mathrm{z}=306.09481[\mathrm{M}+\mathrm{H}]^{+}$

gefunden $\mathrm{m} / \mathrm{z}=306.09518$ (Abweichung $1.14 \mathrm{ppm}$ )

IR $(\mathrm{KBr}): \widetilde{v}=3432,2927,1619,1457,1384,1242,1148,1107,1060,1019,955 \mathrm{~cm}^{-1}$

UV $(\mathrm{MeOH}): \lambda_{\max }(\log \varepsilon)=219$ (3.93), $248(3.43), 340(3.19) \mathrm{nm}$

$(\mathrm{MeOH} / \mathrm{HCl}): \lambda_{\max }(\log \varepsilon)=221(3.83), 248(3.32), 339(3.03) \mathrm{nm}$

$(\mathrm{MeOH} / \mathrm{NaOH}): \lambda_{\max }(\log \varepsilon)=220$ (3.84), 249 (3.31), $340(3.08) \mathrm{nm}$

CD $(\mathrm{MeOH}): \lambda_{\max }([\Theta])=207$ (-2100), 223 (500), $252(-2200), 289(-1800), 345(-1800) \mathrm{nm}$

${ }^{1}$ H-NMR (600 MHz, $\left.\mathrm{CD}_{3} \mathrm{OD}\right): \delta=1.25\left(\mathrm{~d}, J=6.5 \mathrm{~Hz}, 3 \mathrm{H}, 6{ }^{\prime}-\mathrm{H}_{3}\right), 3.71\left(\mathrm{~m}, 1 \mathrm{H}, 4^{\prime}-\mathrm{H}\right), 3.82$ (ddd, $\left.J=3.5,2.0,1.5 \mathrm{~Hz}, 1 \mathrm{H}, 2^{\prime}-\mathrm{H}\right), 3.93$ (t, $\left.J=3.0 \mathrm{~Hz}, 1 \mathrm{H}, 3^{\prime}-\mathrm{H}\right), 4.06$ (q, $J=6.5 \mathrm{~Hz}, 1 \mathrm{H}$, 5'-H), 6.24 (d, $J=2.0 \mathrm{~Hz}, 1 \mathrm{H}, 1$ '-H), 6.56 (ddd, $J=8.0,7.0,1.0 \mathrm{~Hz}, 1 \mathrm{H}, 5-\mathrm{H}), 6.75$ (ddd, $J=8.5,1.0,0.5 \mathrm{~Hz}, 1 \mathrm{H}, 3-\mathrm{H}), 7.25(\mathrm{ddd}, J=8.5,7.0,1.5 \mathrm{~Hz}, 1 \mathrm{H}, 4-\mathrm{H}), 7.74(\mathrm{ddd}, J=8.0$, $1.5,0.5 \mathrm{~Hz}, 1 \mathrm{H}, 6-\mathrm{H})$. 


\subsubsection{1-O-(4-Hydroxyphenyl)-6-desoxy- $\alpha-L-t a l o p y r a n o s i d ~(48)$}

farbloser Feststoff

$\mathrm{C}_{12} \mathrm{H}_{16} \mathrm{O}_{6}(256.25)$

Anfärbeverhalten: braun (Orcin), grau-blau (Ehrlich)

$\mathbf{R}_{\mathbf{f}}=0.15\left(\mathrm{CHCl}_{3} / \mathrm{MeOH} 9: 1\right)$

EI-MS $(70 \mathrm{eV}): \mathrm{m} / \mathrm{z}(\%)=$ nicht erhältlich

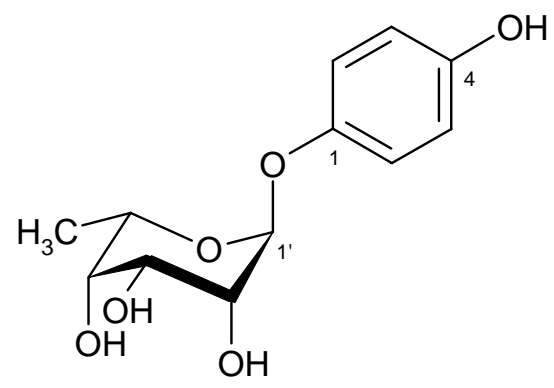

ESI-MS (positive Ionen): $\mathrm{m} / \mathrm{z}=279[\mathrm{M}+\mathrm{Na}]^{+}, 525[2 \mathrm{M}+\mathrm{Na}]^{+}$

HR-ESI-MS: berechnet $\mathrm{m} / \mathrm{z}=279.08391[\mathrm{M}+\mathrm{Na}]^{+}$

gefunden $\mathrm{m} / \mathrm{z}=279.08371$ (Abweichung $0.72 \mathrm{ppm}$ )

IR $(\mathrm{KBr}): \widetilde{v}=3367,3188,2962,2928,2499,2364,1510,1474,1444,1379,1327,1219$, 1102. $1034,973,835,815,772,691,518 \mathrm{~cm}^{-1}$

UV $(\mathrm{MeOH}): \lambda_{\max }(\log \varepsilon)=223(3.93), 286(3.49) \mathrm{nm}$

$(\mathrm{MeOH} / \mathrm{HCl}): \lambda_{\max }(\log \varepsilon)=224(3.85), 287(3.33) \mathrm{nm}$

$(\mathrm{MeOH} / \mathrm{NaOH}): \lambda_{\max }(\log \varepsilon)=236(3.93), 298(3.46) \mathrm{nm}$

CD $(\mathrm{MeOH}): \lambda_{\max }([\Theta])=225(-6800) \mathrm{nm}$

$[\alpha]_{D}^{20}=-108^{\circ}(\mathrm{c}=1$ in Methanol $)$

${ }^{1}$ H-NMR (600 MHz, CD $\left.{ }_{3} \mathrm{OD}\right): \delta=1.21\left(\mathrm{~d}, J=6.5 \mathrm{~Hz}, 3 \mathrm{H}, 6{ }^{\prime}-\mathrm{H}_{3}\right), 3.65\left(\mathrm{~m}, 1 \mathrm{H}, 4^{\prime}-\mathrm{H}\right), 3.86$ (ddd, $\left.J=3.5,2.0,1.5 \mathrm{~Hz}, 2^{\prime}-\mathrm{H}\right), 3.90$ (t, $J=3.5 \mathrm{~Hz}, 1 \mathrm{H}, 3$ '-H), 4.01 (q, $\left.J=6.5 \mathrm{~Hz}, 1 \mathrm{H}, 5^{\prime}-\mathrm{H}\right)$, $5.33\left(\mathrm{~d}, J=2.0 \mathrm{~Hz}, 1 \mathrm{H}, 1^{\prime}-\mathrm{H}\right), 6.70(\mathrm{~d}, J=9.0 \mathrm{~Hz}, 2 \mathrm{H}, 3-\mathrm{H}, 5-\mathrm{H}), 6.89$ (d, $J=9.0 \mathrm{~Hz}, 2 \mathrm{H}$, 2-H, 6-H).

${ }^{1}$ H-NMR (600 MHz, Pyridin-d $\left.{ }_{6}\right): \delta=1.48\left(\mathrm{~d}, J=6.5 \mathrm{~Hz}, 3 \mathrm{H}, 6^{\prime}-\mathrm{H}_{3}\right), 4.09$ (m, 1H, 2'-H)*, 4.31 (q, $\left.J=6.5 \mathrm{~Hz}, 5^{\prime}-\mathrm{H}\right), 4.44\left(\mathrm{~m}, 1 \mathrm{H}, 4^{\prime}-\mathrm{H}\right)^{*}, 4.47$ (m, 1H, 3'-H)*, 5.94 (d, $J=2.0 \mathrm{~Hz}, 1 \mathrm{H}$, 1'-H), 6.39 (br s, 1H, OH), 6.44 (br d, $J=7.0 \mathrm{~Hz}, 1 \mathrm{H}, \mathrm{OH}), 6.76$ (br d, $J=7.0 \mathrm{~Hz}, 1 \mathrm{H}, \mathrm{OH})$, $7.16(\mathrm{~d}, J=9.0 \mathrm{~Hz}, 2 \mathrm{H}, 3-\mathrm{H}, 5-\mathrm{H}), 7.28$ (d, $J=9.0 \mathrm{~Hz}, 2 \mathrm{H}, 2-\mathrm{H}, 6-\mathrm{H})$.

$*=$ Zuordnung austauschbar.

${ }^{13}$ C-NMR (75.5 MHz, CD 3 OD): $\delta=16.9$ (q, C-6'), 67.2 (d, C-3'), 68.9 (d, C-5'), 72.1 (d, C-2'), 74.2 (d, C-4'), 101.7 (d, C-1'), 116.8 (d, C-3, C-5), 119.2 (d, C-2, C-6), 151.1 (s, C-1), 151.7 (s, C-4).

${ }^{1} J_{\mathrm{CH}}$ am anomeren C-Atom: $170.9 \mathrm{~Hz}$ 


\subsubsection{1-O-(4-Hydroxy-2-methoxyphenyl)-6-desoxy- $\alpha-L-t a l o p y r a n o s i d ~(49)$}

farbloser Feststoff

$\mathbf{C}_{13} \mathbf{H}_{18} \mathbf{O}_{7}(286.28)$

Anfärbeverhalten: braun (Orcin)

$\mathbf{R}_{\mathbf{f}}=0.17\left(\mathrm{CHCl}_{3} / \mathrm{MeOH} 9: 1\right)$

ESI-MS (positive Ionen): $\mathrm{m} / \mathrm{z}=309[\mathrm{M}+\mathrm{Na}]^{+}, 595[2 \mathrm{M}+\mathrm{Na}]^{+}$

HR-ESI-MS: berechnet $\mathrm{m} / \mathrm{z}=309.09447[\mathrm{M}+\mathrm{Na}]^{+}$

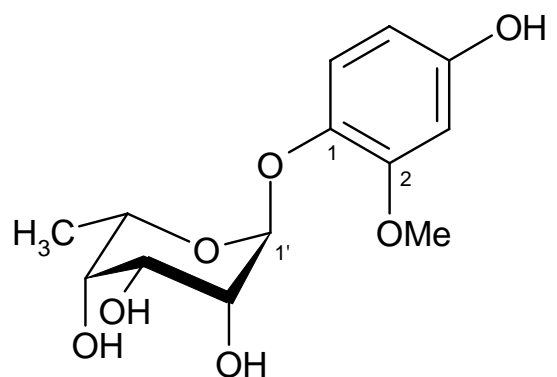

gefunden $\mathrm{m} / \mathrm{z}=309.09441$ (Abweichung $0.06 \mathrm{ppm}$ )

IR $(\mathrm{KBr}): \widetilde{v}=3420,2935,2370,2344,1684,1675,1608,1509,1458,1437,1384,1301$, $1212,1163,1103,1029,976,826,691 \mathrm{~cm}^{-1}$

UV $(\mathrm{MeOH}): \lambda_{\max }(\log \varepsilon)=220(3.55), 282(3.11) \mathrm{nm}$

$(\mathrm{MeOH} / \mathrm{HCl}): \lambda_{\max }(\log \varepsilon)=223(3.31), 283(2.86) \mathrm{nm}$

$(\mathrm{MeOH} / \mathrm{NaOH}): \lambda_{\max }(\log \varepsilon)=239(3.04), 296(2.36) \mathrm{nm}$

CD $(\mathrm{MeOH}): \lambda_{\max }([\Theta])=229(-1800), 254(400), 273(-300) \mathrm{nm}$

${ }^{1}$ H-NMR (600 MHz, CD $\left.{ }_{3} \mathrm{OD}\right): \delta=1.21\left(\mathrm{~d}, J=6.5 \mathrm{~Hz}, 3 \mathrm{H}, 6{ }^{\prime}-\mathrm{H}_{3}\right), 3.67$ (m, 1H, 4'-H), 3.77 (s, 3H, $\mathrm{OCH}_{3}$ ), 3.92 (t, $J=3.0 \mathrm{~Hz}, 1 \mathrm{H}, 3$ '-H), 3.94 (ddd, $\left.J=3.5,2.0,1.5 \mathrm{~Hz}, 1 \mathrm{H}, 2^{\prime}-\mathrm{H}\right), 4.21$ (q, $\left.J=6.5 \mathrm{~Hz}, 1 \mathrm{H}, 5^{\prime}-\mathrm{C}\right), 5.23$ (d, $\left.J=2.0 \mathrm{~Hz}, 1 \mathrm{H}, 1^{\prime}-\mathrm{H}\right), 6.28$ (dd, $\left.J=8.5,2.8 \mathrm{~Hz}, 1 \mathrm{H}, 5-\mathrm{H}\right)$, $6.46(\mathrm{~d}, J=2.8 \mathrm{~Hz}, 1 \mathrm{H}, 3-\mathrm{H}), 6.87$ (d, $J=8.5 \mathrm{~Hz}, 1 \mathrm{H}, 6-\mathrm{H})$.

${ }^{13}$ C-NMR (150.8 MHz, CD $\left.3 \mathrm{OD}\right): \delta=16.8$ (q, C-6'), 56.2 (q, $\mathrm{OCH}_{3}$ ), 67.2 (d, C-3'), 69.2 (d, C-5'), 72.0 (d, C-2'), 74.4 (d, C-4'), 101.7 (d, C-3), 103.3 (d, C-1'), 107.4 (d, C-5), 121.9 (d, C-6), 139.8 (s, C-1), 153.3 (s, C-2), 155.3 (s, C-3).

${ }^{1} \boldsymbol{J}_{\mathrm{CH}}$ am anomeren C-Atom: $174 \mathrm{~Hz}$

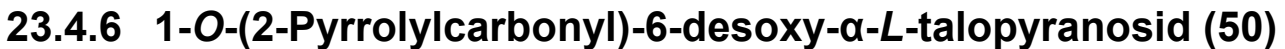

farbloser Feststoff

$\mathrm{C}_{11} \mathrm{H}_{15} \mathrm{NO}_{6}(257.24)$

Anfärbeverhalten: braun (Orcin)

$\mathbf{R}_{\mathbf{f}}=0.19\left(\mathrm{CHCl}_{3} / \mathrm{MeOH} 9: 1\right)$

ESI-MS (positive Ionen): $\mathrm{m} / \mathrm{z}=280[\mathrm{M}+\mathrm{Na}]^{+}, 537[2 \mathrm{M}+\mathrm{Na}]^{+}$

ESI-MS (negative Ionen): nicht erhältlich

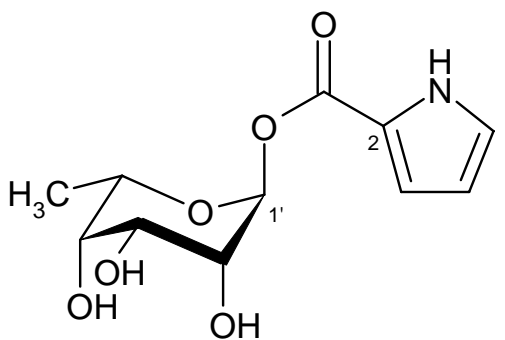


HR-ESI-MS: berechnet $\mathrm{m} / \mathrm{z}=280.07916[\mathrm{M}+\mathrm{Na}]^{+}$

gefunden $\mathrm{m} / \mathrm{z}=280.07912$ (Abweichung $0.14 \mathrm{ppm}$ )

IR $(\mathrm{KBr}): \widetilde{v}=3432,2927,1699,1684,1636,1410,1384,1309,1206,1123,1076,1024$, $961 \mathrm{~cm}^{-1}$

UV $(\mathrm{MeOH}): \lambda_{\max }(\log \varepsilon)=231(3.49), 267(3.96) \mathrm{nm}$

$(\mathrm{MeOH} / \mathrm{HCl}): \lambda_{\max }(\log \varepsilon)=232(3.43), 267(3.95) \mathrm{nm}$

$(\mathrm{MeOH} / \mathrm{NaOH}): \lambda_{\max }(\log \varepsilon)=230(3.33), 267(3.93) \mathrm{nm}$

CD $(\mathrm{MeOH}): \lambda_{\max }([\Theta])=216(1000), 230(1800), 266(800) \mathrm{nm}$

$[\alpha]_{D}^{20}=-60^{\circ}(\mathrm{c}=1$ in Methanol $)$

${ }^{1} \mathbf{H}-\mathrm{NMR}\left(600 \mathrm{MHz}, \mathrm{CD}_{3} \mathrm{OD}\right): \delta=1.24\left(\mathrm{~d}, J=6.5 \mathrm{~Hz}, 3 \mathrm{H}, 6^{\prime}-\mathrm{H}_{3}\right), 3.70$ (m, 1H, 4'-H), 3.79 (ddd, $J=3.5,2.0,1.5 \mathrm{~Hz}, 1 \mathrm{H}, 2$ '-H), 3.97 (dd, $\left.J=3.5,3.0 \mathrm{~Hz}, 1 \mathrm{H}, 3^{\prime}-\mathrm{H}\right), 4.07$ (q, $J=6.5 \mathrm{~Hz}$, $\left.1 \mathrm{H}, 5^{\prime}-\mathrm{H}\right), 6.19\left(\mathrm{~d}, J=2.0 \mathrm{~Hz}, 1 \mathrm{H}, 1^{\prime}-\mathrm{H}\right), 6.21$ (dd, $\left.J=3.5,2.5 \mathrm{~Hz}, 1 \mathrm{H}, 4-\mathrm{H}\right), 6.92$ (dd, $J=3.5,1.5 \mathrm{~Hz}, 1 \mathrm{H}, 3-\mathrm{H}), 7.01(\mathrm{dd}, J=2.5,1.5 \mathrm{~Hz}, 1 \mathrm{H}, 5-\mathrm{H})$.

${ }^{13}$ C-NMR (150.8 MHz, CD 3 OD): $\delta=17.0$ (q, C-6'), 67.2 (d, C-3'), 70.9 (d, C-5'), 71.3 (d, C-2'), 74.0 (d, C-4'), 95.7 (d, C-1'), 111.1 (d, C-4), 117.7 (d, C-3), 122.7 (s, C-2), 125.7 (d, C-5), 160.5 (s, C-6).

\subsubsection{1-O-(4-Hydroxybenzoyl)-6-desoxy- $\alpha-L-t a l o p y r a n o s i d ~(51)$}

farbloser Feststoff

$\mathbf{C}_{13} \mathbf{H}_{16} \mathbf{O}_{7}(284.26)$

Anfärbeverhalten: braun (Orcin)

$\mathbf{R}_{\mathbf{f}}=0.15\left(\mathrm{CHCl}_{3} / \mathrm{MeOH} 9: 1\right)$

ESI-MS (positive Ionen): $\mathrm{m} / \mathrm{z}=307[\mathrm{M}+\mathrm{Na}]^{+}, 591[2 \mathrm{M}+\mathrm{Na}]^{+}$

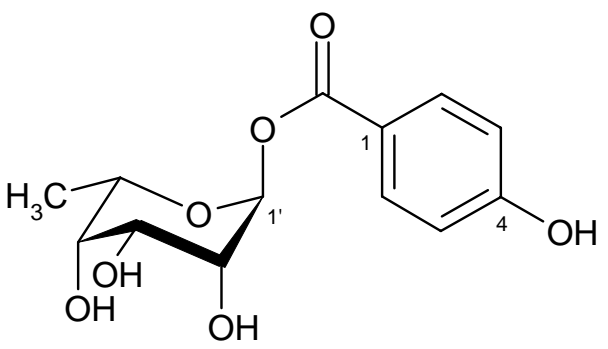

ESI-MS (negative Ionen): $\mathrm{m} / \mathrm{z}=283[\mathrm{M}-\mathrm{H}]^{-}$

HR-ESI-MS: berechnet $\mathrm{m} / \mathrm{z}=307.07882[\mathrm{M}+\mathrm{Na}]^{+}$

$$
\text { gefunden } \mathrm{m} / \mathrm{z}=307.07862 \text { (Abweichung } 0.65 \mathrm{ppm} \text { ) }
$$

IR $(\mathrm{KBr}): \widetilde{v}=3386,2937,1708,1608,1592,1514,1445,1270,1168,1150,1103$ sh, 1082, $1020,956,852,813,770,686 \mathrm{~cm}^{-1}$

UV $(\mathrm{MeOH}): \lambda_{\max }(\log \varepsilon)=211(3.53), 259(3.63) \mathrm{nm}$

$(\mathrm{MeOH} / \mathrm{HCl}): \lambda_{\max }(\log \varepsilon)=209$ (3.69), $260(3.61) \mathrm{nm}$

$(\mathrm{MeOH} / \mathrm{NaOH}): \lambda_{\max }(\log \varepsilon)=221(3.32), 302(3.82) \mathrm{nm}$

CD $(\mathrm{MeOH})$ : kein signifikanter CD-Effekt

$[\alpha]_{D}^{20}=-69^{\circ}(\mathrm{c}=2$ in Methanol $)$ 
${ }^{1} \mathbf{H}-\mathrm{NMR}\left(600 \mathrm{MHz}, \mathrm{CD}_{3} \mathrm{OD}\right): \delta=1.25\left(\mathrm{~d}, J=6.5 \mathrm{~Hz}, 3 \mathrm{H}, 6^{\prime}-\mathrm{H}_{3}\right), 3.72\left(\mathrm{~m}, 1 \mathrm{H}, 4^{\prime}-\mathrm{H}\right), 3.82$ (ddd, $\left.J=3.5,2.0,1.5 \mathrm{~Hz}, 1 \mathrm{H}, 2^{\prime}-\mathrm{H}\right), 3.94$ (t, $\left.J=3.0 \mathrm{~Hz}, 1 \mathrm{H}, 3^{\prime}-\mathrm{H}\right), 4.07$ (q, $J=6.5 \mathrm{~Hz}, 1 \mathrm{H}$, 5'-H), 6.24 (d, $\left.J=2.0 \mathrm{~Hz}, 1 \mathrm{H}, 1^{\prime}-\mathrm{H}\right), 6.84$ (d, $\left.J=9.0 \mathrm{~Hz}, 2 \mathrm{H}, 3-\mathrm{H}, 5-\mathrm{H}\right), 7.88$ (d, $J=9.0 \mathrm{~Hz}$, 2H, 2-H, 6-H).

${ }^{13}$ C-NMR (75.5 MHz, CD 3 OD): $\delta=17.0$ (q, C-6'), 67.3 (d, C-3'), 71.0 (d, C-2'), 71.2 (d, C-5'), 73.9 (d, C-4'), 96.2 (d, C-1'), 116.4 (d, C-3, C-5), 121.5 (s, C-1), 133.1 (d, C-2, C-6), 164.1 (s, C-4), 166.0 (s, C-7).

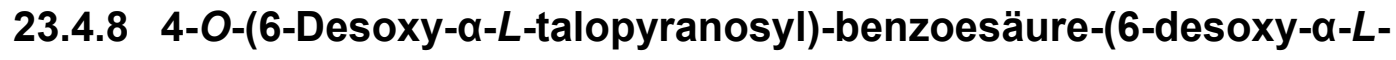 talopyranosyl)ester (54)}

farbloser Feststoff

$\mathbf{C}_{19} \mathbf{H}_{26} \mathbf{O}_{11}$ (430.40)

Anfärbeverhalten: braun (Orcin)

$\mathbf{R}_{\mathbf{f}}=0.13\left(\mathrm{CHCl}_{3} / \mathrm{MeOH} 9: 1\right)$

ESI-MS (positive Ionen): $\mathrm{m} / \mathrm{z}=453[\mathrm{M}+\mathrm{Na}]^{+}, 883[2 \mathrm{M}+\mathrm{Na}]^{+}$

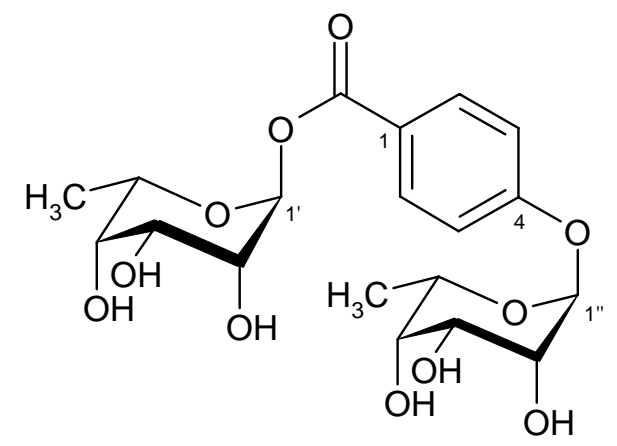

HR-ESI-MS: berechnet $\mathrm{m} / \mathrm{z}=453.13673[\mathrm{M}+\mathrm{Na}]^{+}$ gefunden $\mathrm{m} / \mathrm{z}=453.13677$ (Abweichung $0.09 \mathrm{ppm}$ )

IR $(\mathrm{KBr}): \widetilde{v}=3404,2935,1718,1605,1508,1421,1312,1271,1246,1173,1150,1102$, $1023,974,949 \mathrm{sh}, 912,853,811,771,689 \mathrm{~cm}^{-1}$

UV $(\mathrm{MeOH}): \lambda_{\max }(\log \varepsilon)=252(4.03) \mathrm{nm}$

$(\mathrm{MeOH} / \mathrm{HCl}): \lambda_{\max }(\log \varepsilon)=252(4.01) \mathrm{nm}$

$(\mathrm{MeOH} / \mathrm{NaOH}): \lambda_{\max }(\log \varepsilon)=250(3.99) \mathrm{nm}$

CD $(\mathrm{MeOH}): \lambda_{\max }([\Theta])=252(-6400) \mathrm{nm}$

$[\alpha]_{D}^{20}=-124^{\circ}(\mathrm{c}=2$ in Methanol)

${ }^{1} \mathbf{H}-\mathrm{NMR}\left(600 \mathrm{MHz}, \mathrm{CD}_{3} \mathrm{OD}\right): \delta=1.18\left(\mathrm{~d}, J=6.5 \mathrm{~Hz}, 3 \mathrm{H}, 6{ }^{\prime}{ }^{-}-\mathrm{H}_{3}\right), 1.25(\mathrm{~d}, J=6.5 \mathrm{~Hz}, 3 \mathrm{H}$, 6'- $\left.\mathrm{H}_{3}\right), 3.67$ (m, 1H, 4' '-H), 3.72 (m, 1H, 4'-H), 3.83 (ddd, J=3.5, 2.0, 1.5 Hz, 1H, 2'-H), $3.88(\mathrm{~m}, 1 \mathrm{H}, 5$ '’-H), $3.90(\mathrm{~m}, 1 \mathrm{H}, 2$ ''-H), 3.94 (t, $J=3.0 \mathrm{~Hz}, 1 \mathrm{H}, 3$ ''-H), 3.95 (t, $J=3.0 \mathrm{~Hz}$, $\left.1 \mathrm{H}, 3^{\prime}-\mathrm{H}\right), 4.08$ (q, $J=6.5 \mathrm{~Hz}, 1 \mathrm{H}, 5$ '-H), 5.65 (d, $J=2.0 \mathrm{~Hz}, 1 \mathrm{H}, 1$ ' '-H), 6.24 (d, $J=2.0 \mathrm{~Hz}$, 1H, 1'-H), 7.17 (d, J=9.0 Hz, 2H, 3-H, 5-H), 7.99 (d, $J=9.0$ Hz, 2H, 2-H, 6-H).

${ }^{13}$ C-NMR (75.5 MHz, CD 3 OD): $\delta=16.9$ (q, C-6') 17.0 (q, C-6'), 67.1 (d, C-3'”), 67.3 (d, C-3'), 69.6 (d, C-5'), 71.1 (d, C-2', C-5'), 71.6 (d, C-2'’), 73.9 (d, C-4'’), 96.4 (d, C-1'), 100.2 (d, C-1'’), 117.3 (d, C-3, C-5), 124.3 (s, C-1), 132.8 (d, C-2, C-6), 162.8 (s, C-4), 165.6 (s, C-7). 


\subsection{Häufig aus Pilzstämmen isolierte Metaboliten}

\subsubsection{Ergosterol (58)}

$=(22 E)$-Ergosta-5,7,22-trien-3-ol $=$ Provitamin $\mathrm{D}_{2}$ farbloser Feststoff

$\mathrm{C}_{28} \mathrm{H}_{44} \mathrm{O}$ (396.66)

Anfärbeverhalten: violett (Anisaldehyd) $\mathbf{R}_{\mathbf{f}}=0.70\left(\mathrm{CHCl}_{3} /\right.$ Methanol 9:1)

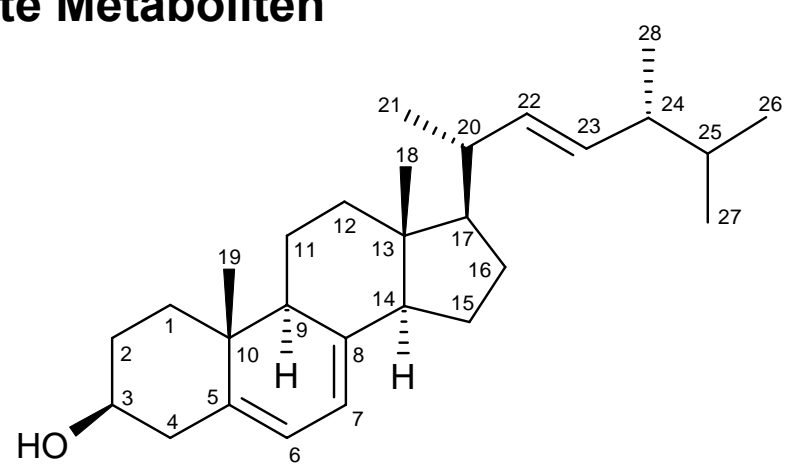

EI-MS (70 eV): m/z (\%) = $396(20)[\mathrm{M}]^{+}, 363(15)\left[\mathrm{M}-\mathrm{CH}_{3}-\mathrm{H}_{2} \mathrm{O}\right]^{+}, 337$ (8), 271 (6), 253 (8), 211 (10), 157 (12), 143 (15), 91 (20), 81 (28), 69 (78), 55 (100), 43 (92)

IR $(\mathrm{KBr}): \widetilde{v}=3420,2954,2871,1711,1653,1458,1372,1333,1242,1160,1056,972,834$, $606 \mathrm{~cm}^{-1}$

UV $(\mathrm{MeOH}): \lambda_{\max }(\log \varepsilon)=263(3.71), 271(3.84), 282(3.86), 293(3.65) \mathrm{nm}$

$(\mathrm{MeOH} / \mathrm{HCl}): \lambda_{\max }(\log \varepsilon)=263$ (3.74), $271(3.85), 281(3.86), 293(3.67) \mathrm{nm}$

$(\mathrm{MeOH} / \mathrm{NaOH}): \lambda_{\max }(\log \varepsilon)=262(3.51), 271(3.70), 281(3.73), 293(3.41) \mathrm{nm}$

CD $(\mathrm{MeOH}): \lambda_{\max }([\Theta])=223$ (16300), 261 (-25700), 270 (-34500), 281 (-33200), 292 $(-16900) \mathrm{nm}$

$[\alpha]_{D}^{20}=-34^{\circ}(\mathrm{c}=1$ in Methanol $)$

${ }^{1}$ H-NMR (600 MHz, $\left.\mathrm{CDCl}_{3}\right): \delta=0.60\left(\mathrm{~s}, 3 \mathrm{H}, 18-\mathrm{H}_{3}\right), 0.80\left(\mathrm{~d}, J=6.5 \mathrm{~Hz}, 3 \mathrm{H}, 26-\mathrm{H}_{3}\right), 0.81$ $\left(\mathrm{d}, J=6.5 \mathrm{~Hz}, 3 \mathrm{H}, 27-\mathrm{H}_{3}\right), 0.89\left(\mathrm{~d}, J=6.5 \mathrm{~Hz}, 3 \mathrm{H}, 28-\mathrm{H}_{3}\right), 0.92\left(\mathrm{~s}, 3 \mathrm{H}, 19-\mathrm{H}_{3}\right), 1.01$ (d, $\left.J=6.5 \mathrm{~Hz}, 3 \mathrm{H}, 21-\mathrm{H}_{3}\right), 1.16-2.07$ (m, 18H, 1- $\mathrm{H}_{2}, 2-\mathrm{H}_{2}, 9-\mathrm{H}, 11-\mathrm{H}_{2}, 12-\mathrm{H}_{2}, 14-\mathrm{H}, 15-\mathrm{H}_{2}$, 16- $\left.\mathrm{H}_{2}, 17-\mathrm{H}, 20-\mathrm{H}, 24-\mathrm{H}, 25-\mathrm{H}\right), 2.26$ (dd, $\left.J=14.0,13.0 \mathrm{~Hz}, 1 \mathrm{H}, 4-\mathrm{H}_{\mathrm{a}}\right), 2.44$ (ddd, $J=14.0$, $\left.3.4,2.0 \mathrm{~Hz}, 1 \mathrm{H}, 4-\mathrm{H}_{\mathrm{b}}\right), 3.61(\mathrm{tt}, J=11.0,4.0 \mathrm{~Hz}, 1 \mathrm{H}, 3-\mathrm{H}), 3.61(\mathrm{tt}, J=11.0,4.0 \mathrm{~Hz}, 1 \mathrm{H}$, 3-H), 5.15 (dd, $J=15.0,8.0 \mathrm{~Hz}, 1 \mathrm{H}, 22-\mathrm{H}), 5.20$ (dd, $J=15.0,7.0 \mathrm{~Hz}, 1 \mathrm{H}, 23-\mathrm{H}), 5.36$ (ddd, $J=8.0,5.5,2.5 \mathrm{~Hz}, 1 \mathrm{H}, 7-\mathrm{H}), 5.54(\mathrm{dd}, J=6.0,2.5 \mathrm{~Hz}, 1 \mathrm{H}, 6-\mathrm{H})$.

${ }^{13}$ C-NMR (150.8 MHz, $\mathrm{CDCl}_{3}$ ): $\delta=12.1$ (q, C-18), 16.3 (q, C-19), 17.3 (q, C-28), 19.7 (q, C-26), 20.0 (q, C-21), 21.1 (q, C-27), 21.1 (t, C-11), 23.0 (t, C-15), 28.3 (t, C-16), 32.0 (t, C-2), 33.1 (d, C-25), 37.0 (s, C-10), 38.4 (t, C-1), 39.1 (t, C-12), 40.5 (d, C-20), 40.8 (t, C-4), 42.8 (s, C-13), 42.8 (d, C-24), 46.3 (d, C-9), 54.6 (d, C-14), 55.7 (d, C-17), 70.5 (d, C-3), 116.3 (d, C-7), 119.6 (d, C-6), 132.0 (d, C-22), 135.6 (d, C-23), 139.8 (s, C-5), 141.4 (s, C-8).

Die Zuordnung der NMR-Daten wird in der Literatur teils widersprüchlich vorgenommen. Die hier wiedergegebene Zuordnung wurde bei einer ausführlichen NMR-Studie erhalten, die INADEQUATE-Messungen beinhaltete und daher als zuverlässig angesehen werden $\operatorname{kann}^{[196]}$. Sie stimmt überein mit den im Handbook of Secondary Fungal Metabolites ${ }^{[179]}$ angegebenen Daten. 


\subsubsection{Cerebrosid C (59)}

farbloses Ö1

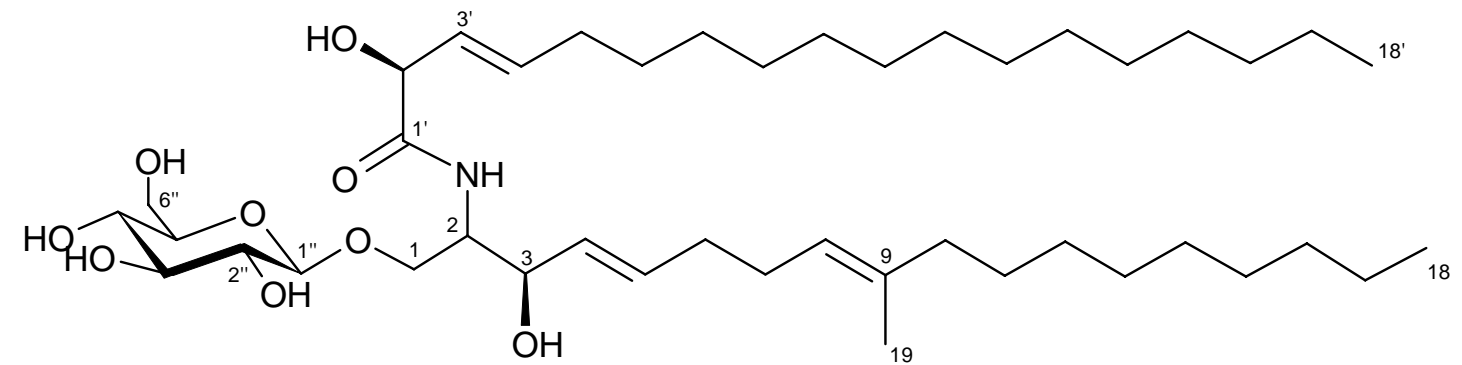

$\mathrm{C}_{43} \mathrm{H}_{79} \mathrm{NO}_{9}$ (754.09)

Anfärbeverhalten: violett (Anisaldehyd)

$\mathbf{R}_{\mathbf{f}}=0.12\left(\mathrm{CHCl}_{3} / \mathrm{MeOH} 9: 1\right)$

EI-MS (70 eV): nicht erhältlich

ESI-MS (positive Ionen): $\mathrm{m} / \mathrm{z}=755[\mathrm{M}+\mathrm{H}]^{+}, 777[\mathrm{M}+\mathrm{Na}]^{+}$

ESI-MS (negative Ionen): $\mathrm{m} / \mathrm{z}=753[\mathrm{M}-\mathrm{H}]^{-}, 798$ [M+HCOO$\left.{ }^{-}\right]^{-}$

IR (KBr): $\widetilde{v}=3426,2923,2860,2364,1727$ (sh), 1639, 1540, 1454, 1383, 1158, 1078, 1033 (sh), $971(\mathrm{sh}) \mathrm{cm}^{-1}$

CD $(\mathrm{MeOH}): \lambda_{\max }([\Theta])=202(-31800) \mathrm{nm}$

$[\alpha]_{D}^{20}=-12^{\circ}(\mathrm{c}=1$ in Methanol $)$

${ }^{1}$ H-NMR $\left(600 \mathrm{MHz}, \mathrm{CD}_{3} \mathrm{OD}\right): \delta=0.89\left(\mathrm{t}, J=7.0 \mathrm{~Hz}, 6 \mathrm{H}, 18-\mathrm{H}_{3}, 18^{\prime}-\mathrm{H}_{3}\right), 1.28(\mathrm{~m}, 36 \mathrm{H}$, $12-\mathrm{H}_{2}$ bis $17-\mathrm{H}_{2}, 6^{\prime}-\mathrm{H}_{2}$ bis $\left.17^{\prime}-\mathrm{H}_{2}\right), 1.38\left(\mathrm{~m}, 2 \mathrm{H}, 11-\mathrm{H}_{2}\right), 1.59$ (s, 3H, 19- $\left.\mathrm{H}_{3}\right), 1.97$ (t, $\left.J=7.5 \mathrm{~Hz}, 2 \mathrm{H}, 10-\mathrm{H}_{2}\right), 2.04\left(\mathrm{~m}, 6 \mathrm{H}, 6-\mathrm{H}_{2}, 7-\mathrm{H}_{2}\right.$ und $\left.5^{\prime}-\mathrm{H}_{2}\right), 3.19(\mathrm{dd}, J=9.0,8.0 \mathrm{~Hz}, 1 \mathrm{H}$, 2"'-H), 3.27 (m, 1H, 4"'-H), $3.29\left(\mathrm{~m}, 1 \mathrm{H}, 5{ }^{\prime},-\mathrm{H}\right)^{*}, 3.35$ (td, $J=9.0,1.0 \mathrm{~Hz}, 1 \mathrm{H}, 3$ '”-H)*, 3.66 $\left(\mathrm{dd}, J=12.0,5.5 \mathrm{~Hz}, 1 \mathrm{H}, 6\right.$ ' $\left.-\mathrm{H}_{\mathrm{a}}\right), 3.70\left(\mathrm{dd}, J=10.5,3.5 \mathrm{~Hz}, 1 \mathrm{H}, 1-\mathrm{H}_{\mathrm{a}}\right), 3.86(\mathrm{dd}, J=12.0$, $1.5 \mathrm{~Hz}, 1 \mathrm{H}, 6$ ' $\left.-\mathrm{H}_{\mathrm{b}}\right), 3.97$ (m, 1H, 2-H), $4.12\left(\mathrm{~m}, 2 \mathrm{H}, 1-\mathrm{H}_{\mathrm{b}}, 3-\mathrm{H}\right), 4.27$ (d, J=7.8 Hz, 1H, 1'’-H), 4.43 (dd, $J=6.0,1.0 \mathrm{~Hz}, 1 \mathrm{H}, 2$ '-H), 5.14 (t, $J=6.8 \mathrm{~Hz}, 1 \mathrm{H}, 8-\mathrm{H}), 5.45$ (ddt, $J=15.0$, 7.2, $1.0 \mathrm{~Hz}, 1 \mathrm{H}, 4-\mathrm{H}), 5.48$ (ddt, $\left.J=15.0,6.0,1.0 \mathrm{~Hz}, 1 \mathrm{H}, 3^{\prime}-\mathrm{H}\right), 5.71$ (dtd, $J=15.0,5.5$, $1.0 \mathrm{~Hz}, 1 \mathrm{H}, 5-\mathrm{H}), 5.83$ (dtd, $\left.J=15.0,6.8,1.0 \mathrm{~Hz}, 1 \mathrm{H}, 4^{\prime}-\mathrm{H}\right)$.

${ }^{13}$ C-NMR (150.5 MHz, CD 3 OD): $\delta=14.5$ (q, C-18, C-18'), 16.2 (q, C-19), 23.8 (t, C-17, C-17'), 28.8 (t, C-7), 29.2 (t, C-11), 30.4 - 30.9 (t, C-12 bis C-15, C-6' bis C-15'), 33.1 (t, C-16, C-16'), 33.5 (t, C-5'), 33.8 (t, C-6), 40.8 (t, C-10), 54.6 (d, C-2), 62.7 (t, C-6’'), 69.7 (t, C-1), 71.6 (d, C-4'’), 72.9 (d, C-3), 74.1 (d, C-2'), 75.0 (d, C-2'’), 77.9 (d, C-5'”)*, 78.0 (d, C-3') ), 104.7 (d, C-1'’), 124.9 (d, C-8), 129.0 (d, C-3'), 131.0 (d, C-4), 134.5 (d, C-5), 134.7 (d, C-4'), 136.7 (s, C-9), 177.2 (s, C-1').

*Zuordnung austauschbar.

Die Zuordnung der NMR-Daten erfolgte nach Literaturangabe ${ }^{[197]}$. 


\subsection{Ascosalipyron aus Diplodia sp. BS 5945}

\subsubsection{Ascosalipyron (60)}

farbloser Feststoff

$\mathrm{C}_{13} \mathrm{H}_{18} \mathrm{O}_{4}$ (238.29)

Anfärbeverhalten: hellbraun (Anisaldehyd)

$\mathbf{R}_{\mathbf{f}}=0.63\left(\mathrm{CHCl}_{3} / \mathrm{MeOH} 9: 1\right)$

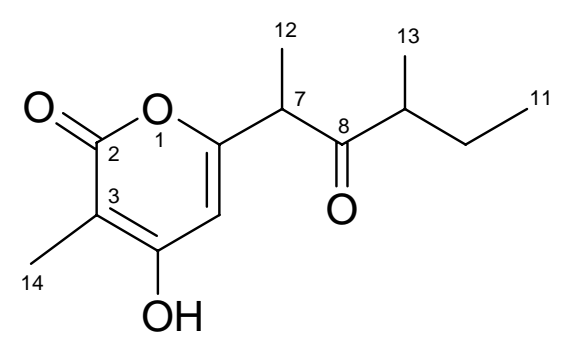

EI-MS $(70 \mathrm{eV}): \mathrm{m} / \mathrm{z}(\%)=238(10)[\mathrm{M}]^{+}, 154(100), 125$ (5), 85 (10), 69 (6), 57 (55), 41 (12)

IR $(\mathrm{KBr}): \widetilde{v}=3427,2968,2934,2370,2343,1717,1700,1684,1654,1646,1636,1587$, $1560,1458,1410,1384,1243,1136,1090,1030 \mathrm{~cm}^{-1}$

UV $(\mathrm{MeOH}): \lambda_{\max }(\log \varepsilon)=213(3.69), 290(3.21) \mathrm{nm}$

$(\mathrm{MeOH} / \mathrm{HCl}): \lambda_{\max }(\log \varepsilon)=287(3.17) \mathrm{nm}$

$(\mathrm{MeOH} / \mathrm{NaOH}): \lambda_{\max }(\log \varepsilon)=215(3.55), 290(3.12) \mathrm{nm}$

CD $(\mathrm{MeOH})$ : kein signifikanter CD-Effekt

$[\alpha]_{D}^{20}=-8^{\circ}(\mathrm{c}=1$ in Methanol $)$

${ }^{1} \mathbf{H}-\mathbf{N M R}\left(300 \mathrm{MHz}, \mathrm{CDCl}_{3}\right): \delta=0.78\left(\mathrm{t}, J=7.5 \mathrm{~Hz}, 3 \mathrm{H}, 11-\mathrm{H}_{3}\right), 1.05(\mathrm{~d}, J=7.0 \mathrm{~Hz}, 3 \mathrm{H}$, $\left.12-\mathrm{H}_{3}\right), 1.33\left(\mathrm{~m}, 1 \mathrm{H}, 10-\mathrm{H}_{\mathrm{a}}\right), 1.34\left(\mathrm{~d}, J=7.0 \mathrm{~Hz}, 3 \mathrm{H}, 13-\mathrm{H}_{3}\right), 1.65(\mathrm{dq}, J=14.0,7.0 \mathrm{~Hz}, 1 \mathrm{H}$, $\left.10-\mathrm{H}_{\mathrm{b}}\right), 1.92\left(\mathrm{~s}, 3 \mathrm{H}, 14-\mathrm{H}_{3}\right), 2.61$ (sext, $\left.J=7.0 \mathrm{~Hz}, 1 \mathrm{H}, 9-\mathrm{H}\right), 3.78$ (q, $\left.J=7.0 \mathrm{~Hz}, 1 \mathrm{H}, 7-\mathrm{H}\right)$, $6.21(\mathrm{~s}, 1 \mathrm{H}, 5-\mathrm{H})$.

${ }^{13}$ C-NMR (75.5 MHz, $\left.\mathrm{CD}_{3} \mathrm{OD}\right): \delta=8.2$ (q, C-14), 11.5 (q, C-11), 14.4 (q, C-12), 16.3 (q, C-13), 25.6 (t, C-10), 46.8 (d, C-9), 49.0 (d, C-7), 99.6 (s, C-3), 101.7 (d, C-5), 160.4 (s, C-6), 166.3 (s, C-4), 167.5 (s, C-2), 211.2 (s, C-8). 


\subsection{Solanapyron C aus Microsphaeropsis sp. BS 6278}

\subsubsection{Solanapyron C (61)}

farbloser Feststoff

$\mathrm{C}_{19} \mathrm{H}_{25} \mathrm{NO}_{4}(331.41)$

Anfärbeverhalten: blau (Anisaldehyd)

$\mathbf{R}_{\mathbf{f}}=0.63\left(\mathrm{CHCl}_{3} / \mathrm{MeOH} 9: 1\right)$

ESI-MS (positive Ionen): $\mathrm{m} / \mathrm{z}=354[\mathrm{M}+\mathrm{Na}]^{+}, 685[2 \mathrm{M}+\mathrm{Na}]^{+}$, $1016[3 \mathrm{M}+\mathrm{Na}]^{+}$

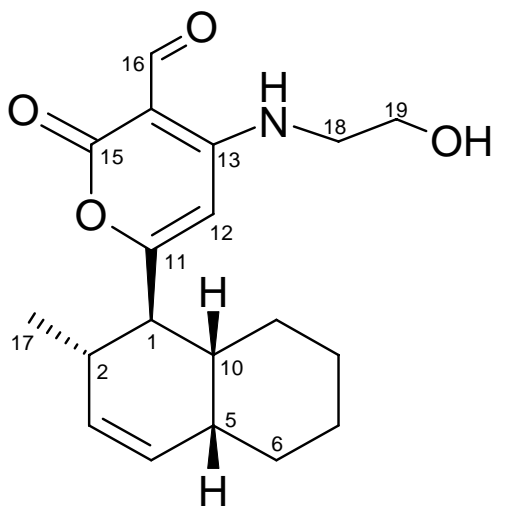

ESI-MS (negative Ionen): $\mathrm{m} / \mathrm{z}=330[\mathrm{M}-\mathrm{H}]^{-}$

UV $(\mathrm{MeOH}): \lambda_{\max }(\log \varepsilon)=203(4.00), 237(4.23), 282(3.72), 318(3.79) \mathrm{nm}$

$(\mathrm{MeOH} / \mathrm{HCl}): \lambda_{\max }(\log \varepsilon)=203$ (3.97), $237(4.22), 282(3.70), 318(3.77) \mathrm{nm}$

$(\mathrm{MeOH} / \mathrm{NaOH}): \lambda_{\max }(\log \varepsilon)=205$ (3.93), 237 (4.20), 281 (3.72), 319 (3.80) nm

CD $(\mathrm{MeOH}): \lambda_{\max }([\Theta])=204$ (18100), 243 (16500), $263(-1000), 313(-4700) \mathrm{nm}$

${ }^{1}$ H-NMR (600 MHz, CD $\left.\mathrm{CD}_{3} \mathrm{OD}\right): \delta=0.97\left(\mathrm{~d}, J=6.5 \mathrm{~Hz}, 3 \mathrm{H}, 17-\mathrm{H}_{3}\right), 1.16-1.51\left(\mathrm{~m}, 6 \mathrm{H}, 6-\mathrm{H}_{\mathrm{ax}}\right.$, 7- $\left.\mathrm{H}_{\mathrm{ax}}, 8-\mathrm{H}_{2}, 9-\mathrm{H}_{2}\right), 1.72\left(\mathrm{~m}, 2 \mathrm{H}, 6-\mathrm{H}_{\mathrm{eq}}, 7-\mathrm{H}_{\mathrm{eq}}\right), 2.14(\mathrm{~m}, 1 \mathrm{H}, 5-\mathrm{H}), 2.22(\mathrm{~m}, 1 \mathrm{H}, 10-\mathrm{H}), 2.54$ (m, 2H, 1-H, 2-H), $3.56\left(\mathrm{t}, J=5.5 \mathrm{~Hz}, 2 \mathrm{H}, 18-\mathrm{H}_{2}\right), 3.73$ (t, $\left.J=5.5 \mathrm{~Hz}, 2 \mathrm{H}, 19-\mathrm{H}_{2}\right), 5.46$ (dt, $J=10.0,1.5 \mathrm{~Hz}, 1 \mathrm{H}, 3-\mathrm{H}), 5.68$ (ddd, $J=10.0,5.0,2.0 \mathrm{~Hz}, 1 \mathrm{H}, 4-\mathrm{H}), 6.48$ (s, 1H, 12-H), 9.89 (s, 1H, 16-H). 


\subsection{Substanzen aus Chaetomium sp. BS 6556}

\subsubsection{Chaetoglobosin A (66)}

gelblicher Feststoff

$\mathrm{C}_{32} \mathbf{H}_{36} \mathbf{N}_{2} \mathbf{O}_{5}(528.65)$

Anfärbeverhalten: braun (Anisaldehyd)

$\mathbf{R}_{\mathbf{f}}=0.57\left(\mathrm{CHCl}_{3} / \mathrm{MeOH} 9: 1\right)$

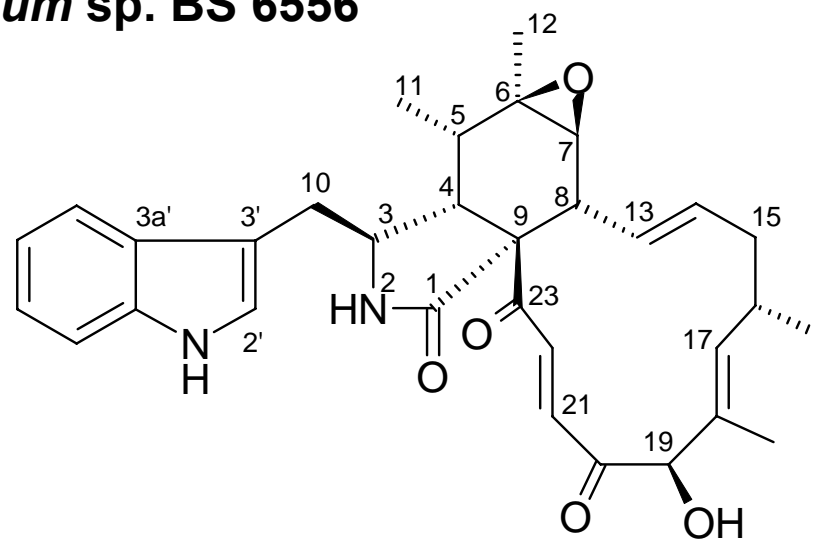

ESI-MS (positive Ionen): $\mathrm{m} / \mathrm{z}=551[\mathrm{M}+\mathrm{Na}]^{+}, 1079[2 \mathrm{M}+\mathrm{Na}]^{+}, 1607[3 \mathrm{M}+\mathrm{Na}]^{+}$

ESI-MS (negative Ionen): $\mathrm{m} / \mathrm{z}=527[\mathrm{M}-\mathrm{H}]^{-}, 1055$ [2M-H]', $1584[3 \mathrm{M}-\mathrm{H}]^{-}$

IR (KBr): $\widetilde{v}=3419,2926,2357,1689,1621$ (sh), 1440, 1383, 1292, 1247, 1157, 1100, 1057, $976,743,670 \mathrm{~cm}^{-1}$

UV (MeOH): $\lambda_{\max }(\log \varepsilon)=220(4.55), 273(3.76), 279(3.76), 290(3.64) \mathrm{nm}$

$(\mathrm{MeOH} / \mathrm{HCl}): \lambda_{\max }(\log \varepsilon)=221$ (4.55), 273 (3.74), 281 (3.73), $288(3.64) \mathrm{nm}$

$(\mathrm{MeOH} / \mathrm{NaOH}): \lambda_{\max }(\log \varepsilon)=220(4.54), 276(3.73), 282(3.75), 289(3.68) \mathrm{nm}$

CD $(\mathrm{MeOH}): \lambda_{\max }([\Theta])=219(-127500) \mathrm{nm}$

$[\alpha]_{D}^{20}=-203^{\circ}(\mathrm{c}=1$ in Methanol)

${ }^{1} \mathbf{H}-\mathrm{NMR}\left(600 \mathrm{MHz}, \mathrm{CDCl}_{3}\right): \delta=0.98\left(\mathrm{~d}, J=6.5 \mathrm{~Hz}, 3 \mathrm{H}, 16-\mathrm{CH}_{3}\right), 1.24(\mathrm{~d}, J=7.0 \mathrm{~Hz}, 3 \mathrm{H}$, $\left.11-\mathrm{H}_{3}\right), 1.28\left(\mathrm{~s}, 3 \mathrm{H}, 12-\mathrm{H}_{3}\right), 1.30\left(\mathrm{~d}, J=1.0 \mathrm{~Hz}, 3 \mathrm{H}, 18-\mathrm{H}_{3}\right), 1.84(\mathrm{qd}, J=7.0,5.0 \mathrm{~Hz}, 1 \mathrm{H}$, 5-H), 2.01 (ddd, $\left.J=13.5,11.0,10.5 \mathrm{~Hz}, 1 \mathrm{H}, 15-\mathrm{H}_{\mathrm{a}}\right), 2.11(\mathrm{dd}, J=10.0,5.0 \mathrm{~Hz}, 1 \mathrm{H}, 8-\mathrm{H})$, $2.25\left(\mathrm{~m}, 1 \mathrm{H}, 15-\mathrm{H}_{\mathrm{b}}\right), 2.43(\mathrm{~m}, 1 \mathrm{H}, 16-\mathrm{H}), 2.61\left(\mathrm{dd}, J=14.5,7.5 \mathrm{~Hz}, 1 \mathrm{H}, 10-\mathrm{H}_{\mathrm{a}}\right), 2.78(\mathrm{~d}, J=$ $5.0 \mathrm{~Hz}, 1 \mathrm{H}, 7-\mathrm{H}), 2.93\left(\mathrm{dd}, J=14.5,4.0 \mathrm{~Hz}, 1 \mathrm{H}, 10-\mathrm{H}_{\mathrm{b}}\right), 3.01(\mathrm{dd}, J=5.0,3.5 \mathrm{~Hz}, 1 \mathrm{H}, 4-\mathrm{H})$, 3.79 (m, 1H, 3-H), 3.85 (br s, 1H, OH), 5.01 (s, 1H, NH), 5.20 (ddd, $J=15.0,10.5,4.0 \mathrm{~Hz}$, $1 \mathrm{H}, 14-\mathrm{H}), 5.58$ (dq, $J=9.0,1.0 \mathrm{~Hz}, 1 \mathrm{H}, 17-\mathrm{H}), 5.84$ (s, 1H, 19-H), 6.04 (ddd, $J=15.0,10.0$, $1.5 \mathrm{~Hz}, 1 \mathrm{H}, 13-\mathrm{H}), 6.46$ (br d, $J=16.5 \mathrm{~Hz}, 1 \mathrm{H}, 21-\mathrm{H}), 6.94$ (d, $J=2.5 \mathrm{~Hz}, 1 \mathrm{H}, 2$ '-H), 7.13 (td, $\left.J=7.5,1.0 \mathrm{~Hz}, 1 \mathrm{H}, 5^{\prime}-\mathrm{H}\right), 7.18\left(\mathrm{td}, J=7.5,1.0 \mathrm{~Hz}, 1 \mathrm{H}, 6{ }^{\prime}-\mathrm{H}\right), 7.34$ (d, $\left.J=8.0 \mathrm{~Hz}, 1 \mathrm{H}, 7^{\prime}-\mathrm{H}\right)$, 7.47 (d, $\left.J=7.5 \mathrm{~Hz}, 1 \mathrm{H}, 4^{\prime}-\mathrm{H}\right), 7.72$ (d, $\left.J=16.5 \mathrm{~Hz}, 22-\mathrm{H}\right), 8.18$ (s, 1H, N1'-H).

${ }^{13}$ C-NMR (150.8 MHz, $\mathrm{CDCl}_{3}$ ): $\delta=10.6$ (q, 18-CH3), 13.6 (q, C-11), 19.9 (q, C-12), 21.0 (q, 16- $\mathrm{CH}_{3}$ ), 32.1 (d, C-16), 34.4 (t, C-10), 36.1 (d, C-5), 41.8 (t, C-15), 47.1 (d, C-4), 48.8 (d, C-8), 52.6 (d, C-3), 58.0 (s, C-6), 62.4 (d, C-7), 63.2 (s, C-9), 81.7 (d, C-19), 110.4 (s, C-3'), 111.6 (d, C-7'), 118.3 (d, C-4'), 120.0 (d, C-5'), 122.5 (d, C-6'), 123.2 (d, C-2'), 126.9 (s, C-3a'), 128.2 (d, C-13), 131.6 (d, C-21), 132.2 (s, C-18), 133.8 (d, C-14), 136.3 (d, C-22), 136.4 (s, C-7a'), 140.4 (d, C-17), 173.0 (s, C-1), 196.7 (s, C-20), 201.7 (s, C-23). 


\subsubsection{9-O-Acetylchaetoglobosin A (65)}

gelblicher Feststoff

$\mathrm{C}_{34} \mathbf{H}_{38} \mathbf{N}_{2} \mathbf{O}_{6}(570.69)$

Anfärbeverhalten: braun (Anisaldehyd)

$\mathbf{R}_{\mathbf{f}}=0.80\left(\mathrm{CHCl}_{3} / \mathrm{MeOH} 9: 1\right)$

ESI-MS (positive Ionen): $\mathrm{m} / \mathrm{z}=593.4[\mathrm{M}+\mathrm{Na}]^{+}, 1163.1[2 \mathrm{M}+\mathrm{Na}]^{+}$

ESI-MS (negative Ionen): $\mathrm{m} / \mathrm{z}=569.2[\mathrm{M}-\mathrm{H}]^{-}, 1139.0[2 \mathrm{M}-\mathrm{H}]^{-}$

HR-ESI-MS: berechnet $\mathrm{m} / \mathrm{z}=593.262208[\mathrm{M}+\mathrm{Na}]^{+}$

$$
\text { gefunden } \mathrm{m} / \mathrm{z}=593.262065 \text { (Abweichung } 0.24 \mathrm{ppm} \text { ) }
$$

IR $(\mathrm{KBr}): \widetilde{v}=3432,2922,2357,1686,1438,1380,1246,1192,1139,1039,974,886,798$, $739,669,545 \mathrm{~cm}^{-1}$

UV $(\mathrm{MeOH}): \lambda_{\max }(\log \varepsilon)=221(4.43), 279(3.70), 288(3.67) \mathrm{nm}$

$(\mathrm{MeOH} / \mathrm{HCl}): \lambda_{\max }(\log \varepsilon)=222(4.46), 282(3.69), 289(3.64) \mathrm{nm}$

$(\mathrm{MeOH} / \mathrm{NaOH}): \lambda_{\max }(\log \varepsilon)=218(4.54), 282(3.80), 290(3.77) \mathrm{nm}$

CD $(\mathrm{MeOH}): \lambda_{\max }([\Theta])=218(-103100), 293(900), 333(-6700) \mathrm{nm}$

$[\alpha]_{D}^{20}=-149^{\circ}(\mathrm{c}=1$ in Methanol $)$

${ }^{1}$ H-NMR $\left(600 \mathrm{MHz}, \mathrm{CD}_{3} \mathrm{OD}\right): \delta=0.94\left(\mathrm{~d}, J=6.5 \mathrm{~Hz}, 3 \mathrm{H}, 16-\mathrm{CH}_{3}\right), 1.13(\mathrm{~d}, J=7.0 \mathrm{~Hz}, 3 \mathrm{H}$, $\left.11-\mathrm{H}_{3}\right), 1.22\left(\mathrm{~s}, 3 \mathrm{H}, 12-\mathrm{H}_{3}\right), 1.39\left(\mathrm{~s}, 3 \mathrm{H}, 18-\mathrm{CH}_{3}\right), 1.76(\mathrm{qd}, J=7.0,5.5 \mathrm{~Hz}, 1 \mathrm{H}, 5-\mathrm{H}), 1.97$ (dt, $\left.J=13.5,11.0 \mathrm{~Hz}, 1 \mathrm{H}, 15-\mathrm{H}_{\mathrm{a}}\right), 2.06$ (dd, $\left.J=10.0,5.0 \mathrm{~Hz}, 1 \mathrm{H}, 8-\mathrm{H}\right), 2.12$ (s, 3H, Acetyl$\left.\mathrm{CH}_{3}\right), 2.21\left(\mathrm{~m}, 1 \mathrm{H}, 15-\mathrm{H}_{\mathrm{b}}\right), 2.40(\mathrm{~m}, 1 \mathrm{H}, 16-\mathrm{H}), 2.63\left(\mathrm{dd}, J=14.5,7.0 \mathrm{~Hz}, 1 \mathrm{H}, 10-\mathrm{H}_{\mathrm{a}}\right), 2.74$ $(\mathrm{d}, J=5.0 \mathrm{~Hz}, 1 \mathrm{H}, 7-\mathrm{H}), 2.83\left(\mathrm{dd}, J=14.5,4.5 \mathrm{~Hz}, 1 \mathrm{H}, 10-\mathrm{H}_{\mathrm{b}}\right), 2.85(\mathrm{~m}, 1 \mathrm{H}, 4-\mathrm{H}), 3.74(\mathrm{~m}$, 1H, 3-H), 5.11 (ddd, $J=15.0,11.0,4.0 \mathrm{~Hz}, 1 \mathrm{H}, 14-\mathrm{H}), 5.62$ (d, $J=9.0 \mathrm{~Hz}, 1 \mathrm{H}, 17-\mathrm{H}), 5.85$ (s, 1H, 19-H), 6.03 (dd, $J=15.0,10.0 \mathrm{~Hz}, 1 \mathrm{H}, 13-\mathrm{H}), 6.30$ (d, $J=16.5 \mathrm{~Hz}, 1 \mathrm{H}, 21-\mathrm{H}), 6.93$ (s, 1H, 2'-H), 7.07 (t, $\left.J=7.5 \mathrm{~Hz}, 1 \mathrm{H}, 5^{\prime}\right), 7.12$ (t, $\left.J=7.5 \mathrm{~Hz}, 1 \mathrm{H}, 6^{\prime}-\mathrm{H}\right), 7.29$ (d, $J=8.0 \mathrm{~Hz}, 1 \mathrm{H}$, 7'-H), 7.43 (d, $\left.J=7.5 \mathrm{~Hz}, 1 \mathrm{H}, 4^{\prime}-\mathrm{H}\right), 7.50$ (d, $\left.J=16.5 \mathrm{~Hz}, 1 \mathrm{H}, 22-\mathrm{H}\right), 8.84$ (s, 1H, N1'-H).

${ }^{13}$ C-NMR (150.8 MHz, $\left.\mathrm{CDCl}_{3}\right): \delta=11.5\left(\mathrm{q}, 18-\mathrm{CH}_{3}\right), 13.2$ (q, C-11), 19.7 (q, C-12), 20.6 (q, Acetyl- $\mathrm{CH}_{3}$ ), 20.7 (q, 16- $\mathrm{CH}_{3}$ ), 32.0 (d, C-16), 33.7 (t, C-10), 36.1 (d, C-5), 41.3 (t, C-15), 47.0 (br d, C-4), 48.3 (d, C-8), 52.4 (d, C-3), 57.8 (s, C-6), 62.2 (d, C-7), 63.2 (br s, C-9), 83.2 (d, C-19), 109.7 (s, C-3'), 111.6 (d, C-7'), 118.1 (d, C-4'), 119.6 (d, C-5'), 122.1 (d, C-6'), 123.6 (d, C-2'), 127.0 (s, C-3a'), 127.6 (s, C-18), 128.3 (d, C-13), 132.8 (d, C-21), 133.1 (d, C-14), ,134.8 (d, C-22), 136.2 (s, C-7a'), 142.4 (d, C-17), 170.0 (s, Acetyl-CO), 173.1 (s, C-1), 194.8 (s, C-20), 196.8 (s, C-23). 


\subsubsection{Chaetoglobosin C (67)}

gelblicher Feststoff

$\mathrm{C}_{32} \mathrm{H}_{36} \mathbf{N}_{2} \mathbf{O}_{5}(528.65)$

Anfärbeverhalten: braun (Anisaldehyd) $\mathbf{R}_{\mathbf{f}}=0.69\left(\mathrm{CHCl}_{3} / \mathrm{MeOH} 9: 1\right)$

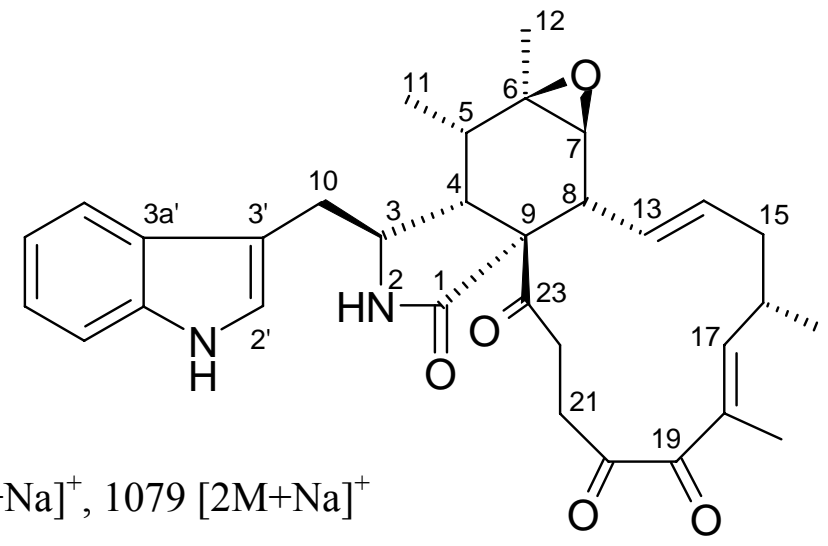

ESI-MS (positive Ionen): $\mathrm{m} / \mathrm{z}=551[\mathrm{M}+\mathrm{Na}]^{+}, 1079[2 \mathrm{M}+\mathrm{Na}]^{+}$

ESI-MS (negative Ionen): $\mathrm{m} / \mathrm{z}=527[\mathrm{M}-\mathrm{H}]^{-}$

IR $(\mathrm{KBr}): \tilde{v}=3427,1925,1698,1649,1447,1385,1335,1245,1100,1025,746,571 \mathrm{~cm}^{-1}$

UV $\quad(\mathrm{MeOH}): \lambda_{\max }(\log \varepsilon)=219(4.33), 281(3.63), 289(3.61) \mathrm{nm}$

$(\mathrm{MeOH} / \mathrm{HCl}): \lambda_{\max }(\log \varepsilon)=220$ (4.27), $283(3.46), 289(3.39) \mathrm{nm}$

$(\mathrm{MeOH} / \mathrm{NaOH}): \lambda_{\max }(\log \varepsilon)=219(4.38), 284(3.76), 290(3.72) \mathrm{nm}$

CD $(\mathrm{MeOH}): \lambda_{\max }([\Theta])=225(-10900), 243(-15900) \mathrm{nm}$

$[\alpha]_{D}^{20}=-20^{\circ}(\mathrm{c}=1$ in Methanol $)$

${ }^{1}$ H-NMR (600 MHz, DMSO-d ${ }_{6}$ ): 0.92 (d, $\left.J=7.0 \mathrm{~Hz}, 3 \mathrm{H}, 11-\mathrm{H}_{3}\right), 0.96(\mathrm{~d}, J=6.5 \mathrm{~Hz}, 3 \mathrm{H}$, $\left.12-\mathrm{H}_{3}\right), 1.17\left(\mathrm{~s}, 3 \mathrm{H}, 16-\mathrm{CH}_{3}\right), 1.61(\mathrm{~m}, 1 \mathrm{H}, 5-\mathrm{H}), 1.64\left(\mathrm{~m}, 1 \mathrm{H}, 22-\mathrm{H}_{\mathrm{a}}\right), 1.69$ (d, J=1.0 Hz, 3H, $\left.18-\mathrm{CH}_{3}\right), 1.75$ (ddd, $\left.J=13.0,10.0,2.5 \mathrm{~Hz}, 1 \mathrm{H}, 21-\mathrm{H}_{\mathrm{a}}\right), 1.82\left(\mathrm{~m}, 1 \mathrm{H}, 15-\mathrm{H}_{\mathrm{a}}\right), 2.15$ $(\mathrm{dd}, J=10.0,6.0 \mathrm{~Hz}, 1 \mathrm{H}, 8-\mathrm{H}), 2.26(\mathrm{dd}, J=5.0,2.5 \mathrm{~Hz}, 1 \mathrm{H}, 4-\mathrm{H}), 2.30$ (m, 1H, 15- $\left.\mathrm{H}_{\mathrm{b}}\right), 2.46$ $\left(\mathrm{td}, J=10.0,2.5 \mathrm{~Hz}, 1 \mathrm{H}, 22-\mathrm{H}_{\mathrm{b}}\right), 2.62\left(\mathrm{ddd}, J=13.0,11.0,2.0 \mathrm{~Hz}, 1 \mathrm{H}, 21-\mathrm{H}_{\mathrm{b}}\right), 2.64(\mathrm{~d}$, $J=6.0 \mathrm{~Hz}, 1 \mathrm{H}, 7-\mathrm{H}), 2.69$ (m, 1H, 16-H), $2.72\left(\mathrm{dd}, J=14.5,4.0 \mathrm{~Hz}, 1 \mathrm{H}, 10-\mathrm{H}_{\mathrm{a}}\right), 2.84$ (dd, $\left.J=14.5,5.5 \mathrm{~Hz}, 1 \mathrm{H}, 10-\mathrm{H}_{\mathrm{b}}\right), 3.75(\mathrm{~m}, 1 \mathrm{H}, 3-\mathrm{H}), 4.97$ (ddd, $\left.J=15.5,11.0,3.0 \mathrm{~Hz}, 1 \mathrm{H}, 14-\mathrm{H}\right)$, $5.92(\mathrm{dq}, J=10.0,1.0 \mathrm{~Hz}, 1 \mathrm{H}, 17-\mathrm{H}), 6.08$ (ddd, $J=15.5,11.0,3.0 \mathrm{~Hz}, 1 \mathrm{H}, 13-\mathrm{H}), 7.00$ (td, $\left.J=7.5,1.0 \mathrm{~Hz}, 1 \mathrm{H}, 5^{\prime}-\mathrm{H}\right), 7.07$ (td, $\left.J=7.5,1.0 \mathrm{~Hz}, 1 \mathrm{H}, 6{ }^{\prime}-\mathrm{H}\right), 7.13$ (d, $\left.J=2.0 \mathrm{~Hz}, 1 \mathrm{H}, 2^{\prime}-\mathrm{H}\right)$, 7.33 (d, $\left.J=8.0 \mathrm{~Hz}, 1 \mathrm{H}, 7^{\prime}-\mathrm{H}\right), 7.54$ (d, $\left.J=8.0 \mathrm{~Hz}, 1 \mathrm{H}, 4^{\prime}-\mathrm{H}\right), 8.42$ (s, N2-H), 10.92 (s, $\mathrm{N} 1$ '-H).

${ }^{13}$ C-NMR (150.8 MHz, DMSO-d 6 ): 10.0 (q, 18- $\mathrm{CH}_{3}$ ), 12.3 (q, C-11), 19.1 (q, C-12), 19.3 (q, 16- $\mathrm{CH}_{3}$ ), 31.8 (t, C-10), 31.9 (t, C-21), 32.5 (d, C-16), 36.1 (d, C-5), 37.1 (t, C-22), 40.0 (t, C-15), 48.3 (d, C-4), 48.4 (d, C-8), 52.3 (d, C-3), 56.7 (s, C-6), 60.3 (d, C-7), 62.3 (s, C-9), 108.1 (s, C-3'), 111.3 (d, C-7'), 118.3 (d, C-4'), 118.7 (d, C-5'), 120.9 (d, C-6'), 125.1 (d, C-2'), 127.1 (d, C-13), 131.0 (s, C-18), 127.7 (s, C-3a'), 133.2 (d, C-14), 135.9 (s, C-7a'), 155.7 (d, C-17), 173.9 (s, C-1), 196.1 (s, C-23), 205.3 (s, C-20), 208.1 (s, C-19). 


\subsubsection{Chaetospiron (68)}

farbloser Feststoff

$\mathbf{C}_{34} \mathbf{H}_{38} \mathbf{O}_{12}$ (638.66)

Anfärbeverhalten: braun (Anisaldehyd)

$\mathbf{R}_{\mathbf{f}}=0.86\left(\mathrm{CHCl}_{3} / \mathrm{MeOH} 9: 1\right)$

ESI-MS (positive Ionen): $\mathrm{m} / \mathrm{z}=661[\mathrm{M}+\mathrm{Na}]^{+}$, $1299[2 \mathrm{M}+\mathrm{Na}]^{+}$

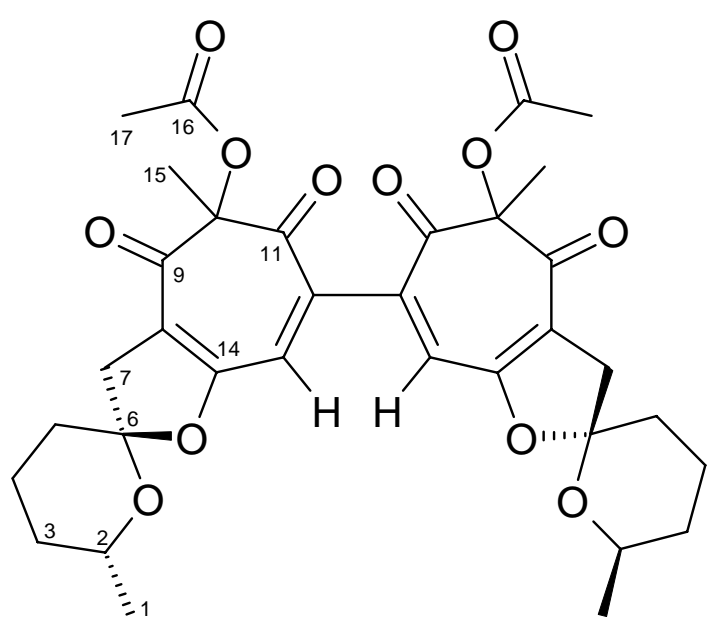

HR-ESI-MS: berechnet $\mathrm{m} / \mathrm{z}=639.243603[\mathrm{M}+\mathrm{H}]^{+}$ gefunden $\mathrm{m} / \mathrm{z}=639.243497$ (Abweichung $0.17 \mathrm{ppm}$ )

IR $(\mathrm{KBr}): \widetilde{v}=3436,2929,1739,1708,1667,1606,1583,1448,1404,1372,1247,1170$, $1129,1080,984,937,902 \mathrm{~cm}^{-1}$

UV $(\mathrm{MeOH}): \lambda_{\max }(\log \varepsilon)=316(4.23) \mathrm{nm}$

$(\mathrm{MeOH} / \mathrm{HCl}): \lambda_{\max }(\log \varepsilon)=318(4.24) \mathrm{nm}$

$(\mathrm{MeOH} / \mathrm{NaOH}): \lambda_{\max }(\log \varepsilon)=224(4.30), 257(3.71), 315(3.81), 454(3.93) \mathrm{nm}$

CD $(\mathrm{MeOH}): \lambda_{\max }([\Theta])=228(-54700), 294(103500), 338(-79400) \mathrm{nm}$

$[\alpha]_{D}^{20}=-217^{\circ}(\mathrm{c}=1$ in Methanol)

${ }^{1} \mathbf{H}-\mathbf{N M R}\left(600 \mathrm{MHz}, \mathrm{CDCl}_{3}\right): 1.06\left(\mathrm{~d}, J=6.0 \mathrm{~Hz}, 3 \mathrm{H}, 1-\mathrm{H}_{3}\right), 1.23\left(\mathrm{~m}, 1 \mathrm{H}, 3-\mathrm{H}_{\mathrm{a}}\right), 1.44(\mathrm{~m}, 1 \mathrm{H}$, $\left.5-\mathrm{H}_{\mathrm{a}}\right), 1.59\left(\mathrm{~s}, 3 \mathrm{H}, 15-\mathrm{H}_{3}\right), 1.62\left(\mathrm{~m}, 1 \mathrm{H}, 3-\mathrm{H}_{\mathrm{b}}\right), 1.66\left(\mathrm{~m}, 1 \mathrm{H}, 4-\mathrm{H}_{\mathrm{a}}\right), 1.84\left(\mathrm{~m}, 1 \mathrm{H}, 4-\mathrm{H}_{\mathrm{b}}\right), 1.87$ $\left(\mathrm{m}, 1 \mathrm{H}, 5-\mathrm{H}_{\mathrm{b}}\right), 2.14\left(\mathrm{~s}, 3 \mathrm{H}, 17-\mathrm{H}_{3}\right), 2.40\left(\mathrm{~d}, J=16.5 \mathrm{~Hz}, 1 \mathrm{H}, 7-\mathrm{H}_{\mathrm{a}}\right), 2.67(\mathrm{~d}, J=16.5 \mathrm{~Hz}, 1 \mathrm{H}$, $\left.7-\mathrm{H}_{\mathrm{b}}\right), 3.83(\mathrm{dqd}, J=12.5,6.0,2.0 \mathrm{~Hz}, 1 \mathrm{H}, 2-\mathrm{H}), 7.76(\mathrm{~s}, 1 \mathrm{H}, 10-\mathrm{H})$.

${ }^{13}$ C-NMR (150.8 MHz, CDCl 3 ): 18.2 (t, C-4), 20.2 (q, C-17), 21.6 (q, C-1), 22.4 (s, C-15), 31.4 (t, C-3), 32.0 (t, C-5), 37.5 (t, C-7), 68.7 (d, C-2), 84.7 (s, C-13), 102.8 (s, C-6), 111.1 (s, C-11), 122.5 (s, C-8), 145.0 (s, C-9), 157.6 (d, C-10), 169.9 (s, C-16), 191.8 (s, C-14), 192.2 (s, C-12). 


\subsection{Sesquiterpene aus Drechs/era sp. BS 6618}

\subsubsection{Helminthosporal (78)}

farbloses Ö1

$\mathrm{C}_{15} \mathbf{H}_{22} \mathbf{O}_{2}$ (234.34)

Anfärbeverhalten: braun (Anisaldehyd)

$\mathbf{R}_{\mathbf{f}}=0.70\left(\mathrm{CHCl}_{3} / \mathrm{MeOH} 9: 1\right)$

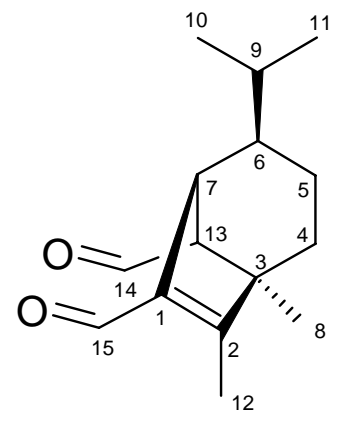

EI-MS (70 eV): m/z (\%) = 234 (2) [M] $]^{+}, 205$ (5), 187 (8), 168 (9), 133 (9), 121 (10), 107 (12), 91 (38), 79 (28), 69 (18), 55 (44), 43 (50), 41 (100)

DCI-MS $(200 \mathrm{eV}): \mathrm{m} / \mathrm{z}=252\left[\mathrm{M}+\mathrm{NH}_{4}\right]^{+}, 486\left[2 \mathrm{M}+\mathrm{NH}_{4}\right]^{+}$

IR (KBr): $\widetilde{v}=3430,2938,2868,2360,1705,1652,1457,1383,1216,1170,1118,1068 \mathrm{~cm}^{-1}$

UV $(\mathrm{MeOH}): \lambda_{\max }(\log \varepsilon)=260(3.38) \mathrm{nm}$

$(\mathrm{MeOH} / \mathrm{HCl}): \lambda_{\max }(\log \varepsilon)=259(3.22) \mathrm{nm}$

$(\mathrm{MeOH} / \mathrm{NaOH}): \lambda_{\max }(\log \varepsilon)=270(3.17) \mathrm{nm}$

CD $(\mathrm{MeOH}): \lambda_{\max }([\Theta])=213(1200), 235(-700), 271(4600), 332(-2900) \mathrm{nm}$

$[\alpha]_{D}^{20}=34^{\circ}(\mathrm{c}=1$ in Methanol $)$

${ }^{1} \mathbf{H}-\mathrm{NMR}\left(300 \mathrm{MHz}, \mathrm{CDCl}_{3}\right): \delta=0.75\left(\mathrm{~d}, J=5.0 \mathrm{~Hz}, 3 \mathrm{H}, 11-\mathrm{H}_{3}\right), 0.88\left(\mathrm{~m}, 1 \mathrm{H}, 5-\mathrm{H}_{\mathrm{a}}\right), 1.04$ $\left(\mathrm{m}, 4 \mathrm{H}, 10-\mathrm{H}_{3}\right.$ und 6-H), $1.04(\mathrm{~m}, 1 \mathrm{H}, 9-\mathrm{H}), 1.14\left(\mathrm{~s}, 3 \mathrm{H}, 8-\mathrm{H}_{3}\right), 1.40\left(\mathrm{~m}, 2 \mathrm{H}, 4-\mathrm{H}_{2}\right), 1.76$ (m, 1H, 5- $\left.\mathrm{H}_{\mathrm{b}}\right), 2.05$ (s, 3H, 12- $\left.\mathrm{H}_{3}\right), 2.14$ (d, $\left.J=4.0 \mathrm{~Hz}, 1 \mathrm{H}, 13-\mathrm{H}\right), 3.36(\mathrm{~s}, 1 \mathrm{H}, 7-\mathrm{H}), 9.48$ (d, $J=4.0 \mathrm{~Hz}, 1 \mathrm{H}, 14-\mathrm{H}), 10.00$ (s, 1H, 15-H).

${ }^{13}$ C-NMR (75.5 MHz, $\left.\mathrm{CDCl}_{3}\right): \delta=10.6$ (q, C-12), 18.9 (q, C-8), 20.6 (q, C-11), 21.5 (q, C-10), 25.0 (t, C-5), 31.4 (d, C-9), 33.5 (t, C-4), 40.1 (d, C-7), 43.6 (d, C-6), 51.2 (s, C-3), 70.7 (d, C-13), 136.9 (s, C-1), 165.0 (s, C-2), 187.3 (d, C-15), 204.0 (d, C-14).

\subsubsection{Helminthosporol (79)}

farbloses Ö1

$\mathrm{C}_{15} \mathrm{H}_{24} \mathrm{O}_{2}$ (236.36)

Anfärbeverhalten: braun (Anisaldehyd)

$\mathbf{R}_{\mathbf{f}}=0.65\left(\mathrm{CHCl}_{3} / \mathrm{MeOH} 9: 1\right)$

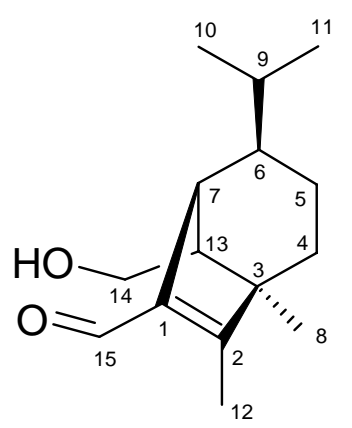

EI-MS $(70 \mathrm{eV}): \mathrm{m} / \mathrm{z}(\%)=236(40)[\mathrm{M}]^{+}, 218(20), 205$ (20), 189 (16), 175 (12), 153 (32), 135 (34), 123 (85), 107 (100), 97 (82), 79 (44), 69 (46), 55 (70), 43 (56), 41 (86) 
ESI-MS (positive Ionen): $\mathrm{m} / \mathrm{z}=237[\mathrm{M}+\mathrm{H}]^{+}$

IR $(\mathrm{KBr}): \widetilde{v}=3433,2935,2866,2359,1702(\mathrm{sh}), 1656,1454,1379,1072,1026,670 \mathrm{~cm}^{-1}$

UV $(\mathrm{MeOH}): \lambda_{\max }(\log \varepsilon)=265(3.83) \mathrm{nm}$

$(\mathrm{MeOH} / \mathrm{HCl}): \lambda_{\max }(\log \varepsilon)=265(3.75) \mathrm{nm}$

$(\mathrm{MeOH} / \mathrm{NaOH}): \lambda_{\max }(\log \varepsilon)=266(3.75) \mathrm{nm}$

CD $(\mathrm{MeOH}): \lambda_{\max }([\Theta])=204$ (8900), 230 (1200), 267 (13300), $333(-7700) \mathrm{nm}$

$[\alpha]_{D}^{20}=-17^{\circ}(\mathrm{c}=1$ in Methanol $)$

${ }^{1} \mathbf{H}-\mathrm{NMR}\left(300 \mathrm{MHz}, \mathrm{CDCl}_{3}\right): \delta=0.72\left(\mathrm{~d}, J=5.5 \mathrm{~Hz}, 3 \mathrm{H}, 11-\mathrm{H}_{3}\right), 0.84\left(\mathrm{~m}, 1 \mathrm{H}, 5-\mathrm{H}_{\mathrm{a}}\right), 1.00$ (s, 3H, 8- $\left.\mathrm{H}_{3}\right), 1.00(\mathrm{~m}, 1 \mathrm{H}, 9-\mathrm{H}), 1.04\left(\mathrm{~d}, J=4.5 \mathrm{~Hz}, 3 \mathrm{H}, 10-\mathrm{H}_{3}\right), 1.01(\mathrm{~m}, 1 \mathrm{H}, 6-\mathrm{H}), 1.37$ $\left(\mathrm{m}, 2 \mathrm{H}, 4-\mathrm{H}_{2}\right), 1.71\left(\mathrm{~m}, 1 \mathrm{H}, 5-\mathrm{H}_{\mathrm{b}}\right), 1.64(\mathrm{dd}, J=9.0,5.5 \mathrm{~Hz}, 1 \mathrm{H}, 13-\mathrm{H}), 1.98\left(\mathrm{~s}, 3 \mathrm{H}, 12-\mathrm{H}_{3}\right)$, $3.16(\mathrm{~s}, 1 \mathrm{H}, 7-\mathrm{H}), 3.28\left(\mathrm{dd}, J=11.0,9.0 \mathrm{~Hz}, 1 \mathrm{H}, 14-\mathrm{H}_{\mathrm{a}}\right), 3.69(\mathrm{dd}, J=11.0,5.0 \mathrm{~Hz}, 1 \mathrm{H}$, $\left.14-\mathrm{H}_{\mathrm{b}}\right), 10.00(\mathrm{~s}, 1 \mathrm{H}, 15-\mathrm{H})$.

${ }^{13}$ C-NMR (75.5 MHz, $\mathrm{CDCl}_{3}$ ): $\delta=10.6$ (q, C-12), 18.3 (q, C-8), 20.7 (q, C-11), 21.6 (q, C-10), 25.2 (t, C-5), 31.7 (d, C-9), 34.1 (t, C-4), 41.1 (d, C-7), 44.8 (d, C-6), 50.7 (s, C-3), 61.1 (d, C-13), 62.2 (t, C-14), 137.2 (s, C-1), 166.3 (s, C-2), 188.3 (d, C-15).

\subsection{3 cis-Sativendiol (80)}

farbloses Ö1

$\mathrm{C}_{15} \mathrm{H}_{24} \mathrm{O}_{2}$ (236.36)

Anfärbeverhalten: hellbraun (Anisaldehyd)

$\mathbf{R}_{\mathbf{f}}=0.72\left(\mathrm{CHCl}_{3} / \mathrm{MeOH} 9: 1\right)$

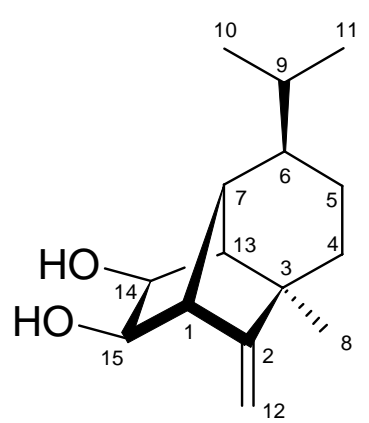

EI-MS $(70 \mathrm{eV}): \mathrm{m} / \mathrm{z}(\%)=219[\mathrm{M}-\mathrm{OH}]^{+}$(70), 207 (28), 201 (92), 177 (45), 159 (24), 145 (28), 133 (24), 119 (26), 105 (38), 91 (35)

DCI-MS $(200 \mathrm{eV}): \mathrm{m} / \mathrm{z}=254(100)\left[\mathrm{M}+\mathrm{NH}_{4}\right]^{+}, 490(2)\left[2 \mathrm{M}+\mathrm{NH}_{4}\right]^{+}$

ESI-MS (positive Ionen): $\mathrm{m} / \mathrm{z}=259[\mathrm{M}+\mathrm{Na}]^{+}, 495[2 \mathrm{M}+\mathrm{Na}]^{+}$

IR (KBr): $\widetilde{v}=3419,2937,2863,2362,2322,1654,1459,1381,1319,1261,1160,1056$, $1022,883,794,669 \mathrm{~cm}^{-1}$

UV $(\mathrm{MeOH}): \lambda_{\max }(\log \varepsilon)=285(3.51) \mathrm{nm}$

$(\mathrm{MeOH} / \mathrm{HCl}): \lambda_{\max }(\log \varepsilon)=287(3.17), 320(3.19) \mathrm{nm}$

$(\mathrm{MeOH} / \mathrm{NaOH}): \lambda_{\max }(\log \varepsilon)=292(3.66) \mathrm{nm}$

CD (MeOH): $201(-36100) \mathrm{nm}$ 
$[\alpha]_{D}^{20}=-77^{\circ}(\mathrm{c}=1$ in Methanol $)$

${ }^{1}$ H-NMR $\left(300 \mathrm{MHz}, \mathrm{CDCl}_{3}\right): \delta=0.85\left(\mathrm{~d}, J=6.0 \mathrm{~Hz}, 3 \mathrm{H}, 11-\mathrm{H}_{3}\right), 0.92(\mathrm{~d}, J=6.0 \mathrm{~Hz}, 3 \mathrm{H}$, $\left.10-\mathrm{H}_{3}\right), 1.03\left(\mathrm{~s}, 3 \mathrm{H}, 8-\mathrm{H}_{3}\right), 1.16\left(\mathrm{dd}, J=12.0,5.0 \mathrm{~Hz}, 1 \mathrm{H}, 5-\mathrm{H}_{\mathrm{a}}\right), 1.30$ (dd, $J=12.5,2.0 \mathrm{~Hz}$, $\left.1 \mathrm{H}, 4-\mathrm{H}_{\mathrm{a}}\right), 1.32(\mathrm{~m}, 1 \mathrm{H}, 6-\mathrm{H}), 1.34(\mathrm{~m}, 1 \mathrm{H}, 9-\mathrm{H}), 1.44\left(\mathrm{dd}, J=12.5,4.5 \mathrm{~Hz}, 1 \mathrm{H}, 4-\mathrm{H}_{\mathrm{b}}\right), 1.56$ $\left(\mathrm{m}, 1 \mathrm{H}, 5-\mathrm{H}_{\mathrm{b}}\right), 1.56$ (br s, 1H, 13-H), 2.40 (br s, 1H, 7-H), 2.63 (br s, 1H, 1-H), 3.63 (d, $J=6.0 \mathrm{~Hz}, 15-\mathrm{H}), 4.02(\mathrm{~d}, J=6.0 \mathrm{~Hz}, 1 \mathrm{H}, 14-\mathrm{H}), 4.58\left(\mathrm{~d}, J=1.0 \mathrm{~Hz}, 1 \mathrm{H}, 12-\mathrm{H}_{\mathrm{a}}\right), 4.89$ (d, $\left.J=1.0 \mathrm{~Hz}, 1 \mathrm{H}, 12-\mathrm{H}_{\mathrm{b}}\right)$.

${ }^{13}$ C-NMR (75.5 MHz, $\mathrm{CDCl}_{3}$ ): $\delta=20.8$ (q, C-8), 20.9 (q, C-11), 21.1 (q, C-10), 25.4 (t, C-5), 33.0 (d, C-9), 39.9 (t, C-4), 41.7 (d, C-7), 41.9 (d, C-6), 42.9 (s, C-3), 54.1 (d, C-1), 58.2 (d, C-13), 69.3 (d, C-14), 74.7 (d, C-15), 103.2 (t, C-12), 157.1 (s, C-2).

\subsection{4 (15R)-Bis(prehelminthosporol) (81A)}

und (15S)-Bis(prehelminthosporol) (81B)
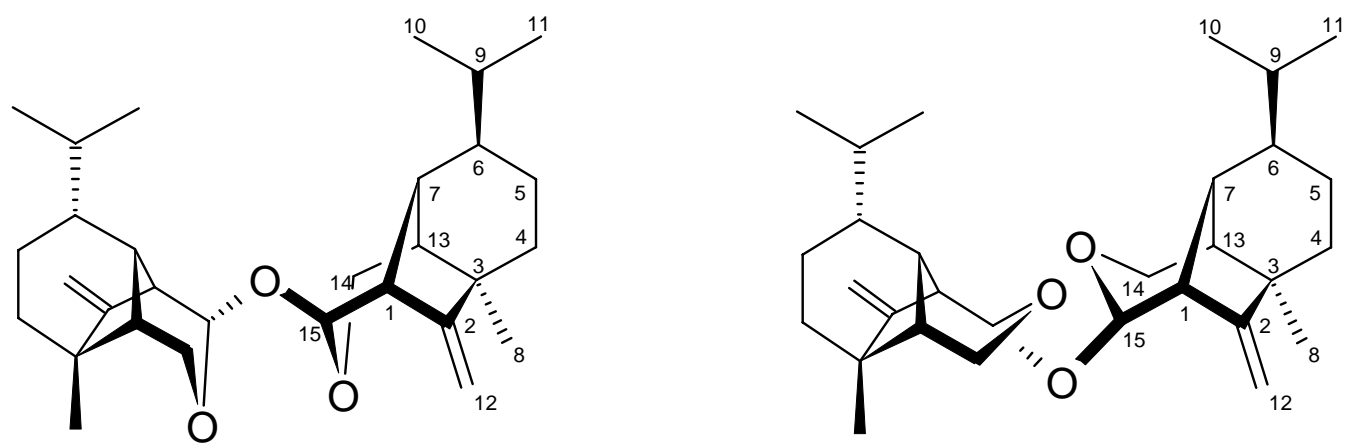

Die Epimere waren unter den verwendeten Bedingungen nicht voneinander trennbar und wurden als farbloses Öl erhalten. Die NMR-Daten konnten der jeweiligen Verbindung zugeordnet werden, alle anderen Daten beziehen sich auf ein 5:3 Gemisch von 81A und 81B.

$\mathrm{C}_{30} \mathrm{H}_{46} \mathrm{O}_{3}$ (454.70)

Anfärbeverhalten: hellbraun (Anisaldehyd)

$\mathbf{R}_{\mathbf{f}}=0.78\left(\mathrm{CHCl}_{3} / \mathrm{MeOH} 9: 1\right)$

EI-MS $(70 \mathrm{eV}): \mathrm{m} / \mathrm{z}(\%)=454(2)[\mathrm{M}]^{+}, 219.3(100)$ [Monomer-Fragment $^{+}$

ESI-MS (positive Ionen): $\mathrm{m} / \mathrm{z}=477.4[\mathrm{M}+\mathrm{Na}]^{+}, 931.3[2 \mathrm{M}+\mathrm{Na}]^{+}$

HR-ESI-MS: berechnet $\mathrm{m} / \mathrm{z}=477.33392[\mathrm{M}+\mathrm{Na}]^{+}$ gefunden $\mathrm{m} / \mathrm{z}=477.33390$ (Abweichung $0.04 \mathrm{ppm}$ ) 


\section{HR-ESI-MS nach Säurezugabe (TFA):}

berechnet $\mathrm{m} / \mathrm{z}=219.17434\left[\mathrm{C}_{15} \mathrm{H}_{22} \mathrm{O}=\text { Monomer-Fragment }\right]^{+}$

gefunden $\mathrm{m} / \mathrm{z}=219.17422$ (Abweichung $0.55 \mathrm{ppm}$ )

IR (KBr): $\widetilde{v}=3412,2930,2880,1657,1456,1376,1334,1279,1249,1222,1169,1114$, $1099,1054,1014,965,924,881,817,730 \mathrm{~cm}^{-1}$

UV $(\mathrm{MeOH}): \lambda_{\max }(\log \varepsilon)=203$ (4.19), 255 (3.55), $280(3.50), 311(3.43) \mathrm{nm}$

$(\mathrm{MeOH} / \mathrm{HCl}): \lambda_{\max }(\log \varepsilon)=204$ (4.32), $324(3.49) \mathrm{nm}$

$(\mathrm{MeOH} / \mathrm{NaOH}): \lambda_{\max }(\log \varepsilon)=206(4.15), 254(3.58), 290(3.64) \mathrm{nm}$

CD $(\mathrm{MeOH}): \lambda_{\max }([\Theta])=202(-59200) \mathrm{nm}$

$[\alpha]_{D}^{20}=-34^{\circ}(\mathrm{c}=1$ in Methanol $)$

(15R)-Bis(prehelminthosporol) (81A):

${ }^{1}$ H-NMR $\left(300 \mathrm{MHz}, \mathrm{CDCl}_{3}\right): \delta=0.85\left(\mathrm{~d}, J=7.0 \mathrm{~Hz}, 3 \mathrm{H}, 11-\mathrm{H}_{3}\right), 0.90(\mathrm{~d}, J=7.0 \mathrm{~Hz}, 3 \mathrm{H}$, $\left.10-\mathrm{H}_{3}\right), 1.13\left(\mathrm{~s}, 3 \mathrm{H}, 8-\mathrm{H}_{3}\right), 1.15\left(\mathrm{~m}, 2 \mathrm{H}, 5-\mathrm{H}_{\mathrm{a}}\right.$ und 6-H), $1.25(\mathrm{~s}, 1 \mathrm{H}, 13-\mathrm{H}), 1.34(\mathrm{~m}, 2 \mathrm{H}, 9-\mathrm{H}$, 4- $\left.\mathrm{H}_{\mathrm{a}}\right), 1.44\left(\mathrm{~m}, 1 \mathrm{H}, 4-\mathrm{H}_{\mathrm{b}}\right), 1.65\left(\mathrm{~m} \mathrm{1H}, 5-\mathrm{H}_{\mathrm{b}}\right), 2.06(\mathrm{~s}, 1 \mathrm{H}, 7-\mathrm{H}), 2.49(\mathrm{~s}, 1 \mathrm{H}, 1-\mathrm{H}), 3.54$ (dd, $\left.J=11.5,1.5 \mathrm{~Hz}, 1 \mathrm{H}, 14-\mathrm{H}_{\mathrm{a}}\right), 3.96\left(\mathrm{dd}, J=11.5,2.0 \mathrm{~Hz}, 1 \mathrm{H}, 14-\mathrm{H}_{\mathrm{b}}\right), 4.64$ (s, 1H, 15-H), 4.77 $\left(\mathrm{s}, 1 \mathrm{H}, 12-\mathrm{H}_{\mathrm{a}}\right), 4.87\left(\mathrm{~s}, 1 \mathrm{H}, 12-\mathrm{H}_{\mathrm{b}}\right)$.

${ }^{13}$ C-NMR (75.5 MHz, $\mathrm{CDCl}_{3}$ ): $\delta=19.0$ (q, C-8), 20.9 (q, C-11), 21.1 (q, C-10), 25.8 (t, C-5), 30.7 (d, C-9), 42.0 (t, C-4), 44.5 (d, C-7), 44.8 (s, C-3), 46.5 (d, C-6), 50.4 (d, C-1), 50.4 (d, C-13), 67.7 (t, C-14), 96.0 (d, C-15), 102.0 (t, C-12), 157.2 (s, C-2).

(15S)-Bis(prehelminthosporol) (81B):

${ }^{1}$ H-NMR $\left(300 \mathrm{MHz}, \mathrm{CDCl}_{3}\right): \delta=0.83\left(\mathrm{~d}, J=6.5 \mathrm{~Hz}, 3 \mathrm{H}, 11-\mathrm{H}_{3}\right), 0.93(\mathrm{~d}, J=6.5 \mathrm{~Hz}, 3 \mathrm{H}$, $\left.10-\mathrm{H}_{3}\right), 1.10(\mathrm{~m}, 1 \mathrm{H}, 6-\mathrm{H}), 1.15\left(\mathrm{~m}, 1 \mathrm{H}, 5-\mathrm{H}_{\mathrm{a}}\right), 1.16\left(\mathrm{~s}, 3 \mathrm{H}, 8-\mathrm{H}_{3}\right), 1.30-1.45\left(\mathrm{~m}, 3 \mathrm{H}, 4-\mathrm{H}_{2}\right.$, 9-H), 1.33 (m, 1H, 13-H), 1.65 (m, 1H, 5- $\left.\mathrm{H}_{\mathrm{b}}\right), 2.57$ (br s, 1H, 1-H), 3.74 (dd, J=11.0, $2.0 \mathrm{~Hz}$, $\left.1 \mathrm{H}, 14-\mathrm{H}_{\mathrm{a}}\right), 3.89$ (d, J=11.0 Hz, 1H, 14- $\left.\mathrm{H}_{\mathrm{b}}\right), 4.70\left(\mathrm{~s}, 1 \mathrm{H}, 12-\mathrm{H}_{\mathrm{a}}\right), 4.80\left(\mathrm{~s}, 1 \mathrm{H}, 12-\mathrm{H}_{\mathrm{b}}\right), 4.92(\mathrm{~d}$, $J=3.0 \mathrm{~Hz}, 1 \mathrm{H}, 15-\mathrm{H})$.

${ }^{13}$ C-NMR (75.5 MHz, $\mathrm{CDCl}_{3}$ ): 20.2 (q, C-8), 21.0 (q, C-11), 21.0 (q, C-10), 25.9 (t, C-5), 30.8 (d, C-9), 36.9 (d, C-7), 41.9 (t, C-4), 43.6 (s, C-3), 46.6 (d, C-6), 48.8 (d, C-1), 51.6 (d, C-13), 64.8 (t, C-14), 97.1 (d, C-15), 101.5 (t, C-12), 157.9 (s, C-2). 


\subsection{Spiroverbindung aus Beauveria sp. BS 6750}

\subsubsection{Beauveriaspirolid (86)}

farbloses Ö1

$\mathbf{C}_{10} \mathbf{H}_{14} \mathbf{O}_{4}(198.22)$

Anfärbeverhalten: gelb (Anisaldehyd)

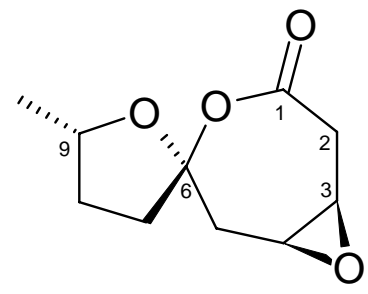

$\mathbf{R}_{\mathbf{f}}=0.58\left(\mathrm{CHCl}_{3} / \mathrm{MeOH} 9: 1\right)$

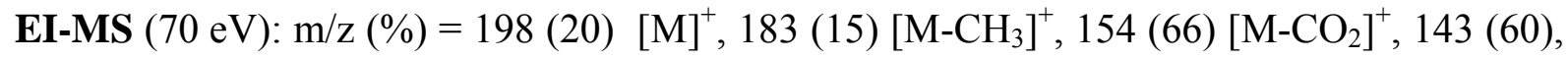
139 (53), 127 (72), 111 (70), 98 (22), 83 (40), 71 (28), 55 (100), 43 (78)

HR-EI-MS: $\mathrm{m} / \mathrm{z}=198.0892$ gefunden wie berechnet.

IR $(\mathrm{KBr}): \widetilde{v}=3430,2963,1774,1636,1560,1457,1353,1195,1157,1098,1054,1026$, $991,921,876,828,661 \mathrm{~cm}^{-1}$

UV $(\mathrm{MeOH}): \lambda_{\max }(\log \varepsilon)=205(3.37) \mathrm{nm}$

$(\mathrm{MeOH} / \mathrm{HCl}): \lambda_{\max }(\log \varepsilon)=203(3.29) \mathrm{nm}$

$(\mathrm{MeOH} / \mathrm{NaOH}): \lambda_{\max }(\log \varepsilon)=273(3.49) \mathrm{nm}$

CD $(\mathrm{MeOH}): \lambda_{\max }([\Theta])=211(-4900) \mathrm{nm}$

$[\alpha]_{D}^{20}=25^{\circ}(\mathrm{c}=1$ in Methanol $)$

${ }^{1}$ H-NMR $\left(300 \mathrm{MHz}, \mathrm{CD}_{3} \mathrm{OD}\right): \delta=1.16\left(\mathrm{~d}, J=6.0 \mathrm{~Hz}, 3 \mathrm{H}, 10-\mathrm{H}_{3}\right), 1.44\left(\mathrm{~m}, 1 \mathrm{H}, 8-\mathrm{H}_{\mathrm{a}}\right), 2.08$ $\left(\mathrm{m}, 1 \mathrm{H}, 8-\mathrm{H}_{\mathrm{b}}\right), 2.00-2.15\left(\mathrm{~m}, 2 \mathrm{H}, 7-\mathrm{H}_{2}\right), 2.19\left(\mathrm{dd}, J=14.5,6.0 \mathrm{~Hz}, 1 \mathrm{H}, 5-\mathrm{H}_{\mathrm{a}}\right), 2.33(\mathrm{~d}$, $\left.J=14.5 \mathrm{~Hz}, 1 \mathrm{H}, 5-\mathrm{H}_{\mathrm{b}}\right), 2.51\left(\mathrm{~d}, J=18.0 \mathrm{~Hz}, 1 \mathrm{H}, 2-\mathrm{H}_{\mathrm{a}}\right), 2.84\left(\mathrm{dd}, J=18.0,7.8 \mathrm{~Hz}, 1 \mathrm{H}, 2-\mathrm{H}_{\mathrm{b}}\right)$, $4.16(\mathrm{~m}, 1 \mathrm{H}, 9-\mathrm{H}), 4.89$ (m, 1H, 3-H), 5.22 (t, $J=5.5 \mathrm{~Hz}, 1 \mathrm{H}, 4-\mathrm{H})$.

${ }^{1}$ H-NMR (600 MHz, $\left.\mathrm{C}_{6} \mathrm{D}_{6}\right): \delta=0.99\left(\mathrm{~d}, J=6.0 \mathrm{~Hz}, 3 \mathrm{H}, 10-\mathrm{H}_{3}\right), 1.02\left(\mathrm{~m}, 1 \mathrm{H}, 7-\mathrm{H}_{\mathrm{a}}\right), 1.35(\mathrm{dd}$, $\left.J=14.0,6.0 \mathrm{~Hz}, 1 \mathrm{H}, 5-\mathrm{H}_{\mathrm{a}}\right), 1.57\left(\mathrm{~m}, 1 \mathrm{H}, 8-\mathrm{H}_{\mathrm{a}}\right), 1.74\left(\mathrm{~m}, 1 \mathrm{H}, 8-\mathrm{H}_{\mathrm{b}}\right), 1.75\left(\mathrm{~m}, 1 \mathrm{H}, 7-\mathrm{H}_{\mathrm{b}}\right), 2.06$ $\left(\mathrm{dd}, J=18.5,8.0 \mathrm{~Hz}, 1 \mathrm{H}, 2-\mathrm{H}_{\mathrm{a}}\right), 2.10\left(\mathrm{~d}, J=14.0 \mathrm{~Hz}, 1 \mathrm{H}, 5-\mathrm{H}_{\mathrm{b}}\right), 2.42(\mathrm{~d}, J=18.5 \mathrm{~Hz}, 1 \mathrm{H}$, 2- $\left.\mathrm{H}_{\mathrm{b}}\right), 3.99$ (m, 1H, 9-H), 4.08 (m, 1H, 3-H), 4.29 (t, $\left.J=6.0 \mathrm{~Hz}, 1 \mathrm{H}, 4-\mathrm{H}\right)$.

${ }^{13}$ C-NMR (75.5 MHz, CD $\left.30 D\right): \delta=21.2$ (q, C-10), 32.2 (t, C-8), 35.1 (t, C-7), 38.6 (t, C-2), 42.4 (t, C-5), 76.2 (d, C-9), 79.1 (d, C-3), 85.7 (d, C-4), 116.6 (s, C-6), 179.1 (s, C-1). 


\subsection{Sekundärmetaboliten aus Phomopsis sp. BS 6769}

\subsubsection{Phomopsolid B (89)}

farbloser Feststoff

$\mathrm{C}_{15} \mathrm{H}_{20} \mathbf{O}_{6}$ (296.32)

Anfärbeverhalten: braun (Anisaldehyd)

$\mathbf{R}_{\mathbf{f}}=0.28\left(\mathrm{CHCl}_{3} / \mathrm{MeOH} 9: 1\right)$

EI-MS $(70 \mathrm{eV})$ : nicht erhältlich

ESI-MS (positive Ionen): $\mathrm{m} / \mathrm{z}=319[\mathrm{M}+\mathrm{Na}]^{+}, 615[2 \mathrm{M}+\mathrm{Na}]^{+}$

IR $(\mathrm{KBr}): \widetilde{v}=3428,2969,2927,1715,1647,1449,1383,1256$,

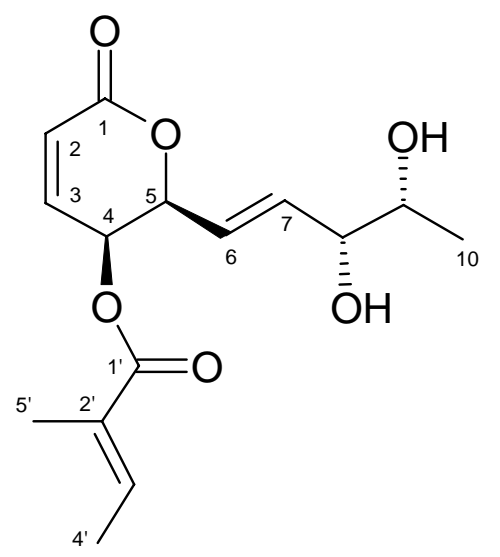

1131, 1074, 1026 (sh), $981(\mathrm{sh}), 829,730 \mathrm{~cm}^{-1}$

UV $(\mathrm{MeOH}): \lambda_{\max }(\log \varepsilon)=217(4.31) \mathrm{nm}$

$(\mathrm{MeOH} / \mathrm{HCl}): \lambda_{\max }(\log \varepsilon)=217(4.31) \mathrm{nm}$

$(\mathrm{MeOH} / \mathrm{NaOH}): \lambda_{\max }(\log \varepsilon)=217(4.20), 318(3.72) \mathrm{nm}$

CD (MeOH): 217 (92900), 264 (4100) nm

$[\alpha]_{D}^{20}=191^{\circ}(\mathrm{c}=1$ in Methanol $)$

${ }^{1} \mathbf{H}-\mathbf{N M R}\left(300 \mathrm{MHz}, \mathrm{CDCl}_{3}\right): \delta=1.06\left(\mathrm{~d}, J=6.2 \mathrm{~Hz}, 3 \mathrm{H}, 10-\mathrm{H}_{3}\right), 1.79\left(\mathrm{~s}, 3 \mathrm{H}, 4{ }^{\prime}-\mathrm{H}_{3}\right), 1.81(\mathrm{~s}$, $\left.3 \mathrm{H}, 5^{\prime}-\mathrm{H}_{3}\right), 3.59$ (qd, $\left.J=6.2,6.0 \mathrm{~Hz}, 1 \mathrm{H}, 9-\mathrm{H}\right), 3.91$ (tt, $\left.J=6.0,1.0 \mathrm{~Hz}, 1 \mathrm{H}, 8-\mathrm{H}\right), 5.21$ (dddd, $J=6.2,3.0,1.0,1.0 \mathrm{~Hz}, 1 \mathrm{H}, 5-\mathrm{H}), 5.39$ (dd, $J=5.7,3.0 \mathrm{~Hz}, 1 \mathrm{H}, 4-\mathrm{H}), 5.87$ (ddd, $J=15.5$, $6.2,1.0 \mathrm{~Hz}, 1 \mathrm{H}, 6-\mathrm{H}), 6.02(\mathrm{ddd}, J=15.5,5.7,1.0 \mathrm{~Hz}, 1 \mathrm{H}, 7-\mathrm{H}), 6.21$ (d, $J=9.8 \mathrm{~Hz}, 1 \mathrm{H}$, 2-H), 6.89 (m, 1H, 3'-H), 7.09 (dd, $J=9.8,5.8 \mathrm{~Hz}, 1 \mathrm{H}, 3-\mathrm{H})$.

${ }^{13}$ C-NMR (75.5 MHz, $\mathrm{CDCl}_{3}$ ): $\delta=12.1$ (q, C-5'), 14.5 (q, C-4'), 18.7 (q, C-10), 65.2 (d, C-4), 71.5 (d, C-5), 76.9 (d, C-9), 80.5 (d, C-8), 125.0 (d, C-2), 125.7 (d, C-6), 128.9 (s, C-2'), 136.4 (d, C-7), 140.4 (d, C-3'), 143.1 (d, C-3), 164.9 (s, C-1), 167.9 (s, C-1'). 


\subsubsection{Phomopsofuran A (90)}

= 5,8-Epoxy-9-hydroxy-4-(2-methyl-(2E)-2-butenoyl)-

5,7-decadiensäuremethylester

farbloser Feststoff

$\mathrm{C}_{16} \mathrm{H}_{22} \mathbf{O}_{6}(310.35)$

Anfärbeverhalten: grün-braun (Anisaldehyd)

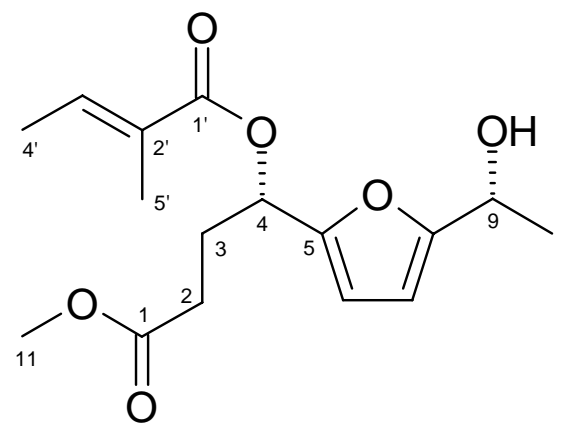

$\mathbf{R}_{\mathbf{f}}=0.56\left(\mathrm{CHCl}_{3} / \mathrm{MeOH} 9: 1\right)$

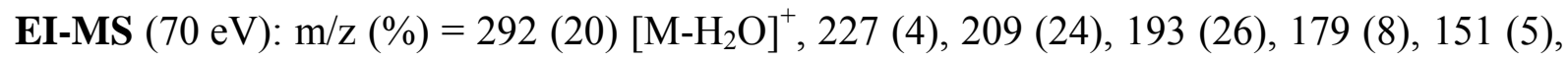
$133(8), 83$ (100), 55 (20), 43 (9)

ESI-MS (positive Ionen): $\mathrm{m} / \mathrm{z}=311[\mathrm{M}+\mathrm{H}]^{+}, 328\left[\mathrm{M}+\mathrm{NH}_{4}\right]^{+}, 333[\mathrm{M}+\mathrm{Na}]^{+}$

HR-ESI-MS: berechnet $\mathrm{m} / \mathrm{z}=333.130860[\mathrm{M}+\mathrm{Na}]^{+}$

$$
\text { gefunden } \mathrm{m} / \mathrm{z}=333.131149 \text { (Abweichung } 0.87 \mathrm{ppm} \text { ) }
$$

IR $(\mathrm{KBr}): \widetilde{v}=3433,2934,2370,1718,1640,1446,1380,1262,1196,1158,1075,813$, $733 \mathrm{~cm}^{-1}$

UV $(\mathrm{MeOH}): \lambda_{\max }(\log \varepsilon)=214(4.18) \mathrm{nm}$

$(\mathrm{MeOH} / \mathrm{HCl}): \lambda_{\max }(\log \varepsilon)=216(4.02) \mathrm{nm}$

$(\mathrm{MeOH} / \mathrm{NaOH}): \lambda_{\max }(\log \varepsilon)=213(4.11), 275(3.62) \mathrm{nm}$

CD $(\mathrm{MeOH}): \lambda_{\max }([\Theta])=210(-11500), 231(3100) \mathrm{nm}$

$[\alpha]_{D}^{20}=-14^{\circ}(\mathrm{c}=1$ in Methanol $)$

${ }^{1} \mathbf{H}-\mathrm{NMR}\left(300 \mathrm{MHz}, \mathrm{CDCl}_{3}\right): \delta=1.46\left(\mathrm{~d}, J=6.5 \mathrm{~Hz}, 3 \mathrm{H}, 10-\mathrm{H}_{3}\right), 1.80$ (s, 3H, 5'- $\left.\mathrm{H}_{3}\right), 1.80$ (s, $\left.3 \mathrm{H}, 4^{\prime}-\mathrm{H}_{3}\right), 2.27\left(\mathrm{~m}, 2 \mathrm{H}, 3-\mathrm{H}_{2}\right), 2.36\left(\mathrm{~m}, 2 \mathrm{H}, 2-\mathrm{H}_{2}\right), 3.63$ (s, 3H, 11- $\left.\mathrm{H}_{3}\right) .4 .76$ (q, J=6.5 Hz, $1 \mathrm{H}, 9-\mathrm{H}), 5.89$ (dd, $J=7.0,6.5 \mathrm{~Hz}, 1 \mathrm{H}, 4-\mathrm{H}), 6.20$ (dd, $J=3.0,1.0 \mathrm{~Hz}, 1 \mathrm{H}, 6-\mathrm{H}), 6.30$ (d, $J=3.0 \mathrm{~Hz}, 1 \mathrm{H}, 7-\mathrm{H}), 6.86$ (m, 1H, 3'-H).

${ }^{13}$ C-NMR (75.5 MHz, $\mathrm{CDCl}_{3}$ ): $\delta=12.1$ (q, C-5'), 14.4 (q, C-4'), 21.7 (q, C-10), 29.0 (t, C-3), 30.8 (t, C-2), 52.2 (q, C-11), 64.1 (d, C-9), 69.3 (d, C-4), 106.7 (d, C-6), 110.2 (d, C-7), 129.5 (s, C-2'), 139.3 (s, C-3'), 152.6 (s, C-5), 159.6 (s, C-8), 168.5 (s, C-1'), 174.8 (s, C-1). 


\subsection{3 p-Menthan-1,2,4-triol (91)}

farbloses Ö1

$\mathrm{C}_{10} \mathrm{H}_{20} \mathrm{O}_{3}$ (188.26)

Anfärbeverhalten: grau-blau (Anisaldehyd)

$\mathbf{R}_{\mathbf{f}}=0.26\left(\mathrm{CHCl}_{3} / \mathrm{MeOH} 9: 1\right)$

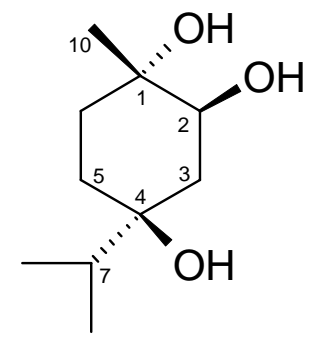

EI-MS $(70 \mathrm{eV}): \mathrm{m} / \mathrm{z}(\%)=170(2)\left[\mathrm{M}-\mathrm{H}_{2} \mathrm{O}\right]^{+}, 145(40), 127(55), 117$ (30), 109 (46), 107 (28), 99 (22), 85 (25), 83 (18), 81 (28), 71 (42), 58 (50), 43 (100), 41 (30)

ESI-MS (positive Ionen): $\mathrm{m} / \mathrm{z}=211[\mathrm{M}+\mathrm{Na}]^{+}, 299(2 \mathrm{M}+\mathrm{Na}]^{+}$

${ }^{1}$ H-NMR $\left(600 \mathrm{MHz}\right.$, DMSO-d $\left.{ }_{6}\right): \delta=0.82\left(\mathrm{~d}, J=7.0 \mathrm{~Hz}, 6 \mathrm{H}, 8-\mathrm{H}_{3}, 9-\mathrm{H}_{3}\right), 1.10(\mathrm{~s}, 3 \mathrm{H}$, $\left.10-\mathrm{H}_{3}\right), 1.24\left(\mathrm{~m}, 1 \mathrm{H}, 6-\mathrm{H}_{\mathrm{eq}}\right), 1.28\left(\mathrm{dq}, J=13.0,3.0 \mathrm{~Hz}, 1 \mathrm{H}, 5-\mathrm{H}_{\mathrm{eq}}\right), 1.40(\mathrm{dt}, J=13.5,3.0 \mathrm{~Hz}$, $1 \mathrm{H}, 3-\mathrm{H}_{\mathrm{eq}}$ ), 1.47 (hept, $\left.J=7.0 \mathrm{~Hz}, 1 \mathrm{H}, 7-\mathrm{H}\right), 1.66$ (td, $J=13.0,4.0 \mathrm{~Hz}, 1 \mathrm{H}, 5-\mathrm{H}_{\mathrm{ax}}$ ), 1.76 (td, $\left.J=13.0,4.0 \mathrm{~Hz}, 1 \mathrm{H}, 6-\mathrm{H}_{\mathrm{ax}}\right), 1.78\left(\mathrm{dd}, J=13.5,3.0 \mathrm{~Hz}, 1 \mathrm{H}, 3-\mathrm{H}_{\mathrm{ax}}\right), 3.35(\mathrm{~m}, 1 \mathrm{H}, 2-\mathrm{H}), 4.10$ (br s, 1H, OH), 4.52 (br s, 1H, OH), 5.10 (br s, 1H, 2-OH).

${ }^{1}$ H-NMR $\left(300 \mathrm{MHz}, \mathrm{CD}_{3} \mathrm{OD}\right): \delta=0.90\left(\mathrm{~d}, J=7.0 \mathrm{~Hz}, 6 \mathrm{H}, 8-\mathrm{H}_{3}, 9-\mathrm{H}_{3}\right), 1.23\left(\mathrm{~s}, 3 \mathrm{H}, 10-\mathrm{H}_{3}\right)$, $1.35-1.47\left(\mathrm{~m}, 2 \mathrm{H}, 5-\mathrm{H}_{\mathrm{eq}}, 6-\mathrm{H}_{\mathrm{eq}}\right), 1.57$ (hept, $\left.J=7.0 \mathrm{~Hz}, 1 \mathrm{H}, 7-\mathrm{H}\right), 1.60(\mathrm{dt}, J=13.0,3.0 \mathrm{~Hz}$, $\left.1 \mathrm{H}, 3-\mathrm{H}_{\mathrm{eq}}\right), 1.82\left(\mathrm{td}, J=13.0,3.5 \mathrm{~Hz}, 1 \mathrm{H}, 5-\mathrm{H}_{\mathrm{ax}}\right), 1.92\left(\mathrm{td}, J=13.0,4.0 \mathrm{~Hz}, 1 \mathrm{H}, 6-\mathrm{H}_{\mathrm{ax}}\right), 1.93$ (dd, $\left.J=13.5,3.0 \mathrm{~Hz}, 1 \mathrm{H}, 3-\mathrm{H}_{\mathrm{ax}}\right), 3.50(\mathrm{td}, J=3.0,1.0 \mathrm{~Hz}, 1 \mathrm{H}, 2-\mathrm{H})$.

${ }^{13}$ C-NMR (150.8 MHz, DMSO-d ${ }_{6}$ ): $\delta=16.8$ (q, C-8, C-9), 27.0 (q, C-10), 29.1 (t, C-5), 29.4 (t, C-6), 34.0 (t, C-3), 37.2 (d, C-7), 69.8 (s, C-1), 73.0 (s, C-4), 73.3 (d, C-2). 


\section{Literaturverzeichnis}

[1] A. L. Demain, in Secondary metabolites: their function and evolution, Ciba Foundation Symposium 171 (Eds.: D. J. Chadwick, J. Whelan), John Wiley \& Sons, Chichester, 1992, pp. 3-16.

[2] W. Piepersberg, A. Zeeck, in Handbuch der Biotechnologie (Eds.: P. Präve, U. Faust, W. Sittig, D. A. Sukatsch), Oldenbourg Verlag, München, 1994, pp. 141-177.

[3] R. A. Maplestone, M. J. Stone, D. H. Williams, Gene 1992, 115, 151-157.

[4] M. Wink, Phytochemistry 2003, 64, 3-19.

[5] M. Engler, T. Anke, O. Sterner, Z. Naturforsch., C: J. Biosci. 1998, 53, 318-324.

[6] F. Yamashita, K. Hotta, S. Kurasawa, Y. Okami, H. Umezawa, J. Antibiot. 1985, 38, 58-63.

[7] M. Kettering, D. Weber, O. Sterner, T. Anke, BIOspektrum 2004, 10, 147-149.

[8] E.-B. Goh, G. Yim, W. Tsui, J. McClure, M. G. Surette, J. Davies, Proc. Natl. Acad. Sci. U. S. A. 2002 , 99, 17025-17030.

[9] J. W. Hastings, E. P. Greenberg, J. Bacteriol. 1999, 181, 2667-2668.

[10] S. Schauder, B. L. Bassler, Genes Dev. 2001, 15, 1468-1480.

[11] M. H. J. Sturme, M. Kleerebezem, J. Nakayama, A. D. L. Akkermans, E. E. Vaughan, W. M. de Vos, Antonie van Leeuwenhoek 2002, 81, 233-243.

[12] R. D. Firn, C. G. Jones, Nat. Prod. Rep. 2003, 20, 382-391.

[13] C. T. Walsh, ChemBioChem 2002, 3, 124-134.

[14] A. Trefzer, G. Blanco, L. Remsing, E. Kunzel, U. Rix, F. Lipata, A. F. Brana, C. Mendez, J. Rohr, A. Bechthold, J. A. Salas, J. Am. Chem. Soc. 2002, 124, 6056-6062.

[15] P. M. Dewick, Nat. Prod. Rep. 2002, 19, 181-222.

[16] S. D. Bentley, K. F. Chater, A. M. Cerdeno-Tarraga, G. L. Challis, N. R. Thomson, K. D. James, D. E. Harris, M. A. Quail, H. Kieser, D. Harper, A. Bateman, S. Brown, G. Chandra, C. W. Chen, M. Collins, A. Cronin, A. Fraser, A. Goble, J. Hidalgo, T. Hornsby, S. Howarth, C. H. Huang, T. Kieser, L. Larke, L. Murphy, K. Oliver, S. O'Neil, E. Rabbinowitsch, M. A. Rajandream, K. Rutherford, S. Rutter, K. Seeger, D. Saunders, S. Sharp, R. Squares, S. Squares, K. Taylor, T. Warren, A. Wietzorrek, J. Woodward, B. G. Barrell, J. Parkhill, D. A. Hopwood, Nature 2002, 417, 141-147.

[17] S. Omura, H. Ikeda, J. Ishikawa, A. Hanamoto, C. Takahashi, M. Shinose, Y. Takahashi, H. Horikawa, H. Nakazawa, T. Osonoe, H. Kikuchi, T. Shiba, Y. Sakaki, M. Hattori, Proc. Natl. Acad. Sci. U. S. A. 2001, 98, 12215-12220.

[18] G. L. Challis, D. A. Hopwood, Proc. Natl. Acad. Sci. U. S. A. 2003, 100, 14555-14561.

[19] H. Ikeda, J. Ishikawa, A. Hanamoto, M. Shinose, H. Kikuchi, T. Shiba, Y. Sakaki, M. Hattori, S. Omura, Nat. Biotechnol. 2003, 21, 526-531.

[20] C. W. Bacon, J. F. White, Microbial Endophytes, Verlag Marcel Dekker, New York, 2000.

[21] G. Strobel, B. Daisy, U. Castillo, J. Harper, J. Nat. Prod. 2004, 67, 257-268.

[22] G. Strobel, B. Daisy, Microbiol. Mol. Biol. Rev. 2003, 67, 491-502.

[23] G. Strobel, R. V. Miller, C. Martinez-Miller, M. M. C. Condron, D. B. Teplow, W. M. Hess, Microbiology 1999, 145, 1919-1926.

[24] J. Y. Li, G. Strobel, J. Harper, E. Lobkovsky, J. Clardy, Org. Lett. 2000, 2, 767-770.

[25] E. Baloglu, D. G. I. Kingston, J. Nat. Prod. 1999, 62, 1448-1472.

[26] W. Fang-ting, C. Dai-jie, Q. Xiu-ping, Chinese Journal of Antibiotics 2004, 29, 184-192.

[27] I. Rizzo, E. Varsavky, M. Haidukowski, H. Frade, Toxicon 1997, 35, 753-757.

[28] P. T. Meinke, M. M. Smitz, W. L. Shoop, Curr. Top. Med. Chem. 2002, 2, 655-674.

[29] M. Schwarz, B. Köpcke, R. W. S. Weber, O. Sterner, H. Anke, Phytochemistry 2004, 65, $2239-2245$.

[30] D. Weber, O. Sterner, T. Anke, S. Gorzalczany, V. Martino, C. Acevedo, J. Antibiot. 2004, 57, 559563.

[31] H. Brockmann, B. Franck, Naturwissenschaften 1954, 51, 451.

[32] H. Brockmann, B. Franck, Chemische Berichte 1954, 87, 1767-1779.

[33] A. B. Mauger, Topics in Antibiotic Chemistry 1980, 5, 229-306.

[34] H. Lackner, Angew. Chem. 1975, 87, 400-411.

[35] M. Schäfer, G. M. Sheldrick, I. Bahner, H. Lackner, Angew. Chem. 1998, 110, 2482-2485.

[36] H. Lackner, I. Bahner, N. Shigematsu, L. K. Pannell, A. B. Mauger, J. Nat. Prod. 2000, 63, 352-356.

[37] H. Friebolin, Ein- und zweidimensionale NMR-Spektroskopie, 3. Aufl., Whiley-VCH, Weinheim, 1999.

[38] H. Lackner, H. Hulsmann, S. Heinze, H. Simon, H. Bar, C. Zimmer, U. Gräfe, J. Antibiot. 2000, 53, 8487.

[39] S. Kamitori, F. Takusagawa, J. Am. Chem. Soc. 1994, 116, 4154-4165.

[40] A. B. Mauger, H. Lackner, in Anticancer Agents from Natural Products (Eds.: D. G. I. Kingston, G. M. Cragg, D. J. Newman), CRC Press, Boca Raton, 2005, pp. 281-298. 
[41] H. Laatsch, AntiBase - a database for the identification of natural products, Chemical Concepts, Weinheim, 2004.

[42] F. A. Badria, W. A. El-Naggar, Sci. Pharm. 1994, 62, 355-362.

[43] K. Pusecker, H. Laatsch, E. Helmke, H. Weyland, J. Antibiot. 1997, 50, 479-483.

[44] S. Kamitori, F. Takusagawa, J. Mol. Biol. 1992, 225, 445-456.

[45] X. Qu, J. Ren, P. V. Riccelli, A. S. Benight, J. B. Chaires, Biochemistry 2003, 42, 11960-11967.

[46] R. M. Wadkins, T. M. Jovin, Biochemistry 1991, 30, 9469-9478.

[47] K. H. Chin, F. M. Chen, S. H. Chou, Nucleic Acids Res. 2003, 31, 2622-2629.

[48] H. Robinson, Y. G. Gao, X. Yang, R. Sanishvili, A. Joachimiak, A. H. Wang, Biochemistry 2001, 40, 5587-5592.

[49] ID:1A7Y, RCSB Protein Data Bank (PDB), www.pdb.org, 2005.

[50] S. Farber, G. D'Anagio, A. Evans, A. Mitus, Journal of Urology 2002, 168, 2560-2562.

[51] F. Takusagawa, R. G. Carlson, R. F. Weaver, Bioorg. Med. Chem. 2001, 9, 719-725.

[52] A. Lifferth, I. Bahner, H. Lackner, M. Schafer, Z. Naturforsch., B: Chem. Sci. 1999, 54, 681-691.

[53] I. Bahner, Dissertation, Universität Göttingen, 1999.

[54] S. A. Waksman, Annals of the New York Academy of Sciences 1960, 89, 285-286.

[55] Dictionary of Natural Products on CD-ROM, Version 13:2, Chapman \& Hall / CRC, Boca Raton (FL), 2004.

[56] H. Brockmann, H. Lackner, Naturwissenschaften 1960, 47, 230.

[57] H. Brockmann, N. Pfennig, Naturwissenschaften 1952, 39, 419-420.

[58] H. Brockmann, B. Franck, Naturwissenschaften 1960, 47, 15.

[59] H. Brockmann, G. Bohnsack, B. Franck, H. Grone, H. Muxfeldt, C. H. Suling, Angew. Chem. 1956, 68, 70-71.

[60] A. W. Johnson, A. B. Mauger, Biochem. J. 1959, 535-538.

[61] G. Schmidt-Kastner, Annals of the New York Academy of Sciences 1960, 89, $299-303$.

[62] H. Brockmann, Annals of the New York Academy of Sciences 1960, 89, 323-335.

[63] A. B. Mauger, O. A. Stuart, J. Antibiot. 1990, 43, 220-221.

[64] H. Brockmann, J. H. Manegold, Chemische Berichte 1962, 95, 1081-1093.

[65] H. Brockmann, J. H. Manegold, Chemische Berichte 1960, 93, 2971-2982.

[66] H. Brockmann, G. Pampus, Angew. Chem. 1955, 67, 519.

[67] M. L. Devan, T. I. Orlova, A. B. Silaev, Antibiotiki 1974, 19, 107-111.

[68] E. Katz, C. R. Waldron, M. L. Meloni, J. Bacteriol. 1961, 82, 600-608.

[69] H. Brockmann, Angew. Chem. 1960, 24, 939-947.

[70] V. S. Kuznetsova, T. I. Orlova, A. B. Silaev, Antibiotiki 1971, 16, 18-21.

[71] V. S. Kuznetsova, T. I. Orlova, A. B. Silaev, Antibiotiki 1974, 19, 295-298.

[72] T. I. Orlova, N. V. Sorokina, A. B. Silaev, Antibiotiki 1972, 17, 409-417.

[73] H. Lackner, unveröffentlichte Ergebnisse.

[74] E. Katz, W. K. Williams, K. T. Mason, A. B. Mauger, Antimicrob. Agents Chemother. 1977, 11, 10561063.

[75] J. V. Formica, E. Katz, J. Biol. Chem. 1973, 248, 2066-2071.

[76] J. S. Nishimura, W. F. Bowers, Biochem. Biophys. Res. Commun. 1967, 28, 665-670.

[77] E. Katz, Annals of the New York Academy of Sciences 1960, 89, 304-322.

[78] J. V. Formica, M. A. Apple, Antimicrob. Agents Chemother. 1976, 9, 214-212.

[79] G. H. Jones, U. Keller, Drugs and the Pharmaceutical Sciences 1997, 82, 335-361.

[80] U. Keller, F. Schauwecker, Prog. Nucleic Acid Res. Mol. Biol. 2001, 70, 233-289.

[81] G. H. Jones, Antimicrob. Agents Chemother. 2000, 44, 1322 - 1327.

[82] L. Liang, Dissertation: Investigation of Secondary Metabolites of North Sea Bacteria: Fermentation, Isolation, Structure Elucidation and Bioactivity, Universität Göttingen, 2003.

[83] T. Große, Diplomarbeit: Untersuchungen zur Naturstoffbildung an neu isolierten Bakterien aus der Nordsee, Hochschule für Angewandte Wissenschaften, Hamburg, 2003.

[84] F. Fdhila, V. Vázquez, J. L. Sánchez, R. Riguera, J. Nat. Prod. 2003, 66, 1299-1301.

[85] V. A. Basyuk, T. Y. Gromovoi, A. A. Chuiko, V. A. Soloshonok, V. P. Kukhar, Synthesis 1992, 5, 449451.

[86] S. D. Bull, S. G. Davies, R. M. Parkin, F. Sánchez-Sancho, J. Chem. Soc., Perkin Trans. 1 1998, 23132320.

[87] H. G. Byun, H. Zhang, M. Mochizuki, K. Adachi, Y. Shizuri, W. J. Lee, S. K. Kim, J. Antibiot. 2003, $56,102-106$.

[88] A. C. Stierle, J. H. Cardellina, G. A. Strobel, Proc. Natl. Acad. Sci. USA 1988, 85, 8008-8011.

[89] G. Degrassi, C. Aguilar, M. Bosco, S. Zahariev, S. Pongor, V. Venturi, Curr. Microbiol. 2002, 45, 250254.

[90] M. D. Fresno, D. Fernandez-Forner, M. Miralpeix, V. Segarra, H. Ryder, M. Royo, F. Albericio, Bioorg. Med. Chem. Lett. 2005, 15, 1659-1664. 
[91] O. S. Kwon, S. H. Park, B.-S. Yun, Y. R. Pyun, C.-J. Kim, J. Antibiot. 2001, 54, 179-181.

[92] G. G. Smith, R. C. Evans, R. Baum, J. Am. Chem. Soc. 1986, 108, 7327-7332.

[93] K. Hasegawa, Y. Ueno, Bull. Chem. Soc. Jpn. 1985, 58, 2832-2839.

[94] R. P. Maskey, F. C. Li, S. Qin, H. H. Fiebig, H. Laatsch, J. Antibiot. 2003, 56, 622-629.

[95] K. Maruyama, T. Moriguchi, T. Mashinio, A. Nishinaga, Chem. Lett. 1996, 819-820.

[96] ACD/CNMR Predictor, Version 6.12, Advanced Chemistry Development Inc., Toronto, 2002.

[97] C. J. Krieger, P. Zhang, L. A. Mueller, A. Wang, S. Paley, M. Arnaud, J. Pick, S. Y. Rhee, P. D. Karp, Nucleic Acids Res. 2004, 32, 438-442.

[98] C. E. Barry, P. G. Nayar, T. P. Begley, Biochemistry 1989, 28, 6323-6333.

[99] Y. Yano, M. Ikuta, Y. Amamiya, T. Nabeshima, Chem. Lett. 1991, 461-464.

[100] M. Zikmundová, K. Drandarov, M. Hesse, C. Werner, Z. Naturforsch., C: J. Biosci. 2002, 57, 660-665.

[101] R. G. Buckley, J. Charalambous, K. Henrick, Acta Cryst., Sect. B: Struct. Sci. 1982, 38, $289-291$.

[102] H. B. Bode, B. Bethe, R. Höfs, A. Zeeck, ChemBioChem 2002, 3, 619-627.

[103] W. D. Celmer, G. N. Chmurny, C. E. Moppett, R. S. Ware, P. C. Watts, E. B. Whipple, J. Am. Chem. Soc. 1980, 102, 4203-4209.

[104] D. E. Cane, C.-C. Yang, J. Am. Chem. Soc. 1984, 106, 784-787.

[105] W. D. Celmer, W. P. Cullen, C. E. Moppett, M. T. Jefferson, L. H. Huang, R. Shibakawa, J. Tone (Pfizer Inc.), U. S. Patent 4148 883, 1977.

[106] J. Kallmerten, in Studies in Natural Products Chemistry, Vol. 17 (Ed.: A.-U. Rahman), 1995, pp. 283310 .

[107] D. E. Cane, W. Tan, W. R. Ott, J. Am. Chem. Soc. 1993, 115, 527-535.

[108] E. M. Stocking, R. M. Williams, Angew. Chem. 2003, 115, 3186-3223.

[109] E. Pretsch, P. Bühlmann, C. Affolter, M. Badertscher, Spektroskopische Daten zur Strukturaufklärung organischer Verbindungen, 4. Auflage, Springer Verlag, Berlin, Heidelberg, New York, 2001.

[110] W. Klyne, Biochem. J. 1950, 47, xli-xlii.

[111] G. O. Aspinall, K. i. Takeo, Carbohydr. Res. 1983, 121, 61-77.

[112] M. Mori, S. Tejima, T. Niwa, Chem. Pharm. Bull. 1986, 34, 4037-4044.

[113] P. M. Collins, W. G. Overend, J. Chem. Soc. 1965, 1912-1918.

[114] D. A. Johnson, H.-w. Liu, in Comprehensive Natural Products Chemistry, Vol. 3 (Ed.: P. M. Pinto), Elsevier, Amsterdam, 1999.

[115] J. Jurenitsch, B. Kopp, I. Gabler-Kolacsek, W. Kubelka, J. Chromatogr. 1981, 210, 337-341.

[116] I. Papastavrou, Dissertation: Entwicklung neuer Methoden zur Vorläufer-dirigierten Biosynthese mikrobieller 1-O-Acyl- $\alpha$-L-rhamnopyranoside sowie Isolierung und Strukturaufklärung neuer Sekundärmetabolite aus Streptomyceten, Universität Göttingen, 2000.

[117] S. Grond, I. Papastavrou, A. Zeeck, Eur. J. Org. Chem. 2000, 1875-1881.

[118] T. Okamoto, K. Yatzaki, M. Tabata, Phytochemistry 1995, 38, 83-88.

[119] S. Kato, K. Shindo, Y. Yamagishi, M. Matsuoka, H. Kawai, J. Mochizuki, J. Antibiot. 1993, 46, 14851493.

[120] T. Reichstein, E. Weiss, Adv. Carbohydr. Chem. Biochem. 1962, 17, 65-120.

[121] A. P. MacLennan, Biochem. J. 1962, 82, 394-400.

[122] J. Weckesser, H. Mayer, I. Fromme, Biochem. J. 1973, 135, 293-297.

[123] B. Lindberg, Adv. Carbohydr. Chem. Biochem. 1990, 48, 279-318.

[124] A. P. MacLennan, Biochim. Biophys. Acta 1961, 48, 600-601.

[125] S. Grond, H.-J. Langer, P. Henne, I. Sattler, R. Thiericke, S. Grabley, H. Zähner, A. Zeeck, Eur. J. Org. Chem. 2000, 929-937.

[126] J.-F. Hu, D. Wunderlich, I. Sattler, A. Härtl, I. Papastavrou, S. Grond, S. Grabley, X.-Z. Feng, R. Thiericke, J. Antibiot. 2000, 53, 944-953.

[127] S. Grond, Dissertation: Die Bildung von 1-O-Acyl- $\alpha$-L-rhamnosiden und anderen Metaboliten durch dirigierte Biosynthese mit Streptomyces griseoviridis (Tü 3634) und Untersuchung ihrer Biogenese, Universität Göttingen, 1997.

[128] C. Milkowski, D. Strack, Phytochemistry 2004, 65, 517-524.

[129] X. M. He, H.-w. Liu, Annu. Rev. Biochem. 2002, 71, 701-754.

[130] H.-w. Liu, J. S. Thorson, Annu. Rev. Microbiol. 1994, 48, 223-256.

[131] C. Hertweck, B. S. Moore, Tetrahedron 2000, 56, 9115-9120.

[132] A. M. Hill, B. L. Thompson, J. P. Harris, R. Segret, Chem. Commun. 2003, 1358-1359.

[133] J. L. Barker, J. W. Frost, Biotechnol. Bioeng. 2001, 76, 376-390.

[134] L. Hoffmann, S. Grond, Eur. J. Org. Chem. 2004, 4771-4777.

[135] M. G. Watve, R. Tickoo, M. M. Jog, B. D. Bhole, Arch. Microbiol. 2001, 176, 386-390.

[136] U. Höller, A. D. Wright, G. F. Matthée, G. M. König, S. Draeger, H.-J. Aust, B. Schulz, Mycol. Res. 2000, 104, 1354-1365. 
[137] J. Edwards, D. Chamberlain, G. Brosnan, D. West, M. S. Stanley, N. J. W. Clipson, P. Hooley, Mycol. Res. 1998, 102, 1998-2002.

[138] R. S. Burden, D. T. Cooke, G. A. Carter, Phytochemistry 1989, 28, 1791-1804.

[139] R. T. Lorenz, W. M. Casey, L. W. Parks, J. Bacteriol. 1989, 171, 6169-6173.

[140] C. Mille-Lindblom, E. von Wachenfeldt, L. J. Tranvik, J. Microbiol. Methods 2004, 59, 253-262.

[141] H. Berndt, Dissertation: Untersuchungen zu einem Verfahren zur Bestimmung von Ergosterol als unspezifischem, quantitativem Parameter für den Pilzbefall von Getreide, Universität Göttingen, 1990.

[142] T. Larsen, J. Axelsen, H. Weber Ravn, J. Chromatogr., A 2004, 1026, 301-304.

[143] H. J. Roth, H. Fenner, Arzneistoffe, Gustav Fischer Verlag, Stuttgart, Jena, Lübeck, Ulm, 1997.

[144] RÖMPP Online, Version 2.6, 2005, http://www.roempp.com

[145] T. Böcking, K. D. Barrow, A. G. Netting, T. C. Chilcott, H. G. L. Coster, M. Höfer, Eur. J. Biochem. 2000, 267, 1607-1618.

[146] G. Kawai, Y. Ikeda, K. Tubaki, Agric. Biol. Chem. 1985, 49, 2137-2146.

[147] J. Koga, T. Yamyuchi, M. Shimura, N. Ogaga, K. Oshima, K. Umemura, M. Kikuchi, N. Ogasawara, $J$. Biol. Chem. 1998, 273, 31985-31991.

[148] C. Osterhage, R. Kaminsky, G. M. König, A. D. Wright, J. Org. Chem. 2000, 65, 6412-6417.

[149] A. Ichihara, H. Tazaki, S. Sakamura, Tetrahedron Lett. 1983, 24, 5373-5376.

[150] H. Oikawa, T. Yokota, C. Sakano, Y. Suzuki, A. Naya, A. Ichihara, Biosci. Biotechnol. Biochem. 1998, 62, 2016-2022.

[151] A. Ichihara, H. Oikawa, Biosci. Biotechnol. Biochem. 1997, 61, 12-18.

[152] G. Pohnert, ChemBioChem 2001, 2, 873-875.

[153] A. Probst, C. Tamm, Helv. Chim. Acta 1981, 64, 2056-2064.

[154] S. Sekita, K. Yoshihira, S. Natori, Chem. Pharm. Bull. 1983, 31, 490-498.

[155] S. Sekita, K. Yoshihira, S. Natori, H. Kuwano, Tetrahedron Lett. 1973, 23, 2109-2112.

[156] S. Sekita, K. Yoshihira, S. Natori, H. Kuwano, Tetrahedron Lett. 1976, 17, 1351-1354.

[157] J. P. Springer, J. Clardy, J. M. Wells, R. J. Cole, J. W. Kirksey, R. D. Macfarlane, D. F. Torgerson, Tetrahedron Lett. 1976, 17, 1355-1358.

[158] W. Jiao, Y. Feng, J. W. Blunt, A. L. J. Cole, M. H. G. Munro, J. Nat. Prod. 2004, 67, 1722-1725.

[159] A. Probst, C. Tamm, Helv. Chim. Acta 1981, 64, 2065-2077.

[160] H. Oikawa, Y. Murakami, A. Ichihara, Tetrahedron Lett. 1991, 32, 4533-4536.

[161] SciFinder Scholar 2004, Chemical Abstracts Service, Columbus (Ohio), 21.04.2005.

[162] R. F. Angawi, D. C. Swenson, J. B. Gloer, D. T. Wicklow, Tetrahedron Lett. 2003, 44, 7593-7596.

[163] R. F. Angawi, D. C. Swenson, J. B. Gloer, D. T. Wicklow, J. Nat. Prod. 2005, 68, 212-216.

[164] A. I. Scott, H. Guilford, E. Lee, J. Am. Chem. Soc. 1971, 93, 3534-3536.

[165] A. I. Scott, K. J. Wiesner, Chem. Commun. 1972, 1075-1077.

[166] M. C. O'Sullivan, J. M. Schwab, Bioorg. Chem. 1995, 23, 131-143.

[167] L. C. Vining, A. G. McInnes, D. G. Smith, L. Johnson, Chem. Commun. 1971, 325-326.

[168] I. Namatame, H. Tomoda, M. Arai, S. Omura, J. Antibiot. 2000, 53, 19-25.

[169] V. Betina, Mycotoxins. Chemical, Biological and Environmental Aspects, Elsevier, Amsterdam, 1989.

[170] A. Evidente, A. Motta, in Bioactive Compounds from Natural Sources (Ed.: C. Tringali), Taylor \& Francis, London, 2001, pp. 473-526.

[171] S. Sekita, K. Yoshihira, S. Natori, S.-i. Udagawa, F. Sakabe, H. Kurata, M. Umeda, Chem. Pharm. Bull. 1982, 30, 1609-1617.

[172] J. G. Kang, K. K. Kim, K. Y. Kang, Agric. Chem. Biotechnol. (Engl. Ed.) 1999, 42, 146-150.

[173] Y. Morita, E. Matsumura, T. Okabe, M. Shibata, M. Sugiura, T. Ohe, H. Tsujibo, N. Ishida, Y. Inamori, Biol. Pharm. Bull. 2003, 26, 1487-1490.

[174] P. Seephonkai, M. Isaka, P. Kittakoop, S. Trakulnaleamsai, R. Rattanajak, M. Tanticharoen, Y. Thebtaranonth, J. Antibiot. 2001, 54, 751-752.

[175] M. Yanagiya, K. Kaneko, T. Kaji, T. Matsumoto, Tetrahedron Lett. 1979, 20, 1761-1764.

[176] C. Osterhage, G. M. König, U. Höller, A. D. Wright, J. Nat. Prod. 2002, 65, 306-313.

[177] M. Nukina, H. Hattori, S. Marumo, J. Am. Chem. Soc. 1975, 97, 2542-2543.

[178] L. M. Pena-Rodriguez, N. A. Armingeon, W. S. Chilton, J. Nat. Prod. 1988, 51, 821-828.

[179] R. J. Cole, M. A. Schweikert, Handbook of Secondary Fungal Metabolites, Vol. I-III, Academic Press, San Diego, London, 2003.

[180] E. Piers, H.-P. Isenring, Can. J. Chem. 1976, 55, 1039-1044.

[181] M. Nukina, S. Marumo, Agric. Biol. Chem. 1976, 40, 2121-2123.

[182] F. Dorn, P. Bernasconi, D. Arigoni, Chimia 1975, 29, 24-25.

[183] W. Francke, W. Kitching, Curr. Org. Chem. 2001, 5, 233-251.

[184] J. F. Grove, J. Chem. Soc., Perkin Trans. I 1985, 865-869.

[185] D. B. Stierle, A. A. Stierle, B. Ganser, J. Nat. Prod. 1997, 60, 1207-1209.

[186] J. A. Dale, H. S. Mosher, J. Am. Chem. Soc. 1973, 95, 512-519.

[187] G. Helmchen, Tetrahedron Lett. 1974, 16, 1427-1530. 
[188] T. Kuzuyama, Biosci. Biotechnol. Biochem. 2002, 66, 1619-1627.

[189] C. P. Gorst-Allmann, V. Puchta, G. Spiteller, Lipids 1988, 23, 1032-1036.

[190] G. Spiteller, J. Lipid. Mediat. 1993, 7, 199-221.

[191] R. K. Thappa, K. L. Dhar, C. K. Atal, Phytochemistry 1976, 15, 1568-1569.

[192] T. Sassa, H. Kenmoku, M. Sato, T. Murayama, N. Kato, Biosci. Biotechnol. Biochem. 2003, 67, 475479.

[193] Merck, Anfärbereagenzien für die Dünnschichtchromatographie, Darmstadt, 1980.

[194] W. C. Still, M. Kahn, A. Mitra, J. Org. Chem 1978, 43, 2923.

[195] U. Höller, G. M. König, A. D. Wright, J. Nat. Prod. 1999, 62, 114-118.

[196] D. N. Kirk, H. C. Toms, C. Douglas, K. A. White, K. E. Smith, S. Latif, R. W. P. Hubbard, J. Chem. Soc., Perkin Trans. II 1990, 1567-1594.

[197] J. Koga, T. Yamyuchi, M. Shimura, N. Ogaga, K. Oshima, K. Umemura, M. Kikuchi, N. Ogasawara, J. Biol. Chem. 1998, 273, 31985-31991. 



\section{Danksagung}

Für ihre unverzichtbare Hilfe beim Kultivieren der Pilzstämme, beim Screening, Säulen und vielen anderen Laborarbeiten geht mein großer Dank an Kerstin Vogel.

Die Aufnahme der NMR-Spektren verdanke ich dem NMR-Team der Abteilung (Stephanie Grond, Sandra Lösgen, Sven Meyer, Inken Plitzko, Oliver Schlörke, Tim Schuhmann, Frank Surup, Diana Wolff) sowie Carola Zolke, Christiane Siebert und Martin Weitemeyer, denen ich herzlich für ihren Einsatz danke. Bei Martin bedanke ich mich insbesondere für das geduldige Bearbeiten, Referenzieren und Ausdrucken zahlreicher Spektren und ihrer Spreizungen. György Udvarnoki, Frau Krökel und Herrn Frauendorf gilt mein Dank für die Aufnahme der Massenspektren. Michaela Klingebiel danke herzlich ich für das Messen der physikalisch-chemischen Daten meiner Substanzen sowie ihre Hilfe bei der Vorläuferdirigierten Biosynthese. Bei technischen Problemen aller Art an der HPLC war die Hilfe von Hans-Peter Kroll unverzichtbar. Bei Nadine Czempinski und Luise Hoffmann bedanke ich mich für die HPLC-MS Messungen.

Für das engagierte und kritische Korrekturlesen dieser Arbeit bedanke ich mich bei Marko Gentzsch, Beatrix Kahle, Sven Meyer, Inken Plitzko, Tim Schuhmann und Frank Surup. Stephanie Grond danke ich für die anregenden und interessanten Diskussionen über Desoxyzucker und deren Eigenarten, für ebenso interessante Diskussionen und Informationen zu den Actinomycinen danke ich Herrn Prof. Lackner.

Für das gute Arbeitsklima in unserem Labor geht ein herzlicher Dank an Hans-Peter, Marco, Michaela und Yee-Yee, Marco danke ich auch für die musikalische Aufwertung des Labors durch Bands mit mehr als drei Buchstaben im Namen.

Vielen, vielen Dank an alle Freunde, die mich während des Studiums begleitet haben: Karin (unvergessen die Partys ebenso wie die Nächte mit PC-Protokollen), Regina, Matthias, Stefan \& Jessica feat. Helge Schneider, Martin \& Isa, Trixi, Christian für die gemeinsame Zeit mit Musik, Proben für die Welttournee, im Freibad oder einer australischen Fischbox, die Emder Pfadis, Pia \& Matthias \& Jona und alle anderen, die zu erwähnen mir hier der Platz fehlt.

Der größte und wichtigste Dank geht an meine Familie und Beate, die mich stets unterstützt haben und dafür verantwortlich sind, dass neben der Chemie mein Blick für die wirklich wichtigen Dinge im Leben nicht verloren ging. 



\section{Lebenslauf}

Ich bin deutscher Staatsbürger und wurde am 02. September 1974 in Ulm als Sohn von Prof. Dr.-Ing. Eberhard Bitzer und seiner Frau Annemarie Bitzer, geb. Nießen, geboren.

Von 1981 bis 1983 besuchte ich die Grundschule in Ulm-Unterweiler, von 1983 bis 1985 die Grundschule in Emden-Larrelt und von 1985 bis 1987 die Orientierungstufe in EmdenWybelsum. 1987 wechselte ich auf das Johannes-Althusius-Gymnasium in Emden, dessen Besuch ich 1994 mit dem Abitur abschloß.

In der Zeit von Juli 1994 bis September 1995 leistete ich Zivildienst in einem Wohnheim für geistig Behinderte der Ostfriesischen Beschützenden Werkstätten (OBW).

Ich begann im Oktober 1995 mit dem Studium der Chemie an der Universität Göttingen und bestand am 04. Mai 1998 die Diplomvorprüfung. Von April 2000 bis Januar 2001 arbeitete fertigte ich im Arbeitskreis von Prof. Dr. A. Zeeck meine Diplomarbeit mit dem Titel „Isolierung und Strukturaufklärung von Sekundärmetaboliten aus neuen Actinomyceten“ an und bestand im Februar 2001 die Diplom-Chemiker-Hauptprüfung.

Seit April 2001 arbeite ich unter der Anleitung von Prof. Dr. A. Zeeck an der vorliegenden Dissertation.

Göttingen, 24. Mai 2005

Jens Bitzer 